\title{
APPENDIX
}

\section{LISTS OF FINDS*}

\section{by Veronica Hinterhuber}

\section{General Remarks}

Over the course of the AcrossBorders 2013-2017 campaigns in SAV1 West and SAV1 East more than 6300 find numbers, often containing several items, were assigned.

The following lists of finds illustrate the rich material discovered in the years 2013-2017. The items are presented in tables which are firstly arranged according to the two excavation areas SAV1 West and SAV1 East and the years of the discovery.

Within this framework, the internal structure of each table consists of the arrangement of the respective finds with their find numbers after their specific location and according to their SU or level numbers. The numbering system of the corpus is based on the find number and the year of discovery. For this system, a clear separation between numbers from SAV1 East and SAV1 West was introduced in 2014, when work started to be conducted at both sectors simultaneously as illustrated in the Table below:

\begin{tabular}{|l|l|l|}
\hline Year & Sector & Numbers \\
\hline 2014 & SAV1 East & $1-499,1500-1522,2000-2425$ \\
\hline 2014 & SAV1 West & $500-1378$ \\
\hline 2015 & SAV1 East & $1000-1684$ \\
\hline 2015 & SAV1 West & $1-851$ \\
\hline 2016 & SAV1 East & $1-512$ \\
\hline 2016 & SAV1 West & $1000-1503$ \\
\hline 2017 & SAV1 East & $500-955$ \\
\hline 2017 & SAV1 West & $1-476$ \\
\hline
\end{tabular}

Numbering system of finds according to sectors

Subsequently, the items were specified alphabetically in the tables according to their various genres such as, e.g., beads, bones, charcoal, faience, pottery or stone tools, with further details concerning amount or material. In case of objects deriving from disturbed contents, fills or sieved material, this column may contain various different object types. Furthermore, the respective quantities in the categories, such as, e.g., textiles, bones, charcoal and leather etc. are not or only partially indicated by additional information like "bag" or "basket". Further data includes the exact date of the discovery.

Finds provided with an additional label "SAV1W" or "SAV1E" and a continuous number dependent on the excavation's chronology are disclosed und fully registered in the AcrossBorders project's database (see Chapter 4.3). At present, a total of 4883 objects have been recorded in this database, including additional data such as detailed descriptions, indication of dimensions, of color tones after Munsell, and photographs.

\footnotetext{
* Based on the original find lists created in the field from 2013 to 2017.
} 


\section{List of Finds SAV1 East 2013-2017}

\begin{tabular}{|c|c|c|c|c|c|}
\hline \multicolumn{6}{|c|}{ Sai Island SAV1 East 2013} \\
\hline Find no. & Location & Description/label/material & Level & Object no. & Date \\
\hline $372 / 2013$ & SQ2B, $4 \mathrm{~m}$ to $\mathrm{E}, \mathrm{S}$ of Feature 28 in debris & $\begin{array}{l}\text { amulet or scaraboid: frog scaraboid/amulet (1 intact piece, } \\
\text { faience, New Kingdom?) }\end{array}$ & $\begin{array}{l}\text { below } \\
\text { Planum } 6\end{array}$ & SAV1E 0294 & 27.02 .2013 \\
\hline $075 / 2013$ & SQ1, cleaning S-baulk & $\begin{array}{l}\text { architecture/window grille (1 fragment, pottery, Post-New } \\
\text { Kingdom) }\end{array}$ & $0-40 \mathrm{~cm}$ & SAV1E 0095 & 24.01 .2013 \\
\hline $052 / 2013$ & SQ1, Feature 3, 0-1.5m N-S & $\begin{array}{l}\text { architecture?/furniture?installation? (1 fragment, clay, Post- } \\
\text { New Kingdom) }\end{array}$ & $30-40 \mathrm{~cm}$ & SAV1E 0091 & 21.01 .2013 \\
\hline $053 / 2013$ & $\begin{array}{l}\text { SQ1, Feature 4, W of Feature 3, up to } 5 \mathrm{~m} \\
\text { to } \mathrm{S}\end{array}$ & $\begin{array}{l}\text { architecture?/furniture?installation? (1 fragment, clay, Post- } \\
\text { New Kingdom) }\end{array}$ & $30-40 \mathrm{~cm}$ & SAV1E 0092 & 21.01 .2013 \\
\hline $260 / 2013$ & $\begin{array}{l}\text { SQ2B, area } 10 \text { of Planum } 1, S \text { of Feature } \\
14, \text { mud brick debris }\end{array}$ & architecture (1 fragment, sandstone) & $\begin{array}{l}\sim 20 \mathrm{~cm}, \\
\text { see } \\
\text { Planum }\end{array}$ & SAV1E 0264 & 06.02 .2013 \\
\hline $352 / 2013$ & SQ2B, SW-corner, debris & architecture (1 fragment, sandstone) & Planum 6 & SAV1E 0267 & 18.02 .2013 \\
\hline $345 / 2013$ & $\mathrm{SQ} 2 \mathrm{~B}, \sim 5.2-5.4 \mathrm{~m}$ to $\mathrm{E}$, at S-baulk & architecture: lintel?/door jamb (1 fragment, sandstone) & $\begin{array}{c}\text { see } \\
\text { Planum } 5\end{array}$ & SAV1E 0265 & 14.02 .2013 \\
\hline $336 / 2013$ & SQ2B, Feature 27, baulk & basket (presumably Ottoman?) & $\begin{array}{c}\text { see } \\
\text { Planum } 5\end{array}$ & & 13.02 .2013 \\
\hline $004 / 2013$ & SQ1, Feature 1 & bead ( 1 tubular bead, faience) & surface & SAV1E 0004 & 06.01 .2013 \\
\hline $355 / 2013$ & $\begin{array}{l}\text { SQ1A, S-baulk } 50 \mathrm{~cm} \text { from N, E of } \\
\text { Feature } 30 \text {, in debris }\end{array}$ & bead ( 1 circular bead, faience) & $20 \mathrm{~cm}$ & SAV1E 0278 & 18.02 .2013 \\
\hline $350 / 2013$ & $\begin{array}{l}\text { SQ1A, } 5 \mathrm{~m} \text { to } \mathrm{E} \text {, from filling of Feature } \\
21\end{array}$ & bead ( 1 circular bead, clay) & \begin{tabular}{|c} 
below \\
Planum 5
\end{tabular} & SAV1E 0255 & 14.02 .2013 \\
\hline $329 / 2013$ & $\begin{array}{l}\text { SQ1A, } 8.5 \mathrm{~m} \text { to E, on top of E extension } \\
\text { of Feature } 21\end{array}$ & bead ( 1 fragment, ring bead, faience) & \begin{tabular}{|c} 
below \\
Planum 5
\end{tabular} & SAV1E 0239 & 13.02 .2013 \\
\hline $147 / 2013$ & $\begin{array}{l}\text { SQ2A, NW-corner, on lowest level of } \\
\text { Feature } 3\end{array}$ & bead ( 1 circular bead, faience) & $\sim 10 \mathrm{~cm}$ & SAV1E 0120 & 29.01 .2013 \\
\hline $370 / 2013$ & $\begin{array}{l}\text { SQ2B, } 2 \mathrm{~m} \text { to } \mathrm{E} \text {, at } \mathrm{N} \text { side of muna } \\
\text { belonging to Feature } 28\end{array}$ & bead (1 ring bead, faience) & $\begin{array}{l}\text { below } \\
\text { Planum } 6\end{array}$ & SAV1E 0286 & 26.02 .2013 \\
\hline $003 / 2013$ & SQ1, Feature 1 & bone (some pieces); seashell (1 fragment) & surface & & 06.01 .2013 \\
\hline $011 / 2013$ & SQ1, Feature 1 & bones (some pieces) & $0-10 \mathrm{~cm}$ & & 07.01 .2013 \\
\hline $025 / 2013$ & SQ1, Feature 1, SE & bone & $10-20 \mathrm{~cm}$ & & 12.01 .2013 \\
\hline $019 / 2013$ & SQ1, Feature 1, SW & bone (1 fragment, skull) & $10-20 \mathrm{~cm}$ & & 09.01 .2013 \\
\hline
\end{tabular}




\begin{tabular}{|c|c|c|c|c|c|}
\hline $222 / 2013$ & SQ1, pit, Feature 6, from SW-corner & bones (some fragments); charcoal (1 bag); doum nut (1 piece) & $\begin{array}{l}\sim 20 \mathrm{~cm} \text { in } \\
\text { pit }\end{array}$ & & 04.02 .2013 \\
\hline $093 / 2013$ & $\begin{array}{l}\text { SQ1/2, S-baulk/N-baulk, 3-4m to E, mud } \\
\text { brick debris }\end{array}$ & bones (some pieces) & $10-15 \mathrm{~cm}$ & & 26.01.2013 \\
\hline $131 / 2013$ & $\begin{array}{l}\text { SQ1/2, baulk } \mathrm{N} \text { of Feature } 15 \text {, mud brick } \\
\text { debris }\end{array}$ & bones & $30-35 \mathrm{~cm}$ & & 28.01.2013 \\
\hline $140 / 2013$ & $\mathrm{SQ} 1 \mathrm{~A}, 0-30 \mathrm{~cm}$ to $\mathrm{E}$ & bone & $\sim 10 \mathrm{~cm}$ & & 29.01 .2013 \\
\hline $074 / 2013$ & $\mathrm{SQ} 2,5 \mathrm{~cm}$ below Feature 18 in Feature 2 & bone & $\begin{array}{l}\sim 25- \\
30 \mathrm{~cm}\end{array}$ & & 24.01.2013 \\
\hline $041 / 2013$ & SQ2, Feature 14 (cleaning) & bones (some fragments) & & & 19.01.2013 \\
\hline $090 / 2013$ & SQ2A, $0-4 \mathrm{~m}$ from $\mathrm{N}, 0.2 \mathrm{~m}$ to $\mathrm{E}$ & bone & $0-5 \mathrm{~cm}$ & & 26.01.2013 \\
\hline $327 / 2013$ & SQ2A, $0-3 \mathrm{~m}$ to $\mathrm{S}$, along $\mathrm{E}$ border & bones; charcoal (1 bag) & $\begin{array}{c}25 \mathrm{~cm}, \\
\text { below } \\
\text { Planum } 5\end{array}$ & & 13.02.2013 \\
\hline $261 / 2013$ & $\begin{array}{l}\text { SQ2B, area 10, Planum 1, between } \\
\text { collapsed bricks }\end{array}$ & bones; charcoal (1 bag) & $\sim 20 \mathrm{~cm}$ & & 06.02 .2013 \\
\hline $365 / 2013$ & $\begin{array}{l}\text { SQ2B, } 6-8 m \text { to } E, 1-2 m \text { to } S \text {, mud brick } \\
\text { debris }\end{array}$ & bones (some fragments); charcoal (1 bag) & $\begin{array}{l}\text { below } \\
\text { Planum } 6\end{array}$ & & 18.02.2013 \\
\hline $368 / 2013$ & SQ2B, Feature 26 , cleaning $7.5-10 \mathrm{~m}$ to E & $\begin{array}{l}\text { bones (some fragments); re-used sherd (1 fragment, New } \\
\text { Kingdom) }\end{array}$ & $\begin{array}{l}\text { before } \\
\text { Planum } 7\end{array}$ & SAV1E 0293 (re-used sherd) & 26.02 .2013 \\
\hline $244 / 2013$ & $\begin{array}{l}\text { SQ1, lowest level of sand filling (Feature } \\
\text { 6) }\end{array}$ & charcoal (1 bag) & $\sim 50 \mathrm{~cm}$ & & 06.02 .2013 \\
\hline $133 / 2013$ & $\begin{array}{l}\text { SQ1/2, baulk } \mathrm{N} \text { of Feature } 15 \text {, mud brick } \\
\text { debris }\end{array}$ & charcoal (1 bag) & $\begin{array}{l}\sim 30- \\
35 \mathrm{~cm}\end{array}$ & & 28.01.2013 \\
\hline $022 / 2013$ & SQ2, Feature 2 & charcoal (2 bags) & $10-20 \mathrm{~cm}$ & & 10.01 .2013 \\
\hline $076 / 2013$ & $\begin{array}{l}\text { SQ2, E of Feature } 18,10 \mathrm{~cm} \text { below lower } \\
\text { edge (below } 74 \text { ) }\end{array}$ & charcoal (1 bag) & $\sim 45 \mathrm{~cm}$ & & 24.01.2013 \\
\hline $046 / 2013$ & SQ2, Feature 14, from E-part & dish (1 piece, pottery) & & & 20.01 .2013 \\
\hline $263 / 2013$ & $\begin{array}{l}\text { SQ1A, Feature } 21 \text {, directly above Feature } \\
3, \text { c. } 8.5 \mathrm{~m} \text { to E }\end{array}$ & glass vessel (1 fragment, Ottoman?) & $\sim 20 \mathrm{~cm}$ & SAV1E 0170 & 06.02 .2013 \\
\hline $151 / 2013$ & $\begin{array}{l}\text { SQ2A, N-half from pebbles, E of Feature } \\
3\end{array}$ & glass vessel (1 fragment) & $\sim 10 \mathrm{~cm}$ & SAV1E 0121 & 29.01.2013 \\
\hline $005 / 2013$ & SQ1, Feature 1 & inlay (1 piece, glazed, clay) & surface & SAV1E 0003 & 06.01 .2013 \\
\hline $050 / 2013$ & SQ2, Feature 14, in find No. 40 & $\begin{array}{l}\text { mud ( } 1 \text { fragment); seal fragments ( } 43 \text { fragments, } \\
\text { uninscribed), contents of vessel } 40 / 2013\end{array}$ & & & 21.01 .2013 \\
\hline $136 / 2013$ & $\mathrm{SQ} 1 \mathrm{~A}, \sim 8 \mathrm{~m}$ to $\mathrm{E}$, above Feature 3 & net weight (1 piece, clay) & $0-10 \mathrm{~cm}$ & SAV1E 0119 & 29.01.2013 \\
\hline
\end{tabular}




\begin{tabular}{|c|c|c|c|c|c|}
\hline $033 / 2013$ & SQ1, Feature 1, SE & Nun bowl (1 fragment, faience) & $20-30 \mathrm{~cm}$ & SAV1E 0085 & 16.01 .2013 \\
\hline $376 / 2013$ & $\mathrm{SQ} 2 \mathrm{~B}, 6 \mathrm{~m}$ to $\mathrm{E}, 1.3 \mathrm{~m}$ to $\mathrm{S}$ & Nun bowl (1 fragment, faience) & $\begin{array}{c}\text { below } \\
\text { Planum } 6\end{array}$ & SAV1E 0317 & 28.02 .2013 \\
\hline $016 / 2013$ & SQ1, Feature 1, NW & organic material (fragment of basket) & $10-20 \mathrm{~cm}$ & & 08.01 .2013 \\
\hline $049 / 2013$ & SQ2, Feature 14, in find No. 40 & organic remains, contents of vessel 40/2013 & & & 20.01 .2013 \\
\hline $083 / 2013$ & SQ1, surface & $\begin{array}{l}\text { pottery, stone and slag: pottery ( } 9 \text { baskets), among them re- } \\
\text { used sherd ( } 1 \text { piece, Post-Meroitic); grindstone ( } 1 \text { fragment, } \\
\text { unspecified stone); slag ( } 1 \text { piece) }\end{array}$ & surface & $\begin{array}{l}\text { SAV1E } 0100 \text { (re-used sherd), SAV1E } 0098 \\
\text { (grindstone), SAV1E } 0209 \text { (slag) }\end{array}$ & 26.01 .2013 \\
\hline $001 / 2013$ & SQ1, Feature 1 & $\begin{array}{l}\text { pottery ( } 40 \text { baskets), among them net weight ( } 1 \text { fragment, } \\
\text { clay), re-used sherds ( } 3 \text { pieces, } 118^{\text {th }} \text { Dynasty, } 1 \text { Post-New } \\
\text { Kingdom, } 1 \text { Christian, pottery) }\end{array}$ & surface & $\begin{array}{l}\text { SAV1E } 0002 \text { (net weight), SAV1E 0007, } \\
\text { SAV1E 0008, SAV1E } 0009 \text { (re-used sherds) }\end{array}$ & 06.01 .2013 \\
\hline $009 / 2013$ & SQ1, Feature 1 & $\begin{array}{l}\text { pottery ( } 28 \text { baskets), among them re-used sherd ( } 1 \text { piece, } \\
\text { from Christian dish, pottery), stopper ( } 1 \text { piece, clay) }\end{array}$ & $0-10 \mathrm{~cm}$ & $\begin{array}{l}\text { SAV1E } 0001 \text { (re-used sherd), SAV1E } 0005 \\
\text { (stopper) }\end{array}$ & 07.01 .2013 \\
\hline $017 / 2013$ & SQ1, Feature 1, NE & pottery (7 baskets) & $10-20 \mathrm{~cm}$ & & 08.01 .2013 \\
\hline $030 / 2013$ & SQ1, Feature 1, NE & $\begin{array}{l}\text { pottery, bones and stone: pottery ( } 4 \text { baskets); bones (some } \\
\text { pieces); pounder ( } 1 \text { piece, quartz) }\end{array}$ & $20-30 \mathrm{~cm}$ & SAV1E 0076 (pounder) & 14.01 .2013 \\
\hline $013 / 2013$ & SQ1, Feature 1, NW & $\begin{array}{l}\text { pottery ( } 13 \text { baskets), among them re-used sherds ( } 2 \text { pieces, } 1 \\
18^{\text {th }} \text { Dynasty, } 1 \text { Christian, pottery), mould ( } 1 \text { fragment, } \\
\text { pottery, Post-New Kingdom), architecture/window grille } \\
\text { (1 fragment, clay, Christian), figurine? ( } 1 \text { fragment, clay, } \\
\text { Post-New Kingdom) }\end{array}$ & $10-20 \mathrm{~cm}$ & $\begin{array}{l}\text { SAV1E 0006, SAV1E } 0010 \text { (re-used sherds), } \\
\text { SAV1E 0011 (mould), SAV1E } 0012 \\
\text { (architecture/ window grille), SAV1E } 0039 \\
\text { (figurine) }\end{array}$ & 08.01 .2013 \\
\hline $027 / 2013$ & SQ1, Feature 1, NW & $\begin{array}{l}\text { pottery ( } 5 \text { baskets), among them re-used sherds ( } 3 \text { pieces, } 2 \\
\text { Post-New Kingdom, } 1 \text { Christian, pottery) }\end{array}$ & $20-30 \mathrm{~cm}$ & $\begin{array}{l}\text { SAV1E 0064, SAV1E 0069, SAV1E } 0070 \\
\text { (re-used sherds) }\end{array}$ & 12.01 .2013 \\
\hline $023 / 2013$ & SQ1, Feature 1, SE & $\begin{array}{l}\text { pottery ( } 5 \text { baskets), among them } \\
\text { architecture?/furniture?installations? ( } 3 \text { pieces, each clay, } \\
\text { each Post-New Kingdom) }\end{array}$ & $10-20 \mathrm{~cm}$ & $\begin{array}{l}\text { SAV1E 0066, SAV1E 0067, SAV1E } 0068 \\
\text { (architecture?/furniture?) }\end{array}$ & 10.01 .2013 \\
\hline $031 / 2013$ & SQ1, Feature 1, SE & $\begin{array}{l}\text { pottery, stone and bones: pottery ( } 3 \text { baskets), among them re- } \\
\text { used sherd ( } 1 \text { piece, Christian), lamp ( } 1 \text { fragment, Post- } \\
\text { Meroitic, pottery), architecture?/furniture?installations? ( } 2 \\
\text { pieces, } 1 \text { clay, } 1 \text { pottery, each Post-Meroitic); stone vessel } \\
\text { (1 fragment, unspecified stone); bones (some pieces) }\end{array}$ & $20-30 \mathrm{~cm}$ & $\begin{array}{l}\text { SAV1E } 0077 \text { (re-used sherd), SAV1E } 0079 \\
\text { (lamp), SAV1E 0078, SAV1E 0080 } \\
\text { (architecture?/ furniture? installations?), } \\
\text { SAV1E } 0081 \text { (stone vessel) }\end{array}$ & 15.01.2013 \\
\hline $015 / 2013$ & SQ1, Feature 1, SW & $\begin{array}{l}\text { pottery ( } 16 \text { baskets), among them re-used sherds ( } 3 \text { pieces, } 1 \\
\text { Post-New Kingdom, } 2 \text { Christian, pottery), } \\
\text { architecture?furniture? ( } 1 \text { fragment, Post-New Kingdom) }\end{array}$ & $10-20 \mathrm{~cm}$ & $\begin{array}{l}\text { SAV1E 0043, SAV1E 0044, SAV1E } 0047 \\
\text { (re-used sherds), SAV1E } 0210 \text { (architecture? } \\
\text { furniture? }\end{array}$ & 08.01 .2013 \\
\hline $029 / 2013$ & SQ1, Feature 1, SW & pottery (11/2 baskets) & $20-30 \mathrm{~cm}$ & & 13.01 .2013 \\
\hline $161 / 2013$ & SQ1, cleaning E-baulk, E of Feature 3 & pottery & $0-30 \mathrm{~cm}$ & & 30.01 .2013 \\
\hline $301 / 2013$ & $\begin{array}{l}\text { SQ1, cleaning N-part of Feature 3, c. 6- } \\
7.5 \mathrm{~m} \text { to E }\end{array}$ & pottery & $\sim 40 \mathrm{~cm}$ & & 10.02 .2013 \\
\hline
\end{tabular}




\begin{tabular}{|c|c|c|c|c|c|}
\hline $224 / 2013$ & SQ1, cleaning W-baulk, $0-5 \mathrm{~m}$ from $\mathrm{N}$ & pottery & $0-40 \mathrm{~cm}$ & & 04.02 .2013 \\
\hline $110 / 2013$ & $\begin{array}{l}\text { SQ1, E-end of Feature 11, from cleaning } \\
\text { of section }\end{array}$ & pottery & $\begin{array}{c}\sim 40 \mathrm{~cm} \\
\text { (section) }\end{array}$ & & 27.01 .2013 \\
\hline $056 / 2013$ & $\mathrm{SQ} 1$, Feature 3, $1.5-3 \mathrm{~m}$ from $\mathrm{N}$ & pottery & $30-40 \mathrm{~cm}$ & & 21.01 .2013 \\
\hline $054 / 2013$ & SQ1, Feature 3, 4-5m N-S & pottery & $30-40 \mathrm{~cm}$ & & 21.01 .2013 \\
\hline $057 / 2013$ & $\mathrm{SQ1}$, Feature 3, 5-6m from $\mathrm{N}$ & pottery & $30-40 \mathrm{~cm}$ & & 21.01 .2013 \\
\hline $058 / 2013$ & SQ1, Feature 3, 7-10m from $\mathrm{N}$ & pottery & $30-40 \mathrm{~cm}$ & & 21.01 .2013 \\
\hline $278 / 2013$ & SQ1, Feature 3, cleaning at N-baulk & pottery & $\sim 40 \mathrm{~cm}$ & & 07.02 .2013 \\
\hline $080 / 2013$ & SQ1, Feature 3, cleaning wall & pottery; charcoal (1 bag) & $20-40 \mathrm{~cm}$ & & 24.01 .2013 \\
\hline $102 / 2013$ & $\begin{array}{l}\text { SQ1, Feature 3, from collapsed brick, } \\
\text { below brick } \sim 7 \mathrm{~m} \text { from } \mathrm{N}\end{array}$ & pottery & $\sim 40 \mathrm{~cm}$ & & 27.01 .2013 \\
\hline $105 / 2013$ & SQ1, Feature 3, SE-corner & pottery & $\sim 40 \mathrm{~cm}$ & & 27.01 .2013 \\
\hline $179 / 2013$ & $\begin{array}{l}\text { SQ1, NE-corner, cleaning of N-baulk up } \\
\text { to Feature } 3\end{array}$ & pottery & $0-35 \mathrm{~cm}$ & & 31.01 .2013 \\
\hline $101 / 2013$ & SQ1, NE-corner, Feature 3 & pottery & $\sim 40 \mathrm{~cm}$ & & 27.01 .2013 \\
\hline $100 / 2013$ & $\begin{array}{l}\text { SQ1, W of Feature 3, in NE-corner of } \\
\text { Feature } 4\end{array}$ & pottery & $\sim 40 \mathrm{~cm}$ & & 27.01 .2013 \\
\hline $079 / 2013$ & $\begin{array}{l}\text { SQ1, Feature } 4 \text { and Feature 1, cleaning } \\
\text { N-baulk W of Feature } 3\end{array}$ & $\begin{array}{l}\text { pottery, among it re-used sherd (1 piece, Post New-Kingdom, } \\
\text { pottery) }\end{array}$ & $0-40 \mathrm{~cm}$ & SAV1E 0094 & 24.01 .2013 \\
\hline $077 / 2013$ & SQ1, Feature 4, cleaning W-baulk & pottery & $0-40 \mathrm{~cm}$ & & 24.01 .2013 \\
\hline $045 / 2013$ & SQ1, Feature 4, NW & pottery & $30-40 \mathrm{~cm}$ & & 20.01 .2013 \\
\hline $043 / 2013$ & SQ1, Feature 4, SW & pottery & $30-40 \mathrm{~cm}$ & & 19.01 .2013 \\
\hline $055 / 2013$ & $\begin{array}{l}\text { SQ1, Feature } 4, \mathrm{~W} \text { of Feature } 3 \text {, up to } 5- \\
10 \mathrm{~m} \text { to } \mathrm{S}\end{array}$ & pottery & $30-40 \mathrm{~cm}$ & & 21.01 .2013 \\
\hline $108 / 2013$ & SQ1, Feature 4, W-half of square & $\begin{array}{l}\text { pottery and bones: pottery, among it } \\
\text { architecture?installation?furniture? ( } 1 \text { fragment, Post-New } \\
\text { Kingdom, clay); bones (some fragments) }\end{array}$ & $\sim 40 \mathrm{~cm}$ & $\begin{array}{l}\text { SAV1E } 0110 \text { (architecture? installation? } \\
\text { furniture?) }\end{array}$ & 27.01 .2013 \\
\hline $103 / 2013$ & SQ1, Feature 6, from sandy filling & pottery & $\sim 40 \mathrm{~cm}$ & & 27.01 .2013 \\
\hline $243 / 2013$ & SQ1, Feature 6, lower sand filling & pottery & $\sim 50 \mathrm{~cm}$ & & 06.02 .2013 \\
\hline $219 / 2013$ & SQ1, Feature 6, from filling & pottery & $>\underset{\text { pit }}{>15 \mathrm{~cm} \text { in }}$ & & 04.02 .2013 \\
\hline $223 / 2013$ & SQ1, Feature 6, pit & pottery; bone; charcoal (1 bag) & $\begin{array}{l}20-40 \mathrm{~cm} \\
\text { in pit }\end{array}$ & & 04.02 .2013 \\
\hline
\end{tabular}




\begin{tabular}{|c|c|c|c|c|c|}
\hline $211 / 2013$ & SQ1, Feature 6, pit, from sandy filling & pottery & $\begin{array}{l}\sim 40- \\
50 \mathrm{~cm}\end{array}$ & & 03.02 .2013 \\
\hline $300 / 2013$ & $\begin{array}{l}\text { SQ1, Feature 6, further cleaning, bottom } \\
\text { of pit }\end{array}$ & pottery & $\sim 50 \mathrm{~cm}$ & & 10.02.2013 \\
\hline $048 / 2013$ & SQ1, Feature 7, from sandy filling & pottery & Pl $1>$ & & 20.01 .2013 \\
\hline $109 / 2013$ & SQ1, NW-corner, debris/Feature 11 & pottery (from section) & $\sim 40 \mathrm{~cm}$ & & 27.01.2013 \\
\hline $051 / 2013$ & SQ1, Feature 13, cleaning & pottery ( 1 basket) & $\begin{array}{c}\text { Pl } 1>, 30- \\
40\end{array}$ & & 21.01 .2013 \\
\hline $177 / 2013$ & $\begin{array}{l}\text { SQ1, N-baulk, cleaning E-part Feature } \\
\text { 13, up to Feature } 3\end{array}$ & $\begin{array}{l}\text { pottery, among it re-used sherd ( } 1 \text { piece, Post New-Kingdom, } \\
\text { pottery) }\end{array}$ & $0-40 \mathrm{~cm}$ & SAV1E 0126 & 31.01 .2013 \\
\hline $178 / 2013$ & SQ1, W-baulk, cleaning S of Feature 13 & pottery & $0-40 \mathrm{~cm}$ & & 31.01 .2013 \\
\hline $217 / 2013$ & $\begin{array}{l}\text { SQ1/1A, cleaning Feature } 21, \mathrm{E} \text { of } \\
\text { Feature } 13\end{array}$ & pottery & $25-30 \mathrm{~cm}$ & & 04.02 .2013 \\
\hline $181 / 2013$ & $\begin{array}{l}\text { SQ1/2, N-baulk between } 8-10 \mathrm{~m} \text { to } \mathrm{E} \\
\text { above Feature } 3\end{array}$ & pottery & $0-10 \mathrm{~cm}$ & & 31.01 .2013 \\
\hline $183 / 2013$ & $\begin{array}{l}\text { SQ1/2, N-baulk of SQ2, between } 8-10 \mathrm{~m} \\
\text { E, above Feature } 3\end{array}$ & pottery & $10-20 \mathrm{~cm}$ & & 31.01 .2013 \\
\hline $213 / 2013$ & $\begin{array}{l}\text { SQ1/2, baulk, NE-corner of Feature } 2 \text {, } \\
\text { above Feature } 3\end{array}$ & pottery & $\sim 40 \mathrm{~cm}$ & & 03.02 .2013 \\
\hline $299 / 2013$ & $\begin{array}{l}\text { SQ1/2, cleaning of Feature } 3 \text {, in baulk } \\
8.5-10 \mathrm{~m} \text { to } \mathrm{E}\end{array}$ & pottery & $\begin{array}{l}\sim 35- \\
40 \mathrm{~cm}\end{array}$ & & 10.02.2013 \\
\hline $232 / 2013$ & SQ1/2, Feature 15, W-baulk cleaning & pottery & $20-40 \mathrm{~cm}$ & & 04.02 .2013 \\
\hline $146 / 2013$ & $\begin{array}{l}\text { SQ1/2, Feature } 15 \text {, from soft-muddy part } \\
\text { in } \mathrm{W} \text { and collapsed brick }\end{array}$ & pottery; bones (some fragments) & $\begin{array}{l}\sim 35- \\
40 \mathrm{~cm}\end{array}$ & & 29.01.2013 \\
\hline $194 / 2013$ & $\begin{array}{l}\text { SQ1/2, W half of Feature } 15 \text {, next to mud } \\
\text { debris }\end{array}$ & pottery; charcoal (1 bag) & $\sim 40 \mathrm{~cm}$ & & 02.02 .2013 \\
\hline $233 / 2013$ & $\mathrm{SQ1} / 2$, Feature $15, \mathrm{~W}$-area & pottery; charcoal ( 5 pieces $)$ & $\sim 40 \mathrm{~cm}$ & & 04.02 .2013 \\
\hline $153 / 2013$ & SQ1/2, Feature 15, from collapsed bricks & pottery & $\sim 40 \mathrm{~cm}$ & & 29.01 .2013 \\
\hline $215 / 2013$ & $\begin{array}{l}\text { SQ1/2, Feature } 15 \text {, from mud debris in } \\
\text { NW-corner }\end{array}$ & pottery & $\begin{array}{l}\sim 40- \\
50 \mathrm{~cm}\end{array}$ & & 03.02 .2013 \\
\hline $295 / 2013$ & SQ1/2, Feature 15 , cleaning Planum 4 & pottery & $\begin{array}{l}\text { below } \\
\text { Planum } 4 \\
\sim 50 \mathrm{~cm}\end{array}$ & & 10.02.2013 \\
\hline $112 / 2013$ & $\begin{array}{l}\mathrm{SQ} 1 / 2 \text {, baulk } \mathrm{N} \text { of Feature } 15, \text { from mud } \\
\text { brick debris }\end{array}$ & pottery & $\sim 25 \mathrm{~cm}$ & & 27.01 .2013 \\
\hline $111 / 2013$ & SQ1/2, baulk $\mathrm{N}$ of Feature 15 , sandy area & pottery & $\sim 25 \mathrm{~cm}$ & & 27.01.2013 \\
\hline
\end{tabular}




\begin{tabular}{|c|c|c|c|c|c|}
\hline $114 / 2013$ & $\begin{array}{l}\text { SQ1/2, baulk } \mathrm{N} \text { of Feature } 15 \text {, mud brick } \\
\text { debris }\end{array}$ & pottery & $\sim 30 \mathrm{~cm}$ & & 27.01.2013 \\
\hline $116 / 2013$ & $\begin{array}{l}\text { SQ1/2, baulk } \mathrm{N} \text { of Feature } 15 \text {, mud brick } \\
\text { debris }\end{array}$ & pottery & $\sim 35 \mathrm{~cm}$ & & 27.01.2013 \\
\hline $130 / 2013$ & $\begin{array}{l}\text { SQ1/2, baulk } \mathrm{N} \text { of Feature } 15 \text {, mud brick } \\
\text { debris }\end{array}$ & pottery & $\begin{array}{l}\sim 30- \\
35 \mathrm{~cm}\end{array}$ & & 28.01.2013 \\
\hline $113 / 2013$ & $\begin{array}{l}\text { SQ1/2, baulk } \mathrm{N} \text { of Feature } 15 \text {, sandy area } \\
\text { to } \mathrm{W}\end{array}$ & pottery & $\sim 30 \mathrm{~cm}$ & & 27.01.2013 \\
\hline $115 / 2013$ & $\begin{array}{l}\mathrm{SQ} 1 / 2 \text {, baulk } \mathrm{N} \text { of Feature } 15 \text {, sandy } \\
\text { area/pit }\end{array}$ & pottery & $\sim 35 \mathrm{~cm}$ & & 27.01.2013 \\
\hline $107 / 2013$ & $\begin{array}{l}\text { SQ1/2, baulk above Feature } 15, \mathrm{~W} \text {-end, } \\
\text { sandy area }\end{array}$ & pottery & $\sim 20 \mathrm{~cm}$ & & 27.01.2013 \\
\hline $106 / 2013$ & $\begin{array}{l}\mathrm{SQ} 1 / 2 \text {, baulk between, above Feature } 15, \\
\text { from mud brick debris }\end{array}$ & pottery & $\sim 20 \mathrm{~cm}$ & & 27.01.2013 \\
\hline $137 / 2013$ & $\begin{array}{l}\text { SQ1/2, baulk, Feature } 15 \text {, mud brick } \\
\text { debris }\end{array}$ & pottery; bones (some pieces); charcoal (3 pieces) & $\sim 40 \mathrm{~cm}$ & & 29.01.2013 \\
\hline $134 / 2013$ & SQ1/2, baulk from mud brick debris & pottery & $30-40 \mathrm{~cm}$ & & 28.01.2013 \\
\hline $143 / 2013$ & $\begin{array}{l}\text { SQ1/2, baulk, Feature 15, from soft part } \\
\text { in W }\end{array}$ & pottery & $\sim 30 \mathrm{~cm}$ & & 29.01.2013 \\
\hline $138 / 2013$ & $\begin{array}{l}\text { SQ1/2, baulk, Feature 15, sandy area to } \\
\text { W }\end{array}$ & $\begin{array}{l}\text { pottery, among it a glazed vessel ( } 1 \text { fragment, glazed on both } \\
\text { sides, Islamic?) }\end{array}$ & $\sim 40 \mathrm{~cm}$ & SAV1E 0122 & 29.01.2013 \\
\hline $129 / 2013$ & SQ1/2, baulk, sandy pit $N$ of Feature 15 & pottery & $\begin{array}{r}\sim 30- \\
35 \mathrm{~cm}\end{array}$ & & 28.01.2013 \\
\hline $195 / 2013$ & $\mathrm{SQ} 1 / 2, \mathrm{E}$ half of Feature 15 in debris & $\begin{array}{l}\text { pottery; among it re-used sherds ( } 2 \text { pieces, each Post-New } \\
\text { Kingdom, pottery) }\end{array}$ & $\sim 40 \mathrm{~cm}$ & SAV1E 0132, SAV1E 0133 (re-used sherds) & 02.02 .2013 \\
\hline $210 / 2013$ & SQ1/2, Feature 17 , from sandy filling & pottery & $20-40 \mathrm{~cm}$ & & 03.02 .2013 \\
\hline $209 / 2013$ & SQ1/2, NW of Feature 17, pebbles & pottery & $\sim 20 \mathrm{~cm}$ & & 03.02 .2013 \\
\hline $208 / 2013$ & $\begin{array}{l}\mathrm{SQ} 1 / 2, \mathrm{NE} \text { of Feature 17, from mud brick } \\
\text { remains }\end{array}$ & pottery & $\sim 20 \mathrm{~cm}$ & & 03.02 .2013 \\
\hline $091 / 2013$ & $\begin{array}{l}\text { SQ1/2, S-baulk/N-baulk, 0-1m E, sandy } \\
\text { pit }\end{array}$ & pottery & $0-10 \mathrm{~cm}$ & & 26.01.2013 \\
\hline $092 / 2013$ & $\begin{array}{l}\mathrm{SQ} 1 / 2, \mathrm{~S}-\mathrm{baulk} / \mathrm{N}-\text { baulk, mud brick } \\
\text { debris, from } 1.5-3 \mathrm{~m} \text { debris area }\end{array}$ & $\begin{array}{l}\text { pottery and bones: pottery, among it architecture/window } \\
\text { grille (1 fragment, Post-New Kingdom, clay); bones (some } \\
\text { pieces) }\end{array}$ & $10-15 \mathrm{~cm}$ & SAV1E 0093 (architecture/window grille) & 26.01.2013 \\
\hline $094 / 2013$ & $\begin{array}{l}\text { SQ1/2, S-baulk/N-baulk, 3-4m to E, mud } \\
\text { brick debris }\end{array}$ & pottery & $10-15 \mathrm{~cm}$ & & 26.01.2013 \\
\hline $089 / 2013$ & SQ1/2, destruction of S-baulk, $0-4 \mathrm{~m}$ to $\mathrm{E}$ & $\begin{array}{l}\text { pottery, among it re-used sherds ( } 2 \text { pieces, each Christian, } \\
\text { pottery) }\end{array}$ & $0-20 \mathrm{~cm}$ & SAV1E 0101, SAV1E 0102 (re-used sherds) & 26.01.2013 \\
\hline
\end{tabular}




\begin{tabular}{|c|c|c|c|c|c|}
\hline $182 / 2013$ & SQ1/2, N-baulk of SQ2, 5-7m to E & pottery ( 1 fragment of overfired pottery) & $0-10 \mathrm{~cm}$ & SAV1E 0129 & 31.01 .2013 \\
\hline $356 / 2013$ & $\begin{array}{l}\text { SQ1+SQ1A, Feature 3, cleaning debris of } \\
\mathrm{N} \text { end of Feature } 3\end{array}$ & pottery; bones (some fragments); slag (3 small fragments) & $\begin{array}{c}\text { below } \\
\text { Planum } 6\end{array}$ & SAV1E 0279 & 18.02.2013 \\
\hline $272 / 2013$ & SQ1A/1, Feature 13, sandy filling & pottery; charcoal (1 bag) & $30-40 \mathrm{~cm}$ & & 07.02 .2013 \\
\hline $128 / 2013$ & SQ1A, Feature $1 \mathrm{~A}, 0-5 \mathrm{~m}$ to $\mathrm{E}$ & $\begin{array}{l}\text { pottery ( } 6 \text { baskets), among them mud object ( } 1 \text { piece, } \\
\text { function unknown) }\end{array}$ & $0-10 \mathrm{~cm}$ & SAV1E 0112 & 28.01.2013 \\
\hline $307 / 2013$ & $\begin{array}{l}\text { SQ1A, Feature } 3, \sim 6-7.5 \mathrm{~m} \text { to } \mathrm{E}, \\
\text { cleaning pit of Feature } 3\end{array}$ & pottery; slag (1 piece) & $\begin{array}{l}\sim 40- \\
50 \mathrm{~cm}\end{array}$ & SAV1E 0216 (slag) & 11.02 .2013 \\
\hline $303 / 2013$ & SQ1A, Feature 3, filling $\sim 6-7.5 \mathrm{~m}$ & pottery & $\begin{array}{l}\sim 30- \\
40 \mathrm{~cm}\end{array}$ & & 11.02 .2013 \\
\hline $304 / 2013$ & SQ1A, E of Feature 3, c. 7.5-9m E & pottery & $\begin{array}{l}\sim 25- \\
30 \mathrm{~cm}\end{array}$ & & 11.02 .2013 \\
\hline $218 / 2013$ & SQ1A, cleaning $\mathrm{E}$ of Feature $3, \sim 9 \mathrm{~m}$ to $\mathrm{E}$ & pottery; slag (1 piece) & $\sim 20 \mathrm{~cm}$ & SAV1E 0208 (slag) & 04.02 .2013 \\
\hline $274 / 2013$ & SQ1A, mud debris, $8-10 \mathrm{~m}$ to $\mathrm{E}$ & pottery & $\begin{array}{l}\sim 25- \\
35 \mathrm{~cm}\end{array}$ & & 07.02 .2013 \\
\hline $173 / 2013$ & $\begin{array}{l}\text { SQ1A, } 7.5-10 \mathrm{~m} \text { to E muddy area, E of } \\
\text { Feature } 3\end{array}$ & pottery & $\sim 15 \mathrm{~cm}$ & & 30.01 .2013 \\
\hline $168 / 2013$ & SQ1A, 7-8m, sandy pit and pebbles & $\begin{array}{l}\text { pottery, among it re-used sherd ( } 1 \text { piece, Post New-Kingdom, } \\
\text { pottery) }\end{array}$ & $\begin{array}{l}\sim 10- \\
15 \mathrm{~cm}\end{array}$ & SAV1E 0125 & 30.01 .2013 \\
\hline $139 / 2013$ & SQ1A, 0-2.5m E & pottery & $\sim 10 \mathrm{~cm}$ & & 29.01 .2013 \\
\hline $331 / 2013$ & $\begin{array}{l}\text { SQ1A, } 0-2 m \text { to E, } 1 \mathrm{~m} \text { to } \mathrm{S} \text {, pit with } \\
\text { debris }\end{array}$ & $\begin{array}{l}\text { pottery, textile and charcoal: pottery, among it re-used sherd } \\
\text { ( } 3 \text { pieces, each Post-New Kingdom, pottery), } \\
\text { architecture/window grille ( } 1 \text { fragment, Post-New Kingdom, } \\
\text { clay); textile ( } 1 \text { piece); charcoal ( } 1 \text { bag) }\end{array}$ & $\begin{array}{l}\text { below } \\
\text { Planum } 5\end{array}$ & $\begin{array}{l}\text { SAV1E } 0236 \text { (architecture/window grille), } \\
\text { SAV1E 0240, SAV1E 0241, SAV1E 0241 } \\
\text { (re-used sherds) }\end{array}$ & 13.02.2013 \\
\hline $357 / 2013$ & $\begin{array}{l}\text { SQ1A, } 2-6 \mathrm{~m} \text { to E, cleaning bottom of } \\
\text { Feature } 21\end{array}$ & pottery & $\begin{array}{l}\text { below } \\
\text { Planum } 6 \\
\text { see } \\
\text { Planum } 7\end{array}$ & & 18.02 .2013 \\
\hline $221 / 2013$ & SQ1A, c. $3 \mathrm{~m}$ to E, cleaning of N-baulk & pottery & $\sim 0-10 \mathrm{~cm}$ & & 04.02 .2013 \\
\hline $166 / 2013$ & $\mathrm{SQ} 1 \mathrm{~A}, \sim 3.5-4 \mathrm{~m}$ to $\mathrm{E}$, muna remains & pottery & $\sim 10 \mathrm{~cm}$ & & 30.01 .2013 \\
\hline $165 / 2013$ & $\begin{array}{l}\text { SQ1A, } 3.5-5 \mathrm{~m} \text { to E, sandy area and } \\
\text { pebbles }\end{array}$ & $\begin{array}{l}\text { pottery, among it re-used sherd ( } 1 \text { piece, Post New-Kingdom, } \\
\text { pottery) }\end{array}$ & $\begin{array}{l}\sim 10- \\
15 \mathrm{~cm}\end{array}$ & SAV1E 0128 & 30.01 .2013 \\
\hline $349 / 2013$ & SQ1A, 3-6m to E, Feature 21 & pottery & $\begin{array}{c}\text { below } \\
\text { Planum } 5\end{array}$ & & 14.02 .2013 \\
\hline $141 / 2013$ & $\begin{array}{l}\text { SQ1A, } 4-5 \mathrm{~m} \text { to E, sandy pit and some } \\
\text { mud remains }\end{array}$ & pottery & $\sim 10 \mathrm{~cm}$ & & 29.01.2013 \\
\hline
\end{tabular}




\begin{tabular}{|c|c|c|c|c|c|}
\hline $205 / 2013$ & $\begin{array}{l}\text { SQ1A, } 4.5-5.5 \mathrm{~m} \text { to E, mud brick debris } \\
\text { in W of Feature } 3\end{array}$ & pottery & $\sim 15-25 \mathrm{~cm}$ & & 03.02 .2013 \\
\hline $135 / 2013$ & $\begin{array}{l}\text { SQ1A, 5-10m to E, first cleaning below } \\
\text { surface }\end{array}$ & pottery & $0-5 / 10 \mathrm{~cm}$ & & 28.01 .2013 \\
\hline $332 / 2013$ & SQ1A, 5-6m to E, N of 21, pit & pottery, charcoal (1 bag) & $\begin{array}{l}\text { below } \\
\text { Planum } 5\end{array}$ & & 13.02 .2013 \\
\hline $347 / 2013$ & SQ1A, 5-6m to E, $N$ of Feature 21 & pottery & $\begin{array}{l}\text { below } \\
\text { Planum } 5\end{array}$ & & 14.02 .2013 \\
\hline $167 / 2013$ & SQ1A, 5-7m to E, sandy pit & pottery & $\begin{array}{l}\sim 10- \\
15 \mathrm{~cm}\end{array}$ & & 30.01 .2013 \\
\hline $172 / 2013$ & $\begin{array}{l}\text { SQ1A, } 5-7.5 \mathrm{~m} \text { to E, sandy area above } \\
\text { Feature } 3\end{array}$ & pottery & $\sim 15 \mathrm{~cm}$ & & 30.01 .2013 \\
\hline $358 / 2013$ & $\begin{array}{l}\text { SQ1A, } 6 \mathrm{~m} \text { to } \mathrm{E} \text {, in pebbles } \mathrm{S} \text { of Feature } \\
21\end{array}$ & pottery, among it rim sherd ( 1 piece, $18^{\text {th }}$ Dynasty) & $\begin{array}{c}\text { see } \\
\text { Planum } \\
7 / \sim 40 \mathrm{~cm} \\
\sim 10 \mathrm{~cm}\end{array}$ & & 18.02 .2013 \\
\hline $321 / 2013$ & $\begin{array}{l}\mathrm{SQ1A}, \sim 6.5-7.5 \mathrm{~m} \text { to } \mathrm{E}, \mathrm{N} \text { corner of } \\
\text { Feature } 3\end{array}$ & $\begin{array}{l}\text { pottery, among it re-used sherds ( } 2 \text { pieces, } 1 \text { Post-New } \\
\text { Kingdom, } 1 \text { unclear, pottery) }\end{array}$ & $\sim 50 \mathrm{~cm}$ & SAV1E 0230, SAV1E 0231 (re-used sherds) & 12.02 .2013 \\
\hline $348 / 2013$ & SQ1A, $6-7.5 \mathrm{~m}$ to E, Feature 3 & $\begin{array}{l}\text { pottery and stone: pottery, among it re-used sherd (1 Christian } \\
\text { ring base, pottery), mud object/seal? (1 piece); grindstone } \\
\text { (1 fragment, sandstone) }\end{array}$ & $\begin{array}{l}\text { below } \\
\text { Planum } 5\end{array}$ & $\begin{array}{l}\text { SAV1E } 0258 \text { (re-used sherd), SAV1E } 0263 \\
\text { (mud object/seal?), SAV1E } 0252 \text { (grindstone) }\end{array}$ & 14.02 .2013 \\
\hline $169 / 2013$ & $\begin{array}{l}\text { SQ1A, c. } 7.5 \mathrm{~m} \text { to E, in } \mathrm{N} \text {-section, } \\
\text { collapsed mud brick }\end{array}$ & pottery & $\sim 10 \mathrm{~cm}$ & & 30.01 .2013 \\
\hline $170 / 2013$ & $\begin{array}{l}\text { SQ1A, } \sim 8-10 \mathrm{~m} \text { to E, muddy area in NE- } \\
\text { corner }\end{array}$ & pottery & $\sim 10 \mathrm{~cm}$ & & 30.01 .2013 \\
\hline $204 / 2013$ & $\begin{array}{l}\text { SQ1A, } 8-10 \mathrm{~m} \text { to E, E of Feature 21, mud } \\
\text { brick debris }\end{array}$ & pottery & $\begin{array}{l}\sim 10- \\
20 \mathrm{~cm}\end{array}$ & & 03.02 .2013 \\
\hline $330 / 2013$ & $\begin{array}{l}\text { SQ1A, 8-9.8m to E, above Feature } 21 \\
\text { extension }\end{array}$ & pottery & $\begin{array}{l}\text { below } \\
\text { Planum } 5\end{array}$ & & 13.02 .2013 \\
\hline $302 / 2013$ & $\begin{array}{l}\text { SQ1A, cleaning } \mathrm{N} \text { of Feature } 21 \text {, sandy } \\
\text { pit, 5-6m to E + Feature } 21\end{array}$ & $\begin{array}{l}\text { pottery, among it re-used sherd ( } 1 \text { handle, Christian, pottery); } \\
\text { slag (1 piece) }\end{array}$ & $\sim 40 \mathrm{~cm}$ & $\begin{array}{l}\text { SAV1E } 0215 \text { (re-used sherd), SAV1E } 0217 \\
\text { (slag) }\end{array}$ & 11.02.2013 \\
\hline $268 / 2013$ & $\begin{array}{l}\text { SQ1A, cleaning of Feature 21, directly } \\
\text { above Feature } 3\end{array}$ & pottery & $\begin{array}{l}\sim 25- \\
30 \mathrm{~cm}\end{array}$ & & 07.02 .2013 \\
\hline $309 / 2013$ & $\begin{array}{l}\text { SQ1A, cleaning debris of Feature 23, see } \\
\text { Planum } 2\end{array}$ & pottery; grindstone (1 fragment, sandstone) & $\sim 10 \mathrm{~cm}$ & SAV1E 0224 (grindstone) & 11.02 .2013 \\
\hline $154 / 2013$ & SQ1A, E-part, 5-7m to E, sandy pit & pottery & $\sim 10 \mathrm{~cm}$ & & 29.01 .2013 \\
\hline $155 / 2013$ & SQ1A, E-part, 8-10m muddy part & pottery & $\sim 10 \mathrm{~cm}$ & & 29.01 .2013 \\
\hline $207 / 2013$ & SQ1A, Feature $21,3-4.5 \mathrm{~m}$ to E, sandy pit & pottery & $\sim 20 \mathrm{~cm}$ & & 03.02 .2013 \\
\hline
\end{tabular}




\begin{tabular}{|c|c|c|c|c|c|}
\hline $322 / 2013$ & $\begin{array}{l}\text { SQ1A, Feature } 21,3-5 \mathrm{~m} \text { to E, sandy } \\
\text { filling }\end{array}$ & pottery & $\begin{array}{r}\sim 40- \\
45 \mathrm{~cm}\end{array}$ & & 12.02 .2013 \\
\hline $203 / 2013$ & SQ1A, Feature $21,4.5-5 \mathrm{~m}$ to E, sandy pit & $\begin{array}{l}\text { pottery, among it re-used sherds ( } 3 \text { pieces, } 1 \text { Post-New } \\
\text { Kingdom, } 218^{\text {th }} \text { Dynasty, pottery) }\end{array}$ & $\sim 10-$ & $\begin{array}{l}\text { SAV1E 0140, SAV1E 0141, SAV1E } 0143 \\
\text { (re-used sherds) }\end{array}$ & 03.02 .2013 \\
\hline $270 / 2013$ & $\begin{array}{l}\text { SQ1A, Feature } 21,5-6.5 \mathrm{~m} \text { to E, sandy } \\
\text { filling W of Feature } 3\end{array}$ & $\begin{array}{l}\text { pottery, among it re-used sherd (1 piece, Christian, pottery); } \\
\text { slag ( } 1 \text { fragment) }\end{array}$ & $\begin{array}{l}\sim 25- \\
30 \mathrm{~cm}\end{array}$ & $\begin{array}{l}\text { SAV1E } 0176 \text { (re-used sherd), SAV1E } 0183 \\
\text { (slag) }\end{array}$ & 07.02 .2013 \\
\hline $277 / 2013$ & $\begin{array}{l}\text { SQ1A, Feature } 21,5-7 \mathrm{~m} \text { to E, sandy } \\
\text { filling }\end{array}$ & pottery & $\begin{array}{l}\sim 30- \\
40 \mathrm{~cm}\end{array}$ & & 07.02 .2013 \\
\hline $276 / 2013$ & $\begin{array}{l}\text { SQ1A, Feature 21, above Feature 3, 7- } \\
8 \mathrm{~m} \text { to E }\end{array}$ & pottery & $\begin{array}{l}\sim 30- \\
40 \mathrm{~cm}\end{array}$ & & 07.02 .2013 \\
\hline $305 / 2013$ & $\begin{array}{l}\text { SQ1A, Feature 21, sandy filling, 3-5m to } \\
\text { E }\end{array}$ & pottery & $\sim 40 \mathrm{~cm}$ & & 11.02 .2013 \\
\hline $306 / 2013$ & $\begin{array}{l}\text { SQ1A, Feature 21, sandy filling, 3-5m to } \\
\text { E }\end{array}$ & pottery & $\begin{array}{l}\sim 40- \\
45 \mathrm{~cm}\end{array}$ & & 11.02 .2013 \\
\hline $273 / 2013$ & $\begin{array}{l}\text { SQ1A, Feature 21, sandy filling, 7-8m to } \\
\text { E }\end{array}$ & pottery & $25-30 \mathrm{~cm}$ & & 07.02.2013 \\
\hline $206 / 2013$ & SQ1A, Feature 21, sandy pit & pottery & $\begin{array}{l}\sim 20- \\
25 \mathrm{~cm}\end{array}$ & & 03.02 .2013 \\
\hline $319 / 2013$ & $\begin{array}{l}\text { SQ1A, N of E extension of Feature 21, at } \\
\text { bottom of Feature } 23\end{array}$ & pottery & $\sim 25 \mathrm{~cm}$ & & 12.02 .2013 \\
\hline $318 / 2013$ & $\begin{array}{l}\text { SQ1A, S of E extension of Feature 21, } \\
9.8 \mathrm{~m} \text { to E }\end{array}$ & pottery & $\sim 40 \mathrm{~cm}$ & & 12.02 .2013 \\
\hline $269 / 2013$ & $\begin{array}{l}\text { SQ1A, sandy back filling of Feature } \\
13 / 21 \text { and mud bricks debris up to } 5 \mathrm{~m}\end{array}$ & $\begin{array}{l}\text { pottery, among it re-used sherd ( } 1 \text { piece, New Kingdom, } \\
\text { pottery) }\end{array}$ & $\begin{array}{l}\sim 30- \\
35 \mathrm{~cm}\end{array}$ & SAV1E 0177 & 07.02 .2013 \\
\hline $361 / 2013$ & SQ1A, from filling of pit, Feature 29 & pottery; bones (some fragments) & $\begin{array}{l}\text { up to } \\
40 \mathrm{~cm}\end{array}$ & & 18.02 .2013 \\
\hline $267 / 2013$ & SQ1A, NE-corner, above mud debris & pottery & $\begin{array}{l}\sim 10- \\
15 \mathrm{~cm}\end{array}$ & & 07.02 .2013 \\
\hline $308 / 2013$ & $\begin{array}{l}\text { SQ1A, NE-corner, } 8-10 \mathrm{~m} \text {, cleaning mud } \\
\text { brick muna and debris }\end{array}$ & pottery & $\begin{array}{l}\sim 30- \\
40 \mathrm{~cm}\end{array}$ & & 11.02 .2013 \\
\hline $354 / 2013$ & $\begin{array}{l}\text { SQ1A, NE-corner Feature 30, cleaning } \\
\text { Feature } 30\end{array}$ & pottery & $\begin{array}{c}\text { after } \\
\text { Planum } 6\end{array}$ & & 18.02 .2013 \\
\hline $163 / 2013$ & SQ1A, NW-corner, mud brick debris & $\begin{array}{l}\text { pottery, among it clay object ( } 1 \text { cylindrical piece, function } \\
\text { unclear) }\end{array}$ & $\sim 10 \mathrm{~cm}$ & SAV1E 0206 & 30.01 .2013 \\
\hline $216 / 2013$ & $\begin{array}{l}\text { SQ1A, NW-corner, cleaning, mud brick } \\
\text { debris }\end{array}$ & pottery; bone (fragments) & $\begin{array}{l}\sim 10- \\
20 \mathrm{~cm}\end{array}$ & & 04.02 .2013 \\
\hline $271 / 2013$ & $\begin{array}{l}\text { SQ1A, NW-corner, cleaning mud brick } \\
\text { debris }\end{array}$ & $\begin{array}{l}\text { pottery, slag, bones and charcoal: pottery, among it re-used } \\
\text { sherds ( } 2 \text { pieces, each Post-New Kingdom); slag ( } 3 \text { pieces); } \\
\text { bones (some fragments); charcoal ( } 1 \text { bag) }\end{array}$ & $\begin{array}{l}\sim 20 \\
25 \mathrm{~cm}\end{array}$ & $\begin{array}{l}\text { SAV1E 0178, SAV1E } 0179 \text { (re-used sherds), } \\
\text { SAV1E 0184, SAV1E 0185, SAV1E } 0186 \\
\text { (slag) }\end{array}$ & 07.02 .2013 \\
\hline
\end{tabular}




\begin{tabular}{|c|c|c|c|c|c|}
\hline $279 / 2013$ & $\begin{array}{l}\text { SQ1A, NW-corner, cleaning mud brick } \\
\text { debris }\end{array}$ & $\begin{array}{l}\text { pottery, stone, bone and charcoal: pottery; hammer (1 piece, } \\
\text { schist); bones (some fragments); charcoal (1 bag) }\end{array}$ & $\begin{array}{r}\sim 25- \\
30 \mathrm{~cm}\end{array}$ & SAV1E 0187 (hammer) & 07.02 .2013 \\
\hline $174 / 2013$ & $\begin{array}{l}\text { SQ1A, NW-corner, second cleaning of } \\
\text { mud brick debris }\end{array}$ & pottery & $\begin{array}{l}\sim 10 \mathrm{~cm} \\
\text { between } \\
\text { bricks }\end{array}$ & & 30.01 .2013 \\
\hline $175 / 2013$ & $\begin{array}{l}\text { SQ1A, NW-corner, second cleaning of } \\
\text { mud brick debris }\end{array}$ & pottery & $\sim 10 \mathrm{~cm}$ & & 31.01 .2013 \\
\hline $320 / 2013$ & $\begin{array}{l}\text { SQ1A, NW-corner, cleaning after } \\
\text { Planum } 3 \text { up to } 2.5 \mathrm{~m} \text { to } \mathrm{E}\end{array}$ & $\begin{array}{l}\text { pottery, among it clay object ( } 1 \text { fragment with } 3 \text { complete } \\
\text { holes) }\end{array}$ & $\begin{array}{l}20- \\
25 \mathrm{~cm}\end{array}$ & SAV1E 0232 & 12.02 .2013 \\
\hline $176 / 2013$ & $\begin{array}{l}\text { SQ1A, NW-corner, cleaning baulk W of } \\
\text { Feature } 13\end{array}$ & pottery & $0-40 \mathrm{~cm}$ & & 31.01 .2013 \\
\hline $164 / 2013$ & $\begin{array}{l}\text { SQ1A, SW-corner and sandy pit above } \\
\text { Feature } 13\end{array}$ & pottery & $\begin{array}{l}\sim 10- \\
15 \mathrm{~cm}\end{array}$ & & 30.01 .2013 \\
\hline $234 / 2013$ & $\begin{array}{l}\text { SQ1A-2B, up to } 1 \mathrm{~m} \text { to } \mathrm{W} \text { of } \mathrm{SQ} 1 \text { and } 2 \text {, } \\
\text { surface cleaning }\end{array}$ & $\begin{array}{l}\text { pottery and slag: pottery (10 baskets), among them re-used } \\
\text { sherds ( } 2 \text { pieces, } 118 \text { th Dynasty, } 1 \text { Post-New Kingdom); slag } \\
\text { ( } 3 \text { fragments) }\end{array}$ & surface & $\begin{array}{l}\text { SAV1E 0163, SAV1E } 0172 \text { (re-used sherds), } \\
\text { SAV1E 0147, SAV1E 0148, SAV1E } 0149 \\
\text { (slag) }\end{array}$ & 05.02 .2013 \\
\hline $156 / 2013$ & $\begin{array}{l}\text { SQ2, SW-corner, cleaning of baulk, at } \\
\text { mud brick }\end{array}$ & pottery; bone (part of skull/teeth - modern?) & $0-20 \mathrm{~cm}$ & & 30.01 .2013 \\
\hline $157 / 2013$ & SQ2, cleaning of baulk, $\mathrm{S}$ & pottery & $0-20 \mathrm{~cm}$ & & 30.01 .2013 \\
\hline $158 / 2013$ & SQ2, cleaning of E-baulk, 6-10m from N & pottery & $0-20 \mathrm{~cm}$ & & 30.01 .2013 \\
\hline $078 / 2013$ & SQ2, cleaning S-part of W-baulk & pottery & $0-40 \mathrm{~cm}$ & & 24.01 .2013 \\
\hline $189 / 2013$ & SQ2, from S section in SE-corner & pottery & $\sim 20 / 25 \mathrm{~cm}$ & & 02.02 .2013 \\
\hline 099/2013 & $\begin{array}{l}\mathrm{SQ} 2 \text {, up to } 2.5 \mathrm{~m} \mathrm{~S} \text { of } \mathrm{SQ} 2+2 \mathrm{~A} \text {, Feature } 2 \text {, } \\
\text { from surface cleaning }\end{array}$ & $\begin{array}{l}\text { pottery ( } 5 \text { baskets), among them re-used sherd ( } 1 \text { piece, } \\
\text { Christian jar?, pottery), scraper ( } 1 \text { re-used body sherd of a jar, } \\
\text { Thutmose III, pottery) }\end{array}$ & surface & $\begin{array}{l}\text { SAV1E } 0109 \text { (re-used sherd), SAV1E } 2902 \\
\text { (scraper }\end{array}$ & 27.01 .2013 \\
\hline $006 / 2013$ & SQ2, Feature 2 & $\begin{array}{l}\text { pottery ( } 18 \text { baskets), among them architecture/window grille } \\
\text { ( } 2 \text { fragments, each clay and Post-Meroitic) }\end{array}$ & surface & $\begin{array}{l}\text { SAV1E 0074, SAV1E } 0075 \text { (architecture/ } \\
\text { window grille) }\end{array}$ & 06.01 .2013 \\
\hline $012 / 2013$ & SQ2, Feature 2 & $\begin{array}{l}\text { pottery and stone: pottery ( } 8 \text { baskets), among them mould } \\
\text { ( } 1 \text { fragment, clay); pounder ( } 1 \text { piece, quartzite) }\end{array}$ & $0-10 \mathrm{~cm}$ & $\begin{array}{l}\text { SAV1E } 0038 \text { (mould), SAV1E } 0040 \\
\text { (pounder) }\end{array}$ & 07.01 .2013 \\
\hline $018 / 2013$ & SQ2, Feature 2 & $\begin{array}{l}\text { pottery and stone: pottery ( } 21 \text { baskets), among them re-used } \\
\text { sherds ( } 4 \text { pieces, } 2 \text { Post-New Kingdom, } 1 \text { Christian, pottery, } \\
118^{\text {th }} \text { Dynasty?), furniture?installations? ( } 3 \text { pieces, } 1 \text { pottery, } \\
2 \text { clay, each Post-New Kingdom); palette ( } 1 \text { fragment, } \\
\text { sandstone), stone tool ( } 1 \text { elongated piece, flat on one side, } \\
\text { widening out at one end, function not specified, stone not } \\
\text { specified), grindstone ( } 1 \text { piece, sandstone) }\end{array}$ & $10-20 \mathrm{~cm}$ & $\begin{array}{l}\text { SAV1E 0045, SAV1E 0046, SAV1E 0063, } \\
\text { SAV1E 0065 (re-used sherds), SAV1E 0048, } \\
\text { SAV1E 0051, SAV1E } 0052 \text { (furniture? } \\
\text { installations?), SAV1E } 0053 \text { (palette), } \\
\text { SAV1E 0050 (grindstone) }\end{array}$ & 09.01 .2013 \\
\hline $032 / 2013$ & SQ2, Feature 2 & $\begin{array}{l}\text { pottery ( } 1 \text { basket), among it re-used sherds ( } 2 \text { pieces, each } \\
18^{\text {th }} \text { Dynasty, pottery) }\end{array}$ & $20-30 \mathrm{~cm}$ & SAV1E 0082, SAV1E 0083 (re-used sherds) & 15.01.2013 \\
\hline
\end{tabular}




\begin{tabular}{|c|c|c|c|c|c|}
\hline $037 / 2013$ & $\begin{array}{l}\text { SQ2, Feature 2, 0-3m to E, cleaning of } \\
\text { wall W-E }\end{array}$ & pottery & $20-30 \mathrm{~cm}$ & & 19.01.2013 \\
\hline 070/2013 & $\begin{array}{l}\text { SQ2, Feature 2, 2-5.5m to E, from gravel } \\
\text { deposit }\end{array}$ & pottery & $\sim 20 \mathrm{~cm}$ & & 23.01 .2013 \\
\hline $044 / 2013$ & $\begin{array}{l}\text { SQ2, Feature } 2,3-6.5 \mathrm{~m} \text { to E, cleaning of } \\
\text { wall W-E }\end{array}$ & pottery & $20-30 \mathrm{~cm}$ & & 19.01.2013 \\
\hline $034 / 2013$ & SQ2, Feature $2,0-6.5 \mathrm{~m}$ to $\mathrm{E}$ & $\begin{array}{l}\text { pottery, stone and bones: pottery ( } 4 \text { baskets), among them re- } \\
\text { used sherd ( } 1 \text { piece, } 18^{\text {th }} \text { Dynasty, pottery), mould/animal } \\
\text { figurine ( } 1 \text { fragment, clay); stone artefact ( } 1 \text { fragment, } \\
\text { quartz?); bones (some pieces) }\end{array}$ & $20-30 \mathrm{~cm}$ & $\begin{array}{l}\text { SAV1E } 0084 \text { (re-used sherd), SAV1E } 0087 \\
\text { (mould), SAV1E } 0088 \text { (stone artefact) }\end{array}$ & 16.01 .2013 \\
\hline $036 / 2013$ & SQ2, Feature 2, cleaning from Feature 14 & pottery (30 pieces) & $20-30 \mathrm{~cm}$ & & 17.01.2013 \\
\hline $042 / 2013$ & SQ2, Feature 2, cleaning S of Feature 14 & $\begin{array}{l}\text { pottery, among it re-used sherd ( } 1 \text { piece, } 18^{\text {th }} \text { Dynasty, } \\
\text { pottery); bone ( } 1 \text { piece) }\end{array}$ & $20-30 \mathrm{~cm}$ & SAV1E 0089 & 19.01.2013 \\
\hline $084 / 2013$ & SQ2, NE-corner above Feature 3 & pottery & $0-10 \mathrm{~cm}$ & & 26.01 .2013 \\
\hline $060 / 2013$ & SQ2, Feature 3 & pottery & $30-40 \mathrm{~cm}$ & & 22.01 .2013 \\
\hline $071 / 2013$ & SQ2, Feature 3, NE-corner, cleaning & pottery & $\begin{array}{l}\sim 30- \\
40 \mathrm{~cm}\end{array}$ & & 23.01 .2013 \\
\hline $159 / 2013$ & $\begin{array}{l}\text { SQ2, cleaning N-baulk in NE-corner, } \\
\text { Feature 3, at wall }\end{array}$ & $\begin{array}{l}\text { pottery, among it re-used sherd ( } 1 \text { piece with hole, pottery, Post- } \\
\text { New Kingdom), scraper ( } 1 \text { re-used } 18^{\text {th }} \text { Dynasty sherd, pottery) }\end{array}$ & $0-30 \mathrm{~cm}$ & $\begin{array}{l}\text { SAV1E } 0127 \text { (re-used sherd), SAV1E } 2901 \\
\text { (scraper) }\end{array}$ & 30.01 .2013 \\
\hline $160 / 2013$ & SQ2, cleaning N-baulk, W of Feature 3 & pottery & $0-30 \mathrm{~cm}$ & & 30.01 .2013 \\
\hline $063 / 2013$ & SQ2, Feature 4, S of Feature 16, E 3.5m & pottery & $30-40 \mathrm{~cm}$ & & 22.01 .2013 \\
\hline $066 / 2013$ & SQ2, Feature 4, SE-corner, up to $6 \mathrm{~m}$ to $\mathrm{E}$ & pottery & $30-40 \mathrm{~cm}$ & & 22.01 .2013 \\
\hline $059 / 2013$ & SQ2, Feature 4, W of Feature 3 & pottery & $30-40 \mathrm{~cm}$ & & 22.01 .2013 \\
\hline $065 / 2013$ & $\mathrm{SQ} 2$, Feature $4, \mathrm{~W}$ part up to $3.5 \mathrm{~m}$ to $\mathrm{E}$ & pottery & $30-40 \mathrm{~cm}$ & & 22.01 .2013 \\
\hline $328 / 2013$ & $\begin{array}{l}\text { SQ2, Feature } 6,0-2.5 \mathrm{~m} \text { to E, cleaning of } \\
\text { mud brick debris }\end{array}$ & pottery & $\begin{array}{l}\sim 40- \\
45 \mathrm{~cm}\end{array}$ & & 13.02 .2013 \\
\hline $145 / 2013$ & SQ2, W-baulk at wall Feature 16 & pottery & $0-40 \mathrm{~cm}$ & & 29.01 .2013 \\
\hline $038 / 2013$ & SQ2, Feature 14, cleaning & pottery, sherds; bones (some pieces) & & & 19.01.2013 \\
\hline $040 / 2013$ & SQ2, Feature 14, NW-corner & pottery: beaker in situ, complete & & & 19.01 .2013 \\
\hline $047 / 2013$ & SQ2, Feature 14, SE-part & pottery: beer jar (1 base, pottery) & & & 20.01 .2013 \\
\hline $072 / 2013$ & SQ2, S of Feature 14 & pottery & $30-40 \mathrm{~cm}$ & & 23.01 .2013 \\
\hline 039/2013 & $\begin{array}{l}\text { SQ2, Feature } 14 \text { (complete vessel in SW- } \\
\text { corner of Feature 14) }\end{array}$ & pottery: vessel, complete & & & 19.01.2013 \\
\hline
\end{tabular}




\begin{tabular}{|c|c|c|c|c|c|}
\hline $162 / 2013$ & SQ2, W-baulk, Feature 15 & pottery & $0-40 \mathrm{~cm}$ & & 30.01 .2013 \\
\hline $142 / 2013$ & $\begin{array}{l}\text { SQ2, Feature 15, from collapsed bricks in } \\
\text { W }\end{array}$ & pottery, among it stopper (1 fragment, mud) & $\begin{array}{l}\sim 20- \\
30 \mathrm{~cm}\end{array}$ & SAV1E 0123 & 29.01.2013 \\
\hline $132 / 2013$ & SQ2, Feature 15, sandy pit in NW-corner & pottery; bones (some pieces) & $\begin{array}{c}30- \\
35 / 40 \mathrm{~cm}\end{array}$ & & 28.01.2013 \\
\hline $062 / 2013$ & SQ2, Feature 15, cleaning of structure & pottery & $30-40 \mathrm{~cm}$ & & 22.01 .2013 \\
\hline $067 / 2013$ & $\mathrm{SQ} 2$, Feature $16>3 \mathrm{~m}$ to $\mathrm{E}$ & pottery & $30-40 \mathrm{~cm}$ & & 23.01 .2013 \\
\hline $061 / 2013$ & $\mathrm{SQ} 2$, Feature $16,10-6.5 \mathrm{~m}$, up to $6.5 \mathrm{~m}$ to $\mathrm{E}$ & pottery & $30-40 \mathrm{~cm}$ & & 22.01 .2013 \\
\hline $088 / 2013$ & $\begin{array}{l}\text { SQ2, wall Feature 16, E-end, from } \\
\text { collapsed brick }\end{array}$ & pottery & $\sim 30 \mathrm{~cm}$ & & 26.01 .2013 \\
\hline $145 / 2013$ & SQ2, W-baulk at wall Feature 16 & pottery & $0-40 \mathrm{~cm}$ & & 29.01 .2013 \\
\hline $144 / 2013$ & SQ2, W-baulk, N of Feature 16 & pottery & $0-40 \mathrm{~cm}$ & & 29.01 .2013 \\
\hline $064 / 2013$ & SQ2, Feature 17, pit & pottery & $30-40 \mathrm{~cm}$ & & 22.01 .2013 \\
\hline $073 / 2013$ & SQ2, Feature 18, from mud brick remains & pottery & $\sim 20 \mathrm{~cm}$ & & 24.01 .2013 \\
\hline $185 / 2013$ & $\begin{array}{l}\text { SQ2, Feature 20, between bricks and in } \\
\text { southern profile }\end{array}$ & pottery & $\sim 20 \mathrm{~cm}$ & & 02.02 .2013 \\
\hline $082 / 2013$ & SQ2A, surface & $\begin{array}{l}\text { pottery ( } 3 \text { baskets), among them re-used sherd ( } 1 \text { piece, } \\
\text { glazed on one side, Islamic?), clay object ( } 1 \text { fragment, clay) }\end{array}$ & surface & $\begin{array}{l}\text { SAV1E } 0107 \text { (re-used sherd), } \\
\text { SAV1E } 0108 \text { (clay object) }\end{array}$ & 26.01 .2013 \\
\hline $201 / 2013$ & SQ2A, $0-2.5 \mathrm{~m}$ to E, S-corner & pottery & $\begin{array}{l}\sim 10- \\
15 \mathrm{~cm}\end{array}$ & & 03.02 .2013 \\
\hline $096 / 2013$ & $\mathrm{SQ} 2 \mathrm{~A}, 0-3 \mathrm{~m}$ from $\mathrm{N}$ & pottery & $\sim 10 \mathrm{~cm}$ & & 26.01 .2013 \\
\hline $086 / 2013$ & $\mathrm{SQ} 2 \mathrm{~A}, 0-4 \mathrm{~m}$ from $\mathrm{N}$ & pottery & $0-50 \mathrm{~cm}$ & & 26.01 .2013 \\
\hline $119 / 2013$ & $\mathrm{SQ} 2 \mathrm{~A}, 1 \mathrm{~m}$ from $\mathrm{N}$ & pottery & $\sim 10 \mathrm{~cm}$ & & 28.01 .2013 \\
\hline $120 / 2013$ & SQ2A, 1-2.5m from N, from sandy debris & pottery & $\sim 10 \mathrm{~cm}$ & & 28.01 .2013 \\
\hline $121 / 2013$ & $\begin{array}{l}\text { SQ2A, } 2.5-4 \mathrm{~m} \text { from N, debris from } \\
\text { Features } 16 \text { and } 3\end{array}$ & pottery & $\sim 10 \mathrm{~cm}$ & & 28.01 .2013 \\
\hline $097 / 2013$ & SQ2A, 3-5m from N, sandy area & pottery & $\sim 10 \mathrm{~cm}$ & & 26.01 .2013 \\
\hline $122 / 2013$ & $\begin{array}{l}\text { SQ2A, } 4.5-6 \mathrm{~m} \text { from } \mathrm{N} \text {, pebbles area and } \\
\text { mud brick debris }\end{array}$ & pottery & $\sim 10 \mathrm{~cm}$ & & 28.01 .2013 \\
\hline $087 / 2013$ & $\mathrm{SQ} 2 \mathrm{~A}, 4-6 \mathrm{~m}$ from $\mathrm{N}$ & pottery & $0-50 \mathrm{~cm}$ & & 26.01 .2013 \\
\hline $098 / 2013$ & $\mathrm{SQ} 2 \mathrm{~A}, 5-6 \mathrm{~m}$ from $\mathrm{N}$ & pottery & $\sim 10 \mathrm{~cm}$ & & 27.01 .2013 \\
\hline
\end{tabular}




\begin{tabular}{|c|c|c|c|c|c|}
\hline $298 / 2013$ & SQ2A, cleaning $0-3 \mathrm{~m}$ to $\mathrm{S}$ along $\mathrm{E}$ border & pottery, among it re-used sherd ( 1 piece, $18^{\text {th }}$ Dynasty) & $\sim 30-35 \mathrm{~cm}$ & SAV1E 0207 & 10.02 .2013 \\
\hline $297 / 2013$ & $\begin{array}{l}\text { SQ2A, cleaning } 4.5-6 \mathrm{~m} \text { to S-end of mud } \\
\text { debris }\end{array}$ & pottery & $\begin{array}{c}\text { below } \\
\text { Planum } 3 \text {, } \\
\sim 25 \mathrm{~cm}\end{array}$ & & 10.02 .2013 \\
\hline $296 / 2013$ & $\begin{array}{l}\text { SQ2A, cleaning Planum 3, E-edge of pit, } \\
0-4 m \text { to } S\end{array}$ & pottery & $\begin{array}{l}\sim 30 \mathrm{~cm} \\
\text { below } \\
\text { Planum } 3\end{array}$ & & 10.02 .2013 \\
\hline $240 / 2013$ & SQ2A, cleaning SE-corner & pottery & $\sim 20-25 \mathrm{~cm}$ & & 05.02 .2013 \\
\hline $239 / 2013$ & SQ2A, E side in sandy depression & pottery & $\sim 10-15 \mathrm{~cm}$ & & 05.02 .2013 \\
\hline $241 / 2013$ & SQ2A, E side, in filling of sandy pit/ridge & pottery, among it figurine (1 fragment, animal figurine?, clay) & $\sim 15-20 \mathrm{~cm}$ & SAV1E 0160 & 05.02 .2013 \\
\hline $242 / 2013$ & SQ2A, E side, in sandy pit, above muna & pottery; grindstone? (1 fragment, sandstone) & $\sim 20 \mathrm{~cm}$ & SAV1E 0171 (grindstone) & 05.02 .2013 \\
\hline $238 / 2013$ & SQ2A, SE-corner, sandy area & pottery; bone fragments & $\sim 20 \mathrm{~cm}$ & & 05.02 .2013 \\
\hline $152 / 2013$ & SQ2A, S-half, mud brick debris & pottery & $\sim 10 \mathrm{~cm}$ & & 29.01 .2013 \\
\hline $323 / 2013$ & $\begin{array}{l}\text { SQ2A, E side up to } 3 \mathrm{~m} \mathrm{~S} \text { below Planum } 5 \text {, } \\
\text { from E pit at bottom of Feature } 3\end{array}$ & pottery & $\sim 25 \mathrm{~cm}$ & & 12.02 .2013 \\
\hline $266 / 2013$ & $\begin{array}{l}\text { SQ2A, along E-ridge, from sandy pit above } \\
\text { muna }\end{array}$ & pottery & $\sim 20 \mathrm{~cm}$ & & 07.02 .2013 \\
\hline $202 / 2013$ & SQ2A, Feature 3, cleaning & pottery & $\sim 10-15 \mathrm{~cm}$ & & 03.02 .2013 \\
\hline $236 / 2013$ & SQ2A, Feature $3,0-3 \mathrm{~m}$ to $\mathrm{S}$ & pottery & $\sim 20 \mathrm{~cm}$ & & 05.02 .2013 \\
\hline $325 / 2013$ & $\begin{array}{l}\text { SQ2A, Feature 3, cleaning between } \\
1-3 \mathrm{~m} \text { to } \mathrm{S}\end{array}$ & pottery & $\begin{array}{c}\sim 20 \mathrm{~cm} \\
\text { below } \\
\text { Planum } 5\end{array}$ & & 12.02 .2013 \\
\hline $324 / 2013$ & $\begin{array}{l}\text { SQ2A, Feature 3, cleaning between brick } \\
\text { and debris, 3-6m to } \mathrm{S}\end{array}$ & $\begin{array}{l}\text { pottery; installation?device for some production? (1 piece, } \\
\text { unspecified material) }\end{array}$ & $\begin{array}{c}\sim 25 \mathrm{~cm} \\
\text { below } \\
\text { Planum } 5\end{array}$ & $\begin{array}{l}\text { SAV1E } 0233 \text { (installation? device for some } \\
\text { production?) }\end{array}$ & 12.02 .2013 \\
\hline $124 / 2013$ & $\begin{array}{l}\text { SQ2A, debris from Feature } 3 \text {, } \\
1-1.5 \mathrm{~m} \text { from } \mathrm{N}\end{array}$ & pottery & $\sim 10-15 \mathrm{~cm}$ & & 28.01 .2013 \\
\hline $125 / 2013$ & $\begin{array}{l}\mathrm{SQ} 2 \mathrm{~A} \text {, debris from Feature } 3, \sim 3-4.5 \mathrm{~m} \\
\text { from } \mathrm{N}\end{array}$ & pottery & $\sim 10-15 \mathrm{~cm}$ & & 28.01 .2013 \\
\hline $148 / 2013$ & SQ2A, N-half of debris, Feature 3 & pottery & $\sim 10-15 \mathrm{~cm}$ & & 29.01.2013 \\
\hline $118 / 2013$ & $\begin{array}{l}\text { SQ2A, NW-corner debris, Feature 3, see } \\
\text { Planum 1, from mud brick debris }\end{array}$ & pottery sherds & $\sim 0-10 \mathrm{~cm}$ & & 28.01 .2013 \\
\hline $123 / 2013$ & $\begin{array}{l}\text { SQ2A, c. } 5 \mathrm{~m} \text { from N, Feature } 3 \text { and } \\
\text { Feature } 16\end{array}$ & pottery (in debris, sherds) & $\sim 10 \mathrm{~cm}$ & & 28.01 .2013 \\
\hline $126 / 2013$ & SQ2A, debris from Feature 3 and & pottery & $\sim 10-15 \mathrm{~cm}$ & & 28.01 .2013 \\
\hline
\end{tabular}




\begin{tabular}{|c|c|c|c|c|c|}
\hline & Feature $16, \sim 4.5-5.5 \mathrm{~m}$ from $\mathrm{N}$ & & & & \\
\hline $150 / 2013$ & SQ2A, N-half of pebbles, E of Feature 3 & pottery & $\sim 10-15 \mathrm{~cm}$ & & 29.01 .2013 \\
\hline $149 / 2013$ & $\begin{array}{l}\text { SQ2A, S-half of debris Feature } 3 \text { and } \\
\text { Feature } 16\end{array}$ & pottery & $\sim 10-15 \mathrm{~cm}$ & & 29.01 .2013 \\
\hline $237 / 2013$ & SQ2A, Feature 3 and Feature $16,3-6 \mathrm{~m}$ to $\mathrm{S}$ & pottery and stone: artefact? (1 fragment, sandstone) & $\sim 20 \mathrm{~cm}$ & SAV1E 0162 (artefact?) & 05.02 .2013 \\
\hline $265 / 2013$ & $\begin{array}{l}\text { SQ2A, SE-corner, cleaning remains of } \\
\text { broken bricks, Feature 3/Feature } 16\end{array}$ & pottery & $\sim 20 \mathrm{~cm}$ & & 07.02 .2013 \\
\hline $256 / 2013$ & SQ2B, $0-1 \mathrm{~m}$ to E, S of Feature 20 & $\begin{array}{l}\text { pottery, among it re-used sherd (1 piece, Post New-Kingdom, } \\
\text { pottery); charcoal (1 bag) }\end{array}$ & $20-25 \mathrm{~cm}$ & SAV1E 0182 (re-used sherd) & 06.02 .2013 \\
\hline $253 / 2013$ & $\begin{array}{l}\text { SQ2B, } 0-1 \mathrm{~m} \text { to E, Feature } 20 \text { and } \\
\text { brick debris }\end{array}$ & pottery & $\sim 20 \mathrm{~cm}$ & & 06.02 .2013 \\
\hline $199 / 2013$ & SQ2B, $0-1 \mathrm{~m}$ to $\mathrm{E}, \mathrm{S}$ of Feature 20 & pottery & $5-10 \mathrm{~cm}$ & & 03.02 .2013 \\
\hline $186 / 2013$ & SQ2B, $0-1 \mathrm{~m}$ to $\mathrm{E}, \mathrm{S}$ of Feature 20 & pottery; slag (1 fragment) & $0-10 \mathrm{~cm}$ & SAV1E 0137 (slag) & 02.02 .2013 \\
\hline $230 / 2013$ & SQ2B, $0-1 \mathrm{~m}$ to $\mathrm{E}, \mathrm{S}$ of Feature 20 & pottery, among it lid (1 fragment, Post-New Kingdom, mud) & $\sim 10-15 \mathrm{~cm}$ & SAV1E 0150 & 04.02 .2013 \\
\hline $338 / 2013$ & $\begin{array}{l}\text { SQ2B, } 0-2.5 \mathrm{~m} \text { to } \mathrm{E} \text {, last cleaning before } \\
\text { Planum } 6\end{array}$ & $\begin{array}{l}\text { pottery, among it re-used sherds ( } 2 \text { pieces, } 118^{\text {th }} \text { Dynasty, } \\
1 \text { Christian, pottery) }\end{array}$ & $\begin{array}{c}\sim 50- \\
55 \mathrm{~cm}, \text { see } \\
\text { Planum } 6\end{array}$ & SAV1E 0257, SAV1E 0261 (re-used sherds) & 14.02 .2013 \\
\hline $310 / 2013$ & SQ2B, $0-2 m$ to E, debris below Planum 4 & $\begin{array}{l}\text { pottery ( } 2 \text { baskets), among them lid? ( } 1 \text { disc-shaped object, } \\
\text { Christian, pottery), mud object (1 piece), stopper ( } 1 \text { piece, mud) }\end{array}$ & $\sim 40-45 \mathrm{~cm}$ & $\begin{array}{l}\text { SAV1E } 0214 \text { (lid?), SAV1E } 0220 \text { (mud } \\
\text { object), SAV1E } 0235 \text { (stopper) }\end{array}$ & 11.02 .2013 \\
\hline $334 / 2013$ & SQ2B, $0-3 \mathrm{~m}$ to E, below Planum 5 & pottery & $\begin{array}{c}\text { below } \\
\text { Planum } 5\end{array}$ & & 13.02 .2013 \\
\hline $371 / 2013$ & $\begin{array}{l}\text { SQ2B, 0-6m to E, cleaning before Planum } \\
\text { 7/SQ2B, 3-6m to E, cleaning debris }\end{array}$ & $\begin{array}{l}\text { pottery, rope and bones: pottery ( } 2 \text { baskets } 26.02 ., 0-3 \mathrm{~m}) \text {, among } \\
\text { them re-used sherd ( } 1 \text { piece, Post-New Kingdom, pottery), re- } \\
\text { used sherds or scrapers }\left(2 \text { pieces, each } 18^{\text {th }} \text { Dynasty, pottery), }\right. \\
\text { pottery ( } 3 \text { baskets } 27.02 ., 3-6.5 \mathrm{~m}) \text {, among them glazed vessel } \\
\text { ( } 1 \text { fragment, glazed, Ottoman?); rope ( } 1 \text { small fragment, organic } \\
\text { fibre); bones }\end{array}$ & $\begin{array}{c}\text { below } \\
\text { Planum } 6\end{array}$ & $\begin{array}{l}\text { SAV1E } 0300 \text { (re-used sherd), SAV1E 0302, } \\
\text { SAV1E } 0303 \text { (re-used sherds or scrapers), } \\
\text { SAV1E } 0298 \text { (glazed vessel), SAV1E } 0299 \\
\text { (rope) }\end{array}$ & $26 . / 27.02 .2013$ \\
\hline $254 / 2013$ & SQ2B, $1-2.5 \mathrm{~m}$ to $\mathrm{E}$ debris & pottery & $\sim 20 \mathrm{~cm}$ & & 06.02 .2013 \\
\hline $257 / 2013$ & SQ2B, $1-2.5 \mathrm{~m}$ to $\mathrm{E}$ debris & pottery & $20-25 \mathrm{~cm}$ & & 06.02 .2013 \\
\hline $291 / 2013$ & SQ2B, $1-3.5 \mathrm{~m}$ to E, before Planum 4 debris & pottery ( 2 baskets), bones & $\sim 30-40 \mathrm{~cm}$ & & 09.02 .2013 \\
\hline $285 / 2013$ & SQ2B, $1-3 m$ to E debris & $\begin{array}{l}\text { pottery, stone, bones and charcoal: pottery ( } 50 \text { baskets), among } \\
\text { them re-used sherd ( } 1 \text { piece, } 18^{\text {th }} \text { Dynasty?, pottery); pounder ( } 1 \\
\text { piece, quartz), bones (some fragments); charcoal ( } 1 \text { bag) }\end{array}$ & $\sim 20-40 \mathrm{~cm}$ & $\begin{array}{l}\text { SAV1E } 0190 \text { (re-used sherd), SAV1E } 0196 \\
\text { (pounder) }\end{array}$ & 09.02 .2013 \\
\hline $229 / 2013$ & $\begin{array}{l}\text { SQ2B, } 1-4 \mathrm{~m} \text { to } \mathrm{E} \text {, from area with mud } \\
\text { bricks }\end{array}$ & pottery & $\sim 10-15 \mathrm{~cm}$ & & 04.02 .2013 \\
\hline
\end{tabular}




\begin{tabular}{|c|c|c|c|c|c|}
\hline $200 / 2013$ & SQ2B, $1-5 \mathrm{~m}$ to $\mathrm{E}$ & pottery & $5-10 \mathrm{~cm}$ & & 03.02 .2013 \\
\hline $187 / 2013$ & SQ2B, $1-5 \mathrm{~m}$ to $\mathrm{E}$ & $\begin{array}{l}\text { pottery ( } 2 \text { baskets), among them re-used sherd (1 disc-shaped } \\
\text { piece with hole, Christian, pottery) }\end{array}$ & $0-10 \mathrm{~cm}$ & SAV1E 0135 & 02.02 .2013 \\
\hline $284 / 2013$ & SQ2B, $1 \mathrm{~m}$ to E, S of Feature 20 & pottery & $\sim 25-30 \mathrm{~cm}$ & & 09.02 .2013 \\
\hline $290 / 2013$ & SQ2B, $1 \mathrm{~m}$ to E, debris $\mathrm{S}$ of Feature 20 & $\begin{array}{l}\text { pottery, slag and stone: pottery; hammer (1 piece, quartzite); slag } \\
\text { ( } 2 \text { pieces) }\end{array}$ & $\begin{array}{c}\sim 30- \\
40 \mathrm{~cm}, \\
\text { before } \\
\text { Planum } 4\end{array}$ & $\begin{array}{l}\text { SAV1E } 0194 \text { (hammer), SAV1E 0198, } \\
\text { SAV1E } 0199 \text { (slag) }\end{array}$ & 09.02 .2013 \\
\hline $258 / 2013$ & SQ2B, $2.5-4.5 \mathrm{~m}$ to E, debris & pottery & $20-25 \mathrm{~cm}$ & & 06.02 .2013 \\
\hline $255 / 2013$ & $\mathrm{SQ} 2 \mathrm{~B}, 2.5-4.5 \mathrm{~m}$ to E, undefined debris & $\begin{array}{l}\text { pottery, among it palette ( } 1 \text { re-used } 18^{\text {th }} \text { Dynasty base sherd, } \\
\text { pottery) }\end{array}$ & $\sim 20 \mathrm{~cm}$ & SAV1E 2834 & 06.02 .2013 \\
\hline $339 / 2013$ & $\begin{array}{l}\text { SQ2B, } 2.5-5 \mathrm{~m} \text { to E, cleaning around } \\
\text { Feature } 28\end{array}$ & $\begin{array}{l}\text { pottery, among it re-used sherds ( } 3 \text { pieces [ } 11 \text { insecure], } 2 \text { Post- } \\
\text { New Kingdom, } 1 \text { Christian, each pottery) }\end{array}$ & $\begin{array}{c}\text { before } \\
\text { Planum } 6 \\
\sim 50-55 \mathrm{~cm}\end{array}$ & $\begin{array}{l}\text { SAV1E 0249, SAV1E 0251, SAV1E } 0260 \\
\text { (re-used sherds) }\end{array}$ & 14.02 .2013 \\
\hline $311 / 2013$ & SQ2B, 2-5m to E, sandy pit and debris & pottery; slag (1 piece) & $\sim 45-50 \mathrm{~cm}$ & SAV1E 0221 (slag) & 11.02 .2013 \\
\hline $288 / 2013$ & $\begin{array}{l}\text { SQ2B, 3.5-5m E, debris and pit of Feature } \\
24\end{array}$ & pottery & $\sim 30-35 \mathrm{~cm}$ & & 09.02 .2013 \\
\hline $335 / 2013$ & SQ2B, 3-5m, cleaning Feature 28 & pottery & \begin{tabular}{|c|} 
below \\
Planum 5
\end{tabular} & & 13.02 .2013 \\
\hline $262 / 2013$ & $\begin{array}{l}\text { SQ2B, } 4-5 m \text { to E, sandy area and some } \\
\text { bricks }\end{array}$ & $\begin{array}{l}\text { pottery and charcoal: pottery ( } 4 \text { baskets), garbage pit, among it } \\
\text { architecture/window grille ( } 1 \text { fragment, pottery); charcoal (1 bag) }\end{array}$ & $\sim 20-40 \mathrm{~cm}$ & SAV1E 0188 & 06.02 .2013 \\
\hline $228 / 2013$ & SQ2B, 4-5m to E, sandy pit & pottery & $\sim 20 \mathrm{~cm}$ & & 04.02 .2013 \\
\hline $249 / 2013$ & $\begin{array}{l}\text { SQ2B, } 4-5 \mathrm{~m} \text { to E, Feature } 24 \text { and muddy } \\
\text { remains }\end{array}$ & pottery & $\sim 25 \mathrm{~cm}$ & & 06.02 .2013 \\
\hline $294 / 2013$ & SQ2B, 4-7.5m to E, further cleaning & pottery & $\sim 30-35 \mathrm{~cm}$ & & 09.02 .2013 \\
\hline $374 / 2013$ & $\begin{array}{l}\text { SQ2B, 5-6.5m to E, cleaning debris } \\
\text { (Feature 25) }\end{array}$ & pottery; bones (some fragments); charcoal (1 bag) & \begin{tabular}{|c|} 
below \\
Planum 6
\end{tabular} & & 28.02 .2013 \\
\hline $341 / 2013$ & SQ2B, $5-6.5 \mathrm{~m}$ to $\mathrm{E}$ & $\begin{array}{l}\text { pottery, among it lid ( } 1 \text { re-used } 18^{\text {th }} \text { Dynasty sherd, pottery), } \\
\text { crucible? ( } 1 \text { fragment, clay) }\end{array}$ & \begin{tabular}{c|} 
before \\
Planum 6
\end{tabular} & SAV1E 0256 (lid), SAV1E 0262 (crucible?) & 14.02 .2013 \\
\hline $196 / 2013$ & SQ2B, 5-6m to E & pottery & $5-10 \mathrm{~cm}$ & & 03.02 .2013 \\
\hline $191 / 2013$ & SQ2B, 5-6m to E & pottery; bone & $0-10 \mathrm{~cm}$ & & 02.02 .2013 \\
\hline $313 / 2013$ & $\begin{array}{l}\text { SQ2B, c. } 5-7 \mathrm{~m} \text { to E, sandy pit, Feature } 25+ \\
\text { extension debris, below Planum } 4\end{array}$ & $\begin{array}{l}\text { pottery, among it re-used sherds ( } 2 \text { pieces, } 118^{\text {th }} \text { Dynasty, } 1 \text { Post- } \\
\text { New Kingdom, pottery) }\end{array}$ & $\sim 40-45 \mathrm{~cm}$ & SAV1E 0218, SAV1E 0219 (re-used sherds) & 11.02 .2013 \\
\hline $342 / 2013$ & $\begin{array}{l}\text { SQ2B, } 6.5-7.8 \mathrm{~m} \text { to E, around Feature } 27 \\
\text { area }\end{array}$ & pottery & \begin{tabular}{|c|} 
before \\
Planum 6
\end{tabular} & & 14.02 .2013 \\
\hline
\end{tabular}




\begin{tabular}{|c|c|c|c|c|c|}
\hline & & & $\begin{array}{c}\text { below } \\
\text { Planum } 5\end{array}$ & & \\
\hline $281 / 2013$ & SQ2B, $6.5-8 \mathrm{~m}$ to $\mathrm{E}$, debris & pottery; bones (some fragments) & $\sim 25 \mathrm{~cm}$ & & 09.02 .2013 \\
\hline $197 / 2013$ & SQ2B, $6-8 \mathrm{~m}$ to $\mathrm{E}$ & pottery & $5-10 \mathrm{~cm}$ & & 03.02 .2013 \\
\hline $192 / 2013$ & SQ2B, 6-8m to E, above brick debris & pottery, among it re-used sherd (1 piece, pottery) & $0-10 \mathrm{~cm}$ & SAV1E 0134 & 02.02 .2013 \\
\hline $363 / 2013$ & $\begin{array}{l}\text { SQ2B, } 6-8 m \text { to } E, 1-2 m \text { to } \mathrm{S} \text {, mud brick } \\
\text { debris }\end{array}$ & $\begin{array}{l}\text { pottery, slag, glass and bead?/quartz artefact?: pottery } \\
\text { ( } 3 \text { baskets); slag ( } 1 \text { fragment), bead?artefact? (1 fragment, quartz, } \\
\text { bluish pigment, painted?, recent?/sub-recent? or Ottoman?); glass } \\
\text { vessel (1 fragment, recent?/sub-recent? or Ottoman?) }\end{array}$ & $\begin{array}{l}\text { below } \\
\text { Planum } 6\end{array}$ & $\begin{array}{l}\text { SAV1E } 0281 \text { (slag), SAV1E } 0287 \text { (bead? } \\
\text { artefact?) SAV1E } 0288 \text { (glass vessel) }\end{array}$ & 18.02.2013 \\
\hline $326 / 2013$ & $\begin{array}{l}\text { SQ2B, 7-8m to E, debris around } \\
\text { Feature 27, before Planum } 5\end{array}$ & $\begin{array}{l}\text { pottery, among it re-used sherds ( } 2 \text { pieces, each Post New- } \\
\text { Kingdom) }\end{array}$ & $\begin{array}{l}\text { below } \\
\text { Planum } 4 \text {, } \\
\sim 20 \mathrm{~cm}\end{array}$ & SAV1E 0225, SAV1E 0226 (re-used sherds) & 12.02.2013 \\
\hline $362 / 2013$ & $\begin{array}{l}\mathrm{SQ} 2 \mathrm{~B}, 7-8 \mathrm{~m} \text { to } \mathrm{E} \text {, up to } 1 \mathrm{~m} \text { to } \mathrm{S}=\text { Feature } \\
27 \text {, cleaning area Feature } 27\end{array}$ & pottery; bones (some fragments) & $\begin{array}{l}\text { below } \\
\text { Planum } 6\end{array}$ & & 18.02 .2013 \\
\hline $293 / 2013$ & $\begin{array}{l}\text { SQ2B, } \sim 7-8.5 \mathrm{~m} \text { to } \mathrm{E} \text {, cleaning mud debris } \\
=\text { area } 10\end{array}$ & pottery & $\sim 30-35 \mathrm{~cm}$ & & 09.02.2013 \\
\hline $193 / 2013$ & SQ2B, $8-10 \mathrm{~m}$ to $\mathrm{E}$ & pottery & $0-10 \mathrm{~cm}$ & & 02.02 .2013 \\
\hline $198 / 2013$ & SQ2B, $8-10 \mathrm{~m}$ to $\mathrm{E}$ & pottery & $5-10 \mathrm{~cm}$ & & 03.02 .2013 \\
\hline $314 / 2013$ & $\begin{array}{l}\text { SQ2B, 8-10m to E, in area of Feature } 26 \text {, } \\
\text { below Planum } 4\end{array}$ & pottery & $\begin{array}{l}\sim 50 \mathrm{~cm}, \\
\text { below } \\
\text { Planum } 4\end{array}$ & & 11.02 .2013 \\
\hline $346 / 2013$ & $\mathrm{SQ} 2 \mathrm{~B}, \sim 8-10 \mathrm{~m}$ to $\mathrm{E}$, above sandy pit & pottery & $\begin{array}{l}\text { below } \\
\text { Planum } 5 \\
\text { before } \\
\text { Planum } 6\end{array}$ & & 14.02 .2013 \\
\hline $292 / 2013$ & $\begin{array}{l}\text { SQ2B, } \sim 8.5-10 \mathrm{~m} \text { to E, cleaning N-line of } \\
\text { SQ2B }\end{array}$ & pottery & $\sim 30 \mathrm{~cm}$ & & 09.02 .2013 \\
\hline $353 / 2013$ & SQ2B, SE-corner, sandy area & pottery & $\begin{array}{c}\text { before } \\
\text { Planum } 6\end{array}$ & & 18.02 .2013 \\
\hline $225 / 2013$ & SQ2B, SE-corner, sandy area from $\mathrm{N}$ & pottery from 11 on Planum 1; charcoal, bones (some fragments) & $20-25 \mathrm{~cm}$ & & 04.02 .2013 \\
\hline $382 / 2013$ & SQ2B, cleaning E-baulk, $0-2 \mathrm{~m}$ to $\mathrm{S}$ & pottery; charcoal (1 bag) & $0-30 \mathrm{~cm}$ & & 02.03 .2013 \\
\hline $351 / 2013$ & SQ2B, S-baulk, 1-6m to E, cleaning baulk & $\begin{array}{l}\text { pottery, stones and charcoal: pottery, among it re-used sherds } \\
\text { ( } 2 \text { pieces, } 118^{\text {th }} \text { Dynasty, } 1 \text { Post-New Kingdom); hammers or } \\
\text { pounders ( } 2 \text { pieces, each quartz), stone artefact ( } 1 \text { fragment, } \\
\text { unspecified stone); charcoal ( } 1 \text { bag) }\end{array}$ & $0-30 \mathrm{~cm}$ & $\begin{array}{l}\text { SAV1E 0270, SAV1E } 0271 \text { (re-used sherds), } \\
\text { SAV1E 0268, SAV1E } 0269 \text { (hammers or } \\
\text { pounders), SAV1E } 0272 \text { (stone artefact) }\end{array}$ & 18.02 .2013 \\
\hline $379 / 2013$ & SQ2B, S-baulk, 8-10m to E, cleaning baulk & pottery & $0-40 \mathrm{~cm}$ & & 02.03 .2013 \\
\hline
\end{tabular}




\begin{tabular}{|c|c|c|c|c|c|}
\hline $259 / 2013$ & $\begin{array}{l}\text { SQ2B, area } 10 \text { Planum } 1, \mathrm{~S} \text { of Feature } 14, \\
\text { mud brick debris, } 7-8 \mathrm{~m} \text { to } \mathrm{E}\end{array}$ & pottery & $\sim 20-30 \mathrm{~cm}$ & & 06.02 .2013 \\
\hline $226 / 2013$ & $\begin{array}{l}\text { SQ2B, mud brick area, S of Feature 14, } \\
\text { from area } 10 \text { on Planum } 1\end{array}$ & $\begin{array}{l}\text { pottery, among it re-used sherd (1 disc-shaped piece with hole, } \\
\text { one half preserved, pottery) }\end{array}$ & $\sim 20-25 \mathrm{~cm}$ & SAV1E 0161 & 04.02 .2013 \\
\hline $231 / 2013$ & $\begin{array}{l}\text { SQ2B, mud brick debris, S of Feature } 14, \\
\text { area } 10 \text { in Planum } 1\end{array}$ & pottery & $15-20 \mathrm{~cm}$ & & 04.02 .2013 \\
\hline $247 / 2013$ & $\begin{array}{l}\text { SQ2B, mud brick remains, } S \text { of Feature } 14, \\
\text { area } 10 \text { in Planum } 2+1\end{array}$ & pottery & $\sim 20-25 \mathrm{~cm}$ & & 06.02 .2013 \\
\hline $289 / 2013$ & $\begin{array}{l}\text { SQ2B, cleaning around basket } 27 \text { in area } \\
10 \text {, mud brick }\end{array}$ & pottery & $\sim 30 \mathrm{~cm}$ & & 09.02 .2013 \\
\hline $316 / 2013$ & SQ2B, cleaning basket 27 & pottery & $\begin{array}{c}\text { see } \\
\text { Planum } 4\end{array}$ & & 11.02 .2013 \\
\hline $227 / 2013$ & $\begin{array}{l}\text { SQ2B, from sandy pit, } \mathrm{W} \text { of area } 10 \text { on } \\
\text { Planum } 1\end{array}$ & pottery & $\sim 20-25 \mathrm{~cm}$ & SAV1E 175 & 04.02 .2013 \\
\hline $188 / 2013$ & $\begin{array}{l}\text { SQ2B, Feature } 16,1-5 \mathrm{~m} \text { to } \mathrm{E} \text {, from } \\
\text { collapsed bricks }\end{array}$ & pottery & $\sim 30 \mathrm{~cm}$ & & 02.02 .2013 \\
\hline $280 / 2013$ & SQ2B, Feature $25,5-6.5 \mathrm{~m}$ to E, debris & $\begin{array}{l}\text { pottery, among it furniture?installation? ( } 2 \text { fragments, each } \\
\text { pottery) }\end{array}$ & $\sim 20-25 \mathrm{~cm}$ & $\begin{array}{l}\text { SAV1E 0191, SAV1E } 0192 \text { (furniture? } \\
\text { installation?) }\end{array}$ & 09.02 .2013 \\
\hline $248 / 2013$ & SQ2B, Feature 25, sandy filling & pottery & $\sim 25 \mathrm{~cm}$ & & 06.02 .2013 \\
\hline $245 / 2013$ & SQ2B, Feature 26, sandy & pottery & $\sim 25 \mathrm{~cm}$ & & 06.02 .2013 \\
\hline $366 / 2013$ & SQ2B, Feature 26 , cleaning $7.5-10 \mathrm{~m}$ to $\mathrm{E}$ & $\begin{array}{l}\text { pottery, slag and charcoal: pottery, among it re-used sherds } \\
\text { ( } 3 \text { pieces, } 118^{\text {th }} \text { Dynasty, } 1 \text { Post-New Kingdom, } 1 \text { Christian, } \\
\text { each pottery); among it slag ( } 1 \text { fragment); charcoal (1 piece) }\end{array}$ & $\begin{array}{l}\text { below } \\
\text { Planum } 6\end{array}$ & $\begin{array}{l}\text { SAV1E 0290, SAV1E 0291, SAV1E } 0292 \\
\text { (re-used sherds), SAV1E } 0289 \text { (slag) }\end{array}$ & 26.02.2013 \\
\hline $282 / 2013$ & SQ2B, Feature $26,8-10 \mathrm{~m}$ to $\mathrm{E}$ & pottery; slag (2 pieces) & $\sim 30-35 \mathrm{~cm}$ & SAV1E 0197, SAV1E 0201 (slag) & 09.02 .2013 \\
\hline $251 / 2013$ & SQ2B, N of Feature 26 , mud brick debris & pottery & $\sim 20-25 \mathrm{~cm}$ & & 06.02 .2013 \\
\hline $246 / 2013$ & SQ2B, S of Feature 26, mud brick remains & pottery & $\sim 25 \mathrm{~cm}$ & & 06.02 .2013 \\
\hline $252 / 2013$ & SQ2B, W of Feature 26, mud brick debris & pottery; pounder? (1 sandstone pebble) & \begin{tabular}{|}
$\sim 10-25 \mathrm{~cm}$ \\
(sloping)
\end{tabular} & SAV1E 0204 (pounder?) & 06.02 .2013 \\
\hline $337 / 2013$ & SQ2B, Feature 27 & pottery; bone (1 fragment) & $\begin{array}{c}\text { see Planum } \\
5\end{array}$ & & 13.02 .2013 \\
\hline $377 / 2013$ & SQ2B, cleaning S of Feature 27 & $\begin{array}{l}\text { pottery, among it re-used sherd/scraper? }\left(118^{\text {th }} \text { Dynasty dish }\right. \\
\text { sherd, pottery); bone fragments }\end{array}$ & \begin{tabular}{|c|} 
below \\
Planum 6
\end{tabular} & SAV1E 0316 (re-used sherd/scraper?) & 28.02 .2013 \\
\hline $383 / 2013$ & $\begin{array}{l}\text { SQ2B, cleaning SW-corner and around } \\
\text { Feature } 28 \text {, before Planum } 7\end{array}$ & pottery & $\begin{array}{c}\text { before } \\
\text { Planum } 7\end{array}$ & & 02.03 .2013 \\
\hline $384 / 2013$ & SQ2B, cleaning section, E of Feature 33 & pottery & $0-60 \mathrm{~cm}$ & & 05.03 .2013 \\
\hline $275 / 2013$ & $\begin{array}{l}\text { SQ1A, Feature 13/21, sandy filling, 3-4.5m } \\
\text { to E }\end{array}$ & pottery, re-used sherd (1 piece, Post-New Kingdom) & $\sim 35-45 \mathrm{~cm}$ & SAV1E 0180 & 07.02 .2013 \\
\hline
\end{tabular}




\begin{tabular}{|c|c|c|c|c|c|}
\hline $360 / 2013$ & SQ1A, $7 \mathrm{~m}$ to E, $\mathrm{N}$ of Feature 30 , in pit & pottery: rim sherd (1 piece) & \begin{tabular}{|c} 
below \\
Planum 6
\end{tabular} & & 18.02 .2013 \\
\hline $359 / 2013$ & $\begin{array}{l}\text { SQ1A, } 8.6 \mathrm{~m} \text { to E, at S-side of Feature } 30 \text { in } \\
\text { pit }\end{array}$ & pottery: rim sherd (1 piece) & \begin{tabular}{|c|} 
below \\
Feature 30
\end{tabular} & & 18.02 .2013 \\
\hline $002 / 2013$ & SQ1, Feature 1 & slag (1 piece) & surface & & 06.01 .2013 \\
\hline $007 / 2013$ & SQ2, Feature 2 & slag (some pieces) & surface & & 06.01 .2013 \\
\hline $085 / 2013$ & SQ1, N of SQ 1 surface dig $<2 \mathrm{~m}$ & stone: grindstone (1 fragment, sandstone) & surface & SAV1E 0105 & 26.01.2013 \\
\hline $010 / 2013$ & SQ1, Feature 1 & $\begin{array}{l}\text { stones: grindstones ( } 13 \text { pieces, each sandstone), pounders } \\
\text { ( } 4 \text { pieces, } 2 \text { sandstone, } 1 \text { quartzite, } 1 \text { quartz), hammers ( } 2 \text { pieces, } \\
1 \text { quartz, } 1 \text { quartzite), hammers or pounders ( } 3 \text { pieces, each } \\
\text { quartz), whetstone ( } 1 \text { piece, sandstone) }\end{array}$ & $0-10 \mathrm{~cm}$ & $\begin{array}{l}\text { SAV1E 0015,SAV1E 0016, SAV1E 0017, } \\
\text { SAV1E 0018, SAV1E 0019, SAV1E 0020, } \\
\text { SAV1E 0021, SAV1E 0022, SAV1E 0023, } \\
\text { SAV1E 0024, SAV1E 0025, SAV1E 0026, } \\
\text { SAV1E 0027 (grindstones), SAV1E 0028, } \\
\text { SAV1E 0029, SAV1E 0030, SAV1E 0031 } \\
\text { (pounders), SAV1E 0035, SAV1E 00330 } \\
\text { (hammers), SAV1E 003, SAV1E 0034, } \\
\text { SAV1E 0036 (hammers or pounders), } \\
\text { SAV1E } 0037 \text { (whetstone) }\end{array}$ & 07.01 .2013 \\
\hline $014 / 2013$ & SQ1, Feature 1, NW & $\begin{array}{l}\text { stones: pounders ( } 2 \text { pieces, } 1 \text { quartz, } 1 \text { schist), hammer and } \\
\text { pounder ( } 1 \text { piece, fossil wood), grindstone ( } 1 \text { piece, sandstone), } \\
\text { pivot stone ( } 1 \text { fragment, sandstone) }\end{array}$ & $10-20 \mathrm{~cm}$ & $\begin{array}{l}\text { SAV1E 0041, SAV1E } 0042 \text { (pounders), } \\
\text { SAV1E } 0056 \text { (hammer and pounder), SAV1E } \\
0057 \text { (grindstone), SAV1E } 0062 \text { (pivot stone) }\end{array}$ & 08.01 .2013 \\
\hline $028 / 2013$ & SQ1, Feature 1, NW & stone: pounder (1 piece, quartz) & $20-30 \mathrm{~cm}$ & SAV1E 0058 & 12.01 .2013 \\
\hline $024 / 2013$ & SQ1, Feature 1, SE & stone: pounder (1 piece, quartz) & $10-20 \mathrm{~cm}$ & SAV1E 0055 & 10.01 .2013 \\
\hline $020 / 2013$ & SQ1, Feature 1, SW & stone: token/gaming piece? (1 piece, rocky crystal) & $10-20 \mathrm{~cm}$ & SAV1E 0054 & 09.01 .2013 \\
\hline $214 / 2013$ & SQ1, Feature 6, from sandy filling & stones: hammers ( 2 pieces, 1 quartz, 1 quartzite?) & $\sim 50 \mathrm{~cm}$ & SAV1E 0144, SAV1E 0145 (hammers) & 03.02 .2013 \\
\hline $104 / 2013$ & $\begin{array}{l}\text { SQ1/2, baulk between SQ1/2, above } \\
\text { Feature } 15\end{array}$ & stone: grindstone (1 fragment, sandstone) & $\sim 20 \mathrm{~cm}$ & SAV1E 0111 & 27.01 .2013 \\
\hline $212 / 2013$ & $\begin{array}{l}\text { SQ1/2, baulk, NE-corner of Feature 2, } \\
\text { above Feature 3, see Planum } 2\end{array}$ & stone: hammer (1 piece, quartzite?) & $\sim 40 \mathrm{~cm}$ & SAV1E 0146 & 03.02 .2013 \\
\hline $171 / 2013$ & $\begin{array}{l}\text { SQ1A, } 7 \mathrm{~m} \text { to E, in sandy pit above } \\
\text { Feature } 3\end{array}$ & stone: pounder (1 piece, quartzite?) & $\sim 15 \mathrm{~cm}$ & SAV1E 0124 & 30.01 .2013 \\
\hline $127 / 2013$ & SQ1A, Feature $19,0-5 \mathrm{~m}$ to $\mathrm{E}$ & $\begin{array}{l}\text { stones, mud and charcoal: hammers ( } 2 \text { pieces, } 1 \text { schist, } \\
1 \text { sandstone), grindstone ( } 1 \text { fragment, sandstone), palette? } \\
\text { (1 piece, sandstone); mud object ( } 1 \text { piece, function unknown); } \\
\text { charcoal ( } 1 \text { bag) }\end{array}$ & surface & $\begin{array}{l}\text { SAV1E 0114, SAV1E } 0115 \text { (hammers), } \\
\text { SAV1E 0116 (grindstone), SAV1E } 0117 \\
\text { (palette?), SAV1E } 0113 \text { (mud object) }\end{array}$ & 28.01 .2013 \\
\hline $220 / 2013$ & $\begin{array}{l}\text { SQ1A, NW-corner, see Planum } 1 \text { between } \\
\text { collapsed mud bricks }\end{array}$ & stone: grindstone (1 fragment, sandstone) & $\sim 10-15 \mathrm{~cm}$ & SAV1E 0173 & 04.02 .2013 \\
\hline
\end{tabular}




\begin{tabular}{|c|c|c|c|c|c|}
\hline $235 / 2013$ & SQ1A-2B, up to $1 \mathrm{~m}$ to $\mathrm{W}$, surface cleaning & $\begin{array}{l}\text { stones: hammers ( } 3 \text { pieces, each quartz), pounders ( } 2 \text { pieces, } \\
1 \text { quartzite?, } 1 \text { quartzite), pounders? ( } 2 \text { pieces, } 1 \text { sandstone, } \\
1 \text { quartz), grindstone ( } 2 \text { pieces, each sandstone), artefact? } \\
\text { (1 piece, sandstone) }\end{array}$ & surface & $\begin{array}{l}\text { SAV1E 0151, SAV1E 0152, SAV1E } 0155 \\
\text { (hammers), SAV1E 0153, SAV1E } 0154 \\
\text { (pounders), SAV1E 0156, SAV1E } 0157 \\
\text { (pounders?), SAV1E 0158, SAV1E } 0159 \\
\text { (grindstones), SAV1E 0168 (artefact?) }\end{array}$ & 05.02 .2013 \\
\hline $180 / 2013$ & SQ2, surface, Feature 2 & stones: grindstones (2 fragments, each sandstone) & surface & SAV1E 0130, SAV1E 0131 grindstones) & 31.01 .2013 \\
\hline $008 / 2013$ & SQ2, Feature 2 & stones: grindstones (2 fragments, each sandstone) & surface & SAV1E 0013, SAV1E 0014 (grindstones) & 06.01 .2013 \\
\hline $021 / 2013$ & SQ2, Feature 2 & $\begin{array}{l}\text { stones: grindstones ( } 2 \text { pieces, } 1 \text { sandstone, } 1 \text { sandstone?), pivot } \\
\text { stone (1 fragment, sandstone) }\end{array}$ & $10-20 \mathrm{~cm}$ & $\begin{array}{l}\text { SAV1E 0049, SAV1E } 0073 \text { (grindstones), } \\
\text { SAV1E } 0072 \text { (pivot stone) }\end{array}$ & 09.01 .2013 \\
\hline $026 / 2013$ & SQ2, Feature 2 & stone: grindstone (1 piece, sandstone) & $10-20 \mathrm{~cm}$ & SAV1E 0059 & 12.01 .2013 \\
\hline $035 / 2013$ & SQ2, Feature 2 & stone: grindstone (1 fragment, sandstone) & $20-30 \mathrm{~cm}$ & SAV1E 0086 & 16.01 .2013 \\
\hline $069 / 2013$ & $\mathrm{SQ} 2$, Feature $2,5.5-6.5 \mathrm{~m}$ to $\mathrm{E}$ & $\begin{array}{l}\text { stone and pottery: pottery (associated with mud brick); } \\
\text { grindstone (1 fragment, unspecified stone) }\end{array}$ & $\sim 20 \mathrm{~cm}$ & SAV1E 0097 (grindstone) & 23.01 .2013 \\
\hline $184 / 2013$ & $\begin{array}{l}\text { SQ2, Feature } 16,1.5 \mathrm{~m} \text { to E, from collapsed } \\
\text { bricks }\end{array}$ & stone: grindstone (1 fragment, sandstone) & $\sim 30 \mathrm{~cm}$ & SAV1E 0139 & 02.02 .2013 \\
\hline $068 / 2013$ & $\begin{array}{l}\mathrm{SQ} 2 \text {, Feature } 16<3 \mathrm{~m} \text { to } \mathrm{E} \text {, from disturbed } \\
\text { area }\end{array}$ & $\begin{array}{l}\text { stone, pottery, bone and charcoal: stone artefact (1 piece, function } \\
\text { not specified, unspecified stone); pottery; bone (1 piece); } \\
\text { charcoal (1 piece) }\end{array}$ & $30-40 \mathrm{~cm}$ & SAV1E 0096 (stone artefact) & 23.01 .2013 \\
\hline $081 / 2013$ & SQ2A, surface & stone: pounder (1 piece, quartzite) & surface & SAV1E 0103 & 26.01 .2013 \\
\hline 095/2013 & $\begin{array}{l}\text { SQ2A, NW-corner, Feature 2, above debris } \\
\text { of Feature } 3\end{array}$ & stone: pounder (1 piece, unspecified stone) & $\sim 10 \mathrm{~cm}$ & SAV1E 0104 & 26.01.2013 \\
\hline $117 / 2013$ & SQ2A, NW-corner, above Feature 3 & stone: grindstone (1 fragment, sandstone) see Planum 1 & $\sim 5-10 \mathrm{~cm}$ & SAV1E 0118 & 28.01 .2013 \\
\hline $286 / 2013$ & $\mathrm{SQ} 2 \mathrm{~B}, 0.5 \mathrm{~m}$ to $\mathrm{E}, 1.5 \mathrm{~m}$ to $\mathrm{S}$ & stone vessel (1 fragment, unspecified stone) & $\sim 20 \mathrm{~cm}$ & SAV1E 0195 & 09.02 .2013 \\
\hline $287 / 2013$ & $\mathrm{SQ} 2 \mathrm{~B}, 1 \mathrm{~m}$ to $\mathrm{S}, 2 \mathrm{~m}$ to $\mathrm{E}$, debris & stone: pounder (1 piece, quartzite) & $\sim 30 \mathrm{~cm}$ & SAV1E 0193 & 09.02 .2013 \\
\hline $333 / 2013$ & $\mathrm{SQ} 2 \mathrm{~B}, 2.3 \mathrm{~m}$ to E, in debris, see Planum 5 & stones: grindstones (2 fragments, each sandstone) & $\begin{array}{l}\text { below } \\
\text { Planum } 5\end{array}$ & SAV1E 0237, SAV1E 0238 (grindstones) & 13.02 .2013 \\
\hline $190 / 2013$ & $\mathrm{SQ} 2 \mathrm{~B}, 2 \mathrm{~m}$ to $\mathrm{E}$ & stone: hammer (1 piece, quartz) & surface & SAV1E 0138 & 02.02 .2013 \\
\hline $373 / 2013$ & $\begin{array}{l}\text { SQ2B, 3-6m to E, cleaning debris (around } \\
\text { Feature 28) }\end{array}$ & $\begin{array}{l}\text { stones: hammer or pounder ( } 1 \text { piece, quartz), pounders ( } 2 \text { pieces, } \\
\text { each quartz), grindstones ( } 2 \text { pieces, } 1 \text { quartzite, } 1 \text { sandstone) }\end{array}$ & $\begin{array}{l}\text { below } \\
\text { Planum } 6\end{array}$ & $\begin{array}{l}\text { SAV1E } 0304 \text { (hammer or pounder), SAV1E } \\
\text { 0305, SAV1E } 0306 \text { (pounders), SAV1E 0307, } \\
\text { SAV1E } 0308 \text { (grindstones) }\end{array}$ & 27.02 .2013 \\
\hline $250 / 2013$ & SQ2B, $4-5 \mathrm{~m}$ to E, S of Feature 24 & stone: grindstone (1 piece, sandstone) & $\sim 25 \mathrm{~cm}$ & SAV1E 0174 & 06.02 .2013 \\
\hline $343 / 2013$ & $\mathrm{SQ} 2 \mathrm{~B}, 5 \mathrm{~m}$ to $\mathrm{E}, 1.8 \mathrm{~m}$ to $\mathrm{S}$, in debris & stone: grindstone (1 fragment, sandstone) & $\begin{array}{l}10 \mathrm{~cm} \\
\text { below } \\
\text { Planum } 5\end{array}$ & SAV1E 0253 & 14.02 .2013 \\
\hline
\end{tabular}




\begin{tabular}{|c|c|c|c|c|c|}
\hline $344 / 2013$ & SQ2B, 5-6m to E, debris & $\begin{array}{l}\text { stones and architecture: hammers ( } 3 \text { pieces, } 1 \text { unspecified stone, } \\
1 \text { quartz, } 1 \text { quartzite?), grindstone ( } 1 \text { piece, sandstone); } \\
\text { architecture (1 fragment, sandstone) }\end{array}$ & \begin{tabular}{|c|}
$10 \mathrm{~cm}$ \\
below \\
Planum 5
\end{tabular} & $\begin{array}{l}\text { SAV1E 0245, SAV1E 0246, SAV1E } 0248 \\
\text { (hammers), SAV1E } 0247 \text { (grindstone), } \\
\text { SAV1E 0266 (architecture) }\end{array}$ & 14.02.2013 \\
\hline $375 / 2013$ & $\begin{array}{l}\text { SQ2B, 5-6.5m to E, cleaning debris } \\
\text { (Feature 25) }\end{array}$ & stone: pestle?/stone tool? (1 piece, quartzite) & $\begin{array}{c}\text { below } \\
\text { Planum } 6\end{array}$ & SAV1E 0319 & 28.02 .2013 \\
\hline $315 / 2013$ & $\begin{array}{l}\mathrm{SQ} 2 \mathrm{~B}, \sim 5 \mathrm{~m} \text { to } \mathrm{E}, 1.5 \mathrm{~m} \text { from } \mathrm{N} \text {, below } \\
\text { Planum } 4\end{array}$ & stone: grindstone (1 fragment, sandstone) & $\begin{array}{l}\sim 40 \mathrm{~cm} \\
\text { see } \\
\text { Planum } 4\end{array}$ & SAV1E 0223 & 11.02.2013 \\
\hline $312 / 2013$ & $\begin{array}{l}\text { SQ2B, c. } 6 \mathrm{~m} \text { to E, in sandy pit Feature } 25, \\
\text { Planum } 4\end{array}$ & stone: grindstone (1 fragment, sandstone?) & $\begin{array}{l}\sim 45 \mathrm{~cm}, \mathrm{~s} \\
\text { Planum } 4\end{array}$ & SAV1E 0222 & 11.02.2013 \\
\hline $364 / 2013$ & $\begin{array}{l}\text { SQ2B, 6-8m to E, 1-2m to S, from mud } \\
\text { brick debris }\end{array}$ & $\begin{array}{l}\text { stones: pounder ( } 1 \text { piece, quartz), grindstones ( } 2 \text { pieces } \\
\text { [1 insecure], } 1 \text { sandstone, } 1 \text { schist?) }\end{array}$ & $\begin{array}{c}\text { below } \\
\text { Planum } 6\end{array}$ & $\begin{array}{l}\text { SAV1E } 0284 \text { (pounder), SAV1E } 0283 \text {, } \\
\text { SAV1E } 0285 \text { (grindstones) }\end{array}$ & 18.02 .2013 \\
\hline $340 / 2013$ & $\begin{array}{l}\text { SQ2B, } 7.5 \mathrm{~m} \text { to } \mathrm{E}, \text { c. } 1.7 \mathrm{~m} \text { to } \mathrm{S} \text {, see Planum } \\
5\end{array}$ & stone: grindstone (1 fragment, sandstone) & $\begin{array}{c}\text { see } \\
\text { Planum } 5\end{array}$ & SAV1E 0254 & 14.02.2013 \\
\hline $264 / 2013$ & $\begin{array}{l}\mathrm{SQ} 2 \mathrm{~B}, \sim 7.5 \mathrm{~m} \text { to } \mathrm{E}, \mathrm{W} \text { of area with mud } \\
\text { bricks debris }\end{array}$ & stone: hammer or pounder (1 piece, quartz) & $\sim 20 \mathrm{~cm}$ & SAV1E 0169 & 06.02 .2013 \\
\hline $317 / 2013$ & SQ2B, cleaning basket 27 & $\begin{array}{l}\text { stone and bone: hammer (1 piece, unspecified stone), bone } \\
\text { (1 fragment, found with the tool) }\end{array}$ & $\begin{array}{c}\text { see } \\
\text { Planum } 4\end{array}$ & SAV1E 0234 & 11.02.2013 \\
\hline $380 / 2013$ & SQ2B, cleaning S-baulk 8-10m to E & stone: grindstone (1 fragment, quartzite) & $0-40 \mathrm{~cm}$ & SAV1E 0321 & 02.03 .2013 \\
\hline $367 / 2013$ & $\begin{array}{l}\text { SQ2B, Feature } 26 \text {, cleaning debris, } \\
7.5-10 \mathrm{~m} \text { to } \mathrm{E}\end{array}$ & $\begin{array}{l}\text { stones: hammer or pounder ( } 1 \text { piece, quartz), grindstones } \\
\text { ( } 2 \text { pieces [ } 1 \text { insecure], each sandstone) }\end{array}$ & $\begin{array}{c}\text { below } \\
\text { Planum } 6\end{array}$ & $\begin{array}{l}\text { SAV1E } 0295 \text { (hammer or pounder), SAV1E } \\
\text { 0297, SAV1E } 0296 \text { (grindstones) }\end{array}$ & 26.02.2013 \\
\hline $369 / 2013$ & SQ2B, Feature 26 , cleaning $7.5-10 \mathrm{~m}$ to $\mathrm{E}$ & $\begin{array}{l}\text { stones: grindstones ( } 3 \text { fragments, each quartzite), hammers or } \\
\text { pounders ( } 2 \text { pieces, each quartz) }\end{array}$ & $\begin{array}{l}\text { before } \\
\text { Planum } 7\end{array}$ & $\begin{array}{l}\text { SAV1E 0309, SAV1E 0310, SAV1E } 0311 \\
\text { (grindstones), SAV1E 0312, SAV1E } 0313 \\
\text { (hammers or pounders) }\end{array}$ & 26.02.2013 \\
\hline $378 / 2013$ & $\begin{array}{l}\text { SQ2B, cleaning SW-corner of Feature 27, } \\
\text { debris }\end{array}$ & stone: pounder or hammer (1 piece, quartz) & $\sim 30 \mathrm{~cm}$ & SAV1E 0318 & 28.02.2013 \\
\hline $283 / 2013$ & $\mathrm{SQ} 2 \mathrm{~B}, 2.5 \mathrm{~m}$ to $\mathrm{E}, 1 \mathrm{~m}$ from $\mathrm{N}$ in debris & stopper (1 piece, mud); bones & $\sim 20 \mathrm{~cm}$ & SAV1E 0205 (stopper) & 09.02 .2013 \\
\hline $381 / 2013$ & SQ2B, cleaning S-baulk 8-10m to E & wood (1 piece); charcoal (3 pieces); bone & $0-40 \mathrm{~cm}$ & & 02.03 .2013 \\
\hline
\end{tabular}

\begin{tabular}{|c|c|c|c|c|c|}
\hline \multicolumn{6}{|c|}{ Sai Island SAV1 East 2014} \\
\hline Find no. & Location & Description/label/material & $\begin{array}{c}\text { SU/ } \\
\text { level }\end{array}$ & Object no. & Date \\
\hline $2144 / 2014$ & $\mathrm{SQ} 3,6 \mathrm{~m} \mathrm{~W}-\mathrm{E} / 4.5 \mathrm{~m} \mathrm{~N}-\mathrm{S}$ & amulet (1 piece, nearly intact, shape unclear, tooth/ivory) & 066 & SAV1E 0971 & 03.02 .2014 \\
\hline $2411 / 2014$ & SQ4 & architecture: lintel (1 large fragment, sandstone) & 110 & SAV1E 1840 & 18.02 .2014 \\
\hline
\end{tabular}




\begin{tabular}{|c|c|c|c|c|c|}
\hline $2145 / 2014$ & $\mathrm{SQ} 3,5.8 \mathrm{~m} \mathrm{~W}-\mathrm{E} / 5 \mathrm{~m} \mathrm{~N}-\mathrm{S}$ & arm ring (1 fragment, wood) & 066 & SAV1E 1000 & 03.02 .2014 \\
\hline $427 / 2014$ & SQ1B, 0-3m W-E/2-3m N-S & basketry (1 bag) & 040 & & 26.01.2014 \\
\hline $2026 / 2014$ & $\mathrm{SQ} 1 \mathrm{~B}, 1.5-4 \mathrm{~m} \mathrm{~W}-\mathrm{E} / 0-2.3 \mathrm{~m} \mathrm{~N}-\mathrm{S}$ & basketry/organic material & 051 & & 29.01.2014 \\
\hline $004 / 2014$ & SQ1B, NE-corner, surface & bead (1 circular bead, bone) & 001 & SAV1E 0358 & 04.01 .2014 \\
\hline $074 / 2014$ & $\mathrm{SQ} 3,0.5 \mathrm{~m} \mathrm{~W}-\mathrm{E} / 7 \mathrm{~m} \mathrm{~N}-\mathrm{S}$ & bead (1 circular bead, faience) & 003 & SAV1E 0353 & 09.01 .2014 \\
\hline $282 / 2014$ & $\mathrm{SQ} 3,0.8 \mathrm{~m} \mathrm{~W}-\mathrm{E} / 0.5 \mathrm{~m} \mathrm{~N}-\mathrm{S}$ & bead (1 fragment, faience) & 023 & SAV1E 0650 & 19.01.2014 \\
\hline $283 / 2014$ & $\mathrm{SQ} 3,1.4 \mathrm{~m} \mathrm{~W}-\mathrm{E} / 0.3 \mathrm{~m} \mathrm{~N}-\mathrm{S}$ & bead (1 piece, stone ?) & 023 & SAV1E 0652 & 19.01.2014 \\
\hline $411 / 2014$ & $\mathrm{SQ} 3,0-1.3 \mathrm{~m} \mathrm{~W}-\mathrm{E} / 7.4-8.7 \mathrm{~m} \mathrm{~N}-\mathrm{S}$ & bead/gaming figure? (1 conical shaped clay object) & 038 & SAV1E 0795 & 25.01.2014 \\
\hline $1515 / 2014$ & $\mathrm{SQ} 3,2.7-5.2 \mathrm{~m} \mathrm{~W}-\mathrm{E} / 7.3-9 \mathrm{~m} \mathrm{~N}-\mathrm{S}$ & bead ( 1 tubular bead, 10 sections, faience) & 046 & SAV1E 0844 (bead) & 27.01.2014 \\
\hline $2041 / 2014$ & SQ1B, $5.9 \mathrm{~m} \mathrm{~W}-\mathrm{E} / 0.4 \mathrm{~m} \mathrm{~N}-\mathrm{S}$ & bead (1 ring bead, faience) & 051 & SAV1E 0895 & 30.01 .2014 \\
\hline $2176 / 2014$ & $\begin{array}{l}\text { SQ3, 8.5-10.5m W-E/0.8-2.3m N-S, } \\
\text { Feature } 7\end{array}$ & bead (1 ring bead, faience) & 071 & SAV1E 0968 & 04.02 .2014 \\
\hline $2275 / 2014$ & SQ4, 10m W-E/1.1m N-S, Feature 15 & bead (1 fragment, cylindrical bead, faience) & 079 & SAV1E 1072 & 08.02 .2014 \\
\hline $2405 / 2014$ & $\mathrm{SQ} 4,7.3-8 \mathrm{~m} \mathrm{~W}-\mathrm{E} / 0-2.5 \mathrm{~m} \mathrm{~N}-\mathrm{S}$ & bead (1 circular bead, bone) & 111 & SAV1E 1129 & 16.02.2014 \\
\hline $087 / 2014$ & $\mathrm{SQ} 3,8.5-9.5 \mathrm{~m} \mathrm{~W}-\mathrm{E} / 2-10 \mathrm{~m} \mathrm{~N}-\mathrm{S}$ & bone & 002 & & 12.01 .2014 \\
\hline $312 / 2014$ & $\mathrm{SQ} 3,9.5-10 \mathrm{~m} \mathrm{~W}-\mathrm{E} / 5.7-8 \mathrm{~m} \mathrm{~N}-\mathrm{S}$ & bone & 002 & & 20.01 .2014 \\
\hline $064 / 2014$ & $\mathrm{SQ} 3,0-2 \mathrm{~m} \mathrm{~W}-\mathrm{E} / 6.5 \mathrm{~m} \mathrm{~N}-\mathrm{S}$ & bone & 003 & & 09.01 .2014 \\
\hline $067 / 2014$ & $\mathrm{SQ} 3,0-2 \mathrm{~m} \mathrm{~W}-\mathrm{E} / 7-10 \mathrm{~m} \mathrm{~N}-\mathrm{S}$ & bone & 003 & & 09.01 .2014 \\
\hline $125 / 2014$ & $\mathrm{SQ} 3,2-5 \mathrm{~m} \mathrm{~W}-\mathrm{E} / 2-4 \mathrm{~m} \mathrm{~N}-\mathrm{S}$ & bone & 003 & & 13.01 .2014 \\
\hline $019 / 2014$ & $\mathrm{SQ} 3,2.3-5 \mathrm{~m} \mathrm{~W} / 5 \mathrm{~m} \mathrm{~S}$ of SP3.2 & bones; teeth (animal) & 003 & & 05.01 .2014 \\
\hline $070 / 2014$ & $\mathrm{SQ} 3,3-5 \mathrm{~m} \mathrm{~W}-\mathrm{E} / 7-10 \mathrm{~m} \mathrm{~N}-\mathrm{S}$ & bone; seashell (1 fragment) & 003 & & 09.01 .2014 \\
\hline $118 / 2014$ & $\mathrm{SQ} 3,5-8.5 \mathrm{~m} \mathrm{~W}-\mathrm{E} / 3-7 \mathrm{~m} \mathrm{~N}-\mathrm{S}$ & bone & 003 & & 13.01 .2014 \\
\hline $032 / 2014$ & $\mathrm{SQ} 4,1 \mathrm{~m} \mathrm{~W}-\mathrm{E} / 8 \mathrm{~m}$ N-S & bone & 003 & & 07.01 .2014 \\
\hline $144 / 2014$ & SQ4, 2.5-5m W-E/0-9m N-S & bone & 003 & & 14.01 .2014 \\
\hline $151 / 2014$ & $\mathrm{SQ} 4,5-8 \mathrm{~m} \mathrm{~W}-\mathrm{E} / 0-10 \mathrm{~m} \mathrm{~N}-\mathrm{S}$ & bone & 003 & & 14.01 .2014 \\
\hline $035 / 2014$ & $\mathrm{SQ} 4,6 \mathrm{~m} \mathrm{~W}-\mathrm{E} / 9.5 \mathrm{~m} \mathrm{~N}-\mathrm{S}$ & bone & 003 & & 07.01 .2014 \\
\hline $038 / 2014$ & SQ3, Feature 7 & bone & 004 & & 08.01 .2014 \\
\hline
\end{tabular}




\begin{tabular}{|c|c|c|c|c|}
\hline $2161 / 2014$ & $\begin{array}{l}\text { SQ3, 8.5-10.5m W-E/0.3-2.3m N-S, } \\
\text { cleaning SU }\end{array}$ & bone & 004 & 04.02 .2014 \\
\hline $048 / 2014$ & $\mathrm{SQ} 3,3-5 \mathrm{~m} \mathrm{~W}-\mathrm{E} / 0-3.5 \mathrm{~m} \mathrm{~N}-\mathrm{S}$ & bone & 005 & 08.01 .2014 \\
\hline $052 / 2014$ & $\mathrm{SQ} 3,3-4.5 \mathrm{~m} \mathrm{~W}-\mathrm{E} / 5-6.5 \mathrm{~m} \mathrm{~N}-\mathrm{S}$ & bone & 006 & 08.01 .2014 \\
\hline $059 / 2014$ & $\mathrm{SQ} 3,3-4.5 \mathrm{~m} \mathrm{~W}-\mathrm{E} / 5-6.5 \mathrm{~m} \mathrm{~N}-\mathrm{S}$ & bone & 006 & 09.01 .2014 \\
\hline $362 / 2014$ & $\mathrm{SQ} 4,0.5-1 \mathrm{~m} \mathrm{~W}-\mathrm{E} / 3-4 \mathrm{~m} \mathrm{~N}-\mathrm{S}$ & bone & 008 & 22.01 .2014 \\
\hline $108 / 2014$ & $\mathrm{SQ} 4,1.5-3 \mathrm{~m} \mathrm{~W}-\mathrm{E} / 2-3 \mathrm{~m} \mathrm{~N}-\mathrm{S}$ & bone & 008 & 12.01.2014 \\
\hline $157 / 2014$ & $\begin{array}{l}\text { SQ4, 2-2.5m W-E/9-10m N-S; SQ4A 1.5- } \\
2.5 \mathrm{~m} \mathrm{~W}-\mathrm{E} / 0-2 \mathrm{~m} \mathrm{~N}-\mathrm{S}\end{array}$ & bone & 009 & 14.01.2014 \\
\hline $114 / 2014$ & $\begin{array}{l}\text { SQ4, 2.5-5.5m W-E/9-10m N-S; SQ4A } \\
2.5-6.5 \mathrm{~m} \mathrm{~W}-\mathrm{E} / 0-2 \mathrm{~m} \mathrm{~N}-\mathrm{S}\end{array}$ & bone & 009 & 13.01.2014 \\
\hline $140 / 2014$ & $\begin{array}{l}\mathrm{SQ} 4,2.5-5.5 \mathrm{~m} \mathrm{~W}-\mathrm{E} / 9-10 \mathrm{~m} \mathrm{~N}-\mathrm{S} ; \mathrm{SQ} 4 \mathrm{~A} \\
2.5-6.5 \mathrm{~m} \mathrm{~W}-\mathrm{E} / 0-2 \mathrm{~m} \mathrm{~N}-\mathrm{S}\end{array}$ & bone & 009 & 14.01 .2014 \\
\hline $131 / 2014$ & $\mathrm{SQ} 4,0-1.5 \mathrm{~m} \mathrm{~W}-\mathrm{E} / 1.5-5 \mathrm{~m} \mathrm{~N}-\mathrm{S}$ & bone & 010 & 13.01.2014 \\
\hline $136 / 2014$ & $\mathrm{SQ} 4,0-1.5 \mathrm{~m} \mathrm{~W}-\mathrm{E} / 7.5-10 \mathrm{~m} \mathrm{~N}-\mathrm{S}$ & bone & 011 & 13.01.2014 \\
\hline $173 / 2014$ & $\mathrm{SQ} 3,3-5 \mathrm{~m} \mathrm{~W}-\mathrm{E} / 0-3.5 \mathrm{~m} \mathrm{~N}-\mathrm{S}$ & bone & 013 & 15.01.2014 \\
\hline $354 / 2014$ & $\mathrm{SQ} 4,0-2.5 \mathrm{~m} \mathrm{~W}-\mathrm{E} / 4.5-5.5 \mathrm{~m} \mathrm{~N}-\mathrm{S}$ & bone & 015 & 22.01 .2014 \\
\hline $208 / 2014$ & $\mathrm{SQ} 4,0-5 \mathrm{~m} \mathrm{~W}-\mathrm{E} / 5-8 \mathrm{~m} \mathrm{~N}-\mathrm{S}$ & bone & 015 & 18.01 .2014 \\
\hline $230 / 2014$ & $\mathrm{SQ} 4,1.5-5 \mathrm{~m} \mathrm{~W}-\mathrm{E} / 3.5-5 \mathrm{~m} \mathrm{~N}-\mathrm{S}$ & bone & 015 & 18.01.2014 \\
\hline $234 / 2014$ & $\mathrm{SQ} 4,1.5-5 \mathrm{~m} \mathrm{~W}-\mathrm{E} / 8-8.5 \mathrm{~m} \mathrm{~N}-\mathrm{S}$ & bone & 015 & 18.01 .2014 \\
\hline $251 / 2014$ & $\mathrm{SQ} 4,1.5-5 \mathrm{~m} \mathrm{~W}-\mathrm{E} / 8-8.5 \mathrm{~m} \mathrm{~N}-\mathrm{S}$ & bone & 015 & 19.01.2014 \\
\hline $263 / 2014$ & $\mathrm{SQ} 4,3.5-5 \mathrm{~m} \mathrm{~W}-\mathrm{E} / 0-3.5 \mathrm{~m} \mathrm{~N}-\mathrm{S}$ & bone & 015 & 19.01.2014 \\
\hline 196/2014 & SQ4A, $10-10.5 \mathrm{~m} \mathrm{~W}-\mathrm{E} / 0-0.5 \mathrm{~m} \mathrm{~N}-\mathrm{S}$ & bone & 017 & 16.01.2014 \\
\hline $213 / 2014$ & SQ1B, 0-3m W-E/0-2m N-S & bone & 018 & 18.01.2014 \\
\hline $2079 / 2014$ & $\mathrm{SQ} 1 \mathrm{~B}, 0-2 \mathrm{~m} \mathrm{~W}-\mathrm{E} / 4-4.7 \mathrm{~m} \mathrm{~N}-\mathrm{S}$ & bone & 020 & 01.02 .2014 \\
\hline $275 / 2014$ & SQ1B, 3-6m W-E/2-3m N-S & bone & 021 & 19.01.2014 \\
\hline $287 / 2014$ & SQ1B, 3-6m W-E/2-3m N-S & bone (naturally mummified lizard) & 021 & 20.01.2014 \\
\hline $268 / 2014$ & $\mathrm{SQ} 1 \mathrm{~B}, 6-7.5 \mathrm{~m} \mathrm{~W}-\mathrm{E} / 0-2.5 \mathrm{~m} \mathrm{~N}-\mathrm{S}$ & bone & 021 & 19.01.2014 \\
\hline $270 / 2014$ & $\mathrm{SQ} 4,0-1.5 \mathrm{~m} \mathrm{~W}-\mathrm{E} / 8-10 \mathrm{~m} \mathrm{~N}-\mathrm{S}$ & bone & 022 & 19.01.2014 \\
\hline
\end{tabular}




\begin{tabular}{|c|c|c|c|c|}
\hline $259 / 2014$ & $\mathrm{SQ} 4 \mathrm{~A}, 0-1.5 \mathrm{~m} \mathrm{~W}-\mathrm{E} / 0-2 \mathrm{~m} \mathrm{~N}-\mathrm{S}$ & bone & 022 & 19.01.2014 \\
\hline $280 / 2014$ & SQ3, 0-2m W-E/0-4.4m N-S & bone & 023 & 19.01.2014 \\
\hline $291 / 2014$ & $\mathrm{SQ} 3,2-3 \mathrm{~m} \mathrm{~W}-\mathrm{E} / 0-4.4 \mathrm{~m} \mathrm{~N}-\mathrm{S}$ & bone & 023 & 20.01 .2014 \\
\hline $2115 / 2014$ & $\begin{array}{l}\text { SQ3, 2.5-3.2m W-E/0-3.5m N-S, } \\
\text { cleaning SU }\end{array}$ & bone & 024 & 02.02 .2014 \\
\hline $332 / 2014$ & $\mathrm{SQ} 3,0-2 \mathrm{~m} \mathrm{~W}-\mathrm{E} / 4.4-10 \mathrm{~m} \mathrm{~N}-\mathrm{S}$ & bone & 025 & 21.01.2014 \\
\hline $318 / 2014$ & SQ3, 8-10.5m W-E/2.5-5.7m N-S & bone & 026 & 21.01.2014 \\
\hline $323 / 2014$ & $\mathrm{SQ} 3,8-10.5 \mathrm{~m} \mathrm{~W}-\mathrm{E} / 5-8 \mathrm{~m} \mathrm{~N}-\mathrm{S}$ & bone; shells & 027 & 21.01.2014 \\
\hline $373 / 2014$ & $\mathrm{SQ} 3,8-10.5 \mathrm{~m} \mathrm{~W}-\mathrm{E} / 2.5-5.7 \mathrm{~m} \mathrm{~N}-\mathrm{S}$ & bone & 032 & 23.01.2014 \\
\hline $376 / 2014$ & $\mathrm{SQ} 4,5-8 \mathrm{~m} \mathrm{~W}-\mathrm{E} / 0-10 \mathrm{~m} \mathrm{~N}-\mathrm{S}$ & bone & 033 & 23.01.2014 \\
\hline $383 / 2014$ & SQ3, 9-10.5m W-E/2.6-5.5m N-S & bone & 034 & 23.01.2014 \\
\hline $394 / 2014$ & SQ4A, 0-2m W-E/0-2m N-S & bone & 035 & 23.01.2014 \\
\hline $403 / 2014$ & SQ3, 0-1.3m W-E/4.4-6.8m N-S & bone & 038 & 25.01.2014 \\
\hline $410 / 2014$ & SQ3, 0-1.3m W-E/7.4-8.7m N-S & bone & 038 & 25.01.2014 \\
\hline $414 / 2014$ & SQ1B, 0-3m W-E/2-3m N-S & bone & 040 & 25.01 .2014 \\
\hline $424 / 2014$ & SQ1B, 0-3m W-E/2-3m N-S & bone & 040 & 26.01.2014 \\
\hline $435 / 2014$ & $\mathrm{SQ} 1 \mathrm{~B}, 0-3 \mathrm{~m} \mathrm{~W}-\mathrm{E} / 2.5-4.5 \mathrm{~m} \mathrm{~N}-\mathrm{S}$ & bone & 040 & 26.01.2014 \\
\hline $440 / 2014$ & SQ1B, 1-3m W-E/3-4m N-S & bone & 040 & 27.01.2014 \\
\hline $1508 / 2014$ & $\mathrm{SQ} 1 \mathrm{~B}, 2.5-8 \mathrm{~m} \mathrm{~W}-\mathrm{E} / 2.5-4 \mathrm{~m} \mathrm{~N}-\mathrm{S}$ & bone & 040 & 27.01.2014 \\
\hline $419 / 2014$ & $\mathrm{SQ} 3,1-5 \mathrm{~m} \mathrm{~W}-\mathrm{E} / 7.2-10 \mathrm{~m} \mathrm{~N}-\mathrm{S}$ & bone & 041 & 25.01 .2014 \\
\hline $431 / 2014$ & $\mathrm{SQ} 3,1.8-5 \mathrm{~m} \mathrm{~W}-\mathrm{E} / 7-10 \mathrm{~m} \mathrm{~N}-\mathrm{S}$ & bone & 041 & 26.01.2014 \\
\hline $444 / 2014$ & $\mathrm{SQ} 4,0.5-3 \mathrm{~m} \mathrm{~W}-\mathrm{E} / 0-4 \mathrm{~m} \mathrm{~N}-\mathrm{S}$ & bone & 042 & 27.01.2014 \\
\hline $448 / 2014$ & SQ1B, 3-10m W-E/3-5m N-S & bone & 043 & 27.01.2014 \\
\hline $1501 / 2014$ & $\mathrm{SQ} 3,0-1.5 \mathrm{~m} \mathrm{~W}-\mathrm{E} / 7.4-8.7 \mathrm{~m} \mathrm{~N}-\mathrm{S}$ & bone & 044 & 27.01.2014 \\
\hline $1514 / 2014$ & $\mathrm{SQ} 3,2.7-5.2 \mathrm{~m} \mathrm{~W}-\mathrm{E} / 7.3-9 \mathrm{~m} \mathrm{~N}-\mathrm{S}$ & bone & 046 & 27.01 .2014 \\
\hline $1521 / 2014$ & $\mathrm{SQ} 3,2.7-5.2 \mathrm{~m} \mathrm{~W}-\mathrm{E} / 7-8.5 \mathrm{~m} \mathrm{~N}-\mathrm{S}$ & bone & 047 & 28.01.2014 \\
\hline $1518 / 2014$ & SQ1B, 8-10m W-E/0-5m N-S & bone & 048 & 28.01 .2014 \\
\hline
\end{tabular}




\begin{tabular}{|c|c|c|c|c|}
\hline $2002 / 2014$ & SQ1B, 0-2m W-E/2.3-3.4m N-S & bone & 049 & 28.01 .2014 \\
\hline $2008 / 2014$ & SQ3, 2.5-5.3m W-E/8.5-10m N-S & bone & 050 & 28.01 .2014 \\
\hline $2019 / 2014$ & $\mathrm{SQ} 3,2.5-5.3 \mathrm{~m} \mathrm{~W}-\mathrm{E} / 8.5-10 \mathrm{~m} \mathrm{~N}-\mathrm{S}$ & bone & 050 & 29.01.2014 \\
\hline $2014 / 2014$ & $\mathrm{SQ} 1 \mathrm{~B}, 0-4 \mathrm{~m} \mathrm{~W}-\mathrm{E} / 0-2.3 \mathrm{~m} \mathrm{~N}-\mathrm{S}$ & bone & 051 & 28.01 .2014 \\
\hline $2023 / 2014$ & SQ1B, $1.5-4 \mathrm{~m} \mathrm{~W}-\mathrm{E} / 0-2.3 \mathrm{~m} \mathrm{~N}-\mathrm{S}$ & bone & 051 & 29.01.2014 \\
\hline $2045 / 2014$ & SQ1B, 4-6.5m W-E/0-1.5m N-S & bone & 051 & 30.01 .2014 \\
\hline $2037 / 2014$ & $\mathrm{SQ} 3,2.3-4 \mathrm{~m} \mathrm{~W}-\mathrm{E} / 3-5.2 \mathrm{~m} \mathrm{~N}-\mathrm{S}$ & bone & 052 & 29.01.2014 \\
\hline $2062 / 2014$ & $\mathrm{SQ} 3,2.3-4.8 \mathrm{~m} \mathrm{~W}-\mathrm{E} / 3-5.2 \mathrm{~m} \mathrm{~N}-\mathrm{S}$ & bone & 053 & 30.01 .2014 \\
\hline $2066 / 2014$ & $\mathrm{SQ} 3,4.8-5.3 \mathrm{~m} \mathrm{~W}-\mathrm{E} / 5.2-7.3 \mathrm{~m} \mathrm{~N}-\mathrm{S}$ & bone & 054 & 01.02 .2014 \\
\hline $2102 / 2014$ & SQ1B, $0.5-1 \mathrm{~m} \mathrm{~W}-\mathrm{E} / 4.2-4.5 \mathrm{~m} \mathrm{~N}-\mathrm{S}$ & bone & 056 & 02.02 .2014 \\
\hline $2083 / 2014$ & $\mathrm{SQ} 3,2-5.5 \mathrm{~m} \mathrm{~W}-\mathrm{E} / 2-3.8 \mathrm{~m} \mathrm{~N}-\mathrm{S}$ & bone & 058 & 01.02 .2014 \\
\hline $2087 / 2014$ & $\mathrm{SQ} 3,2-5.5 \mathrm{~m} \mathrm{~W}-\mathrm{E} / 2-3.8 \mathrm{~m} \mathrm{~N}-\mathrm{S}$ & bone & 059 & 01.02 .2014 \\
\hline $2098 / 2014$ & $\mathrm{SQ} 3,2-5.5 \mathrm{~m} \mathrm{~W}-\mathrm{E} / 2-3.8 \mathrm{~m} \mathrm{~N}-\mathrm{S}$ & bone & 059 & 02.02 .2014 \\
\hline $2107 / 2014$ & $\mathrm{SQ} 3,4.2-6 \mathrm{~m} \mathrm{~W}-\mathrm{E} / 0-3.8 \mathrm{~m} \mathrm{~N}-\mathrm{S}$ & bone & 061 & 02.02 .2014 \\
\hline $2124 / 2014$ & $\mathrm{SQ} 3,4.4-5.6 \mathrm{~m} \mathrm{~W}-\mathrm{E} / 0.8-3 \mathrm{~m} \mathrm{~N}-\mathrm{S}$ & bone & 062 & 03.02 .2014 \\
\hline $2119 / 2014$ & SQ1B, $0-0.8 \mathrm{~m} \mathrm{~W}-\mathrm{E} / 4.2-5 \mathrm{~m} \mathrm{~N}-\mathrm{S}$ & bone & 064 & 03.02 .2014 \\
\hline $2128 / 2014$ & SQ1B, 0-1.1m W-E/4-5m N-S & bone & 065 & 03.02 .2014 \\
\hline $2167 / 2014$ & $\mathrm{SQ} 3,4.8-5.5 \mathrm{~m} \mathrm{~W}-\mathrm{E} / 4-6.8 \mathrm{~m} \mathrm{~N}-\mathrm{S}$ & bone & 066 & 04.02 .2014 \\
\hline $2137 / 2014$ & SQ1B, $0.5-2 \mathrm{~m} \mathrm{~W}-\mathrm{E} / 3.8-4.7 \mathrm{~m} \mathrm{~N}-\mathrm{S}$ & bone & 068 & 03.02 .2014 \\
\hline $2150 / 2014$ & SQ3, 9-10.5m W-E/3.6-5.3m N-S & bone & 069 & 03.02 .2014 \\
\hline $2155 / 2014$ & $\mathrm{SQ} 3,9-10.5 \mathrm{~m} \mathrm{~W}-\mathrm{E} / 3.6-5.3 \mathrm{~m} \mathrm{~N}-\mathrm{S}$ & bone & 069 & 04.02 .2014 \\
\hline $2207 / 2014$ & SQ3, $8.5-10.5 \mathrm{~m} \mathrm{~W}-\mathrm{E} / 5-7.8 \mathrm{~m} \mathrm{~N}-\mathrm{S}$ & bone & 070 & 05.02 .2014 \\
\hline $2220 / 2014$ & $\mathrm{SQ} 3,8.5-10.5 \mathrm{~m} \mathrm{~W}-\mathrm{E} / 5-8.5 \mathrm{~m} \mathrm{~N}-\mathrm{S}$ & bone & 070 & 05.02 .2014 \\
\hline $2228 / 2014$ & $\mathrm{SQ} 3,8.5-10.5 \mathrm{~m} \mathrm{~W}-\mathrm{E} / 5-8.5 \mathrm{~m} \mathrm{~N}-\mathrm{S}$ & bone & 070 & 06.02 .2014 \\
\hline $2254 / 2014$ & SQ3, 8-10.5m W-E/3.6-5.5m N-S & bone & 070 & 06.02 .2014 \\
\hline $2197 / 2014$ & $\mathrm{SQ} 3,9.2-10.5 \mathrm{~m} \mathrm{~W}-\mathrm{E} / 4.7-5.9 \mathrm{~m} \mathrm{~N}-\mathrm{S}$ & bone & 070 & 05.02 .2014 \\
\hline
\end{tabular}




\begin{tabular}{|c|c|c|c|c|}
\hline $2244 / 2014$ & SQ3, $9.2-10.5 \mathrm{~m} \mathrm{~W}-\mathrm{E} / 8-9.2 \mathrm{~m} \mathrm{~N}-\mathrm{S}$ & bone & 070 & 06.02 .2014 \\
\hline $2239 / 2014$ & $\mathrm{SQ} 3,9-10.5 \mathrm{~m} \mathrm{~W}-\mathrm{E} / 2-3.6 \mathrm{~m} \mathrm{~N}-\mathrm{S}$ & bone & 070 & 06.02 .2014 \\
\hline $2250 / 2014$ & SQ3, 9-10.5m W-E/8-9.5m N-S & bone & 070 & 06.02 .2014 \\
\hline $2171 / 2014$ & SQ3, 8.5-10.5m W-E/0.8-2.3m N-S & bone & 071 & 04.02 .2014 \\
\hline $2185 / 2014$ & $\mathrm{SQ} 3,6-8 \mathrm{~m} \mathrm{~W}-\mathrm{E} / 0-7 \mathrm{~m} \mathrm{~N}-\mathrm{S}$ & bone & 072 & 04.02 .2014 \\
\hline $2194 / 2014$ & $\mathrm{SQ} 3,6.6-8 \mathrm{~m} \mathrm{~W}-\mathrm{E} / 1.1-3.2 \mathrm{~m} \mathrm{~N}-\mathrm{S}$ & bone & 073 & 04.02 .2014 \\
\hline $2202 / 2014$ & $\mathrm{SQ} 3,6.6-9 \mathrm{~m} \mathrm{~W}-\mathrm{E} / 1.1-4.5 \mathrm{~m} \mathrm{~N}-\mathrm{S}$ & bone & 073 & 05.02 .2014 \\
\hline $2224 / 2014$ & $\mathrm{SQ} 3,5.7-6.2 \mathrm{~m} \mathrm{~W}-\mathrm{E} / 0.5-2.2 \mathrm{~m} \mathrm{~N}-\mathrm{S}$ & bone & 075 & 05.02 .2014 \\
\hline $2213 / 2014$ & SQ3, 6-8m W-E/0.2-1.1m N-S & bone & 075 & 05.02 .2014 \\
\hline $2232 / 2014$ & SQ3, 8.2-10m W-E/0-2m N-S & bone & 076 & 06.02 .2014 \\
\hline $2235 / 2014$ & SQ3, $8.2-10 \mathrm{~m} \mathrm{~W}-\mathrm{E} / 0.3-1.5 \mathrm{~m} \mathrm{~N}-\mathrm{S}$ & bone & 077 & 06.02 .2014 \\
\hline $2265 / 2014$ & $\mathrm{SQ} 4,9.5-10.5 \mathrm{~m} \mathrm{~W}-\mathrm{E} / 0.6-2 \mathrm{~m} \mathrm{~N}-\mathrm{S}$ & bone & 079 & 08.02 .2014 \\
\hline $2270 / 2014$ & $\mathrm{SQ} 3,6-7.5 \mathrm{~m} \mathrm{~W}-\mathrm{E} / 3.5-5 \mathrm{~m} \mathrm{~N}-\mathrm{S}$ & bone & 080 & 08.02 .2014 \\
\hline $2277 / 2014$ & $\mathrm{SQ} 3,6-8 \mathrm{~m} \mathrm{~W}-\mathrm{E} / 5-7.5 \mathrm{~m} \mathrm{~N}-\mathrm{S}$ & bone & 080 & 08.02 .2014 \\
\hline $2287 / 2014$ & $\mathrm{SQ} 3,6-8.5 \mathrm{~m} \mathrm{~W}-\mathrm{E} / 5-10 \mathrm{~m} \mathrm{~N}-\mathrm{S}$ & bone & 080 & 09.02 .2014 \\
\hline $2282 / 2014$ & $\mathrm{SQ} 4,8-10 \mathrm{~m} \mathrm{~W}-\mathrm{E} / 2-3 \mathrm{~m} \mathrm{~N}-\mathrm{S}$ & bone & 083 & 09.02 .2014 \\
\hline $2285 / 2014$ & $\mathrm{SQ} 4,8-9.5 \mathrm{~m} \mathrm{~W}-\mathrm{E} / 0-2 \mathrm{~m} \mathrm{~N}-\mathrm{S}$ & bone & 084 & 09.02.2014 \\
\hline $2292 / 2014$ & $\mathrm{SQ} 4,8-9.5 \mathrm{~m} \mathrm{~W}-\mathrm{E} / 0-2 \mathrm{~m} \mathrm{~N}-\mathrm{S}$ & bone & 084 & 09.02 .2014 \\
\hline $2305 / 2014$ & SQ3, 9-10.5m W-E/7.5-9.5m N-S & bone & 089 & 10.02 .2014 \\
\hline $2312 / 2014$ & $\mathrm{SQ} 3,8-9.4 \mathrm{~m} \mathrm{~W}-\mathrm{E} / 2.3-3.8 \mathrm{~m} \mathrm{~N}-\mathrm{S}$ & bone & 090 & 10.02 .2014 \\
\hline $2421 / 2014$ & SQ4 & bone; shell & 092 & 24.02 .2014 \\
\hline $2316 / 2014$ & $\mathrm{SQ} 3,7.7-8.8 \mathrm{~m} \mathrm{~W}-\mathrm{E} / 0-3.5 \mathrm{~m} \mathrm{~N}-\mathrm{S}$ & bone & 093 & 10.02 .2014 \\
\hline $2319 / 2014$ & $\mathrm{SQ} 3,7.7-8.8 \mathrm{~m} \mathrm{~W}-\mathrm{E} / 0-3.5 \mathrm{~m} \mathrm{~N}-\mathrm{S}$ & bone & 095 & 11.02 .2014 \\
\hline $2335 / 2014$ & $\mathrm{SQ} 3,7.7-8.8 \mathrm{~m} \mathrm{~W}-\mathrm{E} / 3.5-8 \mathrm{~m} \mathrm{~N}-\mathrm{S}$ & bone & 097 & 11.02 .2014 \\
\hline $2331 / 2014$ & $\mathrm{SQ} 3,7.8-9.6 \mathrm{~m} \mathrm{~W}-\mathrm{E} / 8-9.5 \mathrm{~m} \mathrm{~N}-\mathrm{S}$ & bone & 097 & 11.02 .2014 \\
\hline $2327 / 2014$ & $\mathrm{SQ} 4,9.5-10.5 \mathrm{~m} \mathrm{~W}-\mathrm{E} / 3.5-4.2 \mathrm{~m} \mathrm{~N}-\mathrm{S}$ & bone & 098 & 11.02 .2014 \\
\hline
\end{tabular}




\begin{tabular}{|c|c|c|c|c|}
\hline $2339 / 2014$ & $\mathrm{SQ} 4,8.4-10.5 \mathrm{~m} \mathrm{~W}-\mathrm{E} / 2.5-4.2 \mathrm{~m} \mathrm{~N}-\mathrm{S}$ & bone & 099 & 11.02 .2014 \\
\hline $2351 / 2014$ & $\mathrm{SQ} 4,2-4 \mathrm{~m} \mathrm{~W}-\mathrm{E} / 0-3.6 \mathrm{~m} \mathrm{~N}-\mathrm{S}$ & bone & 100 & 12.02 .2014 \\
\hline $2343 / 2014$ & SQ4, 8.4-10.5m W-E/4.2-5.6m N-S & bone & 101 & 12.02 .2014 \\
\hline $2348 / 2014$ & $\mathrm{SQ} 4,8.5-10 \mathrm{~m} \mathrm{~W}-\mathrm{E} / 4.2-5.6 \mathrm{~m} \mathrm{~N}-\mathrm{S}$ & bone & 102 & 12.02 .2014 \\
\hline $2356 / 2014$ & $\mathrm{SQ} 4,9.2-10.5 \mathrm{~m} \mathrm{~W}-\mathrm{E} / 4.2-5.4 \mathrm{~m} \mathrm{~N}-\mathrm{S}$ & bone & 103 & 12.02 .2014 \\
\hline $2364 / 2014$ & $\mathrm{SQ} 4,8.7-9.5 \mathrm{~m} \mathrm{~W}-\mathrm{E} / 4.2-5.6 \mathrm{~m} \mathrm{~N}-\mathrm{S}$ & bone & 104 & 12.02.2014 \\
\hline $2360 / 2014$ & $\mathrm{SQ} 4,3.5-6 \mathrm{~m} \mathrm{~W}-\mathrm{E} / 0-3.4 \mathrm{~m} \mathrm{~N}-\mathrm{S}$ & bone & 105 & 12.02 .2014 \\
\hline $2387 / 2014$ & $\mathrm{SQ} 4,3.5-6 \mathrm{~m} \mathrm{~W}-\mathrm{E} / 0-3.5 \mathrm{~m} \mathrm{~N}-\mathrm{S}$ & bone & 105 & 13.02 .2014 \\
\hline $2399 / 2014$ & $\mathrm{SQ} 4,3.5-6 \mathrm{~m} \mathrm{~W}-\mathrm{E} / 0-4.7 \mathrm{~m} \mathrm{~N}-\mathrm{S}$ & bone & 105 & 16.02 .2014 \\
\hline $2373 / 2014$ & $\mathrm{SQ} 4,3.5-6 \mathrm{~m} \mathrm{~W}-\mathrm{E} / 2.5-4.7 \mathrm{~m} \mathrm{~N}-\mathrm{S}$ & bone & 105 & 13.02 .2014 \\
\hline $2377 / 2014$ & $\mathrm{SQ} 4,9-10.5 \mathrm{~m} \mathrm{~W}-\mathrm{E} / 5.6-7.2 \mathrm{~m} \mathrm{~N}-\mathrm{S}$ & bone & 108 & 13.02 .2014 \\
\hline $2384 / 2014$ & $\mathrm{SQ} 4,8.5-9.5 \mathrm{~m} \mathrm{~W}-\mathrm{E} / 5.6-7.4 \mathrm{~m} \mathrm{~N}-\mathrm{S}$ & bone & 109 & 13.02 .2014 \\
\hline $2391 / 2014$ & $\mathrm{SQ} 4,8.5-10.5 \mathrm{~m} \mathrm{~W}-\mathrm{E} / 5.6-7 \mathrm{~m} \mathrm{~N}-\mathrm{S}$ & bone & 109 & 15.02 .2014 \\
\hline $2395 / 2014$ & $\mathrm{SQ} 4,7.3-8.7 \mathrm{~m} \mathrm{~W}-\mathrm{E} / 0-2 \mathrm{~m} \mathrm{~N}-\mathrm{S}$ & bone & 110 & 16.02 .2014 \\
\hline $2403 / 2014$ & $\mathrm{SQ} 4,7.3-8 \mathrm{~m} \mathrm{~W}-\mathrm{E} / 0-2.5 \mathrm{~m} \mathrm{~N}-\mathrm{S}$ & bone & 111 & 16.02 .2014 \\
\hline $2416 / 2014$ & SQ 2, Feature 15 & bone; shell & 114 & 24.02 .2014 \\
\hline $029 / 2014$ & $\begin{array}{l}\text { SQ1, cleaning W-section, in area of } \\
\text { Feature } 7\end{array}$ & bone & l & 07.01 .2014 \\
\hline $2181 / 2014$ & $\begin{array}{l}\text { SQ2, 0-2.5m W-E /0.5-1.8m N-S, } \\
\text { Feature } 15\end{array}$ & bone & 1 & 04.02 .2014 \\
\hline $2189 / 2014$ & $\begin{array}{l}\text { SQ2, } 0.5-1.8 \mathrm{~m} \mathrm{~N}-\mathrm{S} \text {, Feature } 15 \text {, cleaning } \\
\text { W-profile }\end{array}$ & bone & / & 04.02 .2014 \\
\hline $308 / 2014$ & $\mathrm{SQ} 1 \mathrm{~B}, 7-8.3 \mathrm{~m} \mathrm{~W}-\mathrm{E} / 0-2.5 \mathrm{~m} \mathrm{~N}-\mathrm{S}$ & botanical material (2 pieces) & 021 & 20.01 .2014 \\
\hline $065 / 2014$ & $\mathrm{SQ} 3,4 \mathrm{~m} \mathrm{~W}-\mathrm{E} / 5.5 \mathrm{~m} \mathrm{~N}-\mathrm{S}$ & chalk & 006 & 09.01 .2014 \\
\hline $095 / 2014$ & $\mathrm{SQ} 4,1-2.5 \mathrm{~m} \mathrm{~W}-\mathrm{E} / 0-1.5 \mathrm{~m} \mathrm{~N}-\mathrm{S}$ & chalk & 008 & 12.01 .2014 \\
\hline $107 / 2014$ & $\mathrm{SQ} 4,1.5-3 \mathrm{~m} \mathrm{~W}-\mathrm{E} / 2-3 \mathrm{~m} \mathrm{~N}-\mathrm{S}$ & chalk & 008 & 12.01 .2014 \\
\hline $117 / 2014$ & $\begin{array}{l}\text { SQ4, 2.5-5.5m W-E/9-10m N-S; SQ4A } \\
\text { 2.5-6.5m W-E/0-2m N-S }\end{array}$ & chalk & 009 & 13.01.2014 \\
\hline $142 / 2014$ & $\begin{array}{l}\text { SQ4, 2.5-5.5m W-E/9-10m N-S; SQ4A } \\
2.5-6.5 \mathrm{~m} \mathrm{~W}-\mathrm{E} / 0-2 \mathrm{~m} \mathrm{~N}-\mathrm{S}\end{array}$ & chalk/lime & 009 & 14.01 .2014 \\
\hline
\end{tabular}




\begin{tabular}{|c|c|c|c|c|}
\hline $2174 / 2014$ & $\begin{array}{l}\text { SQ3, } 8.5-10.5 \mathrm{~m} \mathrm{~W}-\mathrm{E} / 0.8-2.3 \mathrm{~m} \mathrm{~N}-\mathrm{S} \text {, } \\
\text { Feature } 7\end{array}$ & chalk (1 piece) & 071 & 04.02 .2014 \\
\hline $058 / 2014$ & $\mathrm{SQ} 3,0.2 \mathrm{~m} \mathrm{~W}-\mathrm{E} / 0-5 \mathrm{~m} \mathrm{~N}-\mathrm{S}$ & charcoal (1 bag) & 003 & 09.01 .2014 \\
\hline $068 / 2014$ & $\mathrm{SQ} 3,0-2 \mathrm{~m} \mathrm{~W}-\mathrm{E} / 7-10 \mathrm{~m} \mathrm{~N}-\mathrm{S}$ & charcoal (2 pieces) & 003 & 09.01 .2014 \\
\hline $073 / 2014$ & $\mathrm{SQ} 3,3-5 \mathrm{~m} \mathrm{~W}-\mathrm{E} / 7-10 \mathrm{~m} \mathrm{~N}-\mathrm{S}$ & charcoal (1 bag) & 003 & 09.01 .2014 \\
\hline $025 / 2014$ & $\mathrm{SQ} 3,3 \mathrm{~m} \mathrm{~W}-\mathrm{E} / 5.5 \mathrm{~m} \mathrm{~N}-\mathrm{S}$ & charcoal (1 piece) & 003 & 06.01 .2014 \\
\hline $105 / 2014$ & $\mathrm{SQ} 3,5-8.5 \mathrm{~m} \mathrm{~W}-\mathrm{E} / 0-3 \mathrm{~m} \mathrm{~N}-\mathrm{S}$ & charcoal (1 bag) & 003 & 12.01.2014 \\
\hline $119 / 2014$ & $\mathrm{SQ} 3,5-8.5 \mathrm{~m} \mathrm{~W}-\mathrm{E} / 3-7 \mathrm{~m} \mathrm{~N}-\mathrm{S}$ & charcoal (4 pieces) & 003 & 13.01.2014 \\
\hline $096 / 2014$ & $\mathrm{SQ} 3,5-8.5 \mathrm{~m} \mathrm{~W}-\mathrm{E} / 7-10 \mathrm{~m} \mathrm{~N}-\mathrm{S}$ & charcoal (1 bag) & 003 & 12.01 .2014 \\
\hline $091 / 2014$ & $\mathrm{SQ} 3,5-8.5 \mathrm{~m} \mathrm{~W}-\mathrm{E} / 7-10 \mathrm{~m} \mathrm{~N}-\mathrm{S}$ & charcoal (1 piece); bone & 003 & 12.01.2014 \\
\hline $031 / 2014$ & $\mathrm{SQ} 4,0.5 \mathrm{~m} \mathrm{~W}-\mathrm{E} / 7 \mathrm{~m} \mathrm{~N}-\mathrm{S}$ & charcoal (1 bag) & 003 & 07.01 .2014 \\
\hline $143 / 2014$ & $\mathrm{SQ} 4,2.5-5 \mathrm{~m} \mathrm{~W}-\mathrm{E} / 0-9 \mathrm{~m} \mathrm{~N}-\mathrm{S}$ & charcoal (1 bag) & 003 & 14.01.2014 \\
\hline $149 / 2014$ & $\mathrm{SQ} 4,5-8 \mathrm{~m} \mathrm{~W}-\mathrm{E} / 0-10 \mathrm{~m} \mathrm{~N}-\mathrm{S}$ & charcoal (1 bag) & 003 & 14.01 .2014 \\
\hline $043 / 2014$ & SQ3, S-edge, Feature 7 & charcoal (1 bag) & 004 & 08.01 .2014 \\
\hline $040 / 2014$ & $\mathrm{SQ} 3,4 \mathrm{~m} \mathrm{~W}-\mathrm{E} / 0.5 \mathrm{~m} \mathrm{~N}-\mathrm{S}$ & charcoal (1 bag) & 005 & 08.01 .2014 \\
\hline $053 / 2014$ & $\mathrm{SQ} 3,3-4.5 \mathrm{~m} \mathrm{~W}-\mathrm{E} / 5-6.5 \mathrm{~m} \mathrm{~N}-\mathrm{S}$ & charcoal (1 bag) & 006 & 08.01 .2014 \\
\hline $057 / 2014$ & $\mathrm{SQ} 3,3-4.5 \mathrm{~m} \mathrm{~W}-\mathrm{E} / 5-6.5 \mathrm{~m} \mathrm{~N}-\mathrm{S}$ & charcoal (1 bag) & 006 & 09.01 .2014 \\
\hline $078 / 2014$ & SQ1B, $0-10 \mathrm{~m} \mathrm{~W}-\mathrm{E} / 0-5 \mathrm{~m} \mathrm{~N}-\mathrm{S}$ & charcoal (3 pieces) & 007 & 11.01 .2014 \\
\hline $361 / 2014$ & $\mathrm{SQ} 4,0.5-1 \mathrm{~m} \mathrm{~W}-\mathrm{E} / 3-4 \mathrm{~m} \mathrm{~N}-\mathrm{S}$ & charcoal (1 bag) & 008 & 22.01 .2014 \\
\hline $370 / 2014$ & $\mathrm{SQ} 4,0.5-1 \mathrm{~m} \mathrm{~W}-\mathrm{E} / 3-4 \mathrm{~m} \mathrm{~N}-\mathrm{S}$ & charcoal (1 bag) & 008 & 23.01 .2014 \\
\hline $101 / 2014$ & $\mathrm{SQ} 4,1-2.5 \mathrm{~m} \mathrm{~W}-\mathrm{E} / 1.5-2 \mathrm{~m} \mathrm{~N}-\mathrm{S}$ & charcoal (1 bag) & 008 & 12.01.2014 \\
\hline $115 / 2014$ & $\begin{array}{l}\text { SQ4, 2.5-5.5m W-E/9-10m N-S; } \\
\text { SQ4A 2.5-6.5m W-E/0-2m N-S }\end{array}$ & charcoal (1 bag) & 009 & 13.01 .2014 \\
\hline $141 / 2014$ & $\begin{array}{l}\text { SQ4, 2.5-5.5m W-E/9-10m N-S; } \\
\text { SQ4A 2.5-6.5m W-E/0-2m N-S }\end{array}$ & charcoal (1 bag) & 009 & 14.01.2014 \\
\hline $130 / 2014$ & SQ4, 0-1.5m W-E/1.5-5m N-S & charcoal (1 bag) & 010 & 13.01 .2014 \\
\hline $134 / 2014$ & $\mathrm{SQ} 4,0-1.5 \mathrm{~m} \mathrm{~W}-\mathrm{E} / 7.5-10 \mathrm{~m} \mathrm{~N}-\mathrm{S}$ & charcoal (1 bag) & 011 & 13.01.2014 \\
\hline $161 / 2014$ & $\mathrm{SQ} 3,3-5 \mathrm{~m} \mathrm{~W}-\mathrm{E} / 0-3.5 \mathrm{~m} \mathrm{~N}-\mathrm{S}$ & charcoal (1 bag) & 013 & 15.01 .2014 \\
\hline
\end{tabular}




\begin{tabular}{|c|c|c|c|c|}
\hline $167 / 2014$ & $\mathrm{SQ} 4,1-3 \mathrm{~m} \mathrm{~W}-\mathrm{E} / 0-4 \mathrm{~m} \mathrm{~N}-\mathrm{S}$ & charcoal (1 bag) & 014 & 15.01.2014 \\
\hline $353 / 2014$ & $\mathrm{SQ} 4,0-2.5 \mathrm{~m} \mathrm{~W}-\mathrm{E} / 4.5-5.5 \mathrm{~m} \mathrm{~N}-\mathrm{S}$ & charcoal (1 bag) & 015 & 22.01 .2014 \\
\hline $184 / 2014$ & $\mathrm{SQ} 4,0-5 \mathrm{~m} \mathrm{~W}-\mathrm{E} / 5-8 \mathrm{~m} \mathrm{~N}-\mathrm{S}$ & charcoal (1 bag) & 015 & 16.01 .2014 \\
\hline $209 / 2014$ & $\mathrm{SQ} 4,0-5 \mathrm{~m} \mathrm{~W}-\mathrm{E} / 5-8 \mathrm{~m} \mathrm{~N}-\mathrm{S}$ & charcoal (1 bag) & 015 & 18.01 .2014 \\
\hline $229 / 2014$ & $\mathrm{SQ} 4,1.5-5 \mathrm{~m} \mathrm{~W}-\mathrm{E} / 3.5-5 \mathrm{~m} \mathrm{~N}-\mathrm{S}$ & charcoal (1 bag) & 015 & 18.01 .2014 \\
\hline $235 / 2014$ & $\mathrm{SQ} 4,1.5-5 \mathrm{~m} \mathrm{~W}-\mathrm{E} / 8-8.5 \mathrm{~m} \mathrm{~N}-\mathrm{S}$ & charcoal & 015 & 18.01 .2014 \\
\hline $250 / 2014$ & $\mathrm{SQ} 4,1.5-5 \mathrm{~m} \mathrm{~W}-\mathrm{E} / 8-8.5 \mathrm{~m} \mathrm{~N}-\mathrm{S}$ & charcoal (1 bag) & 015 & 19.01.2014 \\
\hline $182 / 2014$ & SQ4A, 9-10.5m W-E/0-2m N-S & charcoal (1 bag) & 016 & 16.01 .2014 \\
\hline $195 / 2014$ & SQ4A, $10-10.5 \mathrm{~m} \mathrm{~W}-\mathrm{E} / 0-0.5 \mathrm{~m} \mathrm{~N}-\mathrm{S}$ & charcoal (1 bag) & 017 & 16.01 .2014 \\
\hline $214 / 2014$ & SQ1B, 0-3m W-E/0-2m N-S & charcoal (1 bag) & 018 & 18.01 .2014 \\
\hline $221 / 2014$ & $\mathrm{SQ1B}, 0.5-2.5 \mathrm{~m} \mathrm{~W}-\mathrm{E} / 4-5 \mathrm{~m} \mathrm{~N}-\mathrm{S}$ & charcoal (1 bag) & 019 & 18.01 .2014 \\
\hline $242 / 2014$ & $\begin{array}{l}\text { SQ1A, } 0.5-2.5 \mathrm{~m} \mathrm{~W}-\mathrm{E} / 0-1 \mathrm{~m} \mathrm{~N}-\mathrm{S} \\
\text { SQ1B, } 0.5-2.5 \mathrm{~m} \mathrm{~W}-\mathrm{E} / 4.5-5 \mathrm{~m} \mathrm{~N}-\mathrm{S}\end{array}$ & charcoal (1 bag) & 019 & 19.01.2014 \\
\hline $2080 / 2014$ & SQ1B, 0-2m W-E/4-4.7m N-S & charcoal (4 pieces) & 020 & 01.02 .2014 \\
\hline $255 / 2014$ & SQ1B, 3-6m W-E/0-2m N-S & charcoal (1 bag) & 021 & 19.01 .2014 \\
\hline $284 / 2014$ & SQ1B, 3-6m W-E/2-3m N-S & charcoal (1 bag) & 021 & 19.01 .2014 \\
\hline $289 / 2014$ & SQ1B, 3-6m W-E/2-3m N-S & charcoal (1 bag) & 021 & 20.01 .2014 \\
\hline $267 / 2014$ & $\mathrm{SQ} 1 \mathrm{~B}, 6-7.5 \mathrm{~m} \mathrm{~W}-\mathrm{E} / 0-2.5 \mathrm{~m} \mathrm{~N}-\mathrm{S}$ & charcoal (1 bag) & 021 & 19.01.2014 \\
\hline $298 / 2014$ & SQ1B, 7-8.3m W-E/0-2.5m N-S & charcoal (1 bag) & 021 & 20.01 .2014 \\
\hline $272 / 2014$ & $\mathrm{SQ} 4,0-1.5 \mathrm{~m} \mathrm{~W}-\mathrm{E} / 8-10 \mathrm{~m} \mathrm{~N}-\mathrm{S}$ & charcoal (1 bag) & 022 & 19.01.2014 \\
\hline $260 / 2014$ & $\mathrm{SQ} 4 \mathrm{~A}, 0-1.5 \mathrm{~m} \mathrm{~W}-\mathrm{E} / 0-2 \mathrm{~m} \mathrm{~N}-\mathrm{S}$ & charcoal (5 pieces) & 022 & 19.01 .2014 \\
\hline $279 / 2014$ & $\mathrm{SQ} 3,0-2 \mathrm{~m} \mathrm{~W}-\mathrm{E} / 0-4.4 \mathrm{~m} \mathrm{~N}-\mathrm{S}$ & charcoal (1 bag) & 023 & 19.01.2014 \\
\hline $292 / 2014$ & $\mathrm{SQ} 3,2-3 \mathrm{~m} \mathrm{~W}-\mathrm{E} / 0-4.4 \mathrm{~m} \mathrm{~N}-\mathrm{S}$ & charcoal (1 bag) & 023 & 20.01 .2014 \\
\hline $303 / 2014$ & $\mathrm{SQ} 3,2.5-3.2 \mathrm{~m} \mathrm{~W}-\mathrm{E} / 0-3.5 \mathrm{~m} \mathrm{~N}-\mathrm{S}$ & charcoal (1 bag) & 024 & 20.01 .2014 \\
\hline $304 / 2014$ & $\mathrm{SQ} 3,2.5-3.2 \mathrm{~m} \mathrm{~W}-\mathrm{E} / 0-3.5 \mathrm{~m} \mathrm{~N}-\mathrm{S}$ & charcoal (1 piece); bone & 024 & 20.01 .2014 \\
\hline $330 / 2014$ & $\mathrm{SQ} 3,0-2 \mathrm{~m} \mathrm{~W}-\mathrm{E} / 4.4-10 \mathrm{~m} \mathrm{~N}-\mathrm{S}$ & charcoal (1 bag) & 025 & 21.01 .2014 \\
\hline $319 / 2014$ & $\mathrm{SQ} 3,8-10.5 \mathrm{~m} \mathrm{~W}-\mathrm{E} / 2.5-5.7 \mathrm{~m} \mathrm{~N}-\mathrm{S}$ & charcoal (1 bag) & 026 & 21.01 .2014 \\
\hline
\end{tabular}




\begin{tabular}{|c|c|c|c|c|}
\hline $324 / 2014$ & $\mathrm{SQ} 3,8-10.5 \mathrm{~m} \mathrm{~W}-\mathrm{E} / 5-8 \mathrm{~m} \mathrm{~N}-\mathrm{S}$ & charcoal (1 bag) & 027 & 21.01.2014 \\
\hline $340 / 2014$ & $\mathrm{SQ} 4,0-3 \mathrm{~m} \mathrm{~W}-\mathrm{E} / 1-5.5 \mathrm{~m} \mathrm{~N}-\mathrm{S}$ & charcoal (1 bag) & 030 & 22.01.2014 \\
\hline $339 / 2014$ & SQ4, 0-3m W-E/1-5.5m N-S & charcoal (1 piece); schist (1 fragment); bone & 030 & 22.01.2014 \\
\hline $348 / 2014$ & $\mathrm{SQ} 3,2.5-9 \mathrm{~m} \mathrm{~W}-\mathrm{E} / 0-10 \mathrm{~m} \mathrm{~N}-\mathrm{S}$ & charcoal (1 bag) & 031 & 22.01.2014 \\
\hline $372 / 2014$ & $\mathrm{SQ} 3,8-10.5 \mathrm{~m} \mathrm{~W}-\mathrm{E} / 2.5-5.7 \mathrm{~m} \mathrm{~N}-\mathrm{S}$ & charcoal (1 bag) & 032 & 23.01.2014 \\
\hline $378 / 2014$ & $\mathrm{SQ} 4,5-8 \mathrm{~m} \mathrm{~W}-\mathrm{E} / 0-10 \mathrm{~m} \mathrm{~N}-\mathrm{S}$ & charcoal (1 bag) & 033 & 23.01.2014 \\
\hline $384 / 2014$ & $\mathrm{SQ} 3,9-10.5 \mathrm{~m} \mathrm{~W}-\mathrm{E} / 2.6-5.5 \mathrm{~m} \mathrm{~N}-\mathrm{S}$ & charcoal (1 bag); doum nuts (2 pieces) & 034 & 23.01.2014 \\
\hline $391 / 2014$ & $\mathrm{SQ} 3,9-10.5 \mathrm{~m} \mathrm{~W}-\mathrm{E} / 3.6-5.3 \mathrm{~m} \mathrm{~N}-\mathrm{S}$ & charcoal (1 bag) & 036 & 23.01.2014 \\
\hline $402 / 2014$ & $\mathrm{SQ} 3,0-1.3 \mathrm{~m} \mathrm{~W}-\mathrm{E} / 4.4-6.8 \mathrm{~m} \mathrm{~N}-\mathrm{S}$ & charcoal (1 bag) & 038 & 25.01.2014 \\
\hline $409 / 2014$ & $\mathrm{SQ} 3,0-1.3 \mathrm{~m} \mathrm{~W}-\mathrm{E} / 7.4-8.7 \mathrm{~m} \mathrm{~N}-\mathrm{S}$ & charcoal (1 bag) & 038 & 25.01.2014 \\
\hline $400 / 2014$ & $\mathrm{SQ} 1 \mathrm{~B}, 0-3 \mathrm{~m} \mathrm{~W}-\mathrm{E} / 2-4.5 \mathrm{~m} \mathrm{~N}-\mathrm{S}$ & charcoal (1 bag) & 039 & 25.01.2014 \\
\hline $436 / 2014$ & $\mathrm{SQ} 1 \mathrm{~B}, 0-3 \mathrm{~m} \mathrm{~W}-\mathrm{E} / 2.5-4.5 \mathrm{~m} \mathrm{~N}-\mathrm{S}$ & charcoal (1 bag) & 040 & 26.01.2014 \\
\hline $415 / 2014$ & SQ1B, 0-3m W-E/2-3m N-S & charcoal (1 bag) & 040 & 25.01 .2014 \\
\hline $425 / 2014$ & SQ1B, $0-3 \mathrm{~m} \mathrm{~W}-\mathrm{E} / 2-3 \mathrm{~m} \mathrm{~N}-\mathrm{S}$ & charcoal (1 bag) & 040 & 26.01 .2014 \\
\hline $441 / 2014$ & SQ1B, 1-3m W-E/3-4m N-S & charcoal (1 bag) & 040 & 27.01 .2014 \\
\hline $1509 / 2014$ & $\mathrm{SQ} 1 \mathrm{~B}, 2.5-8 \mathrm{~m} \mathrm{~W}-\mathrm{E} / 2.5-4 \mathrm{~m} \mathrm{~N}-\mathrm{S}$ & charcoal (1 bag) & 040 & 27.01.2014 \\
\hline $418 / 2014$ & $\mathrm{SQ} 3,1-5 \mathrm{~m} \mathrm{~W}-\mathrm{E} / 7.2-10 \mathrm{~m} \mathrm{~N}-\mathrm{S}$ & charcoal (1 bag) & 041 & 25.01.2014 \\
\hline $432 / 2014$ & $\mathrm{SQ} 3,1.8-5 \mathrm{~m} \mathrm{~W}-\mathrm{E} / 7-10 \mathrm{~m} \mathrm{~N}-\mathrm{S}$ & charcoal (1 bag) & 041 & 26.01 .2014 \\
\hline $445 / 2014$ & $\mathrm{SQ} 4,0.5-3 \mathrm{~m} \mathrm{~W}-\mathrm{E} / 0-4 \mathrm{~m} \mathrm{~N}-\mathrm{S}$ & charcoal (1 bag) & 042 & 27.01.2014 \\
\hline $449 / 2014$ & SQ1B, 3-10m W-E/3-5m N-S & charcoal (1 bag) & 043 & 27.01.2014 \\
\hline $1522 / 2014$ & $\mathrm{SQ} 3,2.7-5.2 \mathrm{~m} \mathrm{~W}-\mathrm{E} / 7-8.5 \mathrm{~m} \mathrm{~N}-\mathrm{S}$ & charcoal (1 bag) & 047 & 28.01 .2014 \\
\hline $2003 / 2014$ & SQ1B, 0-2m W-E/2.3-3.4m N-S & charcoal & 049 & 28.01 .2014 \\
\hline $2009 / 2014$ & $\mathrm{SQ} 3,2.5-5.3 \mathrm{~m} \mathrm{~W}-\mathrm{E} / 8.5-10 \mathrm{~m} \mathrm{~N}-\mathrm{S}$ & charcoal (1 bag) & 050 & 28.01 .2014 \\
\hline $2020 / 2014$ & $\mathrm{SQ} 3,2.5-5.3 \mathrm{~m} \mathrm{~W}-\mathrm{E} / 8.5-10 \mathrm{~m} \mathrm{~N}-\mathrm{S}$ & charcoal (3 pieces) & 050 & 29.01 .2014 \\
\hline $2015 / 2014$ & SQ1B, 0-4m W-E/0-2.3m N-S & charcoal (1 bag) & 051 & 28.01 .2014 \\
\hline $2024 / 2014$ & SQ1B, $1.5-4 \mathrm{~m} \mathrm{~W}-\mathrm{E} / 0-2.3 \mathrm{~m} \mathrm{~N}-\mathrm{S}$ & charcoal (1 bag) & 051 & 29.01 .2014 \\
\hline
\end{tabular}




\begin{tabular}{|c|c|c|c|c|}
\hline $2056 / 2014$ & SQ1B, $1.5-6.5 \mathrm{~m} \mathrm{~W}-\mathrm{E} / 1.3-3 \mathrm{~m} \mathrm{~N}-\mathrm{S}$ & charcoal (1 bag) & 051 & 30.01 .2014 \\
\hline $2032 / 2014$ & SQ1B, 4-6.5m W-E/0-1.5m N-S & charcoal (1 bag) & 051 & 29.01 .2014 \\
\hline $2046 / 2014$ & $\mathrm{SQ} 1 \mathrm{~B}, 4-6.5 \mathrm{~m} \mathrm{~W}-\mathrm{E} / 0-1.5 \mathrm{~m} \mathrm{~N}-\mathrm{S}$ & charcoal (1 bag) & 051 & 30.01 .2014 \\
\hline $2060 / 2014$ & SQ1B, $6.5-7 \mathrm{~m} \mathrm{~W}-\mathrm{E} / 0-1.7 \mathrm{~m} \mathrm{~N}-\mathrm{S}$ & charcoal (1 bag) & 051 & 30.01 .2014 \\
\hline $2039 / 2014$ & $\mathrm{SQ} 3,2.3-4 \mathrm{~m} \mathrm{~W}-\mathrm{E} / 3-5.2 \mathrm{~m} \mathrm{~N}-\mathrm{S}$ & charcoal (1 bag) & 052 & 29.01 .2014 \\
\hline $2052 / 2014$ & $\mathrm{SQ} 3,2.3-4.8 \mathrm{~m} \mathrm{~W}-\mathrm{E} / 3-5.2 \mathrm{~m} \mathrm{~N}-\mathrm{S}$ & charcoal (1 bag) & 052 & 30.01 .2014 \\
\hline $2051 / 2014$ & $\mathrm{SQ} 3,2.3-4.8 \mathrm{~m} \mathrm{~W}-\mathrm{E} / 3-5.2 \mathrm{~m} \mathrm{~N}-\mathrm{S}$ & charcoal (1 piece); bone & 052 & 30.01 .2014 \\
\hline $2063 / 2014$ & $\mathrm{SQ} 3,2.3-4.8 \mathrm{~m} \mathrm{~W}-\mathrm{E} / 3-5.2 \mathrm{~m} \mathrm{~N}-\mathrm{S}$ & charcoal (1 bag) & 053 & 30.01 .2014 \\
\hline $2067 / 2014$ & $\mathrm{SQ} 3,4.8-5.3 \mathrm{~m} \mathrm{~W}-\mathrm{E} / 5.2-7.3 \mathrm{~m} \mathrm{~N}-\mathrm{S}$ & charcoal (1 bag) & 054 & 01.02 .2014 \\
\hline $2094 / 2014$ & $\mathrm{SQ} 1 \mathrm{~B}, 0-1.5 \mathrm{~m} \mathrm{~W}-\mathrm{E} / 2.5-4.5 \mathrm{~m} \mathrm{~N}-\mathrm{S}$ & charcoal (1 bag) & 056 & 01.02 .2014 \\
\hline $2072 / 2014$ & SQ1B, $0-2.8 \mathrm{~m} \mathrm{~W}-\mathrm{E} / 3-4 \mathrm{~m} \mathrm{~N}-\mathrm{S}$, Feature 38 & charcoal (1 bag) & 056 & 01.02 .2014 \\
\hline $2077 / 2014$ & $\mathrm{SQ} 3,2-2.7 \mathrm{~m} \mathrm{~W}-\mathrm{E} / 6.5-7.2 \mathrm{~m} \mathrm{~N}-\mathrm{S}$ & charcoal (1 bag) & 057 & 01.02 .2014 \\
\hline $2085 / 2014$ & $\mathrm{SQ} 3,2-5.5 \mathrm{~m} \mathrm{~W}-\mathrm{E} / 2-3.8 \mathrm{~m} \mathrm{~N}-\mathrm{S}$ & charcoal (1 bag) & 058 & 01.02 .2014 \\
\hline $2088 / 2014$ & $\mathrm{SQ} 3,2-5.5 \mathrm{~m} \mathrm{~W}-\mathrm{E} / 2-3.8 \mathrm{~m} \mathrm{~N}-\mathrm{S}$ & charcoal (1 bag) & 059 & 01.02 .2014 \\
\hline $2099 / 2014$ & $\mathrm{SQ} 3,2-5.5 \mathrm{~m} \mathrm{~W}-\mathrm{E} / 2-3.8 \mathrm{~m} \mathrm{~N}-\mathrm{S}$ & charcoal (1 bag) & 059 & 02.02 .2014 \\
\hline $2106 / 2014$ & $\mathrm{SQ} 3,4.2-6 \mathrm{~m} \mathrm{~W}-\mathrm{E} / 0-3.8 \mathrm{~m} \mathrm{~N}-\mathrm{S}$ & charcoal (1 bag) & 061 & 02.02 .2014 \\
\hline $2125 / 2014$ & $\mathrm{SQ} 3,4.4-5.6 \mathrm{~m} \mathrm{~W}-\mathrm{E} / 0.8-3 \mathrm{~m} \mathrm{~N}-\mathrm{S}$ & charcoal (1 bag) & 062 & 03.02 .2014 \\
\hline $2110 / 2014$ & SQ1B, $0-1.5 \mathrm{~m} \mathrm{~W}-\mathrm{E} / 3.7-4.7 \mathrm{~m} \mathrm{~N}-\mathrm{S}$ & charcoal (3 pieces) & 063 & 02.02 .2014 \\
\hline $2120 / 2014$ & $\mathrm{SQ} 1 \mathrm{~B}, 0-0.8 \mathrm{~m} \mathrm{~W}-\mathrm{E} / 4.2-5 \mathrm{~m} \mathrm{~N}-\mathrm{S}$ & charcoal (1 bag) & 064 & 03.02 .2014 \\
\hline $2129 / 2014$ & SQ1B, 0-1.1m W-E/4-5m N-S & charcoal (1 bag) & 065 & 03.02 .2014 \\
\hline $2168 / 2014$ & $\mathrm{SQ} 3,4.8-5.5 \mathrm{~m} \mathrm{~W}-\mathrm{E} / 4-6.8 \mathrm{~m} \mathrm{~N}-\mathrm{S}$ & charcoal (1 bag) & 066 & 04.02 .2014 \\
\hline $2142 / 2014$ & $\mathrm{SQ} 3,4.8-7 \mathrm{~m} \mathrm{~W}-\mathrm{E} / 4-8.3 \mathrm{~m} \mathrm{~N}-\mathrm{S}$ & charcoal (1 bag); bone & 066 & 03.02 .2014 \\
\hline $2143 / 2014$ & $\mathrm{SQ} 3,4.8-7 \mathrm{~m} \mathrm{~W}-\mathrm{E} / 4-8.3 \mathrm{~m} \mathrm{~N}-\mathrm{S}$ & charcoal (1 bag) & 066 & 03.02 .2014 \\
\hline $2134 / 2014$ & $\mathrm{SQ} 1 \mathrm{~B}, 0-1.1 \mathrm{~m} \mathrm{~W}-\mathrm{E} / 3-4.2 \mathrm{~m} \mathrm{~N}-\mathrm{S}$ & charcoal (1 bag) & 067 & 03.02 .2014 \\
\hline $2138 / 2014$ & SQ1B, $0.5-2 \mathrm{~m} \mathrm{~W}-\mathrm{E} / 3.8-4.7 \mathrm{~m} \mathrm{~N}-\mathrm{S}$ & charcoal (1 bag) & 068 & 03.02 .2014 \\
\hline $2151 / 2014$ & $\mathrm{SQ} 3,9-10.5 \mathrm{~m} \mathrm{~W}-\mathrm{E} / 3.6-5.3 \mathrm{~m} \mathrm{~N}-\mathrm{S}$ & charcoal (1 bag) & 069 & 03.02 .2014 \\
\hline
\end{tabular}




\begin{tabular}{|c|c|c|c|c|}
\hline $2156 / 2014$ & $\mathrm{SQ} 3,9-10.5 \mathrm{~m} \mathrm{~W}-\mathrm{E} / 3.6-5.3 \mathrm{~m} \mathrm{~N}-\mathrm{S}$ & charcoal (1 bag) & 069 & 04.02 .2014 \\
\hline $2255 / 2014$ & $\mathrm{SQ} 3,8-10.5 \mathrm{~m} \mathrm{~W}-\mathrm{E} / 3.6-5.5 \mathrm{~m} \mathrm{~N}-\mathrm{S}$ & charcoal (1 bag) & 070 & 06.02 .2014 \\
\hline $2259 / 2014$ & $\mathrm{SQ} 3,8-10.5 \mathrm{~m} \mathrm{~W}-\mathrm{E} / 3.6-5.5 \mathrm{~m} \mathrm{~N}-\mathrm{S}$ & charcoal (1 bag) & 070 & 08.02 .2014 \\
\hline $2208 / 2014$ & $\mathrm{SQ} 3,8.5-10.5 \mathrm{~m} \mathrm{~W}-\mathrm{E} / 5-7.8 \mathrm{~m} \mathrm{~N}-\mathrm{S}$ & charcoal (1 bag) & 070 & 05.02 .2014 \\
\hline $2229 / 2014$ & $\mathrm{SQ} 3,8.5-10.5 \mathrm{~m} \mathrm{~W}-\mathrm{E} / 5-8.5 \mathrm{~m} \mathrm{~N}-\mathrm{S}$ & charcoal (1 bag) & 070 & 06.02 .2014 \\
\hline $2221 / 2014$ & $\mathrm{SQ} 3,8.5-10.5 \mathrm{~m} \mathrm{~W}-\mathrm{E} / 5-8.5 \mathrm{~m} \mathrm{~N}-\mathrm{S}$ & charcoal ( 2 pieces) & 070 & 05.02 .2014 \\
\hline $2240 / 2014$ & $\mathrm{SQ} 3,9-10.5 \mathrm{~m} \mathrm{~W}-\mathrm{E} / 2-3.6 \mathrm{~m} \mathrm{~N}-\mathrm{S}$ & charcoal (1 bag) & 070 & 06.02 .2014 \\
\hline $2251 / 2014$ & SQ3, 9-10.5m W-E/8-9.5m N-S & charcoal (1 bag) & 070 & 06.02 .2014 \\
\hline $2198 / 2014$ & SQ3, 9.2-10.5m W-E/4.7-5.9m N-S & charcoal (1 bag) & 070 & 05.02 .2014 \\
\hline $2245 / 2014$ & SQ3, $9.2-10.5 \mathrm{~m} \mathrm{~W}-\mathrm{E} / 8-9.2 \mathrm{~m} \mathrm{~N}-\mathrm{S}$ & charcoal (1 bag) & 070 & 06.02 .2014 \\
\hline $2172 / 2014$ & $\begin{array}{l}\mathrm{SQ} 3,8.5-10.5 \mathrm{~m} \mathrm{~W}-\mathrm{E} / 0.8-2.3 \mathrm{~m} \mathrm{~N}-\mathrm{S} \text {, } \\
\text { Feature } 7\end{array}$ & charcoal (1 bag) & 071 & 04.02 .2014 \\
\hline $2186 / 2014$ & $\mathrm{SQ} 3,6-8 \mathrm{~m} \mathrm{~W}-\mathrm{E} / 0-7 \mathrm{~m} \mathrm{~N}-\mathrm{S}$ & charcoal (1 bag) & 072 & 04.02 .2014 \\
\hline $2204 / 2014$ & $\mathrm{SQ} 3,6.6-9 \mathrm{~m} \mathrm{~W}-\mathrm{E} / 1.1-4.5 \mathrm{~m} \mathrm{~N}-\mathrm{S}$ & charcoal (1 bag) & 073 & 05.02 .2014 \\
\hline $2225 / 2014$ & $\mathrm{SQ} 3,5.7-6.2 \mathrm{~m} \mathrm{~W}-\mathrm{E} / 0.5-2.2 \mathrm{~m} \mathrm{~N}-\mathrm{S}$ & charcoal (1 bag) & 075 & 05.02 .2014 \\
\hline $2214 / 2014$ & $\mathrm{SQ} 3,6-8 \mathrm{~m} \mathrm{~W}-\mathrm{E} / 0.2-1.1 \mathrm{~m} \mathrm{~N}-\mathrm{S}$ & charcoal (1 bag) & 075 & 05.02 .2014 \\
\hline $2233 / 2014$ & $\mathrm{SQ} 3,8.2-10 \mathrm{~m} \mathrm{~W}-\mathrm{E} / 0-2 \mathrm{~m} \mathrm{~N}-\mathrm{S}$ & charcoal (1 bag) & 076 & 06.02 .2014 \\
\hline $2236 / 2014$ & $\mathrm{SQ} 3,8.2-10 \mathrm{~m} \mathrm{~W}-\mathrm{E} / 0.3-1.5 \mathrm{~m} \mathrm{~N}-\mathrm{S}$ & charcoal (4 pieces) & 077 & 06.02 .2014 \\
\hline $2266 / 2014$ & $\begin{array}{l}\text { SQ4, } 9.5-10.5 \mathrm{~m} \mathrm{~W}-\mathrm{E} / 0.6-2 \mathrm{~m} \mathrm{~N}-\mathrm{S} \text {, } \\
\text { Feature } 15\end{array}$ & charcoal (1 bag) & 079 & 08.02 .2014 \\
\hline $2271 / 2014$ & $\mathrm{SQ} 3,6-7.5 \mathrm{~m} \mathrm{~W}-\mathrm{E} / 3.5-5 \mathrm{~m} \mathrm{~N}-\mathrm{S}$ & charcoal (1 bag) & 080 & 08.02 .2014 \\
\hline $2279 / 2014$ & $\mathrm{SQ} 3,6-8 \mathrm{~m} \mathrm{~W}-\mathrm{E} / 5-7.5 \mathrm{~m} \mathrm{~N}-\mathrm{S}$ & charcoal (1 bag) & 080 & 08.02 .2014 \\
\hline $2288 / 2014$ & $\mathrm{SQ} 3,6-8.5 \mathrm{~m} \mathrm{~W}-\mathrm{E} / 5-10 \mathrm{~m} \mathrm{~N}-\mathrm{S}$ & charcoal (1 bag) & 080 & 09.02 .2014 \\
\hline $2313 / 2014$ & $\mathrm{SQ} 3,8-9.4 \mathrm{~m} \mathrm{~W}-\mathrm{E} / 2.3-3.8 \mathrm{~m} \mathrm{~N}-\mathrm{S}$ & charcoal (3 pieces) & 090 & 10.02 .2014 \\
\hline $2424 / 2014$ & SQ4, debris in Feature 15 & charcoal (1 piece) & 092 & 24.02 .2014 \\
\hline $2317 / 2014$ & $\mathrm{SQ} 3,7.7-8.8 \mathrm{~m} \mathrm{~W}-\mathrm{E} / 0-3.5 \mathrm{~m} \mathrm{~N}-\mathrm{S}$ & charcoal (1 bag) & 093 & 10.02 .2014 \\
\hline $2320 / 2014$ & $\mathrm{SQ} 3,7.7-8.8 \mathrm{~m} \mathrm{~W}-\mathrm{E} / 0-3.5 \mathrm{~m} \mathrm{~N}-\mathrm{S}$ & charcoal (2 pieces) & 095 & 11.02 .2014 \\
\hline $2328 / 2014$ & $\mathrm{SQ} 4,9.5-10.5 \mathrm{~m} \mathrm{~W}-\mathrm{E} / 3.5-4.2 \mathrm{~m} \mathrm{~N}-\mathrm{S}$ & charcoal (1 bag) & 098 & 11.02 .2014 \\
\hline
\end{tabular}




\begin{tabular}{|c|c|c|c|c|}
\hline $2340 / 2014$ & $\mathrm{SQ} 4,8.4-10.5 \mathrm{~m} \mathrm{~W}-\mathrm{E} / 2.5-4.2 \mathrm{~m} \mathrm{~N}-\mathrm{S}$ & charcoal (1 bag) & 099 & 11.02 .2014 \\
\hline $2352 / 2014$ & $\mathrm{SQ} 4,2-4 \mathrm{~m} \mathrm{~W}-\mathrm{E} / 0-3.6 \mathrm{~m} \mathrm{~N}-\mathrm{S}$ & charcoal (1 bag) & 100 & 12.02 .2014 \\
\hline $2361 / 2014$ & $\mathrm{SQ} 4,3.5-6 \mathrm{~m} \mathrm{~W}-\mathrm{E} / 0-3.4 \mathrm{~m} \mathrm{~N}-\mathrm{S}$ & charcoal (1 bag) & 105 & 12.02 .2014 \\
\hline $2388 / 2014$ & $\mathrm{SQ} 4,3.5-6 \mathrm{~m} \mathrm{~W}-\mathrm{E} / 0-3.5 \mathrm{~m} \mathrm{~N}-\mathrm{S}$ & charcoal (1 bag) & 105 & 13.02 .2014 \\
\hline $2374 / 2014$ & $\mathrm{SQ} 4,3.5-6 \mathrm{~m} \mathrm{~W}-\mathrm{E} / 2.5-4.7 \mathrm{~m} \mathrm{~N}-\mathrm{S}$ & charcoal (1 bag) & 105 & 13.02 .2014 \\
\hline $2378 / 2014$ & $\mathrm{SQ} 4,9-10.5 \mathrm{~m} \mathrm{~W}-\mathrm{E} / 5.6-7.2 \mathrm{~m} \mathrm{~N}-\mathrm{S}$ & charcoal (1 bag) & 108 & 13.02 .2014 \\
\hline $2383 / 2014$ & $\begin{array}{l}\text { SQ4, } 8.5-9.5 \mathrm{~m} \mathrm{~W}-\mathrm{E} / 5.6-7.4 \mathrm{~m} \mathrm{~N}-\mathrm{S} \text {, } \\
\text { Feature } 16\end{array}$ & charcoal (1 bag) & 109 & 13.02 .2014 \\
\hline $2392 / 2014$ & $\begin{array}{l}\text { SQ4, 8.5-10.5m W-E/5.6-7m N-S, } \\
\text { Feature } 16\end{array}$ & charcoal (1 bag) & 109 & 15.02 .2014 \\
\hline $2396 / 2014$ & $\mathrm{SQ} 4,7.3-8.7 \mathrm{~m} \mathrm{~W}-\mathrm{E} / 0-2 \mathrm{~m} \mathrm{~N}-\mathrm{S}$ & charcoal (1 bag) & 110 & 16.02 .2014 \\
\hline $2404 / 2014$ & $\mathrm{SQ} 4,7.3-8 \mathrm{~m} \mathrm{~W}-\mathrm{E} / 0-2.5 \mathrm{~m} \mathrm{~N}-\mathrm{S}$ & charcoal (1 bag) & 111 & 16.02 .2014 \\
\hline $2409 / 2014$ & $\mathrm{SQ} 4,5.7-7.2 \mathrm{~m} \mathrm{~W}-\mathrm{E} / 0.5-10.5 \mathrm{~m} \mathrm{~N}-\mathrm{S}$ & charcoal (1 piece); bone & 112 & 18.02 .2014 \\
\hline $2182 / 2014$ & $\begin{array}{l}\text { SQ2, 0-2.5m W-E/0.5-1.8m N-S, } \\
\text { Feature } 15 \text {, cleaning }\end{array}$ & charcoal (1 bag) & / & 04.02 .2014 \\
\hline $2190 / 2014$ & $\begin{array}{l}\text { SQ2, } 0.5-1.8 \mathrm{~m} \mathrm{~N}-\mathrm{S} \text {, Feature } 15 \text {, cleaning of } \\
\text { W profile }\end{array}$ & charcoal & l & 04.02 .2014 \\
\hline $147 / 2014$ & $\begin{array}{l}\mathrm{SQ} 4,2.5-5.5 \mathrm{~m} \mathrm{~W}-\mathrm{E} / 9-10 \mathrm{~m} \mathrm{~N}-\mathrm{S} ; \mathrm{SQ} 4 \mathrm{~A} \\
2.5-6.5 \mathrm{~m} \mathrm{~W}-\mathrm{E} / 0-2 \mathrm{~m} \mathrm{~N}-\mathrm{S}\end{array}$ & dates (1 bag) & 009 & 14.01.2014 \\
\hline $395 / 2014$ & $\mathrm{SQ} 3,9-10.5 \mathrm{~m} \mathrm{~W}-\mathrm{E} / 3.6-5.3 \mathrm{~m} \mathrm{~N}-\mathrm{S}$ & date seed & 036 & 23.01 .2014 \\
\hline $062 / 2014$ & $\mathrm{SQ} 3,0.2 \mathrm{~mW}-\mathrm{E} / 2.5 \mathrm{~m} \mathrm{~N}-\mathrm{S}$ & doum nut (1 piece) & 003 & 09.01 .2014 \\
\hline $100 / 2014$ & $\mathrm{SQ} 4,1-2.5 \mathrm{~m} \mathrm{~W}-\mathrm{E} / 1.5-2 \mathrm{~m} \mathrm{~N}-\mathrm{S}$ & doum nut (1 piece) & 008 & 12.01.2014 \\
\hline $126 / 2014$ & $\begin{array}{l}\text { SQ4, 2.5-5.5m W-E/9-10m N-S; SQ4A } \\
2.5-6.5 \mathrm{~m} \mathrm{~W}-\mathrm{E} / 0-2 \mathrm{~m} \mathrm{~N}-\mathrm{S}\end{array}$ & doum nut (1 piece) & 009 & 13.01.2014 \\
\hline $177 / 2014$ & $\mathrm{SQ} 3,3-5 \mathrm{~m} \mathrm{~W}-\mathrm{E} / 0-3.5 \mathrm{~m} \mathrm{~N}-\mathrm{S}$ & doum nuts (2 pieces) & 013 & 15.01 .2014 \\
\hline $171 / 2014$ & $\mathrm{SQ} 4,1-3 \mathrm{~m} \mathrm{~W}-\mathrm{E} / 0-4 \mathrm{~m} \mathrm{~N}-\mathrm{S}$ & doum nut (1 piece) & 014 & 15.01.2014 \\
\hline $358 / 2014$ & $\mathrm{SQ} 4,0-2.5 \mathrm{~m} \mathrm{~W}-\mathrm{E} / 4.5-5.5 \mathrm{~m} \mathrm{~N}-\mathrm{S}$ & doum nut (1 piece) & 015 & 22.01 .2014 \\
\hline 199/2014 & $\mathrm{SQ} 4,0-5 \mathrm{~m} \mathrm{~W}-\mathrm{E} / 5-8 \mathrm{~m} \mathrm{~N}-\mathrm{S}$ & doum nut (1 piece) & 015 & 16.01 .2014 \\
\hline $239 / 2014$ & $\mathrm{SQ} 4,1.5-5 \mathrm{~m} \mathrm{~W}-\mathrm{E} / 8-8.5 \mathrm{~m} \mathrm{~N}-\mathrm{S}$ & doum nut (1 piece) & 015 & 18.01 .2014 \\
\hline $188 / 2014$ & SQ4A, 9-10.5m W-E/0-2m N-S & doum nut (1 piece) & 016 & 16.01 .2014 \\
\hline
\end{tabular}




\begin{tabular}{|c|c|c|c|c|c|}
\hline $217 / 2014$ & SQ1B, 0-3m W-E/0-2m N-S & doum nuts ( 2 pieces) & 018 & & 18.01 .2014 \\
\hline $295 / 2014$ & $\mathrm{SQ} 1 \mathrm{~B}, 4.5 \mathrm{~m} \mathrm{~W}-\mathrm{E} / 2.5 \mathrm{~m} \mathrm{~N}-\mathrm{S}$ & doum nuts (2 pieces) & 021 & & 20.01 .2014 \\
\hline $293 / 2014$ & $\mathrm{SQ} 3,2-3 \mathrm{~m} \mathrm{~W}-\mathrm{E} / 0-4.4 \mathrm{~m} \mathrm{~N}-\mathrm{S}$ & doum nut (1 piece) & 023 & & 20.01 .2014 \\
\hline $2410 / 2014$ & $\mathrm{SQ} 4,5.7-7.2 \mathrm{~m} \mathrm{~W}-\mathrm{E} / 0.5-10.5 \mathrm{~m} \mathrm{~N}-\mathrm{S}$ & doum nuts ( 2 pieces) & 112 & & 18.02 .2014 \\
\hline $231 / 2014$ & $\mathrm{SQ} 4,1.5-5 \mathrm{~m} \mathrm{~W}-\mathrm{E} / 3.5-5 \mathrm{~m} \mathrm{~N}-\mathrm{S}$ & dung (1 piece) & 015 & & 18.01.2014 \\
\hline $328 / 2014$ & $\mathrm{SQ} 3,8-10.5 \mathrm{~m} \mathrm{~W}-\mathrm{E} / 5-8 \mathrm{~m} \mathrm{~N}-\mathrm{S}$ & dung (1 piece) & 027 & & 21.01.2014 \\
\hline $390 / 2014$ & $\mathrm{SQ} 3,9-10.5 \mathrm{~m} \mathrm{~W}-\mathrm{E} / 3.6-5.3 \mathrm{~m} \mathrm{~N}-\mathrm{S}$ & dung (1 piece); bone & 036 & & 23.01.2014 \\
\hline $2048 / 2014$ & SQ1B, 4-6.5m W-E/0-1.5m N-S & dung (1 bag) & 051 & & 30.01 .2014 \\
\hline $2274 / 2014$ & SQ4, $9.5-10.5 \mathrm{~m} \mathrm{~W}-\mathrm{E} / 0.6-2 \mathrm{~m} \mathrm{~N}-\mathrm{S}$ & fabric/organic material & 079 & & 08.02 .2014 \\
\hline $314 / 2014$ & $\mathrm{SQ} 3,9.25 \mathrm{~m} \mathrm{~W}-\mathrm{E} / 6.5 \mathrm{~m} \mathrm{~N}-\mathrm{S}$ & faience vessel?/glazed ware? (1 fragment) & 002 & SAV1E 0673 & 20.01 .2014 \\
\hline $049 / 2014$ & $\mathrm{SQ} 3,4.5 \mathrm{~m} \mathrm{~W}-\mathrm{E} / 2 \mathrm{~m} \mathrm{~N}-\mathrm{S}$ & faience vessel (1 rim fragment) & 005 & SAV1E 0351 & 08.01 .2014 \\
\hline $225 / 2014$ & SQ1B, 2m W-E/5m N-S, Feature 29 & faience vessel (1 fragment of rim sherd) & 019 & SAV1E 0635 & 18.01 .2014 \\
\hline $301 / 2014$ & $\mathrm{SQ} 1 \mathrm{~B}, 7.5 \mathrm{~m} \mathrm{~W}-\mathrm{E} / 2 \mathrm{~m} \mathrm{~N}-\mathrm{S}$ & faience vessel? (1 fragment) & 021 & SAV1E 0667 & 20.01.2014 \\
\hline $421 / 2014$ & $\mathrm{SQ} 3,1.3 \mathrm{~m} \mathrm{~W}-\mathrm{E} / 9.8 \mathrm{~m} \mathrm{~N}-\mathrm{S}$ & faience vessel ( 1 body sherd) & 041 & SAV1E 0796 & 25.01.2014 \\
\hline $152 / 2014$ & $\mathrm{SQ} 4 \mathrm{~A}, 5 \mathrm{~m} \mathrm{~W}-\mathrm{E} / 1.30 \mathrm{~m} \mathrm{~N}-\mathrm{S}$ & glass vessel? (1 fragment) & 009 & SAV1E 0596 & 14.01 .2014 \\
\hline $300 / 2014$ & SQ1B, 7.3m W-E/0.5m N-S & glass vessel? (1 fragment) & 021 & SAV1E 0658 & 20.01.2014 \\
\hline $405 / 2014$ & $\mathrm{SQ} 3,0.3 \mathrm{~m} \mathrm{~W}-\mathrm{E} / 5.4 \mathrm{~m} \mathrm{~N}-\mathrm{S}$ & glass vessel? (1 fragment) & 038 & SAV1E 0739 & 25.01 .2014 \\
\hline $2027 / 2014$ & SQ1B, $1.5-4 \mathrm{~m} \mathrm{~W}-\mathrm{E} / 0-2.3 \mathrm{~m} \mathrm{~N}-\mathrm{S}$ & glass vessels? (2 fragments, modern?) & 051 & SAV1E 0855, SAV1E 0856 (glass vessels?) & 29.01.2014 \\
\hline $2035 / 2014$ & $\mathrm{SQ} 1 \mathrm{~B}, 4-6.5 \mathrm{~m} \mathrm{~W}-\mathrm{E} / 0-1.5 \mathrm{~m} \mathrm{~N}-\mathrm{S}$ & glass & 051 & & 29.01.2014 \\
\hline $2042 / 2014$ & SQ1B, 4-6.5m W-E/0-1.5m N-S & glass vessel (1 fragment, modern?) & 051 & SAV1E 0894 & 30.01 .2014 \\
\hline $2406 / 2014$ & $\mathrm{SQ} 4,7.3-8 \mathrm{~m} \mathrm{~W}-\mathrm{E} / 0-2.5 \mathrm{~m} \mathrm{~N}-\mathrm{S}$ & glass vessel? (1 fragment) & 111 & SAV1E 1130 & 16.02 .2014 \\
\hline $2192 / 2014$ & $\begin{array}{l}\text { SQ2, } 0.5-1.8 \mathrm{~m} \mathrm{~N}-\mathrm{S} \text {, Feature 15, cleaning of } \\
\text { W-profile }\end{array}$ & glass vessel (1 fragment) & l & SAV1E 0970 & 04.02 .2014 \\
\hline $050 / 2014$ & $\mathrm{SQ} 3,3 \mathrm{~m} \mathrm{~W}-\mathrm{E} / 0.2 \mathrm{~m} \mathrm{~N}-\mathrm{S}$ & glazed vessel (1 fragment, glazed ware, Ottoman) & 005 & SAV1E 0352 & 08.01 .2014 \\
\hline $237 / 2014$ & $\mathrm{SQ} 4,5 \mathrm{~m} \mathrm{~W}-\mathrm{E} / 8.2 \mathrm{~N}-\mathrm{S}$ & glazed vessel (1 fragment, glazed ware, Ottoman?) & 015 & SAV1E 0636 & 18.01 .2014 \\
\hline $379 / 2014$ & $\mathrm{SQ} 4,5.5 \mathrm{~m} \mathrm{~W}-\mathrm{E} / 4.8 \mathrm{~m} \mathrm{~N}-\mathrm{S}$ & glazed vessel (1 fragment of a rim sherd, glazed ware, Ottoman?) & 033 & SAV1E 0738 & 23.01 .2014 \\
\hline $1519 / 2014$ & $\mathrm{SQ} 3,2.7-5.2 \mathrm{~m} \mathrm{~W}-\mathrm{E} / 7-8.5 \mathrm{~m} \mathrm{~N}-\mathrm{S}$ & glazed vessel (1 fragment, glazed ware), pottery & 047 & SAV1E 0903 (glazed vessel) & 28.01 .2014 \\
\hline
\end{tabular}




\begin{tabular}{|c|c|c|c|c|c|}
\hline $2111 / 2014$ & SQ1B, $6.5-10 \mathrm{~m} \mathrm{~W}-\mathrm{E} / 0-1.7 \mathrm{~m} \mathrm{~N}-\mathrm{S}$ & $\begin{array}{l}\text { glazed vessel (1 fragment, glazed ware/faience?), see also } \\
2075 / 2014 \text { (joint), pottery }\end{array}$ & 048 & SAV1E 0923 & 02.02 .2014 \\
\hline $2075 / 2014$ & SQ1B, 2m W-E/3m N-S & $\begin{array}{l}\text { glazed vessel ( } 1 \text { fragment, glazed ware/faience?), see also } \\
2111 / 2014 \text { (new joint) }\end{array}$ & 056 & SAV1E 0923 & 01.02 .2014 \\
\hline $153 / 2014$ & $\mathrm{SQ} 4,5.5 \mathrm{~m} \mathrm{~W}-\mathrm{E} / 9.5 \mathrm{~m} \mathrm{~N}-\mathrm{S}$ & hair/fur (animal, 1 bunch) & 009 & & 14.01.2014 \\
\hline $169 / 2014$ & $\mathrm{SQ} 4,1-3 \mathrm{~m} \mathrm{~W}-\mathrm{E} / 0-4 \mathrm{~m} \mathrm{~N}-\mathrm{S}$ & hair (1 bag) & 014 & & 15.01 .2014 \\
\hline $273 / 2014$ & SQ4, 0-1.5m W-E/8-10m N-S & hair (1 bag) & 022 & & 19.01.2014 \\
\hline $2121 / 2014$ & SQ1B, $0-0.8 \mathrm{~m} \mathrm{~W}-\mathrm{E} / 4.2-5 \mathrm{~m} \mathrm{~N}-\mathrm{S}$ & hair (1 bag) & 064 & & 03.02 .2014 \\
\hline $2191 / 2014$ & $\begin{array}{l}\text { SQ2, 0.5-1.8m N-S, Feature 15, cleaning of } \\
\text { W-profile }\end{array}$ & hair in gypsum (1 bag) & l & & 04.02 .2014 \\
\hline $2370 / 2014$ & $\mathrm{SQ} 4,9.7 \mathrm{~m} \mathrm{~W}-\mathrm{E} / 7.1 \mathrm{~m} \mathrm{~N}-\mathrm{S}$ & iron object (1 fragment, original function unknown) & 107 & SAV1E 2155 & 13.02 .2014 \\
\hline $192 / 2014$ & SQ4A, 9-10.5m W-E/0-2m N-S & leaf (1 bag) & 016 & & 16.01 .2014 \\
\hline $2163 / 2014$ & $\mathrm{SQ} 3,4.8-7 \mathrm{~m} \mathrm{~W}-\mathrm{E} / 4-8.3 \mathrm{~m} \mathrm{~N}-\mathrm{S}$ & leather (1 piece); bone & 066 & & 04.02 .2014 \\
\hline $360 / 2014$ & $\mathrm{SQ} 4,0.5-1 \mathrm{~m} \mathrm{~W}-\mathrm{E} / 3-4 \mathrm{~m} \mathrm{~N}-\mathrm{S}$ & lime & 008 & & 22.01 .2014 \\
\hline $369 / 2014$ & $\mathrm{SQ} 4,0.5-1 \mathrm{~m} \mathrm{~W}-\mathrm{E} / 3-4 \mathrm{~m} \mathrm{~N}-\mathrm{S}$ & lime & 008 & & 23.01 .2014 \\
\hline $168 / 2014$ & $\mathrm{SQ} 4,1-3 \mathrm{~m} \mathrm{~W}-\mathrm{E} / 0-4 \mathrm{~m} \mathrm{~N}-\mathrm{S}$ & lime & 014 & & 15.01 .2014 \\
\hline $357 / 2014$ & $\mathrm{SQ} 4,0-2.5 \mathrm{~m} \mathrm{~W}-\mathrm{E} / 4.5-5.5 \mathrm{~m} \mathrm{~N}-\mathrm{S}$ & lime & 015 & & 22.01 .2014 \\
\hline $205 / 2014$ & $\mathrm{SQ} 4,0-5 \mathrm{~m} \mathrm{~W}-\mathrm{E} / 5-8 \mathrm{~m} \mathrm{~N}-\mathrm{S}$ & lime & 015 & & 16.01 .2014 \\
\hline $189 / 2014$ & $\mathrm{SQ} 4 \mathrm{~A}, 9-10.5 \mathrm{~m} \mathrm{~W}-\mathrm{E} / 0-2 \mathrm{~m} \mathrm{~N}-\mathrm{S}$ & lime & 016 & & 16.01 .2014 \\
\hline $197 / 2014$ & SQ4A, $10-10.5 \mathrm{~m} \mathrm{~W}-\mathrm{E} / 0-0.5 \mathrm{~m} \mathrm{~N}-\mathrm{S}$ & lime & 017 & & 16.01 .2014 \\
\hline $333 / 2014$ & $\mathrm{SQ} 3,0-2 \mathrm{~m} \mathrm{~W}-\mathrm{E} / 4.4-10 \mathrm{~m} \mathrm{~N}-\mathrm{S}$ & lime & 025 & & 21.01 .2014 \\
\hline $344 / 2014$ & $\mathrm{SQ} 4,0-3 \mathrm{~m} \mathrm{~W}-\mathrm{E} / 1-5.5 \mathrm{~m} \mathrm{~N}-\mathrm{S}$ & lime & 030 & & 22.01 .2014 \\
\hline $350 / 2014$ & $\mathrm{SQ} 3,2.5-9 \mathrm{~m} \mathrm{~W}-\mathrm{E} / 0-10 \mathrm{~m} \mathrm{~N}-\mathrm{S}$ & lime & 031 & & 22.01 .2014 \\
\hline $387 / 2014$ & $\mathrm{SQ} 3,9-10.5 \mathrm{~m} \mathrm{~W}-\mathrm{E} / 2.6-5.5 \mathrm{~m} \mathrm{~N}-\mathrm{S}$ & lime & 034 & & 23.01 .2014 \\
\hline $420 / 2014$ & $\mathrm{SQ} 3,1-5 \mathrm{~m} \mathrm{~W}-\mathrm{E} / 7.2-10 \mathrm{~m} \mathrm{~N}-\mathrm{S}$ & lime & 041 & & 25.01 .2014 \\
\hline $2210 / 2014$ & $\mathrm{SQ} 3,8.5-10.5 \mathrm{~m} \mathrm{~W}-\mathrm{E} / 5-7.8 \mathrm{~m} \mathrm{~N}-\mathrm{S}$ & lime & 070 & & 05.02 .2014 \\
\hline $2247 / 2014$ & SQ3, 9.2-10.5m W-E/8-9.2m N-S & lime & 070 & & 06.02 .2014 \\
\hline $2380 / 2014$ & $\mathrm{SQ} 4,9-10.5 \mathrm{~m} \mathrm{~W}-\mathrm{E} / 5.6-7.2 \mathrm{~m} \mathrm{~N}-\mathrm{S}$ & lime & 108 & & 13.02 .2014 \\
\hline
\end{tabular}




\begin{tabular}{|c|c|c|c|c|c|}
\hline $2040 / 2014$ & $\mathrm{SQ} 3,2.4 \mathrm{~m} \mathrm{~W}-\mathrm{E} / 3.2 \mathrm{~m} \mathrm{~N}-\mathrm{S}$ & metal (2 fragments, 1 fish hook?, each bronze?) & 052 & SAV1E 0887 & 29.01 .2014 \\
\hline $2217 / 2014$ & SQ3, 6-8m W-E/0.2-1.1m N-S & metal object (2 pieces, copper?) & 075 & SAV1E 1010 & 05.02 .2014 \\
\hline $2293 / 2014$ & SQ4, 9.4m W-E/1.1m N-S, Feature 15 & mortar (1 piece, sandstone, with possible content) & 084 & SAV1E 1644 & 09.02.2014 \\
\hline $016 / 2014$ & $\mathrm{SQ} 4,10 \times 10 \mathrm{~m}$, surface cleaning & mould for figurine (1 fragment, pottery) & 001 & SAV1E 0334 & 04.01 .2014 \\
\hline $2268 / 2014$ & $\begin{array}{l}\text { SQ4, } 9.5-10.5 \mathrm{~m} \mathrm{~W}-\mathrm{E} / 0.6-2 \mathrm{~m} \mathrm{~N}-\mathrm{S} \text {, } \\
\text { Feature } 15\end{array}$ & $\begin{array}{l}\text { mud object/architecture?/functional device? (1 piece, mud); } \\
\text { worked bone? (1 piece) }\end{array}$ & 079 & $\begin{array}{l}\text { SAV1E } 1078 \text { (mud } \\
\text { object/architecture?/functional device?), } \\
\text { SAV1E } 1079 \text { (worked bone?) }\end{array}$ & 08.02 .2014 \\
\hline $2011 / 2014$ & $\mathrm{SQ} 3,2.5-5.3 \mathrm{~m} \mathrm{~W}-\mathrm{E} / 8.5-10 \mathrm{~m} \mathrm{~N}-\mathrm{S}$ & $\begin{array}{l}\text { necklace or bracelet ( } 7 \text { smaller beads, } 1 \text { large bead, faience/bone, } \\
\text { ivory?) }\end{array}$ & 050 & SAV1E 0833 & 28.01 .2014 \\
\hline $014 / 2014$ & $\mathrm{SQ} 3,10 \times 10 \mathrm{~m}$, surface cleaning & Nun bowl? (1 rim sherd, faience) & 001 & SAV1E 0350 & 04.01 .2014 \\
\hline $084 / 2014$ & $\mathrm{SQ} 4,8 \mathrm{~m} \mathrm{~W}-\mathrm{E} / 1 \mathrm{~m} \mathrm{~N}-\mathrm{S}$ & Nun bowl? (1 fragment from vessel body, faience) & 002 & SAV1E 0394 & 11.01.2014 \\
\hline $063 / 2014$ & $\mathrm{SQ} 3,4 \mathrm{~m} \mathrm{~W}-\mathrm{E} / 6 \mathrm{~m} \mathrm{~N}-\mathrm{S}$ & organic material (woven - textile?) & 006 & & 09.01.2014 \\
\hline $099 / 2014$ & $\mathrm{SQ} 4,1-2.5 \mathrm{~m} \mathrm{~W}-\mathrm{E} / 0-1.5 \mathrm{~m} \mathrm{~N}-\mathrm{S}$ & organic material (1 bag, sample) & 008 & & 12.01 .2014 \\
\hline $120 / 2014$ & $\begin{array}{l}\text { SQ4, 2.5-5.5m W-E/9-10m N-S; } \\
\text { SQ4A, 2.5-6.5m W-E/0-2m N-S }\end{array}$ & organic material (digested by fox?) & 009 & & 13.01.2014 \\
\hline $132 / 2014$ & SQ4A, 4m W-E/1m N-S & organic material & 009 & & 13.01 .2014 \\
\hline $175 / 2014$ & $\mathrm{SQ} 3,3-5 \mathrm{~m} \mathrm{~W}-\mathrm{E} / 0-3.5 \mathrm{~m} \mathrm{~N}-\mathrm{S}$ & organic material/botanical fragments & 013 & & 15.01 .2014 \\
\hline $216 / 2014$ & SQ1B, 0-3m W-E/0-2m N-S & organic material & 018 & & 18.01 .2014 \\
\hline $281 / 2014$ & SQ3, 0-2m W-E/0-4.4m N-S & organic material & 023 & & 19.01.2014 \\
\hline $327 / 2014$ & $\mathrm{SQ} 3,8-10.5 \mathrm{~m} \mathrm{~W}-\mathrm{E} / 5-8 \mathrm{~m} \mathrm{~N}-\mathrm{S}$ & organic material (1 bag) & 027 & & 21.01 .2014 \\
\hline $386 / 2014$ & SQ3, 9-10.5m W-E/2.6-5.5m N-S & $\begin{array}{l}\text { organic and botanical material (1 bag, wood and modern insect } \\
\text { cocoon) }\end{array}$ & 034 & & 23.01.2014 \\
\hline $2016 / 2014$ & SQ1B, $0-4 \mathrm{~m} \mathrm{~W}-\mathrm{E} / 0-2.3 \mathrm{~m} \mathrm{~N}-\mathrm{S}$ & organic material & 051 & & 28.01 .2014 \\
\hline $2095 / 2014$ & $\mathrm{SQ} 1 \mathrm{~B}, 0-1.5 \mathrm{~m} \mathrm{~W}-\mathrm{E} / 2.5-4.5 \mathrm{~m} \mathrm{~N}-\mathrm{S}$ & organic material (1 bag, sample) & 056 & & 01.02 .2014 \\
\hline $2071 / 2014$ & SQ1B, 0-2.8m W-E/3-4m N-S, Feature 38 & organic material (1 piece, stick); seashell (1 fragment); bone & 056 & & 01.02 .2014 \\
\hline $2135 / 2014$ & SQ1B, $0-1.1 \mathrm{~m} \mathrm{~W}-\mathrm{E} / 3-4.2 \mathrm{~m} \mathrm{~N}-\mathrm{S}$ & organic material (1 bag, sample) & 067 & & 03.02 .2014 \\
\hline $2157 / 2014$ & $\mathrm{SQ} 3,9-10.5 \mathrm{~m} \mathrm{~W}-\mathrm{E} / 3.6-5.3 \mathrm{~m} \mathrm{~N}-\mathrm{S}$ & organic material & 069 & & 04.02 .2014 \\
\hline $2216 / 2014$ & $\mathrm{SQ} 3,6-8 \mathrm{~m} \mathrm{~W}-\mathrm{E} / 0.2-1.1 \mathrm{~m} \mathrm{~N}-\mathrm{S}$ & organic material (1 bag, leather/skin/textile) & 075 & & 05.02 .2014 \\
\hline $2422 / 2014$ & $\begin{array}{l}\text { SQ4, debris from S-wall, Feature } 15 \text { up to } \\
1 \mathrm{~m} \mathrm{N-S} \mathrm{of} \mathrm{square}\end{array}$ & plaque (1 square fragment, copper?) & 092 & SAV1E 1127 & 24.02 .2014 \\
\hline
\end{tabular}




\begin{tabular}{|c|c|c|c|c|c|}
\hline $002 / 2014$ & SQ1B, surface & pottery & 001 & & 04.01 .2014 \\
\hline $013 / 2014$ & $\mathrm{SQ} 1 \mathrm{~B}, 5 \times 10 \mathrm{~m}$, surface & $\begin{array}{l}\text { pottery, among it re-used sherds ( } 3 \text { pieces, } 2 \text { unspecified, } 1 \\
\text { Christian, pottery) }\end{array}$ & 001 & $\begin{array}{l}\text { SAV1E 0338, SAV1E 0339, SAV1E } 0340 \\
\text { (re-used sherds) }\end{array}$ & 04.01 .2014 \\
\hline $007 / 2014$ & SQ4, surface & pottery & 001 & & 04.01 .2014 \\
\hline $005 / 2014$ & $\mathrm{SQ} 3,10 \times 10 \mathrm{~m}$, surface cleaning & $\begin{array}{l}\text { pottery, among it re-used sherd ( } 1 \text { piece, Post-New Kingdom, } \\
\text { pottery), vessel ( } 1 \text { body sherd, pottery) }\end{array}$ & 001 & $\begin{array}{l}\text { SAV1E } 0671 \text { (re-used sherd), SAV1E } 0332 \\
\text { (vessel, body sherd) }\end{array}$ & 04.01 .2014 \\
\hline $015 / 2014$ & $\mathrm{SQ} 4,10 \times 10 \mathrm{~m}$, surface cleaning & $\begin{array}{l}\text { pottery, among it re-used sherds ( } 2 \text { pieces, each Post-New } \\
\text { Kingdom, pottery), weight?/re-used sherd ( } 1 \text { piece, Christian, } \\
\text { pottery) }\end{array}$ & 001 & $\begin{array}{l}\text { SAV1E 0335, SAV1E } 0336 \text { (re-used sherds), } \\
\text { SAV1E } 0337 \text { (weight?/re-used sherd) }\end{array}$ & 04.01 .2014 \\
\hline $021 / 2014$ & $\mathrm{SQ} 3,0-2.3 \mathrm{~m} \mathrm{~W} / 2 \mathrm{~m} \mathrm{~S}$ of SP3.2 & pottery & 002 & & 05.01 .2014 \\
\hline $022 / 2014$ & $\mathrm{SQ} 3,7.7-10 \mathrm{~m} \mathrm{~W}-\mathrm{E} / 8-10 \mathrm{~m} \mathrm{~N}-\mathrm{S}$ & pottery & 002 & & 06.01 .2014 \\
\hline $082 / 2014$ & $\mathrm{SQ} 3,8.5-9.5 \mathrm{~m} \mathrm{~W}-\mathrm{E} / 2-10 \mathrm{~m} \mathrm{~N}-\mathrm{S}$ & pottery & 002 & & 11.01 .2014 \\
\hline $089 / 2014$ & $\mathrm{SQ} 3,8.5-9.5 \mathrm{~m} \mathrm{~W}-\mathrm{E} / 2-10 \mathrm{~m} \mathrm{~N}-\mathrm{S}$ & pottery & 002 & & 12.01 .2014 \\
\hline $310 / 2014$ & $\mathrm{SQ} 3,9.5-10 \mathrm{~m} \mathrm{~W}-\mathrm{E} / 5.7-8 \mathrm{~m} \mathrm{~N}-\mathrm{S}$ & pottery, among it slag (1 piece) & 002 & SAV1E 0668 & 20.01 .2014 \\
\hline $083 / 2014$ & $\mathrm{SQ} 4,8-10 \mathrm{~m} \mathrm{~W}-\mathrm{E} / 0-10 \mathrm{~m} \mathrm{~N}-\mathrm{S}$ & pottery & 002 & & 11.01 .2014 \\
\hline $086 / 2014$ & $\mathrm{SQ} 4,8-10 \mathrm{~m} \mathrm{~W}-\mathrm{E} / 0-10 \mathrm{~m} \mathrm{~N}-\mathrm{S}$ & pottery & 002 & & 12.01 .2014 \\
\hline $037 / 2014$ & SQ4A, 8-10m W-E/0-2m N-S & pottery & 002 & & 08.01 .2014 \\
\hline $155 / 2014$ & SQ4A, 8-10m W-E/0-2m N-S & pottery & 002 & & 14.01 .2014 \\
\hline $055 / 2014$ & SQ3, 0-2m W-E/0-10m N-S & pottery & 003 & & 09.01 .2014 \\
\hline $066 / 2014$ & $\mathrm{SQ} 3,0-2 \mathrm{~m} \mathrm{~W}-\mathrm{E} / 7-10 \mathrm{~m} \mathrm{~N}-\mathrm{S}$ & pottery & 003 & & 09.01 .2014 \\
\hline $024 / 2014$ & $\mathrm{SQ} 3,0-5 \mathrm{~m} \mathrm{~W}-\mathrm{E} / 0-10 \mathrm{~m} \mathrm{~N}-\mathrm{S}$ & pottery; slag (2 pieces); grindstone (1 piece, quartzite) & 003 & $\begin{array}{l}\text { SAV1E } 0327 \text { (slag), SAV1E } 0375 \\
\text { (grindstone) }\end{array}$ & 06.01 .2014 \\
\hline $020 / 2014$ & $\mathrm{SQ} 3,2.30-5 \mathrm{~m} \mathrm{~W} / 5 \mathrm{~m} \mathrm{~S}$ of SP3.2 & pottery & 003 & & 05.01 .2014 \\
\hline $315 / 2014$ & $\begin{array}{l}\mathrm{SQ} 3,2.5-5 \mathrm{~m} \mathrm{~W}-\mathrm{E} / 3.5-7 \mathrm{~m} \mathrm{~N}-\mathrm{S} \text {, } \\
\text { cleaning of SU }\end{array}$ & pottery & 003 & & 20.01 .2014 \\
\hline $123 / 2014$ & $\mathrm{SQ} 3,2-5 \mathrm{~m} \mathrm{~W}-\mathrm{E} / 2-4 \mathrm{~m} \mathrm{~N}-\mathrm{S}$ & pottery & 003 & & 13.01.2014 \\
\hline $069 / 2014$ & $\mathrm{SQ} 3,3-5 \mathrm{~m} \mathrm{~W}-\mathrm{E} / 7-10 \mathrm{~m} \mathrm{~N}-\mathrm{S}$ & $\begin{array}{l}\text { pottery; slag (1 piece); furniture? installation? (1 fragment, } \\
\text { sandstone) }\end{array}$ & 003 & $\begin{array}{l}\text { SAV1E } 0576 \text { (slag), SAV1E } 0904 \text { (sandstone } \\
\text { object) }\end{array}$ & 09.01 .2014 \\
\hline $103 / 2014$ & $\mathrm{SQ} 3,5-8.5 \mathrm{~m} \mathrm{~W}-\mathrm{E} / 0-3 \mathrm{~m} \mathrm{~N}-\mathrm{S}$ & $\begin{array}{l}\text { pottery, among it re-used sherd (1 piece, Post-New Kingdom, } \\
\text { pottery) }\end{array}$ & 003 & SAV1E 0403 & 12.01 .2014 \\
\hline $113 / 2014$ & $\mathrm{SQ} 3,5-8.5 \mathrm{~m} \mathrm{~W}-\mathrm{E} / 3-7 \mathrm{~m} \mathrm{~N}-\mathrm{S}$ & $\begin{array}{l}\text { pottery, among it architecture? ( } 1 \text { cuboid shaped fragment, } \\
\text { pottery) }\end{array}$ & 003 & SAV1E 0397 & 13.01 .2014 \\
\hline
\end{tabular}




\begin{tabular}{|c|c|c|c|c|c|}
\hline $090 / 2014$ & $\mathrm{SQ} 3,5-8.5 \mathrm{~m} \mathrm{~W}-\mathrm{E} / 7-10 \mathrm{~m} \mathrm{~N}-\mathrm{S}$ & $\begin{array}{l}\text { pottery, among it re-used sherd (1 piece, Post-New Kingdom, } \\
\text { pottery) }\end{array}$ & 003 & SAV1E 0402 & 12.01.2014 \\
\hline $028 / 2014$ & $\mathrm{SQ} 4,0-5 \mathrm{~m} \mathrm{~W}-\mathrm{E} / 0-10 \mathrm{~m} \mathrm{~N}-\mathrm{S}$ & $\begin{array}{l}\text { pottery, slag and stone: pottery among it lamp (1 fragment, } \\
\text { Christian, pottery), re-used sherd (1 piece, Post-New Kingdom), } \\
\text { artefact? (1 fragment, cylindrical shape, pottery), vessel } \\
\text { (1 fragment, glazed ware); slag (1 piece), grindstone? (1 piece, } \\
\text { sandstone) }\end{array}$ & 003 & $\begin{array}{l}\text { SAV1E } 0333 \text { (lamp), SAV1E } 0341 \text { (re-used } \\
\text { sherd), SAV1E 0343 (artefact?), } \\
\text { SAV1E } 0349 \text { (vessel), SAV1E } 0325 \text { (slag), } \\
\text { SAV1E } 0376 \text { (grindstone) }\end{array}$ & 07.01 .2014 \\
\hline $137 / 2014$ & $\mathrm{SQ} 4,2.5-5 \mathrm{~m} \mathrm{~W}-\mathrm{E} / 0-9 \mathrm{~m} \mathrm{~N}-\mathrm{S}$ & pottery, among it lid (1 fragment, Christian, pottery) & 003 & SAV1E 0670 & 14.01.2014 \\
\hline $148 / 2014$ & SQ4, 5-8m W-E/0-10m N-S & pottery & 003 & & 14.01.2014 \\
\hline $033 / 2014$ & $\mathrm{SQ} 4,5-8 \mathrm{~m} \mathrm{~W}-\mathrm{E} / 0-10 \mathrm{~m} \mathrm{~N}-\mathrm{S}$ & $\begin{array}{l}\text { pottery, among it re-used sherd (1 piece from Nubian cooking } \\
\text { pot, Post-New Kingdom, pottery) }\end{array}$ & 003 & SAV1E 0322 & 07.01 .2014 \\
\hline $034 / 2014$ & $\mathrm{SQ} 4,8-10 \mathrm{~m} \mathrm{~W}-\mathrm{E} / 0-10 \mathrm{~m} \mathrm{~N}-\mathrm{S}$ & pottery & 003 & & 07.01.2014 \\
\hline $036 / 2014$ & SQ4A, 0-8m W-E/0-2m N-S & pottery & 003 & & 08.01.2014 \\
\hline $039 / 2014$ & SQ3, Feature 7 & pottery & 004 & & 08.01 .2014 \\
\hline $2159 / 2014$ & $\begin{array}{l}\text { SQ3, 8.5-10.5m W-E/0.3-2.3m N-S, } \\
\text { cleaning of SU }\end{array}$ & pottery & 004 & & 04.02 .2014 \\
\hline $041 / 2014$ & $\mathrm{SQ} 3,3-5 \mathrm{~m} \mathrm{~W}-\mathrm{E} / 0-3.5 \mathrm{~m} \mathrm{~N}-\mathrm{S}$ & $\begin{array}{l}\text { pottery and stone: re-used sherds ( } 3 \text { pieces, pottery); grindstone } \\
\text { (1 fragment, quartzite) }\end{array}$ & 005 & $\begin{array}{l}\text { SAV1E 0344, SAV1E 0345, SAV1E } 0346 \\
\text { (re-used sherds), SAV1E } 0377 \text { (grindstone?) }\end{array}$ & 08.01 .2014 \\
\hline $051 / 2014$ & $\mathrm{SQ} 3,3-4.5 \mathrm{~m} \mathrm{~W}-\mathrm{E} / 5-6.5 \mathrm{~m} \mathrm{~N}-\mathrm{S}$ & pottery & 006 & & 08.01 .2014 \\
\hline $056 / 2014$ & $\mathrm{SQ} 3,3-4.5 \mathrm{~m} \mathrm{~W}-\mathrm{E} / 5-6.5 \mathrm{~m} \mathrm{~N}-\mathrm{S}$ & pottery & 006 & & 09.01 .2014 \\
\hline $309 / 2014$ & SQ3, 3-4.5m W-E/5-6.5m N-S, cleaning & pottery & 006 & & 20.01.2014 \\
\hline $076 / 2014$ & SQ1B, 0-10m W-E/0-5m N-S & $\begin{array}{l}\text { pottery, among it re-used sherd ( } 1 \text { piece, Christian, pottery), } \\
\text { moulds for figurines ( } 2 \text { fragments, pottery) }\end{array}$ & 007 & $\begin{array}{l}\text { SAV1E } 0359 \text { (re-used sherd), SAV1E 0361, } \\
\text { SAV1E } 0362 \text { (moulds) }\end{array}$ & 11.01 .2014 \\
\hline $359 / 2014$ & $\mathrm{SQ} 4,0.5-1 \mathrm{~m} \mathrm{~W}-\mathrm{E} / 3-4 \mathrm{~m} \mathrm{~N}-\mathrm{S}$ & $\begin{array}{l}\text { pottery, among it re-used sherd (1 piece, Post-New Kingdom, } \\
\text { pottery) }\end{array}$ & 008 & SAV1E 0736 & 22.01 .2014 \\
\hline $092 / 2014$ & SQ4, 1-2.5m W-E/0-1.5m N-S & pottery & 008 & & 12.01 .2014 \\
\hline $106 / 2014$ & $\mathrm{SQ} 4,1.5-3 \mathrm{~m} \mathrm{~W}-\mathrm{E} / 2-3 \mathrm{~m} \mathrm{~N}-\mathrm{S}$ & pottery & 008 & & 12.01.2014 \\
\hline $365 / 2014$ & $\mathrm{SQ} 4,0.5-1 \mathrm{~m} \mathrm{~W}-\mathrm{E} / 3-4 \mathrm{~m} \mathrm{~N}-\mathrm{S}$ & pottery & 008 & & 23.01.2014 \\
\hline $2298 / 2014$ & $\begin{array}{l}\mathrm{SQ} 4,2.5-5.5 \mathrm{~m} \mathrm{~W}-\mathrm{E} / 9-10 \mathrm{~m} \mathrm{~N}-\mathrm{S} ; \mathrm{SQ} 4 \mathrm{~A}, \\
2.5-6.5 \mathrm{~m} \mathrm{~W}-\mathrm{E} / 0-2 \mathrm{~m} \mathrm{~N}-\mathrm{S} \text {, cleaning of SU }\end{array}$ & pottery & 009 & & 09.02 .2014 \\
\hline $111 / 2014$ & $\begin{array}{l}\mathrm{SQ} 4,2.5-5.5 \mathrm{~m} \mathrm{~W}-\mathrm{E} / 9-10 \mathrm{~m} \mathrm{~N}-\mathrm{S} ; \mathrm{SQ} 4 \mathrm{~A} \\
2.5-6.5 \mathrm{~m} \mathrm{~W}-\mathrm{E} / 0-2 \mathrm{~m} \mathrm{~N}-\mathrm{S}\end{array}$ & $\begin{array}{l}\text { pottery and stone: re-used sherd ( } 1 \text { piece, } 18^{\text {th }} \text { Dynasty, pottery); } \\
\text { grindstone ( } 1 \text { piece, quartzite) }\end{array}$ & 009 & $\begin{array}{l}\text { SAV1E } 2573 \text { (re-used sherd), SAV1E } 0503 \\
\text { (grindstone) }\end{array}$ & 13.01 .2014 \\
\hline $138 / 2014$ & $\begin{array}{l}\text { SQ4, 2.5-5.5m W-E/9-10m N-S; SQ4A, } \\
2.5-6.5 \mathrm{~m} \mathrm{~W}-\mathrm{E} / 0-2 \mathrm{~m} \mathrm{~N}-\mathrm{S}\end{array}$ & $\begin{array}{l}\text { pottery, among it re-used sherds ( } 3 \text { pieces, } 1 \text { Christian, } 2 \\
\text { unspecified, pottery), vessel? ( } 1 \text { fragment, pottery) }\end{array}$ & 009 & $\begin{array}{l}\text { SAV1E 0646, SAV1E 0647, SAV1E } 0648 \\
\text { (re-used sherds), SAV1E } 0735 \text { (vessel?) }\end{array}$ & 14.01 .2014 \\
\hline
\end{tabular}




\begin{tabular}{|c|c|c|c|c|c|}
\hline $156 / 2014$ & $\begin{array}{l}\text { SQ4, 2-2.5m W-E/9-10m N-S; SQ4A, } \\
1.5-2.5 \mathrm{~m} \mathrm{~W}-\mathrm{E} / 0-2 \mathrm{~m} \mathrm{~N}-\mathrm{S}\end{array}$ & $\begin{array}{l}\text { pottery, among it mould for figurine ( } 1 \text { fragment, pottery), re- } \\
\text { used sherd ( } 1 \text { piece, pottery) }\end{array}$ & 009 & $\begin{array}{l}\text { SAV1E } 0591 \text { (mould), SAV1E } 0592 \text { (re-used } \\
\text { sherd) }\end{array}$ & 14.01.2014 \\
\hline $127 / 2014$ & SQ4, 0-1.5m W-E/1.5-5m N-S & pottery & 010 & & 13.01 .2014 \\
\hline $133 / 2014$ & $\mathrm{SQ} 4,0-1.5 \mathrm{~m} \mathrm{~W}-\mathrm{E} / 7.5-10 \mathrm{~m} \mathrm{~N}-\mathrm{S}$ & pottery & 011 & & 13.01.2014 \\
\hline $160 / 2014$ & $\mathrm{SQ} 3,3-5 \mathrm{~m} \mathrm{~W}-\mathrm{E} / 0-3.5 \mathrm{~m} \mathrm{~N}-\mathrm{S}$ & pottery & 013 & & 15.01 .2014 \\
\hline $164 / 2014$ & $\mathrm{SQ} 4,1-3 \mathrm{~m} \mathrm{~W}-\mathrm{E} / 0-4 \mathrm{~m} \mathrm{~N}-\mathrm{S}$ & pottery & 014 & & 15.01 .2014 \\
\hline $352 / 2014$ & $\mathrm{SQ} 4,0-2.5 \mathrm{~m} \mathrm{~W}-\mathrm{E} / 4.5-5.5 \mathrm{~m} \mathrm{~N}-\mathrm{S}$ & $\begin{array}{l}\text { pottery, among it architecture/window grille? (1 fragment, } \\
\text { Christian, pottery) }\end{array}$ & 015 & SAV1E 0737 & 22.01 .2014 \\
\hline $206 / 2014$ & $\mathrm{SQ} 4,0-5 \mathrm{~m} \mathrm{~W}-\mathrm{E} / 5-8 \mathrm{~m} \mathrm{~N}-\mathrm{S}$ & pottery & 015 & & 18.01 .2014 \\
\hline $183 / 2014$ & $\mathrm{SQ} 4,0-5 \mathrm{~m} \mathrm{~W}-\mathrm{E} / 5-8 \mathrm{~m} \mathrm{~N}-\mathrm{S}$ & $\begin{array}{l}\text { pottery, among it re-used sherd (1 piece, Post-New Kingdom, } \\
\text { pottery) }\end{array}$ & 015 & SAV1E 0644 & 16.01.2014 \\
\hline $226 / 2014$ & $\mathrm{SQ} 4,1.5-5 \mathrm{~m} \mathrm{~W}-\mathrm{E} / 3.5-5 \mathrm{~m} \mathrm{~N}-\mathrm{S}$ & pottery & 015 & & 18.01 .2014 \\
\hline $247 / 2014$ & $\mathrm{SQ} 4,1.5-5 \mathrm{~m} \mathrm{~W}-\mathrm{E} / 8-8.5 \mathrm{~m} \mathrm{~N}-\mathrm{S}$ & pottery & 015 & & 19.01.2014 \\
\hline $248 / 2014$ & $\mathrm{SQ} 4,1.5-5 \mathrm{~m} \mathrm{~W}-\mathrm{E} / 8-8.5 \mathrm{~m} \mathrm{~N}-\mathrm{S}$ & $\begin{array}{l}\text { pottery, slag and stone: re-used sherd ( } 1 \text { piece, Post-New } \\
\text { Kingdom?), architecture?/window grille? ( } 1 \text { fragment, Christian, } \\
\text { pottery); slag ( } 1 \text { piece); grindstones ( } 2 \text { pieces, sandstone), } \\
\text { grindstone? (1 piece, sandstone), pounder ( } 1 \text { piece, diorite?) }\end{array}$ & 015 & $\begin{array}{l}\text { SAV1E } 0645 \text { (re-used sherd), SAV1E } 0649 \\
\text { (architecture/window grille?), SAV1E } 0616 \\
\text { (slag), SAV1E 0712, SAV1E 0713 } \\
\text { (grindstone), SAV1E 0714 (grindstone?), } \\
\text { SAV1E 0711 (pounder) }\end{array}$ & 19.01.2014 \\
\hline $232 / 2014$ & $\mathrm{SQ} 4,1.5-5 \mathrm{~m} \mathrm{~W}-\mathrm{E} / 8-8.5 \mathrm{~m} \mathrm{~N}-\mathrm{S}$ & pottery; slag (2 pieces) & 015 & SAV1E 0599 (slag) & 18.01 .2014 \\
\hline $261 / 2014$ & $\mathrm{SQ} 4,3.5-5 \mathrm{~m} \mathrm{~W}-\mathrm{E} / 0-3.5 \mathrm{~m} \mathrm{~N}-\mathrm{S}$ & pottery & 015 & & 19.01.2014 \\
\hline $178 / 2014$ & SQ4A, 9-10.5m W-E/0-2m N-S & pottery, among it mould for figurine? (1 fragment, pottery) & 016 & SAV1E 0593 & 16.01 .2014 \\
\hline $194 / 2014$ & SQ4A, $10-10.5 \mathrm{~m} \mathrm{~W}-\mathrm{E} / 0-0.5 \mathrm{~m} \mathrm{~N}-\mathrm{S}$ & pottery & 017 & & 16.01 .2014 \\
\hline $200 / 2014$ & SQ1B, 0-3m W-E/0-2m N-S & $\begin{array}{l}\text { pottery, among it re-used sherds ( } 3 \text { pieces, } 1 \text { Christian, } 2 \text { Post- } \\
\text { New Kingdom,), architecture/window grille? ( } 1 \text { fragment, } \\
\text { Christian, pottery) }\end{array}$ & 018 & $\begin{array}{l}\text { SAV1E 0637, SAV1E 0638, SAV1E } 0639 \\
\text { (re-used sherds), SAV1E } 0656 \text { (window } \\
\text { grille?) }\end{array}$ & 16.01 .2014 \\
\hline $204 / 2014$ & SQ1B, 0-3m W-E/0-2m N-S & $\begin{array}{l}\text { pottery, re-used sherd (1 piece, Christian, pottery); } \\
\text { charcoal ( } 1 \text { bag) }\end{array}$ & 018 & SAV1E 0595 (re-used sherd) & 16.01 .2014 \\
\hline $211 / 2014$ & SQ1B, 0-3m W-E/0-2m N-S & pottery & 018 & & 18.01 .2014 \\
\hline $218 / 2014$ & $\begin{array}{l}\text { SQ1B, } 0.5-2.5 \mathrm{~m} \mathrm{~W}-\mathrm{E} / 4-5 \mathrm{~m} \mathrm{~N}-\mathrm{S}, \text { Feature } \\
29\end{array}$ & $\begin{array}{l}\text { pottery, among it figurine ( } 1 \text { fragment, probably female figurine, } \\
\text { pottery), scraper (1 re-used sherd, Christian, pottery) }\end{array}$ & 019 & $\begin{array}{l}\text { SAV1E } 0657 \text { (figurine), SAV1E } 2820 \\
\text { (scraper) }\end{array}$ & 18.01.2014 \\
\hline $240 / 2014$ & $\begin{array}{l}\text { SQ1A, } 0.5-2.5 \mathrm{~m} \mathrm{~W}-\mathrm{E} / 0-1 \mathrm{~m} \mathrm{~N}-\mathrm{S} ; \mathrm{SQ} 1 \mathrm{~B} \\
0.5-2.5 \mathrm{~m} \mathrm{~W}-\mathrm{E} / 4.5-5 \mathrm{~m} \mathrm{~N}-\mathrm{S} \text {, lowest filling } \\
\text { of Feature } 29\end{array}$ & pottery, among it re-used sherd ( 1 piece, Christian, pottery) & 019 & SAV1E 0643 & 19.01.2014 \\
\hline $2078 / 2014$ & SQ1B, 0-2m W-E/4 $4.7 \mathrm{~m} \mathrm{N-S}$ & pottery & 020 & & 01.02 .2014 \\
\hline
\end{tabular}




\begin{tabular}{|c|c|c|c|c|c|}
\hline $252 / 2014$ & SQ1B, 3-6m W-E/0-2m N-S & pottery; slag (1 piece) & 021 & SAV1E 0665 (slag) & 19.01.2014 \\
\hline $274 / 2014$ & SQ1B, 3-6m W-E/2-3m N-S & pottery & 021 & & 19.01 .2014 \\
\hline $285 / 2014$ & SQ1B, 3-6m W-E/2-3m N-S & pottery, among it slag (1 piece) & 021 & SAV1E 0655 & 20.01 .2014 \\
\hline $264 / 2014$ & $\mathrm{SQ} 1 \mathrm{~B}, 6-7.5 \mathrm{~m} \mathrm{~W}-\mathrm{E} / 0-2.5 \mathrm{~m} \mathrm{~N}-\mathrm{S}$ & $\begin{array}{l}\text { pottery, among it scraper ( } 1 \text { re-used } 18^{\text {th }} \text { Dynasty rim sherd, } \\
\text { pottery) }\end{array}$ & 021 & SAV1E 0672 & 19.01.2014 \\
\hline $269 / 2014$ & $\mathrm{SQ} 4,0-1.5 \mathrm{~m} \mathrm{~W}-\mathrm{E} / 8-10 \mathrm{~m} \mathrm{~N}-\mathrm{S}$ & pottery & 022 & & 19.01.2014 \\
\hline $258 / 2014$ & SQ4A, 0-1.5m W-E/0-2m N-S & pottery & 022 & & 19.01.2014 \\
\hline $278 / 2014$ & $\mathrm{SQ} 3,0-2 \mathrm{~m} \mathrm{~W}-\mathrm{E} / 0-4.4 \mathrm{~m} \mathrm{~N}-\mathrm{S}$ & pottery & 023 & & 19.01.2014 \\
\hline $290 / 2014$ & $\mathrm{SQ} 3,2-3 \mathrm{~m} \mathrm{~W}-\mathrm{E} / 0-4.4 \mathrm{~m} \mathrm{~N}-\mathrm{S}$ & pottery; slag (1 piece) & 023 & SAV1E 0651 & 20.01 .2014 \\
\hline $302 / 2014$ & $\mathrm{SQ} 3,2.5-3.2 \mathrm{~m} \mathrm{~W}-\mathrm{E} / 0-3.5 \mathrm{~m} \mathrm{~N}-\mathrm{S}$ & pottery & 024 & & 20.01 .2014 \\
\hline $2113 / 2014$ & $\begin{array}{l}\text { SQ3, 2.5-3.2m W-E } / 0-3.5 \mathrm{~m} \mathrm{~N}-\mathrm{S} \text {; cleaning } \\
\text { of SU }\end{array}$ & pottery & 024 & & 02.02 .2014 \\
\hline $2116 / 2014$ & $\begin{array}{l}\mathrm{SQ} 3,2.5-3.2 \mathrm{~m} \mathrm{~W}-\mathrm{E} / 0-3.5 \mathrm{~m} \mathrm{~N}-\mathrm{S} \text {; cleaning } \\
\text { under stone, cleaning of SU }\end{array}$ & pottery & 024 & & 03.02 .2014 \\
\hline $329 / 2014$ & $\mathrm{SQ} 3,0-2 \mathrm{~m} \mathrm{~W}-\mathrm{E} / 4.4-10 \mathrm{~m} \mathrm{~N}-\mathrm{S}$ & $\begin{array}{l}\text { pottery, among it architecture/window grille? (1 fragment, } \\
\text { Christian, pottery) }\end{array}$ & 025 & SAV1E 0677 & 21.01 .2014 \\
\hline $316 / 2014$ & $\mathrm{SQ} 3,8-10.5 \mathrm{~m} \mathrm{~W}-\mathrm{E} / 2.5-5.7 \mathrm{~m} \mathrm{~N}-\mathrm{S}$ & pottery & 026 & & 20.01 .2014 \\
\hline $321 / 2014$ & $\mathrm{SQ} 3,8-10.5 \mathrm{~m} \mathrm{~W}-\mathrm{E} / 5-8 \mathrm{~m} \mathrm{~N}-\mathrm{S}$ & pottery; slag (1 piece) & 027 & SAV1E 0674 (slag) & 21.01 .2014 \\
\hline $336 / 2014$ & $\mathrm{SQ} 3,8-10 \mathrm{~m} \mathrm{~W}-\mathrm{E} / 8-10 \mathrm{~m} \mathrm{~N}-\mathrm{S}$ & pottery & 028 & & 21.01 .2014 \\
\hline $335 / 2014$ & $\mathrm{SQ} 4,8-10 \mathrm{~m} \mathrm{~W}-\mathrm{E} / 0-3.5 \mathrm{~m} \mathrm{~N}-\mathrm{S}$ & pottery & 029 & & 21.01 .2014 \\
\hline $364 / 2014$ & $\mathrm{SQ} 4,8-10 \mathrm{~m} \mathrm{~W}-\mathrm{E} / 3.5-10 \mathrm{~m} \mathrm{~N}-\mathrm{S}$ & pottery & 029 & & 23.01 .2014 \\
\hline $380 / 2014$ & $\begin{array}{l}\mathrm{SQ} 4,0-0.8 \mathrm{~m} \mathrm{~W}-\mathrm{E} / 4-5 \mathrm{~m} \mathrm{~N}-\mathrm{S} \\
\text { cleaning of } \mathrm{SU}\end{array}$ & pottery & 030 & & 23.01 .2014 \\
\hline $338 / 2014$ & $\mathrm{SQ} 4,0-3 \mathrm{~m} \mathrm{~W}-\mathrm{E} / 1-5.5 \mathrm{~m} \mathrm{~N}-\mathrm{S}$ & pottery & 030 & & 22.01 .2014 \\
\hline $345 / 2014$ & $\mathrm{SQ} 3,2.5-9 \mathrm{~m} \mathrm{~W}-\mathrm{E} / 0-10 \mathrm{~m} \mathrm{~N}-\mathrm{S}$ & pottery; slag (1 piece) & 031 & SAV1E 0849 (slag) & 22.01 .2014 \\
\hline $371 / 2014$ & $\mathrm{SQ} 3,8-10.5 \mathrm{~m} \mathrm{~W}-\mathrm{E} / 2.5-5.7 \mathrm{~m} \mathrm{~N}-\mathrm{S}$ & pottery & 032 & & 23.01 .2014 \\
\hline $374 / 2014$ & $\mathrm{SQ} 4,5-8 \mathrm{~m} \mathrm{~W}-\mathrm{E} / 0-10 \mathrm{~m} \mathrm{~N}-\mathrm{S}$ & $\begin{array}{l}\text { pottery and stone: pottery, among it re-used sherd ( } 1 \text { piece, Post- } \\
\text { New Kingdom, pottery), mould for figurine ( } 1 \text { fragment, } \\
\text { Christian, pottery); grindstone (1 piece, sandstone) }\end{array}$ & 033 & $\begin{array}{l}\text { SAV1E } 0768 \text { (re-used sherd), SAV1E } 0769 \\
\text { (mould), SAV1E } 0775 \text { (grindstone) }\end{array}$ & 23.01.2014 \\
\hline $381 / 2014$ & $\mathrm{SQ} 3,9-10.5 \mathrm{~m} \mathrm{~W}-\mathrm{E} / 2.6-5.5 \mathrm{~m} \mathrm{~N}-\mathrm{S}$ & pottery, among it re-used sherd ( 1 piece, $18^{\text {th }}$ Dynasty, pottery) & 034 & SAV1E 0777 & 23.01 .2014 \\
\hline $392 / 2014$ & SQ4A, 0-2m W-E/0-2m N-S & pottery & 035 & & 23.01 .2014 \\
\hline
\end{tabular}




\begin{tabular}{|c|c|c|c|c|c|}
\hline $388 / 2014$ & SQ3, 9-10.5m W-E/3.6-5.3m N-S & pottery & 036 & & 23.01 .2014 \\
\hline $396 / 2014$ & $\mathrm{SQ} 4 \mathrm{~A}, 8-10 \mathrm{~m} \mathrm{~W}-\mathrm{E} / 0-2 \mathrm{~m} \mathrm{~N}-\mathrm{S}$ & pottery & 037 & & 23.01 .2014 \\
\hline $401 / 2014$ & $\mathrm{SQ} 3,0-1.3 \mathrm{~m} \mathrm{~W}-\mathrm{E} / 4.4-6.8 \mathrm{~m} \mathrm{~N}-\mathrm{S}$ & pottery & 038 & & 25.01 .2014 \\
\hline $407 / 2014$ & $\mathrm{SQ} 3,0-1.3 \mathrm{~m} \mathrm{~W}-\mathrm{E} / 7.4-8.7 \mathrm{~m} \mathrm{~N}-\mathrm{S}$ & pottery, among it lid (1 fragment, Christian?, pottery) & 038 & SAV1E 0792 & 25.01 .2014 \\
\hline $434 / 2014$ & $\begin{array}{l}\text { SQ3, 0-1.3m W-E/7.4-8.7m N-S, } \\
\text { cleaning of SU }\end{array}$ & pottery & 038 & & 26.01 .2014 \\
\hline $397 / 2014$ & $\mathrm{SQ1B}, 0-3 \mathrm{~m} \mathrm{~W}-\mathrm{E} / 2-4.5 \mathrm{~m} \mathrm{~N}-\mathrm{S}$ & $\begin{array}{l}\text { pottery and stone: pottery, among it lid or stopper/sealing ( } 1 \text { frag- } \\
\text { ment, mud); grindstone ( } 1 \text { piece, sandstone) }\end{array}$ & 039 & $\begin{array}{l}\text { SAV1E } 0761 \text { (lid or stopper/sealing), } \\
\text { SAV1E } 0781 \text { (grindstone) }\end{array}$ & 25.01 .2014 \\
\hline $428 / 2014$ & $\mathrm{SQ} 1 \mathrm{~B}, 0-3 \mathrm{~m} \mathrm{~W}-\mathrm{E} / 2.5-4.5 \mathrm{~m} \mathrm{~N}-\mathrm{S}$ & pottery; slag (1 piece) & 040 & SAV1E 0789 (slag) & 26.01.2014 \\
\hline $422 / 2014$ & SQ1B, 0-3m W-E/2-3m N-S & pottery & 040 & & 26.01 .2014 \\
\hline $412 / 2014$ & SQ1B, 0-3m W-E/2-3m N-S & $\begin{array}{l}\text { pottery, among it re-used sherd (1 re-used body sherd of Late } \\
\text { Christian plate, pottery) }\end{array}$ & 040 & SAV1E 0794 & 25.01.2014 \\
\hline $439 / 2014$ & $\mathrm{SQ} 1 \mathrm{~B}, 1-3 \mathrm{~m} \mathrm{~W}-\mathrm{E} / 3-4 \mathrm{~m} \mathrm{~N}-\mathrm{S}$ & pottery & 040 & & 27.01 .2014 \\
\hline $1506 / 2014$ & $\mathrm{SQ} 1 \mathrm{~B}, 2.5-8 \mathrm{~m} \mathrm{~W}-\mathrm{E} / 2.5-4 \mathrm{~m} \mathrm{~N}-\mathrm{S}$ & pottery & 040 & & 27.01 .2014 \\
\hline $416 / 2014$ & $\mathrm{SQ} 3,1-5 \mathrm{~m} \mathrm{~W}-\mathrm{E} / 7.2-10 \mathrm{~m} \mathrm{~N}-\mathrm{S}$ & pottery & 041 & & 25.01 .2014 \\
\hline $429 / 2014$ & $\mathrm{SQ} 3,1.8-5 \mathrm{~m} \mathrm{~W}-\mathrm{E} / 7-10 \mathrm{~m} \mathrm{~N}-\mathrm{S}$ & pottery; slag (1 piece) & 041 & SAV1E 0776 (slag) & 26.01 .2014 \\
\hline $442 / 2014$ & SQ4, $0.5-3 \mathrm{~m} \mathrm{~W}-\mathrm{E} / 0-4 \mathrm{~m} \mathrm{~N}-\mathrm{S}$ & $\begin{array}{l}\text { pottery, among it re-used sherd ( } 1 \text { piece, Post-New Kingdom, } \\
\text { pottery), mould for (female?) figurine ( } 1 \text { fragment, pottery) }\end{array}$ & 042 & $\begin{array}{l}\text { SAV1E } 0822 \text { (re-used sherd), } \\
\text { SAV1E } 0823 \text { (mould) }\end{array}$ & 27.01.2014 \\
\hline $447 / 2014$ & SQ1B, 3-10m W-E/3-5m N-S & pottery, among it architecture/window grille (1 fragment, pottery) & 043 & SAV1E 0857 & 27.01.2014 \\
\hline $1500 / 2014$ & $\mathrm{SQ} 3,0-1.5 \mathrm{~m} \mathrm{~W}-\mathrm{E} / 7.4-8.7 \mathrm{~m} \mathrm{~N}-\mathrm{S}$ & pottery & 044 & & 27.01 .2014 \\
\hline $1504 / 2014$ & $\mathrm{SQ} 3,0-2.7 \mathrm{~m} \mathrm{~W}-\mathrm{E} / 8.7-10 \mathrm{~m} \mathrm{~N}-\mathrm{S}$ & pottery; seashell (1 small piece) & 045 & & 27.01 .2014 \\
\hline $1512 / 2014$ & $\mathrm{SQ} 3,2.7-5.2 \mathrm{~m} \mathrm{~W}-\mathrm{E} / 7.3-9 \mathrm{~m} \mathrm{~N}-\mathrm{S}$ & pottery & 046 & & 27.01 .2014 \\
\hline $1516 / 2014$ & $\mathrm{SQ1B}, 8-10 \mathrm{~m} \mathrm{~W}-\mathrm{E} / 0-5 \mathrm{~m} \mathrm{~N}-\mathrm{S}$ & pottery & 048 & & 28.01 .2014 \\
\hline $2000 / 2014$ & SQ1B, 0-2m W-E/2.3-3.4m N-S & pottery & 049 & & 28.01 .2014 \\
\hline $2006 / 2014$ & $\mathrm{SQ} 3,2.5-5.3 \mathrm{~m} \mathrm{~W}-\mathrm{E} / 8.5-10 \mathrm{~m} \mathrm{~N}-\mathrm{S}$ & $\begin{array}{l}\text { pottery, among it re-used sherd (1 piece, Christian, pottery); slag } \\
\text { (1 piece) }\end{array}$ & 050 & $\begin{array}{l}\text { SAV1E } 0897 \text { (re-used sherd), } \\
\text { SAV1E } 0831 \text { (slag) }\end{array}$ & 28.01.2014 \\
\hline $2017 / 2014$ & $\mathrm{SQ} 3,2.5-5.3 \mathrm{~m} \mathrm{~W}-\mathrm{E} / 8.5-10 \mathrm{~m} \mathrm{~N}-\mathrm{S}$ & pottery & 050 & & 29.01 .2014 \\
\hline $2012 / 2014$ & $\mathrm{SQ1B}, 0-4 \mathrm{~m} \mathrm{~W}-\mathrm{E} / 0-2.3 \mathrm{~m} \mathrm{~N}-\mathrm{S}$ & pottery & 051 & & 28.01 .2014 \\
\hline $2021 / 2014$ & $\mathrm{SQ} 1 \mathrm{~B}, 1.5-4 \mathrm{~m} \mathrm{~W}-\mathrm{E} / 0-2.3 \mathrm{~m} \mathrm{~N}-\mathrm{S}$ & $\begin{array}{l}\text { pottery, among it figurines ( } 2 \text { pieces, each fragmented, pottery, } \\
\text { clay); slag ( } 2 \text { pieces) }\end{array}$ & 051 & $\begin{array}{l}\text { SAV1E 2821, SAV1E } 0851 \text { (figurines), } \\
\text { SAV1E } 0850 \text { (slag) }\end{array}$ & 29.01 .2014 \\
\hline
\end{tabular}




\begin{tabular}{|c|c|c|c|c|c|}
\hline $2028 / 2014$ & $\begin{array}{l}\text { SQ1B, } 1.5-4 \mathrm{~m} \mathrm{~W}-\mathrm{E} / 0-2.3 \mathrm{~m} \mathrm{~N}-\mathrm{S} \text {; right } \\
\text { above floor/gravel }\end{array}$ & pottery & 051 & & 29.01.2014 \\
\hline $2053 / 2014$ & SQ1B, $1.5-6.5 \mathrm{~m} \mathrm{~W}-\mathrm{E} / 1.3-3 \mathrm{~m} \mathrm{~N}-\mathrm{S}$ & pottery & 051 & & 30.01 .2014 \\
\hline $2029 / 2014$ & $\mathrm{SQ} 1 \mathrm{~B}, 4-6.5 \mathrm{~m} \mathrm{~W}-\mathrm{E} / 0-1.5 \mathrm{~m} \mathrm{~N}-\mathrm{S}$ & pottery & 051 & & 29.01 .2014 \\
\hline $2043 / 2014$ & $\mathrm{SQ} 1 \mathrm{~B}, 4-6.5 \mathrm{~m} \mathrm{~W}-\mathrm{E} / 0-1.5 \mathrm{~m} \mathrm{~N}-\mathrm{S}$ & pottery & 051 & & 30.01 .2014 \\
\hline $2058 / 2014$ & SQ1B, $6.5-7 \mathrm{~m} \mathrm{~W}-\mathrm{E} / 0-1.7 \mathrm{~m} \mathrm{~N}-\mathrm{S}$ & pottery & 051 & & 30.01 .2014 \\
\hline $2036 / 2014$ & $\mathrm{SQ} 3,2.3-4 \mathrm{~m} \mathrm{~W}-\mathrm{E} / 3-5.2 \mathrm{~m} \mathrm{~N}-\mathrm{S}$ & $\begin{array}{l}\text { pottery, slag and stone: pottery, among it re-used sherds } \\
\text { ( } 3 \text { pieces, } 2 \text { Post-New Kingdom, } 1 \text { unspecified, pottery); } \\
\text { slag ( } 5 \text { pieces); grindstones ( } 2 \text { pieces, each sandstone) }\end{array}$ & 052 & $\begin{array}{l}\text { SAV1E 0899, SAV1E 0900, SAV1E 0901 } \\
\text { (re-used sherds), SAV1E 0888 (slag), SAV1E } \\
\text { 0889, SAV1E 0890 (grindstones) }\end{array}$ & 29.01.2014 \\
\hline $2049 / 2014$ & $\mathrm{SQ} 3,2.3-4.8 \mathrm{~m} \mathrm{~W}-\mathrm{E} / 3-5.2 \mathrm{~m} \mathrm{~N}-\mathrm{S}$ & $\begin{array}{l}\text { pottery, among it figurine ( } 1 \text { lower part of rudimentary female } \\
\text { figurine, clay) }\end{array}$ & 052 & SAV1E 0896 & 30.01 .2014 \\
\hline $2061 / 2014$ & $\mathrm{SQ} 3,2.3-4.8 \mathrm{~m} \mathrm{~W}-\mathrm{E} / 3-5.2 \mathrm{~m} \mathrm{~N}-\mathrm{S}$ & pottery & 053 & & 30.01 .2014 \\
\hline $2064 / 2014$ & $\mathrm{SQ} 3,4.8-5.3 \mathrm{~m} \mathrm{~W}-\mathrm{E} / 5.2-7.3 \mathrm{~m} \mathrm{~N}-\mathrm{S}$ & pottery & 054 & & 01.02 .2014 \\
\hline $2068 / 2014$ & $\mathrm{SQ} 3,4.8-5.3 \mathrm{~m} \mathrm{~W}-\mathrm{E} / 5.2-7.3 \mathrm{~m} \mathrm{~N}-\mathrm{S}$ & pottery & 055 & & 01.02 .2014 \\
\hline $2101 / 2014$ & $\mathrm{SQ1B}, 0.5-1 \mathrm{~m} \mathrm{~W}-\mathrm{E} / 4.2-4.5 \mathrm{~m} \mathrm{~N}-\mathrm{S}$ & pottery & 056 & & 02.02 .2014 \\
\hline $2089 / 2014$ & $\mathrm{SQ} 1 \mathrm{~B}, 0-1.5 \mathrm{~m} \mathrm{~W}-\mathrm{E} / 2.5-4.5 \mathrm{~m} \mathrm{~N}-\mathrm{S}$ & $\begin{array}{l}\text { pottery, among it re-used sherd (1 piece, Post-New Kingdom, } \\
\text { pottery); slag (1 piece) }\end{array}$ & 056 & $\begin{array}{l}\text { SAV1E } 0934 \text { (re-used sherd), } \\
\text { SAV1E } 0919 \text { (slag) }\end{array}$ & 01.02 .2014 \\
\hline $2069 / 2014$ & SQ1B, $0-2.8 \mathrm{~m} \mathrm{~W}-\mathrm{E} / 3-4 \mathrm{~m} \mathrm{~N}-\mathrm{S}$ & pottery & 056 & & 01.02 .2014 \\
\hline $2076 / 2014$ & $\mathrm{SQ} 3,2-2.7 \mathrm{~m} \mathrm{~W}-\mathrm{E} / 6.5-7.2 \mathrm{~m} \mathrm{~N}-\mathrm{S}$ & pottery & 057 & & 01.02 .2014 \\
\hline $2082 / 2014$ & $\mathrm{SQ} 3,2-5.5 \mathrm{~m} \mathrm{~W}-\mathrm{E} / 2-3.8 \mathrm{~m} \mathrm{~N}-\mathrm{S}$ & pottery & 058 & & 01.02 .2014 \\
\hline $2086 / 2014$ & $\mathrm{SQ} 3,2-5.5 \mathrm{~m} \mathrm{~W}-\mathrm{E} / 2-3.8 \mathrm{~m} \mathrm{~N}-\mathrm{S}$ & $\begin{array}{l}\text { pottery, among it re-used sherd (1 piece, Post-New Kingdom, } \\
\text { pottery); slag (1 piece) }\end{array}$ & 059 & $\begin{array}{l}\text { SAV1E } 0920 \text { (slag), } \\
\text { SAV1E } 0935 \text { (re-used sherd) }\end{array}$ & 01.02 .2014 \\
\hline $2097 / 2014$ & $\mathrm{SQ} 3,2-5.5 \mathrm{~m} \mathrm{~W}-\mathrm{E} / 2-3.8 \mathrm{~m} \mathrm{~N}-\mathrm{S}$ & pottery & 059 & & 02.02 .2014 \\
\hline $2103 / 2014$ & $\mathrm{SQ} 1 \mathrm{~B}, 0.6-1.5 \mathrm{~m} \mathrm{~W}-\mathrm{E} / 3.6-4.4 \mathrm{~m} \mathrm{~N}-\mathrm{S}$ & pottery & 060 & & 02.02 .2014 \\
\hline $2104 / 2014$ & $\mathrm{SQ} 3,4.2-6 \mathrm{~m} \mathrm{~W}-\mathrm{E} / 0-3.8 \mathrm{~m} \mathrm{~N}-\mathrm{S}$ & pottery & 061 & & 02.02 .2014 \\
\hline $2122 / 2014$ & $\mathrm{SQ} 3,4.4-5.6 \mathrm{~m} \mathrm{~W}-\mathrm{E} / 0.8-3 \mathrm{~m} \mathrm{~N}-\mathrm{S}$ & pottery & 062 & & 03.02 .2014 \\
\hline $2108 / 2014$ & SQ1B, 0-1.5m W-E/3.7-4.7m N-S & pottery & 063 & & 02.02 .2014 \\
\hline $2117 / 2014$ & SQ1B, $0-0.8 \mathrm{~m} \mathrm{~W}-\mathrm{E} / 4.2-5 \mathrm{~m} \mathrm{~N}-\mathrm{S}$ & pottery & 064 & & 03.02 .2014 \\
\hline $2126 / 2014$ & SQ1B, 0-1.1m W-E/4-5m N-S & pottery; slag?/crucible? (1 piece); faience vessel (1 body sherd) & 065 & $\begin{array}{l}\text { SAV1E } 0954 \text { (slag?/crucible?), } \\
\text { SAV1E } 0978 \text { (faience vessel) }\end{array}$ & 03.02 .2014 \\
\hline $2166 / 2014$ & $\mathrm{SQ} 3,4.8-5.5 \mathrm{~m} \mathrm{~W}-\mathrm{E} / 4-6.8 \mathrm{~m} \mathrm{~N}-\mathrm{S}$ & pottery & 066 & & 04.02 .2014 \\
\hline
\end{tabular}




\begin{tabular}{|c|c|c|c|c|c|}
\hline $2162 / 2014$ & $\mathrm{SQ} 3,4.8-7 \mathrm{~m} \mathrm{~W}-\mathrm{E} / 4-8.3 \mathrm{~m} \mathrm{~N}-\mathrm{S}$ & pottery, among it re-used sherd ( 1 piece, $18^{\text {th }}$ Dynasty) & 066 & SAV1E 1028 & 04.02 .2014 \\
\hline $2140 / 2014$ & $\mathrm{SQ} 3,4.8-7 \mathrm{~m} \mathrm{~W}-\mathrm{E} / 4-8.3 \mathrm{~m} \mathrm{~N}-\mathrm{S}$ & $\begin{array}{l}\text { pottery and slag: window grille ( } 1 \text { fragment, Christian, pottery), } \\
\text { re-used sherds ( } 2 \text { pieces, } 1 \text { Post-New Kingdom, } 1 \text { Christian, } \\
\text { pottery), oil lamp (1 fragment, Christian, pottery); slag ( } 4 \text { pieces })\end{array}$ & 066 & $\begin{array}{l}\text { SAV1E } 0987 \text { (window grille), SAV1E } 0988, \\
\text { SAV1E } 0990 \text { (re-used sherds), SAV1E } 0992 \\
\text { (oil lamp), SAV1E } 0955 \text { (slag) }\end{array}$ & 03.02 .2014 \\
\hline $2146 / 2014$ & $\mathrm{SQ} 3,4.8-7 \mathrm{~m} \mathrm{~W}-\mathrm{E} / 4-8.3 \mathrm{~m} \mathrm{~N}-\mathrm{S}$ & overfired ceramic (1 piece) & 066 & SAV1E 0957 & 03.02 .2014 \\
\hline $2178 / 2014$ & $\begin{array}{l}\text { SQ3, 4.8-7m W-E/4-8.3m N-S, } \\
\text { cleaning of SU }\end{array}$ & pottery, among it stopper/sealing?/lid? (1 fragment, mud) & 066 & SAV1E 0972 & 04.02 .2014 \\
\hline $2131 / 2014$ & SQ1B, $0-1.1 \mathrm{~m} \mathrm{~W}-\mathrm{E} / 3-4.2 \mathrm{~m} \mathrm{~N}-\mathrm{S}$ & pottery, among it window grille ( 1 fragment, Christian, pottery) & 067 & SAV1E 0985 & 03.02 .2014 \\
\hline $2136 / 2014$ & SQ1B, $0.5-2 \mathrm{~m} \mathrm{~W}-\mathrm{E} / 3.8-4.7 \mathrm{~m} \mathrm{~N}-\mathrm{S}$ & $\begin{array}{l}\text { pottery, among it re-used sherds ( } 2 \text { pieces, } 1 \text { Post-New Kingdom, } \\
1 \text { Christian, pottery) }\end{array}$ & 068 & SAV1E 1132, SAV1E 1133 (re-used sherds) & 03.02 .2014 \\
\hline $2148 / 2014$ & SQ3, 9-10.5m W-E/3.6-5.3m N-S & pottery, among it window grille ( 1 fragment, Christian, pottery) & 069 & SAV1E 0986 & 03.02 .2014 \\
\hline $2153 / 2014$ & SQ3, 9-10.5m W-E/3.6-5.3m N-S & pottery & 069 & & 04.02 .2014 \\
\hline $2158 / 2014$ & SQ3, 2.2-2.8m N-S, cleaning of W profile & pottery & 070 & & 04.02 .2014 \\
\hline $2205 / 2014$ & SQ3, 8.5-10.5m W-E/5-7.8m N-S & pottery & 070 & & 05.02 .2014 \\
\hline $2218 / 2014$ & SQ3, 8.5-10.5m W-E/5-8.5m N-S & pottery & 070 & & 05.02 .2014 \\
\hline $2226 / 2014$ & SQ3, 8.5-10.5m W-E/5-8.5m N-S & pottery & 070 & & 06.02 .2014 \\
\hline $2252 / 2014$ & SQ3, 8-10.5m W-E/3.6-5.5m N-S & $\begin{array}{l}\text { pottery, among it re-used sherd ( } 1 \text { piece, Christian, pottery), } \\
\text { window grille ( } 1 \text { fragment, Christian, pottery) }\end{array}$ & 070 & $\begin{array}{l}\text { SAV1E } 1062 \text { (re-used sherd), SAV1E } 1063 \\
\text { (window grille) }\end{array}$ & 06.02 .2014 \\
\hline $2257 / 2014$ & SQ3, 8-10.5m W-E/3.6-5.5m N-S & $\begin{array}{l}\text { pottery, among it re-used sherd (1 piece, Post-New Kingdom, } \\
\text { pottery), lid? (1 fragment, clay); slag (1 piece) }\end{array}$ & 070 & $\begin{array}{l}\text { SAV1E } 1064 \text { (re-used sherd), SAV1E } 1066 \\
\text { (lid), SAV1E } 1012 \text { (slag) }\end{array}$ & 08.02 .2014 \\
\hline $2195 / 2014$ & $\mathrm{SQ} 3,9.2-10.5 \mathrm{~m} \mathrm{~W}-\mathrm{E} / 4.7-5.9 \mathrm{~m} \mathrm{~N}-\mathrm{S}$ & pottery & 070 & & 05.02 .2014 \\
\hline $2242 / 2014$ & $\mathrm{SQ} 3,9.2-10.5 \mathrm{~m} \mathrm{~W}-\mathrm{E} / 8-9.2 \mathrm{~m} \mathrm{~N}-\mathrm{S}$ & $\begin{array}{l}\text { pottery, among it mould for (animal) figurine? (1 fragment, } \\
\text { pottery) }\end{array}$ & 070 & SAV1E 1069 & 06.02 .2014 \\
\hline $2237 / 2014$ & SQ3, 9-10.5m W-E/2-3.6m N-S & pottery & 070 & & 06.02 .2014 \\
\hline $2241 / 2014$ & SQ3, 9-10.5m W-E/2-3.6m N-S & $\begin{array}{l}\text { pottery, among it re-used sherd/gaming piece? (1 re-used body } \\
\text { sherd, glazed ware) }\end{array}$ & 070 & SAV1E 1061 & 06.02 .2014 \\
\hline $2248 / 2014$ & SQ3, 9-10.5m W-E/8-9.5m N-S & pottery & 070 & & 06.02 .2014 \\
\hline $2169 / 2014$ & $\mathrm{SQ} 3,8.5-10.5 \mathrm{~m} \mathrm{~W}-\mathrm{E} / 0.8-2.3 \mathrm{~m} \mathrm{~N}-\mathrm{S}$ & pottery & 071 & & 04.02 .2014 \\
\hline $2184 / 2014$ & $\mathrm{SQ} 3,6-8 \mathrm{~m} \mathrm{~W}-\mathrm{E} / 0-7 \mathrm{~m} \mathrm{~N}-\mathrm{S}$ & pottery, slag (1 piece) & 072 & SAV1E 0966 (slag) & 04.02 .2014 \\
\hline $2193 / 2014$ & $\mathrm{SQ} 3,6.6-8 \mathrm{~m} \mathrm{~W}-\mathrm{E} / 1.1-3.2 \mathrm{~m} \mathrm{~N}-\mathrm{S}$ & pottery & 073 & & 04.02 .2014 \\
\hline $2200 / 2014$ & $\mathrm{SQ} 3,6.6-9 \mathrm{~m} \mathrm{~W}-\mathrm{E} / 1.1-4.5 \mathrm{~m} \mathrm{~N}-\mathrm{S}$ & $\begin{array}{l}\text { pottery, among it re-used sherd (1 piece, Post-New Kingdom, } \\
\text { pottery) }\end{array}$ & 073 & SAV1E 1032 & 05.02 .2014 \\
\hline
\end{tabular}




\begin{tabular}{|c|c|c|c|c|c|}
\hline $2183 / 2014$ & $\mathrm{SQ} 2,1.1-1.8 \mathrm{~m} \mathrm{~W}-\mathrm{E} / 0-1 \mathrm{~m} \mathrm{~N}-\mathrm{S}$ & pottery & 074 & & 04.02 .2014 \\
\hline $2223 / 2014$ & $\mathrm{SQ} 3,5.7-6.2 \mathrm{~m} \mathrm{~W}-\mathrm{E} / 0.5-2.2 \mathrm{~m} \mathrm{~N}-\mathrm{S}$ & pottery, among it window grille ( 1 fragment, Christian, pottery) & 075 & SAV1E 1040 & 05.02 .2014 \\
\hline $2211 / 2014$ & $\mathrm{SQ} 3,6-8 \mathrm{~m} \mathrm{~W}-\mathrm{E} / 0.2-1.1 \mathrm{~m} \mathrm{~N}-\mathrm{S}$ & $\begin{array}{l}\text { pottery, among it re-used sherds ( } 2 \text { pieces, } 1 \text { Post-New Kingdom, } \\
1 \text { unspecified, pottery), lid? (1 fragmented mud object) }\end{array}$ & 075 & $\begin{array}{l}\text { SAV1E 1031, SAV1E } 1035 \text { (re-used sherds), } \\
\text { SAV1E } 1041 \text { (lid?) }\end{array}$ & 05.02 .2014 \\
\hline $2231 / 2014$ & $\mathrm{SQ} 3,8.2-10 \mathrm{~m} \mathrm{~W}-\mathrm{E} / 0-2 \mathrm{~m} \mathrm{~N}-\mathrm{S}$ & $\begin{array}{l}\text { pottery, among it re-used sherds ( } 3 \text { pieces, } 1 \text { unclear, } 1 \text { Christian, } \\
1 \text { Post-New Kingdom, pottery), re-used sherd/weight? (1 piece, } \\
\text { pottery) }\end{array}$ & 076 & $\begin{array}{l}\text { SAV1E 1025, SAV1E 1026, SAV1E } 1037 \\
\text { (re-used sherds), SAV1E } 1034 \text { (re-used } \\
\text { sherd/weight?) }\end{array}$ & 06.02 .2014 \\
\hline $2234 / 2014$ & $\mathrm{SQ} 3,8.2-10 \mathrm{~m} \mathrm{~W}-\mathrm{E} / 0.3-1.5 \mathrm{~m} \mathrm{~N}-\mathrm{S}$ & pottery & 077 & & 06.02 .2014 \\
\hline $2261 / 2014$ & $\mathrm{SQ} 4,8-10 \mathrm{~m} \mathrm{~W}-\mathrm{E} / 0-2 \mathrm{~m} \mathrm{~N}-\mathrm{S}$ & pottery & 078 & & 08.02 .2014 \\
\hline $2263 / 2014$ & $\mathrm{SQ} 4,9.5-10.5 \mathrm{~m} \mathrm{~W}-\mathrm{E} / 0.6-2 \mathrm{~m} \mathrm{~N}-\mathrm{S}$ & pottery & 079 & & 08.02 .2014 \\
\hline $2269 / 2014$ & SQ3, 6-7.5m W-E/3.5-5m N-S & $\begin{array}{l}\text { pottery, among it re-used sherds ( } 2 \text { pieces, } 118^{\text {th }} \text { Dynasty, } 1 \text { Post- } \\
\text { New Kingdom, pottery); slag ( } 1 \text { piece) }\end{array}$ & 080 & $\begin{array}{l}\text { SAV1E 1074, SAV1E } 1075 \text { (re-used sherds), } \\
\text { SAV1E } 1011 \text { (slag) }\end{array}$ & 08.02 .2014 \\
\hline $2276 / 2014$ & $\mathrm{SQ} 3,6-8 \mathrm{~m} \mathrm{~W}-\mathrm{E} / 5-7.5 \mathrm{~m} \mathrm{~N}-\mathrm{S}$ & $\begin{array}{l}\text { pottery, among it re-used sherd/scraper? (1 piece, Post-New } \\
\text { Kingdom, pottery); slag (1 piece) }\end{array}$ & 080 & $\begin{array}{l}\text { SAV1E } 1073 \text { (re-used sherd/scraper?), } \\
\text { SAV1E } 1018 \text { (slag) }\end{array}$ & 08.02 .2014 \\
\hline $2286 / 2014$ & $\mathrm{SQ} 3,6-8.5 \mathrm{~m} \mathrm{~W}-\mathrm{E} / 5-10 \mathrm{~m} \mathrm{~N}-\mathrm{S}$ & pottery & 080 & & 09.02 .2014 \\
\hline $2272 / 2014$ & $\mathrm{SQ} 3,6-6.7 \mathrm{~m} \mathrm{~W}-\mathrm{E} / 3.5-4.4 \mathrm{~m} \mathrm{~N}-\mathrm{S}$ & pottery & 081 & & 08.02 .2014 \\
\hline $2273 / 2014$ & $\mathrm{SQ} 3,6-6.4 \mathrm{~m} \mathrm{~W}-\mathrm{E} / 3.6-4 \mathrm{~m} \mathrm{~N}-\mathrm{S}$ & pottery & 082 & & 08.02 .2014 \\
\hline $2280 / 2014$ & $\mathrm{SQ} 4,8-10 \mathrm{~m} \mathrm{~W}-\mathrm{E} / 2-3 \mathrm{~m} \mathrm{~N}-\mathrm{S}$ & pottery, slag (1 piece) & 083 & SAV1E 1044 (slag) & 09.02 .2014 \\
\hline $2290 / 2014$ & $\mathrm{SQ} 4,8-9.5 \mathrm{~m} \mathrm{~W}-\mathrm{E} / 0-2 \mathrm{~m} \mathrm{~N}-\mathrm{S}$ & pottery, slag (1 piece) & 084 & SAV1E 1045 (slag) & 09.02 .2014 \\
\hline $2283 / 2014$ & SQ4, 8-9.5m W-E/0-2m N-S, Feature 15 & pottery, among it window grille ( 1 fragment, Christian, pottery) & 084 & SAV1E 1077 & 09.02 .2014 \\
\hline $2294 / 2014$ & $\mathrm{SQ} 3,5-7.5 \mathrm{~m} \mathrm{~W}-\mathrm{E} / 8.5-10 \mathrm{~m} \mathrm{~N}-\mathrm{S}$ & pottery & 085 & & 09.02 .2014 \\
\hline $2295 / 2014$ & SQ4, 8.4-9.5m W-E/0-1m N-S & pottery & 086 & & 09.02 .2014 \\
\hline $2299 / 2014$ & $\begin{array}{l}\text { SQ4, } 9.2-9.6 \mathrm{~m} \mathrm{~W}-\mathrm{E} / 0-1.1 \mathrm{~m} \mathrm{~N}-\mathrm{S} \text {, } \\
\text { Feature } 15\end{array}$ & pottery, among it scraper (1 re-used $18^{\text {th }}$ Dynasty sherd, pottery) & 087 & SAV1E 1091 & 09.02 .2014 \\
\hline $2300 / 2014$ & $\mathrm{SQ} 4,8-10.5 \mathrm{~m} \mathrm{~W}-\mathrm{E} / 2-3 \mathrm{~m} \mathrm{~N}-\mathrm{S}$ & pottery & 088 & & 10.02 .2014 \\
\hline $2303 / 2014$ & SQ3, 9-10.5m W-E/7.5-9.5m N-S & pottery, among it slag ( 2 pieces $)$ & 089 & SAV1E 1068 & 10.02 .2014 \\
\hline $2310 / 2014$ & $\mathrm{SQ} 3,8-9.4 \mathrm{~m} \mathrm{~W}-\mathrm{E} / 2.3-3.8 \mathrm{~m} \mathrm{~N}-\mathrm{S}$ & pottery & 090 & & 10.02 .2014 \\
\hline $2306 / 2014$ & $\mathrm{SQ} 4,9.4-9.8 \mathrm{~m} \mathrm{~W}-\mathrm{E} / 2-2.5 \mathrm{~m} \mathrm{~N}-\mathrm{S}$ & pottery & 091 & & 10.02 .2014 \\
\hline $2308 / 2014$ & SQ4, 9.8-10.5m W-E/2-2.4m N-S & pottery & 092 & & 10.02 .2014 \\
\hline $2419 / 2014$ & $\begin{array}{l}\text { SQ4, debris from S-wall Feature } 15 \text { up to } \\
1 \mathrm{~m} \mathrm{N-S} \text { of square }\end{array}$ & $\begin{array}{l}\text { pottery, among it re-used sherd/scraper? (1 piece, Post-New } \\
\text { Kingdom, pottery) }\end{array}$ & 092 & SAV1E 1125 & 24.02 .2014 \\
\hline
\end{tabular}




\begin{tabular}{|c|c|c|c|c|c|}
\hline $2314 / 2014$ & $\mathrm{SQ} 3,7.7-8.8 \mathrm{~m} \mathrm{~W}-\mathrm{E} / 0-3.5 \mathrm{~m} \mathrm{~N}-\mathrm{S}$ & pottery & 093 & & 10.02 .2014 \\
\hline $2318 / 2014$ & $\mathrm{SQ} 3,7.7-8.8 \mathrm{~m} \mathrm{~W}-\mathrm{E} / 0-3.5 \mathrm{~m} \mathrm{~N}-\mathrm{S}$ & pottery & 095 & & 11.02 .2014 \\
\hline $2323 / 2014$ & $\mathrm{SQ} 4,8-10.5 \mathrm{~m} \mathrm{~W}-\mathrm{E} / 3-4.2 \mathrm{~m} \mathrm{~N}-\mathrm{S}$ & pottery & 096 & & 11.02 .2014 \\
\hline $2333 / 2014$ & $\mathrm{SQ} 3,7.7-8.8 \mathrm{~m} \mathrm{~W}-\mathrm{E} / 3.5-8 \mathrm{~m} \mathrm{~N}-\mathrm{S}$ & $\begin{array}{l}\text { pottery, among it re-used sherd (1 piece, } 18^{\text {th }} \text { Dynasty, pottery); } \\
\text { slag (1 piece) }\end{array}$ & 097 & $\begin{array}{l}\text { SAV1E } 1104 \text { (re-used sherd), } \\
\text { SAV1E } 1096 \text { (slag) }\end{array}$ & 11.02.2014 \\
\hline $2330 / 2014$ & $\mathrm{SQ} 3,7.8-9.6 \mathrm{~m} \mathrm{~W}-\mathrm{E} / 8-9.5 \mathrm{~m} \mathrm{~N}-\mathrm{S}$ & $\begin{array}{l}\text { pottery, among it architecture/window grille (1 fragment, } \\
\text { Christian, clay) }\end{array}$ & 097 & SAV1E 1122 & 11.02 .2014 \\
\hline $2326 / 2014$ & $\mathrm{SQ} 4,9.5-10.5 \mathrm{~m} \mathrm{~W}-\mathrm{E} / 3.5-4.2 \mathrm{~m} \mathrm{~N}-\mathrm{S}$ & pottery, slag (1 piece) & 098 & SAV1E 1092 (slag) & 11.02 .2014 \\
\hline $2337 / 2014$ & $\mathrm{SQ} 4,8.4-10.5 \mathrm{~m} \mathrm{~W}-\mathrm{E} / 2.5-4.2 \mathrm{~m} \mathrm{~N}-\mathrm{S}$ & pottery & 099 & & 11.02 .2014 \\
\hline $2349 / 2014$ & $\mathrm{SQ} 4,2-4 \mathrm{~m} \mathrm{~W}-\mathrm{E} / 0-3.6 \mathrm{~m} \mathrm{~N}-\mathrm{S}$ & pottery & 100 & & 12.02 .2014 \\
\hline $2341 / 2014$ & $\mathrm{SQ} 4,8.4-10.5 \mathrm{~m} \mathrm{~W}-\mathrm{E} / 4.2-5.6 \mathrm{~m} \mathrm{~N}-\mathrm{S}$ & pottery, slag (1 piece) & 101 & SAV1E 1100 (slag) & 12.02 .2014 \\
\hline $2346 / 2014$ & $\mathrm{SQ} 4,8.5-10 \mathrm{~m} \mathrm{~W}-\mathrm{E} / 4.2-5.6 \mathrm{~m} \mathrm{~N}-\mathrm{S}$ & pottery & 102 & & 12.02 .2014 \\
\hline $2354 / 2014$ & SQ4, 9.2-10.5m W-E/4.2-5.4m N-S & pottery, slag (1 piece) & 103 & SAV1E 1094 (slag) & 12.02 .2014 \\
\hline $2363 / 2014$ & $\mathrm{SQ} 4,8.7-9.5 \mathrm{~m} \mathrm{~W}-\mathrm{E} / 4.2-5.6 \mathrm{~m} \mathrm{~N}-\mathrm{S}$ & pottery, slag (1 piece) & 104 & SAV1E 1095 (slag) & 12.02 .2014 \\
\hline $2398 / 2014$ & $\mathrm{SQ} 4,3.3-3.6 \mathrm{~m} \mathrm{~W}-\mathrm{E} / 2.5-2.8 \mathrm{~m} \mathrm{~N}-\mathrm{S}$ & pottery (bowl) & 105 & & 16.02 .2014 \\
\hline $2359 / 2014$ & $\mathrm{SQ} 4,3.5-6 \mathrm{~m} \mathrm{~W}-\mathrm{E} / 0-3.4 \mathrm{~m} \mathrm{~N}-\mathrm{S}$ & pottery & 105 & & 12.02 .2014 \\
\hline $2385 / 2014$ & $\mathrm{SQ} 4,3.5-6 \mathrm{~m} \mathrm{~W}-\mathrm{E} / 0-3.5 \mathrm{~m} \mathrm{~N}-\mathrm{S}$ & $\begin{array}{l}\text { pottery, among it stopper/sealing ( } 1 \text { piece, clay), vessel ( } 1 \text { frag- } \\
\text { ment, glazed ware, Ottoman), re-used sherd/scraper? (1 piece, } \\
\text { Post-New Kingdom, pottery), figurine ( } 1 \text { fragment, animal figu- } \\
\text { rine, Christian?, clay), architecture/window grille (1 fragment, } \\
\text { Christian, clay) }\end{array}$ & 105 & $\begin{array}{l}\text { SAV1E } 1107 \text { (stopper/sealing), SAV1E } 1109 \\
\text { (vessel), SAV1E } 1115 \text { (re-used sherd), } \\
\text { SAV1E } 1120 \text { (figurine), SAV1E } 1123 \\
\text { (architecture/window grille) }\end{array}$ & 13.02.2014 \\
\hline $2397 / 2014$ & $\mathrm{SQ} 4,3.5-6 \mathrm{~m} \mathrm{~W}-\mathrm{E} / 0-4.7 \mathrm{~m} \mathrm{~N}-\mathrm{S}$ & pottery & 105 & & 16.02 .2014 \\
\hline $2371 / 2014$ & $\mathrm{SQ} 4,3.5-6 \mathrm{~m} \mathrm{~W}-\mathrm{E} / 2.5-4.7 \mathrm{~m} \mathrm{~N}-\mathrm{S}$ & $\begin{array}{l}\text { pottery, among it window grille ( } 1 \text { fragment, Christian, pottery); } \\
\text { slag (1 piece) }\end{array}$ & 105 & $\begin{array}{l}\text { SAV1E } 1103 \text { (window grille?), SAV1E } 1102 \\
\text { (slag) }\end{array}$ & 13.02.2014 \\
\hline $2365 / 2014$ & $\mathrm{SQ} 4,8.4-9.5 \mathrm{~m} \mathrm{~W}-\mathrm{E} / 3.5-4.2 \mathrm{~m} \mathrm{~N}-\mathrm{S}$ & pottery & 106 & & 13.02 .2014 \\
\hline $2366 / 2014$ & SQ4, 8-10.5m W-E/5.6-7.7m N-S & pottery & 107 & & 13.02 .2014 \\
\hline $2375 / 2014$ & $\begin{array}{l}\text { SQ4, 9-10.5m W-E/5.6-7.2m N-S, } \\
\text { Feature } 16\end{array}$ & $\begin{array}{l}\text { pottery, among it scrapers? ( } 2 \text { pieces, each re-used Post-New } \\
\text { Kingdom sherds, pottery), }\end{array}$ & 108 & SAV1E 1114, SAV1E 1116 (scrapers?) & 13.02.2014 \\
\hline $2389 / 2014$ & $\begin{array}{l}\text { SQ4, } 8.5-10.5 \mathrm{~m} \mathrm{~W}-\mathrm{E} / 5.6-7 \mathrm{~m} \mathrm{~N}-\mathrm{S} \text {, } \\
\text { Feature } 16\end{array}$ & pottery, among it re-used sherd (1 piece, Christian, pottery) & 109 & SAV1E 1110 & 15.02.2014 \\
\hline $2381 / 2014$ & $\begin{array}{l}\mathrm{SQ} 4,8.5-9.5 \mathrm{~m} \mathrm{~W}-\mathrm{E} / 5.6-7.4 \mathrm{~m} \mathrm{~N}-\mathrm{S} \text {, } \\
\text { Feature } 16\end{array}$ & $\begin{array}{l}\text { pottery, among it re-used sherd/lid? ( } 1 \text { re-used complete base of a } \\
\text { qadus/Saqia vessel, Christian, pottery) }\end{array}$ & 109 & SAV1E 1118 & 13.02 .2014 \\
\hline
\end{tabular}




\begin{tabular}{|c|c|c|c|c|c|}
\hline $2412 / 2014$ & SQ4, below 2411/2014 & pottery (few sherds) & 110 & & 18.02 .2014 \\
\hline $2393 / 2014$ & SQ4, 7.3-8.7m W-E/0-2m N-S & pottery & 110 & & 16.02 .2014 \\
\hline $2425 / 2014$ & SQ 4, Feature 15, W-wall & pottery (1 rim sherd) & 111 & & 26.02.2014 \\
\hline $2401 / 2014$ & $\mathrm{SQ} 4,7.3-8 \mathrm{~m} \mathrm{~W}-\mathrm{E} / 0-2.5 \mathrm{~m} \mathrm{~N}-\mathrm{S}$ & $\begin{array}{l}\text { pottery, among it re-used sherd ( } 1 \text { piece, } 18^{\text {th }} \text { Dynasty, pottery), } \\
\text { architecture/window grille ( } 1 \text { fragment, Christian, clay) }\end{array}$ & 111 & $\begin{array}{l}\text { SAV1E } 1112 \text { (re-used sherd), SAV1E } 1121 \\
\text { (architecture/ window grill?) }\end{array}$ & 16.02 .2014 \\
\hline $2407 / 2014$ & SQ4, 5.7-7.2m W-E/0.5-10.5m N-S & $\begin{array}{l}\text { pottery ( } 2 \text { baskets), among them re-used sherds ( } 3 \text { pieces, } 1 \\
\text { unclear, } 1 \text { Post-New Kingdom, } 1 \text { Christian, pottery) }\end{array}$ & 112 & $\begin{array}{l}\text { SAV1E 1111, SAV1E 1113, SAV1E } 1119 \\
\text { (re-used sherds) }\end{array}$ & 18.02 .2014 \\
\hline $2414 / 2014$ & SQ 2, Feature 15 & pottery & 113 & & 24.02 .2014 \\
\hline $2418 / 2014$ & SQ2/4, Feature 15 & pottery & 114 & & 24.02 .2014 \\
\hline $026 / 2014$ & $\begin{array}{l}\text { SQ1, cleaning of W-profile of SQ1 in area } \\
\text { of Feature } 7\end{array}$ & pottery & l & & 07.01 .2014 \\
\hline $2187 / 2014$ & $\begin{array}{l}\text { SQ2, } 0.5-1.8 \mathrm{~m} \mathrm{~N}-\mathrm{S} \text {, Feature } 15 \text {, cleaning of } \\
\text { W profile }\end{array}$ & pottery & l & & 04.02 .2014 \\
\hline $2180 / 2014$ & $\begin{array}{l}\mathrm{SQ} 2,0-2.5 \mathrm{~m} \mathrm{~W}-\mathrm{E} / 0.5-1.8 \mathrm{~m} \mathrm{~N}-\mathrm{S}, \mathrm{W}- \\
\text { profile, Feature } 15\end{array}$ & pottery; charcoal (1 bag) & l & & 04.02 .2014 \\
\hline $001 / 2014$ & $\begin{array}{l}\text { SQ2, Feature } 16 \text {, between broken bricks, } \\
1.5 \mathrm{~m} \text { from } \mathrm{E}\end{array}$ & pottery (sherds) & $\begin{array}{l}\text { height: } \\
\text { 159.63; } \\
159.35 \mathrm{~m}\end{array}$ & & 02.01 .2014 \\
\hline $2413 / 2014$ & SQ3, from muna of Feature 13 & pottery ( 2 sherds, $18^{\text {th }}$ Dynasty) & l & & 20.02 .2014 \\
\hline $102 / 2014$ & $\mathrm{SQ} 4,1-2.5 \mathrm{~m} \mathrm{~W}-\mathrm{E} / 1.5-2 \mathrm{~m} \mathrm{~N}-\mathrm{S}$ & rope (1 piece, organic fibre) & 008 & SAV1E 0398 & 12.01 .2014 \\
\hline $174 / 2014$ & $\mathrm{SQ} 3,3-5 \mathrm{~m} \mathrm{~W}-\mathrm{E} / 0-3.5 \mathrm{~m} \mathrm{~N}-\mathrm{S}$ & rope (1 piece, organic fibre) & 013 & SAV1E 0573 & 15.01 .2014 \\
\hline $176 / 2014$ & $\mathrm{SQ} 4,2.5 \mathrm{~m} \mathrm{~W}-\mathrm{E} / 3 \mathrm{~m} \mathrm{~N}-\mathrm{S}$ & rope (1 piece, organic fibre) & 014 & SAV1E 0574 & 15.01 .2014 \\
\hline $2100 / 2014$ & $\mathrm{SQ} 3,2-5.5 \mathrm{~m} \mathrm{~W}-\mathrm{E} / 2-3.8 \mathrm{~m} \mathrm{~N}-\mathrm{S}$ & rope (1 piece, organic fibre) & 059 & SAV1E 0933 & 02.02 .2014 \\
\hline $2325 / 2014$ & $\mathrm{SQ} 4,10 \mathrm{~m} \mathrm{~W}-\mathrm{E} / 3.8 \mathrm{~m} \mathrm{~N}-\mathrm{S}$ & rope (1 piece, with mud attached, organic fibre) & 096 & SAV1E 1099 & 11.02 .2014 \\
\hline $2358 / 2014$ & $\mathrm{SQ} 4,9.2-10.5 \mathrm{~m} \mathrm{~W}-\mathrm{E} / 4.2-5.4 \mathrm{~m} \mathrm{~N}-\mathrm{S}$ & ropes (3 pieces, organic fibre) & 103 & SAV1E 1098 & 12.02 .2014 \\
\hline $368 / 2014$ & $\mathrm{SQ} 4,0.5-1 \mathrm{~m} \mathrm{~W}-\mathrm{E} / 3-4 \mathrm{~m} \mathrm{~N}-\mathrm{S}$ & schist & 008 & & 23.01 .2014 \\
\hline $170 / 2014$ & $\mathrm{SQ} 4,1-3 \mathrm{~m} \mathrm{~W}-\mathrm{E} / 0-4 \mathrm{~m} \mathrm{~N}-\mathrm{S}$ & schist & 014 & & 15.01 .2014 \\
\hline $356 / 2014$ & $\mathrm{SQ} 4,0-2.5 \mathrm{~m} \mathrm{~W}-\mathrm{E} / 4.5-5.5 \mathrm{~m} \mathrm{~N}-\mathrm{S}$ & schist & 015 & & 22.01 .2014 \\
\hline $187 / 2014$ & $\mathrm{SQ} 4,0-5 \mathrm{~m} \mathrm{~W}-\mathrm{E} / 5-8 \mathrm{~m} \mathrm{~N}-\mathrm{S}$ & schist & 015 & & 16.01 .2014 \\
\hline $210 / 2014$ & $\mathrm{SQ} 4,0-5 \mathrm{~m} \mathrm{~W}-\mathrm{E} / 5-8 \mathrm{~m} \mathrm{~N}-\mathrm{S}$ & schist & 015 & & 18.01 .2014 \\
\hline $228 / 2014$ & $\mathrm{SQ} 4,1.5-5 \mathrm{~m} \mathrm{~W}-\mathrm{E} / 3.5-5 \mathrm{~m} \mathrm{~N}-\mathrm{S}$ & schist & 015 & & 18.01 .2014 \\
\hline
\end{tabular}




\begin{tabular}{|c|c|c|c|c|}
\hline $236 / 2014$ & $\mathrm{SQ} 4,1.5-5 \mathrm{~m} \mathrm{~W}-\mathrm{E} / 8-8.5 \mathrm{~m} \mathrm{~N}-\mathrm{S}$ & schist & 015 & 18.01.2014 \\
\hline $249 / 2014$ & $\mathrm{SQ} 4,1.5-5 \mathrm{~m} \mathrm{~W}-\mathrm{E} / 8-8.5 \mathrm{~m} \mathrm{~N}-\mathrm{S}$ & schist & 015 & 19.01.2014 \\
\hline $262 / 2014$ & $\mathrm{SQ} 4,3.5-5 \mathrm{~m} \mathrm{~W}-\mathrm{E} / 0-3.5 \mathrm{~m} \mathrm{~N}-\mathrm{S}$ & schist & 015 & 19.01.2014 \\
\hline $246 / 2014$ & $\begin{array}{l}\text { SQ1A, } 0.5-2.5 \mathrm{~m} \mathrm{~W}-\mathrm{E} / 0-1 \mathrm{~m} \mathrm{~N} \text { N-S; } \\
\text { SQ1B } 0.5-2.5 \mathrm{~m} \mathrm{~W}-\mathrm{E} / 4.5-5 \mathrm{~m} \mathrm{~N}-\mathrm{S}\end{array}$ & schist & 019 & 19.01.2014 \\
\hline $222 / 2014$ & SQ1B, $0.5-2.5 \mathrm{~m} \mathrm{~W}-\mathrm{E} / 4-5 \mathrm{~m} \mathrm{~N}-\mathrm{S}$ & schist & 019 & 18.01 .2014 \\
\hline $257 / 2014$ & SQ1B, 3-6m W-E/0-2m N-S & schist & 021 & 19.01.2014 \\
\hline $271 / 2014$ & $\mathrm{SQ} 4,0-1.5 \mathrm{~m} \mathrm{~W}-\mathrm{E} / 8-10 \mathrm{~m} \mathrm{~N}-\mathrm{S}$ & schist & 022 & 19.01.2014 \\
\hline $305 / 2014$ & $\mathrm{SQ} 3,2.5-3.2 \mathrm{~m} \mathrm{~W}-\mathrm{E} / 0-3.5 \mathrm{~m} \mathrm{~N}-\mathrm{S}$ & schist & 024 & 20.01 .2014 \\
\hline $331 / 2014$ & $\mathrm{SQ} 3,0-2 \mathrm{~m} \mathrm{~W}-\mathrm{E} / 4.4-10 \mathrm{~m} \mathrm{~N}-\mathrm{S}$ & schist & 025 & 21.01 .2014 \\
\hline $342 / 2014$ & $\mathrm{SQ} 4,0-3 \mathrm{~m} \mathrm{~W}-\mathrm{E} / 1-5.5 \mathrm{~m} \mathrm{~N}-\mathrm{S}$ & schist & 030 & 22.01 .2014 \\
\hline $351 / 2014$ & $\mathrm{SQ} 3,2.5-9 \mathrm{~m} \mathrm{~W}-\mathrm{E} / 0-10 \mathrm{~m} \mathrm{~N}-\mathrm{S}$ & schist & 031 & 22.01 .2014 \\
\hline $377 / 2014$ & $\mathrm{SQ} 4,5-8 \mathrm{~m} \mathrm{~W}-\mathrm{E} / 0-10 \mathrm{~m} \mathrm{~N}-\mathrm{S}$ & schist & 033 & 23.01 .2014 \\
\hline $393 / 2014$ & SQ4A, 0-2m W-E/0-2m N-S & schist & 035 & 23.01 .2014 \\
\hline $446 / 2014$ & $\mathrm{SQ} 3,0-1.5 \mathrm{~m} \mathrm{~W}-\mathrm{E} / 7.4-8.7 \mathrm{~m} \mathrm{~N}-\mathrm{S}$ & schist plate & 044 & 27.01.2014 \\
\hline $104 / 2014$ & $\mathrm{SQ} 3,5-8.5 \mathrm{~m} \mathrm{~W}-\mathrm{E} / 0-3 \mathrm{~m} \mathrm{~N}-\mathrm{S}$ & seashell (1 fragment); bone & 003 & 12.01.2014 \\
\hline $079 / 2014$ & SQ1B, 0-10m W-E/0-5m N-S & seashell (2 fragments) & 007 & 11.01 .2014 \\
\hline $366 / 2014$ & $\mathrm{SQ} 4,0.5-1 \mathrm{~m} \mathrm{~W}-\mathrm{E} / 3-4 \mathrm{~m} \mathrm{~N}-\mathrm{S}$ & seashell (1 fragment); bone & 008 & 23.01 .2014 \\
\hline $093 / 2014$ & $\mathrm{SQ} 4,1-2.5 \mathrm{~m} \mathrm{~W}-\mathrm{E} / 0-1.5 \mathrm{~m}$ N-S & seashell (1 fragment); bone & 008 & 12.01.2014 \\
\hline $166 / 2014$ & $\mathrm{SQ} 4,1-3 \mathrm{~m} \mathrm{~W}-\mathrm{E} / 0-4 \mathrm{~m} \mathrm{~N}-\mathrm{S}$ & seashell (1 fragment); bone & 014 & 15.01 .2014 \\
\hline $181 / 2014$ & SQ4A, 9-10.5m W-E/0-2m N-S & seashell (1 fragment); bone & 016 & 16.01 .2014 \\
\hline $241 / 2014$ & $\begin{array}{l}\text { SQ1A, } 0.5-2.5 \mathrm{~m} \mathrm{~W}-\mathrm{E} / 0-1 \mathrm{~m} \mathrm{~N}-\mathrm{S} \\
\text { SQ1B } 0.5-2.5 \mathrm{~m} \mathrm{~W}-\mathrm{E} / 4.5-5 \mathrm{~m} \mathrm{~N}-\mathrm{S}\end{array}$ & seashell (1 fragment); bone & 019 & 19.01.2014 \\
\hline $220 / 2014$ & SQ1B, $0.5-2.5 \mathrm{~m} \mathrm{~W}-\mathrm{E} / 4-5 \mathrm{~m} \mathrm{~N}-\mathrm{S}$ & seashell (1 small piece); bone & 019 & 18.01 .2014 \\
\hline $254 / 2014$ & SQ1B, 3-6m W-E/0-2m N-S & seashell (2 fragments); bone & 021 & 19.01.2014 \\
\hline $297 / 2014$ & SQ1B, 7-8.3m W-E/0-2.5m N-S & seashell (1 fragment); bone & 021 & 20.01 .2014 \\
\hline $347 / 2014$ & $\mathrm{SQ} 3,2.5-9 \mathrm{~m} \mathrm{~W}-\mathrm{E} / 0-10 \mathrm{~m} \mathrm{~N}-\mathrm{S}$ & seashell (1 fragment); bone & 031 & 22.01 .2014 \\
\hline $399 / 2014$ & $\mathrm{SQ} 1 \mathrm{~B}, 0-3 \mathrm{~m} \mathrm{~W}-\mathrm{E} / 2-4.5 \mathrm{~m} \mathrm{~N}-\mathrm{S}$ & seashell (1 fragment); bone & 039 & 25.01 .2014 \\
\hline
\end{tabular}




\begin{tabular}{|c|c|c|c|c|c|}
\hline $1505 / 2014$ & $\mathrm{SQ} 3,0-2.7 \mathrm{~m} \mathrm{~W}-\mathrm{E} / 8.7-10 \mathrm{~m} \mathrm{~N}-\mathrm{S}$ & seashell (1 large fragment) & 045 & & 27.01 .2014 \\
\hline $2055 / 2014$ & SQ1B, $1.5-6.5 \mathrm{~m} \mathrm{~W}-\mathrm{E} / 1.3-3 \mathrm{~m} \mathrm{~N}-\mathrm{S}$ & seashell (2 small pieces) & 051 & & 30.01 .2014 \\
\hline $2031 / 2014$ & $\mathrm{SQ} 1 \mathrm{~B}, 4-6.5 \mathrm{~m} \mathrm{~W}-\mathrm{E} / 0-1.5 \mathrm{~m} \mathrm{~N}-\mathrm{S}$ & seashell (1 small piece); bone & 051 & & 29.01 .2014 \\
\hline $2093 / 2014$ & $\mathrm{SQ} 1 \mathrm{~B}, 0-1.5 \mathrm{~m} \mathrm{~W}-\mathrm{E} / 2.5-4.5 \mathrm{~m} \mathrm{~N}-\mathrm{S}$ & seashell (1 large fragment) & 056 & & 01.02 .2014 \\
\hline $2179 / 2014$ & $\begin{array}{l}\mathrm{SQ} 3,4.8-7 \mathrm{~m} \mathrm{~W}-\mathrm{E} / 4-8.3 \mathrm{~m} \mathrm{~N}-\mathrm{S} \text {, } \\
\text { cleaning of SU }\end{array}$ & seashell (1 small piece) & 066 & & 04.02 .2014 \\
\hline $2133 / 2014$ & SQ1B, $0-1.1 \mathrm{~m} \mathrm{~W}-\mathrm{E} / 3-4.2 \mathrm{~m} \mathrm{~N}-\mathrm{S}$ & seashell (1 fragment) & 067 & & 03.02 .2014 \\
\hline $2296 / 2014$ & SQ4, 8.4-9.5m W-E/0-1m N-S, Feature 15 & seashell (1 small piece); bone & 086 & & 09.02 .2014 \\
\hline $2302 / 2014$ & $\mathrm{SQ} 4,8-10.5 \mathrm{~m}$ W-E/2-3m N-S, Feature 15 & seashell (1 small piece); bone & 088 & & 10.02 .2014 \\
\hline $2415 / 2014$ & SQ2, Feature 15, ashy deposit & seashell (1 bag, fragments); bone & 113 & & 24.02 .2014 \\
\hline $238 / 2014$ & $\mathrm{SQ} 4,4.8 \mathrm{~m} \mathrm{~W}-\mathrm{E} / 8.4 \mathrm{~m} \mathrm{~N}-\mathrm{S}$ & shell & 015 & & 18.01 .2014 \\
\hline $1503 / 2014$ & SQ1B, 3-10m W-E/3-5m N-S & skin (1 piece) & 043 & & 27.01.2014 \\
\hline $012 / 2014$ & $\mathrm{SQ} 1 \mathrm{~B}, 5 \times 10 \mathrm{~m}$, surface & $\operatorname{slag}(2$ pieces $)$ & 001 & SAV1E 0326 & 04.01 .2014 \\
\hline $011 / 2014$ & $\mathrm{SQ} 3,10 \times 10 \mathrm{~m}$, surface cleaning & slag (8 pieces) & 001 & SAV1E 0330 & 04.01 .2014 \\
\hline $010 / 2014$ & $\mathrm{SQ} 4,10 \times 10 \mathrm{~m}$, surface cleaning & slag (6 pieces) & 001 & SAV1E 0328 & 04.01 .2014 \\
\hline $017 / 2014$ & SQ4A, $2 \times 10 \mathrm{~m}$, surface cleaning & slag (3 pieces) & 001 & SAV1E 0324 & 05.01 .2014 \\
\hline $313 / 2014$ & $\mathrm{SQ} 3,9.5-10 \mathrm{~m} \mathrm{~W}-\mathrm{E} / 5.7-8 \mathrm{~m} \mathrm{~N}-\mathrm{S}$ & slag (2 pieces) & 002 & SAV1E 0660 & 20.01 .2014 \\
\hline $085 / 2014$ & $\mathrm{SQ} 4,8-10 \mathrm{~m} \mathrm{~W}-\mathrm{E} / 0-10 \mathrm{~m} \mathrm{~N}-\mathrm{S}$ & slag (2 pieces) & 002 & SAV1E 0393 & 12.01 .2014 \\
\hline $124 / 2014$ & $\mathrm{SQ} 3,2-5 \mathrm{~m} \mathrm{~W}-\mathrm{E} / 2-4 \mathrm{~m} \mathrm{~N}-\mathrm{S}$ & slag (1 piece) & 003 & SAV1E 0404 & 13.01 .2014 \\
\hline $072 / 2014$ & $\mathrm{SQ} 3,3-5 \mathrm{~m} \mathrm{~W}-\mathrm{E} / 7-10 \mathrm{~m} \mathrm{~N}-\mathrm{S}$ & slag (2 pieces) & 003 & SAV1E 0598 & 09.01 .2014 \\
\hline $150 / 2014$ & $\mathrm{SQ} 4,5-8 \mathrm{~m} \mathrm{~W}-\mathrm{E} / 0-10 \mathrm{~m} \mathrm{~N}-\mathrm{S}$ & slag (2 pieces) & 003 & SAV1E 0527 & 14.01 .2014 \\
\hline $045 / 2014$ & SQ3, Feature 7 & slag (3 pieces) & 004 & SAV1E 0329 & 08.01 .2014 \\
\hline $047 / 2014$ & $\mathrm{SQ} 3,3-5 \mathrm{~m} \mathrm{~W}-\mathrm{E} / 0-3.5 \mathrm{~m} \mathrm{~N}-\mathrm{S}$ & slag (2 pieces) & 005 & SAV1E 0323 & 08.01 .2014 \\
\hline $061 / 2014$ & $\mathrm{SQ} 3,3-4.5 \mathrm{~m} \mathrm{~W}-\mathrm{E} / 5-6.5 \mathrm{~m} \mathrm{~N}-\mathrm{S}$ & slag (1 piece) & 006 & SAV1E 0348 & 09.01 .2014 \\
\hline $077 / 2014$ & SQ1B, 0-10m W-E/0-5m N-S & slag (16 pieces) & 007 & SAV1E 0356 & 11.01 .2014 \\
\hline $110 / 2014$ & $\mathrm{SQ} 4,1.5-3 \mathrm{~m} \mathrm{~W}-\mathrm{E} / 2-3 \mathrm{~m} \mathrm{~N}-\mathrm{S}$ & slag (3 pieces) & 008 & SAV1E 0395 & 12.01 .2014 \\
\hline $094 / 2014$ & $\mathrm{SQ} 4,1-2.5 \mathrm{~m} \mathrm{~W}-\mathrm{E} / 0-1.5 \mathrm{~m} \mathrm{~N}-\mathrm{S}$ & slag (5 pieces) & 008 & SAV1E 0396 & 12.01 .2014 \\
\hline
\end{tabular}




\begin{tabular}{|c|c|c|c|c|c|}
\hline $116 / 2014$ & $\begin{array}{l}\text { SQ4, 2.5-5.5m W-E/9-10m N-S; } \\
\text { SQ4A 2.5-6.5m W-E/0-2m N-S }\end{array}$ & slag (11 pieces) & 009 & SAV1E 0372 & 13.01.2014 \\
\hline $145 / 2014$ & $\begin{array}{l}\mathrm{SQ} 4,2.5-5.5 \mathrm{~m} \mathrm{~W}-\mathrm{E} / 9-10 \mathrm{~m} \mathrm{~N}-\mathrm{S} \\
\text { SQ4A, 2.5-6.5m W-E/0-2m N-S }\end{array}$ & slag (12 pieces) & 009 & SAV1E 0529 & 14.01.2014 \\
\hline $159 / 2014$ & $\begin{array}{l}\text { SQ4, 2-2.5m W-E/9-10m N-S; } \\
\text { SQ4A 1.50-2.50m W-E/0-2m N-S }\end{array}$ & slag (1 piece) & 009 & SAV1E 0526 & 14.01 .2014 \\
\hline $129 / 2014$ & SQ4, 0-1.5m W-E/1.5-5m N-S & slag (1 piece) & 010 & SAV1E 0400 & 13.01 .2014 \\
\hline $162 / 2014$ & $\mathrm{SQ} 3,3-5 \mathrm{~m} \mathrm{~W}-\mathrm{E} / 0-3.5 \mathrm{~m} \mathrm{~N}-\mathrm{S}$ & slag (1 piece) & 013 & SAV1E 0525 & 15.01 .2014 \\
\hline $172 / 2014$ & $\mathrm{SQ} 4,1-3 \mathrm{~m} \mathrm{~W}-\mathrm{E} / 0-4 \mathrm{~m} \mathrm{~N}-\mathrm{S}$ & slag (6 pieces) & 014 & SAV1E 0528 & 15.01 .2014 \\
\hline $191 / 2014$ & $\mathrm{SQ} 4,0-5 \mathrm{~m} \mathrm{~W}-\mathrm{E} / 5-8 \mathrm{~m} \mathrm{~N}-\mathrm{S}$ & slag (1 piece) & 015 & SAV1E 0569 & 16.01 .2014 \\
\hline $180 / 2014$ & SQ4A, 9-10.5m W-E/0-2m N-S & slag (3 pieces) & 016 & SAV1E 0571 & 16.01 .2014 \\
\hline $203 / 2014$ & SQ1B, 0-3m W-E/0-2m N-S & slag & 018 & & 16.01 .2014 \\
\hline $215 / 2014$ & SQ1B, 0-3m W-E/0-2m N-S & slag (3 pieces), slag (12 pieces) & 018 & SAV1E 0570, SAV1E 0572 (slag) & 18.01 .2014 \\
\hline $243 / 2014$ & $\begin{array}{l}\text { SQ1A, } 0.5-2.5 \mathrm{~m} \mathrm{~W}-\mathrm{E} / 0-1 \mathrm{~m} \text { N-S; SQ1B } \\
0.5-2.5 \mathrm{~m} \mathrm{~W}-\mathrm{E} / 4.5-5 \mathrm{~m} \mathrm{~N} \text {-S, Feature } 29\end{array}$ & slag (4 pieces); charcoal (1 piece) & 019 & SAV1E 0664 & 19.01.2014 \\
\hline $223 / 2014$ & $\begin{array}{l}\text { SQ1B, } 0.5-2.5 \mathrm{~m} \mathrm{~W}-\mathrm{E} / 4-5 \mathrm{~m} \mathrm{~N}-\mathrm{S} \text {, } \\
\text { Feature } 29\end{array}$ & slag (8 pieces) & 019 & SAV1E 0584 & 18.01.2014 \\
\hline $2081 / 2014$ & $\mathrm{SQ} 1 \mathrm{~B}, 0-2 \mathrm{~m} \mathrm{~W}-\mathrm{E} / 4-4.7 \mathrm{~m} \mathrm{~N}-\mathrm{S}$ & slag (2 pieces) & 020 & SAV1E 1134 & 01.02 .2014 \\
\hline $256 / 2014$ & SQ1B, 3-6m W-E/0-2m N-S & slag & 021 & & 19.01.2014 \\
\hline $277 / 2014$ & SQ1B, 3-6m W-E/2-3m N-S & slag (3 pieces) & 021 & & 19.01.2014 \\
\hline $288 / 2014$ & SQ1B, 3-6m W-E/2-3m N-S & slag (8 pieces) & 021 & SAV1E 0659 & 20.01 .2014 \\
\hline $266 / 2014$ & $\mathrm{SQ} 1 \mathrm{~B}, 6-7.5 \mathrm{~m} \mathrm{~W}-\mathrm{E} / 0-2.5 \mathrm{~m} \mathrm{~N}-\mathrm{S}$ & slag (3 pieces) & 021 & SAV1E 0666 & 19.01 .2014 \\
\hline $299 / 2014$ & SQ1B, 7-8.3m W-E/0-2.5m N-S & slag (5 pieces) & 021 & SAV1E 0661 & 20.01 .2014 \\
\hline $334 / 2014$ & $\mathrm{SQ} 3,0-2 \mathrm{~m} \mathrm{~W}-\mathrm{E} / 4.4-10 \mathrm{~m} \mathrm{~N}-\mathrm{S}$ & slag (1 piece) & 025 & SAV1E 0676 & 21.01 .2014 \\
\hline $325 / 2014$ & $\mathrm{SQ} 3,8-10.5 \mathrm{~m} \mathrm{~W}-\mathrm{E} / 5-8 \mathrm{~m} \mathrm{~N}-\mathrm{S}$ & slag (5 pieces) & 027 & SAV1E 0675 & 21.01 .2014 \\
\hline $341 / 2014$ & $\mathrm{SQ} 4,0-3 \mathrm{~m} \mathrm{~W}-\mathrm{E} / 1-5.5 \mathrm{~m} \mathrm{~N}-\mathrm{S}$ & slag (2 pieces) & 030 & SAV1E 0702 & 22.01 .2014 \\
\hline $349 / 2014$ & $\mathrm{SQ} 3,2.5-9 \mathrm{~m} \mathrm{~W}-\mathrm{E} / 0-10 \mathrm{~m} \mathrm{~N}-\mathrm{S}$ & slag (10 pieces) & 031 & SAV1E 0703 & 22.01 .2014 \\
\hline $385 / 2014$ & $\mathrm{SQ} 3,9-10.5 \mathrm{~m} \mathrm{~W}-\mathrm{E} / 2.6-5.5 \mathrm{~m} \mathrm{~N}-\mathrm{S}$ & slag (2 pieces) & 034 & SAV1E 0734 & 23.01 .2014 \\
\hline $404 / 2014$ & $\mathrm{SQ} 3,0-1.3 \mathrm{~m} \mathrm{~W}-\mathrm{E} / 4.4-6.8 \mathrm{~m} \mathrm{~N}-\mathrm{S}$ & slag (3 pieces) & 038 & SAV1E 0758 & 25.01 .2014 \\
\hline $437 / 2014$ & $\mathrm{SQ} 1 \mathrm{~B}, 0-3 \mathrm{~m} \mathrm{~W}-\mathrm{E} / 2.5-4.5 \mathrm{~m} \mathrm{~N}-\mathrm{S}$ & slag (10 pieces) & 040 & SAV1E 0791 & 26.01.2014 \\
\hline
\end{tabular}




\begin{tabular}{|c|c|c|c|c|c|}
\hline $426 / 2014$ & SQ1B, 0-3m W-E/2-3m N-S & slag (2 pieces) & 040 & SAV1E 0770 & 26.01 .2014 \\
\hline $1510 / 2014$ & $\mathrm{SQ} 1 \mathrm{~B}, 2.5-8 \mathrm{~m} \mathrm{~W}-\mathrm{E} / 2.5-4 \mathrm{~m} \mathrm{~N}-\mathrm{S}$ & slag (5 pieces) & 040 & SAV1E 0820 & 27.01.2014 \\
\hline $433 / 2014$ & SQ3, 1.8-5m W-E/7-10m N-S & slag (2 pieces) & 041 & SAV1E 0790 & 26.01 .2014 \\
\hline $2004 / 2014$ & SQ1B, $0-2 \mathrm{~m} \mathrm{~W}-\mathrm{E} / 2.3-3.4 \mathrm{~m} \mathrm{~N}-\mathrm{S}$ & slag (3 pieces) & 049 & SAV1E 0819 & 28.01 .2014 \\
\hline $2010 / 2014$ & $\mathrm{SQ} 3,2.5-5.3 \mathrm{~m} \mathrm{~W}-\mathrm{E} / 8.5-10 \mathrm{~m} \mathrm{~N}-\mathrm{S}$ & slag (2 pieces) & 050 & SAV1E 0832 & 28.01 .2014 \\
\hline $2025 / 2014$ & SQ1B, $1.5-4 \mathrm{~m} \mathrm{~W}-\mathrm{E} / 0-2.3 \mathrm{~m} \mathrm{~N}-\mathrm{S}$ & slag (3 pieces), slag (2 pieces) & 051 & SAV1E 0853, SAV1E 0854 (slag) & 29.01.2014 \\
\hline $2057 / 2014$ & SQ1B, $1.5-6.5 \mathrm{~m} \mathrm{~W}-\mathrm{E} / 1.3-3 \mathrm{~m} \mathrm{~N}-\mathrm{S}$ & slag (3 pieces) & 051 & SAV1E 0892 & 30.01 .2014 \\
\hline $2033 / 2014$ & SQ1B, 4-6.5m W-E/0-1.5m N-S & slag (4 pieces) & 051 & SAV1E 0852 & 29.01.2014 \\
\hline $2047 / 2014$ & $\mathrm{SQ} 1 \mathrm{~B}, 4-6.5 \mathrm{~m} \mathrm{~W}-\mathrm{E} / 0-1.5 \mathrm{~m} \mathrm{~N}-\mathrm{S}$ & slag (6 pieces) & 051 & SAV1E 0893 & 30.01 .2014 \\
\hline $2073 / 2014$ & SQ1B, 0-2.8m W-E/3-4m N-S & slag (2 pieces) & 056 & & 01.02 .2014 \\
\hline $2090 / 2014$ & $\mathrm{SQ} 3,2-5.5 \mathrm{~m} \mathrm{~W}-\mathrm{E} / 2-3.8 \mathrm{~m} \mathrm{~N}-\mathrm{S}$ & slag? (1 piece) & 059 & SAV1E 0922 & 01.02 .2014 \\
\hline $2147 / 2014$ & $\mathrm{SQ} 3,4.8-7 \mathrm{~m} \mathrm{~W}-\mathrm{E} / 4-8.3 \mathrm{~m} \mathrm{~N}-\mathrm{S}$ & slag (2 pieces) & 066 & SAV1E 0956 & 03.02 .2014 \\
\hline $2165 / 2014$ & $\mathrm{SQ} 3,4.8-7 \mathrm{~m} \mathrm{~W}-\mathrm{E} / 4-8.3 \mathrm{~m} \mathrm{~N}-\mathrm{S}$ & slag (6 pieces) & 066 & SAV1E 0969 & 04.02 .2014 \\
\hline $2209 / 2014$ & $\mathrm{SQ} 3,8.5-10.5 \mathrm{~m} \mathrm{~W}-\mathrm{E} / 5-7.8 \mathrm{~m} \mathrm{~N}-\mathrm{S}$ & slag (8 pieces) & 070 & SAV1E 0997 & 05.02 .2014 \\
\hline $2230 / 2014$ & $\mathrm{SQ} 3,8.5-10.5 \mathrm{~m} \mathrm{~W}-\mathrm{E} / 5-8.5 \mathrm{~m} \mathrm{~N}-\mathrm{S}$ & slag (3 pieces) & 070 & SAV1E 1004 & 06.02 .2014 \\
\hline $2256 / 2014$ & $\mathrm{SQ} 3,8-10.5 \mathrm{~m} \mathrm{~W}-\mathrm{E} / 3.6-5.5 \mathrm{~m} \mathrm{~N}-\mathrm{S}$ & slag (1 piece) & 070 & SAV1E 1005 & 06.02 .2014 \\
\hline $2258 / 2014$ & $\mathrm{SQ} 3,8-10.5 \mathrm{~m} \mathrm{~W}-\mathrm{E} / 3.6-5.5 \mathrm{~m} \mathrm{~N}-\mathrm{S}$ & slag (2 pieces); seashell (1 fragment) & 070 & SAV1E 2275 (slag) & 08.02 .2014 \\
\hline $2199 / 2014$ & SQ3, 9.2-10.5m W-E/4.7-5.9m N-S & slag (3 pieces) & 070 & SAV1E 0996 & 05.02 .2014 \\
\hline $2246 / 2014$ & $\mathrm{SQ} 3,9.2-10.5 \mathrm{~m} \mathrm{~W}-\mathrm{E} / 8-9.2 \mathrm{~m} \mathrm{~N}-\mathrm{S}$ & slag (4 pieces) & 070 & SAV1E 1003 & 06.02 .2014 \\
\hline $2173 / 2014$ & $\mathrm{SQ} 3,8.5-10.5 \mathrm{~m} \mathrm{~W}-\mathrm{E} / 0.8-2.3 \mathrm{~m} \mathrm{~N}-\mathrm{S}$ & slag (5 pieces) & 071 & SAV1E 0967 & 04.02 .2014 \\
\hline $2203 / 2014$ & $\mathrm{SQ} 3,6.6-9 \mathrm{~m} \mathrm{~W}-\mathrm{E} / 1.1-4.5 \mathrm{~m} \mathrm{~N}-\mathrm{S}$ & slag (1 piece) & 073 & SAV1E 0995 & 05.02 .2014 \\
\hline $2267 / 2014$ & SQ4, $9.5-10.5 \mathrm{~m} \mathrm{~W}-\mathrm{E} / 0.6-2 \mathrm{~m} \mathrm{~N}-\mathrm{S}$ & slag (3 pieces) & 079 & SAV1E 1030 & 08.02 .2014 \\
\hline $2309 / 2014$ & $\begin{array}{l}\text { SQ4, } 9.8-10.5 \mathrm{~m} \mathrm{~W}-\mathrm{E} / 2-2.4 \mathrm{~m} \mathrm{~N}-\mathrm{S} \text {, } \\
\text { Feature } 15\end{array}$ & $\operatorname{slag}(1$ piece $)$ & 092 & SAV1E 1067 & 10.02.2014 \\
\hline $2336 / 2014$ & $\mathrm{SQ} 3,7.7-8.8 \mathrm{~m} \mathrm{~W}-\mathrm{E} / 3.5-8 \mathrm{~m} \mathrm{~N}-\mathrm{S}$ & slag (1 piece) & 097 & SAV1E 1097 & 11.02 .2014 \\
\hline $2357 / 2014$ & SQ4, 9.2-10.5m W-E/4.2-5.4m N-S & slag (1 piece) & 103 & SAV1E 1093 & 12.02 .2014 \\
\hline $2368 / 2014$ & $\mathrm{SQ} 4,8-10.5 \mathrm{~m} \mathrm{~W}-\mathrm{E} / 5.6-7.7 \mathrm{~m} \mathrm{~N}-\mathrm{S}$ & slag (1 piece) & 107 & SAV1E 1105 & 13.02.2014 \\
\hline
\end{tabular}




\begin{tabular}{|c|c|c|c|c|c|}
\hline $2379 / 2014$ & SQ4, 9-10.5m W-E/5.6-7.2m N-S & $\operatorname{slag}(3$ pieces $)$ & 108 & SAV1E 1106 & 13.02 .2014 \\
\hline $2322 / 2014$ & $\mathrm{SQ} 3,8.4 \mathrm{~m} \mathrm{~W}-\mathrm{E} / 0.7 \mathrm{~m} \mathrm{~N}-\mathrm{S}$ & $\begin{array}{l}\text { stamp ( } 1 \text { intact piece, probably Second Intermediate Period or } \\
\text { early } 18^{\text {th }} \text { Dynasty, clay) }\end{array}$ & 095 & SAV1E 1089 & 11.02 .2014 \\
\hline $2369 / 2014$ & $\mathrm{SQ} 4,9.6 \mathrm{~m} \mathrm{~W}-\mathrm{E} / 6,9 \mathrm{~m} \mathrm{~N}-\mathrm{S}$ & $\begin{array}{l}\text { stela ( } 1 \text { fragment, preserved } w 3 s \text {-sceptre, } 18^{\text {th }} \text { Dynasty, } \\
\text { sandstone) }\end{array}$ & 107 & SAV1E 1124 & 13.02.2014 \\
\hline $003 / 2014$ & SQ1B, surface & stone: grindstone (1 piece, quartzite) & 001 & SAV1E 0483 & 04.01 .2014 \\
\hline $006 / 2014$ & $\mathrm{SQ} 3,10 \times 10 \mathrm{~m}$, surface cleaning & $\begin{array}{l}\text { stones: pounders ( } 3 \text { pieces, } 2 \text { quartzite, } 1 \text { sandstone), hammer or } \\
\text { pounder ( } 1 \text { piece, sandstone), hand mills ( } 2 \text { pieces, each } \\
\text { sandstone), grindstones ( } 13 \text { pieces, } 6 \text { sandstone, } 7 \text { quartzite), } \\
\text { grindstones? ( } 3 \text { pieces, } 1 \text { sandstone, } 2 \text { quartzite) }\end{array}$ & 001 & $\begin{array}{l}\text { SAV1E 0436, SAV1E 0437, SAV1E } 0438 \\
\text { (pounders), SAV1E 0443 (hammer or } \\
\text { pounder), SAV1E 0440, SAV1E 0427 (hand } \\
\text { mills), SAV1E 0428, SAV1E 0430, SAV1E } \\
\text { 0431, SAV1E 0432, SAV1E 0434, SAV1E } \\
\text { 0435, SAV1E 0445, SAV1E 0459, SAV1E } \\
\text { 0462, SAV1E 0464, SAV1E 0465, SAV1E } \\
\text { 0439, SAV1E 0441 (grindstones), SAV1E } \\
\text { 0433, SAV1E 0461, SAV1E 0463 } \\
\text { (grindstones?) }\end{array}$ & 04.01 .2014 \\
\hline $008 / 2014$ & $\mathrm{SQ} 4,10 \times 10 \mathrm{~m}$, surface cleaning & $\begin{array}{l}\text { stones: pounders ( } 15 \text { pieces, } 6 \text { quartzite, } 6 \text { quartz, } 1 \text { schist, } \\
1 \text { sandstone, } 1 \text { unspecified stone), pounder? ( } 1 \text { piece, granite), } \\
\text { hammer or pounder ( } 1 \text { piece, quartz), grindstones ( } 5 \text { pieces, } \\
3 \text { sandstone, } 2 \text { quartzite), grindstones? ( } 5 \text { pieces, } 4 \text { quartzite, } \\
1 \text { sandstone), pivot stone ( } 1 \text { piece, sandstone) }\end{array}$ & 001 & $\begin{array}{l}\text { SAV1E 0466, SAV1E 0467, SAV1E 0468, } \\
\text { SAV1E 0470, SAV1E 0471, SAV1E 0472, } \\
\text { SAV1E 0474, SAV1E 0475, SAV1E 0476, } \\
\text { SAV1E 0477, SAV1E 0478, SAV1E 0479, } \\
\text { SAV1E 0480, SAV1E 0481, SAV1E 0482 } \\
\text { (pounders), SAV1E 0473 (pounder?), } \\
\text { SAV1E 0469 (hammer or pounder), } \\
\text { SAV1E 0514, SAV1E 0515, SAV1E 0518, } \\
\text { SAV1E 0519, SAV1E 0520 (grindstones), } \\
\text { SAV1E 0517, SAV1E 0521, SAV1E 0522, } \\
\text { SAV1E 0523, SAV1E 0524 (grindstones?), } \\
\text { SAV1E 0516 (pivot stone) }\end{array}$ & 04.01 .2014 \\
\hline $018 / 2014$ & SQ4A, $2 \times 10$, surface cleaning & $\begin{array}{l}\text { stones: grindstones ( } 9 \text { pieces, } 5 \text { sandstone, } 4 \text { quartzite), } \\
\text { grindstones? ( } 3 \text { pieces, } 2 \text { sandstone, } 1 \text { quartzite), pounders } \\
\text { ( } 2 \text { pieces, } 1 \text { calcite, } 1 \text { calcite?), hammers or pounders ( } 2 \text { pieces, } \\
1 \text { quartzite, } 1 \text { granite?), whetstone ( } 1 \text { piece, sandstone), hand mill } \\
\text { (1 piece, quartzite) }\end{array}$ & 001 & $\begin{array}{l}\text { SAV1E 0451, SAV1E 0453, SAV1E 0455, } \\
\text { SAV1E 0456, SAV1E 0457, SAV1E 0424, } \\
\text { SAV1E 0447, SAV1E 0449, SAV1E 0422 } \\
\text { (grindstones), SAV1E 0452, SAV1E 0454, } \\
\text { SAV1E 0458 (grindstones?), SAV1E 0421, } \\
\text { SAV1E } 0419 \text { (pounders), SAV1E 0413, } \\
\text { SAV1E 0423 (hammers or pounders), SAV1E } \\
\text { 0450 (whetstone), SAV1E 0420 (hand mill) }\end{array}$ & 05.01 .2014 \\
\hline $088 / 2014$ & $\mathrm{SQ} 3,8.5-9.5 \mathrm{~m} \mathrm{~W}-\mathrm{E} / 2-10 \mathrm{~m} \mathrm{~N}-\mathrm{S}$ & stones: pounders ( 4 pieces, 3 quartzite, 1 sandstone) & 002 & $\begin{array}{l}\text { SAV1E 0507, SAV1E 0508, SAV1E 0509, } \\
\text { SAV1E } 0510 \text { (pounders) }\end{array}$ & 12.01 .2014 \\
\hline $311 / 2014$ & $\mathrm{SQ} 3,9.5-10 \mathrm{~m} \mathrm{~W}-\mathrm{E} / 5.7-8 \mathrm{~m} \mathrm{~N}-\mathrm{S}$ & $\begin{array}{l}\text { stones: pounder ( } 1 \text { piece, sandstone), grindstones ( } 4 \text { pieces, } \\
3 \text { sandstone, } 1 \text { granite?) }\end{array}$ & 002 & $\begin{array}{l}\text { SAV1E } 0748 \text { (pounder), SAV1E 0749, } \\
\text { SAV1E 0750, SAV1E 0751, SAV1E } 0752 \\
\text { (grindstones) }\end{array}$ & 20.01.2014 \\
\hline
\end{tabular}




\begin{tabular}{|c|c|c|c|c|c|}
\hline $054 / 2014$ & SQ3, 0-2m W-E/0-10m N-S & stone: pounder (1 piece, quartz) & 003 & SAV1E 0379 & 09.01 .2014 \\
\hline $075 / 2014$ & SQ3, 0-2m W-E/7-10m N-S & stones: grindstones? (2 fragments, each quartzite) & 003 & SAV1E 0380, SAV1E 0381 (grindstones?) & 09.01 .2014 \\
\hline $071 / 2014$ & $\mathrm{SQ} 3,3-5 \mathrm{~m} \mathrm{~W}-\mathrm{E} / 7-10 \mathrm{~m} \mathrm{~N}-\mathrm{S}$ & $\begin{array}{l}\text { stones: pounder (1 piece, quartzite), grindstone (1 piece, } \\
\text { sandstone), grindstone? (1 piece, quartzite) }\end{array}$ & 003 & $\begin{array}{l}\text { SAV1E } 0384 \text { (pounder), SAV1E } 0385 \\
\text { (grindstone), SAV1E } 0386 \text { (grindstone?) }\end{array}$ & 09.01 .2014 \\
\hline $023 / 2014$ & $\mathrm{SQ} 3,5-7.7 \mathrm{~m}$ from $\mathrm{W} / 7.50-10 \mathrm{~m} \mathrm{~N}-\mathrm{S}$ & stone: grindstone? (1 piece, quartzite) & 003 & SAV1E 0374 & 06.01 .2014 \\
\hline $121 / 2014$ & $\mathrm{SQ} 3,5-8.5 \mathrm{~m} \mathrm{~W}-\mathrm{E} / 3-7 \mathrm{~m} \mathrm{~N}-\mathrm{S}$ & stones: grindstones (2 pieces, each sandstone) & 003 & SAV1E 0399, SAV1E 0401 (grindstones) & 13.01 .2014 \\
\hline 098/2014 & $\mathrm{SQ} 3,5-8.5 \mathrm{~m} \mathrm{~W}-\mathrm{E} / 7-10 \mathrm{~m} \mathrm{~N}-\mathrm{S}$ & stones: grindstones (2 pieces, each sandstone) & 003 & SAV1E 0425, SAV1E 0426 (grindstones) & 12.01.2014 \\
\hline $146 / 2014$ & $\mathrm{SQ} 4,2.5-5 \mathrm{~m} \mathrm{~W}-\mathrm{E} / 0-9 \mathrm{~m} \mathrm{~N}-\mathrm{S}$ & $\begin{array}{l}\text { stones: grindstones ( } 2 \text { pieces, } 1 \text { sandstone, } 1 \text { quartzite), artefact? } \\
\text { (1 cylindrical shaped fragment, sandstone) }\end{array}$ & 003 & $\begin{array}{l}\text { SAV1E 0715, SAV1E } 0716 \text { (grindstones), } \\
\text { SAV1E } 0718 \text { (artefact?) }\end{array}$ & 14.01.2014 \\
\hline $154 / 2014$ & $\mathrm{SQ} 4,5-8 \mathrm{~m} \mathrm{~W}-\mathrm{E} / 0-10 \mathrm{~m} \mathrm{~N}-\mathrm{S}$ & $\begin{array}{l}\text { stones: pounder (1 piece, schist?), grindstone or pounder (1 piece, } \\
\text { quartzite) }\end{array}$ & 003 & $\begin{array}{l}\text { SAV1E } 0505 \text { (pounder), SAV1E } 0506 \\
\text { (grindstone or pounder) }\end{array}$ & 14.01.2014 \\
\hline $044 / 2014$ & SQ3, Feature 7, S-edge & $\begin{array}{l}\text { stones: grindstone? ( } 1 \text { piece, sandstone), grindstone (1 piece, } \\
\text { quartzite) }\end{array}$ & 004 & $\begin{array}{l}\text { SAV1E } 0382 \text { (grindstone?), SAV1E } 0383 \\
\text { (grindstone) }\end{array}$ & 08.01 .2014 \\
\hline $2160 / 2014$ & $\begin{array}{l}\text { SQ3, 8.5-10.5m W-E/0.3-2.3m N-S, } \\
\text { cleaning of SU }\end{array}$ & $\begin{array}{l}\text { stones: grindstone (1 piece, sandstone), pounder (1 piece, } \\
\text { quartzite) }\end{array}$ & 004 & $\begin{array}{l}\text { SAV1E } 1047 \text { (grindstone), SAV1E } 1046 \\
\text { (pounder) }\end{array}$ & 04.02 .2014 \\
\hline $046 / 2014$ & SQ3, 3-5m W-E/0-3.5m N-S & stone: grindstone? (1 piece, sandstone) & 005 & SAV1E 0378 & 08.01 .2014 \\
\hline $042 / 2014$ & $\mathrm{SQ} 3,4.5 \mathrm{~m} \mathrm{~W}-\mathrm{E} / 0.5 \mathrm{~m} \mathrm{~N}-\mathrm{S}$ & stone: blade or scraper (1 silex flake) & 005 & SAV1E 0357 & 08.01 .2014 \\
\hline $060 / 2014$ & $\mathrm{SQ} 3,3-4.5 \mathrm{~m} \mathrm{~W}-\mathrm{E} / 5-6.5 \mathrm{~m} \mathrm{~N}-\mathrm{S}$ & $\begin{array}{l}\text { stones: pounders ( } 3 \text { pieces, each quartzite), grindstone ( } 1 \text { piece, } \\
\text { sandstone?), whetstone ( } 1 \text { piece, sandstone) }\end{array}$ & 006 & $\begin{array}{l}\text { SAV1E 0387, SAV1E 0389, SAV1E } 0390 \\
\text { (pounders), SAV1E } 0391 \text { (grindstone), } \\
\text { SAV1E 0388 (whetstone) }\end{array}$ & 09.01 .2014 \\
\hline $080 / 2014$ & SQ1B, 0-10m W-E/0-5m N-S & $\begin{array}{l}\text { stones: pounders ( } 2 \text { pieces, } 1 \text { granite, } 1 \text { schist?), hand mills } \\
\text { ( } 2 \text { pieces, each sandstone), grindstones ( } 5 \text { pieces, } 2 \text { sandstone, } \\
3 \text { quartzite) }\end{array}$ & 007 & $\begin{array}{l}\text { SAV1E 0412, SAV1E } 0405 \text { (pounders), } \\
\text { SAV1E 0407, SAV1E } 0411 \text { (hand mills), } \\
\text { SAV1E 0408, SAV1E 0409, SAV1E 0410, } \\
\text { SAV1E 0417, SAV1E } 0418 \text { (grindstones) }\end{array}$ & 11.01 .2014 \\
\hline $367 / 2014$ & $\mathrm{SQ} 4,0.5-1 \mathrm{~m} \mathrm{~W}-\mathrm{E} / 3-4 \mathrm{~m} \mathrm{~N}-\mathrm{S}$ & stone: grindstone (1 piece, quartzite) & 008 & SAV1E 0762 & 23.01.2014 \\
\hline $109 / 2014$ & $\mathrm{SQ} 4,1.5-3 \mathrm{~m} \mathrm{~W}-\mathrm{E} / 2-3 \mathrm{~m} \mathrm{~N}-\mathrm{S}$ & stone: pounder (1 piece, quartzite) & 008 & SAV1E 0597 & 12.01 .2014 \\
\hline $097 / 2014$ & SQ4, 1-2.5m W-E/0-1.5m N-S & stones: grindstones ( 5 pieces, 4 sandstone, 1 quartzite) & 008 & $\begin{array}{l}\text { SAV1E 0414, SAV1E 0415, SAV1E 0416, } \\
\text { SAV1E 0511, SAV1E } 0512 \text { (grindstones) }\end{array}$ & 12.01.2014 \\
\hline $112 / 2014$ & $\begin{array}{l}\text { SQ4, 2.5-5.5m W-E/9-10m N-S, } \\
\text { SQ4A, 2.5-6.5m W-E/0-2m N-S }\end{array}$ & $\begin{array}{l}\text { stones: pounders ( } 6 \text { pieces, } 1 \text { quartz, } 1 \text { quartzite, } 4 \text { sandstone), } \\
\text { grindstones ( } 16 \text { pieces, } 10 \text { sandstone, } 6 \text { quartzite), grindstones? } \\
\text { ( } 3 \text { pieces, each sandstone), raw material/imported stone } \\
\text { (1 fragment, unspecified stone) }\end{array}$ & 009 & $\begin{array}{l}\text { SAV1E 0567, SAV1E 0580, SAV1E 0585, } \\
\text { SAV1E 0586, SAV1E 0587, SAV1E 0588 } \\
\text { (pounders), SAV1E 0600, SAV1E 0601, } \\
\text { SAV1E 0602, SAV1E 0603, SAV1E 0604, } \\
\text { SAV1E 0605, SAV1E 0905, SAV1E 0561, } \\
\text { SAV1E 0562, SAV1E 0563, SAV1E 0564, } \\
\text { SAV1E 0566, SAV1E 0578, SAV1E 0579, } \\
\text { SAV1E 0568, SAV1E 0589 (grindstones), }\end{array}$ & 13.01.2014 \\
\hline
\end{tabular}




\begin{tabular}{|c|c|c|c|c|c|}
\hline & & & & $\begin{array}{l}\text { SAV1E 0606, SAV1E 0607, SAV1E } 0565 \\
\text { (grindstones?), SAV1E } 0906 \text { (raw } \\
\text { material/imported stone) }\end{array}$ & \\
\hline $139 / 2014$ & $\begin{array}{l}\text { SQ4, 2.5-5.5m W-E/9-10m N-S, } \\
\text { SQ4A, 2.5-6.5m W-E/0-2m N-S }\end{array}$ & $\begin{array}{l}\text { stones: grindstones ( } 5 \text { pieces, } 3 \text { quartzite, } 2 \text { sandstone), } \\
\text { grindstone? ( } 1 \text { piece, sandstone), pounders ( } 6 \text { pieces, each } \\
\text { quartzite) }\end{array}$ & 009 & $\begin{array}{l}\text { SAV1E 0558, SAV1E 0611, SAV1E 0612, } \\
\text { SAV1E 0613, SAV1E 0614 (grindstones), } \\
\text { SAV1E 0615 (grindstone?), SAV1E 0581, } \\
\text { SAV1E 0582, SAV1E 0583, SAV1E 0585, } \\
\text { SAV1E 0586, SAV1E } 0587 \text { (pounders) }\end{array}$ & 14.01.2014 \\
\hline $158 / 2014$ & $\begin{array}{l}\mathrm{SQ} 4,2-2.5 \mathrm{~m} \mathrm{~W}-\mathrm{E} / 9-10 \mathrm{~m} \mathrm{~N}-\mathrm{S} \\
\text { SQ4A, } 1.50-2.50 \mathrm{~m} \mathrm{~W}-\mathrm{E} / 0-2 \mathrm{~m} \mathrm{~N}-\mathrm{S}\end{array}$ & $\begin{array}{l}\text { stones: pounder (1 piece, quartzite), grindstone? (1 piece, } \\
\text { sandstone) }\end{array}$ & 009 & $\begin{array}{l}\text { SAV1E } 0559 \text { (pounder), SAV1E } 0560 \\
\text { (grindstone?) }\end{array}$ & 14.01.2014 \\
\hline $122 / 2014$ & SQ4A, 3m W-E/0.5m N-S & $\begin{array}{l}\text { stone: raw material (1 fragment of unworked stone, traces of red } \\
\text { pigment) }\end{array}$ & 009 & SAV1E 0504 & 13.01.2014 \\
\hline $128 / 2014$ & $\mathrm{SQ} 4,0-1.5 \mathrm{~m} \mathrm{~W}-\mathrm{E} / 1.5-5 \mathrm{~m} \mathrm{~N}-\mathrm{S}$ & stone: grindstone (1 piece, sandstone) & 010 & SAV1E 0446 & 13.01.2014 \\
\hline $163 / 2014$ & $\mathrm{SQ} 3,3-5 \mathrm{~m} \mathrm{~W}-\mathrm{E} / 0-3.5 \mathrm{~m} \mathrm{~N}-\mathrm{S}$ & $\begin{array}{l}\text { stones: pounders ( } 2 \text { pieces, each quartzite), grindstones ( } 4 \text { pieces, } \\
2 \text { quartzite, } 2 \text { sandstone), grindstone? ( } 1 \text { piece, sandstone), } \\
\text { installation/furniture/architecture? ( } 1 \text { fragment, sandstone) }\end{array}$ & 013 & $\begin{array}{l}\text { SAV1E 0617, SAV1E } 0618 \text { (pounders), } \\
\text { SAV1E 0627, SAV1E 0628, SAV1E 0629, } \\
\text { SAV1E 0630 (grindstones), SAV1E 0625 } \\
\text { (grindstone?), SAV1E 0626 } \\
\text { (installation/furniture/architecture?) }\end{array}$ & 15.01 .2014 \\
\hline $165 / 2014$ & $\mathrm{SQ} 4,1-3 \mathrm{~m} \mathrm{~W}-\mathrm{E} / 0-4 \mathrm{~m} \mathrm{~N}-\mathrm{S}$ & $\begin{array}{l}\text { stones: pounders ( } 5 \text { pieces, each quartzite), grindstones ( } 8 \text { pieces, } \\
1 \text { quartzite, } 7 \text { sandstone), grindstones? ( } 2 \text { pieces, each sandstone), } \\
\text { whetstone (1 piece, sandstone) }\end{array}$ & 014 & $\begin{array}{l}\text { SAV1E 0619, SAV1E 0620, SAV1E 0621, } \\
\text { SAV1E 0622, SAV1E 0623, SAV1E 0704, } \\
\text { SAV1E 0706 (pounders), SAV1E 0707, } \\
\text { SAV1E 0708, SAV1E 0709, SAV1E 0710, } \\
\text { SAV1E 0631, SAV1E 0632, SAV1E 0633, } \\
\text { SAV1E 0634 (grindstones), SAV1E 0705, } \\
\text { SAV1E 0624 (grindstones?), SAV1E } 2668 \\
\text { (whetstone) }\end{array}$ & 15.01 .2014 \\
\hline $227 / 2014$ & $\mathrm{SQ} 4,1.5-5 \mathrm{~m} \mathrm{~W}-\mathrm{E} / 3.5-5 \mathrm{~m} \mathrm{~N}-\mathrm{S}$ & stone: pounder (1 piece, quartz) & 015 & SAV1E 0727 & 18.01.2014 \\
\hline $207 / 2014$ & $\mathrm{SQ} 4,0-5 \mathrm{~m} \mathrm{~W}-\mathrm{E} / 5-8 \mathrm{~m} \mathrm{~N}-\mathrm{S}$ & stones: grindstones ( 3 pieces, each quartzite) & 015 & $\begin{array}{l}\text { SAV1E 0728, SAV1E 0729, SAV1E } 0730 \\
\text { (grindstones) }\end{array}$ & 18.01 .2014 \\
\hline $185 / 2014$ & $\mathrm{SQ} 4,0-5 \mathrm{~m} \mathrm{~W}-\mathrm{E} / 5-8 \mathrm{~m} \mathrm{~N}-\mathrm{S}$ & $\begin{array}{l}\text { stones: grindstones ( } 10 \text { pieces, } 8 \text { sandstone, } 2 \text { quartzite), } \\
\text { grindstones? ( } 2 \text { pieces, each sandstone), pounder ( } 2 \text { pieces, } \\
1 \text { basalt?, } 1 \text { quartzite), architecture/crapaudine? ( } 1 \text { fragment, } \\
\text { sandstone) }\end{array}$ & 015 & $\begin{array}{l}\text { SAV1E 0740, SAV1E 0741, SAV1E 0742, } \\
\text { SAV1E 0678, SAV1E 0679, SAV1E 0680, } \\
\text { SAV1E 0684, SAV1E 0685, SAV1E 0687, } \\
\text { SAV1E } 0688 \text { (grindstones), SAV1E 0689, } \\
\text { SAV1E } 0686 \text { (grindstones?), SAV1E 0690, } \\
\text { SAV1E } 0681 \text { (pounders), SAV1E } 0682 \\
\text { (architecture/crapaudine?) }\end{array}$ & 16.01.2014 \\
\hline $355 / 2014$ & $\mathrm{SQ} 4,0-2.5 \mathrm{~m} \mathrm{~W}-\mathrm{E} / 4.5-5.5 \mathrm{~m} \mathrm{~N}-\mathrm{S}$ & stones: grindstones ( 2 pieces, each quartzite) & 015 & SAV1E 0801, SAV1E 0802 (grindstones) & 22.01.2014 \\
\hline $233 / 2014$ & SQ4, 1.5-5m W-E/8-8.5m N-S & stones: grindstones (3 pieces, each sandstone) & 015 & $\begin{array}{l}\text { SAV1E 0771, SAV1E 0772, SAV1E } 0774 \\
\text { (grindstones) }\end{array}$ & 18.01 .2014 \\
\hline
\end{tabular}




\begin{tabular}{|c|c|c|c|c|c|}
\hline $179 / 2014$ & SQ4A, 9-10.5m W-E/0-2m N-S & $\begin{array}{l}\text { stones: grindstones ( } 3 \text { pieces, } 2 \text { sandstone, } 1 \text { quartzite), } \\
\text { grindstone? (1 piece, sandstone) }\end{array}$ & 016 & $\begin{array}{l}\text { SAV1E 0743, SAV1E 0744, SAV1E } 0745 \\
\text { (grindstones), SAV1E } 0746 \text { (grindstone?) }\end{array}$ & 16.01.2014 \\
\hline $201 / 2014$ & SQ1B, 0-3m W-E/0-2m N-S & $\begin{array}{l}\text { stones: whetstone ( } 1 \text { piece, sandstone), pounders ( } 2 \text { pieces, } \\
1 \text { quartz, } 1 \text { quartzite); seashell ( } 1 \text { small piece); bone }\end{array}$ & 018 & $\begin{array}{l}\text { SAV1E } 1682 \text { (whetstone), SAV1E 1683, } \\
\text { SAV1E } 1684 \text { (pounders) }\end{array}$ & 16.01.2014 \\
\hline $202 / 2014$ & SQ1B, 0-3m W-E/0-2m N-S & stones: grindstones ( 6 pieces, 5 sandstone, 1 quartzite) & 018 & $\begin{array}{l}\text { SAV1E 0695, SAV1E 0696, SAV1E 0698, } \\
\text { SAV1E 0699, SAV1E 0700, SAV1E } 0701 \\
\text { (grindstones) }\end{array}$ & 16.01.2014 \\
\hline $212 / 2014$ & SQ1B, 0-3m W-E/0-2m N-S & stones: grindstones (2 pieces, each sandstone) & 018 & SAV1E 0719, SAV1E 0720 (grindstones) & 18.01.2014 \\
\hline $244 / 2014$ & $\begin{array}{l}\text { SQ1A, } 0.5-2.5 \mathrm{~m} \mathrm{~W}-\mathrm{E} / 0-1 \mathrm{~m} \mathrm{~N}-\mathrm{S} \\
\text { SQ1B } 0.5-2.5 \mathrm{~m} \mathrm{~W}-\mathrm{E} / 4.5-5 \mathrm{~m} \mathrm{~N}-\mathrm{S} \\
\text { Feature } 29\end{array}$ & stones: grindstone? (1 piece, sandstone) & 019 & SAV1E 0691 & 19.01 .2014 \\
\hline $219 / 2014$ & $\begin{array}{l}\mathrm{SQ} 1 \mathrm{~B}, 0.5-2.5 \mathrm{~m} \mathrm{~W}-\mathrm{E} / 4-5 \mathrm{~m} \mathrm{~N}-\mathrm{S} \\
\text { Feature } 29\end{array}$ & $\begin{array}{l}\text { stones: grindstone ( } 3 \text { pieces, } 1 \text { sandstone, } 2 \text { quartzite), } \\
\text { grindstone? (1 piece, sandstone), pounder (1 piece, quartz) }\end{array}$ & 019 & $\begin{array}{l}\text { SAV1E 0721, SAV1E 0722, SAV1E } 0725 \\
\text { (grindstones), SAV1E } 0726 \text { (grindstone?), } \\
\text { SAV1E } 0723 \text { (pounder) }\end{array}$ & 18.01.2014 \\
\hline $245 / 2014$ & $\mathrm{SQ} 1 \mathrm{~B}, 1.5 \mathrm{~m} \mathrm{~W}-\mathrm{E} / 5 \mathrm{~m} \mathrm{~N}-\mathrm{S}$ & stone tool? (1 piece, gneiss, lithic) & 019 & SAV1E 0654 & 19.01.2014 \\
\hline $253 / 2014$ & SQ1B, 3-6m W-E/0-2m N-S & stones: grindstones ( 3 pieces, 2 sandstone, 1 quartzite) & 021 & $\begin{array}{l}\text { SAV1E 0692, SAV1E 0693, SAV1E } 0694 \\
\text { (grindstones) }\end{array}$ & 19.01 .2014 \\
\hline $276 / 2014$ & $\mathrm{SQ} 1 \mathrm{~B}, 3-6 \mathrm{~m} \mathrm{~W}-\mathrm{E} / 2-3 \mathrm{~m} \mathrm{~N}-\mathrm{S}$ & stone: pounder? (1 fragment, basalt?) & 021 & SAV1E 0754 & 19.01.2014 \\
\hline $286 / 2014$ & SQ1B, 3-6m W-E/2-3m N-S & $\begin{array}{l}\text { stones: pounder ( } 1 \text { piece, quartzite), grindstones ( } 3 \text { pieces, } \\
2 \text { sandstone, } 1 \text { granite?) }\end{array}$ & 021 & $\begin{array}{l}\text { SAV1E } 0763 \text { (pounder), SAV1E 0764, } \\
\text { SAV1E } 0765 \text {, SAV1E } 0766 \text { (grindstones) }\end{array}$ & 20.01.2014 \\
\hline $265 / 2014$ & SQ1B, 6-7.5m W-E/0-2.5m N-S & stone: pounder (1 piece, quartzite) & 021 & SAV1E 0724 & 19.01.2014 \\
\hline $296 / 2014$ & $\mathrm{SQ} 1 \mathrm{~B}, 7-8.3 \mathrm{~m} \mathrm{~W}-\mathrm{E} / 0-2.5 \mathrm{~m} \mathrm{~N}-\mathrm{S}$ & stone: grindstone? (1 piece, sandstone) & 021 & SAV1E 0759 & 20.01 .2014 \\
\hline $307 / 2014$ & SQ1B, 7-8.3m W-E/0-2.5m N-S & stone: grindstone (1 piece, quartzite) & 021 & SAV1E 0774 & 20.01 .2014 \\
\hline $294 / 2014$ & $\mathrm{SQ} 3,2-3 \mathrm{~m} \mathrm{~W}-\mathrm{E} / 0-4.4 \mathrm{~m} \mathrm{~N}-\mathrm{S}$ & stone: grindstone? (1 piece, sandstone) & 023 & SAV1E 0753 & 20.01 .2014 \\
\hline $306 / 2014$ & $\mathrm{SQ} 3,2.5-3.2 \mathrm{~m} \mathrm{~W}-\mathrm{E} / 0-3.5 \mathrm{~m} \mathrm{~N}-\mathrm{S}$ & stone: grindstone (1 piece, sandstone) & 024 & SAV1E 0760 & 20.01.2014 \\
\hline $2114 / 2014$ & $\begin{array}{l}\text { SQ3, } 2.5-3.2 \mathrm{~m} \mathrm{~W}-\mathrm{E} / 0-3.5 \mathrm{~m} \mathrm{~N}-\mathrm{S} \text {, } \\
\text { cleaning of SU }\end{array}$ & $\begin{array}{l}\text { stones: hand mill (1 fragment, sandstone), grindstone? } \\
\text { (1 fragment, sandstone) }\end{array}$ & 024 & $\begin{array}{l}\text { SAV1E } 0993 \text { (hand mill), SAV1E } 0994 \\
\text { (grindstone?) }\end{array}$ & 02.02 .2014 \\
\hline $317 / 2014$ & $\mathrm{SQ} 3,8-10.5 \mathrm{~m} \mathrm{~W}-\mathrm{E} / 2.5-5.7 \mathrm{~m} \mathrm{~N}-\mathrm{S}$ & stones: grindstones (3 pieces, each sandstone) & 026 & $\begin{array}{l}\text { SAV1E 0755, SAV1E 0756, SAV1E } 0757 \\
\text { (grindstones) }\end{array}$ & 21.01 .2014 \\
\hline $322 / 2014$ & $\mathrm{SQ} 3,8-10.5 \mathrm{~m} \mathrm{~W}-\mathrm{E} / 5-8 \mathrm{~m} \mathrm{~N}-\mathrm{S}$ & stones: grindstones (2 pieces, each sandstone) & 027 & SAV1E 0778, SAV1E 0779 (grindstones) & 21.01 .2014 \\
\hline $337 / 2014$ & $\mathrm{SQ} 3,8-10 \mathrm{~m} \mathrm{~W}-\mathrm{E} / 8-10 \mathrm{~m} \mathrm{~N}-\mathrm{S}$ & stone: grindstone (1 piece, sandstone) & 028 & SAV1E 0780 & 21.01 .2014 \\
\hline $343 / 2014$ & $\mathrm{SQ} 4,0-3 \mathrm{~m} \mathrm{~W}-\mathrm{E} / 1-5.5 \mathrm{~m} \mathrm{~N}-\mathrm{S}$ & stones: grindstones (2 pieces, each sandstone) & 030 & SAV1E 0803, SAV1E 0804 (grindstones) & 22.01.2014 \\
\hline $346 / 2014$ & $\mathrm{SQ} 3,2.5-9 \mathrm{~m} \mathrm{~W}-\mathrm{E} / 0-10 \mathrm{~m} \mathrm{~N}-\mathrm{S}$ & $\begin{array}{l}\text { stones: grindstones ( } 2 \text { pieces, each quartzite), } \\
\text { pounders ( } 4 \text { pieces, } 2 \text { schist, } 1 \text { quartzite?, } 1 \text { schist?) }\end{array}$ & 031 & $\begin{array}{l}\text { SAV1E 0812, SAV1E } 0813 \text { (grindstones), } \\
\text { SAV1E } 0815 \text {, SAV1E } 0816 \text {, SAV1E 0817, } \\
\text { SAV1E } 0818 \text { (pounders) }\end{array}$ & 22.01 .2014 \\
\hline
\end{tabular}




\begin{tabular}{|c|c|c|c|c|c|}
\hline $375 / 2014$ & $\mathrm{SQ} 4,5-8 \mathrm{~m} \mathrm{~W}-\mathrm{E} / 0-10 \mathrm{~m} \mathrm{~N}-\mathrm{S}$ & $\begin{array}{l}\text { stones: grindstones ( } 3 \text { pieces, each sandstone), } \\
\text { pounders ( } 2 \text { pieces, } 1 \text { quartzite, } 1 \text { basalt?), } \\
\text { stone vessel ( } 1 \text { fragment, unspecified stone) }\end{array}$ & 033 & $\begin{array}{l}\text { SAV1E 0834, SAV1E 0835, SAV1E } 0836 \\
\text { (grindstones), SAV1E 0837, SAV1E } 0838 \\
\text { (pounders), SAV1E } 0834 \text { (stone vessel) }\end{array}$ & 23.01.2014 \\
\hline $382 / 2014$ & SQ3, 9-10.5m W-E/2.6-5.5m N-S & $\begin{array}{l}\text { stones: grindstones ( } 3 \text { pieces, } 2 \text { sandstone, } 1 \text { quartzite), } \\
\text { pounder ( } 1 \text { piece, unspecified stone) }\end{array}$ & 034 & $\begin{array}{l}\text { SAV1E 0797, SAV1E 0798, SAV1E } 0800 \\
\text { (grindstones), SAV1E 0799 (pounder) }\end{array}$ & 23.01.2014 \\
\hline $389 / 2014$ & SQ3, 9-10.5m W-E/3.6-5.3m N-S & $\begin{array}{l}\text { stones: pounders ( } 3 \text { pieces, } 1 \text { sandstone, } 1 \text { quartzite, } 1 \text { unspecified } \\
\text { stone) }\end{array}$ & 036 & $\begin{array}{l}\text { SAV1E 0786, SAV1E 0787, SAV1E } 0788 \\
\text { (pounders) }\end{array}$ & 23.01.2014 \\
\hline $406 / 2014$ & $\mathrm{SQ} 3,0-1.3 \mathrm{~m} \mathrm{~W}-\mathrm{E} / 4.4-6.8 \mathrm{~m} \mathrm{~N}-\mathrm{S}$ & $\begin{array}{l}\text { stones: grindstone ( } 1 \text { piece, quartzite), } \\
\text { pounder ( } 1 \text { piece, sandstone) }\end{array}$ & 038 & $\begin{array}{l}\text { SAV1E } 0784 \text { (grindstone), SAV1E } 0785 \\
\text { (pounder) }\end{array}$ & 25.01.2014 \\
\hline $408 / 2014$ & SQ3, 0-1.3m W-E/7.4-8.7m N-S & stone: grindstone (1 piece, quartzite) & 038 & SAV1E 0805 & 25.01.2014 \\
\hline $398 / 2014$ & SQ1B, $0-3 \mathrm{~m} \mathrm{~W}-\mathrm{E} / 2-4.5 \mathrm{~m} \mathrm{~N}-\mathrm{S}$ & $\begin{array}{l}\text { stones: grindstone (1 piece, sandstone), } \\
\text { pounder (1 piece, quartzite) }\end{array}$ & 039 & $\begin{array}{l}\text { SAV1E } 0806 \text { (grindstone), SAV1E } 0807 \\
\text { (pounder) }\end{array}$ & 25.01.2014 \\
\hline $413 / 2014$ & SQ1B, 0-3m W-E/2-3m N-S & stones: grindstones (2 pieces, each sandstone) & 040 & SAV1E 0782, SAV1E 0783 (grindstones) & 25.01 .2014 \\
\hline $423 / 2014$ & SQ1B, 0-3m W-E/2-3m N-S & $\begin{array}{l}\text { stones: grindstones ( } 3 \text { pieces, each sandstone), } \\
\text { grindstone? ( } 1 \text { piece, basalt) }\end{array}$ & 040 & $\begin{array}{l}\text { SAV1E 0840, SAV1E 0841, SAV1E } 0842 \\
\text { (grindstones), SAV1E } 0843 \text { (grindstone?) }\end{array}$ & 26.01.2014 \\
\hline $1507 / 2014$ & $\mathrm{SQ} 1 \mathrm{~B}, 2.5-8 \mathrm{~m} \mathrm{~W}-\mathrm{E} / 2.5-4 \mathrm{~m} \mathrm{~N}-\mathrm{S}$ & stone: grindstone (1 fragment, quartzite) & 040 & SAV1E 0845 & 27.01.2014 \\
\hline $417 / 2014$ & $\mathrm{SQ} 3,1-5 \mathrm{~m} \mathrm{~W}-\mathrm{E} / 7.2-10 \mathrm{~m} \mathrm{~N}-\mathrm{S}$ & stones: grindstones ( 2 pieces, each quartzite) & 041 & SAV1E 0810, SAV1E 0811 (grindstones) & 25.01 .2014 \\
\hline $430 / 2014$ & $\mathrm{SQ} 3,1.8-5 \mathrm{~m}$ W-E/7-10m N-S & $\begin{array}{l}\text { stones: grindstones ( } 6 \text { pieces, each sandstone), pounder ( } 1 \text { piece, } \\
\text { quartzite) }\end{array}$ & 041 & $\begin{array}{l}\text { SAV1E 0825, SAV1E 0826, SAV1E 0827, } \\
\text { SAV1E 0828, SAV1E 0829, SAV1E } 0830 \\
\text { (grindstones), SAV1E 0824 (pounder) }\end{array}$ & 26.01 .2014 \\
\hline $443 / 2014$ & $\mathrm{SQ} 4,0.5-3 \mathrm{~m} \mathrm{~W}-\mathrm{E} / 0-4 \mathrm{~m} \mathrm{~N}-\mathrm{S}$ & stones: grindstone (1 piece, quartzite), pounder (1 piece, quartz) & 042 & $\begin{array}{l}\text { SAV1E } 0808 \text { (grindstone), SAV1E } 0809 \\
\text { (pounder) }\end{array}$ & 27.01.2014 \\
\hline $1502 / 2014$ & SQ1B, 3-10m W-E/3-5m N-S & $\begin{array}{l}\text { stones: grindstone ( } 1 \text { piece, sandstone), pounder (1 piece, quartz), } \\
\text { hammer or pounder (1 piece, granite) }\end{array}$ & 043 & $\begin{array}{l}\text { SAV1E } 0846 \text { (grindstone), SAV1E } 0847 \\
\text { (pounder), SAV1E } 0848 \text { (hammer or } \\
\text { pounder) }\end{array}$ & 27.01.2014 \\
\hline $1520 / 2014$ & $\mathrm{SQ} 3,2.7-5.2 \mathrm{~m} \mathrm{~W}-\mathrm{E} / 7-8.5 \mathrm{~m} \mathrm{~N}-\mathrm{S}$ & $\begin{array}{l}\text { stones: grindstones ( } 2 \text { pieces, each sandstone), pounder (1 piece, } \\
\text { sandstone) }\end{array}$ & 047 & $\begin{array}{l}\text { SAV1E 0860, SAV1E } 0861 \text { (grindstones), } \\
\text { SAV1E } 0862 \text { (pounder) }\end{array}$ & 28.01.2014 \\
\hline $2112 / 2014$ & SQ1B, $6.5-10 \mathrm{~m} \mathrm{~W}-\mathrm{E} / 0-1.7 \mathrm{~m} \mathrm{~N}-\mathrm{S}$ & stones: grindstones (2 pieces, each sandstone) & 048 & SAV1E 1001, SAV1E 1002 (grindstones) & 02.02 .2014 \\
\hline $1517 / 2014$ & SQ1B, 8-10m W-E/0-5m N-S & stone: pounder (1 piece, sandstone) & 048 & SAV1E 0859 (pounder) & 28.01 .2014 \\
\hline $2001 / 2014$ & SQ1B, 0-2m W-E/2.3-3.4m N-S & stones: grindstones ( 4 pieces, 3 sandstone, 1 quartzite) & 049 & $\begin{array}{l}\text { SAV1E 0870, SAV1E 0871, SAV1E 0872, } \\
\text { SAV1E } 0874 \text { (grindstones) }\end{array}$ & 28.01.2014 \\
\hline $2018 / 2014$ & $\mathrm{SQ} 3,2.5-5.3 \mathrm{~m} \mathrm{~W}-\mathrm{E} / 8.5-10 \mathrm{~m} \mathrm{~N}-\mathrm{S}$ & stone: grindstone (1 fragment, sandstone) & 050 & SAV1E 0918 & 29.01.2014 \\
\hline $2007 / 2014$ & $\mathrm{SQ} 3,2.5-5.3 \mathrm{~m} \mathrm{~W}-\mathrm{E} / 8.5-10 \mathrm{~m} \mathrm{~N}-\mathrm{S}$ & $\begin{array}{l}\text { stones: grindstones ( } 6 \text { pieces, each sandstone), pounder ( } 1 \text { piece, } \\
\text { granite?) }\end{array}$ & 050 & $\begin{array}{l}\text { SAV1E 0863, SAV1E 0864, SAV1E 0865, } \\
\text { SAV1E 0866, SAV1E 0867, SAV1E } 0868 \\
\text { (grindstones), SAV1E 0869 (pounder) }\end{array}$ & 28.01.2014 \\
\hline
\end{tabular}




\begin{tabular}{|c|c|c|c|c|c|}
\hline $2013 / 2014$ & SQ1B, $0-4 \mathrm{~m} \mathrm{~W}-\mathrm{E} / 0-2.3 \mathrm{~m} \mathrm{~N}-\mathrm{S}$ & $\begin{array}{l}\text { stones: pounders ( } 3 \text { pieces, } 2 \text { quartz, } 1 \text { basalt), grindstones } \\
\text { ( } 8 \text { pieces, } 3 \text { quartzite, } 5 \text { sandstone), weight (1 piece, basalt?) }\end{array}$ & 051 & $\begin{array}{l}\text { SAV1E 0875, SAV1E 0876, SAV1E } 0877 \\
\text { (pounders), SAV1E 0878, SAV1E 0879, } \\
\text { SAV1E 0880, SAV1E 0881, SAV1E 0882, } \\
\text { SAV1E 0883, SAV1E 0885, SAV1E } 0886 \\
\text { (grindstones), SAV1E 0884 (weight) }\end{array}$ & 28.01 .2014 \\
\hline $2022 / 2014$ & $\mathrm{SQ} 1 \mathrm{~B}, 1.5-4 \mathrm{~m} \mathrm{~W}-\mathrm{E} / 0-2.3 \mathrm{~m} \mathrm{~N}-\mathrm{S}$ & $\begin{array}{l}\text { stones: grindstones ( } 8 \text { pieces, } 1 \text { quartzite, } 7 \text { sandstone), pounders } \\
\text { (3 pieces, } 1 \text { quartzite, } 2 \text { sandstone), pounder or grindstone } \\
\text { (1 piece, sandstone), worked stone (1 piece, sandstone) }\end{array}$ & 051 & $\begin{array}{l}\text { SAV1E 0908, SAV1E 0909, SAV1E 0943, } \\
\text { SAV1E 0944, SAV1E 0945, SAV1E 0946, } \\
\text { SAV1E 0948, SAV1E 0949 (grindstones), } \\
\text { SAV1E 0940, SAV1E 0941, SAV1E 0942 } \\
\text { (pounders), SAV1E } 0947 \text { (pounder or } \\
\text { grindstone), SAV1E } 0950 \text { (worked stone) }\end{array}$ & 29.01 .2014 \\
\hline $2054 / 2014$ & SQ1B, $1.5-6.5 \mathrm{~m} \mathrm{~W}-\mathrm{E} / 1.3-3 \mathrm{~m} \mathrm{~N}-\mathrm{S}$ & $\begin{array}{l}\text { stones: hand mills ( } 2 \text { pieces, each sandstone), grindstone (1 piece, } \\
\text { sandstone), pounder (1 piece, sandstone) }\end{array}$ & 051 & $\begin{array}{l}\text { SAV1E 0958, SAV1E } 0960 \text { (hand mills), } \\
\text { SAV1E } 0959 \text { (grindstone), SAV1E } 0961 \\
\text { (pounder) }\end{array}$ & 30.01 .2014 \\
\hline $2044 / 2014$ & $\mathrm{SQ} 1 \mathrm{~B}, 4-6.5 \mathrm{~m} \mathrm{~W}-\mathrm{E} / 0-1.5 \mathrm{~m} \mathrm{~N}-\mathrm{S}$ & $\begin{array}{l}\text { stones: pounder (1 piece, quartz), pounder or hammer (1 piece, } \\
\text { quartz) }\end{array}$ & 051 & $\begin{array}{l}\text { SAV1E } 0926 \text { (pounder), SAV1E } 0927 \\
\text { (pounder or hammer) }\end{array}$ & 30.01 .2014 \\
\hline $2030 / 2014$ & $\mathrm{SQ} 1 \mathrm{~B}, 4-6.5 \mathrm{~m} \mathrm{~W}-\mathrm{E} / 0-1.5 \mathrm{~m} \mathrm{~N}-\mathrm{S}$ & stones: grindstones (2 pieces, each sandstone); charcoal (1 bag) & 051 & SAV1E 0916, SAV1E 0917 (grindstones) & 29.01 .2014 \\
\hline $2059 / 2014$ & SQ1B, $6.5-7 \mathrm{~m} \mathrm{~W}-\mathrm{E} / 0-1.7 \mathrm{~m} \mathrm{~N}-\mathrm{S}$ & $\begin{array}{l}\text { stones: pounder ( } 1 \text { piece, sandstone), pounder or hammer } \\
\text { (1 piece, fossilised wood) }\end{array}$ & 051 & $\begin{array}{l}\text { SAV1E } 0929 \text { (pounder), SAV1E } 0930 \\
\text { (pounder or hammer) }\end{array}$ & 30.01 .2014 \\
\hline $2038 / 2014$ & $\mathrm{SQ} 3,2.3-4 \mathrm{~m} \mathrm{~W}-\mathrm{E} / 3-5.2 \mathrm{~m} \mathrm{~N}-\mathrm{S}$ & $\begin{array}{l}\text { stones: pounders ( } 5 \text { pieces, } 3 \text { quartzite, } 1 \text { quartz, } 1 \text { basalt), } \\
\text { grindstone ( } 1 \text { piece, sandstone) }\end{array}$ & 052 & $\begin{array}{l}\text { SAV1E 0910, SAV1E 0911, SAV1E 0913, } \\
\text { SAV1E 0914 (pounders), SAV1E } 0915 \\
\text { (grindstone) }\end{array}$ & 29.01 .2014 \\
\hline $2050 / 2014$ & $\mathrm{SQ} 3,2.3-4.8 \mathrm{~m} \mathrm{~W}-\mathrm{E} / 3-5.2 \mathrm{~m} \mathrm{~N}-\mathrm{S}$ & $\begin{array}{l}\text { stones: grindstones ( } 2 \text { pieces, each sandstone), pounder (1 piece, } \\
\text { quartzite) }\end{array}$ & 052 & $\begin{array}{l}\text { SAV1E 0936, SAV1E } 0937 \text { (grindstones), } \\
\text { SAV1E } 0938 \text { (pounder) }\end{array}$ & 30.01 .2014 \\
\hline $2065 / 2014$ & $\mathrm{SQ} 3,4.8-5.3 \mathrm{~m} \mathrm{~W}-\mathrm{E} / 5.2-7.3 \mathrm{~m} \mathrm{~N}-\mathrm{S}$ & $\begin{array}{l}\text { stones: pounders ( } 2 \text { pieces, } 1 \text { quartzite, } 1 \text { silicified stone), } \\
\text { grindstone ( } 1 \text { piece, sandstone) }\end{array}$ & 054 & $\begin{array}{l}\text { SAV1E 0976, SAV1E } 0977 \text { (pounders), } \\
\text { SAV1E } 0974 \text { (grindstone) }\end{array}$ & 01.02 .2014 \\
\hline $2092 / 2014$ & SQ1B, 0-1.5m W-E/2.5-4.5m N-S & stone: grindstone (1 piece, sandstone) & 056 & SAV1E 1052 & 01.02 .2014 \\
\hline $2070 / 2014$ & $\mathrm{SQ1B}, 0-2.8 \mathrm{~m} \mathrm{~W}-\mathrm{E} / 3-4 \mathrm{~m} \mathrm{~N}-\mathrm{S}$ & $\begin{array}{l}\text { stones: pounders ( } 3 \text { pieces, } 2 \text { quartzite, } 1 \text { sandstone), grindstone } \\
\text { (1 piece, sandstone?) }\end{array}$ & 056 & $\begin{array}{l}\text { SAV1E 0963, SAV1E 0964, SAV1E } 0965 \\
\text { (pounders), SAV1E } 0962 \text { (grindstone) }\end{array}$ & 01.02 .2014 \\
\hline $2084 / 2014$ & $\mathrm{SQ} 3,2-5.5 \mathrm{~m} \mathrm{~W}-\mathrm{E} / 2-3.8 \mathrm{~m} \mathrm{~N}-\mathrm{S}$ & stones: pounders ( 2 pieces, each quartz) & 058 & SAV1E 0924, SAV1E 0925 (pounders) & 01.02 .2014 \\
\hline $2091 / 2014$ & $\mathrm{SQ} 3,2-5.5 \mathrm{~m} \mathrm{~W}-\mathrm{E} / 2-3.8 \mathrm{~m} \mathrm{~N}-\mathrm{S}$ & stones: pounders ( 2 pieces, each quartz) & 059 & SAV1E 0931, SAV1E 0932 (pounders) & 01.02 .2014 \\
\hline $2105 / 2014$ & $\mathrm{SQ} 3,4.2-6 \mathrm{~m} \mathrm{~W}-\mathrm{E} / 0-3.8 \mathrm{~m} \mathrm{~N}-\mathrm{S}$ & stones: grindstones (2 pieces, each sandstone) & 061 & SAV1E 0998, SAV1E 0999 (grindstones) & 02.02 .2014 \\
\hline $2123 / 2014$ & $\mathrm{SQ} 3,4.4-5.6 \mathrm{~m} \mathrm{~W}-\mathrm{E} / 0.8-3 \mathrm{~m} \mathrm{~N}-\mathrm{S}$ & stones: hand mill (1 piece, quartzite), pounder (1 piece, quartzite) & 062 & $\begin{array}{l}\text { SAV1E } 1675 \text { (hand mill), SAV1E } 1676 \\
\text { (pounder) }\end{array}$ & 03.02 .2014 \\
\hline $2130 / 2014$ & $\mathrm{SQ} 3,4.4-5.6 \mathrm{~m} \mathrm{~W}-\mathrm{E} / 0.8-3 \mathrm{~m} \mathrm{~N}-\mathrm{S}$ & stone, worked (sandstone) & 062 & & 03.02 .2014 \\
\hline $2109 / 2014$ & SQ1B, $0-1.5 \mathrm{~m} \mathrm{~W}-\mathrm{E} / 3.7-4.7 \mathrm{~m} \mathrm{~N}-\mathrm{S}$ & stone: grindstone? (1 piece, sandstone) & 063 & SAV1E 0979 & 02.02 .2014 \\
\hline $2118 / 2014$ & SQ1B, $0-0.8 \mathrm{~m} \mathrm{~W}-\mathrm{E} / 4.2-5 \mathrm{~m} \mathrm{~N}-\mathrm{S}$ & stone & 064 & & 03.02 .2014 \\
\hline
\end{tabular}




\begin{tabular}{|c|c|c|c|c|c|}
\hline $2127 / 2014$ & SQ1B, $0-1.1 \mathrm{~m} \mathrm{~W}-\mathrm{E} / 4-5 \mathrm{~m} \mathrm{~N}-\mathrm{S}$ & stone: grindstone (1 piece, sandstone) & 065 & SAV1E 0982 & 03.02 .2014 \\
\hline $2164 / 2014$ & $\mathrm{SQ} 3,4.8-7 \mathrm{~m} \mathrm{~W}-\mathrm{E} / 4-8.3 \mathrm{~m} \mathrm{~N}-\mathrm{S}$ & $\begin{array}{l}\text { stones: hand mill (1 piece, sandstone), pounder (1 piece, } \\
\text { quartzite), polishing stone (1 piece, sandstone), grindstone } \\
\text { (1 piece, sandstone), worked stone? (1 piece, sandstone) }\end{array}$ & 066 & $\begin{array}{l}\text { SAV1E } 1056 \text { (hand mill), SAV1E } 1057 \\
\text { (pounder), SAV1E } 1058 \text { (polishing stone), } \\
\text { SAV1E } 1059 \text { (grindstone), SAV1E } 1060 \\
\text { (worked stone?) }\end{array}$ & 04.02 .2014 \\
\hline $2141 / 2014$ & $\mathrm{SQ} 3,4.8-7 \mathrm{~m} \mathrm{~W}-\mathrm{E} / 4-8.3 \mathrm{~m} \mathrm{~N}-\mathrm{S}$ & stones: grindstones (2 pieces, each sandstone) & 066 & SAV1E 0980, SAV1E 0981 (grindstones) & 03.02 .2014 \\
\hline $2132 / 2014$ & $\mathrm{SQ} 1 \mathrm{~B}, 0-1.1 \mathrm{~m} \mathrm{~W}-\mathrm{E} / 3-4.2 \mathrm{~m} \mathrm{~N}-\mathrm{S}$ & $\begin{array}{l}\text { stones: hand mill ( } 2 \text { pieces, } 1 \text { quartzite, } 1 \text { sandstone), pounder } \\
\text { (1 piece, quartz), whetstones ( } 2 \text { pieces, each sandstone) }\end{array}$ & 067 & $\begin{array}{l}\text { SAV1E 1597, SAV1E } 1598 \text { (hand mills), } \\
\text { SAV1E } 1601 \text { (pounder), SAV1E 1599, } \\
\text { SAV1E } 1600 \text { (whetstones) }\end{array}$ & 03.02 .2014 \\
\hline $2149 / 2014$ & SQ3, 9-10.5m W-E/3.6-5.3m N-S & stones: grindstone (1 piece, quartzite), pounder (1 piece, basalt?) & 069 & $\begin{array}{l}\text { SAV1E } 0983 \text { (grindstone), SAV1E } 0984 \\
\text { (pounder) }\end{array}$ & 03.02 .2014 \\
\hline $2154 / 2014$ & SQ3, 9-10.5m W-E/3.6-5.3m N-S & $\begin{array}{l}\text { stones: grindstone (1 piece, sandstone), pounder (1 piece, } \\
\text { quartzite) }\end{array}$ & 069 & $\begin{array}{l}\text { SAV1E } 1008 \text { (grindstone), SAV1E } 1007 \\
\text { (pounder) }\end{array}$ & 04.02 .2014 \\
\hline $2206 / 2014$ & $\mathrm{SQ} 3,8.5-10.5 \mathrm{~m} \mathrm{~W}-\mathrm{E} / 5-7.8 \mathrm{~m} \mathrm{~N}-\mathrm{S}$ & $\begin{array}{l}\text { stones: grindstones ( } 7 \text { pieces, } 4 \text { sandstone, } 3 \text { quartzite), polishing } \\
\text { stones? ( } 2 \text { pieces, } 1 \text { flint, } 1 \text { siliceous shale?), pounders ( } 3 \text { pieces, } \\
1 \text { unspecified stone, } 1 \text { basalt?, } 1 \text { quartzite?), hammer or pounder } \\
\text { (1 piece, quartzite), whetstone (1 piece, sandstone), worked stone } \\
\text { (1 piece, sandstone) }\end{array}$ & 070 & $\begin{array}{l}\text { SAV1E 1082, SAV1E 1083, SAV1E 1088, } \\
\text { SAV1E 1090, SAV1E 1552, SAV1E 1080, } \\
\text { SAV1E } 1081 \text { (grindstones), SAV1E 1554, } \\
\text { SAV1E } 1555 \text { (polishing stones?), SAV1E 1084, } \\
\text { SAV1E 1085, SAV1E 1086 (pounders), SAV1E } \\
\text { 1087 (hammer or pounder), SAV1E 1553 } \\
\text { (whetstone), SAV1E 1009 (worked stone) }\end{array}$ & 05.02 .2014 \\
\hline $2227 / 2014$ & $\mathrm{SQ} 3,8.5-10.5 \mathrm{~m} \mathrm{~W}-\mathrm{E} / 5-8.5 \mathrm{~m} \mathrm{~N}-\mathrm{S}$ & $\begin{array}{l}\text { stones: whetstones ( } 3 \text { pieces, } 2 \text { sandstone, } 1 \text { quartzite), hand mills } \\
\text { ( } 2 \text { pieces, } 1 \text { sandstone, } 1 \text { quartzite) }\end{array}$ & 070 & $\begin{array}{l}\text { SAV1E 1677, SAV1E 1678, SAV1E } 1679 \\
\text { (whetstones), SAV1E 1680, SAV1E } 1681 \\
\text { (hand mills) }\end{array}$ & 06.02 .2014 \\
\hline $2219 / 2014$ & $\mathrm{SQ} 3,8.5-10.5 \mathrm{~m} \mathrm{~W}-\mathrm{E} / 5-8.5 \mathrm{~m} \mathrm{~N}-\mathrm{S}$ & $\begin{array}{l}\text { stones: worked stones ( } 2 \text { pieces, } 1 \text { basalt, } 1 \text { sandstone), } \\
\text { grindstones ( } 2 \text { pieces, each sandstone) }\end{array}$ & 070 & $\begin{array}{l}\text { SAV1E 1048, SAV1E } 1051 \text { (worked stones), } \\
\text { SAV1E 1049, SAV1E } 1050 \text { (grindstones) }\end{array}$ & 05.02 .2014 \\
\hline $2253 / 2014$ & $\mathrm{SQ} 3,8-10.5 \mathrm{~m} \mathrm{~W}-\mathrm{E} / 3.6-5.5 \mathrm{~m} \mathrm{~N}-\mathrm{S}$ & $\begin{array}{l}\text { stones: hand mill (1 piece, quartzite), abrasive stone (1 piece, } \\
\text { sandstone) }\end{array}$ & 070 & $\begin{array}{l}\text { SAV1E } 1517 \text { (hand mill), SAV1E } 1518 \\
\text { (abrasive stone) }\end{array}$ & 06.02 .2014 \\
\hline $2260 / 2014$ & SQ3, 8-10.5m W-E/3.6-5.5m N-S & $\begin{array}{l}\text { stones: pounder ( } 1 \text { piece, quartz?), grindstone (1 piece, quartzite), } \\
\text { abrasive stone (1 piece, sandstone) }\end{array}$ & 070 & $\begin{array}{l}\text { SAV1E } 1528 \text { (pounder), SAV1E } 1529 \\
\text { (grindstone), SAV1E } 1530 \text { (abrasive stone) }\end{array}$ & 08.02 .2014 \\
\hline $2196 / 2014$ & $\mathrm{SQ} 3,9.2-10.5 \mathrm{~m} \mathrm{~W}-\mathrm{E} / 4.7-5.9 \mathrm{~m} \mathrm{~N}-\mathrm{S}$ & $\begin{array}{l}\text { stones: pounders ( } 2 \text { pieces, each quartz), whetstones ( } 3 \text { pieces, } \\
\text { each sandstone), grindstone ( } 1 \text { piece, quartzite), hand mill } \\
\text { (1 piece, quartzite), architecture? (1 piece, unspecified stone), } \\
\text { stone vessel? (1 piece, unspecified stone), worked stone } \\
\text { (1 fragment, sandstone) }\end{array}$ & 070 & $\begin{array}{l}\text { SAV1E 1654, SAV1E } 1655 \text { (pounders), } \\
\text { SAV1E 1656, SAV1E 1657, SAV1E 1658 } \\
\text { (whetstones), SAV1E 1659 (grindstone), } \\
\text { SAV1E 1660 (hand mill), SAV1E 1662 } \\
\text { (architecture?), SAV1E } 1663 \text { (stone vessel?), } \\
\text { SAV1E 1664 (worked stone) }\end{array}$ & 05.02 .2014 \\
\hline $2243 / 2014$ & SQ3, 9.2-10.5m W-E/8-9.2m N-S & $\begin{array}{l}\text { stones: pounders ( } 3 \text { pieces, } 2 \text { quartz, } 1 \text { quartzite?), hand mill? } \\
\text { (1 piece, sandstone), whetstones ( } 2 \text { pieces, each sandstone) }\end{array}$ & 070 & $\begin{array}{l}\text { SAV1E 1546, SAV1E 1547, SAV1E } 1548 \\
\text { (pounders), SAV1E } 1549 \text { (hand mill?), } \\
\text { SAV1E 1550, SAV1E } 1551 \text { (whetstones) }\end{array}$ & 06.02 .2014 \\
\hline $2238 / 2014$ & SQ3, 9-10.5m W-E/2-3.6m N-S & stones: pounders (3 pieces, each quartzite) & 070 & $\begin{array}{l}\text { SAV1E 1021, SAV1E 1022, SAV1E } 1023 \\
\text { (pounders) }\end{array}$ & 06.02 .2014 \\
\hline
\end{tabular}




\begin{tabular}{|c|c|c|c|c|c|}
\hline $2249 / 2014$ & SQ3, 9-10.5m W-E/8-9.5m N-S & stone: whetstone (1 piece, sandstone) & 070 & SAV1E 1536 & 06.02 .2014 \\
\hline $2177 / 2014$ & $\mathrm{SQ} 3,8.5-10.5 \mathrm{~m} \mathrm{~W}-\mathrm{E} / 0.8-2.3 \mathrm{~m} \mathrm{~N}-\mathrm{S}$ & $\begin{array}{l}\text { stone: worked stone/ammunition/front-loader } \\
\text { bullet?/token/gaming piece? (1 spherical shaped stone, } \\
\text { sandstone) }\end{array}$ & 071 & SAV1E 1042 & 04.02 .2014 \\
\hline $2170 / 2014$ & $\begin{array}{l}\text { SQ3, } 8.5-10.5 \mathrm{~m} \mathrm{~W}-\mathrm{E} / 0.8-2.3 \mathrm{~m} \mathrm{~N}-\mathrm{S} \text {, } \\
\text { Feature } 7\end{array}$ & $\begin{array}{l}\text { stones: pounders ( } 2 \text { pieces, quartzite), hand mill (1 piece, } \\
\text { sandstone), grindstone (1 piece, sandstone) }\end{array}$ & 071 & $\begin{array}{l}\text { SAV1E 1014, SAV1E } 1016 \text { (pounders), hand } \\
\text { mill (SAV1E 1015), grindstone (SAV1E } \\
\text { 1017) }\end{array}$ & 04.02 .2014 \\
\hline $2201 / 2014$ & $\mathrm{SQ} 3,6.6-9 \mathrm{~m} \mathrm{~W}-\mathrm{E} / 1.1-4.5 \mathrm{~m} \mathrm{~N}-\mathrm{S}$ & $\begin{array}{l}\text { stones: stone vessel? ( } 1 \text { fragment, quartzite), worked stones } \\
(2 \text { pieces, each fragmented, no stone tools, } 1 \text { quartzite, } \\
1 \text { sandstone) }\end{array}$ & 073 & $\begin{array}{l}\text { SAV1E } 1686 \text { (stone vessel?), SAV1E 1685, } \\
\text { SAV1E } 1687 \text { (worked stones) }\end{array}$ & 05.02 .2014 \\
\hline $2212 / 2014$ & SQ3, 6-8m W-E/0.2-1.1m N-S & $\begin{array}{l}\text { stones: grindstone (1 piece, sandstone), pounder (1 piece, } \\
\text { quartzite) }\end{array}$ & 075 & $\begin{array}{l}\text { SAV1E } 1020 \text { (grindstone), SAV1E } 1019 \\
\text { (pounder) }\end{array}$ & 05.02 .2014 \\
\hline $2215 / 2014$ & $\mathrm{SQ} 3,6-8 \mathrm{~m} \mathrm{~W}-\mathrm{E} / 0.2-1.1 \mathrm{~m} \mathrm{~N}-\mathrm{S}$ & stone: pumice stone? (1 piece) & 075 & SAV1E 1006 & 05.02 .2014 \\
\hline $2262 / 2014$ & $\mathrm{SQ} 4,8-10 \mathrm{~m} \mathrm{~W}-\mathrm{E} / 0-2 \mathrm{~m} \mathrm{~N}-\mathrm{S}$ & stone: pounder (1 piece, quartz) & 078 & SAV1E 1556 & 08.02 .2014 \\
\hline $2264 / 2014$ & $\begin{array}{l}\text { SQ4, 9.5-10.5m W-E/0.6-2m N-S, Feature } \\
15\end{array}$ & $\begin{array}{l}\text { stones: pounders ( } 6 \text { pieces, } 4 \text { quartz, } 1 \text { siliceous shale?, } \\
1 \text { silicified wood?), whetstones ( } 3 \text { pieces, each sandstone), } \\
\text { grindstone? ( } 1 \text { piece, quartzite), hand mill ( } 1 \text { piece, quartzite), } \\
\text { abrasive stone (1 piece, quartzite), raw material (1 piece, } \\
\text { quartzite) }\end{array}$ & 079 & $\begin{array}{l}\text { SAV1E 1665, SAV1E 1666, SAV1E 1667, } \\
\text { SAV1E 1668, SAV1E 1566, SAV1E } 1567 \\
\text { (pounders), SAV1E 1568, SAV1E } 1671, \\
\text { SAV1E 1672 (whetstones), SAV1E 1669 } \\
\text { (grindstone), SAV1E 1670 (hand mill), } \\
\text { SAV1E 1673 (abrasive stone), SAV1E } 1569 \\
\text { (raw material) }\end{array}$ & 08.02 .2014 \\
\hline $2278 / 2014$ & $\mathrm{SQ} 3,6-8 \mathrm{~m} \mathrm{~W}-\mathrm{E} / 5-7.5 \mathrm{~m} \mathrm{~N}-\mathrm{S}$ & $\begin{array}{l}\text { stones: pounder (1 piece, quartz), grindstone (1 piece, quartzite), } \\
\text { hand mill (1 piece, quartzite) }\end{array}$ & 080 & $\begin{array}{l}\text { SAV1E } 1570 \text { (pounder), SAV1E } 1571 \\
\text { (grindstone), SAV1E } 1572 \text { (hand mill) }\end{array}$ & 08.02 .2014 \\
\hline $2289 / 2014$ & $\mathrm{SQ} 3,6-8.5 \mathrm{~m} \mathrm{~W}-\mathrm{E} / 5-10 \mathrm{~m} \mathrm{~N}-\mathrm{S}$ & $\begin{array}{l}\text { stones: grindstone ( } 1 \text { piece, quartzite), pounder (1 piece, quartz), } \\
\text { abrasive stones ( } 2 \text { pieces, each quartzite) }\end{array}$ & 080 & $\begin{array}{l}\text { SAV1E } 1519 \text { (grindstone), SAV1E } 1520 \\
\text { (pounder), SAV1E 1521, SAV1E } 1522 \\
\text { (abrasive stones) }\end{array}$ & 09.02 .2014 \\
\hline $2281 / 2014$ & $\mathrm{SQ} 4,8-10 \mathrm{~m} \mathrm{~W}-\mathrm{E} / 2-3 \mathrm{~m} \mathrm{~N}-\mathrm{S}$ & $\begin{array}{l}\text { stones: pounders ( } 2 \text { pieces, } 1 \text { quartz, } 1 \text { siliceous shale?), hand } \\
\text { mills ( } 2 \text { pieces, each quartzite), grindstones ( } 3 \text { pieces, each } \\
\text { quartzite), abrasive stone ( } 1 \text { piece, quartzite), whetstone ( } 1 \text { piece, } \\
\text { sandstone) }\end{array}$ & 083 & $\begin{array}{l}\text { SAV1E 1557, SAV1E } 1558 \text { (pounders), } \\
\text { SAV1E 1560, SAV1E } 1561 \text { (hand mills), } \\
\text { SAV1E 1562, SAV1E 1563, SAV1E 1564 } \\
\text { (grindstones), SAV1E 1565 (abrasive stone), } \\
\text { SAV1E 1559 (whetstone) }\end{array}$ & 09.02 .2014 \\
\hline $2284 / 2014$ & SQ4, 8-9.5m W-E/0-2m N-S, Feature 15 & $\begin{array}{l}\text { stones: pounder (1 piece, siliceous shale?), hand mill (1 piece, } \\
\text { quartzite) }\end{array}$ & 084 & $\begin{array}{l}\text { SAV1E } 1544 \text { (pounder), SAV1E } 1545 \text { (hand } \\
\text { mill) }\end{array}$ & 09.02 .2014 \\
\hline $2291 / 2014$ & SQ4, 8-9.5m W-E/0-2m N-S, Feature 15 & $\begin{array}{l}\text { stones: pounders ( } 2 \text { pieces, } 1 \text { quartzite, } 1 \text { quartz), whetstones } \\
\text { ( } 2 \text { pieces, each sandstone), hand mills ( } 3 \text { pieces, } 1 \text { sandstone, } 2 \\
\text { quartzite), grindstones ( } 2 \text { pieces, each quartzite) }\end{array}$ & 084 & $\begin{array}{l}\text { SAV1E 1645, SAV1E } 1646 \text { (pounders), SAV1E } \\
\text { 1647, SAV1E 1648 (whetstones), SAV1E 1649, } \\
\text { SAV1E 1650, SAV1E } 1651 \text { (hand mills), } \\
\text { SAV1E 1652, SAV1E } 1653 \text { (grindstones) }\end{array}$ & 09.02 .2014 \\
\hline $2297 / 2014$ & SQ4, 8.4-9.5m W-E/0-1m N-S, Feature 15 & $\begin{array}{l}\text { stones and architecture?: whetstone? ( } 1 \text { piece, sandstone), hand } \\
\text { mill (1 piece, quartzite), worked stone ( } 2 \text { fragments, quartzite); } \\
\text { worked stone/architecture? (1 fragment, limestone) }\end{array}$ & 086 & $\begin{array}{l}\text { SAV1E } 1512 \text { (whetstone?), SAV1E } 1513 \\
\text { (hand mill), SAV1E } 1515 \text { (worked stone), } \\
\text { SAV1E } 1514 \text { (worked stone/architecture?) }\end{array}$ & 09.02 .2014 \\
\hline
\end{tabular}




\begin{tabular}{|c|c|c|c|c|c|}
\hline $2301 / 2014$ & SQ4, 8-10.5m W-E/2-3m N-S, Feature 15 & $\begin{array}{l}\text { stones: pounders ( } 3 \text { pieces, each quartz), hand mills ( } 2 \text { pieces, } \\
\text { each quartzite), grindstone ( } 1 \text { piece, quartzite), raw material } \\
\text { (1 fragment, quartz), stone vessel? (1 fragment, quartzite) }\end{array}$ & 088 & $\begin{array}{l}\text { SAV1E 1618, SAV1E 1619, SAV1E } 1620 \\
\text { (pounders), SAV1E 1622, SAV1E } 1623 \text { (hand } \\
\text { mills), SAV1E } 1621 \text { (grindstone), SAV1E } 1625 \\
\text { (raw material), SAV1E } 1624 \text { (stone vessel?) }\end{array}$ & 10.02 .2014 \\
\hline $2304 / 2014$ & $\mathrm{SQ} 3,9-10.5 \mathrm{~m} \mathrm{~W}-\mathrm{E} / 7.5-9.5 \mathrm{~m} \mathrm{~N}-\mathrm{S}$ & $\begin{array}{l}\text { stones: hand mill (1 piece, quartzite), whetstone (1 piece, } \\
\text { sandstone) }\end{array}$ & 089 & $\begin{array}{l}\text { SAV1E } 1610 \text { (hand mill), SAV1E } 1611 \\
\text { (whetstone) }\end{array}$ & 10.02 .2014 \\
\hline $2311 / 2014$ & $\mathrm{SQ} 3,8-9.4 \mathrm{~m} \mathrm{~W}-\mathrm{E} / 2.3-3.8 \mathrm{~m} \mathrm{~N}-\mathrm{S}$ & stone, schist & 090 & & 10.02 .2014 \\
\hline $2307 / 2014$ & $\mathrm{SQ} 4,9.4-9.8 \mathrm{~m} \mathrm{~W}-\mathrm{E} / 2-2.5 \mathrm{~m} \mathrm{~N}-\mathrm{S}$ & $\begin{array}{l}\text { stones: pounder (1 piece, quartz), abrasive stone?/hand mill? } \\
\text { (1 piece, sandstone) }\end{array}$ & 091 & $\begin{array}{l}\text { SAV1E } 1604 \text { (pounder), SAV1E } 1605 \\
\text { (abrasive stone?/hand mill?) }\end{array}$ & 10.02 .2014 \\
\hline $2420 / 2014$ & SQ4, debris in Feature 15 & stones: grindstones ( 2 pieces, each quartzite) & 092 & SAV1E 1523, SAV1E 1524 (grindstones) & 24.02.2014 \\
\hline $2315 / 2014$ & $\mathrm{SQ} 3,7.7-8.8 \mathrm{~m} \mathrm{~W}-\mathrm{E} / 0-3.5 \mathrm{~m} \mathrm{~N}-\mathrm{S}$ & $\begin{array}{l}\text { stones: pounders ( } 3 \text { pieces, } 2 \text { quartz, } 1 \text { siliceous shale?), } \\
\text { whetstone? ( } 1 \text { piece, sandstone), grindstones ( } 3 \text { pieces, each } \\
\text { quartzite) }\end{array}$ & 093 & $\begin{array}{l}\text { SAV1E 1500, SAV1E 1501, SAV1E } 1502 \\
\text { (pounders), SAV1E } 1503 \text { (whetstone), } \\
\text { SAV1E 1504, SAV1E 1505, SAV1E } 1506 \\
\text { (grindstones) }\end{array}$ & 10.02.2014 \\
\hline $2321 / 2014$ & $\mathrm{SQ} 3,7.7-8.8 \mathrm{~m} \mathrm{~W}-\mathrm{E} / 0-3.5 \mathrm{~m} \mathrm{~N}-\mathrm{S}$ & stones: pounder (1 piece, silex?) & 095 & SAV1E 1593 & 11.02 .2014 \\
\hline $2324 / 2014$ & $\mathrm{SQ} 4,8-10.5 \mathrm{~m} \mathrm{~W}-\mathrm{E} / 3-4.2 \mathrm{~m} \mathrm{~N}-\mathrm{S}$ & $\begin{array}{l}\text { stones: pounders ( } 2 \text { pieces, each quartz), grindstones ( } 2 \text { pieces, } \\
\text { each quartzite), hand mill ( } 1 \text { piece, quartzite), hand mills? } \\
\text { ( } 3 \text { pieces, } 2 \text { quartzite, } 1 \text { sandstone), worked stone ( } 1 \text { piece, } \\
\text { unspecified stone) }\end{array}$ & 096 & $\begin{array}{l}\text { SAV1E 1698, SAV1E } 1699 \text { (pounders), } \\
\text { SAV1E 1700, SAV1E } 1701 \text { (grindstones), } \\
\text { SAV1E 1702 (hand mill), SAV1E 1703, } \\
\text { SAV1E 1704, SAV1E } 1705 \text { (hand mills?), } \\
\text { SAV1E } 1706 \text { (worked stone) }\end{array}$ & 11.02 .2014 \\
\hline $2334 / 2014$ & $\mathrm{SQ} 3,7.7-8.8 \mathrm{~m} \mathrm{~W}-\mathrm{E} / 3.5-8 \mathrm{~m} \mathrm{~N}-\mathrm{S}$ & $\begin{array}{l}\text { stones: pounders ( } 2 \text { pieces, each quartz), hand mill (1 piece, } \\
\text { quartzite) }\end{array}$ & 097 & $\begin{array}{l}\text { SAV1E 1574, SAV1E } 1575 \text { (pounders), } \\
\text { SAV1E } 1576 \text { (hand mill) }\end{array}$ & 11.02 .2014 \\
\hline $2332 / 2014$ & $\mathrm{SQ} 3,7.8-9.6 \mathrm{~m} \mathrm{~W}-\mathrm{E} / 8-9.5 \mathrm{~m} \mathrm{~N}-\mathrm{S}$ & stones: hammer (1 piece, quartzite), pounder (1 piece, quartz) & 097 & $\begin{array}{l}\text { SAV1E } 1602 \text { (hammer), SAV1E } 1603 \\
\text { (pounder) }\end{array}$ & 11.02.2014 \\
\hline $2338 / 2014$ & $\mathrm{SQ} 4,8.4-10.5 \mathrm{~m} \mathrm{~W}-\mathrm{E} / 2.5-4.2 \mathrm{~m} \mathrm{~N}-\mathrm{S}$ & $\begin{array}{l}\text { stones: grindstone (1 piece, quartzite), whetstones ( } 7 \text { pieces, each } \\
\text { sandstone) }\end{array}$ & 099 & $\begin{array}{l}\text { SAV1E } 1585 \text { (grindstone), SAV1E 1586, } \\
\text { SAV1E 1587, SAV1E 1588, SAV1E 1589, } \\
\text { SAV1E 1590, SAV1E 1591, SAV1E } 1592 \\
\text { (whetstones) }\end{array}$ & 11.02.2014 \\
\hline $2350 / 2014$ & $\mathrm{SQ} 4,2-4 \mathrm{~m} \mathrm{~W}-\mathrm{E} / 0-3.6 \mathrm{~m} \mathrm{~N}-\mathrm{S}$ & $\begin{array}{l}\text { stones: pounders ( } 2 \text { pieces, each quartz), abrasive stone (1 piece, } \\
\text { sandstone?), polishing stone? ( } 1 \text { piece, unclear, slate?), } \\
\text { grindstone (1 piece, quartzite) }\end{array}$ & 100 & $\begin{array}{l}\text { SAV1E 1507, SAV1E } 1508 \text { (pounders), } \\
\text { SAV1E } 1509 \text { (abrasive stone), SAV1E } 15010 \\
\text { (polishing stone?), SAV1E } 1511 \text { (grindstone) }\end{array}$ & 12.02.2014 \\
\hline $2344 / 2014$ & $\mathrm{SQ} 4,10 \mathrm{~m} \mathrm{~W}-\mathrm{E} / 5 \mathrm{~m} \mathrm{~N}-\mathrm{S}$ & stone vessel (1 fragment, calcite) & 101 & SAV1E 1101 & 12.02.2014 \\
\hline $2345 / 2014$ & $\mathrm{SQ} 4,10.2 \mathrm{~m} \mathrm{~W}-\mathrm{E} / 4.3 \mathrm{~m} \mathrm{~N}-\mathrm{S}$ & $\begin{array}{l}\text { stone: worked sandstone (1 large rectangular? fragment, original } \\
\text { function unknown, sandstone) }\end{array}$ & 101 & SAV1E 1516 & 12.02 .2014 \\
\hline $2342 / 2014$ & $\mathrm{SQ} 4,8.4-10.5 \mathrm{~m} \mathrm{~W}-\mathrm{E} / 4.2-5.6 \mathrm{~m} \mathrm{~N}-\mathrm{S}$ & $\begin{array}{l}\text { stones: worked stone ( } 1 \text { piece, sandstone), grindstone ( } 1 \text { piece, } \\
\text { sandstone), abrasive stone/whetstone? ( } 1 \text { piece, sandstone), } \\
\text { whetstones ( } 4 \text { pieces, each sandstone) }\end{array}$ & 101 & $\begin{array}{l}\text { SAV1E } 1584 \text { (worked stone, SAV1E } 1577 \\
\text { (grindstone), SAV1E } 1579 \text { (abrasive } \\
\text { stone/whetstone?), SAV1E 1580, SAV1E } \\
\text { 1581, SAV1E 1582, SAV1E 1583 } \\
\text { (whetstones) }\end{array}$ & 12.02.2014 \\
\hline
\end{tabular}




\begin{tabular}{|c|c|c|c|c|c|}
\hline $2347 / 2014$ & $\mathrm{SQ} 4,8.5-10 \mathrm{~m} \mathrm{~W}-\mathrm{E} / 4.2-5.6 \mathrm{~m} \mathrm{~N}-\mathrm{S}$ & stone: pounder (1 piece, quartz) & 102 & SAV1E 1594 & 12.02 .2014 \\
\hline $2355 / 2014$ & SQ4, 9.2-10.5m W-E/4.2-5.4m N-S & $\begin{array}{l}\text { stones: pounders ( } 2 \text { pieces, } 1 \text { siliceous shale?, } 1 \text { quartz), } \\
\text { whetstone ( } 1 \text { piece, sandstone), grindstone (1 piece, quartzite) }\end{array}$ & 103 & $\begin{array}{l}\text { SAV1E 1640, SAV1E } 1641 \text { (pounders), } \\
\text { SAV1E } 1642 \text { (whetstone), SAV1E } 1643 \\
\text { (grindstone) }\end{array}$ & 12.02 .2014 \\
\hline $2362 / 2014$ & $\mathrm{SQ} 4,3.5-6 \mathrm{~m} \mathrm{~W}-\mathrm{E} / 0-3.4 \mathrm{~m} \mathrm{~N}-\mathrm{S}$ & stones: pounder (1 piece, quartz), grindstone? (1 piece, quartzite) & 105 & $\begin{array}{l}\text { SAV1E } 1608 \text { (pounder), SAV1E } 1609 \\
\text { (grindstone?) }\end{array}$ & 12.02 .2014 \\
\hline $2386 / 2014$ & $\mathrm{SQ} 4,3.5-6 \mathrm{~m} \mathrm{~W}-\mathrm{E} / 0-3.5 \mathrm{~m} \mathrm{~N}-\mathrm{S}$ & $\begin{array}{l}\text { stones: hand mills ( } 3 \text { pieces, each quartzite), grindstones } \\
\text { ( } 2 \text { pieces, each quartzite) }\end{array}$ & 105 & $\begin{array}{l}\text { SAV1E 1688, SAV1E 1690, SAV1E } 1689 \\
\text { (hand mills), SAV1E 1691, SAV1E } 1692 \\
\text { (grindstones) }\end{array}$ & 13.02.2014 \\
\hline $2400 / 2014$ & $\mathrm{SQ} 4,3.5-6 \mathrm{~m} \mathrm{~W}-\mathrm{E} / 0-4.7 \mathrm{~m} \mathrm{~N}-\mathrm{S}$ & stone: pounder (1 piece, quartz) & 105 & SAV1E 1541 & 16.02 .2014 \\
\hline $2372 / 2014$ & $\mathrm{SQ} 4,3.5-6 \mathrm{~m} \mathrm{~W}-\mathrm{E} / 2.5-4.7 \mathrm{~m} \mathrm{~N}-\mathrm{S}$ & stones: pounder (1 piece, quartz), hammer (1 piece, quartzite) & 105 & $\begin{array}{l}\text { SAV1E } 1607 \text { (pounder), SAV1E } 1606 \\
\text { (hammer) }\end{array}$ & 13.02.2014 \\
\hline $2367 / 2014$ & SQ4, 8-10.5m W-E/5.6-7.7m N-S & $\begin{array}{l}\text { stones: grindstone or hand mill ( } 1 \text { piece, quartzite), hand mill } \\
\text { (1 piece, quartzite), whetstones ( } 4 \text { pieces, each sandstone) }\end{array}$ & 107 & $\begin{array}{l}\text { SAV1E } 1612 \text { (grindstone or hand mill), } \\
\text { SAV1E } 1613 \text { (hand mill), SAV1E 1614, } \\
\text { SAV1E } 1615 \text {, SAV1E 1616, SAV1E } 1617 \\
\text { (whetstones) }\end{array}$ & 13.02.2014 \\
\hline $2376 / 2014$ & $\begin{array}{l}\text { SQ4, 9-10.5m W-E/5.6-7.2m N-S, } \\
\text { Feature } 16\end{array}$ & $\begin{array}{l}\text { stones: hammer ( } 1 \text { piece, quartzite), pounders ( } 5 \text { pieces, } 1 \text { quartz, } \\
2 \text { siliceous shale?, } 2 \text { silex), hand mills ( } 2 \text { pieces, each quartzite), } \\
\text { grindstones ( } 5 \text { pieces, each quartzite) }\end{array}$ & 108 & $\begin{array}{l}\text { SAV1E } 1626 \text { (hammer), SAV1E 1627, } \\
\text { SAV1E 1628, SAV1E 1629, SAV1E 1630, } \\
\text { SAV1E } 1631 \text { (pounders), SAV1E 1632, } \\
\text { SAV1E } 1634 \text { (hand mills), SAV1E 1635, } \\
\text { SAV1E 1636, SAV1E 1637, SAV1E 1638, } \\
\text { SAV1E } 1633 \text { (grindstones) }\end{array}$ & 13.02.2014 \\
\hline $2390 / 2014$ & $\begin{array}{l}\text { SQ4, } 8.5-10.5 \mathrm{~m} \mathrm{~W}-\mathrm{E} / 5.6-7 \mathrm{~m} \mathrm{~N}-\mathrm{S} \text {, } \\
\text { Feature } 16\end{array}$ & $\begin{array}{l}\text { stones: whetstones ( } 2 \text { pieces, each sandstone), grindstone? } \\
\text { (1 piece, quartzite), pounder (1 piece, quartz) }\end{array}$ & 109 & $\begin{array}{l}\text { SAV1E 1532, SAV1E } 1534 \text { (whetstones), } \\
\text { SAV1E } 1535 \text { (grindstone), SAV1E } 1534 \\
\text { (pounder) }\end{array}$ & 15.02.2014 \\
\hline $2382 / 2014$ & $\begin{array}{l}\mathrm{SQ} 4,8.5-9.5 \mathrm{~m} \mathrm{~W}-\mathrm{E} / 5.6-7.4 \mathrm{~m} \mathrm{~N}-\mathrm{S} \text {, } \\
\text { Feature } 16\end{array}$ & $\begin{array}{l}\text { stones: hand mill ( } 1 \text { piece, quartzite), whetstones ( } 2 \text { pieces, } \\
1 \text { sandstone, } 1 \text { quartzite), worked stones ( } 2 \text { pieces, each } \\
\text { sandstone) }\end{array}$ & 109 & $\begin{array}{l}\text { SAV1E } 1693 \text { (hand mill), SAV1E 1694, } \\
\text { SAV1E } 1695 \text { (whetstones), SAV1E 1696, } \\
\text { SAV1E } 1697 \text { (worked stones) }\end{array}$ & 13.02.2014 \\
\hline $2394 / 2014$ & $\mathrm{SQ} 4,7.3-8.7 \mathrm{~m} \mathrm{~W}-\mathrm{E} / 0-2 \mathrm{~m} \mathrm{~N}-\mathrm{S}$ & $\begin{array}{l}\text { stones: pounder (1 piece, quartz), whetstones ( } 2 \text { pieces, each } \\
\text { sandstone), hand mill (1 piece, quartzite), grindstone (1 fragment, } \\
\text { sandstone) }\end{array}$ & 110 & $\begin{array}{l}\text { SAV1E } 1537 \text { (pounder), SAV1E 1538, } \\
\text { SAV1E } 1539 \text { (whetstones), SAV1E } 1540 \\
\text { (hand mill), SAV1E } 1108 \text { (grindstone) }\end{array}$ & 16.02 .2014 \\
\hline $2402 / 2014$ & $\mathrm{SQ} 4,7.3-8 \mathrm{~m} \mathrm{~W}-\mathrm{E} / 0-2.5 \mathrm{~m} \mathrm{~N}-\mathrm{S}$ & $\begin{array}{l}\text { stones: hand mill (1 piece, quartzite), grindstone (1 piece, } \\
\text { quartzite) }\end{array}$ & 111 & $\begin{array}{l}\text { SAV1E } 1542 \text { ( hand mill), SAV1E } 1543 \\
\text { (grindstone) }\end{array}$ & 16.02.2014 \\
\hline $2408 / 2014$ & $\mathrm{SQ} 4,5.7-7.2 \mathrm{~m} \mathrm{~W}-\mathrm{E} / 0.5-10.5 \mathrm{~m} \mathrm{~N}-\mathrm{S}$ & $\begin{array}{l}\text { stones: polishing stone or hand mill ( } 1 \text { piece, unknown stone), } \\
\text { abrasive stone ( } 1 \text { piece, quartzite), pounders ( } 2 \text { pieces, each } \\
\text { quartz) }\end{array}$ & 112 & $\begin{array}{l}\text { SAV1E } 2903 \text { (polishing stone or hand mill), } \\
\text { SAV1W } 0629 \text { (abrasive stone), SAV1E 0641, } \\
\text { SAV1E } 0642 \text { (pounders) }\end{array}$ & 18.02 .2014 \\
\hline $2417 / 2014$ & $\begin{array}{l}\text { SQ4, debris from S-wall Feature } 15 \text {, up to } \\
1 \mathrm{~m} \text { NS of square }\end{array}$ & stone: tool?/blade? (1 chip of quartz) & 114 & SAV1E 1126 & 24.02 .2014 \\
\hline
\end{tabular}




\begin{tabular}{|c|c|c|c|c|c|}
\hline $027 / 2014$ & $\begin{array}{l}\text { SQ1, cleaning W-profile of SQ1 in area of } \\
\text { Feature } 7\end{array}$ & $\begin{array}{l}\text { stones: grindstones ( } 2 \text { pieces, each sandstone), grindstones? } \\
\text { ( } 4 \text { pieces, } 3 \text { quartzite, } 1 \text { sandstone), hand mill (1 piece, quartzite), } \\
\text { artefact? ( } 1 \text { fragment, circular/oval shaped with hole, schist) }\end{array}$ & l & $\begin{array}{l}\text { SAV1E 0365, SAV1E } 0366 \text { (grindstones), } \\
\text { SAV1E 0368, SAV1E 0369, SAV1E 0364, } \\
\text { SAV1E 0370 (grindstones?), SAV1E } 0367 \\
\text { (hand mill), SAV1E 0371 (artefact?) }\end{array}$ & 07.01 .2014 \\
\hline $2188 / 2014$ & $\begin{array}{l}\text { SQ2, } 0.5-1.8 \mathrm{~m} \mathrm{~N}-\mathrm{S} \text {, Feature } 15 \text {, cleaning of } \\
\text { W profile }\end{array}$ & stones: grindstone (1 piece, sandstone), blade (1 piece, silex) & / & $\begin{array}{l}\text { SAV1E } 1525 \text { (grindstone), SAV1E } 1527 \\
\text { (silex blade) }\end{array}$ & 04.02 .2014 \\
\hline $2423 / 2014$ & $\begin{array}{l}\text { SQ4, debris from S-wall, Feature } 15 \text { up to } \\
1 \mathrm{~m} \text { NS of square }\end{array}$ & stopper/sealing? (2 fragments, mud) & 092 & SAV1E 1128 & 24.02.2014 \\
\hline $135 / 2014$ & $\mathrm{SQ} 4,0-1.5 \mathrm{~m} \mathrm{~W}-\mathrm{E} / 7.5-10 \mathrm{~m} \mathrm{~N}-\mathrm{S}$ & textile (1 piece of linen/cloth) & 011 & SAV1E 0577 & 13.01.2014 \\
\hline $190 / 2014$ & $\mathrm{SQ} 4,0-5 \mathrm{~m} \mathrm{~W}-\mathrm{E} / 5-8 \mathrm{~m} \mathrm{~N}-\mathrm{S}$ & textile (1 piece of linen/cloth) & 015 & SAV1E 0575 & 16.01 .2014 \\
\hline $224 / 2014$ & SQ1B, 1m W-E/5m N-S, Feature 29 & textile (1 piece of linen/cloth) & 019 & SAV1E 0590 & 18.01 .2014 \\
\hline $2096 / 2014$ & $\mathrm{SQ} 1 \mathrm{~B}, 0-1.5 \mathrm{~m} \mathrm{~W}-\mathrm{E} / 2.5-4.5 \mathrm{~m} \mathrm{~N}-\mathrm{S}$ & textile (1 bag) & 056 & & 01.02 .2014 \\
\hline $2222 / 2014$ & $\mathrm{SQ} 3,10 \mathrm{~m} \mathrm{~W}-\mathrm{E} / 8 \mathrm{~m} \mathrm{~N}-\mathrm{S}$ & textile (1 bag) & 070 & & 05.02 .2014 \\
\hline $2353 / 2014$ & $\mathrm{SQ} 4,2-4 \mathrm{~m} \mathrm{~W}-\mathrm{E} / 0-3.6 \mathrm{~m} \mathrm{~N}-\mathrm{S}$ & textile (1 bag) & 100 & & 12.02 .2014 \\
\hline $030 / 2014$ & $\mathrm{SQ} 4,5 \mathrm{~m} \mathrm{~W}-\mathrm{E} / 9 \mathrm{~m}$ N-S & token/gaming piece (1 piece, sandstone) & 003 & SAV1E 0373 & 07.01 .2014 \\
\hline $193 / 2014$ & $\mathrm{SQ} 4,0.5 \mathrm{~m} \mathrm{~W}-\mathrm{E} / 6,7 \mathrm{~m} \mathrm{~N}-\mathrm{S}$ & token/gaming piece (1 re-used sherd?, Christian, pottery) & 015 & SAV1E 0653 & 16.01 .2014 \\
\hline $320 / 2014$ & $\mathrm{SQ} 3,8-10.5 \mathrm{~m} \mathrm{~W}-\mathrm{E} / 2.5-5.7 \mathrm{~m} \mathrm{~N}-\mathrm{S}$ & wall plaster? & 026 & & 21.01 .2014 \\
\hline $009 / 2014$ & $\begin{array}{l}\mathrm{SQ} 4,6 \mathrm{~m} \mathrm{~N} / 2.5 \mathrm{~m} \mathrm{~W} \text { from SP } 4.4, \\
\text { surface }\end{array}$ & weight/loom weight? (1 spherical pottery object) & 001 & SAV1E 0331 & 04.01 .2014 \\
\hline $081 / 2014$ & SQ1B, 7m W-E/1m N-S & wood (3 pieces) & 007 & & 11.01 .2014 \\
\hline $363 / 2014$ & $\mathrm{SQ} 4,0.5-1 \mathrm{~m} \mathrm{~W}-\mathrm{E} / 3-4 \mathrm{~m} \mathrm{~N}-\mathrm{S}$ & wood (1 bag) & 008 & & 22.01 .2014 \\
\hline $186 / 2014$ & $\mathrm{SQ} 4,0-5 \mathrm{~m} \mathrm{~W}-\mathrm{E} / 5-8 \mathrm{~m} \mathrm{~N}-\mathrm{S}$ & wood (2 pieces), charcoal (1 piece), bone & 015 & & 16.01 .2014 \\
\hline $198 / 2014$ & SQ4A, $10-10.5 \mathrm{~m} \mathrm{~W}-\mathrm{E} / 0-0.5 \mathrm{~m} \mathrm{~N}-\mathrm{S}$ & wood (4 pieces) & 017 & & 16.01 .2014 \\
\hline $326 / 2014$ & $\mathrm{SQ} 3,8-10.5 \mathrm{~m} \mathrm{~W}-\mathrm{E} / 5-8 \mathrm{~m} \mathrm{~N}-\mathrm{S}$ & wood (1 piece) & 027 & & 21.01 .2014 \\
\hline $438 / 2014$ & SQ1B, 0-3m W-E/2.5-4.5m N-S & wood (1 bag) & 040 & & 26.01 .2014 \\
\hline $1511 / 2014$ & $\mathrm{SQ} 1 \mathrm{~B}, 2.5-8 \mathrm{~m} \mathrm{~W}-\mathrm{E} / 2.5-4 \mathrm{~m} \mathrm{~N}-\mathrm{S}$ & wood & 040 & & 27.01 .2014 \\
\hline $1513 / 2014$ & $\mathrm{SQ} 3,2.7-5.2 \mathrm{~m} \mathrm{~W}-\mathrm{E} / 7.3-9 \mathrm{~m} \mathrm{~N}-\mathrm{S}$ & wood & 046 & & 27.01 .2014 \\
\hline $2005 / 2014$ & SQ1B, 0-2m W-E $/ 2.3-3.4 \mathrm{~m} \mathrm{~N}-\mathrm{S}$ & wood/organic material & 049 & & 28.01 .2014 \\
\hline $2034 / 2014$ & $\mathrm{SQ} 1 \mathrm{~B}, 4-6.5 \mathrm{~m} \mathrm{~W}-\mathrm{E} / 0-1.5 \mathrm{~m} \mathrm{~N}-\mathrm{S}$ & wood/organic material & 051 & & 29.01 .2014 \\
\hline $2074 / 2014$ & SQ1B, 0-2.8m W-E/3-4m N-S, & wood (1 bag) & 056 & & 01.02 .2014 \\
\hline
\end{tabular}




\begin{tabular}{|c|c|c|c|c|}
\hline & Feature 38 & & & \\
\hline $2139 / 2014$ & SQ1B, $0.5-2 \mathrm{~m} \mathrm{~W}-\mathrm{E} / 3.8-4.7 \mathrm{~m} \mathrm{~N}-\mathrm{S}$ & wood (2 pieces) & 068 & 03.02 .2014 \\
\hline $2152 / 2014$ & SQ3, 9-10.5m W-E/3.6-5.3m N-S & wood (3 pieces) & 069 & 03.02 .2014 \\
\hline $2175 / 2014$ & $\mathrm{SQ} 3,8.5-10.5 \mathrm{~m} \mathrm{~W}-\mathrm{E} / 0.8-2.3 \mathrm{~m} \mathrm{~N}-\mathrm{S}$ & wood (1 bag) & 071 & 04.02 .2014 \\
\hline $2329 / 2014$ & SQ4, 9.5-10.5m W-E/3.5-4.2m N-S & wood (1 bag) & 098 & 11.02 .2014 \\
\hline
\end{tabular}

\begin{tabular}{|c|c|c|c|c|c|}
\hline \multicolumn{6}{|c|}{ Sai Island SAV1 East 2015} \\
\hline Find no. & Location & Description/label/material & $\begin{array}{l}\text { SU/ } \\
\text { level }\end{array}$ & Object no. & Date \\
\hline $1322 / 2015$ & $\mathrm{SQ} 4,1-2.7 \mathrm{~m} \mathrm{~W}-\mathrm{E} / 3.7-5 \mathrm{~m} \mathrm{~N}-\mathrm{S}$ & $\begin{array}{l}\text { architecture: pivot stone? (1 large fragment, sandstone), archi- } \\
\text { tecture? (1 large fragment, worked stone, unspecified stone) }\end{array}$ & 223 & $\begin{array}{l}\text { SAV1E } 1963 \text { (pivot stone?), SAV1E } 1964 \\
\text { (architecture?) }\end{array}$ & 24.01.2015 \\
\hline $1384 / 2015$ & SQ4A, 5.1-7m W-E/0-2m N-S & architecture?/grindstone? (1 large fragment, quartzite) & 230 & SAV1E 2009 & 31.01 .2015 \\
\hline $1450 / 2015$ & SQ4A, 8.2m W-E/2m N-S (section) & architecture: door lintel (1 large fragment, relief, sandstone) & 238 & SAV1E 2179 & 04.02 .2015 \\
\hline $1606 / 2015$ & SQ2, Feature 15 & basin (1 fragment, clay) & 1321 & SAV1E 2347 & 09.02 .2015 \\
\hline $1064 / 2015$ & $\mathrm{SQ} 4,4 \mathrm{~m}$ W-E/6m N-S & bead (1 roughly triangular bead, unknown stone) & 205 & SAV1E 1210 & 07.01 .2015 \\
\hline $1088 / 2015$ & $\mathrm{SQ} 4,1.2 \mathrm{~m} \mathrm{~W}-\mathrm{E} / 5.4 \mathrm{~m} \mathrm{~N}-\mathrm{S}$ & bead ( 1 circular/disc bead, quartz) & 205 & SAV1E 1286 & 10.01 .2015 \\
\hline $1136 / 2015$ & $\mathrm{SQ} 4 \mathrm{~A}, 2 \mathrm{~m} \mathrm{~W}-\mathrm{E} / 0.2 \mathrm{~m} \mathrm{~N}-\mathrm{S}$ & bead (1 circular bead, glass?) & 205 & SAV1E 1409 & 13.01 .2015 \\
\hline $1231 / 2015$ & $\mathrm{SQ} 4,6.5 \mathrm{~m} \mathrm{~W}-\mathrm{E} / 1.8 \mathrm{~m} \mathrm{~N}-\mathrm{S}$ & bead ( 1 circular bead, faience) & 213 & SAV1E 1876 & 19.01 .2015 \\
\hline $1269 / 2015$ & SQ4, 0-1m W-E/8.8-9.7m N-S & bead ( 1 cylindrical bead, faience) & 215 & SAV1E 1929 & 22.01 .2015 \\
\hline $1468 / 2015$ & SQ4, E-W-wall, NW-part of SQ & bead (1 circular bead, ostrich eggshell?) & 223 & SAV1E 2579 & 05.02 .2015 \\
\hline $1344 / 2015$ & $\mathrm{SQ} 4,2 \mathrm{~m} \mathrm{~W}-\mathrm{E} / 6.4 \mathrm{~m} \mathrm{~N}-\mathrm{S}$ & beads ( 2 ring beads, faience) & 226 & SAV1E 1780 & 27.01 .2015 \\
\hline $1356 / 2015$ & $\mathrm{SQ} 4+4 \mathrm{~A}, 0.4-5 \mathrm{~m} \mathrm{~W}-\mathrm{E} / 7.5-12 \mathrm{~m} \mathrm{~N}-\mathrm{S}$ & bead (1 spherical bead, clay) & 227 & SAV1E 1789 & 28.01 .2015 \\
\hline $1370 / 2015$ & $\mathrm{SQ} 4+4 \mathrm{~A}, 0.4-5 \mathrm{~m} \mathrm{~W}-\mathrm{E} / 7.5-12 \mathrm{~m} \mathrm{~N}-\mathrm{S}$ & bead (1 tubular bead, faience) & 228 & SAV1E 2003 & 29.01 .2015 \\
\hline $1377 / 2015$ & $\mathrm{SQ} 4 \mathrm{~A}, 3.5-5.1 \mathrm{~m} \mathrm{~W}-\mathrm{E} / 0-2 \mathrm{~m} \mathrm{~N}-\mathrm{S}$ & bead (1 spherical bead, clay) & 229 & SAV1E 2117 & 31.01 .2015 \\
\hline $1545 / 2015$ & SQ4, NE-corner of SU 310 & bead (1 circular bead, faience) & 1310 & SAV1E 2102 & 04.02 .2015 \\
\hline $1564 / 2015$ & SQ2, Feature 15 & $\begin{array}{l}\text { bead ( } 1 \text { small and long tube, perhaps manufacturing product of } \\
\text { bead making, faience) }\end{array}$ & 1316 & SAV1E 2115 & 05.02 .2015 \\
\hline
\end{tabular}




\begin{tabular}{|c|c|c|c|c|c|}
\hline $1566 / 2015$ & SQ2, Feature 15 & bead ( 1 circular bead, faience) & 1316 & SAV1E 2114 & 05.02 .2015 \\
\hline $1579 / 2015$ & SQ2, Feature 15 & bead (1 ring bead, faience) & 1317 & SAV1E 2261 & 08.02 .2015 \\
\hline $1584 / 2015$ & SQ2+4, Feature 15 & bead (1 circular bead, faience) & 1317 & SAV1E 2263 & 08.02 .2015 \\
\hline $1495 / 2015$ & SQ4, NE-corner & bead (1 spherical bead, clay) & 1319 & SAV1E 2217 & 08.02 .2015 \\
\hline $1605 / 2015$ & SQ2, Feature 15 & bead (1 circular bead, faience) & 1321 & SAV1E 2262 & 09.02 .2015 \\
\hline $1632 / 2015$ & SQ2, Feature 15 & bead (1 disc bead, faience) & 1321 & SAV1E 2232 & 10.02.2015 \\
\hline $1667 / 2015$ & SQ2, Feature 15 & & 1321 & $\begin{array}{l}\text { SAV1E } 2265 \text { (3 circular beads), SAV1E } 2266 \\
\text { (disc bead) }\end{array}$ & 10.02 .2015 \\
\hline $1614 / 2015$ & $\mathrm{SQ} 2$, Feature $15,20 \mathrm{~cm}$ from N-wall & $\begin{array}{l}\text { beads ( } 1 \text { and } 1 / 2 \text { circular beads, faience); Canaanite amphora, } \\
\text { almost complete?; seal impressions ( } 4 \text { fragments, each clay); } \\
\text { seals ( } 9 \text { fragments, uninscribed); wood }(1 \text { bag); bone ( } 1 \text { bag); } \\
\text { organic material }(1 \text { bag); seashell ( } 1 \text { fragment); } \\
\text { seal }(1 \text { fragment, uninscribed); charcoal }(1 \text { bag); } \\
\text { worked stone ( } 2 \text { pieces, function unclear) }\end{array}$ & 1321 & $\begin{array}{l}\text { SAV1E } 2264 \text { (beads), SAV1E 2489, SAV1E } \\
\text { 2490, SAV1E 2491, SAV1E } 2492 \text { (seal } \\
\text { impressions) }\end{array}$ & 10.02 .2015 \\
\hline $1672 / 2015$ & SQ2, Feature 15, sieved material & $\begin{array}{l}\text { beads ( } 2 \text { pieces, } 1 \text { tubular, } 1 \text { circular, each faience); metal object } \\
\text { (1 fragment, function unknown); stone vessel (1 fragment with } \\
\text { part of rim, unspecified stone); seal impression (1 piece, clay); } \\
\text { seals ( } 54 \text { fragments, uninscribed); organic material ( } 1 \text { bag); } \\
\text { seashell ( } 1 \text { bag, fragments); charcoal ( } 1 \text { bag); worked clay } \\
\text { ( } 3 \text { fragment, function unclear); bone ( } 1 \text { bag) }\end{array}$ & 1322 & $\begin{array}{l}\text { SAV1E } 2286 \text { (beads), SAV1E } 2287 \text { (metal } \\
\text { object), SAV1E } 2288 \text { (stone vessel), SAV1E } \\
2572 \text { (seal impression) }\end{array}$ & 11.02 .2015 \\
\hline $1039 / 2015$ & $\mathrm{SQ} 4,3.7-5.8 \mathrm{~m} \mathrm{~W}-\mathrm{E} / 2.8-4.2 \mathrm{~m} \mathrm{~N}-\mathrm{S}$ & bone (1 bag) & 105 & & 06.01 .2015 \\
\hline $1047 / 2015$ & $\mathrm{SQ} 4,3.7-5.8 \mathrm{~m} \mathrm{~W}-\mathrm{E} / 4.2-5.1 \mathrm{~m} \mathrm{~N}-\mathrm{S}$ & bone (1 bag); seashell (1 fragment) & 105 & & 07.01 .2015 \\
\hline $1176 / 2015$ & $\mathrm{SQ} 4,4-5.7 \mathrm{~m} \mathrm{~W}-\mathrm{E} / 4.5-6.5 \mathrm{~m} \mathrm{~N}-\mathrm{S}$ & bone (1 bag) & 105 & & 15.01.2015 \\
\hline $1186 / 2015$ & $\mathrm{SQ} 4,4-5.7 \mathrm{~m} \mathrm{~W}-\mathrm{E} / 4.5-6.5 \mathrm{~m} \mathrm{~N}-\mathrm{S}$ & bone (1 bag) & 105 & & 17.01.2015 \\
\hline $1002 / 2015$ & $\mathrm{SQ} 4+4 \mathrm{~A}, 0-7.5 \mathrm{~m} \mathrm{~W}-\mathrm{E} / 7.1-12 \mathrm{~m} \mathrm{~N}-\mathrm{S}$ & bone (1 bag) & 200 & & 04.01 .2015 \\
\hline $1011 / 2015$ & $\mathrm{SQ} 4,0-7.5 \mathrm{~m} \mathrm{~W}-\mathrm{E} / 7.1-10 \mathrm{~m} \mathrm{~N}-\mathrm{S}$ & bone (1 bag) & 202 & & 04.01 .2015 \\
\hline $1019 / 2015$ & $\begin{array}{l}\text { SQ4, 0-7.5m W-E/7.1-10m N-S (12m N- } \\
\text { S) }\end{array}$ & bone (1 bag) & 202 & & 05.01 .2015 \\
\hline $1023 / 2015$ & SQ4, 5-8m N-S, surface & bone (1 bag) & 203 & & 05.01 .2015 \\
\hline $1027 / 2015$ & $\mathrm{SQ} 4,3.5-6.5 \mathrm{~m} \mathrm{~W}-\mathrm{E} / 5-6.2 \mathrm{~m} \mathrm{~N}-\mathrm{S}$ & bone (1 bag) & 204 & & 06.01 .2015 \\
\hline $1075 / 2015$ & $\mathrm{SQ} 4,0-1.5 \mathrm{~m} \mathrm{~W}-\mathrm{E} / 0-2 \mathrm{~m} \mathrm{~N}-\mathrm{S}$ & bone (1 bag) & 205 & & 08.01 .2015 \\
\hline
\end{tabular}




\begin{tabular}{|c|c|c|c|c|}
\hline $1058 / 2015$ & SQ4, 0-4m W-E/4.7-7.1m N-S & bone (1 bag) & 205 & 07.01 .2015 \\
\hline $1104 / 2015$ & $\mathrm{SQ} 4,0-4 \mathrm{~m} \mathrm{~W}-\mathrm{E} / 6-10 \mathrm{~m} \mathrm{~N}-\mathrm{S}$ & bone (1 bag) & 205 & 11.01.2015 \\
\hline $1110 / 2015$ & SQ4, 0-4m W-E/6-10m N-S & bone (1 piece) & 205 & 11.01 .2015 \\
\hline $1066 / 2015$ & SQ4, 0-4m W-E/7.1-9.7m N-S & bone (1 bag) & 205 & 08.01 .2015 \\
\hline $1095 / 2015$ & $\mathrm{SQ} 4,2-2.8 \mathrm{~m} \mathrm{~W}-\mathrm{E} / 6-7 \mathrm{~m} \mathrm{~N}-\mathrm{S}$ & bone (1 piece) & 205 & 11.01.2015 \\
\hline $1097 / 2015$ & $\mathrm{SQ} 4,3.1-4 \mathrm{~m} \mathrm{~W}-\mathrm{E} / 6-7.5 \mathrm{~m} \mathrm{~N}-\mathrm{S}$ & bone (1 bag) & 205 & 11.01 .2015 \\
\hline $1157 / 2015$ & $\mathrm{SQ} 4,3.5-5 \mathrm{~m} \mathrm{~W}-\mathrm{E} / 9-10 \mathrm{~m} \mathrm{~N}-\mathrm{S}$ & bone (1 bag) & 205 & 14.01.2015 \\
\hline $1162 / 2015$ & $\mathrm{SQ} 4,3.5-5 \mathrm{~m} \mathrm{~W}-\mathrm{E} / 9-10 \mathrm{~m} \mathrm{~N}-\mathrm{S}$ & bone (1 bag) & 205 & 15.01.2015 \\
\hline $1150 / 2015$ & $\mathrm{SQ} 4,4.5-5.2 \mathrm{~m} \mathrm{~W}-\mathrm{E} / 7.6-8.2 \mathrm{~m} \mathrm{~N}-\mathrm{S}$ & bone (1 bag) & 205 & 14.01 .2015 \\
\hline $1128 / 2015$ & SQ4A, 0-3m W-E/0-2m N-S & bone ( 2 bags) & 205 & 13.01.2015 \\
\hline $1140 / 2015$ & SQ4A, $1.7-3.2 \mathrm{~m} \mathrm{~W}-\mathrm{E} / 0-1.5 \mathrm{~m} \mathrm{~N}-\mathrm{S}$ & bone (1 bag) & 205 & 14.01.2015 \\
\hline $1168 / 2015$ & SQ4A, 4-5m W-E/1.4-2m N-S & bone (1 bag) & 205 & 15.01 .2015 \\
\hline $1146 / 2015$ & SQ4, 3.9-5.1m W-E/7.3-8.8m N-S & bone (1 bag) & 206 & 14.01.2015 \\
\hline $1195 / 2015$ & $\mathrm{SQ} 4,5-5.9 \mathrm{~m} \mathrm{~W}-\mathrm{E} / 4.2-5.8 \mathrm{~m} \mathrm{~N}-\mathrm{S}$ & bone & 207 & 17.01.2015 \\
\hline $1995 / 2015$ & $\mathrm{SQ} 4,5-5.9 \mathrm{~m} \mathrm{~W}-\mathrm{E} / 4.2-5.8 \mathrm{~m} \mathrm{~N}-\mathrm{S}$ & bone (1 piece) & 207 & 17.01 .2015 \\
\hline $1197 / 2015$ & $\mathrm{SQ} 4,4.2-5.9 \mathrm{~m} \mathrm{~W}-\mathrm{E} / 6.5-8 \mathrm{~m} \mathrm{~N}-\mathrm{S}$ & bone (1 bag) & 209 & 17.01 .2015 \\
\hline $1202 / 2015$ & SQ4+4A, 6-7.5m W-E/8.5-12m N-S & bone (1 bag) & 210 & 17.01 .2015 \\
\hline $1208 / 2015$ & SQ4A, 3.7-7m W-E/0-2m N-S & bone (1 bag) & 211 & 17.01.2015 \\
\hline $1211 / 2015$ & $\mathrm{SQ} 4,7-9.2 \mathrm{~m} \mathrm{~W}-\mathrm{E} / 6.8-8 \mathrm{~m} \mathrm{~N}-\mathrm{S}$ & bone (1 bag) & 212 & 18.01.2015 \\
\hline $1221 / 2015$ & $\mathrm{SQ} 4,7-9.2 \mathrm{~m} \mathrm{~W}-\mathrm{E} / 6.8-8 \mathrm{~m} \mathrm{~N}-\mathrm{S}$ & bone (1 bag) & 212 & 19.01.2015 \\
\hline $1250 / 2015$ & SQ4+4A, 5-6.5m W-E/8.2-12m N-S & bone (1 bag); seashell (1 fragment); charcoal (1 piece) & 213 & 20.01 .2015 \\
\hline $1240 / 2015$ & $\mathrm{SQ} 4+4 \mathrm{~A}, 6.5-8 \mathrm{~m} \mathrm{~W}-\mathrm{E} / 8.2-12 \mathrm{~m} \mathrm{~N}-\mathrm{S}$ & bone (1 bag) & 213 & 20.01 .2015 \\
\hline $1226 / 2015$ & $\mathrm{SQ} 4+4 \mathrm{~A}, 6-10 \mathrm{~m} \mathrm{~W}-\mathrm{E} / 8.2-12 \mathrm{~m} \mathrm{~N}-\mathrm{S}$ & bone (1 bag) & 213 & 19.01 .2015 \\
\hline $1256 / 2015$ & $\mathrm{SQ} 4+4 \mathrm{~A}, 3.5-5.5 \mathrm{~m} \mathrm{~W}-\mathrm{E} / 8.3-11.3 \mathrm{~m} \mathrm{~N}-\mathrm{S}$ & bone (1 bag) & 214 & 21.01 .2015 \\
\hline $1266 / 2015$ & SQ4, 0-1m W-E/8.8-9.7m N-S & bone (1 bag) & 215 & 22.01 .2015 \\
\hline $1272 / 2015$ & SQ4A, 0-1m W-E/0-2m N-S & bone (1 bag) & 216 & 22.01 .2015 \\
\hline
\end{tabular}




\begin{tabular}{|c|c|c|c|c|}
\hline $1276 / 2015$ & SQ4A, 0-1m W-E/0-2m N-S & bone (1 bag) & 217 & 22.01 .2015 \\
\hline $1279 / 2015$ & SQ4A, 0-1m W-E/0-2m N-S & bone (1 bag) & 218 & 22.01 .2015 \\
\hline $1286 / 2015$ & $\begin{array}{l}\mathrm{SQ} 4,5-8.8 \mathrm{~m} \mathrm{~N}-\mathrm{S} \\
\text { section cleaning }\end{array}$ & bone (1 bag) & 219 & 22.01 .2015 \\
\hline $1294 / 2015$ & $\mathrm{SQ} 4,0-1.2 \mathrm{~m} \mathrm{~W}-\mathrm{E} / 0-4.5 \mathrm{~m} \mathrm{~N}-\mathrm{S}$ & bone (1 bag) & 220 & 22.01 .2015 \\
\hline $1300 / 2015$ & $\mathrm{SQ} 4,0-1.2 \mathrm{~m} \mathrm{~W}-\mathrm{E} / 0-4.5 \mathrm{~m} \mathrm{~N}-\mathrm{S}$ & bone (1 bag) & 220 & 24.01 .2015 \\
\hline $1309 / 2015$ & $\mathrm{SQ} 4,0-0.8 \mathrm{~m} \mathrm{~W}-\mathrm{E} / 4.7-5.1 \mathrm{~m} \mathrm{~N}-\mathrm{S}$ & bone (1 bag) & 221 & 24.01 .2015 \\
\hline $1316 / 2015$ & $\mathrm{SQ} 4,0-0.8 \mathrm{~m} \mathrm{~W}-\mathrm{E} / 4.4-5.1 \mathrm{~m} \mathrm{~N}-\mathrm{S}$ & bone (1 bag) & 222 & 24.01 .2015 \\
\hline $1321 / 2015$ & $\mathrm{SQ} 4,1-2.7 \mathrm{~m} \mathrm{~W}-\mathrm{E} / 3.7-5 \mathrm{~m} \mathrm{~N}-\mathrm{S}$ & bone (1 bag) & 223 & 24.01 .2015 \\
\hline $1326 / 2015$ & $\mathrm{SQ} 4,2.7-4 \mathrm{~m} \mathrm{~W}-\mathrm{E} / 3.7-5 \mathrm{~m} \mathrm{~N}-\mathrm{S}$ & bone (1 bag) & 223 & 27.01 .2015 \\
\hline $1467 / 2015$ & SQ4, E-W-wall, NW-part of SQ & bone (1 piece) & 223 & 05.02 .2015 \\
\hline $1333 / 2015$ & $\mathrm{SQ} 4,1-1.5 \mathrm{~m} \mathrm{~W}-\mathrm{E} / 6.8-7.1 \mathrm{~m} \mathrm{~N}-\mathrm{S}$ & bone (1 bag) & 225 & 27.01 .2015 \\
\hline $1335 / 2015$ & SQ4, $0.4-3.3 \mathrm{~m} \mathrm{~W}-\mathrm{E} / 6-7.5 \mathrm{~m} \mathrm{~N}-\mathrm{S}$ & bone (1 bag) & 226 & 27.01 .2015 \\
\hline $1346 / 2015$ & $\mathrm{SQ} 4+4 \mathrm{~A}, 0.4-5 \mathrm{~m} \mathrm{~W}-\mathrm{E} / 7.5-12 \mathrm{~m} \mathrm{~N}-\mathrm{S}$ & bones (2 bags) & 227 & 28.01 .2015 \\
\hline $1361 / 2015$ & $\mathrm{SQ} 4+4 \mathrm{~A}, 0.4-5 \mathrm{~m} \mathrm{~W}-\mathrm{E} / 7.5-12 \mathrm{~m} \mathrm{~N}-\mathrm{S}$ & bone (1 bag) & 228 & 29.01 .2015 \\
\hline $1374 / 2015$ & SQ4A, 3.5-5.1m W-E/0-2m N-S & bone (1 bag) & 229 & 31.01 .2015 \\
\hline $1385 / 2015$ & SQ4A, 5.1-7m W-E/0-2m N-S & bone (1 bag) & 230 & 31.01 .2015 \\
\hline $1396 / 2015$ & SQ4A, 6-7.7m W-E/0-2m N-S & bone (1 bag) & 232 & 01.02 .2015 \\
\hline $1405 / 2015$ & $\mathrm{SQ} 4+4 \mathrm{~A}, 8.8-10 \mathrm{~m} \mathrm{~W}-\mathrm{E} / 9-12 \mathrm{~m} \mathrm{~N}-\mathrm{S}$ & bone (1 bag) & 234 & 01.02 .2015 \\
\hline $1409 / 2015$ & $\mathrm{SQ} 4+4 \mathrm{~A}, 9.2-10.5 \mathrm{~m} \mathrm{~W}-\mathrm{E} / 9-12 \mathrm{~m} \mathrm{~N}-\mathrm{S}$ & bone (1 bag) & 235 & 02.02 .2015 \\
\hline $1416 / 2015$ & $\mathrm{SQ} 4+4 \mathrm{~A}, 8.8-10.5 \mathrm{~m} \mathrm{~W}-\mathrm{E} / 9.3-12 \mathrm{~m} \mathrm{~N}-\mathrm{S}$ & bone (1 bag) & 236 & 02.02 .2015 \\
\hline $1451 / 2015$ & $\mathrm{SQ} 4 \mathrm{~A}, 6-8.5 \mathrm{~m} \mathrm{~W}-\mathrm{E} / 1.5-2 \mathrm{~m} \mathrm{~N}-\mathrm{S}$ & bone (1 bag) & 238 & 04.02 .2015 \\
\hline $1458 / 2015$ & $\mathrm{SQ} 4 \mathrm{~A}+2 \mathrm{~A}, 7-11 \mathrm{~m} \mathrm{~W}-\mathrm{E} / 1-2 \mathrm{~m} \mathrm{~N}-\mathrm{S}$ & bone (1 bag) & 238 & 04.02 .2015 \\
\hline $1430 / 2015$ & $\mathrm{SQ} 4 \mathrm{~A}+2 \mathrm{~A}, 8.8-11 \mathrm{~m} \mathrm{~W}-\mathrm{E} / 1-2 \mathrm{~m} \mathrm{~N}-\mathrm{S}$ & bone (1 bag) & 238 & 03.02 .2015 \\
\hline $1437 / 2015$ & SQ4+4A, 8-9m W-E/9.4-11.4m N-S & bone (1 bag) & 239 & 03.02 .2015 \\
\hline $1443 / 2015$ & SQ4A, 7.2-8.6m W-E/1-2m N-S & bone (1 bag) & 240 & 03.02 .2015 \\
\hline $1445 / 2015$ & SQ4A, 5.4-7m W-E/1-2m N-S & bone (1 bag) & 241 & 04.02 .2015 \\
\hline
\end{tabular}




\begin{tabular}{|c|c|c|c|c|}
\hline $1463 / 2015$ & $\mathrm{SQ} 4,5.2-6.5 \mathrm{~m} \mathrm{~W}-\mathrm{E} / 7-8.2 \mathrm{~m} \mathrm{~N}-\mathrm{S}$ & bone (1 bag) & 242 & 04.02 .2015 \\
\hline $1504 / 2015$ & SQ3, SE-corner & bone (1 bag) & 1302 & 02.02 .2015 \\
\hline $1509 / 2015$ & SQ3-4, baulk & bone (1 bag) & 1303 & 02.02 .2015 \\
\hline $1512 / 2015$ & SQ3-4, baulk & bone (1 bag) & 1304 & 02.02 .2015 \\
\hline $1517 / 2015$ & SQ3-4, baulk & bone (1 bag) & 1306 & 03.02 .2015 \\
\hline $1520 / 2015$ & SQ4-3, baulk & bone (1 bag) & 1307 & 03.02 .2015 \\
\hline $1527 / 2015$ & SQ4, N-baulk & bone (1 bag) & 1308 & 03.02 .2015 \\
\hline $1532 / 2015$ & SQ4, N-baulk & bone (1 bag) & 1309 & 03.02 .2015 \\
\hline $1540 / 2015$ & SQ4, Feature 15, W-side & bone (1 bag) & 1310 & 03.02 .2015 \\
\hline $1544 / 2015$ & SQ4, Feature 15, W-part & bone (1 bag) & 1310 & 04.02 .2015 \\
\hline $1547 / 2015$ & SQ4, along Feature 43 in Feature 15 & bone (1 bag) & 1311 & 04.02 .2015 \\
\hline $1554 / 2015$ & SQ4, Feature 15 & bone (1 bag) & 1314 & 04.02 .2015 \\
\hline $1560 / 2015$ & SQ2, Feature 15 & bone (1 bag) & 1316 & 05.02 .2015 \\
\hline $1568 / 2015$ & SQ4, Feature 15 & bone (1 bag) & 1316 & 05.02 .2015 \\
\hline $1573 / 2015$ & SQ2, Feature 15 & bone (1 bag) & 1316 & 08.02 .2015 \\
\hline $1589 / 2015$ & SQ2, Feature 15, E of Wall 44 & bone (1 bag) & 1316 & 09.02 .2015 \\
\hline $1578 / 2015$ & SQ2, Feature 15 & bone (1 bag) & 1317 & 08.02 .2015 \\
\hline $1497 / 2015$ & SQ2, Feature 15 & bone (1 bag) & 1318 & 08.02 .2015 \\
\hline $1648 / 2015$ & SQ2, E of wall 44 in Feature 15 & bones (2 pieces) & 1318 & 11.02 .2015 \\
\hline $1586 / 2015$ & $\mathrm{SQ} 2+4$ & bone (1 bag) & 1319 & 08.02 .2015 \\
\hline $1598 / 2015$ & SQ2, Feature 15, NE-corner & bone (1 bag) & 1320 & 09.02 .2015 \\
\hline $1600 / 2015$ & SQ2, Feature 15 & bone (1 bag) & 1321 & 09.02 .2015 \\
\hline $1611 / 2015$ & SQ2, Feature 15 & bone (1 bag) & 1321 & 10.02 .2015 \\
\hline $1641 / 2015$ & SQ2, Feature 15 & bone (1 bag) & 1321 & 11.02 .2015 \\
\hline $1669 / 2015$ & SQ2, Feature 15 & bone & 1321 & 12.02 .2015 \\
\hline $1651 / 2015$ & SQ2, Feature 15 & bone (1 bag) & 1323 & 12.02 .2015 \\
\hline
\end{tabular}




\begin{tabular}{|c|c|c|c|c|}
\hline $1658 / 2015$ & SQ4, wall 44 & bone (1 bag) & 1324 & 12.02.2015 \\
\hline $1455 / 2015$ & SQ4A, cleaning 8-10m W-E, S section & bone (1 bag) & l & 04.02 .2015 \\
\hline $1179 / 2015$ & $\mathrm{SQ} 4,4-5.7 \mathrm{~m} \mathrm{~W}-\mathrm{E} / 4.5-6.5 \mathrm{~m} \mathrm{~N}-\mathrm{S}$ & botanical material (1 bag) & 105 & 15.01 .2015 \\
\hline $1107 / 2015$ & $\mathrm{SQ} 4,0-4 \mathrm{~m} \mathrm{~W}-\mathrm{E} / 6-10 \mathrm{~m} \mathrm{~N}-\mathrm{S}$ & botanical material (1 bag) & 205 & 11.01 .2015 \\
\hline $1154 / 2015$ & SQ4, 3.5-5m W-E/9-10m N-S & botanical material (1 bag) & 205 & 14.01.2015 \\
\hline $1171 / 2015$ & SQ4A, 4-5m W-E/1.4-2m N-S & botanical material (1 bag) & 205 & 15.01.2015 \\
\hline $1147 / 2015$ & $\mathrm{SQ} 4,3.9-5.1 \mathrm{~m} \mathrm{~W}-\mathrm{E} / 7.3-8.8 \mathrm{~m} \mathrm{~N}-\mathrm{S}$ & botanical material (1 bag) & 206 & 14.01.2015 \\
\hline $1307 / 2015$ & $\mathrm{SQ} 4,0-1.2 \mathrm{~m} \mathrm{~W}-\mathrm{E} / 0-4.5 \mathrm{~m} \mathrm{~N}-\mathrm{S}$ & botanical material (1 bag); chalk (1 bag) & 220 & 24.01 .2015 \\
\hline $1330 / 2015$ & SQ4, 2.4-3.3m W-E/7.7-8.5m N-S & botanical material (1 bag, termite eaten) & 224 & 27.01 .2015 \\
\hline $1387 / 2015$ & SQ4A, 5.1-7m W-E/0-2m N-S & botanical material (1 bag) & 230 & 31.01 .2015 \\
\hline $1042 / 2015$ & $\mathrm{SQ} 4,3.7-5.8 \mathrm{~m} \mathrm{~W}-\mathrm{E} / 2.8-4.2 \mathrm{~m} \mathrm{~N}-\mathrm{S}$ & chalk (1 bag) & 105 & 06.01 .2015 \\
\hline $1051 / 2015$ & $\mathrm{SQ} 4,3.7-5.8 \mathrm{~m} \mathrm{~W}-\mathrm{E} / 4.2-5.1 \mathrm{~m} \mathrm{~N}-\mathrm{S}$ & chalk (1 bag) & 105 & 07.01 .2015 \\
\hline $1182 / 2015$ & $\mathrm{SQ} 4,4-5.7 \mathrm{~m} \mathrm{~W}-\mathrm{E} / 4.5-6.5 \mathrm{~m} \mathrm{~N}-\mathrm{S}$ & chalk (1 bag) & 105 & 15.01.2015 \\
\hline $1033 / 2015$ & $\begin{array}{l}\text { SQ4, collapse of SU } 105,4-5 \mathrm{~m} \mathrm{~W}-\mathrm{E} / 3.5 \mathrm{~m} \\
\text { N-S }\end{array}$ & chalk (1 large piece) & \begin{tabular}{|c|}
105, \\
collapse of \\
SU
\end{tabular} & 06.01 .2015 \\
\hline $1024 / 2015$ & $\mathrm{SQ} 4,5-8 \mathrm{~m} \mathrm{~N}-\mathrm{S}$, surface & chalk (1 bag) & 203 & 05.01 .2015 \\
\hline $1063 / 2015$ & $\mathrm{SQ} 4,0-4 \mathrm{~m} \mathrm{~W}-\mathrm{E} / 4.7-7.1 \mathrm{~m} \mathrm{~N}-\mathrm{S}$ & chalk (1 bag) & 205 & 07.01 .2015 \\
\hline $1084 / 2015$ & SQ4, 0-4m W-E/5-10m N-S & chalk (1 bag) & 205 & 10.01.2015 \\
\hline $1112 / 2015$ & $\mathrm{SQ} 4,0-4 \mathrm{~m} \mathrm{~W}-\mathrm{E} / 6-10 \mathrm{~m} \mathrm{~N}-\mathrm{S}$ & chalk (1 bag) & 205 & 11.01 .2015 \\
\hline $1121 / 2015$ & $\mathrm{SQ} 4,0-4 \mathrm{~m} \mathrm{~W}-\mathrm{E} / 7-9 \mathrm{~m} \mathrm{~N}-\mathrm{S} / 9-10 \mathrm{~m} \mathrm{~N}-\mathrm{S}$ & chalk (2 bags) & 205 & 12.01 .2015 \\
\hline $1135 / 2015$ & SQ4A, 0-3m W-E/0-2m N-S & chalk (1 bag) & 205 & 13.01.2015 \\
\hline $1298 / 2015$ & $\mathrm{SQ} 4,0-1.2 \mathrm{~m} \mathrm{~W}-\mathrm{E} / 0-4.5 \mathrm{~m} \mathrm{~N}-\mathrm{S}$ & chalk (1 bag) & 220 & 22.01 .2015 \\
\hline $1319 / 2015$ & $\mathrm{SQ} 4,0-0.8 \mathrm{~m} \mathrm{~W}-\mathrm{E} / 4.4-5.1 \mathrm{~m} \mathrm{~N}-\mathrm{S}$ & chalk (1 bag) & 222 & 24.01 .2015 \\
\hline $1328 / 2015$ & $\mathrm{SQ} 4,2.7-4 \mathrm{~m} \mathrm{~W}-\mathrm{E} / 3.7-5 \mathrm{~m} \mathrm{~N}-\mathrm{S}$ & chalk (1 bag) & 223 & 27.01.2015 \\
\hline $1337 / 2015$ & $\mathrm{SQ} 4,0.4-3.3 \mathrm{~m} \mathrm{~W}-\mathrm{E} / 6-7.5 \mathrm{~m} \mathrm{~N}-\mathrm{S}$ & chalk (1 bag) & 226 & 27.01 .2015 \\
\hline $1352 / 2015$ & $\mathrm{SQ} 4+4 \mathrm{~A}, 0.4-5 \mathrm{~m} \mathrm{~W}-\mathrm{E} / 7.5-12 \mathrm{~m} \mathrm{~N}-\mathrm{S}$ & chalk (1 bag) & 227 & 28.01.2015 \\
\hline
\end{tabular}




\begin{tabular}{|c|c|c|c|c|}
\hline $1365 / 2015$ & $\mathrm{SQ} 4+4 \mathrm{~A}, 0.4-5 \mathrm{~m} \mathrm{~W}-\mathrm{E} / 7.5-12 \mathrm{~m} \mathrm{~N}-\mathrm{S}$ & chalk (1 bag) & 228 & 29.01 .2015 \\
\hline $1380 / 2015$ & SQ4A, 3.5-5.1m W-E/0-2m N-S & chalk (1 bag) & 229 & 31.01 .2015 \\
\hline $1036 / 2015$ & $\mathrm{SQ} 4,3.7-5.8 \mathrm{~m} \mathrm{~W}-\mathrm{E} / 2.8-4.2 \mathrm{~m} \mathrm{~N}-\mathrm{S}$ & charcoal (1 bag) & 105 & 06.01 .2015 \\
\hline $1046 / 2015$ & $\mathrm{SQ} 4,3.7-5.8 \mathrm{~m} \mathrm{~W}-\mathrm{E} / 4.2-5.1 \mathrm{~m} \mathrm{~N}-\mathrm{S}$ & charcoal (1 bag) & 105 & 07.01 .2015 \\
\hline $1177 / 2015$ & $\mathrm{SQ} 4,4-5.7 \mathrm{~m} \mathrm{~W}-\mathrm{E} / 4.5-6.5 \mathrm{~m} \mathrm{~N}-\mathrm{S}$ & charcoal (1 bag) & 105 & 15.01 .2015 \\
\hline $1187 / 2015$ & $\mathrm{SQ} 4,4-5.7 \mathrm{~m} \mathrm{~W}-\mathrm{E} / 4.5-6.5 \mathrm{~m} \mathrm{~N}-\mathrm{S}$ & charcoal (1 bag) & 105 & 17.01 .2015 \\
\hline $1003 / 2015$ & $\mathrm{SQ} 4+4 \mathrm{~A}, 0-7.5 \mathrm{~m} \mathrm{~W}-\mathrm{E} / 7.1-12 \mathrm{~m} \mathrm{~N}-\mathrm{S}$ & charcoal (1 bag) & 200 & 04.01 .2015 \\
\hline $1012 / 2015$ & $\mathrm{SQ} 4,0-7.5 \mathrm{~m} \mathrm{~W}-\mathrm{E} / 7.1-10 \mathrm{~m} \mathrm{~N}-\mathrm{S}$ & charcoal (1 bag) & 202 & 04.01 .2015 \\
\hline $1020 / 2015$ & $\mathrm{SQ} 4,0-7.5 \mathrm{~m} \mathrm{~W}-\mathrm{E} / 7.1-10 \mathrm{~m} \mathrm{~N}-\mathrm{S}$ & charcoal (1 bag) & 202 & 05.01 .2015 \\
\hline $1028 / 2015$ & $\mathrm{SQ} 4,3.5-6.5 \mathrm{~m} \mathrm{~W}-\mathrm{E} / 5-6.2 \mathrm{~m} \mathrm{~N}-\mathrm{S}$ & charcoal (1 bag) & 204 & 06.01 .2015 \\
\hline $1074 / 2015$ & $\mathrm{SQ} 4,0-1.5 \mathrm{~m} \mathrm{~W}-\mathrm{E} / 0-2 \mathrm{~m} \mathrm{~N}-\mathrm{S}$ & charcoal (1 bag) & 205 & 08.01 .2015 \\
\hline $1057 / 2015$ & $\mathrm{SQ} 4,0-4 \mathrm{~m} \mathrm{~W}-\mathrm{E} / 4.7-7.1 \mathrm{~m} \mathrm{~N}-\mathrm{S}$ & charcoal (1 bag) & 205 & 07.01 .2015 \\
\hline $1082 / 2015$ & $\mathrm{SQ} 4,0-4 \mathrm{~m} \mathrm{~W}-\mathrm{E} / 5-10 \mathrm{~m} \mathrm{~N}-\mathrm{S}$ & charcoal (1 bag) & 205 & 10.01 .2015 \\
\hline $1067 / 2015$ & SQ4, 0-4m W-E/7.1-9.7m N-S & charcoal (1 bag) & 205 & 08.01 .2015 \\
\hline $1117 / 2015$ & $\mathrm{SQ} 4,0-4 \mathrm{~m} \mathrm{~W}-\mathrm{E} / 7-9 \mathrm{~m} \mathrm{~N}-\mathrm{S} / 9-10 \mathrm{~m} \mathrm{~N}-\mathrm{S}$ & charcoal (2 bags) & 205 & 12.01 .2015 \\
\hline $1098 / 2015$ & $\mathrm{SQ} 4,3.1-4 \mathrm{~m} \mathrm{~W}-\mathrm{E} / 6-7.5 \mathrm{~m} \mathrm{~N}-\mathrm{S}$ & charcoal (1 piece) & 205 & 11.01 .2015 \\
\hline $1158 / 2015$ & $\mathrm{SQ} 4,3.5-5 \mathrm{~m} \mathrm{~W}-\mathrm{E} / 9-10 \mathrm{~m} \mathrm{~N}-\mathrm{S}$ & charcoal (1 bag) & 205 & 14.01 .2015 \\
\hline $1163 / 2015$ & $\mathrm{SQ} 4,3.5-5 \mathrm{~m} \mathrm{~W}-\mathrm{E} / 9-10 \mathrm{~m} \mathrm{~N}-\mathrm{S}$ & charcoal (1 bag) & 205 & 15.01 .2015 \\
\hline $1151 / 2015$ & $\mathrm{SQ} 4,4.5-5.2 \mathrm{~m} \mathrm{~W}-\mathrm{E} / 7.6-8.2 \mathrm{~m} \mathrm{~N}-\mathrm{S}$ & charcoal (1 bag) & 205 & 14.01 .2015 \\
\hline $1127 / 2015$ & SQ4A, 0-3m W-E/0-2m N-S & charcoal (1 bag); seashell (1 fragment) & 205 & 13.01 .2015 \\
\hline $1144 / 2015$ & SQ4A, 1.7-3.2m W-E/0-1.5m N-S & charcoal & 205 & 14.01 .2015 \\
\hline $1169 / 2015$ & SQ4A, 4-5m W-E/1.4-2m N-S & charcoal (1 bag) & 205 & 15.01 .2015 \\
\hline $1191 / 2015$ & $\mathrm{SQ} 4,5-5.7 \mathrm{~m} \mathrm{~W}-\mathrm{E} / 7-8.5 \mathrm{~m} \mathrm{~N}-\mathrm{S}$ & charcoal (1 bag) & 208 & 17.01 .2015 \\
\hline $1198 / 2015$ & $\mathrm{SQ} 4,4.2-5.9 \mathrm{~m} \mathrm{~W}-\mathrm{E} / 6.5-8 \mathrm{~m} \mathrm{~N}-\mathrm{S}$ & charcoal (1 bag) & 209 & 17.01 .2015 \\
\hline $1205 / 2015$ & $\mathrm{SQ} 4+4 \mathrm{~A}, 6-7.5 \mathrm{~m} \mathrm{~W}-\mathrm{E} / 8.5-12 \mathrm{~m} \mathrm{~N}-\mathrm{S}$ & charcoal (1 bag) & 210 & 17.01 .2015 \\
\hline $1216 / 2015$ & $\mathrm{SQ} 4,7-9.2 \mathrm{~m} \mathrm{~W}-\mathrm{E} / 6.8-8 \mathrm{~m} \mathrm{~N}-\mathrm{S}$ & charcoal (1 bag) & 212 & 18.01.2015 \\
\hline
\end{tabular}




\begin{tabular}{|c|c|c|c|c|}
\hline $1220 / 2015$ & $\mathrm{SQ} 4,7-9.2 \mathrm{~m} \mathrm{~W}-\mathrm{E} / 6.8-8 \mathrm{~m} \mathrm{~N}-\mathrm{S}$ & charcoal (1 bag) & 212 & 19.01.2015 \\
\hline $1249 / 2015$ & $\mathrm{SQ} 4+4 \mathrm{~A}, 5-6.5 \mathrm{~m} \mathrm{~W}-\mathrm{E} / 8.2-12 \mathrm{~m} \mathrm{~N}-\mathrm{S}$ & charcoal (1 bag) & 213 & 20.01 .2015 \\
\hline $1239 / 2015$ & $\mathrm{SQ} 4+4 \mathrm{~A}, 6.5-8 \mathrm{~m} \mathrm{~W}-\mathrm{E} / 8.2-12 \mathrm{~m} \mathrm{~N}-\mathrm{S}$ & charcoal (1 bag) & 213 & 20.01 .2015 \\
\hline $1227 / 2015$ & $\mathrm{SQ} 4+4 \mathrm{~A}, 6-10 \mathrm{~m} \mathrm{~W}-\mathrm{E} / 8.2-12 \mathrm{~m} \mathrm{~N}-\mathrm{S}$ & charcoal (1 bag) & 213 & 19.01.2015 \\
\hline $1257 / 2015$ & $\mathrm{SQ} 4+4 \mathrm{~A}, 3.5-5.5 \mathrm{~m} \mathrm{~W}-\mathrm{E} / 8.3-11.3 \mathrm{~m} \mathrm{~N}-\mathrm{S}$ & charcoal (1 bag) & 214 & 21.01 .2015 \\
\hline $1274 / 2015$ & SQ4A, 0-1m W-E/0-2m N-S & charcoal (2 pieces) & 216 & 22.01 .2015 \\
\hline $1280 / 2015$ & SQ4A, 0-1m W-E/0-2m N-S & charcoal (3 pieces) & 218 & 22.01 .2015 \\
\hline $1291 / 2015$ & SQ4, 5-8.8m N-S, section cleaning & charcoal (1 bag) & 219 & 22.01 .2015 \\
\hline $1295 / 2015$ & $\mathrm{SQ} 4,0-1.2 \mathrm{~m} \mathrm{~W}-\mathrm{E} / 0-4.5 \mathrm{~m} \mathrm{~N}-\mathrm{S}$ & charcoal (1 bag) & 220 & 22.01 .2015 \\
\hline $1302 / 2015$ & $\mathrm{SQ} 4,0-1.2 \mathrm{~m} \mathrm{~W}-\mathrm{E} / 0-4.5 \mathrm{~m} \mathrm{~N}-\mathrm{S}$ & charcoal (1 bag) & 220 & 24.01 .2015 \\
\hline $1310 / 2015$ & $\mathrm{SQ} 4,0-0.8 \mathrm{~m} \mathrm{~W}-\mathrm{E} / 4.7-5.1 \mathrm{~m} \mathrm{~N}-\mathrm{S}$ & charcoal (1 bag) & 221 & 24.01 .2015 \\
\hline $1314 / 2015$ & $\mathrm{SQ} 4,0-0.8 \mathrm{~m} \mathrm{~W}-\mathrm{E} / 4.4-5.1 \mathrm{~m} \mathrm{~N}-\mathrm{S}$ & charcoal (1 bag) & 222 & 24.01 .2015 \\
\hline $1336 / 2015$ & SQ4, 0.4-3.3m W-E/6-7.5m N-S & charcoal (1 bag) & 226 & 27.01 .2015 \\
\hline $1347 / 2015$ & $\mathrm{SQ} 4+4 \mathrm{~A}, 0.4-5 \mathrm{~m} \mathrm{~W}-\mathrm{E} / 7.5-12 \mathrm{~m} \mathrm{~N}-\mathrm{S}$ & charcoal (2 bags) & 227 & 28.01 .2015 \\
\hline $1362 / 2015$ & $\mathrm{SQ} 4+4 \mathrm{~A}, 0.4-5 \mathrm{~m} \mathrm{~W}-\mathrm{E} / 7.5-12 \mathrm{~m} \mathrm{~N}-\mathrm{S}$ & charcoal (1 bag) & 228 & 29.01 .2015 \\
\hline $1364 / 2015$ & $\mathrm{SQ} 4+4 \mathrm{~A}, 0.4-5 \mathrm{~m} \mathrm{~W}-\mathrm{E} / 7.5-12 \mathrm{~m} \mathrm{~N}-\mathrm{S}$ & charcoal (1 bag) & 228 & 29.01 .2015 \\
\hline $1375 / 2015$ & $\mathrm{SQ} 4 \mathrm{~A}, 3.5-5.1 \mathrm{~m} \mathrm{~W}-\mathrm{E} / 0-2 \mathrm{~m} \mathrm{~N}-\mathrm{S}$ & charcoal (1 bag) & 229 & 31.01 .2015 \\
\hline $1386 / 2015$ & $\mathrm{SQ} 4 \mathrm{~A}, 5.1-7 \mathrm{~m} \mathrm{~W}-\mathrm{E} / 0-2 \mathrm{~m} \mathrm{~N}-\mathrm{S}$ & charcoal (1 bag) & 230 & 31.01 .2015 \\
\hline $1393 / 2015$ & SQ4A, 4.4-5.4m W-E/1-1.7m N-S & charcoal (1 bag) & 231 & 31.01 .2015 \\
\hline $1397 / 2015$ & SQ4A, 6-7.7m W-E/0-2m N-S & charcoal (1 bag) & 232 & 01.02 .2015 \\
\hline $1402 / 2015$ & $\mathrm{SQ} 4,7.8-8.8 \mathrm{~m} \mathrm{~W}-\mathrm{E} / 8-9.4 \mathrm{~m} \mathrm{~N}-\mathrm{S}$ & charcoal (1 bag) & 233 & 01.02 .2015 \\
\hline $1406 / 2015$ & $\mathrm{SQ} 4+4 \mathrm{~A}, 8.8-10 \mathrm{~m} \mathrm{~W}-\mathrm{E} / 9-12 \mathrm{~m} \mathrm{~N}-\mathrm{S}$ & charcoal (1 bag) & 234 & 01.02 .2015 \\
\hline $1410 / 2015$ & $\mathrm{SQ} 4+4 \mathrm{~A}, 9.2-10.5 \mathrm{~m} \mathrm{~W}-\mathrm{E} / 9-12 \mathrm{~m} \mathrm{~N}-\mathrm{S}$ & charcoal (1 bag) & 235 & 02.02 .2015 \\
\hline $1417 / 2015$ & $\mathrm{SQ} 4+4 \mathrm{~A}, 8.8-10.5 \mathrm{~m} \mathrm{~W}-\mathrm{E} / 9.3-12 \mathrm{~m} \mathrm{~N}-\mathrm{S}$ & charcoal (1 bag) & 236 & 02.02 .2015 \\
\hline $1459 / 2015$ & $\mathrm{SQ} 4 \mathrm{~A}+2 \mathrm{~A}, 7-11 \mathrm{~m} \mathrm{~W}-\mathrm{E} / 1-2 \mathrm{~m} \mathrm{~N}-\mathrm{S}$ & charcoal (1 bag) & 238 & 04.02 .2015 \\
\hline $1431 / 2015$ & $\mathrm{SQ} 4 \mathrm{~A}+2 \mathrm{~A}, 8.8-11 \mathrm{~m} \mathrm{~W}-\mathrm{E} / 1-2 \mathrm{~m} \mathrm{~N}-\mathrm{S}$ & charcoal (1 bag) & 238 & 03.02 .2015 \\
\hline
\end{tabular}




\begin{tabular}{|c|c|c|c|c|}
\hline $1438 / 2015$ & SQ4+4A, 8-9m W-E/9.4-11.4m N-S & charcoal (1 bag) & 239 & 03.02 .2015 \\
\hline $1446 / 2015$ & SQ4A, $5.4-7 \mathrm{~m} \mathrm{~W}-\mathrm{E} / 1-2 \mathrm{~m} \mathrm{~N}-\mathrm{S}$ & charcoal (1 bag) & 241 & 04.02 .2015 \\
\hline $1462 / 2015$ & $\mathrm{SQ} 4,5.2-6.5 \mathrm{~m} \mathrm{~W}-\mathrm{E} / 7-8.2 \mathrm{~m} \mathrm{~N}-\mathrm{S}$ & charcoal (1 bag) & 242 & 04.02 .2015 \\
\hline $1505 / 2015$ & SQ3, SE-corner & charcoal (1 bag) & 1302 & 02.02 .2015 \\
\hline $1518 / 2015$ & SQ3-4, baulk & charcoal (1 bag) & 1306 & 03.02 .2015 \\
\hline $1524 / 2015$ & SQ4-3, baulk & charcoal (2 pieces) & 1307 & 03.02 .2015 \\
\hline $1529 / 2015$ & SQ4, N-baulk & charcoal (1 bag) & 1308 & 03.02 .2015 \\
\hline $1537 / 2015$ & SQ4, N-baulk, Feature 15 & charcoal (1 bag) & 1309 & 03.02 .2015 \\
\hline $1543 / 2015$ & SQ4, Feature 15, W-part & charcoal (1 bag) & 1310 & 04.02 .2015 \\
\hline $1548 / 2015$ & SQ4, along Feature 43 in Feature 15 & charcoal (1 bag) & 1311 & 04.02 .2015 \\
\hline $1555 / 2015$ & SQ4, Feature 15 & charcoal (1 bag) & 1314 & 04.02 .2015 \\
\hline $1558 / 2015$ & SQ2, Feature 15 & charcoal (1 bag) & 1316 & 05.02 .2015 \\
\hline $1574 / 2015$ & SQ2, Feature 15 & charcoal (1 bag) & 1316 & 08.02 .2015 \\
\hline $1590 / 2015$ & SQ2, Feature 15, E of Wall 44 & charcoal (1 bag) & 1316 & 09.02 .2015 \\
\hline $1567 / 2015$ & SQ4, Feature 15 & charcoal (1 bag) & 1316 & 05.02 .2015 \\
\hline $1577 / 2015$ & SQ2, Feature 15 & charcoal (1 bag) & 1317 & 08.02 .2015 \\
\hline $1594 / 2015$ & SQ2, Feature 15, NE-corner & charcoal (1 bag) & 1317 & 09.02 .2015 \\
\hline $1498 / 2015$ & SQ2, Feature 15 & charcoal (1 piece) & 1318 & 08.02 .2015 \\
\hline $1646 / 2015$ & SQ2, E of wall 44 in Feature 15 & charcoal (1 bag) & 1318 & 11.02 .2015 \\
\hline $1587 / 2015$ & $\mathrm{SQ} 2+4$ & charcoal (1 bag) & 1319 & 08.02 .2015 \\
\hline $1596 / 2015$ & SQ2, Feature 15 & charcoal & 1320 & 09.02 .2015 \\
\hline $1601 / 2015$ & SQ2, Feature 15 & charcoal (1 bag) & 1321 & 09.02 .2015 \\
\hline $1610 / 2015$ & SQ2, Feature 15 & charcoal (1 bag) & 1321 & 10.02 .2015 \\
\hline $1640 / 2015$ & SQ2, Feature 15 & charcoal (1 bag) & 1321 & 11.02 .2015 \\
\hline $1654 / 2015$ & SQ2, Feature 15, E of Wall 44 & charcoal (1 bag) & 1321 & 12.02 .2015 \\
\hline $1652 / 2015$ & SQ2, Feature 15 & charcoal (1 bag) & 1323 & 12.02 .2015 \\
\hline
\end{tabular}




\begin{tabular}{|c|c|c|c|c|c|}
\hline $1657 / 2015$ & SQ4, wall 44 & charcoal (1 bag) & 1324 & & 12.02 .2015 \\
\hline $1476 / 2015$ & $\begin{array}{l}\text { SQ4, Feature } 49, \mathrm{SW} \text {, removal of mud brick } \\
\text { and material below }\end{array}$ & charcoal (1 bag) & / & & 09.02 .2015 \\
\hline $1105 / 2015$ & $\mathrm{SQ} 4,0-4 \mathrm{~m} \mathrm{~W}-\mathrm{E} / 6-10 \mathrm{~m} \mathrm{~N}-\mathrm{S}$ & $\begin{array}{l}\text { clay objects ( } 2 \text { fragments, function unknown, } 1 \text { Post-New } \\
\text { Kingdom); scraper ( } 1 \text { re-used Post-New Kingdom sherd, } \\
\text { pottery); charcoal (1 bag) }\end{array}$ & 205 & $\begin{array}{l}\text { SAV1E 1378, SAV1E } 1380 \text { (clay objects), } \\
\text { SAV1E } 1381 \text { (scraper) }\end{array}$ & 11.01 .2015 \\
\hline $1613 / 2015$ & SQ2, Feature 15 & $\begin{array}{l}\text { disc-shaped object/used as weight or jewellery? } \\
\text { (1 piece, clay) }\end{array}$ & 1321 & SAV1E 2231 & 10.02 .2015 \\
\hline $1627 / 2015$ & SQ2, Feature 15 & $\begin{array}{l}\text { disc-shaped object/used as weight or jewellery? } \\
\text { (1 piece, clay) }\end{array}$ & 1321 & SAV1E 2230 & 10.02 .2015 \\
\hline $1006 / 2015$ & $\mathrm{SQ} 4+4 \mathrm{~A}, 0-7.5 \mathrm{~m} \mathrm{~W}-\mathrm{E} / 7.1-12 \mathrm{~m} \mathrm{~N}-\mathrm{S}$ & doum nuts (4 pieces) & 200 & & 04.01 .2015 \\
\hline $1062 / 2015$ & $\mathrm{SQ} 4,0-4 \mathrm{~m} \mathrm{~W}-\mathrm{E} / 4.7-7.1 \mathrm{~m} \mathrm{~N}-\mathrm{S}$ & doum nut (1 piece) & 205 & & 07.01 .2015 \\
\hline $1143 / 2015$ & $\mathrm{SQ} 4 \mathrm{~A}, 1.7-3.2 \mathrm{~m} \mathrm{~W}-\mathrm{E} / 0-1.5 \mathrm{~m} \mathrm{~N}-\mathrm{S}$ & doum nut? (1 piece) & 205 & & 14.01 .2015 \\
\hline $1200 / 2015$ & SQ4, 4.2-5.9m W-E/6.5-8m N-S & doum nut (1 piece) & 209 & & 17.01.2015 \\
\hline $1252 / 2015$ & $\mathrm{SQ} 4+4 \mathrm{~A}, 5-6.5 \mathrm{~m} \mathrm{~W}-\mathrm{E} / 8.2-12 \mathrm{~m} \mathrm{~N}-\mathrm{S}$ & doum nuts (1 bag) & 213 & & 20.01 .2015 \\
\hline $1238 / 2015$ & $\mathrm{SQ} 4+4 \mathrm{~A}, 6.5-8 \mathrm{~m} \mathrm{~W}-\mathrm{E} / 8.2-12 \mathrm{~m} \mathrm{~N}-\mathrm{S}$ & doum nuts (1 bag) & 213 & & 20.01 .2015 \\
\hline $1296 / 2015$ & $\mathrm{SQ} 4,0-1.2 \mathrm{~m} \mathrm{~W}-\mathrm{E} / 0-4.5 \mathrm{~m} \mathrm{~N}-\mathrm{S}$ & doum nut (1 piece) & 220 & & 22.01 .2015 \\
\hline $1327 / 2015$ & $\mathrm{SQ} 4,2.7-4 \mathrm{~m} \mathrm{~W}-\mathrm{E} / 3.7-5 \mathrm{~m} \mathrm{~N}-\mathrm{S}$ & doum nuts (3 pieces) & 223 & & 27.01 .2015 \\
\hline $1366 / 2015$ & $\mathrm{SQ} 4+4 \mathrm{~A}, 0.4-5 \mathrm{~m} \mathrm{~W}-\mathrm{E} / 7.5-12 \mathrm{~m} \mathrm{~N}-\mathrm{S}$ & doum nuts (1 bag); wood ( 2 pieces) & 228 & & 29.01 .2015 \\
\hline $1556 / 2015$ & SQ4, Feature 15 & doum nut (1 piece) & 1314 & & 04.02 .2015 \\
\hline $1575 / 2015$ & SQ2, Feature 15 & doum nuts (1 bag) & 1316 & & 08.02 .2015 \\
\hline $1682 / 2015$ & SQ2, Feature 15, along E-wall & doum nuts (1 bag); charcoal (1 bag) & 1316 & & 09.02 .2015 \\
\hline $1581 / 2015$ & SQ2, Feature 15 & doum nuts (1 bag) & 1317 & & 08.02 .2015 \\
\hline $1591 / 2015$ & SQ2, Feature 15, SE-corner & doum nuts (1 bag) & 1317 & & 09.02 .2015 \\
\hline $1593 / 2015$ & SQ2, Feature 15, NE-corner & doum nuts (1 bag) & 1317 & & 09.02 .2015 \\
\hline $1597 / 2015$ & SQ2, Feature 15, NE-corner & doum nuts (1 bag) & 1320 & & 09.02 .2015 \\
\hline $1602 / 2015$ & SQ2, Feature 15 & doum nuts (1 bag) & 1321 & & 09.02 .2015 \\
\hline $1612 / 2015$ & SQ2, Feature 15 & doum nuts (1 bag) & 1321 & & 10.02 .2015 \\
\hline $1639 / 2015$ & SQ2, Feature 15 & doum nuts (1 bag) & 1321 & & 11.02.2015 \\
\hline
\end{tabular}




\begin{tabular}{|c|c|c|c|c|c|}
\hline $1534 / 2015$ & SQ4, N-baulk, Feature 15 & dung (1 bag) & 1309 & & 03.02 .2015 \\
\hline $1625 / 2015$ & SQ2, Feature 15, SE-corner of Feature & faience vessel (1 small fragment) & 1321 & SAV1E 2229 & 10.02 .2015 \\
\hline $1118 / 2015$ & $\mathrm{SQ} 4,0-4 \mathrm{~m} \mathrm{~W}-\mathrm{E} / 7-9 \mathrm{~m} \mathrm{~N}-\mathrm{S} / 9-10 \mathrm{~m} \mathrm{~N}-\mathrm{S}$ & $\begin{array}{l}\text { figurine ( } 1 \text { fragment, animal head, camel?, clay); } \\
\text { charcoal ( } 1 \text { bag); bones ( } 2 \text { bags) }\end{array}$ & 205 & SAV1E 2355 (figurine) & 12.01 .2015 \\
\hline $1618 / 2015$ & $\begin{array}{l}\text { SQ2, Feature 15, along E-wall } \\
\text { (NE-corner) }\end{array}$ & figurine (1 fragment, animal figurine, quadruped, pottery) & 1321 & SAV1E 2276 & 10.02.2015 \\
\hline $1608 / 2015$ & $\begin{array}{l}\text { SQ2, Feature } 15,70 \mathrm{~cm} \text { to } E \text { in SU } 1321 \text {, } \\
20 \mathrm{~cm} \text { from S-wall Feature } 15\end{array}$ & $\begin{array}{l}\text { figurine ( } 1 \text { piece, obelisk shaped with square base, decorated on } 4 \\
\text { sides with black paint, mid- } 18^{\text {th }} \text { Dynasty, clay) }\end{array}$ & 1321 & SAV1E 2226 & 10.02 .2015 \\
\hline $1234 / 2015$ & $\mathrm{SQ} 4+4 \mathrm{~A}, 6-10 \mathrm{~m} \mathrm{~W}-\mathrm{E} / 8.2-12 \mathrm{~m} \mathrm{~N}-\mathrm{S}$ & glass object (1 small fragment, function unknown) & 213 & SAV1E 1877 & 19.01 .2015 \\
\hline $1439 / 2015$ & SQ4+4A, 8-9m W-E/9.4-11.4m N-S & glass (1 fragment, original function unknown) & 239 & SAV1E 2062 & 03.02 .2015 \\
\hline $1507 / 2015$ & $\mathrm{SQ} 3,9 \mathrm{~m} \mathrm{~W}-\mathrm{E} / 0.5 \mathrm{~m} \mathrm{~N}-\mathrm{S}$ & glass (1 fragment, original function unknown) & 1302 & SAV1E 2074 & 02.02 .2015 \\
\hline $1053 / 2015$ & $\mathrm{SQ} 4,3.7-5.8 \mathrm{~m} \mathrm{~W}-\mathrm{E} / 4.2-5.1 \mathrm{~m} \mathrm{~N}-\mathrm{S}$ & glazed sherds (Islamic) & 105 & & 07.01 .2015 \\
\hline $1174 / 2015$ & $\mathrm{SQ} 4,5.3 \mathrm{~m} \mathrm{~W}-\mathrm{E} / 6 \mathrm{~m} \mathrm{~N}-\mathrm{S}$ & glazed vessel (1 fragment, Islamic glazed sherd, pottery) & 105 & SAV1E 1847 & 15.01 .2015 \\
\hline $1285 / 2015$ & SQ4, 5-8.8m N-S, section cleaning & glazed vessel (1 fragment, glazed ware) & 219 & SAV1E 2077 & 22.01 .2015 \\
\hline $1077 / 2015$ & $\mathrm{SQ} 4,0-1.5 \mathrm{~m} \mathrm{~W}-\mathrm{E} / 0-2 \mathrm{~m} \mathrm{~N}-\mathrm{S}$ & hair (1 bag) & 205 & & 08.01 .2015 \\
\hline $1466 / 2015$ & SQ4, E-W-wall, NW-part of SQ & leather (1 piece) & 223 & & 05.02 .2015 \\
\hline $1350 / 2015$ & $\mathrm{SQ} 4+4 \mathrm{~A}, 0.4-5 \mathrm{~m} \mathrm{~W}-\mathrm{E} / 7.5-12 \mathrm{~m} \mathrm{~N}-\mathrm{S}$ & leather (1 piece) & 227 & & 28.01 .2015 \\
\hline $1369 / 2015$ & $\mathrm{SQ} 4+4 \mathrm{~A}, 0.4-5 \mathrm{~m} \mathrm{~W}-\mathrm{E} / 7.5-12 \mathrm{~m} \mathrm{~N}-\mathrm{S}$ & lid (1 piece, clay) & 228 & SAV1E 2120 & 29.01 .2015 \\
\hline $1607 / 2015$ & SQ2, Feature 15, along N-wall & lid or stopper (1 piece, clay) & 1321 & SAV1E 2349 & 09.02 .2015 \\
\hline $1628 / 2015$ & SQ2, Feature 15 & lid or token/gaming piece? (1 piece, complete, unspecified stone) & 1321 & SAV1E 2227 & 10.02 .2015 \\
\hline $1301 / 2015$ & $\mathrm{SQ} 4,0-1.2 \mathrm{~m} \mathrm{~W}-\mathrm{E} / 0-4.5 \mathrm{~m} \mathrm{~N}-\mathrm{S}$ & lime & 220 & & 24.01 .2015 \\
\hline $1331 / 2015$ & $\mathrm{SQ} 4,2.4-3.3 \mathrm{~m} \mathrm{~W}-\mathrm{E} / 7.7-8.5 \mathrm{~m} \mathrm{~N}-\mathrm{S}$ & lime & 224 & & 27.01 .2015 \\
\hline $1055 / 2015$ & $\mathrm{SQ} 4,0.7 \mathrm{~m} \mathrm{~W}-\mathrm{E} / 5.6 \mathrm{~m} \mathrm{~N}-\mathrm{S}$ & metal copper alloy & 205 & & 07.01 .2015 \\
\hline $1093 / 2015$ & $\mathrm{SQ} 4,0-4 \mathrm{~m} \mathrm{~W}-\mathrm{E} / 5-12 \mathrm{~m} \mathrm{~N}-\mathrm{S}$ & $\begin{array}{l}\text { metal objects ( } 1 \text { fragment copper, } 2 \text { fragments iron, original } \\
\text { function unknown) }\end{array}$ & 205 & SAV1E 1288 & 10.01.2015 \\
\hline $1400 / 2015$ & SQ4A, 6-7.7m W-E/0-2m N-S & metal object (1 fragment, original function unknown) & 232 & SAV1E 2122 & 01.02 .2015 \\
\hline $1559 / 2015$ & SQ2, Feature 15 & metal (1 small fragment, original function unknown) & 1316 & SAV1E 2116 & 05.02 .2015 \\
\hline $1624 / 2015$ & SQ2, Feature 15 & $\begin{array}{l}\text { metal ( } 2 \text { small fragments, } 1 \text { possible nail, } 1 \text { original function } \\
\text { unknown, copper?) }\end{array}$ & 1321 & SAV1E 2228 & 10.02 .2015 \\
\hline
\end{tabular}




\begin{tabular}{|c|c|c|c|c|c|}
\hline $1236 / 2015$ & $\mathrm{SQ} 4+4 \mathrm{~A}, 6.5-7.2 \mathrm{~m} \mathrm{~W}-\mathrm{E} / 8.2-12 \mathrm{~m} \mathrm{~N}-\mathrm{S}$ & mud brick (1 piece, with semi-circular impression) & 213 & & 19.01.2015 \\
\hline $1404 / 2015$ & SQ4A, $9.6 \mathrm{~m} \mathrm{~W}-\mathrm{E} / 1.8 \mathrm{~m} \mathrm{~N}-\mathrm{S}$ & net weight (1 piece, pottery) & 234 & SAV1E 2110 & 01.02 .2015 \\
\hline $1070 / 2015$ & $\mathrm{SQ} 4,4 \mathrm{~m} \mathrm{~W}-\mathrm{E} / 8 \mathrm{~m}$ N-S & Nun bowl? (1 small fragment, faience) & 205 & SAV1E 1252 & 08.01 .2015 \\
\hline $1041 / 2015$ & $\mathrm{SQ} 4,3.7-5.8 \mathrm{~m} \mathrm{~W}-\mathrm{E} / 2.8-4.2 \mathrm{~m} \mathrm{~N}-\mathrm{S}$ & organic material (4 pieces) & 105 & & 06.01 .2015 \\
\hline $1086 / 2015$ & SQ4, 0-4m W-E/5-10m N-S & organic material (1 bag) & 205 & & 10.01.2015 \\
\hline $1123 / 2015$ & $\mathrm{SQ} 4,0-4 \mathrm{~m}$ W-E/7-9m N-S/9-10m N-S & organic material (1 piece, fur, with skin?); organic material (1 bag) & 205 & & 12.01 .2015 \\
\hline $1152 / 2015$ & $\mathrm{SQ} 4,4.5-5.2 \mathrm{~m} \mathrm{~W}-\mathrm{E} / 7.6-8.2 \mathrm{~m} \mathrm{~N}-\mathrm{S}$ & organic material (animal?, 1 bag) & 205 & & 14.01 .2015 \\
\hline $1134 / 2015$ & SQ4A, 0-3m W-E/0-2m N-S & organic material (1 bag) & 205 & & 13.01 .2015 \\
\hline $1194 / 2015$ & $\mathrm{SQ} 4,5-5.7 \mathrm{~m} \mathrm{~W}-\mathrm{E} / 7-8.5 \mathrm{~m} \mathrm{~N}-\mathrm{S}$ & organic material (1 bag, unclear) & 208 & & 17.01.2015 \\
\hline $1213 / 2015$ & $\mathrm{SQ} 4,7-9.2 \mathrm{~m} \mathrm{~W}-\mathrm{E} / 6.8-8 \mathrm{~m} \mathrm{~N}-\mathrm{S}$ & organic material (1 bag) & 212 & & 18.01 .2015 \\
\hline $1222 / 2015$ & $\mathrm{SQ} 4,7-9.2 \mathrm{~m} \mathrm{~W}-\mathrm{E} / 6.8-8 \mathrm{~m} \mathrm{~N}-\mathrm{S}$ & organic material (1 bag) & 212 & & 19.01 .2015 \\
\hline $1229 / 2015$ & $\mathrm{SQ} 4+4 \mathrm{~A}, 6-10 \mathrm{~m} \mathrm{~W}-\mathrm{E} / 8.2-12 \mathrm{~m} \mathrm{~N}-\mathrm{S}$ & organic material (1 bag, including soil sample) & 213 & & 19.01.2015 \\
\hline $1259 / 2015$ & $\mathrm{SQ} 4+4 \mathrm{~A}, 3.5-5.5 \mathrm{~m} \mathrm{~W}-\mathrm{E} / 8.3-11.3 \mathrm{~m} \mathrm{~N}-\mathrm{S}$ & organic material (1 bag) & 214 & & 21.01 .2015 \\
\hline $1311 / 2015$ & $\mathrm{SQ} 4,0-0.8 \mathrm{~m} \mathrm{~W}-\mathrm{E} / 4.7-5.1 \mathrm{~m} \mathrm{~N}-\mathrm{S}$ & organic material (1 bag, unclear) & 221 & & 24.01 .2015 \\
\hline $1317 / 2015$ & $\mathrm{SQ} 4,0-0.8 \mathrm{~m} \mathrm{~W}-\mathrm{E} / 4.4-5.1 \mathrm{~m} \mathrm{~N}-\mathrm{S}$ & organic material (1 piece) & 222 & & 24.01 .2015 \\
\hline $1338 / 2015$ & $\mathrm{SQ} 4,0.4-3.3 \mathrm{~m} \mathrm{~W}-\mathrm{E} / 6-7.5 \mathrm{~m} \mathrm{~N}-\mathrm{S}$ & organic material (1 bag) & 226 & & 27.01 .2015 \\
\hline $1376 / 2015$ & SQ4A, 3.5-5.1m W-E/0-2m N-S & organic material (1 bag) & 229 & & 31.01 .2015 \\
\hline $1399 / 2015$ & SQ4A, 6-7.7m W-E/0-2m N-S & organic material (1 bag) & 232 & & 01.02 .2015 \\
\hline $1411 / 2015$ & $\mathrm{SQ} 4+4 \mathrm{~A}, 9.2-10.5 \mathrm{~m} \mathrm{~W}-\mathrm{E} / 9-12 \mathrm{~m} \mathrm{~N}-\mathrm{S}$ & organic material (2 pieces) & 235 & & 02.02 .2015 \\
\hline $1421 / 2015$ & $\mathrm{SQ} 4+4 \mathrm{~A}, 8.8-10.5 \mathrm{~m} \mathrm{~W}-\mathrm{E} / 9.3-12 \mathrm{~m} \mathrm{~N}-\mathrm{S}$ & organic material (1 bag) & 236 & & 02.02 .2015 \\
\hline $1428 / 2015$ & $\mathrm{SQ} 4 \mathrm{~A}+2 \mathrm{~A}, 8.8-11 \mathrm{~m} \mathrm{~W}-\mathrm{E} / 1-2 \mathrm{~m} \mathrm{~N}-\mathrm{S}$ & organic material (1 bag) & 238 & & 03.02 .2015 \\
\hline $1441 / 2015$ & $\mathrm{SQ} 4+4 \mathrm{~A}, 8-9 \mathrm{~m} \mathrm{~W}-\mathrm{E} / 9.4-11.4 \mathrm{~m} \mathrm{~N}-\mathrm{S}$ & organic material (1 bag) & 239 & & 03.02 .2015 \\
\hline $1447 / 2015$ & SQ4A, 5.4-7m W-E/1-2m N-S & organic material (1 bag) & 241 & & 04.02 .2015 \\
\hline $1523 / 2015$ & SQ4-3, baulk & organic material (1 bag) & 1307 & & 03.02 .2015 \\
\hline $1562 / 2015$ & SQ2, Feature 15 & \multirow{2}{*}{$\begin{array}{l}\text { organic material (1 bag) } \\
\text { organic material (1 bag) }\end{array}$} & 1316 & & 05.02 .2015 \\
\hline $1649 / 2015$ & SQ2, E of wall 44 in Feature 15 & & 1318 & & 11.02 .2015 \\
\hline
\end{tabular}




\begin{tabular}{|c|c|c|c|c|c|}
\hline $1660 / 2015$ & SQ4, wall 44 & organic material (1 bag) & 1324 & & 12.02 .2015 \\
\hline $1661 / 2015$ & SQ4, wall 44 & organic material (1 bag) & 1324 & & 12.02 .2015 \\
\hline $1474 / 2015$ & $\begin{array}{l}\text { SQ4, Feature 49, NE cleaning small debris } \\
\text { area, corner Feature } 49 / \text { Feature } 36\end{array}$ & organic material $(1 \mathrm{bag})$ & / & & 09.02 .2015 \\
\hline $1192 / 2015$ & $\mathrm{SQ} 4,5-5.7 \mathrm{~m} \mathrm{~W}-\mathrm{E} / 7-8.5 \mathrm{~m} \mathrm{~N}-\mathrm{S}$ & $\begin{array}{l}\text { pipe ( } 1 \text { fragment, clay, Ottoman); } \\
\text { bone ( } 1 \text { bag) }\end{array}$ & 208 & SAV1E 2661 (pipe) & 17.01 .2015 \\
\hline $1035 / 2015$ & $\mathrm{SQ} 4,3.7-5.8 \mathrm{~m} \mathrm{~W}-\mathrm{E} / 2.8-4.2 \mathrm{~m} \mathrm{~N}-\mathrm{S}$ & pottery & 105 & & 06.01 .2015 \\
\hline $1045 / 2015$ & $\mathrm{SQ} 4,3.7-5.8 \mathrm{~m} \mathrm{~W}-\mathrm{E} / 4.2-5.1 \mathrm{~m} \mathrm{~N}-\mathrm{S}$ & pottery & 105 & & 07.01 .2015 \\
\hline $1185 / 2015$ & $\mathrm{SQ} 4,4-5.7 \mathrm{~m} \mathrm{~W}-\mathrm{E} / 4.5-6.5 \mathrm{~m} \mathrm{~N}-\mathrm{S}$ & pottery & 105 & & 17.01 .2015 \\
\hline $1173 / 2015$ & $\mathrm{SQ} 4,4-5.7 \mathrm{~m} \mathrm{~W}-\mathrm{E} / 4.5-6.5 \mathrm{~m} \mathrm{~N}-\mathrm{S}$ & $\begin{array}{l}\text { pottery, among it unidentified object (1 re-used Post-New } \\
\text { Kingdom pot sherd, pottery) }\end{array}$ & 105 & SAV1E 1866 & 15.01 .2015 \\
\hline $1032 / 2015$ & $\begin{array}{l}\text { SQ4, 4-5m W-E/3.5m N-S, collapse of SU } \\
105\end{array}$ & pottery & $\begin{array}{l}105, \\
\text { collapse of } \\
\text { SU }\end{array}$ & & 06.01 .2015 \\
\hline $1000 / 2015$ & $\mathrm{SQ} 4+4 \mathrm{~A}, 0-7.5 \mathrm{~m} \mathrm{~W}-\mathrm{E} / 7.1-12 \mathrm{~m} \mathrm{~N}-\mathrm{S}$ & $\begin{array}{l}\text { pottery and stone: pottery ( } 5 \text { baskets), among them weight? } \\
\text { ( } 1 \text { re-used sherd of a Canaanite amphora), re-used sherd ( } 1 \text { re- } \\
\text { used pottery fragment, secondary use unclear), window grille? } \\
\text { ( } 2 \text { fragments of pottery figurines, possibly from a window grille, } \\
\text { Christian); overfired ceramic ( } 1 \text { piece) }\end{array}$ & 200 & $\begin{array}{l}\text { SAV1E } 1138 \text { (weight?), SAV1E } 1139 \text { (re- } \\
\text { used sherd), SAV1E } 1140 \text { (window grille?), } \\
\text { SAV1E } 1137 \text { (overfired ceramic) }\end{array}$ & 04.01 .2015 \\
\hline $1008 / 2015$ & $\mathrm{SQ} 4+4 \mathrm{~A}, 2-7.5 \mathrm{~m} \mathrm{~W}-\mathrm{E} / 9-12 \mathrm{~m} \mathrm{~N}-\mathrm{S}$ & pottery (1 basket) & 201 & & 04.01 .2015 \\
\hline $1009 / 2015$ & $\mathrm{SQ} 4,0-7.5 \mathrm{~m} \mathrm{~W}-\mathrm{E} / 7.1-10 \mathrm{~m} \mathrm{~N}-\mathrm{S}$ & $\begin{array}{l}\text { pottery and slag: pottery ( } 5 \text { baskets), among it figurine ( } 1 \text { small } \\
\text { fragment of a pottery figurine, possibly a leg); slag ( } 3 \text { fragments) }\end{array}$ & 202 & SAV1E 2355 (figurine), SAV1E 1141 (slag) & 04.01 .2015 \\
\hline $1016 / 2015$ & $\begin{array}{l}\text { SQ4, 0-7.5m W-E/7.1-10m N-S } \\
(12 \mathrm{~m} \mathrm{~N}-\mathrm{S})\end{array}$ & $\begin{array}{l}\text { pottery, slag and brick, bones and stone: slag ( } 4 \text { pieces); bones ( } 4 \\
\text { pieces); grindstone ( } 1 \text { small fragment) }\end{array}$ & 202 & SAV1E 1171 (slag and brick) & 05.01 .2015 \\
\hline $1022 / 2015$ & $\mathrm{SQ} 4,5-8 \mathrm{~m} \mathrm{~N}-\mathrm{S}$, surface & pottery & 203 & & 05.01 .2015 \\
\hline $1026 / 2015$ & $\mathrm{SQ} 4,3.5-6.5 \mathrm{~m} \mathrm{~W}-\mathrm{E} / 5-6.2 \mathrm{~m} \mathrm{~N}-\mathrm{S}$ & $\begin{array}{l}\text { pottery, among it scraper (1 re-used sherd, Post-New Kingdom, } \\
\text { pottery) }\end{array}$ & 204 & SAV1E 1180 & 06.01 .2015 \\
\hline $1080 / 2015$ & $\mathrm{SQ} 4,0-4 \mathrm{~m} \mathrm{~W}-\mathrm{E} / 5-10 \mathrm{~m} \mathrm{~N}-\mathrm{S}$ & $\begin{array}{l}\text { pottery, slag and stone: pottery ( } 5 \text { baskets), among them } \\
\text { token/gaming pieces ( } 2 \text { fragments, } 1 \text { pottery, late Christian, } \\
1 \text { unspecified stone), oil lamp ( } 1 \text { fragment, pottery, Christian), } \\
\text { scraper ( } 2 \text { pieces, } 1 \text { re-used Post-New Kingdom sherd, pottery, } \\
1 \text { re-used } 18^{\text {th }} \text { Dynasty pot sherd, pottery); } \\
\text { slag ( } 6 \text { fragments); overfired ceramic ( } 1 \text { small fragment) }\end{array}$ & 205 & $\begin{array}{l}\text { SAV1E 1301, SAV1E } 1305 \text { (token/gaming } \\
\text { pieces), SAV1E 1302 (oil lamp), } \\
\text { SAV1E 1303, SAV1E } 1304 \text { (scrapers), } \\
\text { SAV1E 1290 (slag), SAV1E } 1289 \text { (overfired } \\
\text { ceramic) }\end{array}$ & 10.01 .2015 \\
\hline $1101 / 2015$ & $\mathrm{SQ} 4,0-4 \mathrm{~m} \mathrm{~W}-\mathrm{E} / 6-10 \mathrm{~m} \mathrm{~N}-\mathrm{S}$ & $\begin{array}{l}\text { pottery, slag and brick: pottery ( } 7 \text { baskets), among them clay } \\
\text { object ( } 1 \text { fragment, function unknown), window frame ( } 2 \text { pieces, } \\
1 \text { fragment, pottery, Christian, } 1 \text { fragment, clay, Christian), lid or }\end{array}$ & 205 & $\begin{array}{l}\text { SAV1E } 1376 \text { (clay object), SAV1E 1377, } \\
\text { SAV1E } 1388 \text { (window frame?), SAV1E } 1385\end{array}$ & 11.01 .2015 \\
\hline
\end{tabular}




\begin{tabular}{|c|c|c|c|c|c|}
\hline & & $\begin{array}{l}\text { token ( } 1 \text { re-used sherd, pottery), figurine/ application } \\
\text { ( } 1 \text { fragment, pottery), mould?/unidentified object ( } 1 \text { fragment, } \\
\text { pottery), scrapers ( } 2 \text { pieces, each re-used sherds, pottery), stand } \\
\text { ( } 1 \text { fragment, pottery); slag and brick ( } 5 \text { fragments) }\end{array}$ & & $\begin{array}{l}\text { (lid or token), SAV1E } 1386 \text { (figurine), } \\
\text { SAV1E } 1387 \text { (mould?), } \\
\text { SAV1E } 1389 \text {, SAV1E } 1390 \text { (scrapers), } \\
\text { SAV1E } 1391 \text { (pottery stand), } \\
\text { SAV1E } 1365 \text { (slag) }\end{array}$ & \\
\hline $1065 / 2015$ & $\mathrm{SQ} 4,0-4 \mathrm{~m} \mathrm{~W}-\mathrm{E} / 7.1-9.7 \mathrm{~m} \mathrm{~N}-\mathrm{S}$ & $\begin{array}{l}\text { pottery ( } 5 \text { baskets), among them window grille ( } 2 \text { fragments, } \\
\text { each clay), scraper? ( } 1 \text { fragment of re-used pot sherd, pottery), } \\
\text { unidentified object ( } 1 \text { fragment, clay) }\end{array}$ & 205 & $\begin{array}{l}\text { SAV1E 1263, SAV1E } 1265 \text { (window } \\
\text { frames?), SAV1E } 1264 \text { (scraper?), } \\
\text { SAV1E } 1266 \text { (unidentified object) }\end{array}$ & 08.01 .2015 \\
\hline $1114 / 2015$ & SQ4, 0-4m W-E/7-9m N-S/9-10m N-S & $\begin{array}{l}\text { pottery, slag, stone architecture: pottery ( } 7 \text { baskets), among them } \\
\text { window grilles ( } 3 \text { fragments, each clay, Christian), lid (1 re-used } \\
\text { sherd, Christian), lid or stopper ( } 1 \text { fragment, clay), scraper (1 re- } \\
\text { used Post-New Kingdom pot sherd, pottery), clay object (1 frag- } \\
\text { ment, original function unknown); slag and brick ( } 6 \text { fragments); } \\
\text { unidentified stone object (1 fragment, unspecified stone), } \\
\text { whetstone (1 fragment, sandstone); architecture (1 fragment, } \\
\text { sandstone) }\end{array}$ & 205 & $\begin{array}{l}\text { SAV1E 1412, SAV1E 1413, SAV1E } 1414 \\
\text { (window grilles), SAV1E 1416 (lid), SAV1E } \\
1417 \text { (lid or stopper), SAV1E } 1419 \text { (scraper), } \\
\text { SAV1E } 1421 \text { (clay object), SAV1E } 1392 \\
\text { (slag and brick), SAV1E 1383 (unidentified } \\
\text { object), SAV1E 1384 (whetstone), SAV1E } \\
1382 \text { (architecture) }\end{array}$ & 12.01.2015 \\
\hline $1094 / 2015$ & SQ4, 2-2.8m W-E/6-7m N-S & pottery (1 basket) & 205 & & 11.01 .2015 \\
\hline $1096 / 2015$ & $\mathrm{SQ} 4,3.1-4 \mathrm{~m} \mathrm{~W}-\mathrm{E} / 6-7.5 \mathrm{~m} \mathrm{~N}-\mathrm{S}$ & pottery & 205 & & 11.01 .2015 \\
\hline $1161 / 2015$ & $\mathrm{SQ} 4,3.5-5 \mathrm{~m} \mathrm{~W}-\mathrm{E} / 9-10 \mathrm{~m} \mathrm{~N}-\mathrm{S}$ & $\begin{array}{l}\text { pottery ( } 2 \text { baskets), among them scraper ( } 1 \text { re-used sherd, } \\
\text { pottery), lid or stopper ( } 1 \text { fragment, clay) }\end{array}$ & 205 & $\begin{array}{l}\text { SAV1E } 1813 \text { (scraper), SAV1E } 1814 \text { (lid or } \\
\text { stopper) }\end{array}$ & 15.01.2015 \\
\hline $1160 / 2015$ & $\mathrm{SQ} 4,3.5-5 \mathrm{~m} \mathrm{~W}-\mathrm{E} / 9-10 \mathrm{~m} \mathrm{~N}-\mathrm{S}$ & $\begin{array}{l}\text { pottery ( } 2 \text { baskets), among them token/gaming piece? (1 re-used } \\
\text { Christian sherd, pottery) }\end{array}$ & 205 & SAV1E 1816 & 14.01.2015 \\
\hline $1149 / 2015$ & $\mathrm{SQ} 4,4.5-5.2 \mathrm{~m} \mathrm{~W}-\mathrm{E} / 7.6-8.2 \mathrm{~m} \mathrm{~N}-\mathrm{S}$ & $\begin{array}{l}\text { pottery ( } 1 \text { basket), among it window grille ( } 1 \text { fragment, pottery, } \\
\text { Christian) }\end{array}$ & 205 & SAV1E 1818 & 14.01.2015 \\
\hline $1091 / 2015$ & SQ4A, 0-1.5m W-E/0-2m N-S & $\begin{array}{l}\text { pottery and slag: pottery ( } 1 \text { basket), among it clay object } \\
\text { (1 fragment, original function unknown); slag ( } 3 \text { fragments) }\end{array}$ & 205 & SAV1E 1321 (clay object), SAV1E 1308 (slag) & 10.01.2015 \\
\hline $1125 / 2015$ & SQ4A, 0-3m W-E/0-2m N-S & $\begin{array}{l}\text { pottery, slag and stones: pottery ( } 23 \text { baskets), among them } \\
\text { window grilles ( } 2 \text { fragments, each clay, Christian), lid ( } 1 \text { re-used } \\
\text { sherd, pottery), clay object ( } 1 \text { fragment, original function } \\
\text { unknown), seal impression? (1 fragment, clay), scrapers } \\
\text { ( } 4 \text { pieces, } 3 \text { re-used Post-New Kingdom sherds, } 1 \text { re-used sherd, } \\
\text { Christian, pottery), re-used sherd ( } 1 \text { fragment, use unknown, } \\
\text { Christian, pottery), lid ( } 1 \text { piece, clay), stela ( } 2 \text { fragments, } \\
\text { Christian, pottery), lid ( } 1 \text { re-used } 18^{\text {th }} \text { Dynasty spinning bowl), } \\
\text { pottery ( } 1 \text { piece, overfired); slag ( } 12 \text { fragments); hand mill } \\
\text { (1 fragment, quartzite), whetstone ( } 1 \text { fragment, sandstone) }\end{array}$ & 205 & $\begin{array}{l}\text { SAV1E 1415, SAV1E } 1451 \text { (window grilles), } \\
\text { SAV1E 1418 (lid), SAV1E } 1425 \text { (clay } \\
\text { object), SAV1E 1449 (seal impression?), } \\
\text { SAV1E 1452, SAV1E 1454, SAV1E 1452, } \\
\text { SAV1E 1455, SAV1E 1458 (scrapers), } \\
\text { SAV1E 1456 (re-used sherd), SAV1E } 1459 \\
\text { (lid), SAV1E 1460 (stela), SAV1E 2629 (lid), } \\
\text { SAV1E 1423 (slag), SAV1E 1427 (hand } \\
\text { mill), SAV1E 1428 (whetstone) }\end{array}$ & 13.01.2015 \\
\hline $1137 / 2015$ & SQ4A, $1.7-3.2 \mathrm{~m} \mathrm{~W}-\mathrm{E} / 0-1.5 \mathrm{~m} \mathrm{~N}-\mathrm{S}$ & $\begin{array}{l}\text { pottery, slag and stone: pottery ( } 8 \text { baskets), among them weight? } \\
\text { ( } 1 \text { re-used sherd, Christian, pottery), scrapers ( } 4 \text { pieces, } 2 \text { re-used } \\
\text { sherds, Christian, } 2 \text { re-used } 18^{\text {th }} \text { Dynasty sherds, pottery), } \\
\text { unidentified object ( } 1 \text { fragment, pottery), window grilles ( } 5\end{array}$ & 205 & $\begin{array}{l}\text { SAV1E } 1462 \text { (weight?), SAV1E 1463, } \\
\text { SAV1E 1464, SAV1E 1465, SAV1E } 1466 \\
\text { (scrapers), SAV1E 1467 (unidentified object), } \\
\text { SAV1E 1468, SAV1E 1469, SAV1E 1470, }\end{array}$ & 14.01.2015 \\
\hline
\end{tabular}




\begin{tabular}{|c|c|c|c|c|c|}
\hline & & $\begin{array}{l}\text { fragments, each pottery, Christian), seal impression ( } 1 \text { fragment, } \\
\text { clay); slag ( } 4 \text { fragments), whetstone (1 piece, sandstone), hand } \\
\text { mill (1 piece, quartzite) }\end{array}$ & & $\begin{array}{l}\text { SAV1E 1471, SAV1E } 1472 \text { (window grilles), } \\
\text { SAV1E 1483 (seal impression?), SAV1E } \\
1482 \text { (slag), SAV1E } 1447 \text { (whetstone), } \\
\text { SAV1E } 1448 \text { (hand mill) }\end{array}$ & \\
\hline $1167 / 2015$ & SQ4A, 4-5m W-E/1.4-2m N-S & $\begin{array}{l}\text { pottery, among it scrapers ( } 2 \text { pieces, } 1 \text { re-used Post-New } \\
\text { Kingdom pot sherd, } 1 \text { re-used fragment of a Canaanite amphora, } \\
18^{\text {th }} \text { Dynasty, pottery) }\end{array}$ & 205 & SAV1E 1819, SAV1E 1820 (scrapers) & 15.01 .2015 \\
\hline $1189 / 2015$ & $\mathrm{SQ} 4,5-5.9 \mathrm{~m} \mathrm{~W}-\mathrm{E} / 4.2-5.8 \mathrm{~m} \mathrm{~N}-\mathrm{S}$ & $\begin{array}{l}\text { pottery and stone: scraper ( } 1 \text { re-used } 18^{\text {th }} \text { Dynasty pot sherd, } \\
\text { pottery), net weight ( } 1 \text { fragment, pottery); hand mill ( } 1 \text { fragment, } \\
\text { quartzite) }\end{array}$ & 207 & $\begin{array}{l}\text { SAV1E } 1874 \text { (scraper), SAV1E } 1873 \\
\text { (weight), SAV1E } 1821 \text { (hand mill) }\end{array}$ & 17.01.2015 \\
\hline $1190 / 2015$ & $\mathrm{SQ} 4,5-5.7 \mathrm{~m} \mathrm{~W}-\mathrm{E} / 7-8.5 \mathrm{~m} \mathrm{~N}-\mathrm{S}$ & $\begin{array}{l}\text { pottery, among it scraper (1 re-used Post-New Kingdom pot } \\
\text { sherd, pottery) }\end{array}$ & 208 & SAV1E 1915 & 17.01.2015 \\
\hline $1470 / 2015$ & SQ4, N-S trench & pottery & 209 & & 05.02 .2015 \\
\hline $1201 / 2015$ & $\mathrm{SQ} 4+4 \mathrm{~A}, 6-7.5 \mathrm{~m} \mathrm{~W}-\mathrm{E} / 8.5-12 \mathrm{~m} \mathrm{~N}-\mathrm{S}$ & pottery, among it window grille? (1 fragment, Christian, pottery) & 210 & SAV1E 2367 & 17.01.2015 \\
\hline $1207 / 2015$ & SQ4A, 3.7-7m W-E/0-2m N-S & $\begin{array}{l}\text { pottery, among it window grilles ( } 2 \text { pieces, each fragments, } \\
\text { Christian, pottery) }\end{array}$ & 211 & SAV1E 2368, SAV1E 2369 & 17.01 .2015 \\
\hline $1219 / 2015$ & $\mathrm{SQ} 4,4.7-9.2 \mathrm{~m} \mathrm{~W}-\mathrm{E} / 6.8-8 \mathrm{~m} \mathrm{~N}-\mathrm{S}$ & $\begin{array}{l}\text { pottery, among it slag and brick ( } 2 \text { fragments), window grille } \\
\text { (1 fragment, Christian, pottery) }\end{array}$ & 212 & SAV1E 1872, SAV1E 1902 & 19.01.2015 \\
\hline $1210 / 2015$ & SQ4, 7-9.2m W-E/6.8-8m N-S & $\begin{array}{l}\text { pottery ( } 6 \text { baskets), among them disc-shaped object ( } 1 \text { frag-ment, } \\
\text { Christian, pottery), scrapers ( } 2 \text { pieces, each re-used Post-New } \\
\text { Kingdom pot sherds, pottery), window grille ( } 1 \text { fragment, } \\
\text { Christian, pottery), unidentified object ( } 1 \text { re-used sherd, use } \\
\text { unknown) }\end{array}$ & 212 & $\begin{array}{l}\text { SAV1E } 1898 \text { (disc shaped object), SAV1E } \\
\text { 1899, SAV1E } 1900 \text { (scrapers), SAV1E } 1901 \\
\text { (window grille), SAV1E } 2626 \text { (unidentified } \\
\text { object) }\end{array}$ & 18.01.2015 \\
\hline $1245 / 2015$ & E of SU 213, surface cleaning & pottery & 213 & & 20.01.2015 \\
\hline $1246 / 2015$ & $\begin{array}{l}\mathrm{SQ} 4+4 \mathrm{~A}, 5-6.5 \mathrm{~m} \mathrm{~W}-\mathrm{E} / 8.2-12 \mathrm{~m} \mathrm{~N}-\mathrm{S} \text {, } \\
\text { surface cleaning }\end{array}$ & pottery & 213 & & 20.01 .2015 \\
\hline $1247 / 2015$ & $\mathrm{SQ} 4+4 \mathrm{~A}, 5-6.5 \mathrm{~m} \mathrm{~W}-\mathrm{E} / 8.2-12 \mathrm{~m} \mathrm{~N}-\mathrm{S}$ & $\begin{array}{l}\text { pottery, faience and slag: pottery ( } 6 \text { baskets), among them } \\
\text { stopper/sealing? ( } 1 \text { fragment, mud), window grille ( } 1 \text { frag-ment, } \\
\text { Christian, pottery); faience vessel (1 fragment); slag and brick } \\
\text { ( } 2 \text { fragments) }\end{array}$ & 213 & $\begin{array}{l}\text { SAV1E } 1912 \text { (stopper/sealing?, SAV1E } 2152 \\
\text { (window grille), SAV1E } 1910 \text { (faience } \\
\text { vessel), SAV1E } 1911 \text { (slag and brick) }\end{array}$ & 20.01.2015 \\
\hline $1235 / 2015$ & $\mathrm{SQ} 4+4 \mathrm{~A}, 6.5-7.2 \mathrm{~m} \mathrm{~W}-\mathrm{E} / 8.2-12 \mathrm{~m} \mathrm{~N}-\mathrm{S}$ & $\begin{array}{l}\text { pottery, among it weight? (1 fragment of a re-used late Christian } \\
\text { dish, pottery), window grille (1 fragment, Christian, pottery) }\end{array}$ & 213 & $\begin{array}{l}\text { SAV1E } 1970 \text { (weight), SAV1E } 2371 \\
\text { (window grille) }\end{array}$ & 19.01.2015 \\
\hline $1243 / 2015$ & $\mathrm{SQ} 4+4 \mathrm{~A}, 6.5-8 \mathrm{~m} \mathrm{~W}-\mathrm{E} / 8.2-12 \mathrm{~m} \mathrm{~N}-\mathrm{S}$ & pottery, among it oil lamps ( 2 fragments, each pottery, Christian) & 213 & SAV1E 1943, SAV1E 2577 (oil lamps) & 20.01.2015 \\
\hline $1225 / 2015$ & $\mathrm{SQ} 4+4 \mathrm{~A}, 6-10 \mathrm{~m} \mathrm{~W}-\mathrm{E} / 8.2-12 \mathrm{~m} \mathrm{~N}-\mathrm{S}$ & $\begin{array}{l}\text { pottery, among it scrapers ( } 2 \text { pieces, each re-used } 18^{\text {th }} \text { Dynasty } \\
\text { sherds, pottery), lid or scraper ( } 1 \text { re-used sherd, Christian, } \\
\text { pottery), window grille ( } 1 \text { fragment, Christian, pottery) }\end{array}$ & 213 & $\begin{array}{l}\text { SAV1E 1930, SAV1E } 1931 \text { (scrapers), } \\
\text { SAV1E } 1933 \text { (lid or scraper), SAV1E } 2370 \\
\text { (window grille) }\end{array}$ & 19.01.2015 \\
\hline $1471 / 2015$ & SQ4, S & pottery & 214 & & 05.02 .2015 \\
\hline
\end{tabular}




\begin{tabular}{|c|c|c|c|c|c|}
\hline $1255 / 2015$ & $\begin{array}{l}\mathrm{SQ} 4+4 \mathrm{~A}, \\
3.5-5.5 \mathrm{~m} \mathrm{~W}-\mathrm{E} / 8.3-11.3 \mathrm{~m} \mathrm{~N}-\mathrm{S}\end{array}$ & $\begin{array}{l}\text { pottery, stones and ochre: pottery ( } 15 \text { baskets), among them } \\
\text { scrapers ( } 5 \text { pieces, each re-used sherds, pottery), } \\
\text { lids ( } 4 \text { pieces, each re-used sherds, pottery), } \\
\text { window grille ( } 4 \text { fragments, each Christian, pottery), } \\
\text { stopper/sealings ( } 2 \text { fragments, each clay), discs ( } 2 \text { pieces, each } \\
\text { pottery), weight? ( } 1 \text { re-used } 18^{\text {th }} \text { Dynasty sherd, pottery), } \\
\text { unknown object ( } 1 \text { re-used } 18^{\text {th }} \text { Dynasty sherd, pottery), figurine } \\
\text { ( } 1 \text { fragment, animal figurine, pottery), } \\
\text { token/gaming piece ( } 1 \text { piece, pottery), } \\
\text { overfired ceramic ( } 1 \text { piece); } \\
\text { grindstone or hand mill }(1 \text { piece, quartzite), pounder (1 piece, } \\
\text { quartz), worked stone (1 piece, limestone), token/gaming piece } \\
\text { (1 small piece, worked stone, unspecified stone); } \\
\text { ochre ( } 1 \text { piece, yellow) }\end{array}$ & 214 & $\begin{array}{l}\text { SAV1E 1797, SAV1E 1798, SAV1E 2631, } \\
\text { SAV1E 1941, SAV1E 1942 (scrapers), SAV1E } \\
\text { 1968, SAV1E 1794. SAV1E 1795, SAV1E } \\
\text { 2630 (lids), SAV1E 1966, SAV1E 1967, } \\
\text { SAV1E 1771, SAV1E 1772 (window grilles), } \\
\text { SAV1E 2001, SAV1E } 1923 \text { (stopper/sealings), } \\
\text { SAV1E 1799, SAV1E } 2000 \text { (discs), SAV1E } \\
\text { 1965 (weight?), SAV1E 1940 (unknown object), } \\
\text { SAV1E 1939 (figurine), SAV1E 1793 } \\
\text { (token/gaming piece), SAV1E } 1924 \text { (overfired } \\
\text { ceramic), SAV1E 1928 (grindstone or hand } \\
\text { mill), SAV1E 1927 (pounder), SAV1E 1748 } \\
\text { (worked stone), SAV1E 1925 (token/gaming } \\
\text { piece) }\end{array}$ & 21.01 .2015 \\
\hline $1265 / 2015$ & SQ4, 0-1m W-E/8.8-9.7m N-S & $\begin{array}{l}\text { pottery ( } 2 \text { baskets), among it figurine ( } 1 \text { fragment, animal } \\
\text { figurine, pottery) }\end{array}$ & 215 & SAV1E 2016 & 22.01 .2015 \\
\hline $1275 / 2015$ & SQ4A, 0-1m W-E/0-2m N-S & $\begin{array}{l}\text { pottery ( } 1 \text { basket), among it scraper ( } 1 \text { re-used } 18^{\text {th }} \text { Dynasty dish } \\
\text { sherd, pottery) }\end{array}$ & 216 & SAV1E 2632 & 22.01 .2015 \\
\hline $1271 / 2015$ & SQ4A, 0-1m W-E/0-2m N-S & $\begin{array}{l}\text { pottery ( } 1 \text { basket), among it scraper ( } 1 \text { re-used Post-New } \\
\text { Kingdom sherd, pottery) }\end{array}$ & 216 & SAV1E 2118 & 22.01 .2015 \\
\hline $1277 / 2015$ & SQ4A, 0-1m W-E/0-2m N-S & pottery (1 basket) & 218 & & 22.01 .2015 \\
\hline $1284 / 2015$ & SQ4, 5-8.8m N-S, section cleaning & $\begin{array}{l}\text { pottery ( } 31 / 2 \text { baskets), among them scraper ( } 1 \text { re-used Post-New } \\
\text { Kingdom sherd, pottery) }\end{array}$ & 219 & SAV1E 2633 & 22.01.2015 \\
\hline $1469 / 2015$ & SQ4, NW-corner & pottery & 220 & & 05.02 .2015 \\
\hline $1299 / 2015$ & $\mathrm{SQ} 4,0-1.2 \mathrm{~m} \mathrm{~W}-\mathrm{E} / 0-4.5 \mathrm{~m} \mathrm{~N}-\mathrm{S}$ & pottery (6 baskets) & 220 & & 24.01.2015 \\
\hline $1308 / 2015$ & $\mathrm{SQ} 4,0-0.8 \mathrm{~m} \mathrm{~W}-\mathrm{E} / 4.7-5.1 \mathrm{~m} \mathrm{~N}-\mathrm{S}$ & pottery (1 basket) & 221 & & 24.01 .2015 \\
\hline $1313 / 2015$ & $\mathrm{SQ} 4,0-0.8 \mathrm{~m} \mathrm{~W}-\mathrm{E} / 4.4-5.1 \mathrm{~m} \mathrm{~N}-\mathrm{S}$ & pottery ( 2 baskets) & 222 & & 24.01 .2015 \\
\hline $1465 / 2015$ & SQ4, E-W-wall, NW-part & pottery & 223 & & 05.02 .2015 \\
\hline $1320 / 2015$ & $\mathrm{SQ} 4,1-2.7 \mathrm{~m} \mathrm{~W}-\mathrm{E} / 3.7-5 \mathrm{~m} \mathrm{~N}-\mathrm{S}$ & $\begin{array}{l}\text { pottery ( } 1 \text { basket), among it figurine ( } 1 \text { fragment, head of animal, } \\
\text { bull?, pottery), token/gaming piece ( } 1 \text { re-used Post-New } \\
\text { Kingdom sherd, pottery), scrapers ( } 2 \text { pieces, each re-used Post- } \\
\text { New Kingdom sherds, pottery), oil lamp (1 frag-ment, Christian, } \\
\text { pottery) }\end{array}$ & 223 & $\begin{array}{l}\text { SAV1E } 2002 \text { (figurine), SAV1E } 2013 \\
\text { (token/gaming piece), SAV1E 2014, SAV1E } \\
2015 \text { (scrapers), SAV1E } 2575 \text { (oil lamp) }\end{array}$ & 24.01 .2015 \\
\hline $1325 / 2015$ & $\mathrm{SQ} 4,2.7-4 \mathrm{~m} \mathrm{~W}-\mathrm{E} / 3.7-5 \mathrm{~m} \mathrm{~N}-\mathrm{S}$ & $\begin{array}{l}\text { pottery ( } 1 \text { basket), among it lid (1 re-used Post New Kingdom } \\
\text { sherd, pottery) }\end{array}$ & 223 & SAV1E 2125 & 27.01.2015 \\
\hline $1329 / 2015$ & SQ4, 2.4-3.3m W-E/7.7-8.5m N-S & pottery & 224 & & 27.01.2015 \\
\hline $1332 / 2015$ & $\mathrm{SQ} 4,1-1.5 \mathrm{~m} \mathrm{~W}-\mathrm{E} / 6.8-7.1 \mathrm{~m} \mathrm{~N}-\mathrm{S}$ & pottery, among it stopper/sealing (1 fragment, mud) & 225 & $1332 / 2015$ & 27.01.2015 \\
\hline
\end{tabular}




\begin{tabular}{|c|c|c|c|c|c|}
\hline $1334 / 2015$ & $\mathrm{SQ} 4,0.4-3.3 \mathrm{~m} \mathrm{~W}-\mathrm{E} / 6-7.5 \mathrm{~m} \mathrm{~N}-\mathrm{S}$ & $\begin{array}{l}\text { pottery, stone, slag: pottery ( } 8 \text { baskets), among them scrapers } \\
\text { ( } 2 \text { pieces, each re-used Post-New Kingdom sherds, pottery), } \\
\text { weight? ( } 1 \text { re-used sherd, pottery), window grille ( } 1 \text { fragment, } \\
\text { Christian, pottery), qadus, part of base for a water wheel pot? } \\
\text { ( } 1 \text { piece, pottery); whetstone ( } 1 \text { fragment, sandstone); } \\
\text { slag ( } 1 \text { fragment) }\end{array}$ & 226 & $\begin{array}{l}\text { SAV1E 2004, SAV1E } 2007 \text { (scrapers), } \\
\text { SAV1E 2005 (weight?), SAV1E 2006 } \\
\text { (window grille), SAV1E 2008 (qadus, part of } \\
\text { base for a water wheel pot?), SAV1E } 1782 \\
\text { (whetstone), SAV1E 1781 (slag) }\end{array}$ & 27.01 .2015 \\
\hline $1345 / 2015$ & $\mathrm{SQ} 4+4 \mathrm{~A}, 0.5-3.5 \mathrm{~m} \mathrm{~W}-\mathrm{E} / 7.5-10.3 \mathrm{~m} \mathrm{~N}-\mathrm{S}$ & $\begin{array}{l}\text { pottery ( } 9 \text { baskets), among them token/gaming piece (1 re-used } \\
\text { pot sherd, pottery), scrapers ( } 5 \text { pieces, each re-used sherds, } \\
\text { pottery) }\end{array}$ & 227 & $\begin{array}{l}\text { SAV1E } 2070 \text { (token/gaming piece), SAV1E } \\
\text { 2071, SAV1E 2072, SAV1E 2119, SAV1E } \\
\text { 2222, SAV1E 2634 (scrapers) }\end{array}$ & 28.01 .2015 \\
\hline $1357 / 2015$ & $\mathrm{SQ} 4+4 \mathrm{~A}, 0.7-5 \mathrm{~m} \mathrm{~W}-\mathrm{E} / 10.3-12 \mathrm{~m} \mathrm{~N}-\mathrm{S}$ & $\begin{array}{l}\text { pottery ( } 16 \text { baskets), among them scrapers ( } 7 \text { pieces, each re-used } \\
\text { sherds, pottery), lids ( } 3 \text { pieces, each re-used sherds, pottery), } \\
\text { qadus ( } 2 \text { pieces, each pottery), weight? ( } 1 \text { re-used sherd, pottery), } \\
\text { figurine ( } 1 \text { fragment, pottery), unidentified object/mould? } \\
\text { ( } 1 \text { fragment, pottery); slag ( } 4 \text { fragments) }\end{array}$ & 227 & $\begin{array}{l}\text { SAV1E 2635, SAV1E 2636, SAV1E 2637, } \\
\text { SAV1E 2638, SAV1E 2641, SAV1E 2643, } \\
\text { SAV1E 2278 (scrapers), SAV1E 2619, } \\
\text { SAV1E 2620, SAV1E 2281 (lids), } \\
\text { SAV1E 2363, SAV1E 2364 (qadus), SAV1E } \\
\text { 2277 (weight?), } \\
\text { SAV1E } 2361 \text { (figurine), } \\
\text { SAV1E } 2627 \text { (unidentified object/mould?), } \\
\text { SAV1E } 1791 \text { (slag) }\end{array}$ & 28.01 .2015 \\
\hline $1358 / 2015$ & $\mathrm{SQ} 4+4 \mathrm{~A}, 0.7-5 \mathrm{~m} \mathrm{~W}-\mathrm{E} / 10.3-12 \mathrm{~m} \mathrm{~N}-\mathrm{S}$ & pottery, among it net weight (1 piece, pottery) & 227 & SAV1E 2068 & 28.01 .2015 \\
\hline $1360 / 2015$ & $\mathrm{SQ} 4+4 \mathrm{~A}, 0.4-5 \mathrm{~m} \mathrm{~W}-\mathrm{E} / 7.5-12 \mathrm{~m} \mathrm{~N}-\mathrm{S}$ & $\begin{array}{l}\text { pottery ( } 16 \text { baskets), among them scrapers ( } 7 \text { pieces, each re-used } \\
\text { sherds, pottery), lid (1 re-used sherd, pottery), stopper/sealing } \\
\text { ( } 1 \text { piece, clay), unidentified objects ( } 2 \text { re-used sherds, pottery), } \\
\text { window grille ( } 1 \text { fragment, Christian, pottery), disc-shaped } \\
\text { object/token? ( } 1 \text { re-used sherd, pottery) }\end{array}$ & 228 & $\begin{array}{l}\text { SAV1E 2259, SAV1E 2290, SAV1E 2291, } \\
\text { SAV1E 2292, SAV1E 22903, SAV1E 2623, } \\
\text { SAV1E 2624 (scrapers), SAV1E 2625 (lid), } \\
\text { SAV1E } 2059 \text { (stopper/sealing), SAV1E 2258, } \\
\text { SAV1E } 2289 \text { (unidentified objects), SAV1E } \\
\text { 2282(window grille), SAV1E 2295 (disc- } \\
\text { shaped object/token?) }\end{array}$ & 29.01 .2015 \\
\hline $1372 / 2015$ & SQ4A, $3.5-5.1 \mathrm{~m} \mathrm{~W}-\mathrm{E} / 0-2 \mathrm{~m} \mathrm{~N}-\mathrm{S}$ & $\begin{array}{l}\text { pottery and faience: pottery ( } 6 \text { baskets), among them scrapers } \\
\text { ( } 3 \text { pieces, each re-used sherds, pottery), oil lamp (1 fragment, } \\
\text { Christian, pottery), disc-shaped object ( } 1 \text { re-used sherd, pottery, } \\
\text { architecture/basin/oven/crucible? ( } 1 \text { fragment, clay), window } \\
\text { grilles ( } 2 \text { fragments, Christian, pottery); faience vessel } \\
\text { (1 fragment, faience), Nun bowl? (1 fragment, faience) }\end{array}$ & 229 & $\begin{array}{l}\text { SAV1E 2267, SAV1E 2268, SAV1E 2270 } \\
\text { (scrapers), SAV1E 2269 (oil lamp), SAV1E } \\
2271 \text { (disc-shaped object), SAV1E 2272 } \\
\text { (architecture/basin/oven/crucible?), SAV1E } \\
\text { 2273, SAV1E 2274 (window grilles), SAV1E } \\
2075 \text { (faience vessel), SAV1E 2076 (Nun } \\
\text { bowl?) }\end{array}$ & 31.01 .2015 \\
\hline $1383 / 2015$ & SQ4A, 5.1-7m W-E/0-2m N-S & $\begin{array}{l}\text { pottery ( } 2 \text { baskets), among them stoppers/sealings? ( } 3 \text { frag- } \\
\text { ments, each clay), crucible? ( } 1 \text { fragment, clay) }\end{array}$ & 230 & $\begin{array}{l}\text { SAV1E 2112, SAV1E 2283, SAV1E } 2284 \\
\text { (stoppers/sealings), SAV1E } 2285 \text { (crucible?) }\end{array}$ & 31.01 .2015 \\
\hline $1392 / 2015$ & SQ4A, $4.4-5.4 \mathrm{~m} \mathrm{~W}-\mathrm{E} / 1-1.7 \mathrm{~m} \mathrm{~N}-\mathrm{S}$ & pottery (1 basket) & 231 & & 31.01 .2015 \\
\hline $1391 / 2015$ & SQ4A, 5.1-5.9m W-E/0-2m N-S & $\begin{array}{l}\text { pottery ( } 1 \text { basket), among it scraper ( } 1 \text { re-used } 18^{\text {th }} \text { Dynasty } \\
\text { sherd, pottery) }\end{array}$ & 231 & SAV1E 2257 & 31.01 .2015 \\
\hline $1395 / 2015$ & SQ4A, 6-7.7m W-E/0-2m N-S & $\begin{array}{l}\text { pottery ( } 1 \text { basket), among it scrapers ( } 2 \text { pieces, each re-used } \\
\text { sherds, pottery) }\end{array}$ & 232 & SAV1E 2108, SAV1E 2108 & 01.02 .2015 \\
\hline
\end{tabular}




\begin{tabular}{|c|c|c|c|c|c|}
\hline $1401 / 2015$ & SQ4, 7.8-8.8m W-E/8-9.4m N-S & pottery (1 basket) & 233 & & 01.02 .2015 \\
\hline $1403 / 2015$ & SQ4+4A, 8.8-10m W-E/9-12m N-S & $\begin{array}{l}\text { pottery ( } 3 \text { baskets), among them scraper ( } 1 \text { re-used Post-New } \\
\text { Kingdom sherd, pottery), mould ( } 1 \text { fragment, pottery) }\end{array}$ & 234 & SAV1E 2106, SAV1E 2107 & 01.02 .2015 \\
\hline $1415 / 2015$ & $\mathrm{SQ} 4+4 \mathrm{~A}, 8.8-10.5 \mathrm{~m} \mathrm{~W}-\mathrm{E} / 9.3-12 \mathrm{~m} \mathrm{~N}-\mathrm{S}$ & $\begin{array}{l}\text { pottery ( } 4 \text { baskets), among them figurine ( } 1 \text { fragment, animal } \\
\text { figurine, ram, pottery), scraper ( } 1 \text { re-used sherd, pottery) }\end{array}$ & 236 & SAV1E 2218, SAV1E 2645 & 02.02 .2015 \\
\hline $1425 / 2015$ & $\mathrm{SQ} 4 \mathrm{~A}+2 \mathrm{~A}, 9.4-10.4 \mathrm{~m} \mathrm{~W}-\mathrm{E} / 1-2 \mathrm{~m} \mathrm{~N}-\mathrm{S}$ & $\begin{array}{l}\text { pottery and bone; pottery, among it token/gaming piece (1 re- } \\
\text { used sherd, pottery); bone (1 piece) }\end{array}$ & 237 & SAV1E 2365 & 03.02 .2015 \\
\hline $1449 / 2015$ & SQ4A, 6-8.5m W-E/1.5-2m N-S & pottery & 238 & & 04.02 .2015 \\
\hline $1457 / 2015$ & $\mathrm{SQ} 4 \mathrm{~A}+2 \mathrm{~A}, 7-11 \mathrm{~m} \mathrm{~W}-\mathrm{E} / 1-2 \mathrm{~m} \mathrm{~N}-\mathrm{S}$ & pottery (1 basket) & 238 & & 04.02 .2015 \\
\hline $1427 / 2015$ & $\mathrm{SQ} 4 \mathrm{~A}+2 \mathrm{~A}, 8.8-11 \mathrm{~m} \mathrm{~W}-\mathrm{E} / 1-2 \mathrm{~m} \mathrm{~N}-\mathrm{S}$ & pottery ( 2 baskets) & 238 & & 03.02 .2015 \\
\hline $1435 / 2015$ & SQ4+4A, 8-9m W-E/9.4-11.4m N-S & $\begin{array}{l}\text { pottery, slag and brick: pottery ( } 3 \text { baskets), among them cru- } \\
\text { cible? ( } 1 \text { fragment, pottery), figurine ( } 1 \text { fragment, animal } \\
\text { figurine, pottery), window grille ( } 1 \text { fragment, Christian, pot-tery), } \\
\text { oil lamp ( } 1 \text { fragment, Christian, pottery), scraper ( } 1 \text { re-used } \\
\text { sherd, pottery, Christian); slag and brick ( } 1 \text { fragment })\end{array}$ & 239 & $\begin{array}{l}\text { SAV1E } 2104 \text { (crucible?), SAV1E } 2224 \\
\text { (figurine), SAV1E 1773 (window grille), } \\
\text { SAV1E 2576 (oil lamp), SAV1E 2650 } \\
\text { (scraper),SAV1E 2103 (slag and brick) }\end{array}$ & 03.02 .2015 \\
\hline $1442 / 2015$ & SQ4A, 7.2-8.6m W-E/1-2m N-S & pottery (1 basket) & 240 & & 03.02 .2015 \\
\hline $1444 / 2015$ & SQ4A, 5.4-7m W-E/1-2m N-S & $\begin{array}{l}\text { pottery and stone: pottery (1 basket); hand mill (1 fragment, } \\
\text { sandstone) }\end{array}$ & 241 & SAV1E 2130 (hand mill) & 04.02 .2015 \\
\hline $1464 / 2015$ & SQ4, E-bulk, $\mathrm{N}$ of terracing wall & pottery & 242 & & 05.02 .2015 \\
\hline $1461 / 2015$ & $\mathrm{SQ} 4,5.2-6.5 \mathrm{~m} \mathrm{~W}-\mathrm{E} / 7-8.2 \mathrm{~m} \mathrm{~N}-\mathrm{S}$ & pottery ( 1 basket); slag and brick ( 1 fragment) & 242 & SAV1E 2256 (slag and brick) & 04.02 .2015 \\
\hline $1500 / 2015$ & SQ4+2, Feature 15 & pottery & 1300 & & 02.02 .2015 \\
\hline $1502 / 2015$ & SQ4, NE-corner & pottery & 1301 & & 02.02 .2015 \\
\hline $1508 / 2015$ & SQ3-4, baulk & pottery & 1303 & & 02.02 .2015 \\
\hline $1511 / 2015$ & SQ3-4, baulk & pottery & 1304 & & 02.02 .2015 \\
\hline $1514 / 2015$ & SQ4, along former N-baulk & pottery (16 baskets) & 1305 & & 02.02 .2015 \\
\hline $1515 / 2015$ & SQ3-4, baulk & pottery & 1306 & & 03.02 .2015 \\
\hline $1519 / 2015$ & SQ3-4, baulk & $\begin{array}{l}\text { pottery, among it token/gaming pieces ( } 2 \text { pieces, each re-used } \\
\text { sherds, pottery), figurine? ( } 1 \text { fragment, pottery), window grille } \\
\text { ( } 1 \text { fragment, Christian, pottery) }\end{array}$ & 1307 & $\begin{array}{l}\text { SAV1E 2220, SAV1E } 2221 \text { (token/gaming } \\
\text { pieces), SAV1E } 2362 \text { (figurine?), SAV1E } \\
1774 \text { (window grille) }\end{array}$ & 03.02 .2015 \\
\hline $1525 / 2015$ & SQ4, N-baulk & pottery, among it window grille (1 fragment, Christian, pottery) & 1308 & SAV1E 2578 & 03.02 .2015 \\
\hline $1531 / 2015$ & SQ4, N-baulk, Feature 15 & $\begin{array}{l}\text { pottery, among it scrapers ( } 2 \text { pieces, } 1 \text { re-used } 18^{\text {th }} \text { Dynasty } \\
\text { sherd, } 1 \text { re-used Post-New Kingdom pot sherd, pottery) }\end{array}$ & 1309 & SAV1E 2651, SAV1E 2652 & 03.02 .2015 \\
\hline
\end{tabular}




\begin{tabular}{|c|c|c|c|c|c|}
\hline $1539 / 2015$ & SQ4, Feature 15, W-side & pottery & 1310 & & 03.02 .2015 \\
\hline $1542 / 2015$ & SQ4, Feature 15, W-part & pottery & 1310 & & 04.02 .2015 \\
\hline $1546 / 2015$ & SQ4, along Feature 43 in Feature 15 & pottery & 1311 & & 04.02 .2015 \\
\hline $1550 / 2015$ & SQ4, above N-wall of Feature 15 & pottery & 1312 & & 04.02 .2015 \\
\hline $1551 / 2015$ & SQ4, Feature 15 & pottery & 1313 & & 04.02 .2015 \\
\hline $1552 / 2015$ & SQ4, Feature 15 & pottery: vessel, complete & 1313 & & 04.02 .2015 \\
\hline $1553 / 2015$ & SQ4, Feature 15 & pottery & 1314 & & 04.02 .2015 \\
\hline $1557 / 2015$ & SQ2, Feature 15 & pottery & 1316 & & 05.02 .2015 \\
\hline $1565 / 2015$ & SQ4, Feature 15 & pottery & 1316 & & 05.02 .2015 \\
\hline $1572 / 2015$ & SQ2, Feature 15 & pottery & 1316 & & 08.02 .2015 \\
\hline $1588 / 2015$ & SQ2, Feature 15 & pottery & 1316 & & 09.02 .2015 \\
\hline $1569 / 2015$ & SQ2, Feature 15, in pebble, along E-wall & pottery & 1316 & & 05.02 .2015 \\
\hline $1570 / 2015$ & SQ2, Feature 15, sieved material & pottery ( 7 bags of sieved material) & 1316 & & 05.02 .2015 \\
\hline $1576 / 2015$ & SQ2, Feature 15 & pottery & 1317 & & 08.02 .2015 \\
\hline $1592 / 2015$ & SQ2, Feature 15, NE-corner & pottery & 1317 & & 09.02 .2015 \\
\hline $1582 / 2015$ & SQ2, Feature 15 & pottery & 1318 & & 08.02 .2015 \\
\hline $1645 / 2015$ & SQ2, E of wall 44 in Feature 15 & pottery & 1318 & & 11.02 .2015 \\
\hline $1662 / 2015$ & $\begin{array}{l}\text { SQ2, along wall 44, lowest layer of } \\
\text { SU } 1318\end{array}$ & pottery & 1318 & & 12.02 .2015 \\
\hline $1585 / 2015$ & SQ2+4, Feature 15 & pottery & 1319 & & 08.02 .2015 \\
\hline $1595 / 2015$ & $\begin{array}{l}\text { SQ2, Feature } 15 \text { (E P112 content) and SQ2, } \\
\text { Feature 15, NE-corner (E P114 content) }\end{array}$ & $\begin{array}{l}\text { pottery; seal impression (1 fragment, mud); organic material (1 } \\
\text { bag); charcoal ( } 1 \text { bag), charcoal (1 bag); wood (1 bag); bone (1 } \\
\text { bag); doum nut (1 piece) }\end{array}$ & 1320 & SAV1E 2335 (seal impression) & 09.02 .2015 \\
\hline $1638 / 2015$ & SQ2, Feature 15 & pottery ( 1 basket) & 1321 & & 11.02 .2015 \\
\hline $1634 / 2015$ & SQ2, Feature 15 & pottery (2 baskets) & 1321 & & 10.02 .2015 \\
\hline $1609 / 2015$ & SQ2, Feature 15 & $\begin{array}{l}\text { pottery and architecture/stone: pottery ( } 8 \text { baskets); } \\
\text { architecture/later used as whetstone? ( } 1 \text { fragment, sandstone) }\end{array}$ & 1321 & $\begin{array}{l}\text { SAV1E } 2428 \text { (architecture/later used as } \\
\text { whetstone?) }\end{array}$ & 10.02 .2015 \\
\hline $1631 / 2015$ & $\begin{array}{l}\text { SQ2, Feature } 15 \text { (E P107 content) und SQ2, } \\
\text { Feature } 15 \text { (E P108 content) }\end{array}$ & $\begin{array}{l}\text { pottery; charcoal (1 bag), charcoal (1 bag); organic material (1 } \\
\text { bag), bone (1 bag) }\end{array}$ & 1321 & & 10.02 .2015 \\
\hline
\end{tabular}




\begin{tabular}{|c|c|c|c|c|c|}
\hline $1599 / 2015$ & SQ2, Feature 15 (E P113 content) & $\begin{array}{l}\text { pottery, among it scrapers }\left(2 \text { pieces, each re-used } 18^{\text {th }} \text { Dynasty }\right. \\
\text { sherds, pottery); token/gaming piece? }\left(1 \text { re-used } 18^{\text {th }} \text { Dynasty }\right. \\
\text { sherd); seashell ( } 2 \text { fragments); charcoal }(1 \text { bag })\end{array}$ & 1321 & $\begin{array}{l}\text { SAV1E 1309, SAV1E } 2656 \text { (scrapers), } \\
\text { SAV1E } 2655 \text { (token/gaming piece) }\end{array}$ & 09.02 .2015 \\
\hline $1671 / 2015$ & SQ2, Feature 15 , above floor & pottery (sieved) & 1321 & & 11.02 .2015 \\
\hline $1630 / 2015$ & SQ2, Feature 15, along S-wall & pottery, complete vessels & 1321 & & 10.02 .2015 \\
\hline $1664 / 2015$ & SQ2, Feature 15, contents of P 127 & $\begin{array}{l}\text { pottery; charcoal ( } 1 \text { bag); organic material ( } 1 \text { bag); } \\
\text { bone ( } 1 \text { bag); seals ( } 7 \text { fragments, uninscribed })\end{array}$ & 1321 & & 12.02 .2015 \\
\hline $1644 / 2015$ & SQ2, Feature 15, directly on floor & pottery & 1321 & & 11.02 .2015 \\
\hline $1653 / 2015$ & SQ2, Feature 15, E of wall 44 & pottery & 1321 & & 12.02 .2015 \\
\hline $1615 / 2015$ & $\begin{array}{l}\text { SQ2, Feature } 15, \mathrm{~N} \text { of } 1614 \text {, at } \mathrm{N} \text {-wall of } \\
\text { Feature } 15 \text { (vessel E P110 + content) }\end{array}$ & $\begin{array}{l}\text { pottery vessel; charcoal (1 bag); organic material (1 piece); bone } \\
\text { (1 piece) }\end{array}$ & 1321 & SAV1E P110 & 10.02 .2015 \\
\hline $1619 / 2015$ & $\begin{array}{l}\text { SQ2, Feature 15, N of S-wall, } \\
\text { along E-wall (E P111 content) }\end{array}$ & $\begin{array}{l}\text { pottery: dish ( } 1 \text { complete piece, pottery); shell (1 piece); doum } \\
\text { nut (1 piece); charcoal (1 bag); bone (1 bag) }\end{array}$ & 1321 & & 10.02.2015 \\
\hline $1622 / 2015$ & SQ2, Feature 15, SW-corner SU 321 & pottery vessel (carinated jar, Nile clay, complete) & 1321 & & 10.02 .2015 \\
\hline $1623 / 2015$ & SQ2, Feature 15, SW-corner SU 1321 & pottery: dish, complete, DP9 & 1321 & & 10.02 .2015 \\
\hline $1655 / 2015$ & SQ2, Feature $15,55 \mathrm{~cm} \mathrm{E}$ of wall 44 & pottery: dish, complete & 1321 & & 12.02.2015 \\
\hline $1636 / 2015$ & $\begin{array}{l}\text { SQ2, Feature } 15 \text { (E P101 content), (E P102 } \\
\text { content), SQ2, Feature } 15 \text { (E P103 content), } \\
\text { SQ2, Feature } 15 \text { (E P104 content), SQ2, } \\
\text { Feature } 15 \text { (E P105 content) }\end{array}$ & $\begin{array}{l}\text { pottery, stones, charcoal, bone: pottery ( } 9 \text { baskets), among them } \\
\text { unidentified object ( } 2 \text { chunks of pottery/brick, original function } \\
\text { unknown); whetstones ( } 2 \text { pieces, each sandstone); bone ( } 2 \text { bags); } \\
\text { charcoal ( } 5 \text { bags) }\end{array}$ & 1322 & $\begin{array}{l}\text { SAV1E 2414, SAV1E } 2443 \text { (whetstones), } \\
\text { SAV1E } 2443 \text { (unidentified object) }\end{array}$ & 11.02.2015 \\
\hline $1673 / 2015$ & SQ2, Feature 15, sieved material & pottery (sieved) & 1322 & & 11.02 .2015 \\
\hline $1650 / 2015$ & SQ2, Feature 15 & pottery, among it scraper? ( 1 re-used $18^{\text {th }}$ Dynasty sherd, pottery $)$ & 1323 & SAV1E 2657 & 12.02 .2015 \\
\hline $1656 / 2015$ & SQ4, wall 44 & $\begin{array}{l}\text { pottery, among it scraper ( } 1 \text { re-used fragment of a Nubian storage } \\
\text { vessel?, pottery), unidentified object (1 fragment, pottery) }\end{array}$ & 1324 & $\begin{array}{l}\text { SAV1E } 2653 \text { (scraper), SAV1E } 2654 \\
\text { (unidentified pottery object) }\end{array}$ & 12.02 .2015 \\
\hline $1580 / 2015$ & SQ2, Feature 15 & pottery from debris above $\mathrm{N}$-wall & / & & 08.02 .2015 \\
\hline $1424 / 2015$ & $\begin{array}{l}\text { SQ2, S-part/limit of excavation, cleaning of } \\
\text { windblown sand }\end{array}$ & pottery & l & & 02.02 .2015 \\
\hline $1472 / 2015$ & SQ3, Feature 35 trench, cleaning & pottery & / & & 05.02 .2015 \\
\hline $1148 / 2015$ & SQ3, surface cleaning N-section & pottery & I & & 14.01 .2015 \\
\hline $1473 / 2015$ & $\begin{array}{l}\text { SQ4, Feature 49, NE-corner, cleaning small } \\
\text { debris area, corner Feature } 49 / \text { Feature36 }\end{array}$ & pottery & I & & 09.02 .2015 \\
\hline $1475 / 2015$ & $\begin{array}{l}\text { SQ4, Feature } 49, \mathrm{SW} \text {, removal of mud brick } \\
\text { and material below }\end{array}$ & pottery & / & & 09.02 .2015 \\
\hline
\end{tabular}




\begin{tabular}{|c|c|c|c|c|c|}
\hline $1477 / 2015$ & $\begin{array}{l}\text { SQ4+4A, Feature 51, SW-corner, cleaning } \\
\text { on top of wall }\end{array}$ & pottery & / & & 09.02 .2015 \\
\hline $1414 / 2015$ & $\begin{array}{l}\text { SQ4+4A, southern limit of excavation, } \\
\text { cleaning of windblown sand }\end{array}$ & pottery & / & & 02.02 .2015 \\
\hline $1454 / 2015$ & SQ4A, 8-10m W-E, south section & $\begin{array}{l}\text { pottery ( } 1 \text { basket), among it window grille ( } 1 \text { fragment, pottery, } \\
\text { Christian) }\end{array}$ & / & SAV1E 2225 & 04.02 .2015 \\
\hline $1126 / 2015$ & $\mathrm{SQ} 4 \mathrm{~A}, 1.2 \mathrm{~m} \mathrm{~W}-\mathrm{E} / 1.7 \mathrm{~m} \mathrm{~N}-\mathrm{S}$ & $\begin{array}{l}\text { re-used sherd ( } 1 \text { fragment, used for spinning/making ropes?, } \\
\text { Post-New Kingdom, pottery) }\end{array}$ & 205 & SAV1E 1410 & 13.01 .2015 \\
\hline $1111 / 2015$ & $\mathrm{SQ} 4,4 \mathrm{~m} \mathrm{~W}-\mathrm{E} / 7.35 \mathrm{~m}$ N-S & rope (1 short piece) & 205 & SAV1E 1306 & 11.01 .2015 \\
\hline $1371 / 2015$ & $\mathrm{SQ} 4+4 \mathrm{~A}, 0.4-5 \mathrm{~m} \mathrm{~W}-\mathrm{E} / 7.5-12 \mathrm{~m} \mathrm{~N}-\mathrm{S}$ & rope (3 pieces) & 228 & SAV1E 2010 & 29.01 .2015 \\
\hline $1378 / 2015$ & $\mathrm{SQ} 4 \mathrm{~A}, 3.5-5.1 \mathrm{~m} \mathrm{~W}-\mathrm{E} / 0-2 \mathrm{~m} \mathrm{~N}-\mathrm{S}$ & rope (1 small fragment) & 229 & & 31.01 .2015 \\
\hline $1044 / 2015$ & $\mathrm{SQ} 4,3.7-5.8 \mathrm{~m} \mathrm{~W}-\mathrm{E} / 2.8-4.2 \mathrm{~m} \mathrm{~N}-\mathrm{S}$ & schist (3 fragments) & 105 & & 06.01 .2015 \\
\hline $1049 / 2015$ & $\mathrm{SQ} 4,3.7-5.8 \mathrm{~m} \mathrm{~W}-\mathrm{E} / 4.2-5.1 \mathrm{~m} \mathrm{~N}-\mathrm{S}$ & schist (6 fragments) & 105 & & 07.01 .2015 \\
\hline $1175 / 2015$ & $\mathrm{SQ} 4,4-5.7 \mathrm{~m} \mathrm{~W}-\mathrm{E} / 4.5-6.5 \mathrm{~m} \mathrm{~N}-\mathrm{S}$ & schist (29 fragments) & 105 & & 15.01 .2015 \\
\hline $1013 / 2015$ & $\mathrm{SQ} 4,0-7.5 \mathrm{~m} \mathrm{~W}-\mathrm{E} / 7.1-10 \mathrm{~m} \mathrm{~N}-\mathrm{S}$ & schist (7 fragments) & 202 & & 04.01 .2015 \\
\hline $1018 / 2015$ & $\begin{array}{l}\text { SQ4, 0-7.5m W-E/7.1-10m N-S }(12 \mathrm{~m} \mathrm{~N}- \\
\text { S) }\end{array}$ & schist (21 fragments) & 202 & & 05.01 .2015 \\
\hline $1071 / 2015$ & $\mathrm{SQ} 4,0-1.5 \mathrm{~m} \mathrm{~W}-\mathrm{E} / 0-2 \mathrm{~m} \mathrm{~N}-\mathrm{S}$ & schist (4 fragments) & 205 & & 08.01 .2015 \\
\hline $1078 / 2015$ & $\mathrm{SQ} 4,0-1.5 \mathrm{~m} \mathrm{~W}-\mathrm{E} / 0-2 \mathrm{~m} \mathrm{~N}-\mathrm{S}$ & schist (3 fragments) & 205 & & 08.01 .2015 \\
\hline $1090 / 2015$ & $\mathrm{SQ} 4,0-4 \mathrm{~m} \mathrm{~W}-\mathrm{E} / 5-12 \mathrm{~m} \mathrm{~N}-\mathrm{S}$ & schist (8 fragments) & 205 & & 10.01 .2015 \\
\hline $1103 / 2015$ & SQ4, 0-4m W-E/6-10m N-S & schist (48 fragments, 2 bags) & 205 & & 11.01 .2015 \\
\hline $1116 / 2015$ & $\mathrm{SQ} 4,0-4 \mathrm{~m} \mathrm{~W}-\mathrm{E} / 7-9 \mathrm{~m} \mathrm{~N}-\mathrm{S} / 9-10 \mathrm{~m} \mathrm{~N}-\mathrm{S}$ & schist (12 fragments), schist (15 fragments) & 205 & & 12.01 .2015 \\
\hline $1100 / 2015$ & $\mathrm{SQ} 4,3.1-4 \mathrm{~m} \mathrm{~W}-\mathrm{E} / 6-7.5 \mathrm{~m} \mathrm{~N}-\mathrm{S}$ & schist (5 fragments, 1 large) & 205 & & 11.01 .2015 \\
\hline $1166 / 2015$ & $\mathrm{SQ} 4,3.5-5 \mathrm{~m} \mathrm{~W}-\mathrm{E} / 9-10 \mathrm{~m} \mathrm{~N}-\mathrm{S}$ & schist (6 fragments) & 205 & & 15.01 .2015 \\
\hline $1129 / 2015$ & SQ4A, 0-3m W-E/0-2m N-S & schist (63 fragments, 3 bags) & 205 & & 13.01 .2015 \\
\hline $1139 / 2015$ & SQ4A, $1.7-3.2 \mathrm{~m} \mathrm{~W}-\mathrm{E} / 0-1.5 \mathrm{~m} \mathrm{~N}-\mathrm{S}$ & schist (31 fragments) & 205 & & 14.01 .2015 \\
\hline $1217 / 2015$ & $\mathrm{SQ} 4,7-9.2 \mathrm{~m} \mathrm{~W}-\mathrm{E} / 6.8-8 \mathrm{~m} \mathrm{~N}-\mathrm{S}$ & schist (15 fragments) & 212 & & 18.01 .2015 \\
\hline $1224 / 2015$ & $\mathrm{SQ} 4,7-9.2 \mathrm{~m} \mathrm{~W}-\mathrm{E} / 6.8-8 \mathrm{~m} \mathrm{~N}-\mathrm{S}$ & schist (5 fragments) & 212 & & 19.01.2015 \\
\hline $1253 / 2015$ & $\mathrm{SQ} 4+4 \mathrm{~A}, 5-6.5 \mathrm{~m} \mathrm{~W}-\mathrm{E} / 8.2-12 \mathrm{~m} \mathrm{~N}-\mathrm{S}$ & schist (12 fragments) & 213 & & 20.01 .2015 \\
\hline
\end{tabular}




\begin{tabular}{|c|c|c|c|c|c|}
\hline $1244 / 2015$ & $\mathrm{SQ} 4+4 \mathrm{~A}, 6.5-8 \mathrm{~m} \mathrm{~W}-\mathrm{E} / 8.2-12 \mathrm{~m} \mathrm{~N}-\mathrm{S}$ & schist (9 fragments) & 213 & & 20.01 .2015 \\
\hline $1232 / 2015$ & $\mathrm{SQ} 4+4 \mathrm{~A}, 6-10 \mathrm{~m} \mathrm{~W}-\mathrm{E} / 8.2-12 \mathrm{~m} \mathrm{~N}-\mathrm{S}$ & schist (21 fragments) & 213 & & 19.01.2015 \\
\hline $1261 / 2015$ & $\mathrm{SQ} 4+4 \mathrm{~A}, 3.5-5.5 \mathrm{~m} \mathrm{~W}-\mathrm{E} / 8.3-11.3 \mathrm{~m} \mathrm{~N}-\mathrm{S}$ & schist (26 fragments) & 214 & & 21.01 .2015 \\
\hline $1282 / 2015$ & SQ4A, 0-1m W-E/0-2m N-S & schist (3 fragments), plaster (1 bag) & 218 & & 22.01 .2015 \\
\hline $1323 / 2015$ & $\mathrm{SQ} 4,1-2.7 \mathrm{~m} \mathrm{~W}-\mathrm{E} / 3.7-5 \mathrm{~m} \mathrm{~N}-\mathrm{S}$ & schist (15 fragments, 2 bags) & 223 & & 24.01 .2015 \\
\hline $1343 / 2015$ & $\mathrm{SQ} 4,0.4-3.3 \mathrm{~m} \mathrm{~W}-\mathrm{E} / 6-7.5 \mathrm{~m} \mathrm{~N}-\mathrm{S}$ & schist (17 fragments, 2 bags) & 226 & & 27.01 .2015 \\
\hline $1354 / 2015$ & $\mathrm{SQ} 4+4 \mathrm{~A}, 0.4-5 \mathrm{~m} \mathrm{~W}-\mathrm{E} / 7.5-12 \mathrm{~m} \mathrm{~N}-\mathrm{S}$ & schist (51 fragments, 3 bags) & 227 & & 28.01 .2015 \\
\hline $1368 / 2015$ & $\mathrm{SQ} 4+4 \mathrm{~A}, 0.4-5 \mathrm{~m} \mathrm{~W}-\mathrm{E} / 7.5-12 \mathrm{~m} \mathrm{~N}-\mathrm{S}$ & schist (29 fragments, 2 bags) & 228 & & 29.01 .2015 \\
\hline $1382 / 2015$ & $\mathrm{SQ} 4 \mathrm{~A}, 3.5-5.1 \mathrm{~m} \mathrm{~W}-\mathrm{E} / 0-2 \mathrm{~m} \mathrm{~N}-\mathrm{S}$ & schist (23 fragments) & 229 & & 31.01 .2015 \\
\hline $1389 / 2015$ & SQ4A, $5.1-7 \mathrm{~m} \mathrm{~W}-\mathrm{E} / 0-2 \mathrm{~m} \mathrm{~N}-\mathrm{S}$ & schist (10 fragments) & 230 & & 31.01 .2015 \\
\hline $1422 / 2015$ & $\mathrm{SQ} 4+4 \mathrm{~A}, 8.8-10.5 \mathrm{~m} \mathrm{~W}-\mathrm{E} / 9.3-12 \mathrm{~m} \mathrm{~N}-\mathrm{S}$ & schist (20 fragments) & 236 & & 02.02 .2015 \\
\hline $1426 / 2015$ & $\mathrm{SQ} 4 \mathrm{~A}+2 \mathrm{~A}, 9.4-10.4 \mathrm{~m} \mathrm{~W}-\mathrm{E} / 1-2 \mathrm{~m} \mathrm{~N}-\mathrm{S}$ & schist (1 fragment) & 237 & & 03.02 .2015 \\
\hline $1434 / 2015$ & $\mathrm{SQ} 4 \mathrm{~A}+2 \mathrm{~A}, 8.8-11 \mathrm{~m} \mathrm{~W}-\mathrm{E} / 1-2 \mathrm{~m} \mathrm{~N}-\mathrm{S}$ & schist (7 fragments) & 238 & & 03.02 .2015 \\
\hline $1440 / 2015$ & $\mathrm{SQ} 4+4 \mathrm{~A}, 8-9 \mathrm{~m} \mathrm{~W}-\mathrm{E} / 9.4-11.4 \mathrm{~m} \mathrm{~N}-\mathrm{S}$ & schist (7 fragments) & 239 & & 03.02 .2015 \\
\hline $1506 / 2015$ & SQ3, SE-corner & schist (2 fragments) & 1302 & & 02.02 .2015 \\
\hline $1633 / 2015$ & SQ2, Feature 15 & scraper (1 re-used $18^{\text {th }}$ Dynasty pot sherd, pottery) & 1321 & SAV1E 2236 & 10.02 .2015 \\
\hline $1571 / 2015$ & SQ2, Feature 15, sieved material (7 bags) & $\begin{array}{l}\text { seal impressions ( } 25 \text { pieces, clay); worked wood ( } 2 \text { pieces); } \\
\text { figurine ( } 1 \text { fragment, head, animal? human?, clay); lid or stopper } \\
\text { ( } 1 \text { fragment, clay); token/gaming piece (1 piece, clay); stoppers } \\
\text { ( } 2 \text { fragments, } 1 \text { fragment, clay); } \\
\text { slag ( } 1 \text { fragment); bone ( } 1 \text { bag); charcoal ( } 1 \text { bag); clay fragments } \\
\text { ( } 4 \text { bags); seals ( } 36 \text { fragments, uninscribed); } \\
\text { wood ( } 1 \text { piece); seashell ( } 2 \text { fragments); ostrich (?) } \\
\text { egg ( } 1 \text { piece) }\end{array}$ & 1316 & $\begin{array}{l}\text { SAV1E 2470, SAV1E 2471, SAV1E 2472, } \\
\text { SAV1E 2473, SAV1E 2474, SAV1E 2475, } \\
\text { SAV1E 2476, SAV1E 2477, SAV1E 2478, } \\
\text { SAV1E 2479, SAV1E 2480, SAV1E 2481, } \\
\text { SAV1E 2482, SAV1E 2483, SAV1E 2484, } \\
\text { SAV1E 2485, SAV1E 2486, SAV1E 2488, } \\
\text { SAV1E 2336, SAV1E 2337, SAV1E 2338, } \\
\text { SAV1E 2339, SAV1E 2340, SAV1E 2341, } \\
\text { SAV1E 2342 (seal impressions), SAV1E } \\
\text { 2356, SAV1E 2357 (worked wood), SAV1E } \\
\text { 2358 (figurine), SAV1E 2359 (lid), SAV1E } \\
\text { 2360 (token/gaming piece), SAV1E 2613, } \\
\text { SAV1E 2614 (stoppers), SAV1E 2615 (slag) }\end{array}$ & 05.02 .2015 \\
\hline $1681 / 2015$ & SQ2, Feature 15 , sieved material & $\begin{array}{l}\text { seal impression ( } 1 \text { piece, clay); worked stone/whetstone? } \\
\text { ( } 1 \text { fragment, sandstone); wood ( } 1 \text { bag); bone ( } 1 \text { bag); seashell } \\
\text { (fragments, } 1 \text { bag); charcoal ( } 1 \text { bag); ochre ( } 2 \text { pieces red and } \\
1 \text { piece yellow) }\end{array}$ & 1316 & $\begin{array}{l}\text { SAV1E } 2512 \text { (seal impression), SAV1E } 2616 \\
\text { (worked stone/whetstone?) }\end{array}$ & 09.02 .2015 \\
\hline
\end{tabular}




\begin{tabular}{|c|c|c|c|c|c|}
\hline $1683 / 2015$ & $\begin{array}{l}\text { SQ2, Feature 15, W-part of SU, sieved } \\
\text { material }\end{array}$ & $\begin{array}{l}\text { seal impressions ( } 6 \text { pieces, each clay); stopper (1 piece, clay); } \\
\text { seals ( } 11 \text { fragments, uninscribed); charcoal ( } 1 \text { bag) }\end{array}$ & 1316 & $\begin{array}{l}\text { SAV1E 2497, SAV1E 2498, SAV1E 2499, } \\
\text { SAV1E 2500, SAV1E 2501, SAV1E 2502 } \\
\text { (seal impressions), SAV1E } 2617 \text { (stopper) }\end{array}$ & 08.02 .2015 \\
\hline $1680 / 2015$ & SQ2, Feature 15, sieved material & $\begin{array}{l}\text { seal impressions ( } 2 \text { pieces, clay); seals ( } 8 \text { fragments, unin- } \\
\text { scribed); doum nuts ( } 1 \text { bag); bone ( } 1 \text { bag); charcoal ( } 1 \text { bag) }\end{array}$ & 1317 & $\begin{array}{l}\text { SAV1E 2503, SAV1E } 2504 \text { (seal } \\
\text { impressions) }\end{array}$ & 08.02 .2015 \\
\hline $1679 / 2015$ & $\begin{array}{l}\text { SQ2, Feature 15, NE-corner, sieved } \\
\text { material }\end{array}$ & $\begin{array}{l}\text { seal impression ( } 1 \text { fragment, clay); figurine? ( } 1 \text { fragment, clay); } \\
\text { seals ( } 4 \text { fragments, uninscribed); wood ( } 1 \text { bag); } \\
\text { bone ( } 1 \text { bag); doum nuts ( } 1 \text { bag); charcoal ( } 1 \text { bag })\end{array}$ & 1320 & $\begin{array}{l}\text { SAV1E } 2496 \text { (seal impression?), SAV1E } \\
2618 \text { (figurine?) }\end{array}$ & 09.02 .2015 \\
\hline $1670 / 2015$ & $\begin{array}{l}\text { SQ2, Feature 15, above floor, sieved } \\
\text { material }\end{array}$ & $\begin{array}{l}\text { seal impressions ( } 14 \text { pieces, each mud); unidentified object } \\
\text { ( } 1 \text { piece, clay); tokens/gaming pieces ( } 2 \text { pieces, clay); } \\
\text { bone }(1 \mathrm{bag}) \text {; doum nuts }(1 \mathrm{bag}) \text {; seals ( } 25 \text { fragments, } \\
\text { uninscribed); charcoal ( } 1 \text { bag); organic material (fur, } 1 \text { bag); } \\
\text { worked clay ( } 1 \text { fragment, function unclear) }\end{array}$ & 1321 & $\begin{array}{l}\text { SAV1E 2299, SAV1E 2300, SAV1E 2301, } \\
\text { SAV1E 2302, SAV1E 2303, SAV1E 2304, } \\
\text { SAV1E 2305, SAV1E 2306, SAV1E 2307, } \\
\text { SAV1E 2308, SAV1E 2309, SAV1E 2310, } \\
\text { SAV1E 2311, SAV1E 2312 (seal } \\
\text { impressions), SAV1E 2296 (unidentified } \\
\text { object), SAV1E 2297, SAV1E } 2298 \\
\text { (tokens/gaming pieces) }\end{array}$ & 11.02 .2015 \\
\hline $1675 / 2015$ & SQ2, Feature 15, sieved material & $\begin{array}{l}\text { seal impressions ( } 11 \text { pieces, mud); lid? ( } 1 \text { piece, clay); } \\
\text { token/gaming piece? ( } 1 \text { re-used sherd, pottery); stopper/sealing } \\
\text { ( } 1 \text { fragment, clay); slag ( } 1 \text { fragment); } \\
\text { charcoal ( } 2 \text { bags); organic material (1 bag); bone ( } 1 \text { bag); wood } \\
\text { (1 bag); seashell (fragments, } 1 \text { bag); } \\
\text { seals ( } 26 \text { fragments, uninscribed); chalk ( } 4 \text { pieces) }\end{array}$ & 1321 & $\begin{array}{l}\text { SAV1E 2322, SAV1E 2323, SAV1E 2324, } \\
\text { SAV1E 2325, SAV1E 2326, SAV1E 2327, } \\
\text { SAV1E 2328, SAV1E 2329, SAV1E 2330, } \\
\text { SAV1E 2331, SAV1E 2332 (seal impres- } \\
\text { sions), SAV1E 2350 (lid?), SAV1E 2351 } \\
\text { (token/gaming piece), SAV1E 2352 } \\
\text { (stopper/sealing), SAV1E 2353 (slag) }\end{array}$ & 09.02 .2015 \\
\hline $1478 / 2015$ & SQ2, Feature 15, sieved material & $\begin{array}{l}\text { seals/clay fragments used for sealing/stoppers ( } 22 \text { pieces, clay); } \\
\text { beads ( } 11 \text { circular beads, faience); bead ( } 1 \text { circular bead, } \\
\text { carnelian?); re-used sherds }\left(2 \text { re-used } 18^{\text {th }} \text { Dynasty sherds, }\right. \\
\text { pottery); worked stones ( } 2 \text { fragments, } 1 \text { sandstone, } 1 \text { unspecified } \\
\text { stone); slag ( } 1 \text { piece); figurine? ( } 1 \text { fragment, pottery); metal ( } 6 \\
\text { small fragments, original function unknown); clay ball ( } 1 \text { piece, } \\
\text { original function unknown); token/gaming piece ( } 1 \text { clay ball })\end{array}$ & 1321 & $\begin{array}{l}\text { SAV1E 2580, SAV1E 2581, SAV1E 2582, } \\
\text { SAV1E 2583, SAV1E 2584, SAV1E 2585, } \\
\text { SAV1E 2586, SAV1E 2587, SAV1E 2588, } \\
\text { SAV1E 2589, SAV1E 2590, SAV1E 2591, } \\
\text { SAV1E 2592, SAV1E 2593, SAV1E 2594, } \\
\text { SAV1E 2595, SAV1E 2596, SAV1E 2597, } \\
\text { SAV1E 2598, SAV1E 2599, SAV1E 2600, } \\
\text { (seals/clay fragments used for } \\
\text { sealing/stoppers), SAV1E 2610 (beads), } \\
\text { SAV1E 2611 (bead), SAV1E 2608, SAV1E } \\
\text { 2609 (re-used sherds), SAV1E 2607, SAV1E } \\
\text { 2604 (worked stones), SAV1E 2606 (slag), } \\
\text { SAV1E 2605 (figurine), SAV1E 2603 } \\
\text { (metal), SAV1E 2602 (clay ball), SAV1E } \\
\text { 2601 (token/gaming piece) }\end{array}$ & 11.02 .2015 \\
\hline $1678 / 2015$ & $\begin{array}{l}\text { SQ2, Feature } 15, \\
\text { sieved material } \\
\text { (16 bags) }\end{array}$ & $\begin{array}{l}\text { seal impressions ( } 77 \text { pieces, each clay); clay object ( } 1 \text { piece, } \\
\text { function unknown); raw material for sealing? ( } 2 \text { pieces, clay); } \\
\text { token/gaming piece ( } 1 \text { piece, clay ball); charcoal ( } 2 \text { bags); bone }\end{array}$ & 1321 & $\begin{array}{l}\text { SAV1E 2532, SAV1E 2533, SAV1E } 2534 \\
\text { SAV1E 2535, SAV1E 2536, SAV1E 2537, } \\
\text { SAV1E 2538, SAV1E 2539 SAV1E 2540, } \\
\text { SAV1E 2541, SAV1E 2542, SAV1E 2543, }\end{array}$ & 11.02 .2015 \\
\hline
\end{tabular}




\begin{tabular}{|c|c|c|c|c|c|}
\hline & & $\begin{array}{l}(1 \mathrm{bag}) \text {; organic material }(1 \mathrm{bag}) \text {; seashell (fragments, } 1 \text { bag); } \\
\text { wood }(1 \mathrm{bag}) \text {, ochre }(2 \text { pieces, yellow); } \\
\text { plaster }(1 \mathrm{bag}) ; \text { seals (146 fragments, uninscribed); } \\
\text { pigment }(1 \mathrm{bag})\end{array}$ & & $\begin{array}{l}\text { SAV1E 2544, SAV1E 2545 SAV1E 2546, } \\
\text { SAV1E 2547, SAV1E 2548, SAV1E 2549, } \\
\text { SAV1E 2550, SAV1E 2551 SAV1E 2552, } \\
\text { SAV1E 2553, SAV1E 2554 SAV1E 2555, } \\
\text { SAV1E 2556, SAV1E 2557, SAV1E 2558, } \\
\text { SAV1E 2559, SAV1E 2560 SAV1E 2561, } \\
\text { SAV1E 2562, SAV1E 2563, SAV1E 2564, } \\
\text { SAV1E 2565, SAV1E 2566, SAV1E 2567, } \\
\text { SAV1E 2568, SAV1E 2569 SAV1E 2570, } \\
\text { SAV1E 2571, SAV1E 2455, SAV1E 2456, } \\
\text { SAV1E 2457, SAV1E 2458 SAV1E 2459, } \\
\text { SAV1E 2460, SAV1E 2461, SAV1E 2462, } \\
\text { SAV1E 2463, SAV1E 2464 SAV1E 2465, } \\
\text { SAV1E 2466, SAV1E 2467, SAV1E 2468, } \\
\text { SAV1E 2469, SAV1E 2505, SAV1E 2506 } \\
\text { SAV1E 2507, SAV1E 2512, SAV1E 2513, } \\
\text { SAV1E 2514, SAV1E 2515, SAV1E 2516, } \\
\text { SAV1E 2517, SAV1E 2518, SAV1E 2519 } \\
\text { SAV1E 2520, SAV1E 2521, SAV1E 2522, } \\
\text { SAV1E 2523, SAV1E 2524, SAV1E 2525, } \\
\text { SAV1E 2526, SAV1E 2527, SAV1E 2528, } \\
\text { SAV1E 2529, SAV1E 2530, SAV1E 2531 } \\
\text { (seal impressions), SAV1E 2508 (clay } \\
\text { object), SAV1E 2509, SAV1E 2510 (raw } \\
\text { material for sealing?), SAV1E 2511 } \\
\text { (token/gaming piece) }\end{array}$ & \\
\hline $1677 / 2015$ & $\begin{array}{l}\text { SQ2, Feature } 15, \\
\text { sieved material ( } 8 \text { bags) }\end{array}$ & $\begin{array}{l}\text { seal impressions ( } 14 \text { pieces, each clay); stoppers ( } 3 \text { pieces, each } \\
\text { clay); worked stones ( } 2 \text { pieces, each sandstone); unidentified } \\
\text { objects ( } 2 \text { pieces, each pottery); re-used sherd } \\
\text { (1 piece, original function unknown, pottery); token/gaming } \\
\text { piece ( } 1 \text { piece, pottery); hand mill (1 piece, sandstone); stela? (1 } \\
\text { small fragment, sandstone); bead (1 ring bead, faience); faience } \\
\text { vessel ( } 1 \text { fragment); charcoal ( } 3 \text { bags); organic material ( } 1 \text { bag); } \\
\text { bone ( } 1 \text { bag); seals ( } 29 \text { fragments, uninscribed); chalk (1 piece); } \\
\text { stone flakes ( } 2 \text { pieces, reference); shell ( } 1 \text { bag); organic material } \\
\text { (seeds, } 1 \text { bag); seashell (fragments, } 1 \text { bag); shell (1 bag) }\end{array}$ & 1321 & $\begin{array}{l}\text { SAV1E 2429, SAV1E 2430, SAV1E 2431, } \\
\text { SAV1E 2432, SAV1E 2433, SAV1E 2434 } \\
\text { SAV1E 2435, SAV1E 2436, SAV1E 2437, } \\
\text { SAV1E 2438, SAV1E 2439, SAV1E 2440, } \\
\text { SAV1E 2441, SAV1E 2442 (seal } \\
\text { impressions), SAV1E 2445, SAV1E 2446, } \\
\text { SAV1E 2447 (stoppers), SAV1E 2450, } \\
\text { SAV1E } 2453 \text { (worked stones), SAV1E 2660, } \\
\text { SAV1E 2448 (unidentified objects), SAV1E } \\
\text { 2659 (re-used sherd), SAV1E 2658 } \\
\text { (token/gaming piece), SAV1E 2454 (hand } \\
\text { mill), SAV1E 2452 (stela?), SAV1E 2451 } \\
\text { (bead), SAV1E 2449 (faience vessel) }\end{array}$ & 10.02 .2015 \\
\hline $1676 / 2015$ & $\begin{array}{l}\text { SQ2, Feature 15, W-part of SU, sieved } \\
\text { material }\end{array}$ & $\begin{array}{l}\text { seal impressions ( } 44 \text { pieces, clay); stoppers ( } 5 \text { pieces, clay); } \\
\text { token/gaming pieces ( } 2 \text { pieces, } 1 \text { pottery, } 3 \text { re-used sherds, } \\
\text { pottery); figurines ( } 2 \text { pieces, } 1 \text { pottery, } 1 \text { clay, animal? human?); } \\
\text { bead ( } 1 \text { circular bead, faience); clay objects ( } 3 \text { pieces, original }\end{array}$ & 1321 & $\begin{array}{l}\text { SAV1E 2333, SAV1E 2334, SAV1E 2372, } \\
\text { SAV1E 2373, SAV1E 2374, SAV1E 2375, } \\
\text { SAV1E 2376, SAV1E 2377, SAV1E 2378, } \\
\text { SAV1E 2379, SAV1E 2380, SAV1E 2381, }\end{array}$ & 12.02 .2015 \\
\hline
\end{tabular}




\begin{tabular}{|c|c|c|c|c|c|}
\hline & & $\begin{array}{l}\text { function unknown); metal object ( } 2 \text { fragments, original function } \\
\text { unknown); faience vessel? ( } 1 \text { small fragment); charcoal ( } 2 \text { bags); } \\
\text { doum nuts ( } 1 \text { bag); bone ( } 1 \text { bag); seashell (fragments, } 1 \text { bag); } \\
\text { chalk ( } 4 \text { pieces); seals ( } 163 \text { fragments, uninscribed); stone flake } \\
\text { (1 piece, agate, reference) }\end{array}$ & & $\begin{array}{l}\text { SAV1E 2382, SAV1E 2383, SAV1E 2384, } \\
\text { SAV1E 2385, SAV1E 2386, SAV1E 2387, } \\
\text { SAV1E 2388, SAV1E 2389, SAV1E 2390, } \\
\text { SAV1E 2391, SAV1E 2392, SAV1E 2393, } \\
\text { SAV1E 2394, SAV1E 2395, SAV1E 2396, } \\
\text { SAV1E 2397, SAV1E 2398, SAV1E 2399, } \\
\text { SAV1E 2400, SAV1E 2401, SAV1E 2402, } \\
\text { SAV1E 2403, SAV1E 2404, SAV1E 2405, } \\
\text { SAV1E 2406, SAV1E 2407, SAV1E 2408, } \\
\text { SAV1E 2409, SAV1E 2410, SAV1E 2411, } \\
\text { SAV1E 2412, SAV1E 2413 (seal } \\
\text { impressions), SAV1E 2346, SAV1E 2417, } \\
\text { SAV1E 2418, SAV1E 2419, SAV1E 2420 } \\
\text { (stoppers), SAV1E 2344, SAV1E 2345, } \\
\text { SAV1E 2422, SAV1E 2415 (token/gaming } \\
\text { pieces), SAV1E 2343, SAV1E 2421 } \\
\text { (figurines), SAV1E 2427 (bead), SAV1E } \\
\text { 2423, SAV1E 2424, SAV1E 2425 (clay } \\
\text { objects), SAV1E 2426 (metal object), SAV1E } \\
\text { 2416 (faience vessel?) }\end{array}$ & \\
\hline $1684 / 2015$ & $\begin{array}{l}\text { SQ2, Feature 15, W-part, fine sieved } \\
\text { material }\end{array}$ & $\begin{array}{l}\text { seal impressions ( } 3 \text { pieces, each clay); seals ( } 5 \text { fragments, } \\
\text { uninscribed); bone ( } 1 \text { bag) }\end{array}$ & 1321 & $\begin{array}{l}\text { SAV1E 2493, SAV1E 2493, SAV1E } 2495 \\
\text { (seal impressions) }\end{array}$ & 09.02.2015 \\
\hline $1674 / 2015$ & SQ2, Feature 15 , sieved material & $\begin{array}{l}\text { seal impressions ( } 8 \text { pieces, mud/clay); bead ( } 1 \text { circular bead, } \\
\text { faience); metal object ( } 2 \text { pieces, function unknown); charcoal ( } 1 \\
\text { bag); bone ( } 1 \text { bag); organic material ( } 1 \text { bag); } \\
\text { seals ( } 16 \text { fragments, uninscribed) }\end{array}$ & 1323 & $\begin{array}{l}\text { SAV1E 2315, SAV1E 2316, SAV1E 2317, } \\
\text { SAV1E 2318, SAV1E 2319, SAV1E 2320, } \\
\text { SAV1E 2321, SAV1E } 2487 \text { (seal } \\
\text { impressions), SAV1E } 2314 \text { (bead), SAV1E } \\
2313 \text { (metal object) }\end{array}$ & 12.02 .2015 \\
\hline $1040 / 2015$ & $\mathrm{SQ} 4,3.7-5.8 \mathrm{~m} \mathrm{~W}-\mathrm{E} / 2.8-4.2 \mathrm{~m} \mathrm{~N}-\mathrm{S}$ & seashell (4 fragments) & 105 & & 06.01 .2015 \\
\hline $1004 / 2015$ & $\mathrm{SQ} 4+4 \mathrm{~A}, 0-7.5 \mathrm{~m} \mathrm{~W}-\mathrm{E} / 7.1-12 \mathrm{~m} \mathrm{~N}-\mathrm{S}$ & seashell (2 fragments) & 200 & & 04.01 .2015 \\
\hline $1076 / 2015$ & $\mathrm{SQ} 4,0-1.5 \mathrm{~m} \mathrm{~W}-\mathrm{E} / 0-2 \mathrm{~m} \mathrm{~N}-\mathrm{S}$ & seashell (1 fragment) & 205 & & 08.01 .2015 \\
\hline $1059 / 2015$ & $\mathrm{SQ} 4,0-4 \mathrm{~m} \mathrm{~W}-\mathrm{E} / 4.7-7.1 \mathrm{~m} \mathrm{~N}-\mathrm{S}$ & seashell (1 piece) & 205 & & 07.01 .2015 \\
\hline $1108 / 2015$ & $\mathrm{SQ} 4,0-4 \mathrm{~m} \mathrm{~W}-\mathrm{E} / 6-10 \mathrm{~m} \mathrm{~N}-\mathrm{S}$ & seashell (3 fragments) & 205 & & 11.01.2015 \\
\hline $1124 / 2015$ & $\mathrm{SQ} 4,0-4 \mathrm{~m} \mathrm{~W}-\mathrm{E} / 9-12 \mathrm{~m} \mathrm{~N}-\mathrm{S}$ & seashell (2 fragments) & 205 & & 12.01.2015 \\
\hline $1092 / 2015$ & SQ4A, $0-1.5 \mathrm{~m} \mathrm{~W}-\mathrm{E} / 0-2 \mathrm{~m} \mathrm{~N}-\mathrm{S}$ & seashell (1 fragment) & 205 & & 10.01 .2015 \\
\hline $1130 / 2015$ & SQ4A, 0-3m W-E/0-2m N-S & seashell (fragments, 1 bag) & 205 & & 13.01.2015 \\
\hline $1142 / 2015$ & SQ4A, $1.7-3.2 \mathrm{~m} \mathrm{~W}-\mathrm{E} / 0-1.5 \mathrm{~m} \mathrm{~N}-\mathrm{S}$ & seashell (fragments, 1 bag) & 205 & & 14.01.2015 \\
\hline
\end{tabular}




\begin{tabular}{|c|c|c|c|c|c|}
\hline $1228 / 2015$ & $\mathrm{SQ} 4+4 \mathrm{~A}, 6-10 \mathrm{~m} \mathrm{~W}-\mathrm{E} / 8.2-12 \mathrm{~m} \mathrm{~N}-\mathrm{S}$ & seashell (4 fragments) & 213 & & 19.01.2015 \\
\hline $1264 / 2015$ & $\mathrm{SQ} 4+4 \mathrm{~A}, 3.5-5.5 \mathrm{~m} \mathrm{~W}-\mathrm{E} / 8.3-11.3 \mathrm{~m} \mathrm{~N}-\mathrm{S}$ & seashell (fragments, 1 bag) & 214 & & 21.01 .2015 \\
\hline $1267 / 2015$ & SQ4, 0-1m W-E/8.8-9.7m N-S & seashell (1 large fragment) & 215 & & 22.01 .2015 \\
\hline $1273 / 2015$ & SQ4A, 0-1m W-E/0-2m N-S & seashell (1 fragment) & 216 & & 22.01 .2015 \\
\hline $1287 / 2015$ & SQ4, 5-8.8m N-S, section cleaning & seashell (1 fragment) & 219 & & 22.01 .2015 \\
\hline $1315 / 2015$ & $\mathrm{SQ} 4,0-0.8 \mathrm{~m} \mathrm{~W}-\mathrm{E} / 4.4-5.1 \mathrm{~m} \mathrm{~N}-\mathrm{S}$ & seashell (fragments, 1 bag) & 222 & & 24.01 .2015 \\
\hline $1341 / 2015$ & SQ4, 0.4-3.3m W-E/6-7.5m N-S & seashell (1 fragment) & 226 & & 27.01 .2015 \\
\hline $1348 / 2015$ & $\mathrm{SQ} 4+4 \mathrm{~A}, 0.4-5 \mathrm{~m} \mathrm{~W}-\mathrm{E} / 7.5-12 \mathrm{~m} \mathrm{~N}-\mathrm{S}$ & seashell (fragments, 1 bag) & 227 & & 28.01 .2015 \\
\hline $1419 / 2015$ & $\mathrm{SQ} 4+4 \mathrm{~A}, 8.8-10.5 \mathrm{~m} \mathrm{~W}-\mathrm{E} / 9.3-12 \mathrm{~m} \mathrm{~N}-\mathrm{S}$ & seashell (1 fragment) & 236 & & 02.02 .2015 \\
\hline $1496 / 2015$ & SQ2, Feature 15 & seashell (fragments, 1 bag) & 1316 & & 08.02 .2015 \\
\hline $1563 / 2015$ & SQ2, Feature 15 & seashell (fragments, 1 bag) & 1316 & & 05.02 .2015 \\
\hline $1620 / 2015$ & SQ2, Feature 15 & seashell (1 large fragment) & 1321 & & 10.02 .2015 \\
\hline $1626 / 2015$ & SQ2, Feature 15, along S-wall & seashell (2 large fragments) & 1321 & & 10.02 .2015 \\
\hline $1665 / 2015$ & $\begin{array}{l}\text { SQ2, Feature 15, W-part of SU, } \\
\text { above floor }\end{array}$ & seashell (1 fragment); bone (1 bag) & 1321 & & 12.02 .2015 \\
\hline $1278 / 2015$ & SQ4A, 0-1m W-E/0-2m N-S & skin (1 bag, animal, with fur) & 217 & & 22.01 .2015 \\
\hline $1281 / 2015$ & SQ4A, 0-1m W-E/0-2m N-S & skin (1 bag, animal) & 218 & & 22.01 .2015 \\
\hline $1290 / 2015$ & SQ4, 5-8.8m N-S, section cleaning & skin (1 bag, animal) & 219 & & 22.01 .2015 \\
\hline $1351 / 2015$ & $\mathrm{SQ} 4+4 \mathrm{~A}, 0.4-5 \mathrm{~m} \mathrm{~W}-\mathrm{E} / 7.5-12 \mathrm{~m} \mathrm{~N}-\mathrm{S}$ & skin (with fur, 1 bag, animal); organic material (1 bag) & 227 & & 28.01 .2015 \\
\hline $1037 / 2015$ & $\mathrm{SQ} 4,3.7-5.8 \mathrm{~m} \mathrm{~W}-\mathrm{E} / 2.8-4.2 \mathrm{~m} \mathrm{~N}-\mathrm{S}$ & slag (3 small fragments) & 105 & SAV1E 1197 & 06.01 .2015 \\
\hline $1050 / 2015$ & $\mathrm{SQ} 4,3.7-5.8 \mathrm{~m} \mathrm{~W}-\mathrm{E} / 4.2-5.1 \mathrm{~m} \mathrm{~N}-\mathrm{S}$ & slag (1 small fragment) & 105 & SAV1E 1209 & 07.01 .2015 \\
\hline $1188 / 2015$ & $\mathrm{SQ} 4,4-5.7 \mathrm{~m} \mathrm{~W}-\mathrm{E} / 4.5-6.5 \mathrm{~m} \mathrm{~N}-\mathrm{S}$ & slag (1 fragment) & 105 & & 17.01 .2015 \\
\hline $1183 / 2015$ & $\mathrm{SQ} 4,4-5.7 \mathrm{~m} \mathrm{~W}-\mathrm{E} / 4.5-6.5 \mathrm{~m} \mathrm{~N}-\mathrm{S}$ & slag (4 fragments) & 105 & SAV1E 1474 & 15.01 .2015 \\
\hline $1005 / 2015$ & $\mathrm{SQ} 4+4 \mathrm{~A}, 0-7.5 \mathrm{~m} \mathrm{~W}-\mathrm{E} / 7.1-12 \mathrm{~m} \mathrm{~N}-\mathrm{S}$ & slag & 200 & & 04.01 .2015 \\
\hline $1014 / 2015$ & SQ4, 0-7.5m W-E/7.1-10m N-S & slag (2 small fragments) & 202 & SAV1E 1135 & 04.01 .2015 \\
\hline $1015 / 2015$ & SQ4, 0-7.5m W-E/7.1-10m N-S & slag (6 small fragments); doum nuts (2 pieces) & 202 & SAV1E 1136 (slag) & 04.01 .2015 \\
\hline $1021 / 2015$ & $\mathrm{SQ} 4,0-7.5 \mathrm{~m} \mathrm{~W}-\mathrm{E} / 7.1-10 \mathrm{~m} \mathrm{~N}-\mathrm{S}$ & slag (1 small piece) & 202 & SAV1E 1172 & 05.01 .2015 \\
\hline
\end{tabular}




\begin{tabular}{|c|c|c|c|c|c|}
\hline $1029 / 2015$ & $\mathrm{SQ} 4,3.5-6.5 \mathrm{~m} \mathrm{~W}-\mathrm{E} / 5-6.2 \mathrm{~m} \mathrm{~N}-\mathrm{S}$ & slag (1 small fragment) & 204 & SAV1E 1181 & 06.01 .2015 \\
\hline $1079 / 2015$ & SQ4, 0-1.5m W-E/0-2m N-S & slag (1 small fragment) & 205 & SAV1E 1254 & 08.01 .2015 \\
\hline $1054 / 2015$ & $\mathrm{SQ} 4,0-4 \mathrm{~m} \mathrm{~W}-\mathrm{E} / 4.7-7.1 \mathrm{~m} \mathrm{~N}-\mathrm{S}$ & $\begin{array}{l}\text { slag ( } 2 \text { fragments, } 1 \text { with brick); vessel ( } 2 \text { fragments, glazed } \\
\text { pottery vessel, Islamic/Ottoman); clay object ( } 2 \text { fragments, each } \\
\text { possibly from window grilles, clay) }\end{array}$ & 205 & $\begin{array}{l}\text { SAV1E 1212, SAV1E } 1213 \text { (slag), SAV1E } \\
1214 \text { (glazed vessel), SAV1E 1214, SAV1E } \\
1251 \text { (clay objects/window grilles?) }\end{array}$ & 07.01 .2015 \\
\hline $1085 / 2015$ & $\mathrm{SQ} 4,0-4 \mathrm{~m} \mathrm{~W}-\mathrm{E} / 5-10 \mathrm{~m} \mathrm{~N}-\mathrm{S}$ & slag (5 pieces) & 205 & SAV1E 1284 & 10.01 .2015 \\
\hline $1109 / 2015$ & SQ4, 0-4m W-E/6-10m N-S & slag (1 small fragment) & 205 & SAV1E 1307 & 11.01.2015 \\
\hline $1069 / 2015$ & $\mathrm{SQ} 4,0-4 \mathrm{~m} \mathrm{~W}-\mathrm{E} / 7.1-9.7 \mathrm{~m} \mathrm{~N}-\mathrm{S}$ & slag (2 small fragments) & 205 & SAV1E 1253 & 08.01 .2015 \\
\hline $1119 / 2015$ & $\mathrm{SQ} 4,0-4 \mathrm{~m} \mathrm{~W}-\mathrm{E} / 7-9 \mathrm{~m} \mathrm{~N}-\mathrm{S} / 9-10 \mathrm{~m} \mathrm{~N}-\mathrm{S}$ & slag (1 fragment) & 205 & & 12.01 .2015 \\
\hline $1122 / 2015$ & $\mathrm{SQ} 4,0-4 \mathrm{~m} \mathrm{~W}-\mathrm{E} / 7-9 \mathrm{~m} \mathrm{~N}-\mathrm{S}$ & Slag (1 fragment) & 205 & SAV1E 1374 & 12.01.2015 \\
\hline $1164 / 2015$ & $\mathrm{SQ} 4,3.5-5 \mathrm{~m} \mathrm{~W}-\mathrm{E} / 9-10 \mathrm{~m} \mathrm{~N}-\mathrm{S}$ & slag (2 fragments) & 205 & SAV1E 1473 & 15.01 .2015 \\
\hline $1155 / 2015$ & $\mathrm{SQ} 4,4.4 \mathrm{~m} \mathrm{~W}-\mathrm{E} / 9.8 \mathrm{~m} \mathrm{~N}-\mathrm{S}$ & slag (2 fragments) & 205 & SAV1E 1481 & 14.01 .2015 \\
\hline $1133 / 2015$ & SQ4A, 0-3m W-E/0-2m N-S & slag (13 fragments) & 205 & SAV1E 1422 & 13.01 .2015 \\
\hline $1141 / 2015$ & $\mathrm{SQ} 4 \mathrm{~A}, 1.7-3.2 \mathrm{~m} \mathrm{~W}-\mathrm{E} / 0-1.5 \mathrm{~m} \mathrm{~N}-\mathrm{S}$ & slag (3 fragments) & 205 & SAV1E 1426 & 14.01 .2015 \\
\hline $1145 / 2015$ & $\mathrm{SQ} 4,3.9-5.1 \mathrm{~m} \mathrm{~W}-\mathrm{E} / 7.3-8.8 \mathrm{~m} \mathrm{~N}-\mathrm{S}$ & slag and brick (5 fragments) & 206 & SAV1E 1888 & 14.01 .2015 \\
\hline $1196 / 2015$ & $\mathrm{SQ} 4,4.2-5.9 \mathrm{~m} \mathrm{~W}-\mathrm{E} / 6.5-8 \mathrm{~m} \mathrm{~N}-\mathrm{S}$ & $\begin{array}{l}\text { slag and brick ( } 1 \text { fragment); re-used sherd (Christian/medieval); } \\
\text { lamp ( } 1 \text { fragment, Christian, pottery) }\end{array}$ & 209 & $\begin{array}{l}\text { SAV1E } 1846 \text { (slag), SAV1E } 1914 \text { (re-used } \\
\text { sherd), SAV1E } 2574 \text { (lamp) }\end{array}$ & 17.01.2015 \\
\hline $1204 / 2015$ & $\mathrm{SQ} 4+4 \mathrm{~A}, 6-7.5 \mathrm{~m} \mathrm{~W}-\mathrm{E} / 8.5-12 \mathrm{~m} \mathrm{~N}-\mathrm{S}$ & slag (1 fragment) & 210 & SAV1E 1839 & 17.01.2015 \\
\hline $1214 / 2015$ & $\mathrm{SQ} 4,7-9.2 \mathrm{~m} \mathrm{~W}-\mathrm{E} / 6.8-8 \mathrm{~m} \mathrm{~N}-\mathrm{S}$ & slag (6 fragments) & 212 & SAV1E 1868 & 18.01 .2015 \\
\hline $1251 / 2015$ & $\mathrm{SQ} 4+4 \mathrm{~A}, 5-6.5 \mathrm{~m} \mathrm{~W}-\mathrm{E} / 8.2-12 \mathrm{~m} \mathrm{~N}-\mathrm{S}$ & slag (5 fragments) & 213 & SAV1E 1903 & 20.01 .2015 \\
\hline $1241 / 2015$ & $\mathrm{SQ} 4+4 \mathrm{~A}, 6.5-8 \mathrm{~m} \mathrm{~W}-\mathrm{E} / 8.2-12 \mathrm{~m} \mathrm{~N}-\mathrm{S}$ & slag (5 fragments) & 213 & SAV1E 1904 & 20.01 .2015 \\
\hline $1230 / 2015$ & $\mathrm{SQ} 4+4 \mathrm{~A}, 6-10 \mathrm{~m} \mathrm{~W}-\mathrm{E} / 8.2-12 \mathrm{~m} \mathrm{~N}-\mathrm{S}$ & slag (5 fragments) & 213 & SAV1E 1905 & 19.01 .2015 \\
\hline $1258 / 2015$ & $\mathrm{SQ} 4+4 \mathrm{~A}, 3.5-5.5 \mathrm{~m} \mathrm{~W}-\mathrm{E} / 8.3-11.3 \mathrm{~m} \mathrm{~N}-\mathrm{S}$ & slag and brick (12 pieces) & 214 & SAV1E 1926 & 21.01 .2015 \\
\hline $1268 / 2015$ & $\mathrm{SQ} 4,0-1 \mathrm{~m} \mathrm{~W}-\mathrm{E} / 8.8-9.7 \mathrm{~m} \mathrm{~N}-\mathrm{S}$ & slag (1 fragment) & 215 & SAV1E 1932 & 22.01 .2015 \\
\hline $1288 / 2015$ & SQ4, 5-8.8m N-S, section cleaning & slag (1 fragment) & 219 & SAV1E 1936 & 22.01 .2015 \\
\hline $1283 / 2015$ & SQ4A, 0-1m W-E/0-2m N-S & slag (2 fragments) & 219 & SAV1E 1934 & 22.01 .2015 \\
\hline $1293 / 2015$ & $\mathrm{SQ} 4,0-1.2 \mathrm{~m} \mathrm{~W}-\mathrm{E} / 0-4.5 \mathrm{~m} \mathrm{~N}-\mathrm{S}$ & $\begin{array}{l}\text { slag ( } 1 \text { fragment), } \\
\text { disc ( } 1 \text { re-used Post-New Kingdom sherd, pottery) }\end{array}$ & 220 & SAV1E 1937 (slag), SAV1E 2621 (disc) & 22.01 .2015 \\
\hline
\end{tabular}




\begin{tabular}{|c|c|c|c|c|c|}
\hline $1339 / 2015$ & $\mathrm{SQ} 4,0.4-3.3 \mathrm{~m} \mathrm{~W}-\mathrm{E} / 6-7.5 \mathrm{~m} \mathrm{~N}-\mathrm{S}$ & slag (1 fragment) & 226 & SAV1E 1779 & 27.01.2015 \\
\hline $1359 / 2015$ & $\mathrm{SQ} 4+4 \mathrm{~A}, 0.4-5 \mathrm{~m} \mathrm{~W}-\mathrm{E} / 7.5-12 \mathrm{~m} \mathrm{~N}-\mathrm{S}$ & slag (4 fragments) & 227 & SAV1E 1790 & 28.01 .2015 \\
\hline $1363 / 2015$ & $\mathrm{SQ} 4+4 \mathrm{~A}, 0.4-5 \mathrm{~m} \mathrm{~W}-\mathrm{E} / 7.5-12 \mathrm{~m} \mathrm{~N}-\mathrm{S}$ & slag (7 fragments) & 228 & SAV1E 2049 & 29.01 .2015 \\
\hline $1373 / 2015$ & SQ4A, 3.5-5.1m W-E/0-2m N-S & slag (2 fragments) & 229 & SAV1E 2011 & 31.01 .2015 \\
\hline $1388 / 2015$ & SQ4A, 5.1-7m W-E/0-2m N-S & slag and brick (5 fragments) & 230 & SAV1E 2012 & 31.01 .2015 \\
\hline $1398 / 2015$ & SQ4A, 6-7.7m W-E/0-2m N-S & slag (1 fragment) & 232 & SAV1E 2123 & 01.02 .2015 \\
\hline $1418 / 2015$ & $\mathrm{SQ} 4+4 \mathrm{~A}, 8.8-10.5 \mathrm{~m} \mathrm{~W}-\mathrm{E} / 9.3-12 \mathrm{~m} \mathrm{~N}-\mathrm{S}$ & slag (2 fragments) & 236 & SAV1E 2067 & 02.02 .2015 \\
\hline $1432 / 2015$ & $\mathrm{SQ} 4 \mathrm{~A}+2 \mathrm{~A}, 8.8-11 \mathrm{~m} \mathrm{~W}-\mathrm{E} / 1-2 \mathrm{~m} \mathrm{~N}-\mathrm{S}$ & slag (2 fragments) & 238 & SAV1E 2060 & 03.02 .2015 \\
\hline $1513 / 2015$ & SQ3-4, baulk & slag (1 fragment) & 1304 & SAV1E 2069 & 02.02 .2015 \\
\hline $1528 / 2015$ & SQ4, N-baulk & slag (2 small fragments) & 1308 & SAV1E 2066 & 03.02 .2015 \\
\hline $1535 / 2015$ & SQ4, N-baulk, Feature 15 & slag (1 fragment) & 1309 & SAV1E 2063 & 03.02 .2015 \\
\hline $1541 / 2015$ & SQ4, Feature 15, W-side & slag (1 fragment) & 1310 & SAV1E 2064 & 03.02 .2015 \\
\hline $1043 / 2015$ & $\mathrm{SQ} 4,3.7-5.8 \mathrm{~m} \mathrm{~W}-\mathrm{E} / 2.8-4.2 \mathrm{~m} \mathrm{~N}-\mathrm{S}$ & $\begin{array}{l}\text { stones: grindstones ( } 3 \text { pieces, each quartzite), } \\
\text { whetstone ( } 1 \text { piece, sandstone), pounders }(4 \text { pieces, } \\
3 \text { quartzite, } 1 \text { silicified wood), polishing stone? ( } 1 \text { piece, schist), } \\
\text { raw material ( } 1 \text { small quartz pebble); silicified wood ( } 1 \text { fragment, } \\
\text { unworked) }\end{array}$ & 105 & $\begin{array}{l}\text { SAV1E 1198, SAV1E 1199, SAV1E } 1200 \\
\text { (grindstones), SAV1E } 1201 \text { (whetstone), } \\
\text { SAV1E 1202, SAV1E 1203, SAV1E 1204, } \\
\text { SAV1E 1205 (pounders), SAV1E 1206 } \\
\text { (polishing stones), SAV1E } 1207 \text { (raw } \\
\text { material) }\end{array}$ & 06.01 .2015 \\
\hline $1184 / 2015$ & $\mathrm{SQ} 4,4-5.7 \mathrm{~m} \mathrm{~W}-\mathrm{E} / 4.5-6.5 \mathrm{~m} \mathrm{~N}-\mathrm{S}$ & stone: blade? (1 piece, silex) & 105 & SAV1E 1475 & 15.01 .2015 \\
\hline $1181 / 2015$ & $\mathrm{SQ} 4,4-5.7 \mathrm{~m} \mathrm{~W}-\mathrm{E} / 4.5-6.5 \mathrm{~m} \mathrm{~N}-\mathrm{S}$ & $\begin{array}{l}\text { stones: grindstone ( } 4 \text { pieces, each quartzite), } \\
\text { hand mills ( } 2 \text { pieces, each quartzite), } \\
\text { pounders ( } 2 \text { pieces, } 1 \text { quartzite, } 1 \text { quartz), } \\
\text { whetstone (1 piece, quartzite) }\end{array}$ & 105 & $\begin{array}{l}\text { SAV1E 1919, SAV1E 1920, SAV1E 1921, } \\
\text { SAV1E } 1922 \text { (grindstones), SAV1E 1917, } \\
\text { SAV1E } 1918 \text { (hand mills), SAV1E 1916, } \\
\text { SAV1E } 1875 \text { (pounders), SAV1E } 1840 \\
\text { (whetstone) }\end{array}$ & 15.01 .2015 \\
\hline $1048 / 2015$ & $\mathrm{SQ} 4,3.7-5.8 \mathrm{~m} \mathrm{~W}-\mathrm{E} / 4.2-5.1 \mathrm{~m} \mathrm{~N}-\mathrm{S}$ & $\begin{array}{l}\text { stones: pounders ( } 4 \text { pieces, } 3 \text { quartz pebbles, } 1 \text { quartzite), } \\
\text { whetstones ( } 7 \text { pieces, each sandstone), whetstone or polishing } \\
\text { stone ( } 1 \text { piece, sandstone), grindstone ( } 1 \text { piece, quartzite), } \\
\text { grindstone or hand mill (1 piece, quartzite); } \\
\text { schist (1 fragment) }\end{array}$ & 105 & $\begin{array}{l}\text { SAV1E 1215, SAV1E 1216, SAV1E, SAV1E } \\
\text { (pounders), SAV1E 1219, SAV1E 1220, } \\
\text { SAV1E 1221, SAV1E 1222, SAV1E 1225, } \\
\text { SAV1E 1226, SAV1E 1227 (whetstones), } \\
\text { SAV1E 1228 (whetstone or polishing stone), } \\
\text { SAV1E 1223 (grindstone), SAV1E 1224 } \\
\text { (grindstone or hand mill) }\end{array}$ & 07.01 .2015 \\
\hline $1034 / 2015$ & $\begin{array}{l}\text { SQ4, 4-5m W-E/3.5m N-S, collapse of SU } \\
105\end{array}$ & stones: grindstone ( 1 small fragment, quartzite) & $\begin{array}{c}105, \\
\text { collapse of } \\
\text { SU }\end{array}$ & SAV1E 1194 & 06.01 .2015 \\
\hline
\end{tabular}




\begin{tabular}{|c|c|c|c|c|c|}
\hline $1001 / 2015$ & $\mathrm{SQ} 4+4 \mathrm{~A}, 0-7.5 \mathrm{~m} \mathrm{~W}-\mathrm{E} / 7.1-12 \mathrm{~m} \mathrm{~N}-\mathrm{S}$ & $\begin{array}{l}\text { stones: hand mill ( } 1 \text { half, quartzite), abrasive stone ( } 1 \text { piece, } \\
\text { quartzite), hammer ( } 1 \text { piece, quartzite), pounders ( } 5 \text { pieces, each } \\
\text { quartzite), whetstones ( } 4 \text { pieces, each sandstone), grindstones } \\
\text { ( } 3 \text { pieces, each quartzite); schist ( } 1 \text { fragment), grindstone } \\
\text { ( } 2 \text { fragments), pounder (4 fragments) }\end{array}$ & 200 & $\begin{array}{l}\text { SAV1E } 1156 \text { (hand mill), SAV1E } 1157 \\
\text { (abrasive stone), SAV1E } 1158 \text { (hammer), } \\
\text { SAV1E 1159, SAV1E 1160, SAV1E 1161, } \\
\text { SAV1E 1162, SAV1E 1163 (pounders), } \\
\text { SAV1E 1164, SAV1E 1165, SAV1E 1166, } \\
\text { SAV1E 1167 (whetstones), SAV1E 1168, } \\
\text { SAV1E 1169, SAV1E 1170 (grindstones) }\end{array}$ & 04.01 .2015 \\
\hline $1010 / 2015$ & SQ4, 0-7.5m W-E/7.1-10m N-S & $\begin{array}{l}\text { stones and architecture?: grindstones ( } 2 \text { fragments, each } \\
\text { quartzite), whetstones ( } 5 \text { pieces, each sandstone), pounders ( } 5 \\
\text { pieces, each quartzite), limestone ( } 1 \text { fragment, possibly } \\
\text { architecture) }\end{array}$ & 202 & $\begin{array}{l}\text { SAV1E 1143, SAV1E } 1144 \text { (grindstones), } \\
\text { SAV1E 1145, SAV1E } 1146 \text {, SAV1E 1147, } \\
\text { SAV1E 1148, SAV1E } 1149 \text { (whetstones), } \\
\text { SAV1E 1151, SAV1E 1152, SAV1E 1153, } \\
\text { SAV1E 1154, SAV1E 1155 (pounders), } \\
\text { SAV1E 1150 (limestone, architecture?) }\end{array}$ & 04.01 .2015 \\
\hline $1017 / 2015$ & $\begin{array}{l}\text { SQ4, 0-7.5m W-E/7.1-10m N-S } \\
(12 \mathrm{~m} \mathrm{~N}-\mathrm{S})\end{array}$ & $\begin{array}{l}\text { stones and architecture?: pounder ( } 3 \text { pieces, } 2 \text { quartzite, } \\
1 \text { silicified stone), abrasive stone ( } 1 \text { piece, sandstone), } \\
\text { whetstones ( } 3 \text { pieces, each sandstone), grindstones ( } 3 \text { pie-ces, } \\
\text { each quartzite), pounder ( } 1 \text { fragment), grindstone } \\
\text { ( } 1 \text { fragment), stone ( } 1 \text { fragment, unclear use), limestone } \\
\text { ( } 2 \text { fragments, perhaps parts of architecture) }\end{array}$ & 202 & $\begin{array}{l}\text { SAV1E 1182, SAV1E 1183, SAV1E 1186 } \\
\text { (pounders), SAV1E 1184, SAV1E 1187 } \\
\text { (abrasive stone), SAV1E 1188, SAV1E 1190, } \\
\text { SAV1E 1191 (whetstones), SAV1E 1192, } \\
\text { SAV1E 1193, SAV1E 1189 (grindstones), } \\
\text { SAV1E 1185 (limestones, architecture?) }\end{array}$ & 05.01 .2015 \\
\hline $1025 / 2015$ & SQ4, 5-8m N-S, surface & $\begin{array}{l}\text { stones: pounder ( } 1 \text { piece, quartzite), abrasive stone ( } 1 \text { piece, } \\
\text { quartzite), whetstones ( } 2 \text { pieces, each sandstone), schist } \\
\text { ( } 3 \text { fragments) }\end{array}$ & 203 & $\begin{array}{l}\text { SAV1E } 1176 \text { (pounder), SAV1E } 1177 \\
\text { (abrasive stone), SAV1E 1178, SAV1E } 1179 \\
\text { (whetstones) }\end{array}$ & 05.01 .2015 \\
\hline $1031 / 2015$ & SQ4, 3.5-6.5m W-E/5-6.2m N-S & stones: grindstones (2 small fragments, each quartzite) & 204 & SAV1E 1195 and SAV1E 1196 (grindstones) & 06.01 .2015 \\
\hline $1072 / 2015$ & $\mathrm{SQ} 4,0-1.5 \mathrm{~m} \mathrm{~W}-\mathrm{E} / 0-2 \mathrm{~m} \mathrm{~N}-\mathrm{S}$ & stone: hand mill (1 fragment, quartzite) & 205 & SAV1E 1256 & 08.01 .2015 \\
\hline $1153 / 2015$ & $\mathrm{SQ} 4,3.5-5 \mathrm{~m} \mathrm{~W}-\mathrm{E} / 9-10 \mathrm{~m} \mathrm{~N}-\mathrm{S}$ & stone: pounder (1 fragment of quartz pebble) & 205 & SAV1E 1823 & 14.01 .2015 \\
\hline $1073 / 2015$ & SQ4, 0-1.5m W-E/0-2m N-S & $\begin{array}{l}\text { stones: pounder ( } 2 \text { pieces, } 1 \text { quartz, } 1 \text { unspecified stone), raw } \\
\text { material ( } 1 \text { fragment, quartz), grindstone ( } 1 \text { piece, quartzite), } \\
\text { hand mills ( } 3 \text { pieces, each quartzite) }\end{array}$ & 205 & $\begin{array}{l}\text { SAV1E 1277, SAV1E } 1278 \text { (pounders), } \\
\text { SAV1E 1279 (raw material), SAV1E } 1280 \\
\text { (grindstone), SAV1E 1281, SAV1E 1282, } \\
\text { SAV1E 1283 (hand mills) }\end{array}$ & 08.01 .2015 \\
\hline $1056 / 2015$ & $\mathrm{SQ} 4,0-4 \mathrm{~m} \mathrm{~W}-\mathrm{E} / 4.7-7.1 \mathrm{~m} \mathrm{~N}-\mathrm{S}$ & $\begin{array}{l}\text { stones and architecture?: pounders ( } 8 \text { pieces, } 4 \text { quartz } \\
\text { pebbles, } 3 \text { quartzite, } 1 \text { not specified), polishing stone/ } \\
\text { burnishing tool ( } 1 \text { piece, silex), whetstones ( } 7 \text { pieces, each } \\
\text { sandstone), hand mill ( } 1 \text { piece, quartzite), grindstone, later } \\
\text { pounder? (1 piece, quartzite), grindstones ( } 2 \text { pieces, each } \\
\text { quartzite), grindstone ( } 1 \text { small fragment); architecture? (1 } \\
\text { large fragment, sandstone), floor tile? (1 large fragment, } \\
\text { siliceous shale? traces of gold?) }\end{array}$ & 205 & $\begin{array}{l}\text { SAV1E 1229, SAV1E 1230, SAV1E 1231, } \\
\text { SAV1E 1232, SAV1E 1233, SAV1E 1242, } \\
\text { SAV1E 1243, SAV1E 1244 (pounders), } \\
\text { SAV1E 1234 (polishing stone/burnishing } \\
\text { tool), SAV1E 1235, SAV1E 1236, SAV1E } \\
\text { 1237, SAV1E 1238, SAV1E 1245, SAV1E } \\
\text { 1246 (whetstones), SAV1E 1247 (hand mill), } \\
\text { SAV1E 1248 (grindstone, later pounder?), } \\
\text { SAV1E 1249, SAV1E 1241 (grindstones), } \\
\text { SAV1E 1239 (architecture), SAV1E 1240 } \\
\text { (floor tile?) }\end{array}$ & 07.01 .2015 \\
\hline
\end{tabular}




\begin{tabular}{|c|c|c|c|c|c|}
\hline $1081 / 2015$ & $\mathrm{SQ} 4,0-4 \mathrm{~m} \mathrm{~W}-\mathrm{E} / 5-10 \mathrm{~m} \mathrm{~N}-\mathrm{S}$ & $\begin{array}{l}\text { stones: pounders ( } 3 \text { pieces, each quartz), grindstones } \\
\text { ( } 6 \text { pieces, each quartzite), token/gaming piece ( } 1 \text { piece, } \\
\text { unspecified stone), raw material ( } 1 \text { piece, quartz) }\end{array}$ & 205 & $\begin{array}{l}\text { SAV1E 1293, SAV1E 1294, SAV1E } 1300 \\
\text { (pounders), SAV1E 1287, SAV1E 1295, } \\
\text { SAV1E 1296, SAV1E 1297, SAV1E } 1298, \\
\text { SAV1E 1299 (grindstones), SAV1E } 1291 \\
\text { (token/gaming piece), SAV1E } 1292 \text { (raw } \\
\text { material) }\end{array}$ & 10.01 .2015 \\
\hline $1083 / 2015$ & $\mathrm{SQ} 4,0-4 \mathrm{~m} \mathrm{~W}-\mathrm{E} / 5-10 \mathrm{~m} \mathrm{~N}-\mathrm{S}$ & $\begin{array}{l}\text { stones: whetstones (11 pieces, each sandstone); } \\
\text { bone ( } 1 \mathrm{bag})\end{array}$ & 205 & $\begin{array}{l}\text { SAV1E 1310, SAV1E 1311, SAV1E 1312, } \\
\text { SAV1E 1313, SAV1E 1314, SAV1E 1315, } \\
\text { SAV1E 1316, SAV1E 1317, SAV1E 1318, } \\
\text { SAV1E 1319, SAV1E } 1320 \text { (whetstones) }\end{array}$ & 10.01 .2015 \\
\hline $1102 / 2015$ & SQ4, 0-4m W-E/6-10m N-S & $\begin{array}{l}\text { stones and architecture: polishing stones ( } 5 \text { pieces, } \\
2 \text { quartzite, } 1 \text { quartz, } 1 \text { siliceous shale, } 1 \text { limestone), } \\
\text { grindstones ( } 8 \text { pieces, each sandstone), hand mills ( } 4 \text { pieces, } \\
\text { each quartzite), whetstones ( } 12 \text { pieces, each sandstone), } \\
\text { pounders ( } 11 \text { pieces, } 10 \text { quartz, } 1 \text { quartzite), hammer ( } 1 \text { piece, } \\
\text { quartzite), worked stone ( } 1 \text { piece, unidentified stone), palette } \\
\text { ( } 1 \text { fragment, schist); architecture ( } 1 \text { piece, sandstone), pivot } \\
\text { stone ( } 1 \text { fragment, sandstone), architecture ( } 1 \text { fragment, } \\
\text { unspecified stone) }\end{array}$ & 205 & $\begin{array}{l}\text { SAV1E 1329, SAV1E 1330, SAV1E 1331, } \\
\text { SAV1E 1332, SAV1E 1333 (polishing } \\
\text { stones), SAV1E 1357, SAV1E 1358, SAV1E } \\
\text { 1359, SAV1E 1360, SAV1E 1361, SAV1E } \\
\text { 1362, SAV1E 1363, SAV1E 1364 } \\
\text { (grindstones), SAV1E 1334, SAV1E 1335, } \\
\text { SAV1E 1336, SAV1E 1347 (hand mills), } \\
\text { SAV1E 1348, SAV1E 1349, SAV1E 1350, } \\
\text { SAV1E 1351, SAV1E 1352, SAV1E 1353, } \\
\text { SAV1E 1354, SAV1E 1355, SAV1E 1356, } \\
\text { SAV1E 1371, SAV1E 1372, SAV1E 1373 } \\
\text { (whetstones), SAV1E 1369, SAV1E 1370, } \\
\text { SAV1E 1337, SAV1E 1338, SAV1E 1339, } \\
\text { SAV1E 1340, SAV1E 1341, SAV1E 1342, } \\
\text { SAV1E 1343, SAV1E 1344, SAV1E 1345 } \\
\text { (pounders), SAV1E 1346 (hammer), SAV1E } \\
\text { 1367 (worked stone), SAV1E 1368 (palette), } \\
\text { SAV1E 1366 (architecture), SAV1E 1328 } \\
\text { (pivot stone) }\end{array}$ & 11.01 .2015 \\
\hline $1068 / 2015$ & $\mathrm{SQ} 4,0-4 \mathrm{~m} \mathrm{~W}-\mathrm{E} / 7.1-9.7 \mathrm{~m} \mathrm{~N}-\mathrm{S}$ & $\begin{array}{l}\text { stones and architecture?: pounders ( } 6 \text { pieces, } 2 \text { quartz } \\
\text { pebbles, } 2 \text { siliceous shale?, } 2 \text { quartz), polishing stone? } \\
\text { (1 fragment, unspecified stone), hand mill (1 fragment, } \\
\text { quartzite), grindstones ( } 2 \text { pieces, each quartzite), whetstones } \\
\text { (4 pieces, each sandstone), whetstone ( } 1 \text { fragment); stone } \\
\text { ( } 1 \text { fragment, unspecified stone, original function unknown, } \\
\text { perhaps paving stone), piece of architecture, later re-used as } \\
\text { tool? ( } 1 \text { fragment, quartzite) }\end{array}$ & 205 & $\begin{array}{l}\text { SAV1E 1257, SAV1E 1258, SAV1E 1259, } \\
\text { SAV1E 1260, SAV1E 1261, SAV1E 1270, } \\
\text { (pounders), SAV1E 1262 (polishing stone?), } \\
\text { SAV1E 1269 (hand mill), SAV1E 1271, } \\
\text { SAV1E 1272 (grindstones), SAV1E 1273, } \\
\text { SAV1E 1274, SAV1E 1275, SAV1E 1276 } \\
\text { (whetstones), SAV1E 1267 (stone, original } \\
\text { function unknown, perhaps paving stone), } \\
\text { SAV1E 1268 (piece of architecture, later re- } \\
\text { used as tool?) }\end{array}$ & 08.01 .2015 \\
\hline $1115 / 2015$ & $\mathrm{SQ} 4,0-4 \mathrm{~m} \mathrm{~W}-\mathrm{E} / 7-9 \mathrm{~m} \mathrm{~N}-\mathrm{S} / 9-10 \mathrm{~m} \mathrm{~N}-\mathrm{S}$ & $\begin{array}{l}\text { stones: whetstones ( } 15 \text { pieces, each sandstone), hand mills } \\
\text { ( } 10 \text { pieces, each quartzite), grindstones ( } 14 \text { pieces, each } \\
\text { quartzite), pounders ( } 11 \text { pieces, each quartz), worked stones } \\
\text { ( } 2 \text { pieces, } 1 \text { quartzite, } 1 \text { unspecified stone), polishing stones }\end{array}$ & 205 & $\begin{array}{l}\text { SAV1E 1841, SAV1E 1842, SAV1E } 1843, \\
\text { SAV1E 1844, SAV1E 1845, SAV1E 1428, } \\
\text { SAV1E 1438, SAV1E 1439, SAV1E 1440, } \\
\text { SAV1E 1441, SAV1E 1442, SAV1E 1443, }\end{array}$ & 12.01 .2015 \\
\hline
\end{tabular}




\begin{tabular}{|c|c|c|c|c|c|}
\hline & & $\begin{array}{l}\text { ( } 2 \text { pieces, } 1 \text { siliceous shale?, } 1 \text { quartz), stone vessel } \\
\text { (1 fragment, quartzite), grindstone ( } 3 \text { fragments), hand mill } \\
\text { (1 fragment) }\end{array}$ & & $\begin{array}{l}\text { SAV1E 1444, SAV1E 1445, SAV1E 1446 } \\
\text { (whetstones), SAV1E 1836, SAV1E 1837, } \\
\text { SAV1E 1838, SAV1E 1398, SAV1E 1399, } \\
\text { SAV1E 1400, SAV1E 1401, SAV1E 1402, } \\
\text { SAV1E 1403, SAV1E 1427 (hand mills), } \\
\text { SAV1E 1831, SAV1E 1832, SAV1E 1833, } \\
\text { SAV1E 1834, SAV1E 1835, SAV1E 1429, } \\
\text { SAV1E 1430, SAV1E 1431, SAV1E 1432, } \\
\text { SAV1E 1433, SAV1E 1434, SAV1E 1435, } \\
\text { SAV1E 1436, SAV1E 1437 (grindstones), } \\
\text { SAV1E 1404, SAV1E 1405, SAV1E 1406, } \\
\text { SAV1E 1407, SAV1E 1408, SAV1E 1825, } \\
\text { SAV1E 1826, SAV1E 1827, SAV1E 1828, } \\
\text { SAV1E 1829, SAV1E 1830 (pounders), SAV1E } \\
\text { 1394, SAV1E 1395 (worked stones), SAV1E } \\
\text { 1396, SAV1E 1397 (polishing stones), SAV1E } \\
\text { 1824 (stone vessel) }\end{array}$ & \\
\hline $1099 / 2015$ & $\mathrm{SQ} 4,3.1-4 \mathrm{~m} \mathrm{~W}-\mathrm{E} / 6-7.5 \mathrm{~m} \mathrm{~N}-\mathrm{S}$ & $\begin{array}{l}\text { stones: pounders ( } 2 \text { pieces, } 1 \text { quartz, } 1 \text { quartzite), whetstone } \\
\text { ( } 2 \text { pieces, each sandstone), worked stone (1 piece, quartzite), } \\
\text { mortar ( } 1 \text { fragment, sandstone) }\end{array}$ & 205 & $\begin{array}{l}\text { SAV1E 1322, SAV1E } 1323 \text { (pounders), } \\
\text { SAV1E 1324, SAV1E } 1325 \text { (whetstones), } \\
\text { SAV1E 1326 (worked stone), SAV1E } 1327 \\
\text { (mortar)s }\end{array}$ & 11.01 .2015 \\
\hline $1159 / 2015$ & $\mathrm{SQ} 4,3.5-5 \mathrm{~m} \mathrm{~W}-\mathrm{E} / 9-10 \mathrm{~m} \mathrm{~N}-\mathrm{S}$ & $\begin{array}{l}\text { stones: pounders ( } 2 \text { pieces, each quartz), whetstones } \\
\text { ( } 2 \text { pieces, each sandstone), grindstone (1 piece, quartzite); } \\
\text { schist ( } 5 \text { fragments) }\end{array}$ & 205 & $\begin{array}{l}\text { SAV1E 1476, SAV1E } 1477 \text { (pounders), } \\
\text { SAV1E 1478, SAV1E } 1479 \text { (whetstones), } \\
\text { SAV1E } 1480 \text { (grindstone) }\end{array}$ & 14.01.2015 \\
\hline $1165 / 2015$ & $\mathrm{SQ} 4,3.5-5 \mathrm{~m} \mathrm{~W}-\mathrm{E} / 9-10 \mathrm{~m} \mathrm{~N}-\mathrm{S}$ & $\begin{array}{l}\text { stones: pounders ( } 3 \text { pieces, } 2 \text { quartz, } 1 \text { quartzite), hand mill } \\
\text { ( } 2 \text { pieces, each quartzite), hammer ( } 1 \text { piece, quartzite) }\end{array}$ & 205 & $\begin{array}{l}\text { SAV1E 1889, SAV1E 1891, SAV1E } 1892 \\
\text { (pounders), SAV1E 1893, SAV1E } 1894 \text { (hand } \\
\text { mills), SAV1E } 1890 \text { (hammer) }\end{array}$ & 15.01.2015 \\
\hline $1132 / 2015$ & SQ4A, 0-3m W-E/0-2m N-S & $\begin{array}{l}\text { stones and architecture?: pounders ( } 11 \text { pieces, } 8 \text { quartz, } \\
2 \text { unspecified stone, } 1 \text { siliceous shale?), grindstones ( } 6 \text { pieces, } \\
\text { each quartzite), hand mills ( } 4 \text { pieces, } 3 \text { quartzite, } \\
1 \text { sandstone), whetstones ( } 2 \text { pieces each sandstone), polishing } \\
\text { stone ( } 1 \text { piece, schist), abrasive stone ( } 1 \text { piece, quartzite), } \\
\text { statue?( } 1 \text { small fragment, siliceous shale), stone vessel? } \\
\text { (1 fragment, quartzite); architecture? ( } 2 \text { fragments, each } \\
\text { quartzite) }\end{array}$ & 205 & $\begin{array}{l}\text { SAV1E 1484, SAV1E 1485, SAV1E 1486, } \\
\text { SAV1E 1487, SAV1E 1488, SAV1E 1489, } \\
\text { SAV1E 1490, SAV1E 1491, SAV1E 1492, } \\
\text { SAV1E 1493, SAV1E 1494 (pounders), } \\
\text { SAV1E 1806, SAV1E 1807, SAV1E 1808, } \\
\text { SAV1E 1809, SAV1E 1810, SAV1E 1811 } \\
\text { (grindstones), SAV1E 1822, SAV1E 1800, } \\
\text { SAV1E 1801, SAV1E 1802 (hand mills), } \\
\text { SAV1E 1803, SAV1E } 1804 \text { (whetstones), } \\
\text { SAV1E 1499 (polishing stone), SAV1E 1805 } \\
\text { (abrasive stone), SAV1E 1495 (statue?), } \\
\text { SAV1E } 1496 \text { (stone vessel?), SAV1E 1497, } \\
\text { SAV1E } 1498 \text { (architecture?) }\end{array}$ & 13.01 .2015 \\
\hline
\end{tabular}




\begin{tabular}{|c|c|c|c|c|c|}
\hline $1138 / 2015$ & SQ4A, $1.7-3.2 \mathrm{~m} \mathrm{~W}-\mathrm{E} / 0-1.5 \mathrm{~m} \mathrm{~N}-\mathrm{S}$ & $\begin{array}{l}\text { stones and architecture?: pounders ( } 13 \text { pieces, } 9 \text { quartz, } \\
4 \text { siliceous shale), whetstones ( } 6 \text { pieces, } 1 \text { silicified stone, } 5 \\
\text { sandstone), hand mills ( } 9 \text { pieces, each quartzite); architecture } \\
\text { ( } 2 \text { large fragments, } 1 \text { unspecified stone, } \\
1 \text { sandstone and possibly base of door jamb) }\end{array}$ & 205 & $\begin{array}{l}\text { SAV1E 1848, SAV1E 1849, SAV1E 1850, } \\
\text { SAV1E 1851, SAV1E 1852, SAV1E 1853, } \\
\text { SAV1E 1854, SAV1E 1855, SAV1E 1856, } \\
\text { SAV1E 1895, SAV1E 1896, SAV1E 1897, } \\
\text { SAV1E 1906 (pounders), SAV1E 1982, } \\
\text { SAV1E 1983, SAV1E 1984, SAV1E 1985, } \\
\text { SAV1E 1986, SAV1E 1907 (whetstones), } \\
\text { SAV1E 1857, SAV1E 1858, SAV1E 1859, } \\
\text { SAV1E 1860, SAV1E 1861, SAV1E 1862, } \\
\text { SAV1E 1863, SAV1E 1864, SAV1E } 1865 \\
\text { (hand mills), SAV1E 1908, SAV1E } 1909 \\
\text { (architecture) }\end{array}$ & 14.01 .2015 \\
\hline $1170 / 2015$ & SQ4A, 4-5m W-E/1.4-2m N-S & $\begin{array}{l}\text { stones: pounders ( } 2 \text { pieces, } 1 \text { siliceous shale?, } 1 \text { quartz } \\
\text { pebble), hand mill (1 piece, quartzite); schist ( } 3 \text { fragments) }\end{array}$ & 205 & $\begin{array}{l}\text { SAV1E 1885, SAV1E } 1886 \text { (pounders), } \\
\text { SAV1E } 1887 \text { (hand mill) }\end{array}$ & 15.01 .2015 \\
\hline $1193 / 2015$ & $\mathrm{SQ} 4,5-5.7 \mathrm{~m} \mathrm{~W}-\mathrm{E} / 7-8.5 \mathrm{~m} \mathrm{~N}-\mathrm{S}$ & $\begin{array}{l}\text { stones and bone: hand mills ( } 5 \text { pieces, each quartzite), } \\
\text { pounders ( } 2 \text { pieces, each quartzite), grindstone (1 piece, } \\
\text { quartzite); bone ( } 1 \text { bag) }\end{array}$ & 208 & $\begin{array}{l}\text { SAV1E 1721, SAV1E 1722, SAV1E 1723, } \\
\text { SAV1E 1724, SAV1E } 1725 \text { (hand mills), } \\
\text { SAV1E 1727, SAV1E } 1728 \text { (pounders), } \\
\text { SAV1E } 1726 \text { (grindstone) }\end{array}$ & 17.01.2015 \\
\hline $1199 / 2015$ & SQ4, 4.2-5.9m W-E/6.5-8m N-S & $\begin{array}{l}\text { stones: pounder (1 quartz pebble), grindstone (1 fragment, } \\
\text { quartzite) }\end{array}$ & 209 & SAV1E 1870, SAV1E 1871 & 17.01.2015 \\
\hline $1203 / 2015$ & $\mathrm{SQ} 4+4 \mathrm{~A}, 6-7.5 \mathrm{~m} \mathrm{~W}-\mathrm{E} / 8.5-12 \mathrm{~m} \mathrm{~N}-\mathrm{S}$ & $\begin{array}{l}\text { stones: hand mill ( } 1 \text { fragment, quartzite), pounder } \\
\text { ( } 1 \text { fragment, quartz pebble), whetstone ( } 1 \text { fragment, } \\
\text { sandstone); schist (1 fragment) ( } 2 \text { pieces) }\end{array}$ & 210 & SAV1E 1990, SAV1E 1991, SAV1E 1992 & 17.01.2015 \\
\hline $1209 / 2015$ & SQ4A, 3.7-7m W-E/0-2m N-S & $\begin{array}{l}\text { stones: pounder ( } 1 \text { fragment, quartz pebble), grindstones } \\
\text { ( } 2 \text { fragments, each quartzite); schist ( } 2 \text { fragments) }\end{array}$ & 211 & SAV1E 1987, SAV1E 1988, SAV1E 1989 & 17.01.2015 \\
\hline $1215 / 2015$ & $\mathrm{SQ} 4,7-9.2 \mathrm{~m} \mathrm{~W}-\mathrm{E} / 6.8-8 \mathrm{~m} \mathrm{~N}-\mathrm{S}$ & stone: pumice stone? (1 small fragment) & 212 & SAV1E 1867 & 18.01 .2015 \\
\hline $1223 / 2015$ & $\mathrm{SQ} 4,7-9.2 \mathrm{~m} \mathrm{~W}-\mathrm{E} / 6.8-8 \mathrm{~m} \mathrm{~N}-\mathrm{S}$ & $\begin{array}{l}\text { stones: pounders ( } 2 \text { pieces, } 1 \text { quartzite, } 1 \text { siliceous shale?), } \\
\text { hand mill ( } 1 \text { fragment, quartzite), vessel (1 fragment, } \\
\text { unspecified stone), grindstone or hand mill (1 fragment) }\end{array}$ & 212 & $\begin{array}{l}\text { SAV1E 1993, SAV1E } 1994 \text { (pounders), } \\
\text { SAV1E } 1996 \text { (hand mill), SAV1E } 1995 \\
\text { (vessel) }\end{array}$ & 19.01.2015 \\
\hline $1212 / 2015$ & $\mathrm{SQ} 4,7-9.2 \mathrm{~m} \mathrm{~W}-\mathrm{E} / 6.8-8 \mathrm{~m} \mathrm{~N}-\mathrm{S}$ & $\begin{array}{l}\text { stones: pounders ( } 4 \text { pieces, } 2 \text { quartz, } 2 \text { quartzite, } 1 \text { siliceous } \\
\text { shale), whetstones ( } 4 \text { pieces, each sandstone), hand mill } \\
\text { ( } 1 \text { piece, quartzite), grindstones or hand mills ( } 2 \text { pieces each } \\
\text { quartzite), polishing stone ( } 1 \text { piece, unidentified stone) }\end{array}$ & 212 & $\begin{array}{l}\text { SAV1E 1730, SAV1E 1731, SAV1E 1732, } \\
\text { SAV1E } 1733 \text { (pounders), I SAV1E 1737, } \\
\text { SAV1E 1738, SAV1E 1739, SAV1E } 1740 \\
\text { (whetstones), SAV1E } 1734 \text { (hand mill), } \\
\text { SAV1E 1735, SAV1E } 1736 \text { (grindstones or } \\
\text { hand mills), SAV1E } 1729 \text { (polishing stone) }\end{array}$ & 18.01 .2015 \\
\hline $1248 / 2015$ & $\mathrm{SQ} 4+4 \mathrm{~A}, 5-6.5 \mathrm{~m} \mathrm{~W}-\mathrm{E} / 8.2-12 \mathrm{~m} \mathrm{~N}-\mathrm{S}$ & $\begin{array}{l}\text { stones: pounders ( } 5 \text { pieces, each quartz), hand mills ( } 3 \text { pieces, } \\
\text { each quartzite), grindstone ( } 1 \text { piece, quartzite), polishing } \\
\text { stone ( } 1 \text { piece, quartzite) }\end{array}$ & 213 & $\begin{array}{l}\text { SAV1E 0952, SAV1E 0953, SAV1E 1741, } \\
\text { SAV1E 1742, SAV1E } 1743 \text { (pounders), } \\
\text { SAV1E 1747, SAV1E 1744, SAV1E 1745 } \\
\text { (hand mills), SAV1E } 1746 \text { (grindstone), } \\
\text { SAV1E 0951 (polishing stone) }\end{array}$ & 20.01.2015 \\
\hline
\end{tabular}




\begin{tabular}{|c|c|c|c|c|c|}
\hline $1237 / 2015$ & $\mathrm{SQ} 4+4 \mathrm{~A}, 6.5-8 \mathrm{~m} \mathrm{~W}-\mathrm{E} / 8.2-12 \mathrm{~m} \mathrm{~N}-\mathrm{S}$ & $\begin{array}{l}\text { stones: pounders ( } 3 \text { pieces, } 2 \text { quartz, } 1 \text { unspecified stone), } \\
\text { whetstones ( } 3 \text { pieces, each sandstone), grindstone ( } 1 \text { piece, } \\
\text { quartzite), hand mill ( } 1 \text { piece, quartzite), grindstone or hand } \\
\text { mill ( } 1 \text { piece, unspecified stone) }\end{array}$ & 213 & $\begin{array}{l}\text { SAV1E 1997, SAV1E 1998, SAV1E } 1999 \\
\text { (pounders), SAV1E 1711, SAV1E 1712, } \\
\text { SAV1E 1713 (whetstones), SAV1E 1710 } \\
\text { (grindstone), SAV1E 1708 (hand mill), } \\
\text { SAV1E 1709 (grindstone or hand mill) }\end{array}$ & 20.01.2015 \\
\hline $1233 / 2015$ & $\mathrm{SQ} 4+4 \mathrm{~A}, 6-10 \mathrm{~m} \mathrm{~W}-\mathrm{E} / 8.2-12 \mathrm{~m} \mathrm{~N}-\mathrm{S}$ & $\begin{array}{l}\text { stones and lime: whetstones ( } 3 \text { pieces, each sandstone), } \\
\text { pounders ( } 4 \text { pieces, } 1 \text { siliceous shale?, } 3 \text { quartz), worked } \\
\text { stone ( } 3 \text { fragments, unclear function); lime ( } 1 \text { bag) }\end{array}$ & 213 & $\begin{array}{l}\text { SAV1E 1878, SAV1E 1879, SAV1E 1880, } \\
\text { SAV1E 1881 (whetstones), SAV1E 1882, } \\
\text { SAV1E 1883, SAV1E } 1884 \text { (pounders) }\end{array}$ & 19.01.2015 \\
\hline $1262 / 2015$ & $\mathrm{SQ} 4+4 \mathrm{~A}, 3.5-5.5 \mathrm{~m} \mathrm{~W}-\mathrm{E} / 8.3-11.3 \mathrm{~m} \mathrm{~N}-\mathrm{S}$ & $\begin{array}{l}\text { stones and chalk: pounders ( } 11 \text { pieces, } 5 \text { quartzite, } 6 \text { quartz), } \\
\text { whetstones ( } 3 \text { pieces, each sandstone), grindstones ( } 6 \text { pieces, } \\
5 \text { quartzite, } 1 \text { sandstone), hand mills ( } 4 \text { pieces, each } \\
\text { quartzite), grindstones or hand mills ( } 2 \text { pieces, each } \\
\text { quartzite); chalk ( } 1 \text { piece) }\end{array}$ & 214 & $\begin{array}{l}\text { SAV1E 1749, SAV1E 1750, SAV1E 1751, } \\
\text { SAV1E 1752, SAV1E 1753, SAV1E 1754, } \\
\text { SAV1E 1755, SAV1E 1756, SAV1E 1757, } \\
\text { SAV1E 1758, SAV1E 1759 (pounders), } \\
\text { SAV1E 1760, SAV1E 1761, SAV1E } 1762 \\
\text { (whetstones), SAV1E 1767, SAV1E 1768, } \\
\text { SAV1E 1769, SAV1E 1770, SAV1E 1775, } \\
\text { SAV1E 1777 (grindstones), SAV1E 1776, } \\
\text { SAV1E 1778, SAV1E 1763, SAV1E 1766 } \\
\text { (hand mills), SAV1E 1764, SAV1E } 1765 \\
\text { (grindstones or hand mills) }\end{array}$ & 21.01.2015 \\
\hline $1270 / 2015$ & $\mathrm{SQ} 4,0-1 \mathrm{~m} \mathrm{~W}-\mathrm{E} / 8.8-9.7 \mathrm{~m} \mathrm{~N}-\mathrm{S}$ & $\begin{array}{l}\text { stones: stone vessel (1 fragment, unspecified stone), pounder } \\
\text { (1 piece, quartz) }\end{array}$ & 215 & SAV1E 1869, SAV1E 1935 & 22.01 .2015 \\
\hline $1292 / 2015$ & SQ4, 5-8.8m N-S, section cleaning & $\begin{array}{l}\text { stones and architecture?: pounders ( } 3 \text { pieces, } 1 \text { unspecified } \\
\text { stone, } 1 \text { siliceous shale, } 1 \text { quartzite), grindstones ( } 3 \text { pieces, } \\
\text { each quartzite), hand mills ( } 3 \text { pieces, each quartzite), } \\
\text { whetstone ( } 1 \text { piece, sandstone); schist ( } 3 \text { fragments); } \\
\text { architecture? ( } 1 \text { piece, sandstone) }\end{array}$ & 219 & $\begin{array}{l}\text { SAV1E 1971, SAV1E 1972, SAV1E 1973 } \\
\text { (pounders), SAV1E 1975, SAV1E 1976, SAV1E } \\
1977 \text { (grindstones), SAV1E 1978, SAV1E 1979, } \\
\text { SAV1E } 1980 \text { (hand mills), SAV1E 1981 } \\
\text { (whetstone), SAV1E 1974 (architecture?) }\end{array}$ & 22.01.2015 \\
\hline $1306 / 2015$ & $\mathrm{SQ} 4,0-1.2 \mathrm{~m} \mathrm{~W}-\mathrm{E} / 0-4.5 \mathrm{~m} \mathrm{~N}-\mathrm{S}$ & stone: stela (1 small fragment, limestone, New Kingdom) & 220 & SAV1E 1938 & 24.01 .2015 \\
\hline $1304 / 2015$ & $\mathrm{SQ} 4,0-1.2 \mathrm{~m} \mathrm{~W}-\mathrm{E} / 0-4.5 \mathrm{~m} \mathrm{~N}-\mathrm{S}$ & stone, worked (1 fragment) & 220 & & 24.01 .2015 \\
\hline $1297 / 2015$ & $\mathrm{SQ} 4,0-1.2 \mathrm{~m} \mathrm{~W}-\mathrm{E} / 0-4.5 \mathrm{~m} \mathrm{~N}-\mathrm{S}$ & $\begin{array}{l}\text { stones and architecture?: grindstone ( } 2 \text { pieces, each quartzite), } \\
\text { grindstones or hand mills ( } 3 \text { pieces, each quartzite), pounder } \\
\text { (1 piece, quartz); schist ( } \text { fragments); architecture? } \\
\text { (1 fragment, limestone) }\end{array}$ & 220 & $\begin{array}{l}\text { SAV1E 1719, SAV1E } 1720 \text { (grindstones), } \\
\text { SAV1E 1716, SAV1E 1717, SAV1E 1718 } \\
\text { (grindstone or hand mills), SAV1E 1714 } \\
\text { (pounder), SAV1E 1715 (architecture?) }\end{array}$ & 22.01 .2015 \\
\hline $1305 / 2015$ & $\mathrm{SQ} 4,0-1.2 \mathrm{~m} \mathrm{~W}-\mathrm{E} / 0-4.5 \mathrm{~m} \mathrm{~N}-\mathrm{S}$ & $\begin{array}{l}\text { stones and architecture?: pounders ( } 6 \text { pieces, } 1 \text { unspecified } \\
\text { stone, } 1 \text { siliceous shale, } 4 \text { quartzite), hand mills ( } 4 \text { pieces, } \\
\text { each quartzite), grindstones or hand mill ( } 1 \text { piece, quartzite), } \\
\text { grindstones ( } 3 \text { pieces, each quartzite), hammer (1 piece, } \\
\text { quartzite); schist ( } 12 \text { fragments); architecture? (1 large } \\
\text { fragment, sandstone) }\end{array}$ & 220 & $\begin{array}{l}\text { SAV1E 1945, SAV1E 1946, SAV1E 1948, } \\
\text { SAV1E 1949, SAV1E 1950, SAV1E 1951 } \\
\text { (pounders), SAV1E 1954, SAV1E 1955, } \\
\text { SAV1E 1957, SAV1E 1959 (hand } \\
\text { mills)SAV1E 1952 (grindstone or hand mill), } \\
\text { SAV1E 1958, SAV1E 1960, SAV1E 1953 } \\
\text { (grindstones), SAV1E 1947 (hammer), } \\
\text { SAV1E 1956 (architecture) }\end{array}$ & 24.01.2015 \\
\hline
\end{tabular}




\begin{tabular}{|c|c|c|c|c|c|}
\hline $1318 / 2015$ & $\mathrm{SQ} 4,0-0.8 \mathrm{~m} \mathrm{~W}-\mathrm{E} / 4.4-5.1 \mathrm{~m} \mathrm{~N}-\mathrm{S}$ & $\begin{array}{l}\text { stones: grindstone or hand mill (1 fragment, quartzite); schist } \\
\text { (1 fragment) }\end{array}$ & 222 & & 24.01 .2015 \\
\hline $1324 / 2015$ & $\mathrm{SQ} 4,1-2.7 \mathrm{~m} \mathrm{~W}-\mathrm{E} / 3.7-5 \mathrm{~m} \mathrm{~N}-\mathrm{S}$ & stone: blade (1 piece, silex, New Kingdom?) & 223 & SAV1E 1962 & 24.01 .2015 \\
\hline $1342 / 2015$ & $\mathrm{SQ} 4,0.4-3.3 \mathrm{~m} \mathrm{~W}-\mathrm{E} / 6-7.5 \mathrm{~m} \mathrm{~N}-\mathrm{S}$ & $\begin{array}{l}\text { stones and architecture?: hand mills ( } 6 \text { fragments, each } \\
\text { quartzite), grindstones ( } 2 \text { pieces, each quartzite), } \\
\text { pounders ( } 4 \text { pieces, each quartz); architecture? ( } 1 \text { fragment, } \\
\text { limestone) }\end{array}$ & 226 & $\begin{array}{l}\text { SAV1E 2050, SAV1E 2051, SAV1E 2052, } \\
\text { SAV1E 2053, SAV1E 2054, SAV1E } 2055 \\
\text { (hand mills), SAV1E 2056, SAV1E } 2057 \\
\text { (grindstones), SAV1E 1784, SAV1E } 1785, \\
\text { SAV1E 1786, SAV1E } 1787 \text { (pounders), } \\
\text { SAV1E 1783 (architecture?) }\end{array}$ & 27.01.2015 \\
\hline $1353 / 2015$ & $\mathrm{SQ} 4+4 \mathrm{~A}, 0.4-5 \mathrm{~m} \mathrm{~W}-\mathrm{E} / 7.5-12 \mathrm{~m} \mathrm{~N}-\mathrm{S}$ & stone: grindstone (1 fragment, quartzite) & 227 & SAV1E 1788 & 28.01 .2015 \\
\hline $1367 / 2015$ & $\mathrm{SQ} 4+4 \mathrm{~A}, 0.4-5 \mathrm{~m} \mathrm{~W}-\mathrm{E} / 7.5-12 \mathrm{~m} \mathrm{~N}-\mathrm{S}$ & $\begin{array}{l}\text { stones and architecture: pounders ( } 11 \text { pieces, } 1 \text { quartzite, } \\
1 \text { siliceous shale?, } 9 \text { quartz), grindstones ( } 3 \text { pieces, each } \\
\text { quartzite), hand mills ( } 5 \text { pieces, each sandstone), whetstones } \\
\text { ( } 3 \text { pieces, each sandstone); architecture (1 fragment, } \\
\text { sandstone) }\end{array}$ & 228 & $\begin{array}{l}\text { SAV1E 2079, SAV1E 2080, SAV1E 2081, } \\
\text { SAV1E 2082, SAV1E 2083, SAV1E 2084, } \\
\text { SAV1E 2085, SAV1E 2086, SAV1E 2087, } \\
\text { SAV1E 2088, SAV1E 2089 (pounders), } \\
\text { SAV1E 2090, SAV1E 2095, SAV1E } 2096 \\
\text { (grindstones), SAV1E 2101, SAV1E 2091, } \\
\text { SAV1E 2092, SAV1E 2093, SAV1E 2094 } \\
\text { (hand mills), SAV1E 2097, SAV1E 2098, } \\
\text { SAV1E 2099 (whetstones), SAV1E } 2100 \\
\text { (architecture) }\end{array}$ & 29.01 .2015 \\
\hline $1381 / 2015$ & SQ4A, 3.5-5.1m W-E/0-2m N-S & $\begin{array}{l}\text { stones: pounders ( } 4 \text { pieces, } 2 \text { quartzite, } 2 \text { quartz), hand mills } \\
\text { ( } 5 \text { pieces, } 4 \text { sandstone, } 1 \text { quartzite), worked stones ( } 2 \text { pieces, } \\
\text { each sandstone), whetstone ( } 1 \text { piece, sandstone), polishing } \\
\text { stone ( } 1 \text { piece, sandstone), pounders ( } 3 \text { pieces, barely used })\end{array}$ & 229 & $\begin{array}{l}\text { SAV1E 2173, SAV1E 2174, SAV1E 2175, } \\
\text { SAV1E 2176 (pounders), SAV1E 2180, SAV1E } \\
\text { 2181, SAV1E 2182, SAV1E 2183, SAV1E } \\
\text { 2184 (hand mills), SAV1E 2186, SAV1E } 2187 \\
\text { (worked stones), SAV1E 2185 (whetstone), } \\
\text { SAV1E } 2172 \text { (polishing stone) }\end{array}$ & 31.01 .2015 \\
\hline $1390 / 2015$ & SQ4A, $5.1-7 \mathrm{~m} \mathrm{~W}-\mathrm{E} / 0-2 \mathrm{~m} \mathrm{~N}-\mathrm{S}$ & $\begin{array}{l}\text { stones: pounders ( } 2 \text { pieces, } 1 \text { quartz, } 1 \text { silicified wood), } \\
\text { worked stones ( } 2 \text { pieces, } 1 \text { sandstone, } 1 \text { unspecified stone) }\end{array}$ & 230 & $\begin{array}{l}\text { SAV1E 2146, SAV1E } 2147 \text { (pounders), SAV1E } \\
\text { 2148, SAV1E 2149 (worked stones) }\end{array}$ & 31.01 .2015 \\
\hline $1394 / 2015$ & SQ4A, $4.4-5.4 \mathrm{~m} \mathrm{~W}-\mathrm{E} / 1-1.7 \mathrm{~m} \mathrm{~N}-\mathrm{S}$ & stone, worked (1 fragment, quartzite) & 231 & SAV1E 2058 & 31.01 .2015 \\
\hline $1407 / 2015$ & $\mathrm{SQ} 4+4 \mathrm{~A}, 8.8-10 \mathrm{~m} \mathrm{~W}-\mathrm{E} / 9-12 \mathrm{~m} \mathrm{~N}-\mathrm{S}$ & $\begin{array}{l}\text { stones: pounders ( } 2 \text { pieces, } 1 \text { quartz, } 1 \text { siliceous shale?), } \\
\text { grindstone ( } 1 \text { fragment, sandstone), hand mills ( } 3 \text { fragments } \\
\text { each sandstone), worked stone ( } 1 \text { fragment, sandstone), } \\
\text { worked stone ( } 1 \text { fragment); schist ( } 2 \text { fragments) }\end{array}$ & 234 & $\begin{array}{l}\text { SAV1E 2165, SAV1E } 2166 \text { (pounders), SAV1E } \\
2167 \text { (grindstone), SAV1E 2168, SAV1E 2169, } \\
\text { SAV1E 2170, SAV1E } 2171 \text { (worked stone) }\end{array}$ & 01.02 .2015 \\
\hline $1412 / 2015$ & $\mathrm{SQ} 4+4 \mathrm{~A}, 9.2-10.5 \mathrm{~m} \mathrm{~W}-\mathrm{E} / 9-12 \mathrm{~m} \mathrm{~N}-\mathrm{S}$ & $\begin{array}{l}\text { stones: grindstone ( } 1 \text { fragment, sandstone), pounder ( } 1 \text { piece, } \\
\text { unspecified stone), worked stone ( } 1 \text { fragment quartzite); } \\
\text { textile ( } 1 \text { bag); schist ( } 2 \text { fragments })\end{array}$ & 235 & $\begin{array}{l}\text { SAV1E } 2162 \text { (grindstone), SAV1E } 2163 \\
\text { (worked stone), SAV1E } 2164 \text { (pounder) }\end{array}$ & 02.02 .2015 \\
\hline $1413 / 2015$ & $\mathrm{SQ} 4+4 \mathrm{~A}, 9.2-10.5 \mathrm{~m} \mathrm{~W}-\mathrm{E} / 9-12 \mathrm{~m} \mathrm{~N}-\mathrm{S}$ & stone & 235 & & 02.02 .2015 \\
\hline $1423 / 2015$ & $\mathrm{SQ} 4+4 \mathrm{~A}, 8.8-10.5 \mathrm{~m} \mathrm{~W}-\mathrm{E} / 9.3-12 \mathrm{~m} \mathrm{~N}-\mathrm{S}$ & $\begin{array}{l}\text { stones and architecture?: pounders ( } 2 \text { pieces, } 1 \text { unspecified } \\
\text { stone, } 1 \text { quartzite), grindstones ( } 4 \text { pieces, each sandstone), }\end{array}$ & 236 & $\begin{array}{l}\text { SAV1E 2188, SAV1E } 2190 \text { (pounders), SAV1E } \\
\text { 2196, SAV1E 2197, SAV1E 2198, SAV1E }\end{array}$ & 02.02 .2015 \\
\hline
\end{tabular}




\begin{tabular}{|c|c|c|c|c|c|}
\hline & & $\begin{array}{l}\text { hand mills ( } 4 \text { pieces, } 3 \text { sandstone, } 1 \text { quartzite), worked stones } \\
\text { ( } 2 \text { pieces, each sandstone), whetstone ( } 1 \text { piece, sandstone), } \\
\text { polishing stone ( } 1 \text { piece, quartz); architecture? ( } 1 \text { piece, } \\
\text { sandstone) }\end{array}$ & & $\begin{array}{l}2192 \text { (grindstones), SAV1E 2105, SAV1E 2189, } \\
\text { SAV1E 2199, SAV1E } 2200 \text { (hand mills), } \\
\text { SAV1E 2194, SAV1E } 2193 \text { (worked stones), } \\
\text { SAV1E 2195 (whetstone), SAV1E 2078 } \\
\text { (polishing stone), SAV1E 2191 (architecture?) }\end{array}$ & \\
\hline $1453 / 2015$ & SQ4A, 6-8.5m W-E/1.5-2m N-S & $\begin{array}{l}\text { stones: grindstone (1 fragment, sandstone), worked stone } \\
\text { (1 fragment, sandstone) }\end{array}$ & 238 & SAV1E 2134, SAV1E 2135 & 04.02 .2015 \\
\hline $1433 / 2015$ & $\mathrm{SQ} 4 \mathrm{~A}+2 \mathrm{~A}, 8.8-11 \mathrm{~m} \mathrm{~W}-\mathrm{E} / 1-2 \mathrm{~m} \mathrm{~N}-\mathrm{S}$ & $\begin{array}{l}\text { stones and architecture?: pounder ( } 1 \text { piece, quartz), } \\
\text { grindstone ( } 3 \text { pieces, each sandstone), hammer ( } 1 \text { piece, } \\
\text { quartzite), whetstones ( } 2 \text { pieces, sandstone), grindstone } \\
\text { (1 fragment, possibly edge piece); architecture? (1 fragment, } \\
\text { sandstone) }\end{array}$ & 238 & $\begin{array}{l}\text { SAV1E } 2129 \text { (pounder), SAV1E 2160, SAV1E } \\
\text { 2131, SAV1E } 2132 \text { (grindstones), SAV1E } 2161 \\
\text { (hammer), SAV1E 2177, SAV1E 2159 } \\
\text { (whetstones), SAV1E } 2178 \text { (architecture) }\end{array}$ & 03.02 .2015 \\
\hline $1460 / 2015$ & $\mathrm{SQ} 4 \mathrm{~A}+2 \mathrm{~A}, 7-11 \mathrm{~m} \mathrm{~W}-\mathrm{E} / 1-2 \mathrm{~m} \mathrm{~N}-\mathrm{S}$ & $\begin{array}{l}\text { stones: pounders ( } 3 \text { pieces, } 2 \text { quartz, } 1 \text { sandstone); schist } \\
\text { ( } 2 \text { fragments) }\end{array}$ & 238 & SAV1E 2143, SAV1E 2144, SAV1E 2145 & 04.02 .2015 \\
\hline $1436 / 2015$ & $\mathrm{SQ} 4+4 \mathrm{~A}, 8-9 \mathrm{~m} \mathrm{~W}-\mathrm{E} / 9.4-11.4 \mathrm{~m} \mathrm{~N}-\mathrm{S}$ & $\begin{array}{l}\text { stones: pounders ( } 8 \text { pieces, } 1 \text { siliceous shale?, } 2 \text { unspecified } \\
\text { stone, } 5 \text { quartz), grindstone ( } 1 \text { piece, sandstone), hand mill } \\
\text { ( } 1 \text { piece, sandstone), worked stone ( } 1 \text { piece, sandstone), pivot } \\
\text { stone ( } 1 \text { piece, sandstone), grindstone ( } 1 \text { fragment, possibly } \\
\text { edge piece) }\end{array}$ & 239 & $\begin{array}{l}\text { SAV1E 2201, SAV1E 2202, SAV1E 2203, } \\
\text { SAV1E 2204, SAV1E 2205, SAV1E 2206, } \\
\text { SAV1E 2207, SAV1E 2127 (pounders), SAV1E } \\
\text { 2133 (grindstone), SAV1E 2208 (hand mil), } \\
\text { SAV1E 2209 (worked stone), SAV1E 2210 } \\
\text { (pivot stone) }\end{array}$ & 03.02 .2015 \\
\hline $1448 / 2015$ & SQ4A, 5.4-7m W-E/1-2m N-S & stone: pounder (1 piece, siliceous shale?) & 241 & SAV1E 2128 & 04.02 .2015 \\
\hline $1501 / 2015$ & $\begin{array}{l}\text { SQ4+2, Feature 15, from windblown } \\
\text { sand/surface }\end{array}$ & stones: pounders ( 2 pieces, each quartzite) & 1300 & SAV1E 2150, SAV1E 2151 (pounders) & 02.02 .2015 \\
\hline $1503 / 2015$ & SQ3, SE-corner & stone, worked (1 piece, unclear function) & 1302 & & 02.02 .2015 \\
\hline $1510 / 2015$ & SQ3-4, baulk & stone: pounder (1 fragment, unspecified stone) & 1303 & SAV1E 2126 & 02.02 .2015 \\
\hline $1516 / 2015$ & SQ3-4, baulk & $\begin{array}{l}\text { stones: pounder ( } 1 \text { piece, quartzite), grindstone ( } 1 \text { fragment, } \\
\text { sandstone), whetstone ( } 1 \text { fragment, sandstone) }\end{array}$ & 1306 & $\begin{array}{l}\text { SAV1E } 2156 \text { (pounder), SAV1E } 2157 \\
\text { (grindstone), SAV1E } 2158 \text { (whetstone) }\end{array}$ & 03.02 .2015 \\
\hline $1521 / 2015$ & SQ4-3, baulk & $\begin{array}{l}\text { stones: hand mills ( } 2 \text { pieces, each sandstone, } 1 \text { eventually also } \\
\text { grindstone) }\end{array}$ & 1307 & SAV1E 2153, SAV1E 2154 (hand mills) & 03.02 .2015 \\
\hline $1526 / 2015$ & SQ4, N-baulk & $\begin{array}{l}\text { stones: pounder ( } 1 \text { piece, quartz), grindstone ( } 1 \text { fragment, } \\
\text { sandstone), whetstone ( } 1 \text { fragment, sandstone) }\end{array}$ & 1308 & SAV1E 2140, SAV1E 2141, SAV1E 2142 & 03.02 .2015 \\
\hline $1533 / 2015$ & SQ4, N-baulk, Feature 15 & $\begin{array}{l}\text { stones: pounder ( } 1 \text { piece, unspecified stone), worked stone } \\
\text { (1 fragment, sandstone) }\end{array}$ & 1309 & SAV1E 2138, SAV1E 2139 & 03.02 .2015 \\
\hline $1549 / 2015$ & SQ4 (along Feature 43 in Feature 15) & stone: hand mill (1 fragment, sandstone) & 1311 & SAV1E 2113 & 04.02 .2015 \\
\hline $1666 / 2015$ & SQ2, Feature 15 & stone: hand mill or grindstone ( 1 fragment, sandstone) & 1316 & SAV1E 2241 & 08.02 .2015 \\
\hline $1583 / 2015$ & SQ2, Feature 15, along E-wall & stone: hand mill (1 fragment, sandstone) & 1317 & SAV1E 2215 & 08.02 .2015 \\
\hline
\end{tabular}




\begin{tabular}{|c|c|c|c|c|c|}
\hline $1499 / 2015$ & SQ2, Feature 15 & stone: tool? (1 small fragment, silex flake) & 1318 & SAV1E 2348 & 08.02 .2015 \\
\hline $1647 / 2015$ & SQ2, E of wall 44 in Feature 15 & stone & 1318 & & 11.02 .2015 \\
\hline $1494 / 2015$ & SQ4, NE-corner & $\begin{array}{l}\text { stone, worked (1 fragment, sandstone, original function } \\
\text { unknown) }\end{array}$ & 1319 & SAV1E 2216 & 08.02 .2015 \\
\hline $1629 / 2015$ & SQ2, Feature 15 & stone: polishing stone? (1 piece, unspecified stone) & 1321 & SAV1E 2233 & 10.02 .2015 \\
\hline $1642 / 2015$ & SQ2, Feature 15 & $\begin{array}{l}\text { stones: hand mills ( } 3 \text { fragments, each sandstone), pounder } \\
\text { ( } 1 \text { fragment, quartz) }\end{array}$ & 1321 & $\begin{array}{l}\text { SAV1E 2240, SAV1E 2237, SAV1E } 2238 \\
\text { (hand mills), SAV1E } 2239 \text { (pounder) }\end{array}$ & 11.02 .2015 \\
\hline $1604 / 2015$ & SQ2, Feature 15 & $\begin{array}{l}\text { stones: pounders ( } 2 \text { pieces, each quartzite), grindstone } \\
\text { (1 piece, sandstone), worked stone ( } 1 \text { piece, sandstone) }\end{array}$ & 1321 & $\begin{array}{l}\text { SAV1E 2211, SAV1E } 2212 \text { (pounders), SAV1E } \\
2213 \text { (grindstone), SAV1E } 2214 \text { (worked stone) }\end{array}$ & 09.02 .2015 \\
\hline $1616 / 2015$ & SQ2, Feature 15 & $\begin{array}{l}\text { stones and architecture?: pounders ( } 3 \text { pieces, } 1 \text { unspecified } \\
\text { stone, } 2 \text { siliceous shale?), polishing stone ( } 1 \text { piece, siliceous } \\
\text { shale?), worked stones ( } 2 \text { pieces, each sandstone); } \\
\text { architecture? ( } 1 \text { fragment, sandstone) }\end{array}$ & 1321 & $\begin{array}{l}\text { SAV1E 2243, SAV1E 2244, SAV1E } 2245 \\
\text { (pounders), SAV1E } 2246 \text { (polishing stone) } \\
\text { SAV1E 2248, SAV1E } 2249 \text { (worked stones), } \\
\text { SAV1E 2247 (architecture) }\end{array}$ & 10.02.2015 \\
\hline $1659 / 2015$ & SQ4, Wall 44 & $\begin{array}{l}\text { stones: worked stones ( } 2 \text { pieces, each sandstone), } \\
\text { pounders ( } 4 \text { pieces, } 1 \text { unspecified stone, } 1 \text { quartz, } 1 \text { siliceous } \\
\text { shale, } 1 \text { sandstone) }\end{array}$ & 1324 & $\begin{array}{l}\text { SAV1E 2250, SAV1E } 2251 \text { (worked stones), } \\
\text { SAV1E 2252, SAV1E 2253, SAV1E 2254, } \\
\text { SAV1E 2255 (pounders) }\end{array}$ & 12.02.2015 \\
\hline $1456 / 2015$ & SQ4A, cleaning 8-10m W-E, S section & $\begin{array}{l}\text { stones: worked stone ( } 1 \text { fragment, sandstone), whetstone? } \\
\text { (1 fragment, sandstone), grindstone/hand mill (1 fragment); } \\
\text { schist ( } 1 \text { fragment) }\end{array}$ & l & $\begin{array}{l}\text { SAV1E } 2136 \text { (worked stone), SAV1E } 2137 \\
\text { (whetstone) }\end{array}$ & 04.02 .2015 \\
\hline $1530 / 2015$ & SQ4, N-baulk & stopper/sealing (1 fragment, clay) & 1308 & SAV1E 2061 & 03.02 .2015 \\
\hline $1536 / 2015$ & SQ4, N-baulk, Feature 15 & stopper/sealing? (4 fragments, clay) & 1309 & SAV1E 2065 & 03.02 .2015 \\
\hline $1635 / 2015$ & SQ2, Feature 15 & stopper (1 piece, conical, clay) & 1321 & SAV1E 2242 & 10.02 .2015 \\
\hline $1643 / 2015$ & SQ2, Feature 15 & stopper/sealing (1 fragment, mud) & 1321 & SAV1E 2235 & 11.02 .2015 \\
\hline $1617 / 2015$ & SQ2, Feature 15, NE-corner & stopper/sealing? (1 fragment, mud) & 1321 & SAV1E 2234 & 10.02 .2015 \\
\hline $1052 / 2015$ & $\mathrm{SQ} 4,3.7-5.8 \mathrm{~m} \mathrm{~W}-\mathrm{E} / 4.2-5.1 \mathrm{~m} \mathrm{~N}-\mathrm{S}$ & textile (1 bag) & 105 & & 07.01 .2015 \\
\hline $1178 / 2015$ & $\mathrm{SQ} 4,4-5.7 \mathrm{~m} \mathrm{~W}-\mathrm{E} / 4.5-6.5 \mathrm{~m} \mathrm{~N}-\mathrm{S}$ & textile (1 bag) & 105 & & 15.01 .2015 \\
\hline $1060 / 2015$ & $\mathrm{SQ} 4,0-4 \mathrm{~m} \mathrm{~W}-\mathrm{E} / 4.7-7.1 \mathrm{~m} \mathrm{~N}-\mathrm{S}$ & textile (1 bag) & 205 & & 07.01 .2015 \\
\hline $1113 / 2015$ & SQ4, 0-4m W-E/6-10m N-S & textile (1 small piece) & 205 & & 11.01 .2015 \\
\hline $1172 / 2015$ & SQ4A, 4-5m W-E/1.4-2m N-S & textile (1 bag) & 205 & & 15.01 .2015 \\
\hline $1218 / 2015$ & SQ4, 7-9.2m W-E/6.8-8m N-S & textile (1 bag) & 212 & & 18.01 .2015 \\
\hline $1242 / 2015$ & $\mathrm{SQ} 4+4 \mathrm{~A}, 6.5-8 \mathrm{~m} \mathrm{~W}-\mathrm{E} / 8.2-12 \mathrm{~m} \mathrm{~N}-\mathrm{S}$ & textile (1 piece, fibre) & 213 & & 20.01 .2015 \\
\hline $1260 / 2015$ & $\mathrm{SQ} 4+4 \mathrm{~A}, 3.5-5.5 \mathrm{~m} \mathrm{~W}-\mathrm{E} / 8.3-11.3 \mathrm{~m} \mathrm{~N}-\mathrm{S}$ & textile (1 bag) & 214 & & 21.01 .2015 \\
\hline
\end{tabular}




\begin{tabular}{|c|c|c|c|c|c|}
\hline $1303 / 2015$ & $\mathrm{SQ} 4,0-1.2 \mathrm{~m} \mathrm{~W}-\mathrm{E} / 0-4.5 \mathrm{~m} \mathrm{~N}-\mathrm{S}$ & textile ( 1 bag) & 220 & & 24.01.2015 \\
\hline $1312 / 2015$ & $\mathrm{SQ} 4,0-0.8 \mathrm{~m} \mathrm{~W}-\mathrm{E} / 4.7-5.1 \mathrm{~m} \mathrm{~N}-\mathrm{S}$ & textile (1 bag) & 221 & & 24.01 .2015 \\
\hline $1349 / 2015$ & $\mathrm{SQ} 4+4 \mathrm{~A}, 0.4-5 \mathrm{~m} \mathrm{~W}-\mathrm{E} / 7.5-12 \mathrm{~m} \mathrm{~N}-\mathrm{S}$ & textile (1 bag) & 227 & & 28.01 .2015 \\
\hline $1379 / 2015$ & $\mathrm{SQ} 4 \mathrm{~A}, 3.5-5.1 \mathrm{~m} \mathrm{~W}-\mathrm{E} / 0-2 \mathrm{~m} \mathrm{~N}-\mathrm{S}$ & textile (1 piece) & 229 & & 31.01 .2015 \\
\hline $1420 / 2015$ & $\mathrm{SQ} 4+4 \mathrm{~A}, 8.8-10.5 \mathrm{~m} \mathrm{~W}-\mathrm{E} / 9.3-12 \mathrm{~m} \mathrm{~N}-\mathrm{S}$ & textile (1 bag) & 236 & & 02.02 .2015 \\
\hline $1429 / 2015$ & $\mathrm{SQ} 4 \mathrm{~A}+2 \mathrm{~A}, 8.8-11 \mathrm{~m} \mathrm{~W}-\mathrm{E} / 1-2 \mathrm{~m} \mathrm{~N}-\mathrm{S}$ & textile (1 bag) & 238 & & 03.02 .2015 \\
\hline $1156 / 2015$ & $\mathrm{SQ} 4,4 \mathrm{~m}$ to $\mathrm{E}, 9.8 \mathrm{~m}$ to $\mathrm{S}$ & token/gaming piece? (1 re-used Christian sherd, pottery) & 205 & SAV1E 1461 & 14.01.2015 \\
\hline $1668 / 2015$ & SQ2, Feature 15 & token/gaming piece ( 1 piece, conical, clay) & 1321 & SAV1E 2260 & 10.02 .2015 \\
\hline $1089 / 2015$ & $\mathrm{SQ} 4,1 \mathrm{~m} \mathrm{~W}-\mathrm{E} / 9 \mathrm{~m} \mathrm{~N}-\mathrm{S}$ & weight (1 small piece, loom weight, sandstone) & 205 & SAV1E 1285 & 10.01 .2015 \\
\hline $1038 / 2015$ & $\mathrm{SQ} 4,3.7-5.8 \mathrm{~m} \mathrm{~W}-\mathrm{E} / 2.8-4.2 \mathrm{~m} \mathrm{~N}-\mathrm{S}$ & wood (4 pieces) & 105 & & 06.01 .2015 \\
\hline $1180 / 2015$ & $\mathrm{SQ} 4,4-5.7 \mathrm{~m} \mathrm{~W}-\mathrm{E} / 4.5-6.5 \mathrm{~m} \mathrm{~N}-\mathrm{S}$ & wood (1 piece) & 105 & & 15.01 .2015 \\
\hline $1007 / 2015$ & $\mathrm{SQ} 4+4 \mathrm{~A}, 0-7.5 \mathrm{~m} \mathrm{~W}-\mathrm{E} / 7.1-12 \mathrm{~m} \mathrm{~N}-\mathrm{S}$ & wood (3 fragments) & 200 & & 04.01 .2015 \\
\hline $1030 / 2015$ & $\mathrm{SQ} 4,3.5-6.5 \mathrm{~m} \mathrm{~W}-\mathrm{E} / 5-6.2 \mathrm{~m} \mathrm{~N}-\mathrm{S}$ & wood (1 piece) & 204 & & 06.01 .2015 \\
\hline $1061 / 2015$ & $\mathrm{SQ} 4,0-4 \mathrm{~m} \mathrm{~W}-\mathrm{E} / 4.7-7.1 \mathrm{~m} \mathrm{~N}-\mathrm{S}$ & wood (1 bag) & 205 & & 07.01 .2015 \\
\hline $1087 / 2015$ & $\mathrm{SQ} 4,0-4 \mathrm{~m} \mathrm{~W}-\mathrm{E} / 5-10 \mathrm{~m} \mathrm{~N}-\mathrm{S}$ & wood (1 bag) & 205 & & 10.01 .2015 \\
\hline $1106 / 2015$ & $\mathrm{SQ} 4,0-4 \mathrm{~m} \mathrm{~W}-\mathrm{E} / 6-10 \mathrm{~m} \mathrm{~N}-\mathrm{S}$ & wood (1 bag) & 205 & & 11.01 .2015 \\
\hline $1120 / 2015$ & $\mathrm{SQ} 4,0-4 \mathrm{~m} \mathrm{~W}-\mathrm{E} / 7-9 \mathrm{~m} \mathrm{~N}-\mathrm{S}$ & wood (1 bag) & 205 & & 12.01 .2015 \\
\hline $1131 / 2015$ & SQ4A, 0-3m W-E/0-2m N-S & wood (1 bag) & 205 & & 13.01 .2015 \\
\hline $1206 / 2015$ & $\mathrm{SQ} 4,4.2-5.9 \mathrm{~m} \mathrm{~W}-\mathrm{E} / 6.5-8 \mathrm{~m} \mathrm{~N}-\mathrm{S}$ & wood (1 piece) & 209 & & 17.01 .2015 \\
\hline $1254 / 2015$ & $\mathrm{SQ} 4 \mathrm{~A}, 6.8 \mathrm{~m} \mathrm{~W}-\mathrm{E} / 0.5 \mathrm{~m} \mathrm{~N}-\mathrm{S}$ & wood, worked (1 fragment, lid of khol pot?) & 213 & SAV1E 1913 & 20.01 .2015 \\
\hline $1263 / 2015$ & $\mathrm{SQ} 4+4 \mathrm{~A}, 3.5-5.5 \mathrm{~m} \mathrm{~W}-\mathrm{E} / 8.3-11.3 \mathrm{~m} \mathrm{~N}-\mathrm{S}$ & wood (1 bag) & 214 & & 21.01 .2015 \\
\hline $1289 / 2015$ & SQ4, 5-8.8m N-S, section cleaning & wood (1 bag); hair/fur (1 clump) & 219 & & 22.01 .2015 \\
\hline $1340 / 2015$ & $\mathrm{SQ} 4,0.4-3.3 \mathrm{~m} \mathrm{~W}-\mathrm{E} / 6-7.5 \mathrm{~m} \mathrm{~N}-\mathrm{S}$ & wood (1 piece) & 226 & & 27.01 .2015 \\
\hline $1355 / 2015$ & $\mathrm{SQ} 4+4 \mathrm{~A}, 0.4-5 \mathrm{~m} \mathrm{~W}-\mathrm{E} / 7.5-12 \mathrm{~m} \mathrm{~N}-\mathrm{S}$ & wood (1 bag) & 227 & & 28.01 .2015 \\
\hline $1408 / 2015$ & $\mathrm{SQ} 4+4 \mathrm{~A}, 9.2-10.5 \mathrm{~m} \mathrm{~W}-\mathrm{E} / 9-12 \mathrm{~m} \mathrm{~N}-\mathrm{S}$ & wood, worked (1 fragment); slag (1 fragment) & 235 & $\begin{array}{l}\text { SAV1E } 2073 \text { (worked wood), SAV1E } 2111 \\
\text { (slag) }\end{array}$ & 02.02 .2015 \\
\hline $1452 / 2015$ & SQ4A, $6-8.5 \mathrm{~m} \mathrm{~W}-\mathrm{E} / 1.5-2 \mathrm{~m} \mathrm{~N}-\mathrm{S}$ & wood (1 piece) & 238 & & 04.02 .2015 \\
\hline
\end{tabular}




\begin{tabular}{|c|c|c|c|c|}
\hline $1522 / 2015$ & SQ4-3, baulk & wood (2 pieces) & 1307 & 03.02 .2015 \\
\hline $1538 / 2015$ & SQ4, N-baulk, Feature 15 & wood (1 piece) & 1309 & 03.02 .2015 \\
\hline $1561 / 2015$ & SQ2, Feature 15 & wood (1 bag) & 1316 & 05.02 .2015 \\
\hline $1621 / 2015$ & SQ2, Feature 15 & wood & 1321 & 10.02 .2015 \\
\hline $1603 / 2015$ & SQ2, Feature 15 & wood (1 bag) & 1321 & 09.02 .2015 \\
\hline $1663 / 2015$ & SQ2, Feature 15 & wood/organic material & 1321 & 12.02.2015 \\
\hline $1637 / 2015$ & SQ2, Feature 15 & wood (1 bag) & 1322 & 11.02 .2015 \\
\hline
\end{tabular}

\begin{tabular}{|c|c|c|c|c|c|}
\hline \multicolumn{6}{|c|}{ Sai Island SAV1 East 2016} \\
\hline Find no. & Location & Description/label/material & $\begin{array}{l}\text { SU/ } \\
\text { level }\end{array}$ & Object no. & Date \\
\hline $773 / 2016$ & SQ4B, sifted & agate (1 piece, sample) & 451 & & 03.02 .2016 \\
\hline $842 / 2016$ & SQ4, Feature 75 & agate (2 pieces) & 462 & & 06.02 .2016 \\
\hline $949 / 2016$ & $\begin{array}{l}\text { SQ4, Feature 15, lower part of SU, W of } \\
\text { Feature } 44\end{array}$ & agate (5 pieces, sample) & 1329 & & 09.02 .2016 \\
\hline $068 / 2016$ & SQ4B & amulet (1 piece, faience) & 317 & SAV1E 0531 & 09.01 .2016 \\
\hline $259 / 2016$ & SQ4A & amulet? (1 piece, steatite) & 352 & SAV1E 2730 & 16.01 .2016 \\
\hline $732 / 2016$ & SQ4C & $\begin{array}{l}\text { amulet/pendant (1 piece, shaped like a pomegranate seed, } \\
\text { faience) }\end{array}$ & 441 & SAV1E 2852 & 02.02 .2016 \\
\hline $309 / 2016$ & SQ4C & $\begin{array}{l}\text { architecture: lateral door frame ( } 2 \text { adjoining pieces, sandstone, } \\
\text { re-used as tethering stone) }\end{array}$ & 351 & SAV1E 0717 & 17.01.2016 \\
\hline $004 / 2016$ & SQ4B, surface & bead (1 spherical bead, clay) & 300 & SAV1E 2662 & 03.01 .2016 \\
\hline $459 / 2016$ & SQ4B1 & bead (1 ring bead, faience) & 301 & SAV1E 2777 & 24.01 .2016 \\
\hline $096 / 2016$ & SQ4C & bead (1 ring bead, faience) & 322 & SAV1E 0928 & 10.01 .2016 \\
\hline $123 / 2016$ & SQ4C & bead (1 tubular bead, faience) & 328 & SAV1E 0891 & 10.01 .2016 \\
\hline $153 / 2016$ & SQ4B & bead? weight? (1 large cylindrical-shaped piece, clay/mud) & 336 & SAV1E 2683 & 11.01 .2016 \\
\hline $220 / 2016$ & SQ4C & bead (1 tubular bead, pottery) & 341 & SAV1E 2720 & 14.01.2016 \\
\hline $340 / 2016$ & SQ4C & bead (1 fragment, disc bead, faience) & 370 & SAV1E 0697 & 18.01.2016 \\
\hline
\end{tabular}




\begin{tabular}{|c|c|c|c|c|c|}
\hline $360 / 2016$ & SQ4C & bead (1 spherical bead, clay) & 375 & SAV1E 1661 & 19.01.2016 \\
\hline $369 / 2016$ & SQ4B & bead (1 ring bead, faience) & 377 & SAV1E 1596 & 19.01.2016 \\
\hline $452 / 2016$ & SQ4C & bead (1 tubular bead, faience) & 384 & SAV1E 2768 & 23.01 .2016 \\
\hline $492 / 2016$ & SQ4B1 & bead (1 ring bead with fluted outer edge, faience) & 398 & SAV1E 2783 & 25.01 .2016 \\
\hline $529 / 2016$ & SQ4B1, N-part of SU & beads (1 ring bead, faience, 1 ring bead, bone) & 405 & $\begin{array}{l}\text { SAV1E } 2807 \text { (ring bead, faience), SAV1E } \\
2808 \text { (ring bead, bone) }\end{array}$ & 27.01 .2016 \\
\hline $540 / 2016$ & SQ4B1, S-part of SU & bead (1 fragment, cylindrical bead, broken in half, glass) & 405 & SAV1E 2806 & 27.01.2016 \\
\hline $585 / 2016$ & SQ4B1 & bead (1 tubular bead, faience) & 414 & SAV1E 2811 & 28.01 .2016 \\
\hline $600 / 2016$ & SQ4B1 & bead (1 tubular bead, faience) & 414 & SAV1E 2831 & 30.01 .2016 \\
\hline $656 / 2016$ & SQ4B1 & bead (1 ring bead, white faience) & 426 & SAV1E 2847 & 31.01 .2016 \\
\hline $700 / 2016$ & SQ4C & bead (1 disc bead, possibly a sequin, faience) & 435 & SAV1E 2844 & 01.02 .2016 \\
\hline $712 / 2016$ & SQ4C & bead (1 tubular bead, faience) & 439 & SAV1E 2845 & 01.02 .2016 \\
\hline $713 / 2016$ & SQ4C & bead on leather string ( 1 ring bead, glass?, Ottoman? Christian?) & 439 & SAV1E 2850 & 01.02 .2016 \\
\hline $915 / 2016$ & SQ4C & beads (3 ring beads, faience) & 449 & SAV1E 2889 & 08.02 .2016 \\
\hline $892 / 2016$ & SQ4C, directly at wall & bead (1 ring bead, faience) & 449 & SAV1E 2888 & 08.02 .2016 \\
\hline $844 / 2016$ & SQ4B, sifted material & bead (1 ring bead, white faience) & 451 & SAV1E 1070 & 06.02 .2016 \\
\hline $845 / 2016$ & SQ4B, sifted material & beads ( 1 ring bead, faience, 1 ring bead, bone) & 452 & $\begin{array}{l}\text { SAV1E } 1039 \text { (bead, faience), SAV1E } 1055 \\
\text { (bead, bone) }\end{array}$ & 06.02 .2016 \\
\hline $800 / 2016$ & SQ4C & bead (1 ring bead, blue glass) & 456 & SAV1E 2871 & 04.02 .2016 \\
\hline $866 / 2016$ & SQ4C & bead (1 ring bead, faience) & 466 & SAV1E 2881 & 07.02 .2016 \\
\hline $008 / 2016$ & SQ4B & bone (1 bag) & 301 & & 05.01 .2016 \\
\hline $460 / 2016$ & SQ4B1 & bone (1 bag) & 301 & & 24.01 .2016 \\
\hline $011 / 2016$ & SQ4C & bone (1 bag) & 301 & & 05.01 .2016 \\
\hline $019 / 2016$ & SQ4B & bone (1 bag) & 303 & & 06.01 .2016 \\
\hline $018 / 2016$ & SQ4B & bone (1 bag) & 304 & & 06.01 .2016 \\
\hline $024 / 2016$ & SQ4B & bone (1 bag) & 306 & & 06.01 .2016 \\
\hline $026 / 2016$ & SQ4B & bone (1 bag) & 307 & & 06.01 .2016 \\
\hline $028 / 2016$ & SQ4B & bone (1 bag) & 308 & & 06.01 .2016 \\
\hline
\end{tabular}




\begin{tabular}{|c|c|c|c|c|}
\hline $027 / 2016$ & SQ4B & bone (1 bag) & 309 & 06.01 .2016 \\
\hline $033 / 2016$ & SQ4C & bone (1 bag) & 310 & 06.01 .2016 \\
\hline $035 / 2016$ & SQ4C & bone (1 bag) & 310 & 07.01 .2016 \\
\hline $037 / 2016$ & SQ4B & bone (1 bag) & 311 & 07.01 .2016 \\
\hline $046 / 2016$ & SQ4B & bone (1 bag) & 312 & 07.01 .2016 \\
\hline $048 / 2016$ & SQ4B & bone (1 bag) & 313 & 07.01 .2016 \\
\hline $051 / 2016$ & SQ4C & bone (1 bag) & 314 & 07.01 .2016 \\
\hline $053 / 2016$ & SQ4C & bone (1 bag) & 315 & 07.01 .2016 \\
\hline $072 / 2016$ & SQ4C & bone (1 bag) & 315 & 09.01 .2016 \\
\hline $092 / 2016$ & SQ4C & bones ( 2 pieces) & 315 & 10.01.2016 \\
\hline $088 / 2016$ & SQ4C & bones ( 2 pieces) & 315 & 09.01 .2016 \\
\hline $057 / 2016$ & SQ4B & bone (1 bag) & 316 & 07.01 .2016 \\
\hline $087 / 2016$ & SQ4B & bone (1 bag) & 316 & 09.01 .2016 \\
\hline $107 / 2016$ & SQ4B & bone (1 piece, animal) & 316 & 10.01.2016 \\
\hline $112 / 2016$ & SQ4B & bone & 316 & 10.01 .2016 \\
\hline $061 / 2016$ & SQ4B & bone (1 bag) & 317 & 09.01 .2016 \\
\hline $064 / 2016$ & SQ4B & bone (1 bag) & 318 & 09.01 .2016 \\
\hline $085 / 2016$ & SQ4C & bone (1 bag) & 319 & 09.01 .2016 \\
\hline $082 / 2016$ & SQ4B & bone (1 bag) & 321 & 09.01 .2016 \\
\hline $090 / 2016$ & SQ4C & bone (1 bag) & 322 & 09.01 .2016 \\
\hline $094 / 2016$ & SQ4C & bone (1 bag) & 322 & 10.01 .2016 \\
\hline $101 / 2016$ & SQ4C & bone (1 bag) & 322 & 10.01 .2016 \\
\hline $104 / 2016$ & SQ4B & bone (1 bag) & 324 & 10.01.2016 \\
\hline $110 / 2016$ & SQ4B & bone (1 bag) & 325 & 10.01 .2016 \\
\hline $114 / 2016$ & SQ4B & bone (1 bag) & 326 & 10.01 .2016 \\
\hline $125 / 2016$ & SQ4B & bone (1 bag) & 326 & 11.01 .2016 \\
\hline
\end{tabular}




\begin{tabular}{|c|c|c|c|c|}
\hline $118 / 2016$ & SQ4C & bone (1 bag) & 327 & 10.01 .2016 \\
\hline $127 / 2016$ & SQ4C & bone (1 bag) & 327 & 11.01 .2016 \\
\hline $121 / 2016$ & SQ4C & bone (1 bag) & 328 & 10.01 .2016 \\
\hline $129 / 2016$ & SQ4C & bone (1 bag) & 328 & 11.01 .2016 \\
\hline $133 / 2016$ & SQ4C & bones ( 3 pieces) & 329 & 11.01 .2016 \\
\hline $136 / 2016$ & SQ4C & bone (1 bag) & 330 & 11.01 .2016 \\
\hline $139 / 2016$ & SQ4B & bone (1 bag) & 332 & 11.01 .2016 \\
\hline $142 / 2016$ & SQ4B & bone (1 bag) & 333 & 11.01 .2016 \\
\hline $145 / 2016$ & SQ4B & bone (1 bag) & 334 & 11.01 .2016 \\
\hline $157 / 2016$ & SQ4C & bone (1 bag) & 335 & 11.01 .2016 \\
\hline $179 / 2016$ & SQ4C & bone (1 bag) & 335 & 13.01 .2016 \\
\hline $165 / 2016$ & SQ4C & bone (1 large bag) & 335 & 12.01 .2016 \\
\hline $156 / 2016$ & SQ4B & bone (1 bag) & 336 & 11.01 .2016 \\
\hline $163 / 2016$ & SQ4B & bone (1 bag) & 336 & 12.01 .2016 \\
\hline $171 / 2016$ & SQ4B & bone (1 bag) & 337 & 12.01 .2016 \\
\hline $184 / 2016$ & SQ4B & bone (1 bag) & 338 & 13.01 .2016 \\
\hline $188 / 2016$ & SQ4B & bone (1 bag) & 339 & 13.01 .2016 \\
\hline $190 / 2016$ & SQ4B & bone (1 bag) & 339 & 13.01 .2016 \\
\hline $193 / 2016$ & SQ4C & bone (1 bag) & 340 & 13.01 .2016 \\
\hline $196 / 2016$ & SQ4C & bone (1 bag) & 341 & 13.01 .2016 \\
\hline $211 / 2016$ & SQ4C & bone (1 bag) & 341 & 14.01 .2016 \\
\hline $199 / 2016$ & SQ4B & bone (1 bag) & 342 & 13.01 .2016 \\
\hline $204 / 2016$ & SQ4C & bone (1 bag) & 343 & 13.01 .2016 \\
\hline $208 / 2016$ & SQ4C & bone (1 bag) & 344 & 13.01 .2016 \\
\hline $218 / 2016$ & SQ4C & bone (1 bag) & 344 & 14.01 .2016 \\
\hline $213 / 2016$ & SQ4B & bone (1 bag) & 345 & 14.01 .2016 \\
\hline
\end{tabular}




\begin{tabular}{|c|c|c|c|c|}
\hline $224 / 2016$ & SQ4B & bone (1 bag) & 346 & 14.01 .2016 \\
\hline $230 / 2016$ & SQ4B & bone (1 bag) & 347 & 14.01 .2016 \\
\hline $235 / 2016$ & SQ4C & bone (1 bag) & 348 & 14.01.2016 \\
\hline $252 / 2016$ & SQ4C & bone (1 bag) & 348 & 16.01 .2016 \\
\hline $238 / 2016$ & SQ4C & bone (1 bag) & 349 & 14.01.2016 \\
\hline $243 / 2016$ & SQ4B & bone (1 bag) & 350 & 14.01 .2016 \\
\hline $248 / 2016$ & SQ4B & bone (1 bag) & 350 & 16.01.2016 \\
\hline $255 / 2016$ & SQ4C & bone (1 bag) & 351 & 16.01.2016 \\
\hline $257 / 2016$ & SQ4A & bone (1 bag) & 352 & 16.01 .2016 \\
\hline $263 / 2016$ & SQ4C & bone (1 bag) & 353 & 16.01 .2016 \\
\hline $267 / 2016$ & SQ4B & bone (1 bag) & 354 & 16.01.2016 \\
\hline $271 / 2016$ & SQ4B & bone (1 bag) & 355 & 16.01.2016 \\
\hline $278 / 2016$ & SQ4B & bone (1 bag) & 356 & 16.01.2016 \\
\hline $284 / 2016$ & SQ4B & bone (1 bag) & 357 & 16.01.2016 \\
\hline $290 / 2016$ & SQ4B & bone (1 bag) & 357 & 17.01.2016 \\
\hline $285 / 2016$ & SQ4B & bone (1 bag) & 358 & 16.01.2016 \\
\hline $293 / 2016$ & SQ4C & bone (1 bag) & 359 & 17.01.2016 \\
\hline $296 / 2016$ & SQ4C & bone (1 bag) & 360 & 17.01.2016 \\
\hline $304 / 2016$ & SQ4B & bone (1 bag) & 361 & 17.01.2016 \\
\hline $302 / 2016$ & SQ4C & bone (1 bag) & 362 & 17.01.2016 \\
\hline $307 / 2016$ & SQ4B & bone (1 bag) & 363 & 17.01 .2016 \\
\hline $310 / 2016$ & SQ4B & bone (1 bag) & 364 & 17.01.2016 \\
\hline $314 / 2016$ & SQ4C & bone (1 bag) & 365 & 17.01.2016 \\
\hline $316 / 2016$ & SQ4B & bone (1 bag) & 366 & 17.01.2016 \\
\hline $319 / 2016$ & SQ4C & bone (1 bag) & 367 & 17.01.2016 \\
\hline $327 / 2016$ & SQ4C & bone (1 bag) & 369 & 18.01.2016 \\
\hline
\end{tabular}




\begin{tabular}{|c|c|c|c|c|}
\hline $329 / 2016$ & SQ4C & bone (1 bag) & 370 & 18.01 .2016 \\
\hline $332 / 2016$ & SQ4B & bone (1 bag) & 371 & 18.01 .2016 \\
\hline $335 / 2016$ & SQ4C & bone (1 bag) & 372 & 18.01 .2016 \\
\hline $338 / 2016$ & SQ4B & bone (1 bag) & 373 & 18.01 .2016 \\
\hline $348 / 2016$ & SQ4B & bone (1 bag) & 374 & 19.01 .2016 \\
\hline $352 / 2016$ & SQ4C & bone (1 bag) & 375 & 19.01 .2016 \\
\hline $357 / 2016$ & SQ4C & bone (1 bag) & 376 & 19.01 .2016 \\
\hline $367 / 2016$ & SQ4B & bone (1 bag) & 377 & 19.01 .2016 \\
\hline $375 / 2016$ & SQ4C & bone (1 bag) & 378 & 19.01 .2016 \\
\hline $379 / 2016$ & SQ4B & bone (1 bag) & 379 & 19.01 .2016 \\
\hline $384 / 2016$ & SQ4C & bone (1 bag) & 380 & 20.01 .2016 \\
\hline $387 / 2016$ & SQ4C & bone (1 bag) & 381 & 20.01 .2016 \\
\hline $390 / 2016$ & SQ4C & bone (1 bag) & 382 & 20.01 .2016 \\
\hline $401 / 2016$ & SQ4C & bone (1 bag) & 383 & 20.01 .2016 \\
\hline $403 / 2016$ & SQ4C & bone (1 bag) & 383 & 21.01 .2016 \\
\hline $425 / 2016$ & SQ4C & bone (1 bag) & 384 & 21.01 .2016 \\
\hline $429 / 2016$ & SQ4C & bone (1 bag) & 384 & 23.01 .2016 \\
\hline $406 / 2016$ & SQ4B & bone (1 bag) & 385 & 21.01 .2016 \\
\hline $412 / 2016$ & SQ4B & bone (1 bag) & 386 & 21.01 .2016 \\
\hline $420 / 2016$ & SQ4B & bone (1 bag) & 387 & 21.01 .2016 \\
\hline $418 / 2016$ & SQ4B & bone (1 bag) & 388 & 21.01 .2016 \\
\hline $432 / 2016$ & SQ4C & bone (1 bag) & 390 & 23.01 .2016 \\
\hline $439 / 2016$ & SQ4B & bone (1 bag) & 391 & 23.01 .2016 \\
\hline $463 / 2016$ & SQ4C & bone (1 bag) & 393 & 24.01 .2016 \\
\hline $466 / 2016$ & SQ4C & bone (1 bag) & 393 & 25.01 .2016 \\
\hline $470 / 2016$ & SQ4B1 & bone (1 bag) & 394 & 25.01 .2016 \\
\hline
\end{tabular}




\begin{tabular}{|c|c|c|c|c|}
\hline $472 / 2016$ & SQ4B1 & bone (1 bag) & 395 & 25.01 .2016 \\
\hline $475 / 2016$ & SQ4B & bone (1 bag) & 396 & 25.01 .2016 \\
\hline $487 / 2016$ & SQ4C & bone (1 bag) & 397 & 25.01 .2016 \\
\hline $498 / 2016$ & SQ4B1 & bone (1 bag) & 398 & 25.01 .2016 \\
\hline $500 / 2016$ & SQ4C & bone (1 bag) & 399 & 26.01.2016 \\
\hline $508 / 2016$ & SQ4C & bone (1 bag) & 400 & 26.01.2016 \\
\hline $506 / 2016$ & SQ4B1 & bone (1 bag) & 401 & 26.01.2016 \\
\hline $511 / 2016$ & SQ4C & bone (1 bag) & 402 & 26.01.2016 \\
\hline $517 / 2016$ & SQ4B1 & bone (1 bag) & 403 & 26.01 .2016 \\
\hline $523 / 2016$ & SQ4C & bone (1 bag) & 404 & 26.01 .2016 \\
\hline $527 / 2016$ & SQ4B1, S-part of SU & bone (1 bag) & 405 & 27.01.2016 \\
\hline $567 / 2016$ & SQ4B1, S-part of SU & bone (1 bag) & 405 & 28.01 .2016 \\
\hline $531 / 2016$ & SQ4B1, N-part of SU & bone (1 bag) & 405 & 27.01.2016 \\
\hline $572 / 2016$ & SQ4B1, N-part of SU & bone (1 bag) & 405 & 28.01.2016 \\
\hline $533 / 2016$ & SQ4B & bone (1 bag) & 406 & 27.01 .2016 \\
\hline $546 / 2016$ & SQ4C & bone (1 bag) & 407 & 27.01.2016 \\
\hline $549 / 2016$ & SQ4C & bone (1 bag) & 408 & 27.01.2016 \\
\hline $561 / 2016$ & SQ4C & bone (1 bag) & 409 & 28.01 .2016 \\
\hline $578 / 2016$ & SQ4 & bone (1 bag) & 411 & 28.01 .2016 \\
\hline $580 / 2016$ & SQ4B & bone (1 bag) & 412 & 28.01 .2016 \\
\hline $582 / 2016$ & SQ4B1 & bone (1 bag) & 413 & 28.01 .2016 \\
\hline $586 / 2016$ & SQ4B1 & bone (1 bag) & 414 & 28.01 .2016 \\
\hline $598 / 2016$ & SQ4B1 & bone (1 bag) & 414 & 30.01 .2016 \\
\hline $592 / 2016$ & SQ4B & bone (1 bag) & 415 & 28.01.2016 \\
\hline $603 / 2016$ & SQ4B & bone (1 bag) & 415 & 30.01 .2016 \\
\hline $606 / 2016$ & SQ4B1 & bone (1 bag) & 416 & 30.01 .2016 \\
\hline
\end{tabular}




\begin{tabular}{|c|c|c|c|c|}
\hline $610 / 2016$ & SQ4/4B & bone (1 bag) & 417 & 30.01 .2016 \\
\hline $614 / 2016$ & SQ4B1 & bone (1 bag) & 418 & 30.01 .2016 \\
\hline $617 / 2016$ & SQ4B1 & bone (1 bag) & 419 & 30.01 .2016 \\
\hline $628 / 2016$ & SQ4B1 & bone (1 bag) & 420 & 30.01 .2016 \\
\hline $635 / 2016$ & SQ4B1 & bone (1 bag) & 420 & 31.01 .2016 \\
\hline $631 / 2016$ & SQ4C & bone (1 bag) & 421 & 30.01 .2016 \\
\hline $642 / 2016$ & SQ4B & bone (1 bag) & 422 & 31.01 .2016 \\
\hline $645 / 2016$ & SQ4B1 & bone (1 piece) & 423 & 31.01 .2016 \\
\hline $638 / 2016$ & SQ4/4B & bone (1 bag) & 424 & 31.01 .2016 \\
\hline $648 / 2016$ & SQ4C & bone (1 bag) & 425 & 31.01 .2016 \\
\hline $651 / 2016$ & SQ4B1 & bone (1 bag) & 426 & 31.01 .2016 \\
\hline $654 / 2016$ & SQ4C & bone (1 bag) & 427 & 31.01 .2016 \\
\hline $661 / 2016$ & SQ4B1 & bone (1 bag) & 428 & 31.01 .2016 \\
\hline $665 / 2016$ & SQ4B & bone (1 bag) & 429 & 31.01 .2016 \\
\hline $671 / 2016$ & SQ4C & bone (1 bag) & 430 & 31.01 .2016 \\
\hline $675 / 2016$ & SQ4C & bone (1 bag) & 430 & 01.02 .2016 \\
\hline $678 / 2016$ & SQ4B1 & bone (1 bag) & 433 & 01.02 .2016 \\
\hline $682 / 2016$ & SQ4B & bone (1 bag) & 434 & 01.02 .2016 \\
\hline $685 / 2016$ & SQ4C & bone (1 bag) & 435 & 01.02 .2016 \\
\hline $721 / 2016$ & SQ4C & bone (1 bag) & 435 & 02.02 .2016 \\
\hline $688 / 2016$ & SQ4C & bone & 436 & 01.02 .2016 \\
\hline $695 / 2016$ & SQ4B & bone (1 bag) & 437 & 01.02 .2016 \\
\hline $698 / 2016$ & SQ4B1 & bone (1 bag) & 438 & 01.02 .2016 \\
\hline $706 / 2016$ & SQ4C & bone (1 bag) & 439 & 01.02 .2016 \\
\hline $724 / 2016$ & SQ4C & bone (1 bag) & 440 & 02.02 .2016 \\
\hline $727 / 2016$ & SQ4C & bone (1 bag) & 441 & 02.02 .2016 \\
\hline
\end{tabular}




\begin{tabular}{|c|c|c|c|c|}
\hline $730 / 2016$ & SQ4 & bone (1 bag) & 442 & 02.02 .2016 \\
\hline $738 / 2016$ & SQ4B & bone & 443 & 02.02 .2016 \\
\hline $741 / 2016$ & $\mathrm{SQ} 4 / 4 \mathrm{~B}$ & bone (1 bag) & 444 & 02.02 .2016 \\
\hline $744 / 2016$ & $\mathrm{SQ} 4 / 4 \mathrm{~B}$ & bone (1 bag) & 445 & 02.02 .2016 \\
\hline $749 / 2016$ & SQ4B & bone (1 bag) & 446 & 02.02 .2016 \\
\hline $756 / 2016$ & SQ4C & bone (1 bag) & 447 & 02.02 .2016 \\
\hline $759 / 2016$ & SQ4B & bone (1 bag) & 448 & 03.02 .2016 \\
\hline $909 / 2016$ & SQ4C & bone (1 bag) & 449 & 08.02 .2016 \\
\hline $765 / 2016$ & SQ4B & bone (1 bag) & 451 & 03.02 .2016 \\
\hline $769 / 2016$ & SQ4B & bone (1 bag) & 452 & 03.02 .2016 \\
\hline $777 / 2016$ & SQ4 & bone (1 bag) & 453 & 03.02 .2016 \\
\hline $782 / 2016$ & SQ4 & bone (1 bag) & 454 & 03.02 .2016 \\
\hline $785 / 2016$ & SQ4B & bone (1 bag) & 455 & 04.02 .2016 \\
\hline $788 / 2016$ & SQ4C & bone (1 bag) & 456 & 04.02 .2016 \\
\hline $812 / 2016$ & SQ4C & bone (1 bag) & 456 & 06.02 .2016 \\
\hline $793 / 2016$ & $\mathrm{SQ} 4 \mathrm{~B} / \mathrm{C}$ & bone (1 bag) & 457 & 04.02 .2016 \\
\hline $798 / 2016$ & SQ4 & bone (1 bag) & 458 & 04.02 .2016 \\
\hline $816 / 2016$ & SQ4 & bone & 458 & 06.02 .2016 \\
\hline $822 / 2016$ & SQ4A/C & bone (1 bag) & 460 & 06.02 .2016 \\
\hline $826 / 2016$ & SQ4, Feature 75 & bone (1 bag) & 461 & 06.02 .2016 \\
\hline $838 / 2016$ & SQ4, Feature 75 & bone (1 bag) & 462 & 06.02 .2016 \\
\hline $853 / 2016$ & SQ4, Feature 75 & bone (1 bag) & 462 & 07.02 .2016 \\
\hline $837 / 2016$ & SQ4C & bone (1 bag) & 463 & 06.02 .2016 \\
\hline $848 / 2016$ & SQ4C & bone (1 bag) & 463 & 07.02 .2016 \\
\hline $851 / 2016$ & SQ4C & bone (1 bag) & 464 & 07.02 .2016 \\
\hline $859 / 2016$ & SQ4, Feature 75 & bones (1 tiny bag) & 465 & 07.02 .2016 \\
\hline
\end{tabular}




\begin{tabular}{|c|c|c|c|c|}
\hline $865 / 2016$ & SQ4C & bone (1 bag) & 466 & 07.02 .2016 \\
\hline $869 / 2016$ & $\mathrm{SQ} 4 \mathrm{C} / 4 \mathrm{~A}$ & bone (1 bag) & 467 & 07.02 .2016 \\
\hline $876 / 2016$ & SQ4C & bone (1 bag) & 469 & 07.02 .2016 \\
\hline $887 / 2016$ & SQ4B1 & bone (1 bag) & 470 & 08.02 .2016 \\
\hline $880 / 2016$ & SQ4B1 & bone (1 piece) & 471 & 08.02 .2016 \\
\hline $884 / 2016$ & SQ4B1 & bone (1 piece) & 472 & 08.02 .2016 \\
\hline $925 / 2016$ & SQ4C & bone (1 bag) & 473 & 08.02 .2016 \\
\hline $898 / 2016$ & SQ4C & bone (1 bag) & 474 & 08.02 .2016 \\
\hline $901 / 2016$ & SQ4C & bone (1 bag) & 475 & 08.02 .2016 \\
\hline $921 / 2016$ & SQ4B & bone (1 bag) & 477 & 08.02 .2016 \\
\hline $933 / 2016$ & SQ4, Feature 15 & bone (1 bag) & 1325 & 09.02 .2016 \\
\hline $942 / 2016$ & SQ4, Feature 15 & bones (1 tiny bag) & 1327 & 09.02 .2016 \\
\hline $947 / 2016$ & SQ4, Feature $15, \mathrm{~W}$ of Feature 44 & bones (1 tiny bag) & 1329 & 09.02 .2016 \\
\hline $952 / 2016$ & SQ4, Feature $15, \mathrm{~W}$ of Feature 44 & bone (1 bag) & 1331 & 10.02 .2016 \\
\hline $955 / 2016$ & $\begin{array}{l}\text { SQ4, Feature } 15 / \text { Feature } 44, W+\text { directly in } \\
\text { Wall } 44\end{array}$ & bone (1 bag) & 1332 & 10.02 .2016 \\
\hline $957 / 2016$ & SQ4, Feature 15 & bones (1 tiny bag) & 1332 & 10.02 .2016 \\
\hline $959 / 2016$ & SQ4, Feature 44, from inside Wall 44 & bone (1 bag) & 1333 & 10.02 .2016 \\
\hline $962 / 2016$ & SQ4, Feature 44 & bones ( 3 pieces) & 1334 & 10.02 .2016 \\
\hline $966 / 2016$ & SQ4, Feature $15,0-1,6 \mathrm{~m}$ to $\mathrm{E}$ & bones ( 5 pieces $)$ & 1335 & 10.02 .2016 \\
\hline $969 / 2016$ & SQ4, Feature $15,1.4-1 \mathrm{~m}$ to $\mathrm{E}$ & bone (1 bag) & 1336 & 10.02 .2016 \\
\hline $971 / 2016$ & SQ4, Feature 15 & bone (1 tiny piece) & 1337 & 10.02 .2016 \\
\hline $976 / 2016$ & SQ4, Feature 15 & bone (1 bag) & 1338 & 11.02 .2016 \\
\hline $978 / 2016$ & SQ4, Feature 15 & bone (1 bag) & 1339 & 11.02 .2016 \\
\hline $981 / 2016$ & SQ4, Feature 15 & bone (1 bag) & 1340 & 11.02 .2016 \\
\hline $987 / 2016$ & SQ4, Feature 15 & bone (1 bag) & 1341 & 11.02 .2016 \\
\hline $079 / 2016$ & SQ4C & botanical material (1 bag) & 315 & 09.01 .2016 \\
\hline
\end{tabular}




\begin{tabular}{|c|c|c|c|c|}
\hline $066 / 2016$ & SQ4C & botanical material (1 piece, stick) & 319 & 09.01 .2016 \\
\hline $158 / 2016$ & SQ4C & botanical material (2 pieces) & 335 & 11.01 .2016 \\
\hline $182 / 2016$ & SQ4C & botanical material (1 piece) & 335 & 13.01.2016 \\
\hline $160 / 2016$ & $\begin{array}{l}\text { SQ4B, found } \mathrm{W} \text { adjacent to remains of mud } \\
\text { brick wall }\end{array}$ & botanical material (2 pieces) & 336 & 11.01.2016 \\
\hline $174 / 2016$ & SQ4B & botanical material (2 pieces) & 337 & 12.01.2016 \\
\hline $205 / 2016$ & SQ4C & botanical material (1 piece) & 343 & 13.01.2016 \\
\hline $240 / 2016$ & SQ4C & botanical material (2 pieces) & 349 & 14.01 .2016 \\
\hline $277 / 2016$ & SQ4B & botanical material (1 bag, fruit?) & 355 & 16.01 .2016 \\
\hline $422 / 2016$ & SQ4B & botanical material (2 pieces) & 388 & 21.01.2016 \\
\hline $515 / 2016$ & SQ4B1 & botanical material (1 bag) & 401 & 26.01.2016 \\
\hline $555 / 2016$ & SQ4B1, S-part of SU & botanical material (1 bag, mixed) & 405 & 27.01 .2016 \\
\hline $619 / 2016$ & SQ4B1 & botanical material (1 bag, mixed) & 419 & 30.01 .2016 \\
\hline $630 / 2016$ & SQ4B1 & botanical material (1 bag, mixed) & 420 & 30.01 .2016 \\
\hline $646 / 2016$ & SQ4B1 & botanical material (1 bag, mixed) & 423 & 31.01 .2016 \\
\hline $833 / 2016$ & SQ4, Feature 75 & botanical material (2 pieces) & 461 & 06.02.2016 \\
\hline $883 / 2016$ & SQ4B1 & botanical material (5 pieces, reed?) & 472 & 08.02 .2016 \\
\hline $927 / 2016$ & SQ4C & botanical material (1 bag, mixed) & 473 & 08.02 .2016 \\
\hline $897 / 2016$ & SQ4C & botanical material (1 bag) & 474 & 08.02 .2016 \\
\hline $949 / 2016$ & $\begin{array}{l}\text { SQ4, Feature 15, lower part of SU, W of } \\
\text { Feature } 44\end{array}$ & botanical material (1 bag, mixed) & 1329 & 09.02.2016 \\
\hline $437 / 2016$ & SQ4B1 & charcoal (4 pieces) & 301 & 24.01 .2016 \\
\hline $041 / 2016$ & SQ4B & charcoal (1 bag) & 311 & 07.01 .2016 \\
\hline $047 / 2016$ & SQ4B & charcoal (1 bag) & 312 & 07.01 .2016 \\
\hline $049 / 2016$ & SQ4B & charcoal (1 bag) & 313 & 07.01 .2016 \\
\hline $071 / 2016$ & SQ4C & charcoal ( 2 pieces) & 315 & 09.01 .2016 \\
\hline $073 / 2016$ & SQ4C & charcoal (1 bag) & 319 & 09.01 .2016 \\
\hline $077 / 2016$ & SQ4B & charcoal (3 pieces) & 320 & 09.01 .2016 \\
\hline
\end{tabular}




\begin{tabular}{|c|c|c|c|c|}
\hline $083 / 2016$ & SQ4B & charcoal (2 pieces) & 321 & 09.01 .2016 \\
\hline $095 / 2016$ & SQ4C & charcoal (1 bag) & 322 & 10.01 .2016 \\
\hline $100 / 2016$ & $\mathrm{SQ} 4 \mathrm{~B}+\mathrm{C}$ & charcoal (1 bag) & 323 & 10.01 .2016 \\
\hline $111 / 2016$ & SQ4B & charcoal (1 bag) & 325 & 10.01 .2016 \\
\hline $115 / 2016$ & SQ4B & charcoal (1 bag) & 326 & 10.01 .2016 \\
\hline $119 / 2016$ & SQ4C & charcoal (1 bag) & 327 & 10.01.2016 \\
\hline $130 / 2016$ & SQ4C & charcoal (1 piece) & 328 & 11.01 .2016 \\
\hline $137 / 2016$ & SQ4C & charcoal (1 piece) & 331 & 11.01.2016 \\
\hline $151 / 2016$ & SQ4C & charcoal (1 piece) & 335 & 11.01 .2016 \\
\hline $159 / 2016$ & SQ4C & charcoal (1 bag) & 335 & 11.01 .2016 \\
\hline $166 / 2016$ & SQ4C & charcoal (1 bag) & 335 & 12.01.2016 \\
\hline $180 / 2016$ & SQ4C & charcoal (1 bag) & 335 & 13.01.2016 \\
\hline $161 / 2016$ & $\begin{array}{l}\text { SQ4B, found } \mathrm{W} \text { adjacent to remains of mud } \\
\text { brick wall }\end{array}$ & charcoal (1 bag) & 336 & 11.01 .2016 \\
\hline $172 / 2016$ & SQ4B & charcoal (1 bag) & 337 & 12.01.2016 \\
\hline $185 / 2016$ & SQ4B & charcoal (1 bag) & 338 & 13.01.2016 \\
\hline $189 / 2016$ & SQ4B & charcoal (1 bag) & 339 & 13.01.2016 \\
\hline $194 / 2016$ & SQ4C & charcoal (1 bag) & 340 & 13.01.2016 \\
\hline $197 / 2016$ & SQ4C & charcoal (1 bag) & 341 & 13.01.2016 \\
\hline $216 / 2016$ & SQ4C & charcoal (1 piece) & 341 & 14.01 .2016 \\
\hline $214 / 2016$ & SQ4B & charcoal (1 bag) & 345 & 14.01.2016 \\
\hline $225 / 2016$ & SQ4B & charcoal ( 1 bag, 1 piece with clay/mud attached) & 346 & 14.01.2016 \\
\hline $231 / 2016$ & SQ4B & charcoal ( 5 pieces $)$ & 347 & 14.01.2016 \\
\hline $236 / 2016$ & SQ4C & charcoal (1 bag) & 348 & 14.01.2016 \\
\hline $253 / 2016$ & SQ4C & charcoal (1 bag) & 348 & 16.01 .2016 \\
\hline $239 / 2016$ & SQ4C & charcoal (1 bag) & 349 & 14.01.2016 \\
\hline $251 / 2016$ & SQ4B & charcoal (1 bag) & 350 & 16.01.2016 \\
\hline
\end{tabular}




\begin{tabular}{|c|c|c|c|c|}
\hline $258 / 2016$ & SQ4A & charcoal (1 bag) & 352 & 16.01.2016 \\
\hline $265 / 2016$ & SQ4C & charcoal (1 bag) & 353 & 16.01.2016 \\
\hline $268 / 2016$ & SQ4B & charcoal (1 bag) & 354 & 16.01.2016 \\
\hline $276 / 2016$ & SQ4B & charcoal (1 bag) & 355 & 16.01.2016 \\
\hline $279 / 2016$ & SQ4B & charcoal (1 bag) & 356 & 16.01.2016 \\
\hline $291 / 2016$ & SQ4B & charcoal (1 bag) & 357 & 17.01.2016 \\
\hline $286 / 2016$ & SQ4B & charcoal (4 pieces) & 358 & 16.01.2016 \\
\hline $294 / 2016$ & SQ4C & charcoal (1 bag) & 359 & 17.01.2016 \\
\hline $297 / 2016$ & SQ4C & charcoal (1 bag) & 360 & 17.01.2016 \\
\hline $299 / 2016$ & SQ4B & charcoal (1 bag) & 361 & 17.01.2016 \\
\hline $303 / 2016$ & SQ4C & charcoal (1 bag) & 362 & 17.01.2016 \\
\hline $311 / 2016$ & SQ4B & charcoal (1 bag) & 364 & 17.01.2016 \\
\hline $317 / 2016$ & SQ4B & charcoal (3 pieces) & 366 & 17.01.2016 \\
\hline $320 / 2016$ & SQ4C & charcoal (1 bag) & 367 & 17.01.2016 \\
\hline $330 / 2016$ & SQ4C & charcoal (1 bag) & 370 & 18.01.2016 \\
\hline $333 / 2016$ & SQ4B & charcoal (1 bag) & 371 & 18.01.2016 \\
\hline $336 / 2016$ & SQ4C & charcoal (1 bag) & 372 & 18.01.2016 \\
\hline $346 / 2016$ & SQ4B & charcoal (1 bag) & 373 & 18.01.2016 \\
\hline $349 / 2016$ & SQ4B & charcoal (1 bag) & 374 & 19.01.2016 \\
\hline $354 / 2016$ & SQ4C & charcoal (1 bag) & 375 & 19.01.2016 \\
\hline $373 / 2016$ & SQ4B & charcoal (1 bag) & 377 & 19.01.2016 \\
\hline $376 / 2016$ & SQ4C & charcoal (1 bag) & 378 & 19.01.2016 \\
\hline $380 / 2016$ & SQ4B & charcoal (1 bag) & 379 & 19.01.2016 \\
\hline $385 / 2016$ & SQ4C & charcoal (1 bag) & 380 & 20.01 .2016 \\
\hline $388 / 2016$ & SQ4C & charcoal (1 bag) & 381 & 20.01.2016 \\
\hline $397 / 2016$ & SQ4C & charcoal (1 bag) & 382 & 20.01 .2016 \\
\hline
\end{tabular}




\begin{tabular}{|c|c|c|c|c|}
\hline $404 / 2016$ & SQ4C & charcoal (1 bag) & 383 & 21.01 .2016 \\
\hline $426 / 2016$ & SQ4C & charcoal (3 pieces) & 384 & 21.01 .2016 \\
\hline $454 / 2016$ & SQ4C & charcoal (1 bag) & 384 & 23.01 .2016 \\
\hline $407 / 2016$ & SQ4B & charcoal (1 bag) & 385 & 21.01 .2016 \\
\hline $413 / 2016$ & SQ4B & charcoal (1 bag) & 386 & 21.01 .2016 \\
\hline $419 / 2016$ & SQ4B & charcoal (1 bag) & 388 & 21.01 .2016 \\
\hline $433 / 2016$ & SQ4C & charcoal (4 pieces) & 390 & 23.01 .2016 \\
\hline $440 / 2016$ & SQ4B & charcoal (1 bag) & 391 & 23.01 .2016 \\
\hline $449 / 2016$ & SQ4C & charcoal (4 pieces) & 392 & 23.01 .2016 \\
\hline $464 / 2016$ & SQ4C & charcoal (3 pieces) & 393 & 24.01 .2016 \\
\hline $467 / 2016$ & SQ4C & charcoal (1 bag) & 393 & 25.01 .2016 \\
\hline $473 / 2016$ & SQ4B1 & charcoal (1 bag) & 395 & 25.01 .2016 \\
\hline $476 / 2016$ & SQ4B & charcoal (1 bag) & 396 & 25.01 .2016 \\
\hline $488 / 2016$ & SQ4C & charcoal (1 bag) & 397 & 25.01 .2016 \\
\hline $501 / 2016$ & SQ4C & charcoal (1 bag) & 399 & 26.01 .2016 \\
\hline $504 / 2016$ & SQ4C & charcoal (1 bag) & 400 & 26.01 .2016 \\
\hline $507 / 2016$ & SQ4B1 & charcoal (1 bag) & 401 & 26.01 .2016 \\
\hline $520 / 2016$ & SQ4C & charcoal (1 bag) & 402 & 26.01 .2016 \\
\hline $518 / 2016$ & SQ4B1 & charcoal (1 bag) & 403 & 26.01 .2016 \\
\hline $552 / 2016$ & SQ4B1, N-part of SU & charcoal (1 bag) & 405 & 27.01 .2016 \\
\hline $573 / 2016$ & SQ4B1, N-part of SU & charcoal (3 pieces) & 405 & 28.01 .2016 \\
\hline $534 / 2016$ & SQ4B & charcoal (1 bag) & 406 & 27.01.2016 \\
\hline $557 / 2016$ & SQ4C & charcoal (1 bag) & 408 & 27.01 .2016 \\
\hline $562 / 2016$ & SQ4C & charcoal (1 bag) & 409 & 28.01 .2016 \\
\hline $594 / 2016$ & SQ4B1 & charcoal (1 bag) & 413 & 28.01 .2016 \\
\hline $590 / 2016$ & SQ4B1 & charcoal (1 bag) & 414 & 28.01 .2016 \\
\hline
\end{tabular}




\begin{tabular}{|c|c|c|c|c|}
\hline $599 / 2016$ & SQ4B1 & charcoal (4 pieces) & 414 & 30.01 .2016 \\
\hline $595 / 2016$ & SQ4B & charcoal (1 bag) & 415 & 28.01 .2016 \\
\hline $604 / 2016$ & SQ4B & charcoal (1 bag) & 415 & 30.01 .2016 \\
\hline $607 / 2016$ & SQ4B1 & charcoal (1 bag) & 416 & 30.01 .2016 \\
\hline $611 / 2016$ & SQ4/4B & charcoal (1 bag) & 417 & 30.01 .2016 \\
\hline $626 / 2016$ & SQ4B1 & charcoal (1 bag) & 418 & 30.01 .2016 \\
\hline $618 / 2016$ & SQ4B1 & charcoal (1 bag) & 419 & 30.01 .2016 \\
\hline $629 / 2016$ & SQ4B1 & charcoal (1 bag) & 420 & 30.01 .2016 \\
\hline $636 / 2016$ & SQ4B1 & charcoal (1 bag) & 420 & 31.01 .2016 \\
\hline $632 / 2016$ & SQ4C & charcoal (1 bag) & 421 & 30.01 .2016 \\
\hline $643 / 2016$ & SQ4B & charcoal (1 bag) & 422 & 31.01 .2016 \\
\hline $639 / 2016$ & SQ4/4B & charcoal (1 bag) & 424 & 31.01 .2016 \\
\hline $652 / 2016$ & SQ4B1 & charcoal (1 bag) & 426 & 31.01 .2016 \\
\hline $662 / 2016$ & SQ4B1 & charcoal (1 bag) & 428 & 31.01 .2016 \\
\hline $666 / 2016$ & SQ4B & charcoal (1 bag) & 429 & 31.01 .2016 \\
\hline $672 / 2016$ & SQ4C & charcoal (3 pieces) & 430 & 31.01 .2016 \\
\hline $676 / 2016$ & SQ4C & charcoal (1 bag) & 430 & 01.02 .2016 \\
\hline $669 / 2016$ & SQ4B1 & charcoal (5 pieces) & 432 & 31.01 .2016 \\
\hline $679 / 2016$ & SQ4B1 & charcoal (1 bag) & 433 & 01.02 .2016 \\
\hline $690 / 2016$ & SQ4B & charcoal (1 bag) & 434 & 01.02 .2016 \\
\hline $686 / 2016$ & SQ4C & charcoal (1 bag) & 435 & 01.02 .2016 \\
\hline $722 / 2016$ & SQ4C & charcoal (1 bag) & 435 & 02.02 .2016 \\
\hline $689 / 2016$ & SQ4C & charcoal (1 bag) & 436 & 01.02 .2016 \\
\hline $696 / 2016$ & SQ4B & charcoal (1 bag) & 437 & 01.02 .2016 \\
\hline $699 / 2016$ & SQ4B1 & charcoal (1 bag) & 438 & 01.02 .2016 \\
\hline $707 / 2016$ & SQ4C & charcoal (1 bag) & 439 & 01.02 .2016 \\
\hline
\end{tabular}




\begin{tabular}{|c|c|c|c|c|}
\hline $709 / 2016$ & SQ4C & charcoal (1 bag) & 440 & 01.02 .2016 \\
\hline $725 / 2016$ & SQ4C & charcoal (1 bag) & 440 & 02.02 .2016 \\
\hline $728 / 2016$ & SQ4C & charcoal (1 bag) & 441 & 02.02 .2016 \\
\hline $731 / 2016$ & SQ4 & charcoal (1 bag) & 442 & 02.02 .2016 \\
\hline $739 / 2016$ & SQ4B & charcoal (1 bag) & 443 & 02.02 .2016 \\
\hline $742 / 2016$ & $\mathrm{SQ} 4 / 4 \mathrm{~B}$ & charcoal (1 bag) & 444 & 02.02 .2016 \\
\hline $745 / 2016$ & $\mathrm{SQ} 4 / 4 \mathrm{~B}$ & charcoal (1 bag) & 445 & 02.02 .2016 \\
\hline $750 / 2016$ & SQ4B & charcoal (1 bag) & 446 & 02.02 .2016 \\
\hline $757 / 2016$ & SQ4C & charcoal (1 bag) & 447 & 02.02 .2016 \\
\hline $760 / 2016$ & SQ4B & charcoal (1 bag) & 448 & 03.02 .2016 \\
\hline $908 / 2016$ & SQ4C & charcoal (1 bag) & 449 & 08.02 .2016 \\
\hline $766 / 2016$ & SQ4B & charcoal (1 bag) & 451 & 03.02 .2016 \\
\hline $770 / 2016$ & SQ4B & charcoal (1 bag) & 452 & 03.02 .2016 \\
\hline $778 / 2016$ & SQ4 & charcoal (1 bag) & 453 & 03.02 .2016 \\
\hline $783 / 2016$ & SQ4 & charcoal (1 bag) & 454 & 03.02 .2016 \\
\hline $786 / 2016$ & SQ4B & charcoal (1 bag) & 455 & 04.02 .2016 \\
\hline $789 / 2016$ & SQ4C & charcoal (1 bag) & 456 & 04.02 .2016 \\
\hline $813 / 2016$ & SQ4C & charcoal (1 bag) & 456 & 06.02 .2016 \\
\hline $794 / 2016$ & SQ4B/C & charcoal (1 bag) & 457 & 04.02 .2016 \\
\hline $799 / 2016$ & SQ4 & charcoal (1 bag) & 458 & 04.02 .2016 \\
\hline $817 / 2016$ & SQ4 & charcoal (4 pieces) & 458 & 06.02 .2016 \\
\hline $806 / 2016$ & $\mathrm{SQ} 4 / 4 \mathrm{~B}$ & charcoal (1 bag) & 459 & 04.02 .2016 \\
\hline $823 / 2016$ & $\mathrm{SQ} 4 \mathrm{~A} / \mathrm{C}$ & charcoal (1 bag) & 460 & 06.02 .2016 \\
\hline $827 / 2016$ & SQ4, Feature 75 & charcoal (1 bag) & 461 & 06.02 .2016 \\
\hline $839 / 2016$ & SQ4, Feature 75 & charcoal (1 bag) & 462 & 06.02 .2016 \\
\hline $854 / 2016$ & SQ4, Feature 75 & charcoal (1 bag) & 462 & 07.02 .2016 \\
\hline
\end{tabular}




\begin{tabular}{|c|c|c|c|c|c|}
\hline $849 / 2016$ & SQ4C & charcoal (1 bag) & 463 & & 07.02 .2016 \\
\hline $860 / 2016$ & SQ4, Feature 75 & charcoal (1 bag) & 465 & & 07.02 .2016 \\
\hline $864 / 2016$ & $\mathrm{SQ} 4 \mathrm{C}$ & charcoal (1 bag) & 466 & & 07.02 .2016 \\
\hline $868 / 2016$ & $\mathrm{SQ} 4 \mathrm{C} / 4 \mathrm{~A}$ & charcoal (1 bag) & 467 & & 07.02 .2016 \\
\hline $875 / 2016$ & $\mathrm{SQ} 4 \mathrm{C}$ & charcoal (1 bag) & 469 & & 07.02 .2016 \\
\hline $886 / 2016$ & SQ4B1 & charcoal (1 bag) & 470 & & 08.02 .2016 \\
\hline $879 / 2016$ & SQ4B1 & charcoal (3 pieces) & 471 & & 08.02 .2016 \\
\hline $882 / 2016$ & SQ4B1 & charcoal (1 bag) & 472 & & 08.02 .2016 \\
\hline $922 / 2016$ & $\mathrm{SQ} 4 \mathrm{C}$ & charcoal (1 bag) & 473 & & 08.02 .2016 \\
\hline $896 / 2016$ & SQ4C & charcoal (1 bag) & 474 & & 08.02 .2016 \\
\hline $905 / 2016$ & $\begin{array}{l}\text { SQ4B/4B1, N-part of SU between } \\
\text { Feature } 68 / 67 \text { and Feature } 69 / 70\end{array}$ & charcoal (2 pieces) & 476 & & 08.02 .2016 \\
\hline $932 / 2016$ & SQ4, Feature 15 & charcoal (1 bag) & 1325 & & 09.02 .2016 \\
\hline $941 / 2016$ & SQ4, Feature 15, W of Feature 44 & charcoal (1 bag) & 1327 & & 09.02 .2016 \\
\hline $946 / 2016$ & SQ4, Feature 15, W of Feature 44 & charcoal (1 bag) & 1329 & & 09.02 .2016 \\
\hline $953 / 2016$ & SQ4, Feature 15, W of Feature 44 & charcoal (1 bag) & 1331 & & 10.02 .2016 \\
\hline $957 / 2016$ & SQ4, Feature 15 & charcoal (1 bag) & 1332 & & 10.02 .2016 \\
\hline $960 / 2016$ & SQ4, Feature 44 & charcoal (3 pieces) & 1333 & & 10.02 .2016 \\
\hline $963 / 2016$ & SQ4, Feature 44 & charcoal (1 piece) & 1334 & & 10.02 .2016 \\
\hline $965 / 2016$ & SQ4, Feature $15,0-1,6 \mathrm{~m}$ to $\mathrm{E}$ & charcoal (1 bag) & 1335 & & 10.02 .2016 \\
\hline $968 / 2016$ & SQ4, Feature 15, 1,4-1,6m & charcoal (1 bag) & 1336 & & 10.02 .2016 \\
\hline $972 / 2016$ & SQ4, Feature 15 & charcoal (3 pieces) & 1337 & & 10.02 .2016 \\
\hline $976 / 2016$ & SQ4, Feature 15 & charcoal (1 bag) & 1338 & & 11.02 .2016 \\
\hline $979 / 2016$ & SQ4, Feature 15 & charcoal (1 bag) & 1339 & & 11.02 .2016 \\
\hline $982 / 2016$ & SQ4, Feature 15 & charcoal (1 bag) & 1340 & & 11.02 .2016 \\
\hline $986 / 2016$ & SQ4, Feature 15 & charcoal (1 bag) & 1341 & & 11.02 .2016 \\
\hline $222 / 2016$ & SQ4C & crucible (1 fragment with traces of oxidation) & 344 & SAV1E 2721 & 14.01.2016 \\
\hline
\end{tabular}




\begin{tabular}{|c|c|c|c|c|c|}
\hline $341 / 2016$ & SQ4C & crucible? (1 fragment, pottery with slag attached) & 370 & SAV1E 1526 & 18.01 .2016 \\
\hline $443 / 2016$ & SQ4C & crystal with plaster inside; gypsum for mortar (1 piece) & 392 & & 23.01 .2016 \\
\hline $548 / 2016$ & SQ4C & crystal with plaster inside; gypsum for mortar (1 piece) & 408 & & 27.01 .2016 \\
\hline $904 / 2016$ & SQ4B, S-part of SU, E of Feature 70 & crystal? stone?; gypsum for mortar (1 piece) & 476 & & 08.02 .2016 \\
\hline $045 / 2016$ & SQ4B & date seed (1 piece) & 312 & & 07.01 .2016 \\
\hline $423 / 2016$ & SQ4B & date seeds ( 2 pieces, worked with small holes) & 388 & SAV1E 2749 & 21.01 .2016 \\
\hline $809 / 2016$ & $\mathrm{SQ} 4 / 4 \mathrm{~B}$ & date seed (1 piece) & 459 & & 04.02 .2016 \\
\hline $450 / 2016$ & SQ4C, found in E half of SU & dice (1 piece, clay) & 384 & SAV1E 2771 & 23.01 .2016 \\
\hline $039 / 2016$ & SQ4B & doum nuts (1 bag) & 311 & & 07.01 .2016 \\
\hline $150 / 2016$ & SQ4C & doum nut (1 piece, burned) & 335 & & 11.01 .2016 \\
\hline $186 / 2016$ & SQ4B & doum nut (1 piece) & 338 & & 13.01.2016 \\
\hline $273 / 2016$ & SQ4B & doum nuts ( 1 bag, burned) & 354 & & 16.01 .2016 \\
\hline $287 / 2016$ & SQ4B & doum nuts (3 pieces, burned) & 358 & & 16.01 .2016 \\
\hline $321 / 2016$ & SQ4C & doum nuts (1 bag) & 367 & & 17.01 .2016 \\
\hline $350 / 2016$ & SQ4B & doum nuts (1 bag) & 374 & & 19.01 .2016 \\
\hline $468 / 2016$ & SQ4C & doum nuts (2 pieces) & 393 & & 25.01 .2016 \\
\hline $502 / 2016$ & SQ4C & doum nuts (1 bag) & 399 & & 26.01 .2016 \\
\hline $551 / 2016$ & SQ4B1, S-part of SU & doum nut (1 piece) & 405 & & 27.01 .2016 \\
\hline $553 / 2016$ & SQ4B1, N-part of SU & doum nut (1 piece) & 405 & & 27.01 .2016 \\
\hline $558 / 2016$ & SQ4C & doum nut (1 piece) & 408 & & 27.01 .2016 \\
\hline $612 / 2016$ & $\mathrm{SQ} 4 / 4 \mathrm{~B}$ & doum nuts (2 pieces) & 417 & & 30.01 .2016 \\
\hline $627 / 2016$ & SQ4B1 & doum nut (1 piece) & 418 & & 30.01 .2016 \\
\hline $633 / 2016$ & SQ4B1 & doum nut (1 piece) & 420 & & 30.01 .2016 \\
\hline $658 / 2016$ & SQ4B1 & doum nuts (2 pieces) & 420 & & 31.01 .2016 \\
\hline $649 / 2016$ & SQ4C & doum nuts (1 bag) & 425 & & 31.01 .2016 \\
\hline $710 / 2016$ & SQ4C & doum nuts (1 bag) & 440 & & 01.02 .2016 \\
\hline
\end{tabular}




\begin{tabular}{|c|c|c|c|c|c|}
\hline $779 / 2016$ & SQ4 & doum nuts (1 bag) & 453 & & 03.02 .2016 \\
\hline $802 / 2016$ & SQ4C & doum nuts (1 bag) & 456 & & 04.02 .2016 \\
\hline $818 / 2016$ & SQ4C & doum nuts ( 2 pieces) & 456 & & 06.02 .2016 \\
\hline $807 / 2016$ & SQ4/4B & doum nuts ( 2 pieces) & 459 & & 04.02 .2016 \\
\hline $824 / 2016$ & $\mathrm{SQ} 4 \mathrm{~A} / \mathrm{C}$ & doum nuts (1 bag) & 460 & & 06.02 .2016 \\
\hline $829 / 2016$ & SQ4, Feature 75 & doum nut (1 piece) & 461 & & 06.02 .2016 \\
\hline $857 / 2016$ & SQ4, Feature 75 & doum nuts ( 2 pieces) & 462 & & 07.02 .2016 \\
\hline $934 / 2016$ & SQ4, Feature 15 & doum nuts (1 bag) & 1325 & & 09.02 .2016 \\
\hline $936 / 2016$ & SQ4, Feature 15, from sieving & doum nuts (1 bag) & 1325 & & 09.02 .2016 \\
\hline $954 / 2016$ & SQ4, Feature 15, W of Wall 44 & doum nuts (4 pieces) & 1331 & & 10.02 .2016 \\
\hline $957 / 2016$ & SQ4, Feature 15 & doum nut (1 piece) & 1332 & & 10.02 .2016 \\
\hline $974 / 2016$ & SQ4, Feature 15 & doum nuts (1 bag) & 1336 & & 10.02 .2016 \\
\hline $979 / 2016$ & SQ4, Feature 15 & doum nuts (1 bag) & 1339 & & 11.02 .2016 \\
\hline $983 / 2016$ & SQ4, Feature 15 & doum nuts (1 bag) & 1340 & & 11.02 .2016 \\
\hline $988 / 2016$ & SQ4, Feature 15 & doum nuts (1 bag) & 1341 & & 11.02 .2016 \\
\hline $261 / 2016$ & SQ4B & ear ring (1 fragment, faience) & 354 & SAV1E 2729 & 16.01.2016 \\
\hline $275 / 2016$ & SQ4B & $\begin{array}{l}\text { faience artefact (1 small fragment, original form and function } \\
\text { unclear) }\end{array}$ & 355 & SAV1E 2731 & 16.01 .2016 \\
\hline $615 / 2016$ & SQ4B1 & $\begin{array}{l}\text { faience object (1 small fragment, original form and function } \\
\text { unclear) }\end{array}$ & 418 & SAV1E 2832 & 30.01 .2016 \\
\hline $701 / 2016$ & $\begin{array}{l}\text { SQ4B, found on the bottom surface of } \\
\text { the SU }\end{array}$ & faience bowl? (1 rim sherd, re-used as scraper) & 437 & SAV1E 2846 & 01.02 .2016 \\
\hline $458 / 2016$ & SQ4B1 & $\begin{array}{l}\text { figurine ( } 1 \text { fragment, head part of a human figure, probably } \\
\text { male, pottery) }\end{array}$ & 301 & SAV1E 2779 & 24.01 .2016 \\
\hline $014 / 2016$ & $\mathrm{SQ} 4 \mathrm{~A} / 4$ & $\begin{array}{l}\text { figurine of a horse (1 fragment, head part and front legs, } \\
\text { pottery) }\end{array}$ & 302 & SAV1E 2667 & 05.01 .2016 \\
\hline $042 / 2016$ & SQ4B & figurine (1 fragment, probably horse figurine, pottery, Christian) & 311 & SAV1E 0406 & 07.01 .2016 \\
\hline $097 / 2016$ & SQ4C & figurine ( 1 fragment, rudimentary female figurine, clay) & 322 & SAV1E 0939 & 10.01 .2016 \\
\hline $556 / 2016$ & $\begin{array}{l}\text { SQ4B1, S-part of SU, directly W of mud } \\
\text { brick wall }\end{array}$ & figurine (1 small female figurine, complete, clay) & 405 & SAV1E 2801 & 27.01 .2016 \\
\hline
\end{tabular}




\begin{tabular}{|c|c|c|c|c|c|}
\hline $913 / 2016$ & SQ4C & figurine? stopper? (1 conical fragment of a clay object) & 449 & SAV1E 2891 & 08.02 .2016 \\
\hline $973 / 2016$ & SQ4 & figurine ( 1 seated ram, probably small lid, clay) & 1337 & SAV1E 0181 & 10.02 .2016 \\
\hline $870 / 2016$ & SQ4C/4A, SE of SU in mud brick debris & $\begin{array}{l}\text { finger ring/ring bezel ( } 1 \text { fragment with wedjat-eye as central } \\
\text { element, faience) }\end{array}$ & 467 & SAV1E 2882 & 07.02 .2016 \\
\hline $177 / 2016$ & SQ4C & glass (1 fragment, perhaps from a vessel) & 335 & SAV1E 2701 & 12.01 .2016 \\
\hline $364 / 2016$ & SQ4B & $\begin{array}{l}\text { glass ( } 1 \text { fragment, perhaps from a vessel/bottle, perhaps } \\
\text { modern) }\end{array}$ & 374 & SAV1E 1639 & 19.01.2016 \\
\hline $430 / 2016$ & SQ4C & glass (1 fragment, original form and function unclear) & 384 & SAV1E 2769 & 23.01 .2016 \\
\hline $494 / 2016$ & SQ4C & glass (1 small rounded fragment) & 397 & SAV1E 2784 & 25.01 .2016 \\
\hline $569 / 2016$ & SQ4B1, S-part of SU & $\begin{array}{l}\text { glass ( } 1 \text { small fragment of aqua coloured glass, original form } \\
\text { and function unclear) }\end{array}$ & 405 & SAV1E 2810 & 28.01.2016 \\
\hline $539 / 2016$ & SQ4B & glass vessel (1 small fragment) & 406 & SAV1E 2803 & 27.01 .2016 \\
\hline $680 / 2016$ & SQ4B1 & glass (1 fragment, original form and function unclear) & 433 & SAV1E 2848 & 01.02 .2016 \\
\hline $753 / 2016$ & SQ4/4B & $\begin{array}{l}\text { glass ( } 1 \text { small fragment of clear glass, original form and } \\
\text { function unclear) }\end{array}$ & 444 & SAV1E 2864 & 02.02 .2016 \\
\hline $819 / 2016$ & SQ4C & $\begin{array}{l}\text { glass ( } 1 \text { small fragment, probably from vessel, original form } \\
\text { and function unclear) }\end{array}$ & 456 & SAV1E 1071 & 06.02 .2016 \\
\hline $907 / 2016$ & SQ4B, N-part of SU & $\begin{array}{l}\text { glass ( } 1 \text { small fragment of blue-green glass, original form and } \\
\text { function unclear, vessel?) }\end{array}$ & 476 & SAV1E 2886 & 08.02 .2016 \\
\hline $920 / 2016$ & SQ4B & $\begin{array}{l}\text { glass/glass paste ( } 1 \text { small fragment, original form and function } \\
\text { unclear) }\end{array}$ & 477 & SAV1E 2885 & 08.02 .2016 \\
\hline $936 / 2016$ & SQ4, Feature 15 from sieving & glass (1 fragment, reference) & 1325 & & 09.02 .2016 \\
\hline $038 / 2016$ & SQ4B & hair/fur (1 bag) & 311 & & 07.01 .2016 \\
\hline $601 / 2016$ & SQ4B1 & hair (1 bag) & 414 & & 30.01 .2016 \\
\hline $181 / 2016$ & $\mathrm{SQ} 4 \mathrm{C}$ & horn (1 piece) & 335 & & 13.01.2016 \\
\hline $227 / 2016$ & SQ4B & horn (1 piece) & 346 & & 14.01 .2016 \\
\hline $372 / 2016$ & SQ4C & horn (1 piece) & 375 & & 19.01.2016 \\
\hline $398 / 2016$ & SQ4C & horn (1 bag) & 380 & & 20.01 .2016 \\
\hline $421 / 2016$ & $\mathrm{SQ} 4 \mathrm{C}$ & horn (1 piece) & 383 & & 21.01 .2016 \\
\hline $486 / 2016$ & SQ4B1 & horn (1 piece) & 395 & & 25.01 .2016 \\
\hline $528 / 2016$ & SQ4B1, S-part of SU & horn (2 large pieces) & 405 & & 27.01 .2016 \\
\hline
\end{tabular}




\begin{tabular}{|c|c|c|c|c|c|}
\hline $790 / 2016$ & SQ4C & horn (1 bag) & 456 & & 04.02 .2016 \\
\hline $105 / 2016$ & SQ4B & jar stopper (1 piece, clay/mud) & 324 & SAV1E 0975 & 10.01 .2016 \\
\hline $108 / 2016$ & SQ4B & leather sandal (1 piece, left - modern ?) & 325 & & 10.01.2016 \\
\hline $146 / 2016$ & SQ4B & leather (1 piece) & 333 & & 11.01 .2016 \\
\hline $169 / 2016$ & SQ4C & leather (1 piece) & 335 & & 12.01.2016 \\
\hline $245 / 2016$ & SQ4C & leather (1 piece) & 349 & & 14.01.2016 \\
\hline $280 / 2016$ & SQ4C & leather (1 piece) & 353 & & 16.01 .2016 \\
\hline $343 / 2016$ & SQ4B & leather (1 piece) & 373 & & 18.01.2016 \\
\hline $353 / 2016$ & SQ4C & leather (1 piece) & 375 & & 19.01.2016 \\
\hline $415 / 2016$ & SQ4B & leather (1 piece) & 386 & & 21.01 .2016 \\
\hline $448 / 2016$ & SQ4C & leather (2 pieces) & 392 & & 23.01 .2016 \\
\hline $477 / 2016$ & SQ4B & leather (1 piece) & 396 & & 25.01 .2016 \\
\hline $550 / 2016$ & SQ4B1, S-part of SU & leather (1 bag) & 405 & & 27.01 .2016 \\
\hline $570 / 2016$ & SQ4B & leather (1 piece) & 406 & & 28.01 .2016 \\
\hline $593 / 2016$ & SQ4B1 & leather (2 pieces) & 413 & & 28.01 .2016 \\
\hline $588 / 2016$ & SQ4B1 & leather (1 piece) & 414 & & 28.01 .2016 \\
\hline $625 / 2016$ & $\mathrm{SQ} 4 / 4 \mathrm{~B}$ & leather (1 bag) & 417 & & 30.01 .2016 \\
\hline $659 / 2016$ & SQ4B1 & leather/animal skin (1 piece) & 420 & & 31.01 .2016 \\
\hline $657 / 2016$ & SQ4B1 & leather (1 piece) & 426 & & 31.01 .2016 \\
\hline $667 / 2016$ & SQ4B & leather/animal skin (1 bag) & 429 & & 31.01 .2016 \\
\hline $791 / 2016$ & SQ4C & leather/animal skin (1 bag) & 456 & & 04.02 .2016 \\
\hline $814 / 2016$ & SQ4C & leather/animal skin (1 piece) & 456 & & 06.02 .2016 \\
\hline $846 / 2016$ & SQ4 & leather/animal skin (1 bag) & 460 & & 06.02 .2016 \\
\hline $872 / 2016$ & $\mathrm{SQ} 4 \mathrm{C} / 4 \mathrm{~A}$ & leather/animal skin (1 piece) & 467 & & 07.02 .2016 \\
\hline $479 / 2016$ & SQ4B & lid (1 piece, re-used base sherd of a $18^{\text {th }}$ Dynasty pottery vessel) & 396 & SAV1E 2788 & 25.01 .2016 \\
\hline $714 / 2016$ & SQ4B & metal (1 piece) & 437 & & 01.02 .2016 \\
\hline
\end{tabular}




\begin{tabular}{|c|c|c|c|c|c|}
\hline $936 / 2016$ & SQ4, Feature 15 from sieving & metal (1 tiny piece, sample) & 1325 & & 09.02.2016 \\
\hline $368 / 2016$ & SQ4C & mud ball, unfired, possible stopper? (1 small piece) & 376 & SAV1E 0211 & 19.01.2016 \\
\hline $536 / 2016$ & SQ4B & nail (1 small point end of a nail, metal) & 406 & SAV1E 2802 & 27.01.2016 \\
\hline $683 / 2016$ & SQ4B & nail? hook? (1 fragment, metal) & 434 & SAV1E 2849 & 01.02 .2016 \\
\hline $069 / 2016$ & SQ4C & net weight (1 nearly complete piece, pottery) & 319 & SAV1E 0608 & 09.01 .2016 \\
\hline $596 / 2016$ & SQ4B1 & net weight ( 1 corner fragment, pottery) & 414 & SAV1E 2818 & 28.01.2016 \\
\hline $623 / 2016$ & SQ4C & net weight (1 piece, pottery) & 421 & SAV1E 2833 & 30.01 .2016 \\
\hline $076 / 2016$ & SQ4B & Nun bowl (1 fragment, faience) & 320 & SAV1E 0513 & 09.01.2016 \\
\hline $451 / 2016$ & SQ4B & Nun bowl (1 rim sherd, faience) & 391 & SAV1E 2767 & 23.01.2016 \\
\hline $485 / 2016$ & SQ4B1 & $\begin{array}{l}\text { Nun bowl (1 fragment containing rim and small part of base, } \\
\text { faience) }\end{array}$ & 394 & SAV1E 2785 & 25.01.2016 \\
\hline $541 / 2016$ & SQ4B1, S-part of SU & Nun bowl? (1 fragment, faience) & 405 & SAV1E 2804 & 27.01.2016 \\
\hline $900 / 2016$ & SQ4C & Nun bowl? (1 rim fragment, faience) & 475 & SAV1E 2884 & 08.02 .2016 \\
\hline $131 / 2016$ & SQ4C & organic material (2 pieces) & 328 & & 11.01.2016 \\
\hline $221 / 2016$ & SQ4C & organic material (1 piece, fruit) & 341 & & 14.01.2016 \\
\hline $232 / 2016$ & SQ4B & organic material (1 bag) & 347 & & 14.01.2016 \\
\hline $300 / 2016$ & SQ4B & organic material ( 3 pieces) & 361 & & 17.01.2016 \\
\hline $399 / 2016$ & SQ4C & organic material (1 piece) & 380 & & 20.01.2016 \\
\hline $490 / 2016$ & SQ4C & organic material (1 bag) & 397 & & 25.01 .2016 \\
\hline $568 / 2016$ & SQ4B1, S-part of SU & organic material ( 2 pieces) & 405 & & 28.01 .2016 \\
\hline $574 / 2016$ & SQ4B1, N-part of SU & organic material (1 piece) & 405 & & 28.01.2016 \\
\hline $535 / 2016$ & SQ4B & organic material (1 bag) & 406 & & 27.01.2016 \\
\hline $563 / 2016$ & SQ4C & organic material (1 piece) & 409 & & 28.01 .2016 \\
\hline $564 / 2016$ & SQ4C & organic material (1 bag) & 409 & & 28.01.2016 \\
\hline $587 / 2016$ & SQ4B1 & organic material (1 bag) & 414 & & 28.01 .2016 \\
\hline $608 / 2016$ & SQ4B1 & organic material (1 bag) & 414 & & 30.01 .2016 \\
\hline $655 / 2016$ & SQ4B1 & organic material ( 1 bag, mixed) & 426 & & 31.01 .2016 \\
\hline
\end{tabular}




\begin{tabular}{|c|c|c|c|c|c|}
\hline $693 / 2016$ & SQ4B1 & organic material ( 1 bag, mixed) & 433 & & 01.02 .2016 \\
\hline $691 / 2016$ & SQ4B & organic material (4 pieces) & 434 & & 01.02 .2016 \\
\hline $717 / 2016$ & SQ4B & organic material & 437 & & 01.02 .2016 \\
\hline $704 / 2016$ & SQ4B1 & organic material ( 1 bag, mixed) & 438 & & 01.02 .2016 \\
\hline $754 / 2016$ & SQ4B & organic material ( 1 bag, mixed) & 443 & & 02.02 .2016 \\
\hline $752 / 2016$ & SQ4/4B & organic material ( 1 bag, mixed) & 444 & & 02.02 .2016 \\
\hline $751 / 2016$ & SQ4B & organic material ( 1 bag, mixed) & 446 & & 02.02 .2016 \\
\hline $762 / 2016$ & SQ4B & organic material ( 1 bag, mixed) & 448 & & 03.02 .2016 \\
\hline $910 / 2016$ & SQ4C & organic material (1 bag, mixed) & 449 & & 08.02 .2016 \\
\hline $771 / 2016$ & SQ4B & organic material ( 1 bag, mixed) & 451 & & 03.02 .2016 \\
\hline $774 / 2016$ & SQ4B & organic material ( 1 bag, mixed) & 452 & & 03.02 .2016 \\
\hline $795 / 2016$ & $\mathrm{SQ} 4 \mathrm{~B} / \mathrm{C}$ & organic material (1 bag, mixed) & 457 & & 04.02 .2016 \\
\hline $926 / 2016$ & SQ4C & organic material (1 piece, fibre with knot) & 473 & & 08.02 .2016 \\
\hline $989 / 2016$ & SQ4, Feature 15 & organic material (1 piece) & 1341 & & 11.02.2016 \\
\hline $175 / 2016$ & SQ4B & pipe (1 piece, clay, Christian/Ottoman) & 337 & SAV1E 2702 & 12.01 .2016 \\
\hline $099 / 2016$ & $\mathrm{SQ} 4 \mathrm{~B}+\mathrm{C}$ & plaster (1 bag) & 323 & & 10.01.2016 \\
\hline $241 / 2016$ & SQ4C, floor surface & plaster and schist fragments & 349 & & 14.01.2016 \\
\hline $249 / 2016$ & SQ4C, floor surface & plaster (1 bag) & 349 & & 16.01.2016 \\
\hline $322 / 2016$ & SQ4C, floor surface & plaster (1 bag) & 362 & & 17.01.2016 \\
\hline $312 / 2016$ & SQ4C, floor surface & plaster (5 pieces) & 364 & & 17.01.2016 \\
\hline $355 / 2016$ & SQ4C, floor surface & plaster (1 bag) & 375 & & 19.01.2016 \\
\hline $377 / 2016$ & SQ4C, floor surface & plaster (1 bag) & 378 & & 19.01.2016 \\
\hline $382 / 2016$ & SQ4C, floor surface & plaster (1 bag) & 378 & & 20.01.2016 \\
\hline $773 / 2016$ & SQ4B, sifted & plaster (4 pieces) & 451 & & 03.02 .2016 \\
\hline $834 / 2016$ & SQ4, Feature 75 & plaster (1 piece) & 461 & & 06.02 .2016 \\
\hline $890 / 2016$ & SQ4B1 & plaster (1 bag) & 470 & & 08.02 .2016 \\
\hline
\end{tabular}




\begin{tabular}{|c|c|c|c|c|c|}
\hline $949 / 2016$ & $\begin{array}{l}\text { SQ4, Feature 15, lower part of SU, W of } \\
\text { Feature } 44\end{array}$ & plaster/chalk (1 piece) & 1329 & & 09.02 .2016 \\
\hline $001 / 2016$ & SQ4B, surface & pottery (21 baskets) & 300 & & 03.01 .2016 \\
\hline $003 / 2016$ & SQ4B, surface & pottery (44 baskets) & 300 & & 03.01 .2016 \\
\hline $006 / 2016$ & $\mathrm{SQ} 4 \mathrm{~B}+\mathrm{C}$, strips around squares $4 \mathrm{~B}+\mathrm{C}$ & pottery (18 baskets) & 300 & & 04.01 .2016 \\
\hline $456 / 2016$ & SQ4B1, surface cleaning & pottery (13 baskets) & 300 & & 24.01 .2016 \\
\hline $007 / 2016$ & SQ4B & pottery ( 4 baskets) & 301 & & 05.01 .2016 \\
\hline $457 / 2016$ & SQ4B1 & pottery ( 9 baskets) & 301 & & 24.01 .2016 \\
\hline $010 / 2016$ & $\mathrm{SQ} 4 \mathrm{C}$ & pottery (4 1/2 baskets) & 301 & & 05.01 .2016 \\
\hline $013 / 2016$ & $\mathrm{SQ} 4 \mathrm{~A} / 4$ & pottery ( 6 baskets) & 302 & & 05.01 .2016 \\
\hline 016/2016 & SQ4B & pottery ( 3 baskets) & 303 & & 06.01 .2016 \\
\hline $017 / 2016$ & SQ4B & pottery (3 baskets) & 304 & & 06.01 .2016 \\
\hline $020 / 2016$ & SQ4B & pottery (1 basket) & 305 & & 06.01 .2016 \\
\hline $021 / 2016$ & SQ4B & pottery (2 baskets) & 306 & & 06.01 .2016 \\
\hline $022 / 2016$ & SQ4B & pottery (1 basket) & 307 & & 06.01 .2016 \\
\hline $025 / 2016$ & SQ4B & pottery (1 basket) & 308 & & 06.01 .2016 \\
\hline $023 / 2016$ & SQ4B & pottery (2 baskets) & 309 & & 06.01 .2016 \\
\hline $030 / 2016$ & $\mathrm{SQ} 4 \mathrm{C}$ & pottery & 310 & & 06.01 .2016 \\
\hline $034 / 2016$ & SQ4C & pottery (1 basket) & 310 & & 07.01 .2016 \\
\hline $036 / 2016$ & SQ4B & pottery (4 baskets) & 311 & & 07.01 .2016 \\
\hline $043 / 2016$ & SQ4B & $\begin{array}{l}\text { pottery ( } 3 \text { baskets), among them clay object (1 large fragment, } \\
\text { original function unknown) }\end{array}$ & 312 & SAV1E 2671 & 07.01 .2016 \\
\hline $044 / 2016$ & SQ4B & pottery ( 2 baskets) & 313 & & 07.01 .2016 \\
\hline $050 / 2016$ & SQ4C & pottery (2 baskets) & 314 & & 07.01 .2016 \\
\hline $052 / 2016$ & $\mathrm{SQ} 4 \mathrm{C}$ & pottery ( 8 baskets) & 315 & & 07.01 .2016 \\
\hline $059 / 2016$ & SQ4C & $\begin{array}{l}\text { pottery ( } 51 / 2 \text { baskets), among them weight? polishing stone? } \\
\text { ( } 1 \text { re-used base fragment of a pot) }\end{array}$ & 315 & SAV1E 0731 & 09.01 .2016 \\
\hline $091 / 2016$ & SQ4C & pottery (1 basket) & 315 & & 10.01 .2016 \\
\hline
\end{tabular}




\begin{tabular}{|c|c|c|c|c|c|}
\hline $056 / 2016$ & SQ4B & pottery ( 2 baskets) & 316 & & 07.01 .2016 \\
\hline $086 / 2016$ & SQ4B & $\begin{array}{l}\text { pottery and charcoal: pottery ( } 11 / 2 \text { baskets), among them } \\
\text { window grille ( } 1 \text { fragment, pottery, Christian), figurine } \\
\text { ( } 1 \text { fragment, head part, perhaps of doll, pottery); charcoal ( } 1 \\
\text { bag) }\end{array}$ & 316 & $\begin{array}{l}\text { SAV1E } 2676 \text { (window grille), SAV1E } 2677 \\
\text { (figurine) }\end{array}$ & 09.01 .2016 \\
\hline $106 / 2016$ & SQ4B & $\begin{array}{l}\text { pottery ( } 2 \text { baskets), among them scraper (1 piece, re-used } \\
\text { Christian dish) }\end{array}$ & 316 & SAV1E 2679 & 10.01.2016 \\
\hline $060 / 2016$ & SQ4B & $\begin{array}{l}\text { pottery ( } 1 \text { basket), among it weight ( } 1 \text { piece, re-used fragment } \\
\text { of a Christian vessel) }\end{array}$ & 317 & SAV1E 2681 & 09.01 .2016 \\
\hline $063 / 2016$ & SQ4B & $\begin{array}{l}\text { pottery ( } 2 \text { baskets), among them oil lamp ( } 1 \text { fragment, } \\
\text { Christian, pottery) }\end{array}$ & 318 & SAV1E 2680 & 09.01 .2016 \\
\hline $065 / 2016$ & SQ4C & $\begin{array}{l}\text { pottery ( } 9 \text { baskets), among them token/gaming piece ( } 1 \text { piece, } \\
\text { re-used } 18^{\text {th }} \text { Dynasty pottery), weight or disc ( } 1 \text { small fragment, } \\
\text { re-used } 18^{\text {th }} \text { Dynasty pottery), scraper ( } 1 \text { piece, re-used dish } \\
\text { base, pottery, Post-New Kingdom), scraper ( } 1 \text { piece, re-used } \\
\text { base sherd, pottery, Christian), scraper ( } 1 \text { piece, re-used pottery) }\end{array}$ & 319 & $\begin{array}{l}\text { SAV1E } 2686 \text { (token/gaming piece), SAV1E } \\
2687 \text { (weight or disc), SAV1E } 2688 \text { (scraper, } \\
\text { Post-New Kingdom), SAV1E } 2689 \text { (scraper, } \\
\text { Christian), } 2699 \text { (scraper) }\end{array}$ & 09.01 .2016 \\
\hline $078 / 2016$ & SQ4B & pottery (1 basket) & 320 & & 09.01 .2016 \\
\hline $080 / 2016$ & SQ4B & pottery ( 1 basket) & 321 & & 09.01 .2016 \\
\hline $089 / 2016$ & SQ4C & pottery and plaster: pottery ( 1 basket); plaster ( 1 bag) & 322 & & 09.01 .2016 \\
\hline $093 / 2016$ & SQ4C & $\begin{array}{l}\text { pottery ( } 21 / 2 \text { baskets), among them scrapers ( } 2 \text { pieces, re-used } \\
\text { pottery, } 18^{\text {th }} \text { Dynasty and Christian), figurine? ( } 1 \text { fragment of a } \\
\text { pottery object) }\end{array}$ & 322 & $\begin{array}{l}\text { SAV1E } 0767 \text { (scraper, Christian), SAV1E } \\
0814 \text { (scraper, } 18^{\text {th }} \text { Dynasty), SAV1E } 0858 \\
\text { (figurine?) }\end{array}$ & 10.01.2016 \\
\hline $098 / 2016$ & $\mathrm{SQ} 4 \mathrm{~B}+\mathrm{C}$ & $\begin{array}{l}\text { pottery ( } 41 / 2 \text { baskets), among them window grille ( } 1 \text { fragment, } \\
\text { pottery, Christian) }\end{array}$ & 323 & SAV1E 2682 (window grille) & 10.01.2016 \\
\hline $103 / 2016$ & SQ4B & $\begin{array}{l}\text { pottery ( } 2 \text { baskets), among them scraper (1 piece, re-used } \\
\text { pottery) }\end{array}$ & 324 & SAV1E 0873 (scraper) & 10.01.2016 \\
\hline $109 / 2016$ & SQ4B & pottery ( $11 / 2$ baskets) & 325 & & 10.01.2016 \\
\hline $113 / 2016$ & SQ4B & $\begin{array}{l}\text { pottery ( } 2 \text { baskets), among them scraper ( } 1 \text { piece, re-used } \\
\text { fragment of a zir jar) }\end{array}$ & 326 & SAV1E 2678 (scraper) & 10.01.2016 \\
\hline $124 / 2016$ & SQ4B & pottery ( 2 baskets) & 326 & & 11.01.2016 \\
\hline $117 / 2016$ & SQ4C & $\begin{array}{l}\text { pottery ( } 4 \text { baskets), among them lids ( } 2 \text { pieces, re-used pottery, } \\
18^{\text {th }} \text { Dynasty and Christian), scraper ( } 1 \text { piece, re-used pottery) }\end{array}$ & 327 & $\begin{array}{l}\text { SAV1E } 1053 \text { (lid, Christian), SAV1E } 2672 \\
\text { (lid, } 18^{\text {th }} \text { Dynasty), SAV1E } 2673 \text { (scraper) }\end{array}$ & 10.01 .2016 \\
\hline $126 / 2016$ & SQ4C & pottery ( 2 baskets) & 327 & & 11.01.2016 \\
\hline $120 / 2016$ & SQ4C & $\begin{array}{l}\text { pottery ( } 11 / 2 \text { baskets), among them token/gaming piece } \\
\text { ( } 1 \text { piece, re-used pottery), figurine ( } 1 \text { fragment of a horse } \\
\text { figurine, pottery) }\end{array}$ & 328 & $\begin{array}{l}\text { SAV1E } 2674 \text { (token/gaming piece), SAV1E } \\
2675 \text { (horse figurine) }\end{array}$ & 10.01.2016 \\
\hline
\end{tabular}




\begin{tabular}{|c|c|c|c|c|c|}
\hline $128 / 2016$ & SQ4C & $\begin{array}{l}\text { pottery ( } 1 \text { basket), among it weight ( } 1 \text { piece, re-used fragment } \\
\text { of a Christian bowl) }\end{array}$ & 328 & SAV1E 2690 & 11.01 .2016 \\
\hline $132 / 2016$ & SQ4C & $\begin{array}{l}\text { pottery ( } 1 / 2 \text { basket), among it weights or tokens/gaming pieces } \\
\text { ( } 2 \text { pieces, re-used Christian pottery), token/gaming piece } \\
\text { ( } 1 \text { piece, re-used pottery, New Kingdom), oil lamp ( } 1 \text { fragment, } \\
\text { pottery, Christian) }\end{array}$ & 329 & $\begin{array}{l}\text { SAV1E 2691, SAV1E } 2692 \text { (weights or } \\
\text { tokens/gaming pieces), SAV1E } 2693 \\
\text { (token/gaming piece), SAV1E } 2694 \text { (oil } \\
\text { lamp) }\end{array}$ & 11.01 .2016 \\
\hline $134 / 2016$ & SQ4C & $\begin{array}{l}\text { pottery ( } 2 \text { baskets), among them scrapers ( } 2 \text { pieces, re-used } \\
\text { pottery, } 18^{\text {th }} \text { Dynasty) }\end{array}$ & 330 & SAV1E 2695, SAV1E 2696 (scrapers) & 11.01 .2016 \\
\hline $135 / 2016$ & SQ4C & pottery (1 basket) & 331 & & 11.01 .2016 \\
\hline $138 / 2016$ & SQ4B & pottery ( 3 baskets) & 332 & & 11.01 .2016 \\
\hline $141 / 2016$ & SQ4B & $\begin{array}{l}\text { pottery ( } 1 \text { basket), among them pottery object ( } 1 \text { small } \\
\text { fragment, original form and function unclear) }\end{array}$ & 333 & SAV1E 2698 (unidentified pottery object) & 11.01 .2016 \\
\hline $144 / 2016$ & SQ4B & pottery (1 basket) & 334 & & 11.01 .2016 \\
\hline $148 / 2016$ & SQ4C & $\begin{array}{l}\text { pottery ( } 4 \text { baskets), among them scraper (1 piece, re-used } \\
\text { pottery, Christian) }\end{array}$ & 335 & SAV1E 2697 & 11.01 .2016 \\
\hline $164 / 2016$ & SQ4C & $\begin{array}{l}\text { pottery ( } 20 \text { baskets), among them } 2 \text { window grilles } \\
\text { ( } 2 \text { fragments, pottery, Christian), } 3 \text { unidentified pieces of } \\
\text { pottery ( } 3 \text { fragments, perhaps originally part of furniture), } \\
4 \text { scrapers ( } 4 \text { pieces, re-used pottery, Post-New Kingdom to } \\
\text { Christian), } \\
1 \text { lid ( } 1 \text { piece, re-used pottery vessel, } 18^{\text {th }} \text { Dynasty) }\end{array}$ & 335 & $\begin{array}{l}\text { SAV1E 2709, SAV1E } 2710 \text { (window grilles), } \\
\text { SAV1E 2711, SAV1E } 2712 \text { and SAV1E } 2712 \\
\text { (unidentified pieces of pottery), SAV1E 2713, } \\
\text { SAV1E 2714, SAV1E 2715, SAV1E } 2717 \\
\text { (scrapers), SAV1E 2716 (lid) }\end{array}$ & 12.01 .2016 \\
\hline $178 / 2016$ & SQ4C & pottery (2 1/2 baskets) & 335 & & 13.01 .2016 \\
\hline $155 / 2016$ & SQ4B & pottery ( 2 baskets) & 336 & & 11.01 .2016 \\
\hline $162 / 2016$ & SQ4B & pottery (3 baskets) & 336 & & 12.01 .2016 \\
\hline $168 / 2016$ & SQ4B & $\begin{array}{l}\text { pottery ( } 6 \text { baskets), among them figurine ( } 1 \text { fragment, } \\
\text { unidentified quadruped, pottery), token/gaming piece ( } 1 \text { piece, } \\
\text { re-used pottery, Christian), scraper ( } 1 \text { piece, re-used pottery, } \\
\text { Christian) }\end{array}$ & 337 & $\begin{array}{l}\text { SAV1E } 2703 \text { (figurine), SAV1E } 2704 \\
\text { (token/gaming piece), SAV1E } 2705 \text { (scraper) }\end{array}$ & 12.01 .2016 \\
\hline $183 / 2016$ & SQ4B & pottery (1 basket) & 338 & & 13.01 .2016 \\
\hline $187 / 2016$ & SQ4B & $\begin{array}{l}\text { pottery ( } 2 \text { baskets), among them oil lamps ( } 2 \text { pieces/fragments, } \\
\text { Christian, pottery), token/gaming piece ( } 1 \text { half, re-used pottery, } \\
\text { Post-New Kingdom/Christian) }\end{array}$ & 339 & $\begin{array}{l}\text { SAV1E 2723, SAV1E } 2724 \text { (oil lamps), } \\
\text { SAV1E } 2725 \text { (token/gaming piece) }\end{array}$ & 13.01 .2016 \\
\hline $192 / 2016$ & SQ4C & pottery (2 1/2 baskets) & 340 & & 13.01 .2016 \\
\hline $195 / 2016$ & SQ4C & $\begin{array}{l}\text { pottery ( } 7 \text { baskets), among them scraper (1 piece, re-used } \\
\text { Christian vessel) }\end{array}$ & 341 & SAV1E 2727 & 13.01 .2016 \\
\hline $210 / 2016$ & SQ4C & pottery ( 3 baskets) & 341 & & 14.01 .2016 \\
\hline
\end{tabular}




\begin{tabular}{|c|c|c|c|c|c|}
\hline $219 / 2016$ & SQ4C & $\begin{array}{l}\text { pottery/artefact (1 triangular shaped fragment, overfired with } \\
\text { traces of use) }\end{array}$ & 341 & SAV1E 2722 & 14.01 .2016 \\
\hline $198 / 2016$ & SQ4B & pottery ( 3 baskets) & 342 & & 13.01.2016 \\
\hline $203 / 2016$ & SQ4C & $\begin{array}{l}\text { pottery ( } 2 \text { baskets), among them unidentified piece of furniture } \\
\text { or installation ( } 1 \text { fragment, pottery) }\end{array}$ & 343 & SAV1E 2719 & 13.01 .2016 \\
\hline $207 / 2016$ & SQ4C & $\begin{array}{l}\text { pottery ( } 3 \text { baskets), among them weight ( } 1 \text { piece, re-used } \\
\text { Christian vessel) }\end{array}$ & 344 & SAV1E 2726 & 13.01 .2016 \\
\hline $217 / 2016$ & SQ4C & $\begin{array}{l}\text { pottery ( } 2 \text { baskets), among them scraper (1 piece, re-used } \\
\text { Christian? vessel) }\end{array}$ & 344 & SAV1E 2728 & 14.01 .2016 \\
\hline $212 / 2016$ & SQ4B & pottery (1 basket) & 345 & & 14.01.2016 \\
\hline $223 / 2016$ & SQ4B & $\begin{array}{l}\text { pottery ( } 3 \text { baskets), among them scraper (1 piece, re-used } \\
\text { Christian pottery) }\end{array}$ & 346 & SAV1E 0907 & 14.01.2016 \\
\hline $229 / 2016$ & SQ4B & pottery ( 2 baskets) & 347 & & 14.01.2016 \\
\hline $234 / 2016$ & SQ4C & $\begin{array}{l}\text { pottery ( } 3 \text { baskets), among them scrapers ( } 3 \text { pieces, re-used } \\
\text { pottery, } 18^{\text {th }} \text { Dynasty to Christian/medieval) }\end{array}$ & 348 & $\begin{array}{l}\text { SAV1E } 1054 \text { (scraper, Christian), SAV1E } \\
0442 \text { (scraper, } 18^{\text {th }} \text { Dynasty), SAV1E } 0444 \\
\text { (scraper, Christian) }\end{array}$ & 14.01 .2016 \\
\hline $246 / 2016$ & SQ4C & pottery ( 2 baskets) & 348 & & 16.01 .2016 \\
\hline $237 / 2016$ & SQ4C & pottery (1 basket) & 349 & & 14.01.2016 \\
\hline $250 / 2016$ & SQ4C & pottery ( $1 / 2$ basket) & 349 & & 16.01.2016 \\
\hline $242 / 2016$ & SQ4B & pottery (1 basket) & 350 & & 14.01 .2016 \\
\hline $247 / 2016$ & SQ4B & pottery ( $1 / 2$ basket) & 350 & & 16.01.2016 \\
\hline $254 / 2016$ & SQ4C & pottery ( 2 baskets) & 351 & & 16.01 .2016 \\
\hline $256 / 2016$ & SQ4A & pottery (1 basket) & 352 & & 16.01 .2016 \\
\hline $262 / 2016$ & SQ4C & pottery ( 6 baskets) & 353 & & 16.01.2016 \\
\hline $266 / 2016$ & SQ4B & pottery (1 basket) & 354 & & 16.01 .2016 \\
\hline $270 / 2016$ & SQ4B & $\begin{array}{l}\text { pottery ( } 21 / 2 \text { baskets), among them scraper ( } 1 \text { piece, re-used } \\
\text { pottery), token/gaming piece ( } 1 \text { piece, re-used pottery) }\end{array}$ & 355 & $\begin{array}{l}\text { SAV1E } 0363 \text { (scraper), SAV1E } 0392 \\
\text { (token/gaming piece) }\end{array}$ & 16.01 .2016 \\
\hline $274 / 2016$ & SQ4B & pottery (1 basket) & 356 & & 16.01 .2016 \\
\hline $283 / 2016$ & SQ4B & pottery ( $1 / 2$ basket) & 357 & & 16.01.2016 \\
\hline $289 / 2016$ & SQ4B & pottery (2 baskets) & 357 & & 17.01 .2016 \\
\hline $282 / 2016$ & SQ4B & pottery ( $1 / 2$ basket) & 358 & & 16.01 .2016 \\
\hline
\end{tabular}




\begin{tabular}{|c|c|c|c|c|c|}
\hline $292 / 2016$ & SQ4C & pottery ( 8 baskets) & 359 & & 17.01 .2016 \\
\hline $295 / 2016$ & SQ4C & pottery (1/2 basket) & 360 & & 17.01 .2016 \\
\hline $298 / 2016$ & SQ4B & pottery (1 basket) & 361 & & 17.01 .2016 \\
\hline $301 / 2016$ & SQ4C & pottery (2 1/2 baskets) & 362 & & 17.01 .2016 \\
\hline $306 / 2016$ & SQ4B & pottery (1 basket) & 363 & & 17.01 .2016 \\
\hline $308 / 2016$ & SQ4B & pottery ( $1 / 2$ basket) & 364 & & 17.01 .2016 \\
\hline $313 / 2016$ & SQ4C & pottery (2 1/2 baskets) & 365 & & 17.01.2016 \\
\hline $315 / 2016$ & SQ4B & pottery ( $1 / 2$ basket) & 366 & & 17.01 .2016 \\
\hline $318 / 2016$ & SQ4C & pottery (1 basket) & 367 & & 17.01 .2016 \\
\hline $323 / 2016$ & SQ4C & pottery ( $1 / 2$ basket) & 368 & & 17.01 .2016 \\
\hline $326 / 2016$ & SQ4C & pottery ( $11 / 2$ baskets) & 369 & & 18.01 .2016 \\
\hline $328 / 2016$ & SQ4C & $\begin{array}{l}\text { pottery ( } 10 \text { baskets), among them } 3 \text { window grilles } \\
\text { ( } 3 \text { fragmented pieces, pottery, Christian), scraper ( } 1 \text { piece, re- } \\
\text { used pottery, Christian) }\end{array}$ & 370 & $\begin{array}{l}\text { SAV1E 1707, SAV1E 0099, SAV1E } 0167 \\
\text { (window grilles), SAV1E } 0202 \text { (scraper) }\end{array}$ & 18.01 .2016 \\
\hline $331 / 2016$ & SQ4B & pottery ( $1 / 2$ basket) & 371 & & 18.01 .2016 \\
\hline $334 / 2016$ & SQ4C & pottery ( 3 baskets) & 372 & & 18.01 .2016 \\
\hline $337 / 2016$ & SQ4B & $\begin{array}{l}\text { pottery ( } 4 \text { baskets), among them figurine ( } 1 \text { fragment, probably } \\
\text { horse figurine, pottery, Christian) }\end{array}$ & 373 & SAV1E 0342 & 18.01 .2016 \\
\hline $347 / 2016$ & SQ4B & $\begin{array}{l}\text { pottery ( } 41 / 2 \text { baskets), among them window grille (1 fragment, } \\
\text { pottery, Christian) }\end{array}$ & 374 & SAV1E 2743 & 19.01 .2016 \\
\hline $351 / 2016$ & SQ4C & $\begin{array}{l}\text { pottery and stone: pottery ( } 4 \text { baskets), among them lid ( } 1 \text { piece, } \\
\text { re-used pottery), scraper ( } 1 \text { piece, re-used pottery dish); } \\
\text { whetstone ( } 1 \text { piece, sandstone) }\end{array}$ & 375 & $\begin{array}{l}\text { SAV1E } 2733 \text { (lid), SAV1E } 2734 \text { (scraper), } \\
\text { SAV1E } 2732 \text { (whetstone) }\end{array}$ & 19.01 .2016 \\
\hline $356 / 2016$ & SQ4C & $\begin{array}{l}\text { pottery ( } 6 \text { baskets), among them window grille ( } 1 \text { fragment, } \\
\text { pottery, Christian) }\end{array}$ & 376 & SAV1E 2742 & 19.01.2016 \\
\hline $366 / 2016$ & SQ4B & pottery ( 2 baskets) & 377 & & 19.01 .2016 \\
\hline $374 / 2016$ & SQ4C & pottery (1 basket) & 378 & & 19.01 .2016 \\
\hline $381 / 2016$ & SQ4C & pottery ( $1 / 2$ basket) & 378 & & 20.01 .2016 \\
\hline $378 / 2016$ & SQ4B & $\begin{array}{l}\text { pottery and stone: pottery ( } 1 / 2 \text { basket); grindstone ( } 1 \text { fragment, } \\
\text { sandstone) }\end{array}$ & 379 & SAV1E 2741 (grindstone) & 19.01.2016 \\
\hline $383 / 2016$ & SQ4C & $\begin{array}{l}\text { pottery (12 baskets), among them lid ( } 1 \text { piece, re-used pottery } \\
\text { vessel, Christian), scraper ( } 1 \text { piece, re-used pottery, Christian) }\end{array}$ & 380 & SAV1E 2738 (lid), SAV1E 2739 (scraper) & 20.01 .2016 \\
\hline
\end{tabular}




\begin{tabular}{|c|c|c|c|c|c|}
\hline $386 / 2016$ & SQ4C & $\begin{array}{l}\text { pottery and stone: pottery ( } 5 \text { baskets); worked stone } \\
\text { (1 fragment, original function unknown, sandstone) }\end{array}$ & 381 & SAV1E 2740 (worked stone) & 20.01.2016 \\
\hline $389 / 2016$ & SQ4C & $\begin{array}{l}\text { pottery and slag/brick: pottery ( } 41 / 2 \text { baskets), among them } \\
\text { scraper (1 piece, re-used Christian vessel), token/gaming piece } \\
\text { (1 piece, re-used Christian vessel), slag and brick ( } 1 \text { fragment) }\end{array}$ & 382 & $\begin{array}{l}\text { SAV1E } 2736 \text { (scraper), SAV1E } 2736 \\
\text { (token/gaming piece), SAV1E } 2735 \text { (slag and } \\
\text { brick) }\end{array}$ & 20.01.2016 \\
\hline $400 / 2016$ & SQ4C & pottery ( $1 / 2$ basket) & 383 & & 20.01 .2016 \\
\hline $402 / 2016$ & SQ4C & $\begin{array}{l}\text { pottery and slag: pottery (11 baskets), among them lid or } \\
\text { token/gaming piece ( } 1 \text { piece, re-used body sherd of a pottery } \\
\text { vessel, Christian), scraper (1 piece, re-used sherd, Post-New } \\
\text { Kingdom), stand for model vessels (1 piece, pottery), figurine } \\
\text { (1 piece, animal figurine?, pottery); slag (min. } 3 \text { sherds molded } \\
\text { together into slag) }\end{array}$ & 383 & $\begin{array}{l}\text { SAV1E } 2756 \text { (lid or token/gaming piece), } \\
\text { SAV1E } 2757 \text { (scraper), SAV1E } 2758 \text { (stand } \\
\text { for model vessels), SAV1E } 2759 \text { (figurine), } \\
\text { SAV1E } 2760 \text { (slag) }\end{array}$ & 21.01.2016 \\
\hline $424 / 2016$ & SQ4C & $\begin{array}{l}\text { pottery and stone: pottery ( } 21 / 2 \text { baskets), among them window } \\
\text { grille ( } 1 \text { fragment, pottery, Christian); worked stone } \\
\text { ( } 1 \text { fragment, original function unclear, limestone) }\end{array}$ & 384 & $\begin{array}{l}\text { SAV1E } 2754 \text { (window grille), SAV1E } 2753 \\
\text { (worked stone) }\end{array}$ & 21.01.2016 \\
\hline $428 / 2016$ & SQ4C & $\begin{array}{l}\text { pottery ( } 19 \text { baskets), among them lids ( } 3 \text { pieces, re-used } \\
\text { pottery), window grilles? ( } 3 \text { fragmented pieces), scraper } \\
\text { ( } 1 \text { piece, re-used pottery) }\end{array}$ & 384 & $\begin{array}{l}\text { SAV1E 2761, SAV1E 2762, SAV1E } 2781 \\
\text { (lids), SAV1E 2764, SAV1E 2765, SAV1E } \\
\text { 2766 (window grilles?), SAV1E } 2763 \\
\text { (scraper) }\end{array}$ & 23.01.2016 \\
\hline $436 / 2016$ & SQ4C & pottery: overfired ceramic (Post-New Kingdom) & 384 & SAV1E 2805 & 23.01 .2016 \\
\hline $405 / 2016$ & SQ4B & $\begin{array}{l}\text { pottery and slag: pottery ( } 2 \text { baskets), among them window grille } \\
\text { ( } 1 \text { fragment, pottery, Christian); slag ( } 1 \text { small fragment) }\end{array}$ & 385 & $\begin{array}{l}\text { SAV1E } 2770 \text { (window grille); SAV1E } 2750 \\
\text { (slag) }\end{array}$ & 21.01.2016 \\
\hline $411 / 2016$ & SQ4B & pottery ( $1 / 2$ basket) & 386 & & 21.01.2016 \\
\hline $414 / 2016$ & SQ4B & pottery ( $11 / 2$ baskets) & 387 & & 21.01 .2016 \\
\hline $417 / 2016$ & SQ4B & pottery ( 2 baskets) & 388 & & 21.01 .2016 \\
\hline $427 / 2016$ & SQ4C & $\begin{array}{l}\text { pottery ( } 1 \text { basket), among it lid ( } 1 \text { piece, re-used body sherd of a } \\
\text { pottery vessel, Post-New Kingdom) }\end{array}$ & 389 & SAV1E 2755 & 21.01 .2016 \\
\hline $431 / 2016$ & SQ4C & pottery ( $11 / 2$ baskets) & 390 & & 23.01 .2016 \\
\hline $438 / 2016$ & SQ4B & pottery ( $11 / 2$ baskets) & 391 & & 23.01 .2016 \\
\hline $583 / 2016$ & SQ4B & pottery & 391 & & 28.01 .2016 \\
\hline $442 / 2016$ & SQ4C & pottery (2 baskets) & 392 & & 23.01 .2016 \\
\hline $462 / 2016$ & SQ4C & $\begin{array}{l}\text { pottery ( } 2 / 12 \text { baskets), among them figurine? ( } 1 \text { fragment of an } \\
\text { irregularly shaped clay object) }\end{array}$ & 393 & SAV1E 2778 & 24.01.2016 \\
\hline $465 / 2016$ & SQ4C & pottery ( 8 baskets) & 393 & & 25.01 .2016 \\
\hline $469 / 2016$ & SQ4B1 & pottery (4 baskets) & 394 & & 25.01 .2016 \\
\hline
\end{tabular}




\begin{tabular}{|c|c|c|c|c|c|}
\hline $471 / 2016$ & SQ4B1 & $\begin{array}{l}\text { pottery ( } 3 \text { baskets), among them token/gaming piece ( } 1 \text { small } \\
\text { conical pottery object), figurine ( } 1 \text { small fragment, probably the } \\
\text { head of a horse, pottery) }\end{array}$ & 395 & $\begin{array}{l}\text { SAV1E } 2786 \text { (token/gaming piece), SAV1E } \\
2787 \text { (figurine) }\end{array}$ & 25.01.2016 \\
\hline $474 / 2016$ & SQ4B & pottery (1 basket) & 396 & & 25.01 .2016 \\
\hline $483 / 2016$ & SQ4C & pottery ( 7 baskets) & 397 & & 25.01.2016 \\
\hline $491 / 2016$ & SQ4B1 & pottery ( 3 baskets) & 398 & & 25.01 .2016 \\
\hline $499 / 2016$ & SQ4C & $\begin{array}{l}\text { pottery ( } 7 \text { baskets), among them token/gaming piece ( } 1 \text { piece, } \\
\text { re-used body sherd of a pottery vessel) }\end{array}$ & 399 & & 26.01.2016 \\
\hline $503 / 2016$ & SQ4C & pottery (1/2 basket) & 400 & & 26.01 .2016 \\
\hline $505 / 2016$ & SQ4B1 & $\begin{array}{l}\text { pottery ( } 5 \text { baskets), among them token/gaming piece ( } 1 \text { piece, } \\
\text { re-used body sherd of a pottery vessel, Christian) }\end{array}$ & 401 & SAV1E 2793 & 26.01 .2016 \\
\hline $510 / 2016$ & SQ4C & pottery (4 baskets) & 402 & & 26.01 .2016 \\
\hline $516 / 2016$ & SQ4B1 & pottery ( 2 baskets) & 403 & & 26.01.2016 \\
\hline $521 / 2016$ & SQ4C & $\begin{array}{l}\text { pottery ( } 1 / 2 \text { basket), among it token/gaming piece ( } 1 \text { piece, re- } \\
\text { used body sherd of a pottery vessel), window grille ( } 1 \text { fragment, } \\
\text { pottery) }\end{array}$ & 404 & $\begin{array}{l}\text { SAV1E } 2791 \text { (token/gaming piece), SAV1E } \\
2792 \text { (window grille) }\end{array}$ & 26.01 .2016 \\
\hline $526 / 2016$ & SQ4B1, S-part of SU & $\begin{array}{l}\text { pottery ( } 6 \text { baskets), among them scrapers ( } 2 \text { pieces, re-used } \\
\text { body sherds of pottery vessels, Post-New Kingdom) }\end{array}$ & 405 & SAV1E 2813, SAV1E 2814 (scrapers) & 27.01.2016 \\
\hline $566 / 2016$ & SQ4B1, S-part of SU & pottery ( 2 baskets) & 405 & & 28.01.2016 \\
\hline $530 / 2016$ & SQ4B1, N-part of SU & $\begin{array}{l}\text { pottery ( } 6 \text { baskets), among them figurine? ( } 1 \text { fragment of a } \\
\text { shaped clay object, original form and function unclear) }\end{array}$ & 405 & SAV1E 2819 & 27.01.2016 \\
\hline $571 / 2016$ & SQ4B1, N-part of SU & pottery ( 2 baskets) & 405 & & 28.01 .2016 \\
\hline $532 / 2016$ & SQ4B & pottery ( 5 baskets) & 406 & & 27.01 .2016 \\
\hline $544 / 2016$ & SQ4C & pottery (1 basket) & 407 & & 27.01.2016 \\
\hline $547 / 2016$ & SQ4C & $\begin{array}{l}\text { pottery ( } 3 \text { baskets), among them scrapers ( } 3 \text { pieces, } 1 \text { re-used } \\
\text { Christian body sherd, } 1 \text { re-used } 18^{\text {th }} \text { Dynasty? pottery vessel, } \\
1 \text { large re-used Christian body sherd) }\end{array}$ & 408 & $\begin{array}{l}\text { SAV1E } 2815 \text { (scraper, Christian), SAV1E } \\
2816 \text { (scraper, } 18^{\text {th }} \text { Dynasty), SAV1E } 2817 \\
\text { (scraper, Christian, large re-used sherd) }\end{array}$ & 27.01.2016 \\
\hline $560 / 2016$ & SQ4C & pottery (1 $1 / 2$ baskets) & 409 & & 28.01 .2016 \\
\hline $575 / 2016$ & SQ4C & pottery (1 basket) & 410 & & 28.01 .2016 \\
\hline $577 / 2016$ & SQ4 & pottery (3 baskets) & 411 & & 28.01 .2016 \\
\hline $579 / 2016$ & SQ4B & pottery (1 basket) & 412 & & 28.01 .2016 \\
\hline $581 / 2016$ & SQ4B1 & pottery ( 2 baskets) & 413 & & 28.01 .2016 \\
\hline
\end{tabular}




\begin{tabular}{|c|c|c|c|c|c|}
\hline $584 / 2016$ & SQ4B1 & pottery ( 2 baskets) & 414 & & 28.01 .2016 \\
\hline $597 / 2016$ & SQ4B1 & pottery (1 basket) & 414 & & 30.01 .2016 \\
\hline $591 / 2016$ & SQ4B & pottery (2 baskets) & 415 & & 28.01 .2016 \\
\hline $602 / 2016$ & SQ4B & $\begin{array}{l}\text { pottery ( } 3 \text { baskets), among them scraper ( } 1 \text { piece, re-used Post- } \\
\text { New Kingdom body sherd), window grille ( } 1 \text { small fragment, } \\
\text { pottery), window grille ( } 1 \text { larger fragment, pottery) }\end{array}$ & 415 & $\begin{array}{l}\text { SAV1E } 2828 \text { (scraper), SAV1E } 2829 \\
\text { (window grille, small fragment), SAV1E } \\
2830 \text { (window grille, larger fragment) }\end{array}$ & 30.01 .2016 \\
\hline $605 / 2016$ & SQ4B1 & pottery (2 baskets) & 416 & & 30.01 .2016 \\
\hline $609 / 2016$ & SQ4/4B & pottery (1 baskets) & 417 & & 30.01 .2016 \\
\hline $613 / 2016$ & SQ4B1 & $\begin{array}{l}\text { pottery (1 basket), among it scraper (1 piece, re-used body } \\
\text { sherd) }\end{array}$ & 418 & SAV1E 2835 & 30.01 .2016 \\
\hline $616 / 2016$ & SQ4B1 & $\begin{array}{l}\text { pottery ( } 1 \text { basket), among it scraper ( } 1 \text { piece, re-used } 18^{\text {th }} \\
\text { Dynasty body sherd from a storage vessel) }\end{array}$ & 419 & SAV1E 2836 & 30.01 .2016 \\
\hline $620 / 2016$ & SQ4B1 & pottery (1 basket) & 420 & & 30.01 .2016 \\
\hline $634 / 2016$ & SQ4B1 & $\begin{array}{l}\text { pottery ( } 3 \text { baskets), among them figurine ( } 1 \text { fragment, } \\
\text { unidentified quadruped, possibly a dog figurine, pottery) }\end{array}$ & 420 & SAV1E 2840 & 31.01 .2016 \\
\hline $621 / 2016$ & SQ4B1, N-part of SU & pottery (1 basket) & 420 & & 30.01 .2016 \\
\hline $622 / 2016$ & SQ4C & $\begin{array}{l}\text { pottery ( } 5 \text { baskets), among them pottery object/artefact } \\
\text { ( } 1 \text { fragment, original function unclear), lid? (1 fragment, } \\
\text { pottery, Christian) }\end{array}$ & 421 & $\begin{array}{l}\text { SAV1E } 2837 \text { (pottery object/artefact), } \\
\text { SAV1E } 2838 \text { (lid) }\end{array}$ & 30.01 .2016 \\
\hline $641 / 2016$ & SQ4B & pottery (1 basket) & 422 & & 31.01 .2016 \\
\hline $644 / 2016$ & SQ4B1 & $\begin{array}{l}\text { pottery ( } 1 \text { basket), among it scraper ( } 1 \text { piece, re-used } 18^{\text {th }} \\
\text { Dynasty rim sherd) }\end{array}$ & 423 & SAV1E 2843 & 31.01 .2016 \\
\hline $637 / 2016$ & SQ4/4B & $\begin{array}{l}\text { pottery ( } 1 / 2 \text { basket), among it object ( } 1 \text { fragment, with two } \\
\text { broad sides, pottery) }\end{array}$ & 424 & SAV1E 2853 & 31.01 .2016 \\
\hline $647 / 2016$ & SQ4C & pottery (5 baskets) & 425 & & 31.01 .2016 \\
\hline $650 / 2016$ & SQ4B1 & pottery (1/2 basket) & 426 & & 31.01 .2016 \\
\hline $653 / 2016$ & SQ4C & pottery (1 basket) & 427 & & 31.01 .2016 \\
\hline $660 / 2016$ & SQ4B1 & pottery (1/2 basket) & 428 & & 31.01 .2016 \\
\hline $663 / 2016$ & SQ4B & pottery (1 basket) & 429 & & 31.01 .2016 \\
\hline $670 / 2016$ & SQ4C & $\begin{array}{l}\text { pottery ( } 31 / 2 \text { baskets), among them window grilles } \\
\text { ( } 2 \text { fragmented pieces, pottery) }\end{array}$ & 430 & SAV1E 2839, SAV1E 2851 (window grilles) & 31.01 .2016 \\
\hline $674 / 2016$ & SQ4C & pottery (3 baskets) & 430 & & 01.02 .2016 \\
\hline
\end{tabular}




\begin{tabular}{|c|c|c|c|c|c|}
\hline $664 / 2016$ & SQ4/4B & pottery ( $1 / 2$ basket) & 431 & & 31.01 .2016 \\
\hline $668 / 2016$ & SQ4B1 & $\begin{array}{l}\text { pottery ( } 1 / 3 \text { basket), among it scraper ( } 1 \text { piece, re-used body } \\
\text { sherd, pottery) }\end{array}$ & 432 & SAV1E 2842 & 31.01 .2016 \\
\hline $677 / 2016$ & SQ4B1 & pottery ( 2 baskets) & 433 & & 01.02 .2016 \\
\hline $702 / 2016$ & SQ4B1 & overfired ceramic (1 small fragment) & 433 & SAV1E 2858 & 01.02 .2016 \\
\hline $681 / 2016$ & SQ4B & $\begin{array}{l}\text { pottery ( } 1 \text { basket), among it basin ( } 1 \text { corner fragment of a } \\
\text { pottery basin) }\end{array}$ & 434 & SAV1E 2841 & 01.02 .2016 \\
\hline $684 / 2016$ & SQ4C & $\begin{array}{l}\text { pottery ( } 7 \text { baskets), among them lid ( } 1 \text { piece, re-used body sherd } \\
\text { of a pottery vessel, Post-New Kingdom) }\end{array}$ & 435 & SAV1E 2854 & 01.02 .2016 \\
\hline $720 / 2016$ & SQ4C & $\begin{array}{l}\text { pottery ( } 4 \text { baskets), among it lid ( } 1 \text { piece, clay), pottery object } \\
\text { ( } 1 \text { fragment, unclear function, possibly some furniture or } \\
\text { installation?) }\end{array}$ & 435 & $\begin{array}{l}\text { SAV1E } 2855 \text { (lid), SAV1E } 2870 \text { (pottery } \\
\text { object) }\end{array}$ & 02.02 .2016 \\
\hline $687 / 2016$ & $\mathrm{SQ} 4 \mathrm{C}$ & pottery (1/2 basket) & 436 & & 01.02 .2016 \\
\hline $694 / 2016$ & SQ4B & pottery ( 2 baskets) & 437 & & 01.02 .2016 \\
\hline $697 / 2016$ & SQ4B1 & pottery (1 basket) & 438 & & 01.02 .2016 \\
\hline $705 / 2016$ & SQ4C & pottery (2 baskets) & 439 & & 01.02 .2016 \\
\hline $719 / 2016$ & $\mathrm{SQ} 4 \mathrm{C}$ & pottery ( $1 / 2$ medium bag) & 439 & & 02.02 .2016 \\
\hline $708 / 2016$ & SQ4C & pottery (1 basket) & 440 & & 01.02 .2016 \\
\hline $723 / 2016$ & SQ4C & pottery ( $1 / 2$ basket) & 440 & & 02.02 .2016 \\
\hline $726 / 2016$ & SQ4C & pottery ( 2 baskets) & 441 & & 02.02 .2016 \\
\hline $729 / 2016$ & SQ4 & pottery (1 basket) & 442 & & 02.02 .2016 \\
\hline $737 / 2016$ & SQ4B & pottery (1/3 basket) & 443 & & 02.02 .2016 \\
\hline $740 / 2016$ & $\mathrm{SQ} 4 / 4 \mathrm{~B}$ & pottery (1/3 basket) & 444 & & 02.02 .2016 \\
\hline $743 / 2016$ & $\mathrm{SQ} 4 / 4 \mathrm{~B}$ & pottery (1 basket) & 445 & & 02.02 .2016 \\
\hline $748 / 2016$ & SQ4B & pottery (1/3 basket) & 446 & & 02.02 .2016 \\
\hline $755 / 2016$ & SQ4C & $\begin{array}{l}\text { pottery ( } 1 \text { basket), among it scraper (1 piece, re-used sherd of a } \\
\text { pottery vessel, Christian) }\end{array}$ & 447 & SAV1E 2868 & 02.02 .2016 \\
\hline $758 / 2016$ & SQ4B & $\begin{array}{l}\text { pottery ( } 4 \text { baskets), among them scraper (1 piece, re-used Post- } \\
\text { New Kingdom body sherd) }\end{array}$ & 448 & SAV1E 2866 & 03.02 .2016 \\
\hline $891 / 2016$ & $\mathrm{SQ} 4 \mathrm{C}$ & pottery & 449 & & 08.02 .2016 \\
\hline $894 / 2016$ & SQ4C & pottery ( $1 / 2$ basket) & 449 & & 08.02 .2016 \\
\hline
\end{tabular}




\begin{tabular}{|c|c|c|c|c|c|}
\hline $889 / 2016$ & SQ4C & pottery: baking plate & 449 & & 08.02 .2016 \\
\hline $764 / 2016$ & SQ4B & pottery (1 basket) & 451 & & 03.02 .2016 \\
\hline $767 / 2016$ & SQ4B & pottery (1 basket) & 452 & & 03.02 .2016 \\
\hline $768 / 2016$ & SQ4B & pottery (cooking pot from installation) & 452 & & 03.02 .2016 \\
\hline $776 / 2016$ & SQ4 & pottery (1 medium bag) & 453 & & 03.02 .2016 \\
\hline $781 / 2016$ & SQ4 & $\begin{array}{l}\text { pottery ( } 1 \text { basket), among it window grille ( } 1 \text { fragment, pottery, } \\
\text { Christian) }\end{array}$ & 454 & SAV1E 2124 & 03.02 .2016 \\
\hline $784 / 2016$ & SQ4B & pottery ( 1 basket) & 455 & & 04.02 .2016 \\
\hline $787 / 2016$ & SQ4C & $\begin{array}{l}\text { pottery ( } 7 \text { baskets), among them lid ( } 1 \text { piece, re-used body sherd } \\
\text { of a pottery vessel, } 18^{\text {th }} \text { Dynasty) }\end{array}$ & 456 & SAV1E 0314 & 04.02 .2016 \\
\hline $810 / 2016$ & SQ4C & overfired ceramic & 456 & SAV1E 2872 & 04.02 .2016 \\
\hline $811 / 2016$ & SQ4C & pottery (3 baskets) & 456 & & 06.02 .2016 \\
\hline $792 / 2016$ & $\mathrm{SQ} 4 \mathrm{~B} / \mathrm{C}$ & pottery (1 basket) & 457 & & 04.02 .2016 \\
\hline $797 / 2016$ & SQ4 & pottery (1 small bag) & 458 & & 04.02 .2016 \\
\hline $815 / 2016$ & SQ4 & pottery (1 small bag) & 458 & & 06.02 .2016 \\
\hline $805 / 2016$ & SQ4/4B & pottery (1 basket) & 459 & & 04.02 .2016 \\
\hline $820 / 2016$ & SQ4A/C & pottery ( $31 / 2$ baskets) & 460 & & 06.02 .2016 \\
\hline $825 / 2016$ & SQ4, Feature 75 & pottery (1 medium bag) & 461 & & 06.02 .2016 \\
\hline $835 / 2016$ & SQ4 & pottery (1 medium bag) & 462 & & 06.02 .2016 \\
\hline $852 / 2016$ & SQ4 & pottery (1 small bag) & 462 & & 07.02 .2016 \\
\hline $836 / 2016$ & SQ4C & pottery (1/2 basket) & 463 & & 06.02 .2016 \\
\hline $847 / 2016$ & SQ4C & pottery (1/2 basket) & 463 & & 07.02 .2016 \\
\hline $850 / 2016$ & SQ4C & $\begin{array}{l}\text { pottery ( } 3 \text { baskets), among them token/gaming piece (1 piece, } \\
\text { re-used body sherd) }\end{array}$ & 464 & SAV1E 1969 & 07.02 .2016 \\
\hline $858 / 2016$ & SQ4 & pottery (1 small bag) & 465 & & 07.02 .2016 \\
\hline $863 / 2016$ & SQ4C & pottery ( $1 / 2$ basket), among it weight ( 1 piece, pottery) & 466 & SAV1E 0747 & 07.02 .2016 \\
\hline $867 / 2016$ & $\mathrm{SQ} 4 \mathrm{C} / 4 \mathrm{~A}$ & pottery (1/2 basket) & 467 & & 07.02 .2016 \\
\hline $871 / 2016$ & SQ4C & pottery (1 small bag) & 468 & & 07.02 .2016 \\
\hline
\end{tabular}




\begin{tabular}{|c|c|c|c|c|c|}
\hline $874 / 2016$ & SQ4C & $\begin{array}{l}\text { pottery and slag: pottery ( } 1 \text { basket), among it scraper ( } 1 \text { piece, } \\
\text { re-used base of a pottery vessel, Post-New Kingdom; slag } \\
\text { (1 piece) }\end{array}$ & 469 & SAV1E 1796 (scraper) & 07.02 .2016 \\
\hline $877 / 2016$ & SQ4B1 & pottery (1 bag) & 470 & & 08.02 .2016 \\
\hline $878 / 2016$ & SQ4B1 & pottery ( 1 medium sized bag) & 471 & & 08.02 .2016 \\
\hline $881 / 2016$ & SQ4B1 & pottery (1 small bag) & 472 & & 08.02 .2016 \\
\hline $885 / 2016$ & SQ4C & pottery (1 basket) & 473 & & 08.02 .2016 \\
\hline $895 / 2016$ & SQ4C & pottery (1 bag) & 474 & & 08.02 .2016 \\
\hline $899 / 2016$ & SQ4C & $\begin{array}{l}\text { pottery ( } 1 / 2 \text { basket), among it window grille ( } 1 \text { fragment, } \\
\text { pottery) }\end{array}$ & 475 & SAV1E 2900 & 08.02 .2016 \\
\hline $902 / 2016$ & SQ4B, S-part of SU, E of Feature 70 & pottery ( $1 \mathrm{bag}$ ), among it window grille? ( 1 fragment, pottery) & 476 & SAV1E 0594 & 08.02 .2016 \\
\hline $903 / 2016$ & $\begin{array}{l}\text { SQ4B/4B1, N-part of SU, between Features } \\
68 / 67 \text { and } 69 / 70\end{array}$ & pottery (1 bag) & 476 & & 08.02 .2016 \\
\hline $919 / 2016$ & SQ4B & pottery ( $1 / 2$ basket) & 477 & & 08.02.2016 \\
\hline $031 / 2016$ & SQ4C & rope (3 strands) & 310 & & 06.01 .2016 \\
\hline $143 / 2016$ & SQ4B & rope (1 bag, fragments) & 333 & & 11.01.2016 \\
\hline $206 / 2016$ & SQ4C & rope (1 piece) & 341 & & 13.01.2016 \\
\hline $200 / 2016$ & SQ4B & rope (1 bag) & 342 & & 13.01 .2016 \\
\hline $233 / 2016$ & SQ4B & rope (1 piece) & 347 & & 14.01.2016 \\
\hline $370 / 2016$ & SQ4B & $\begin{array}{l}\text { scarab ( } 1 \text { piece, scarab beetle hacked off, incised back } \\
\text { decoration, winged cobra/vulture and empty cartouche, steatite) }\end{array}$ & 377 & SAV1E 1595 & 19.01.2016 \\
\hline $116 / 2016$ & SQ4B & seal? (mud with impression of fabric) & 326 & SAV1E 0973 & 10.01.2016 \\
\hline $493 / 2016$ & SQ4B1 & seal (1 fragment, clay) & 398 & SAV1E 2782 & 25.01.2016 \\
\hline $524 / 2016$ & SQ4B1 & seal (1 small fragment, clay) & 403 & SAV1E 2794 & 26.01 .2016 \\
\hline $545 / 2016$ & SQ4B1, N-part of SU & seal ( 2 small pieces, clay) & 405 & SAV1E 2812 & 27.01.2016 \\
\hline $912 / 2016$ & SQ4C & seal (1 small fragment, clay) & 449 & SAV1E 2892 & 08.02 .2016 \\
\hline $914 / 2016$ & SQ4C & seals (3 pieces, unstamped) & 449 & & 08.02 .2016 \\
\hline $918 / 2016$ & SQ4C & seal (1 fragment of a large seal, clay) & 449 & SAV1E 2894 & 08.02 .2016 \\
\hline $856 / 2016$ & SQ4, Feature 75 & seal (1 fragmented piece, uninscribed) & 462 & & 07.02.2016 \\
\hline
\end{tabular}




\begin{tabular}{|c|c|c|c|c|c|}
\hline $862 / 2016$ & SQ4, Feature 75 & seal (1 fragment, clay) & 465 & SAV1E 2883 & 07.02.2016 \\
\hline $930 / 2016$ & SQ4B1 & seal (1 piece, unstamped) & 470 & & 08.02 .2016 \\
\hline $936 / 2016$ & SQ4, Feature 15 from sieving & seal fragments ( 15 fragments, unstamped) & 1325 & & 09.02 .2016 \\
\hline $949 / 2016$ & $\begin{array}{l}\text { SQ4, Feature 15, lower part of SU, W of } \\
\text { Feature } 44\end{array}$ & seal fragments ( 8 fragments, unstamped) & 1329 & & 09.02 .2016 \\
\hline $954 / 2016$ & SQ4, Feature $15, \mathrm{~W}$ of Wall 44 & seal fragment (1 piece) & 1331 & & 10.02.2016 \\
\hline $957 / 2016$ & SQ4, Feature 15 & seal fragments (6 fragments, unstamped) & 1332 & & 10.02 .2016 \\
\hline $974 / 2016$ & SQ4, Feature 15 & $\begin{array}{l}\text { seal fragments ( } 20 \text { fragments, } 1 \text { with stamp of Thutmose III, } 19 \\
\text { unstamped) }\end{array}$ & 1336 & SAV1E 0203 & 10.02 .2016 \\
\hline $979 / 2016$ & SQ4, Feature 15 & seal fragments (6 fragments, unstamped) & 1339 & & 11.02.2016 \\
\hline $983 / 2016$ & SQ4, Feature 15 & seal fragments ( 3 fragments, unstamped) & 1340 & & 11.02 .2016 \\
\hline $989 / 2016$ & SQ4, Feature 15 & seal fragments (20 fragments) & 1341 & & 11.02 .2016 \\
\hline $478 / 2016$ & SQ4B & seeds (4 pieces) & 396 & & 25.01 .2016 \\
\hline $522 / 2016$ & SQ4C & seed (1 piece) & 404 & & 26.01 .2016 \\
\hline $542 / 2016$ & SQ4B1, N-part of SU & seeds (2 pieces) & 405 & & 27.01 .2016 \\
\hline $576 / 2016$ & SQ4C & seeds ( 2 pieces) & 410 & & 28.01.2016 \\
\hline $747 / 2016$ & $\mathrm{SQ} 4 / 4 \mathrm{~B}$ & seeds ( 3 pieces) & 445 & & 02.02 .2016 \\
\hline $801 / 2016$ & SQ4B & seeds (1 bag) & 455 & & 04.02 .2016 \\
\hline $873 / 2016$ & $\mathrm{SQ} 4 \mathrm{C} / 4 \mathrm{~A}$ & seeds ( 2 pieces) & 467 & & 07.02 .2016 \\
\hline $936 / 2016$ & SQ4, Feature 15 from sieving & seeds (6 pieces) & 1325 & & 09.02 .2016 \\
\hline $954 / 2016$ & SQ4, Feature $15, \mathrm{~W}$ of Wall 44 & seed (1 piece) & 1331 & & 10.02 .2016 \\
\hline $957 / 2016$ & SQ4, Feature 15 & seed (2 pieces) & 1332 & & 10.02 .2016 \\
\hline $974 / 2016$ & SQ4, Feature 15 & seeds (6 pieces) & 1336 & & 10.02 .2016 \\
\hline $983 / 2016$ & SQ4, Feature 15 & seed (1 piece) & 1340 & & 11.02 .2016 \\
\hline $989 / 2016$ & SQ4, Feature 15 & seeds (1 bag) & 1341 & & 11.02 .2016 \\
\hline $054 / 2016$ & SQ4C & shell (2 pieces) & 315 & & 07.01 .2016 \\
\hline $067 / 2016$ & SQ4C & shell & 319 & & 09.01 .2016 \\
\hline $170 / 2016$ & SQ4C & shell (1 piece) & 335 & & 12.01.2016 \\
\hline
\end{tabular}




\begin{tabular}{|c|c|c|c|c|}
\hline $173 / 2016$ & SQ4B & shells ( 2 pieces and fragments) & 337 & 12.01.2016 \\
\hline $201 / 2016$ & SQ4B & shell (1 piece) & 342 & 13.01.2016 \\
\hline $215 / 2016$ & SQ4B & shell (1 piece) & 345 & 14.01 .2016 \\
\hline $228 / 2016$ & SQ4B & shell (1 piece) & 346 & 14.01 .2016 \\
\hline $244 / 2016$ & SQ4C & shell (1 piece) & 348 & 14.01.2016 \\
\hline $324 / 2016$ & SQ4C & shell (1 piece) & 365 & 17.01.2016 \\
\hline $359 / 2016$ & SQ4C & shell (2 pieces) & 375 & 19.01.2016 \\
\hline $409 / 2016$ & SQ4C & shell (1 piece) & 383 & 21.01.2016 \\
\hline $447 / 2016$ & SQ4C & shell (1 bag) & 384 & 23.01.2016 \\
\hline $408 / 2016$ & SQ4B & shell (1 piece) & 385 & 21.01.2016 \\
\hline $482 / 2016$ & SQ4B1 & shell (1 piece) & 394 & 25.01.2016 \\
\hline $484 / 2016$ & SQ4C & shell (1 bag) & 397 & 25.01 .2016 \\
\hline $514 / 2016$ & SQ4B1 & shell (1 piece) & 401 & 26.01.2016 \\
\hline $537 / 2016$ & SQ4B & shell (1 piece) & 406 & 27.01.2016 \\
\hline $565 / 2016$ & SQ4C & shell (1 piece) & 409 & 28.01 .2016 \\
\hline $624 / 2016$ & $\mathrm{SQ} 4 / 4 \mathrm{~B}$ & shell (1 piece) & 417 & 30.01 .2016 \\
\hline $692 / 2016$ & SQ4B1 & shell (1 bag) & 433 & 01.02 .2016 \\
\hline $715 / 2016$ & SQ4C & shell (1 piece) & 435 & 01.02 .2016 \\
\hline $735 / 2016$ & SQ4C & shell (1 piece) & 435 & 02.02 .2016 \\
\hline $746 / 2016$ & SQ4/4B & shell (1 piece) & 445 & 02.02 .2016 \\
\hline $761 / 2016$ & SQ4B & shell (1 bag) & 448 & 03.02 .2016 \\
\hline $773 / 2016$ & SQ4B, sifted & shell (1 piece) & 451 & 03.02 .2016 \\
\hline $780 / 2016$ & SQ4 & shell (1 piece) & 453 & 03.02 .2016 \\
\hline $803 / 2016$ & SQ4C & shell & 456 & 04.02 .2016 \\
\hline $888 / 2016$ & SQ4B1 & shell (1 bag) & 470 & 08.02 .2016 \\
\hline $924 / 2016$ & SQ4C & shell (1 piece) & 473 & 08.02 .2016 \\
\hline
\end{tabular}




\begin{tabular}{|c|c|c|c|c|c|}
\hline $916 / 2016$ & SQ4C & $\begin{array}{l}\text { silicified organic material/silicified wood? (1 small conical } \\
\text { piece) }\end{array}$ & 449 & SAV1E 2893 & 08.02 .2016 \\
\hline $002 / 2016$ & SQ4B, surface & slag (5 pieces) & 300 & SAV1E 2663 & 03.01 .2016 \\
\hline $005 / 2016$ & SQ4B, surface & slag (21 pieces, some with traces of pottery or brick attached) & 300 & SAV1E 2664 & 03.01 .2016 \\
\hline $009 / 2016$ & SQ4B & slag (1 small piece) & 301 & SAV1E 2666 & 05.01 .2016 \\
\hline $012 / 2016$ & SQ4C & slag (1 small piece) & 301 & SAV1E 2665 & 05.01 .2016 \\
\hline $029 / 2016$ & SQ4B & slag (2 pieces) & 309 & SAV1E 2670 & 06.01 .2016 \\
\hline $075 / 2016$ & SQ4C & slag (2 pieces) & 315 & SAV1E 0683 & 09.01 .2016 \\
\hline $074 / 2016$ & SQ4C & $\operatorname{slag}(2$ pieces $)$ & 319 & SAV1E 0610 & 09.01 .2016 \\
\hline $102 / 2016$ & SQ4C & slag and crucible: slag (1 piece); crucible (1 fragment, pottery) & 322 & SAV1E 1013 (slag), SAV1E 1033 (crucible) & 10.01 .2016 \\
\hline $772 / 2016$ & SQ4B & stamp (1 piece, small pyramidal stamp, incised design, clay) & 451 & SAV1E 2865 & 03.02 .2016 \\
\hline $015 / 2016$ & SQ4B & stone: whetstone (1 piece, sandstone) & 303 & SAV1E 2669 & 06.01 .2016 \\
\hline $055 / 2016$ & SQ4C & $\begin{array}{l}\text { stone: pounder/grindstone ( } 1 \text { piece, with possible remains of red } \\
\text { colour, quartz) }\end{array}$ & 315 & SAV1E 0487 & 07.01 .2016 \\
\hline $070 / 2016$ & SQ4C & stone: blade (1 piece, silex) & 315 & SAV1E 0609 & 09.01 .2016 \\
\hline $081 / 2016$ & SQ4B & stone: token/gaming piece (1 piece, sandstone) & 321 & SAV1E 0553 & 09.01 .2016 \\
\hline $176 / 2016$ & SQ4C & stone: pumice stone? (1 large fragment) & 335 & SAV1E 2700 & 12.01 .2016 \\
\hline $154 / 2016$ & SQ4B & stone: grindstone (1 piece, sandstone) & 336 & SAV1E 2685 & 11.01.2016 \\
\hline $202 / 2016$ & SQ4C & stone: pounder (1 piece, stone, quartz) & 340 & SAV1E 2706 & 13.01.2016 \\
\hline $272 / 2016$ & SQ4B & stone: pounder (1 piece, quartzite) & 354 & SAV1E 0460 & 16.01 .2016 \\
\hline $281 / 2016$ & SQ4B & stone: pivot stone (1 piece, sandstone) & 358 & SAV1E 0533 & 16.01 .2016 \\
\hline $288 / 2016$ & SQ4B & stone: whetstone (1 piece, sandstone) & 358 & SAV1E 0448 & 16.01 .2016 \\
\hline $325 / 2016$ & SQ4C & $\begin{array}{l}\text { stones: pivot stone (1 piece, sandstone), worked stone } \\
\text { (1 fragment, original function unknown, sandstone) }\end{array}$ & 359 & $\begin{array}{l}\text { SAV1E } 0537 \text { (pivot stone), SAV1E } 0543 \\
\text { (worked stone) }\end{array}$ & 17.01.2016 \\
\hline $305 / 2016$ & SQ4B & stone: hand mill (1 piece, sandstone) & 361 & SAV1E 0552 & 17.01 .2016 \\
\hline $339 / 2016$ & SQ4C & stone: hand mill (1 fragment, quartzite) & 370 & SAV1E 1533 & 18.01 .2016 \\
\hline $362 / 2016$ & SQ4C & $\begin{array}{l}\text { stone, perhaps worked (1 fragment, possibly used as mortar, } \\
\text { sandstone) }\end{array}$ & 375 & SAV1E 2745 & 19.01.2016 \\
\hline $361 / 2016$ & SQ4C & $\begin{array}{l}\text { stone, worked (1 fragment, original function unknown, } \\
\text { sandstone) }\end{array}$ & 375 & SAV1E 2746 & 19.01.2016 \\
\hline
\end{tabular}




\begin{tabular}{|c|c|c|c|c|c|}
\hline $363 / 2016$ & SQ4C & stone, worked (1 fragment, sandstone, with remains of colour) & 376 & SAV1E 2744 & 19.01.2016 \\
\hline $371 / 2016$ & SQ4B & stone, worked (1 half, used as weight?, granite) & 377 & SAV1E 1674 & 19.01 .2016 \\
\hline $392 / 2016$ & SQ4C & stone: grindstone (1 fragment, sandstone) & 381 & SAV1E 2748 & 20.01.2016 \\
\hline $393 / 2016$ & SQ4C & stone: tool or blade (1 fragment, silex) & 381 & SAV1E 0793 & 20.01 .2016 \\
\hline $394 / 2016$ & SQ4C & stone: disc? (1 fragment, schist) & 381 & SAV1E 1578 & 20.01 .2016 \\
\hline $396 / 2016$ & SQ4C & stone: grindstone (1 fragment, sandstone) & 381 & SAV1E 2747 & 20.01.2016 \\
\hline $416 / 2016$ & SQ4C & stone: grindstone (1 fragment, sandstone) & 383 & SAV1E 2752 & 21.01 .2016 \\
\hline $445 / 2016$ & SQ4C & stone: pivot stone (1 fragment, sandstone) & 384 & SAV1E 2774 & 23.01 .2016 \\
\hline $446 / 2016$ & SQ4C & stone: polishing stone or pounder (1 fragment of a stone pebble) & 384 & SAV1E 2772 & 23.01 .2016 \\
\hline $453 / 2016$ & SQ4C & stone: grindstone (1 large fragment, sandstone) & 384 & SAV1E 2775 & 23.01 .2016 \\
\hline $410 / 2016$ & SQ4B & $\begin{array}{l}\text { stone: hand mill (1 fragment, with remains of pigment on it, } \\
\text { sandstone) }\end{array}$ & 385 & SAV1E 2751 & 21.01 .2016 \\
\hline $434 / 2016$ & SQ4C & stone: grindstone (1 large fragment, sandstone) & 390 & SAV1E 2776 & 23.01.2016 \\
\hline $435 / 2016$ & SQ4C & stone: pestle (1 half, granite) & 390 & SAV1E 2773 & 23.01.2016 \\
\hline $481 / 2016$ & SQ4C & stone: grindstone (1 fragment, sandstone) & 393 & SAV1E 2797 & 25.01 .2016 \\
\hline $497 / 2016$ & SQ4B1 & stone: pivot stone? (1 fragment, sandstone) & 395 & SAV1E 2795 & 25.01 .2016 \\
\hline $495 / 2016$ & SQ4B1 & $\begin{array}{l}\text { stone, worked ( } 1 \text { fragment, original form and function unclear, } \\
\text { sandstone) }\end{array}$ & 398 & SAV1E 2790 & 25.01 .2016 \\
\hline $191 / 2016$ & SQ4B & $\begin{array}{l}\text { stones: pounder (1 piece, quartzite), hand mill (1 fragment, } \\
\text { sandstone) }\end{array}$ & 399 & $\begin{array}{l}\text { SAV1E } 2707 \text { (pounder), SAV1E } 2708 \text { (hand } \\
\text { mill) }\end{array}$ & 13.01.2016 \\
\hline $512 / 2016$ & SQ4C & stone: grindstone (1 large fragment, sandstone) & 399 & SAV1E 2798 & 26.01 .2016 \\
\hline $513 / 2016$ & SQ4C & stone: grindstone (1 large fragment, sandstone) & 402 & SAV1E 2799 & 26.01 .2016 \\
\hline $525 / 2016$ & SQ4B1 & stone: grindstone (1 fragment, sandstone) & 403 & SAV1E 2796 & 26.01 .2016 \\
\hline $554 / 2016$ & SQ4B1, N-part of SU & $\begin{array}{l}\text { stones: pounders ( } 6 \text { pieces: pounder/hammer, } 1 \text { piece, quartzite, } \\
\text { pounders, } 3 \text { pieces, quartz, pounder, } 1 \text { piece, quartzite, pounder, } \\
1 \text { piece, stone) }\end{array}$ & 405 & $\begin{array}{l}\text { SAV1E } 2822 \text { (pounder/hammer, quartzite), } \\
\text { SAV1E 2823, SAV1E 2824 and SAV1E } 2825 \\
\text { (pounders, quartz), SAV1E 2826 (pounder, } \\
\text { quartzite), SAV1E 2827 (pounder, stone) }\end{array}$ & 27.01 .2016 \\
\hline $640 / 2016$ & $\mathrm{SQ} 4 / 4 \mathrm{~B}$ & $\begin{array}{l}\text { stone: pounder? (1 pebble, with traces of colour/pigment on it, } \\
\text { quartzite) }\end{array}$ & 424 & SAV1E 2857 & 31.01 .2016 \\
\hline $703 / 2016$ & SQ4B1 & stone: grindstone (1 fragment, sandstone) & 433 & SAV1E 2862 & 01.02 .2016 \\
\hline
\end{tabular}




\begin{tabular}{|c|c|c|c|c|c|}
\hline $711 / 2016$ & SQ4C & $\begin{array}{l}\text { stone: grindstone (1 fragment, with traces of pigment on it, } \\
\text { sandstone) }\end{array}$ & 435 & SAV1E 2863 & 01.02 .2016 \\
\hline $736 / 2016$ & SQ4C & stone: mortar or vessel (1 small base fragment, stone) & 435 & SAV1E 2859 & 02.02 .2016 \\
\hline $718 / 2016$ & SQ4B & stone: whetstone (1 piece, sandstone) & 437 & SAV1E 2860 & 01.02 .2016 \\
\hline $733 / 2016$ & SQ4C & $\begin{array}{l}\text { stone: grindstone ( } 1 \text { fragment, re-used, maybe originally } \\
\text { architecture, sandstone) }\end{array}$ & 441 & SAV1E 2861 & 02.02 .2016 \\
\hline $734 / 2016$ & SQ4C & $\begin{array}{l}\text { stone, worked ( } 1 \text { small fragment, with a single register line } \\
\text { carved on the outer face, sandstone) }\end{array}$ & 441 & SAV1E 2856 & 02.02 .2016 \\
\hline $893 / 2016$ & SQ4C, directly at wall & $\begin{array}{l}\text { stone: pounder or polishing stone (1 irregularly shaped pebble, } \\
\text { stone) }\end{array}$ & 449 & SAV1E 2887 & 08.02 .2016 \\
\hline $917 / 2016$ & SQ4C & stone, worked (1 small piece, schist) & 449 & SAV1E 2890 & 08.02 .2016 \\
\hline $804 / 2016$ & SQ4/4B & stone: pounder (1 piece, quartzite) & 459 & SAV1E 2873 & 04.02 .2016 \\
\hline $821 / 2016$ & $\begin{array}{l}\mathrm{SQ} 4 \mathrm{~A} / \mathrm{C} \text {, found directly in the E-W running } \\
\text { wall }\end{array}$ & $\begin{array}{l}\text { stone, worked (1 fragment, original form and function unclear, } \\
\text { quartzite) }\end{array}$ & 460 & SAV1E 1117 & 06.02 .2016 \\
\hline $830 / 2016$ & SQ4, Feature 75 & stone: pounder (1 fragment of a pebble, quartzite) & 461 & SAV1E 2880 & 06.02 .2016 \\
\hline $831 / 2016$ & SQ4, Feature 75 & stone: blade (1 piece, silex), flake (1 small silex flake) & 461 & SAV1E 2876 (blade), SAV1E 2877 (flake) & 06.02 .2016 \\
\hline $832 / 2016$ & SQ4, Feature 75 & stone: blade? (1 fragment, quartz & 461 & SAV1E 2878 & 06.02 .2016 \\
\hline $841 / 2016$ & SQ4, Feature 75 & stone: blade (1 small flake, silex) & 462 & SAV1E 2875 & 06.02 .2016 \\
\hline $843 / 2016$ & SQ4, Feature 75 & stone: polishing stone (1 fragment, sandstone) & 462 & SAV1E 2879 & 06.02 .2016 \\
\hline $861 / 2016$ & SQ4, Feature 75 & stone: stone (2 small silex flakes, sample) & 465 & & 07.02 .2016 \\
\hline $928 / 2016$ & SQ4C & $\begin{array}{l}\text { stones: blade (1 small flake of quartz pebble), flake ( } 1 \text { small } \\
\text { silex flake) }\end{array}$ & 473 & SAV1E 2895 (blade), SAV1E 2896 (flake) & 08.02 .2016 \\
\hline $954 / 2016$ & SQ4, Feature $15, \mathrm{~W}$ of Wall 44 & stones ( 9 flakes, reference) & 1331 & & 10.02 .2016 \\
\hline $957 / 2016$ & SQ4, Feature 15 & stones ( 5 flakes, reference) & 1332 & & 10.02 .2016 \\
\hline $960 / 2016$ & SQ4, Feature 44 & stones ( 2 flakes, sample) & 1333 & & 10.02 .2016 \\
\hline $974 / 2016$ & SQ4, Feature 15 & stones (3 flakes quartz and agate, sample) & 1336 & & 10.02 .2016 \\
\hline $976 / 2016$ & SQ4, Feature 15 & stones ( 2 flakes, reference) & 1338 & & 11.02 .2016 \\
\hline $979 / 2016$ & SQ4, Feature 15 & stones (6 flakes, reference) & 1339 & & 11.02 .2016 \\
\hline $989 / 2016$ & SQ4, Feature 15 & stone flake (1 piece, reference) & 1341 & & 11.02 .2016 \\
\hline $395 / 2016$ & SQ4C & stopper? (1 small object, clay) & 380 & SAV1E 0347 & 20.01 .2016 \\
\hline
\end{tabular}




\begin{tabular}{|c|c|c|c|c|c|}
\hline $480 / 2016$ & SQ4C & stopper? (1 small object, clay) & 393 & SAV1E 2789 & 25.01 .2016 \\
\hline $543 / 2016$ & SQ4B1, S-part of SU & stopper (1 piece, clay) & 405 & SAV1E 2809 & 27.01 .2016 \\
\hline $763 / 2016$ & SQ4B & stopper (1 piece, clay) & 448 & SAV1E 2869 & 03.02 .2016 \\
\hline $808 / 2016$ & $\mathrm{SQ} 4 / 4 \mathrm{~B}$ & stopper (1 piece, clay) & 459 & SAV1E 2874 & 04.02 .2016 \\
\hline $122 / 2016$ & SQ4C & textile (1 bag) & 328 & & 10.01 .2016 \\
\hline $140 / 2016$ & SQ4B & textile $(1 \mathrm{bag})$ & 332 & & 11.01 .2016 \\
\hline $209 / 2016$ & SQ4C & textile/rope (1 piece) & 341 & & 13.01 .2016 \\
\hline $342 / 2016$ & SQ4B & textile (1 bag) & 373 & & 18.01 .2016 \\
\hline $358 / 2016$ & SQ4C & textile (1 bag) & 375 & & 19.01 .2016 \\
\hline $365 / 2016$ & SQ4C & textile (1 piece) & 376 & & 19.01.2016 \\
\hline $391 / 2016$ & SQ4C & textile (1 bag) & 380 & & 20.01 .2016 \\
\hline $455 / 2016$ & SQ4C & textile (1 piece) & 384 & & 23.01 .2016 \\
\hline $489 / 2016$ & SQ4C & textile (1 bag) & 397 & & 25.01 .2016 \\
\hline $496 / 2016$ & SQ4B1 & textile (1 bag) & 398 & & 25.01 .2016 \\
\hline $519 / 2016$ & SQ4C & textile (1 piece) & 399 & & 26.01 .2016 \\
\hline $509 / 2016$ & SQ4C & textile (1 bag) & 400 & & 26.01 .2016 \\
\hline $538 / 2016$ & SQ4B & textile (1 piece) & 406 & & 27.01 .2016 \\
\hline $559 / 2016$ & SQ4C & textile (1 bag) & 408 & & 27.01 .2016 \\
\hline $589 / 2016$ & SQ4B1 & textile (1 bag) & 414 & & 28.01 .2016 \\
\hline $673 / 2016$ & SQ4C & textile (1 bag) & 430 & & 31.01 .2016 \\
\hline $716 / 2016$ & SQ4C & textile (1 bag) & 435 & & 01.02 .2016 \\
\hline $773 / 2016$ & SQ4B, sifted & textile (1 piece) & 451 & & 03.02 .2016 \\
\hline $775 / 2016$ & SQ4B & textile (1 bag) & 452 & & 03.02 .2016 \\
\hline $796 / 2016$ & SQ4B/C & textile (1 piece) & 457 & & 04.02 .2016 \\
\hline $906 / 2016$ & $\begin{array}{l}\text { SQ4B/4B1, N-part of SU between Feature } \\
68 / 67 \text { and Feature } 69 / 70\end{array}$ & textile (1 piece) & 476 & & 08.02 .2016 \\
\hline $058 / 2016$ & SQ4C & token/gaming piece (1 piece, clay/mud) & 315 & SAV1E 0429 & 07.01 .2016 \\
\hline
\end{tabular}




\begin{tabular}{|c|c|c|c|c|c|}
\hline $152 / 2016$ & SQ4C & token/gaming piece or weight (clay/mud) & 335 & SAV1E 2684 & 11.01.2016 \\
\hline $032 / 2016$ & SQ4C & unknown material (1 piece, dung? burnt pumice stone?) & 310 & & 06.01 .2016 \\
\hline $461 / 2016$ & SQ4B1 & weight (1 piece, pottery) & 301 & SAV1E 2780 & 24.01 .2016 \\
\hline $260 / 2016$ & $\mathrm{SQ} 4 \mathrm{C}$ & weight (1 piece, limestone) & 348 & SAV1E 0485 & 16.01 .2016 \\
\hline $345 / 2016$ & $\mathrm{SQ} 4 \mathrm{C}$ & weight (1 piece, clay) & 370 & SAV1E 1043 & 18.01 .2016 \\
\hline $040 / 2016$ & SQ4B & wood (1 bag) & 311 & & 07.01 .2016 \\
\hline $062 / 2016$ & SQ4B & wood & 317 & & 09.01 .2016 \\
\hline $084 / 2016$ & SQ4B & wood (1 bag) & 321 & & 09.01 .2016 \\
\hline $147 / 2016$ & SQ4B & wood (1 piece) & 334 & & 11.01 .2016 \\
\hline $149 / 2016$ & SQ4C & wood (1 piece) & 335 & & 11.01 .2016 \\
\hline $167 / 2016$ & SQ4B & wood (1 piece) & 336 & & 12.01 .2016 \\
\hline $226 / 2016$ & SQ4B & wood (1 bag) & 346 & & 14.01 .2016 \\
\hline $264 / 2016$ & SQ4C & wood (1 piece) & 353 & & 16.01 .2016 \\
\hline $269 / 2016$ & SQ4B & wood (1 bag) & 354 & & 16.01 .2016 \\
\hline $344 / 2016$ & SQ4B & wood (1 piece) & 373 & & 18.01 .2016 \\
\hline $441 / 2016$ & SQ4B & wood (1 bag) & 391 & & 23.01 .2016 \\
\hline $444 / 2016$ & SQ4C & wood (1 bag) & 392 & & 23.01 .2016 \\
\hline $911 / 2016$ & SQ4C & wood (1 bag) & 449 & & 08.02 .2016 \\
\hline $773 / 2016$ & SQ4B, sifted & wood (1 piece) & 451 & & 03.02 .2016 \\
\hline $828 / 2016$ & SQ4, Feature 75 & wood (2 pieces) & 461 & & 06.02 .2016 \\
\hline $840 / 2016$ & SQ4, Feature 75 & wood (1 bag) & 462 & & 06.02 .2016 \\
\hline $855 / 2016$ & SQ4, Feature 75 & wood (2 pieces) & 462 & & 07.02 .2016 \\
\hline $929 / 2016$ & $\mathrm{SQ} 4 \mathrm{C}$ & wood (1 bag) & 470 & & 08.02 .2016 \\
\hline $923 / 2016$ & SQ4C & wood (1 bag) & 473 & & 08.02 .2016 \\
\hline $954 / 2016$ & SQ4, Feature 15, W of Wall 44 & wood (1 bag) & 1331 & & 10.02 .2016 \\
\hline
\end{tabular}




\begin{tabular}{|c|c|c|c|c|c|}
\hline \multicolumn{6}{|c|}{ Sai Island SAV1 East 2017} \\
\hline Find no. & Location & Description/label/material & Level & Object no. & Date \\
\hline $640 / 2017$ & SQ4C, SW-corner & $\begin{array}{l}\text { architecture (1 large architectural element, sandstone, } \\
\text { reworked from an inscribed royal lintel) }\end{array}$ & 1416 & SAV1E 2904 & 25.02.2017 \\
\hline $501 / 2017$ & SQ4D & architecture (1 fragment, sandstone) & 1400 & SAV1E 1450 & 18.02.2017 \\
\hline $685 / 2017$ & SQ4D, from debris in pit & architecture (1 large fragment, sandstone) & 1420 & SAV1E 2959 & 27.02.2017 \\
\hline $665 / 2017$ & SQ4D & $\begin{array}{l}\text { architecture (1 large fragment, sandstone, reworked as } \\
\text { tethering stone) }\end{array}$ & 1421 & SAV1E 2905 & 26.02.2017 \\
\hline $709 / 2017$ & SQ4D, Feature 83, W-wall & architectural block (1 large sandstone) & 1424 & SAV1E 2906 & 28.02.2017 \\
\hline $747 / 2017$ & SQ4D, Feature 83, SW-corner & architecture: column/drum base (1 large fragment, sandstone) & 1427 & SAV1E 2907 & 01.03.2017 \\
\hline $810 / 2017$ & SQ4D, Feature 85 & $\begin{array}{l}\text { architecture: door jamb (1 large rectangular architectural } \\
\text { fragment, sandstone, re-used for grinding) }\end{array}$ & 1435 & SAV1E 2974 & 04.03 .2017 \\
\hline $816 / 2017$ & SQ4D, Feature 85, SW-corner of cellar & $\begin{array}{l}\text { architecture: door lintel? (1 large fragment of worked } \\
\text { sandstone) }\end{array}$ & 1435 & SAV1E 2977 & 04.03.2017 \\
\hline $838 / 2017$ & SQ4D, Feature 85, NW-corner & architecture: lintel? (1 re-used architectural element) & 1438 & & 05.03 .2017 \\
\hline $923 / 2017$ & SQ4D, Feature 85 & architecture: door lintel (1 large piece, sandstone) & 1445 & SAV1E 2975 & 08.03.2017 \\
\hline $652 / 2017$ & $\mathrm{SQ} 4 \mathrm{C} / 4 \mathrm{D}$ & basketry & 1410 & & 23.02 .2017 \\
\hline $904 / 2017$ & SQ4D, E of Feature 85 & bead (1 ring bead, bone) & 1400 & SAV1E 2926 & 07.03.2017 \\
\hline $505 / 2017$ & SQ4D, N-part & bead (1 ring bead, bone or faience) & 1401 & SAV1E 1379 & 18.02.2017 \\
\hline $526 / 2017$ & SQ4D, from ash-spot/base of SU & bead (1 disc bead, shell) & 1403 & SAV1E 1036 & 19.02.2017 \\
\hline $543 / 2017$ & SQ4D, pebble area & bead ( 1 disc bead, shell) & 1403 & SAV1E 1076 & 20.02.2017 \\
\hline $571 / 2017$ & SQ4D, N-part & bead ( 1 clay ball bead) & 1407 & SAV1E 2219 & 22.02.2017 \\
\hline $586 / 2017$ & SQ4D & bead (1 spherical bead, clay) & 1408 & SAV1E 2294 & 23.02.2017 \\
\hline $583 / 2017$ & SQ4D, S of 'new wall' & bead ( 1 ball bead, glass? stone?) & 1408 & SAV1E 2628 & 23.02.2017 \\
\hline $613 / 2017$ & SQ4D & bead (1 ring bead, faience) & 1412 & SAV1E 2366 & 25.02.2017 \\
\hline $614 / 2017$ & SQ4D & bead ( 1 fragment of a tubular bead, faience) & 1412 & SAV1E 2622 & 25.02.2017 \\
\hline $669 / 2017$ & SQ4D, SE corner SU & bead (1 disc bead, faience) & 1419 & SAV1E 2973 & 26.02.2017 \\
\hline $676 / 2017$ & SQ4D, SW-corner & bead (1 tubular bead, faience) & 1420 & SAV1E 0497 & 27.02.2017 \\
\hline $795 / 2017$ & SQ4D, Feature 85 & bead (stone) & 1433 & & 02.03.2017 \\
\hline
\end{tabular}




\begin{tabular}{|c|c|c|c|c|c|}
\hline $875 / 2017$ & SQ4D, Feature 83, above pavement & beads ( 2 ring beads, faience) & 1441 & SAV1E 2963 & 06.03 .2017 \\
\hline $909 / 2017$ & SQ4D, Feature 83 & bead (1 ring bead, faience) & 1443 & SAV1E 2962 & 07.03 .2017 \\
\hline $949 / 2017$ & SQ4D, Feature 85 & bead ( 1 ring bead, faience), bead? ( 1 small tube, bronze) & 1449 & $\begin{array}{l}\text { SAV1E } 2956 \text { (faience bead), SAV1E } 2957 \\
\text { (bead?/tube) }\end{array}$ & 09.03.2017 \\
\hline $955 / 2017$ & SQ4D, Feature 85 & beads ( 3 ring beads, faience) & 1452 & SAV1E 2955 & 09.03.2017 \\
\hline $840 / 2017$ & SQ4C & bead ( 1 fragment of a thick ring bead, faience) & 1480 & SAV1E 2640 & 05.03 .2017 \\
\hline $506 / 2017$ & SQ4D & bone & 1401 & & 18.02 .2017 \\
\hline $512 / 2017$ & SQ4D & bone & 1402 & & 18.02 .2017 \\
\hline $518 / 2017$ & SQ4D & bone & 1402 & & 19.02.2017 \\
\hline $530 / 2017$ & SQ4D & bones & 1403 & & 19.02 .2017 \\
\hline $536 / 2017$ & SQ4D & bone & 1403 & & 19.02 .2017 \\
\hline $545 / 2017$ & SQ4D & bone & 1403 & & 20.02.2017 \\
\hline $539 / 2017$ & SQ4D, ashy area & bone & 1403 & & 20.02.2017 \\
\hline $523 / 2017$ & SQ4D, from ash-spot/base of SU & bone & 1403 & & 19.02.2017 \\
\hline $542 / 2017$ & SQ4D, pebble area & bone & 1403 & & 20.02 .2017 \\
\hline $560 / 2017$ & SQ4D & bone & 1404 & & 20.02 .2017 \\
\hline $557 / 2017$ & SQ4D, W-edge SU & bone & 1404 & & 20.02 .2017 \\
\hline $562 / 2017$ & SQ4D & bone & 1405 & & 20.02 .2017 \\
\hline $566 / 2017$ & SQ4D & bone & 1406 & & 20.02 .2017 \\
\hline $575 / 2017$ & SQ4D & bone & 1407 & & 22.02 .2017 \\
\hline $569 / 2017$ & SQ4D, N-part & bone & 1407 & & 20.02.2017 \\
\hline $579 / 2017$ & SQ4D & bone & 1408 & & 23.02.2017 \\
\hline $600 / 2017$ & SQ4D & bone & 1409 & & 23.02.2017 \\
\hline $595 / 2017$ & $\mathrm{SQ} 4 \mathrm{C} / 4 \mathrm{D}$ & bone & 1410 & & 23.02 .2017 \\
\hline $609 / 2017$ & SQ4C/4D, outside, $S$ of $4 C$ & bone & 1411 & & 23.02 .2017 \\
\hline $607 / 2017$ & SQ4D & bone & 1412 & & 23.02.2017 \\
\hline $619 / 2017$ & SQ4D & bone & 1412 & & 25.02 .2017 \\
\hline
\end{tabular}




\begin{tabular}{|c|c|c|c|c|}
\hline $624 / 2017$ & $\mathrm{SQ} 4 \mathrm{C} / 4 \mathrm{D}$ & bone & 1413 & 25.02.2017 \\
\hline $632 / 2017$ & SQ4D & bone & 1414 & 25.02.2017 \\
\hline $638 / 2017$ & SQ4D, NE-corner of SU & bone & 1414 & 25.02.2017 \\
\hline $644 / 2017$ & SQ4D & bone & 1415 & 25.02.2017 \\
\hline $651 / 2017$ & $\mathrm{SQ} 4 \mathrm{C} / 4 \mathrm{D}$ & bone & 1416 & 25.02.2017 \\
\hline $641 / 2017$ & SQ4C/4D, from SE-edge of SU 1416) & bone & 1416 & 25.02.2017 \\
\hline $660 / 2017$ & SQ4D & bone & 1417 & 26.02.2017 \\
\hline $657 / 2017$ & SQ4D & bone & 1418 & 26.02.2017 \\
\hline $674 / 2017$ & SQ4D & bone & 1419 & 26.02.2017 \\
\hline $678 / 2017$ & SQ4D, S-part & bone & 1420 & 27.02.2017 \\
\hline $689 / 2017$ & SQ4D, from pit & bone & 1420 & 27.02.2017 \\
\hline $694 / 2017$ & SQ4D & bone & 1421 & 27.02.2017 \\
\hline $702 / 2017$ & SQ4D & bone & 1422 & 28.02 .2017 \\
\hline $738 / 2017$ & SQ4D & bone & 1423 & 28.02.2017 \\
\hline $715 / 2017$ & SQ4D, Feature 83 & bone & 1424 & 28.02.2017 \\
\hline $712 / 2017$ & SQ4D & bone & 1425 & 28.02.2017 \\
\hline $725 / 2017$ & SQ4D & bone & 1426 & 28.02 .2017 \\
\hline $733 / 2017$ & SQ4D, Feature 83 & bone & 1427 & 28.02 .2017 \\
\hline $755 / 2017$ & SQ4D, Feature 83 & bone & 1427 & 01.03 .2017 \\
\hline $743 / 2017$ & SQ4D & bone & 1428 & 28.02 .2017 \\
\hline $750 / 2017$ & SQ4D & bone & 1429 & 01.03 .2017 \\
\hline $761 / 2017$ & SQ4D & bone & 1430 & 01.03 .2017 \\
\hline $769 / 2017$ & SQ4D, Feature 83 & bone & 1431 & 01.03 .2017 \\
\hline $777 / 2017$ & SQ4D, Feature 83 & bone & 1431 & 02.03 .2017 \\
\hline $788 / 2017$ & SQ4D, Feature 83, lower part & bone & 1431 & 02.03 .2017 \\
\hline $766 / 2017$ & SQ4D & bone & 1432 & 01.03 .2017 \\
\hline
\end{tabular}




\begin{tabular}{|c|c|c|c|c|}
\hline $782 / 2017$ & SQ4D, Feature 85 & bone & 1432 & 02.03 .2017 \\
\hline $792 / 2017$ & SQ4D, Feature 85 & bone & 1433 & 02.03.2017 \\
\hline $808 / 2017$ & SQ4D, Feature 85 & bone & 1433 & 04.03 .2017 \\
\hline $799 / 2017$ & SQ4D, Feature 83 & bone & 1434 & 02.03.2017 \\
\hline $804 / 2017$ & SQ4D, Feature 83 & bone & 1434 & 04.03.2017 \\
\hline $818 / 2017$ & SQ4D, Feature 85 & bone & 1435 & 04.03.2017 \\
\hline $814 / 2017$ & SQ4D, Feature 83 & bone & 1436 & 04.03 .2017 \\
\hline $824 / 2017$ & SQ4D, Feature 83 & bone & 1437 & 04.03.2017 \\
\hline $856 / 2017$ & $\begin{array}{l}\text { SQ4D, Feature 83, from bottom surface } \\
\text { of SU }\end{array}$ & bone & 1437 & 05.03.2017 \\
\hline $831 / 2017$ & SQ4D, Feature 85 & bone & 1438 & 04.03.2017 \\
\hline $842 / 2017$ & SQ4D, Feature 85 & bone & 1438 & 05.03.2017 \\
\hline $833 / 2017$ & SQ4D, Feature 66/Feature 85 & bone & 1439 & 05.03.2017 \\
\hline $848 / 2017$ & SQ4D, Feature 85, W-part & bone & 1440 & 05.03.2017 \\
\hline $861 / 2017$ & SQ4D, Feature 85, E-part & bone & 1440 & 05.03.2017 \\
\hline $881 / 2017$ & SQ4D, Feature 83 & bone (animal) & 1441 & 06.03.2017 \\
\hline $896 / 2017$ & SQ4D, Feature 85 & bone & 1442 & 07.03 .2017 \\
\hline $922 / 2017$ & SQ4D, Feature 85 & bone & 1444 & 08.03.2017 \\
\hline $927 / 2017$ & SQ4D, Feature 85 & bone & 1445 & 08.03 .2017 \\
\hline $932 / 2017$ & SQ4D, Feature 85 & bone & 1446 & 08.03.2017 \\
\hline $935 / 2017$ & SQ4D, Feature 85 & bone & 1447 & 08.03.2017 \\
\hline $939 / 2017$ & SQ4D, Feature 85 & bone & 1448 & 09.03.2017 \\
\hline $944 / 2017$ & SQ4D, Feature 85 & bone & 1449 & 09.03.2017 \\
\hline $951 / 2017$ & SQ4D, Feature 85 & bone & 1452 & 09.03.2017 \\
\hline $852 / 2017$ & SQ4C & bone & 1480 & 05.03 .2017 \\
\hline $884 / 2017$ & SQ4C & bone & 1481 & 06.03.2017 \\
\hline $886 / 2017$ & SQ4C & bone & 1482 & 06.03.2017 \\
\hline
\end{tabular}




\begin{tabular}{|c|c|c|c|c|c|}
\hline $901 / 2017$ & SQ4C & bone & 1483 & & 07.03.2017 \\
\hline $917 / 2017$ & SQ4C & bone & 1484 & & 07.03.2017 \\
\hline $906 / 2017$ & SQ4C & bone & 1485 & & 07.03.2017 \\
\hline $914 / 2017$ & SQ4C & bone & 1486 & & 07.03.2017 \\
\hline $533 / 2017$ & SQ4D & botanical material & 1403 & & 19.02.2017 \\
\hline $529 / 2017$ & SQ4D, from ash-spot/base of SU & botanical material (1 tiny piece of branch) & 1403 & & 19.02.2017 \\
\hline $611 / 2017$ & $\mathrm{SQ} 4 \mathrm{C} / 4 \mathrm{D}$ & botanical material & 1413 & & 25.02 .2017 \\
\hline $683 / 2017$ & SQ4D, S-part & botanical material & 1420 & & 27.02.2017 \\
\hline $773 / 2017$ & SQ4D, Feature 83 & botanical material/chaff & 1431 & & 01.03 .2017 \\
\hline $924 / 2017$ & SQ4D, Feature 85, E-part & bracelet? (1 fragment, blue glaze, faience) & 1445 & SAV1E 2967 & 08.03 .2017 \\
\hline $877 / 2017$ & SQ4D, Feature 83 & chalk & 1441 & & 06.03 .2017 \\
\hline $507 / 2017$ & SQ4D & charcoal & 1401 & & 18.02 .2017 \\
\hline $510 / 2017$ & SQ4D & charcoal & 1402 & & 18.02 .2017 \\
\hline $517 / 2017$ & SQ4D & charcoal & 1402 & & 19.02.2017 \\
\hline $531 / 2017$ & SQ4D & charcoal & 1403 & & 19.02.2017 \\
\hline $547 / 2017$ & SQ4D & charcoal & 1403 & & 20.02.2017 \\
\hline $538 / 2017$ & SQ4D, ashy area & charcoal & 1403 & & 20.02 .2017 \\
\hline $522 / 2017$ & SQ4D, from ash-spot/base of SU & charcoal & 1403 & & 19.02.2017 \\
\hline $541 / 2017$ & SQ4D, pebble area & charcoal & 1403 & & 20.02.2017 \\
\hline $559 / 2017$ & SQ4D & charcoal & 1404 & & 20.02.2017 \\
\hline $554 / 2017$ & SQ4D, W-edge SU & charcoal & 1404 & & 20.02.2017 \\
\hline $563 / 2017$ & SQ4D & charcoal & 1405 & & 20.02.2017 \\
\hline $565 / 2017$ & SQ4D & charcoal & 1406 & & 20.02.2017 \\
\hline $574 / 2017$ & SQ4D & charcoal & 1407 & & 22.02.2017 \\
\hline $568 / 2017$ & SQ4D, N-part & charcoal & 1407 & & 20.02.2017 \\
\hline $580 / 2017$ & SQ4D & charcoal & 1408 & & 23.02.2017 \\
\hline
\end{tabular}




\begin{tabular}{|c|c|c|c|c|}
\hline $599 / 2017$ & SQ4D & charcoal & 1409 & 23.02.2017 \\
\hline $594 / 2017$ & $\mathrm{SQ} 4 \mathrm{C} / 4 \mathrm{D}$ & charcoal & 1410 & 23.02.2017 \\
\hline $605 / 2017$ & SQ4D & charcoal & 1411 & 23.02.2017 \\
\hline $608 / 2017$ & SQ4D & charcoal & 1412 & 23.02.2017 \\
\hline $623 / 2017$ & SQ4D & charcoal & 1412 & 25.02.2017 \\
\hline $626 / 2017$ & $\mathrm{SQ} 4 \mathrm{C} / 4 \mathrm{D}$ & charcoal & 1413 & 25.02 .2017 \\
\hline $633 / 2017$ & SQ4D & charcoal & 1414 & 25.02.2017 \\
\hline $646 / 2017$ & SQ4D & charcoal & 1415 & 25.02.2017 \\
\hline $648 / 2017$ & $\mathrm{SQ} 4 \mathrm{C} / 4 \mathrm{D}$ & charcoal & 1416 & 25.02 .2017 \\
\hline $662 / 2017$ & SQ4D & charcoal & 1417 & 26.02.2017 \\
\hline $656 / 2017$ & SQ4D & charcoal & 1418 & 26.02.2017 \\
\hline $673 / 2017$ & SQ4D & charcoal & 1419 & 26.02 .2017 \\
\hline $679 / 2017$ & SQ4D, S-part & charcoal & 1420 & 27.02.2017 \\
\hline $688 / 2017$ & SQ4D, from pit & charcoal & 1420 & 27.02.2017 \\
\hline $695 / 2017$ & SQ4D & charcoal & 1421 & 27.02.2017 \\
\hline $703 / 2017$ & SQ4D & charcoal & 1422 & 28.02.2017 \\
\hline $737 / 2017$ & SQ4D & charcoal & 1423 & 28.02 .2017 \\
\hline $719 / 2017$ & SQ4D, Feature 83 & charcoal & 1424 & 28.02 .2017 \\
\hline $714 / 2017$ & SQ4D & charcoal & 1425 & 28.02 .2017 \\
\hline $726 / 2017$ & SQ4D & charcoal & 1426 & 28.02 .2017 \\
\hline $732 / 2017$ & SQ4D, Feature 83 & charcoal & 1427 & 28.02 .2017 \\
\hline $756 / 2017$ & SQ4D, Feature 83 & charcoal & 1427 & 01.03 .2017 \\
\hline $742 / 2017$ & SQ4D & charcoal & 1428 & 28.02 .2017 \\
\hline $751 / 2017$ & SQ4D & charcoal & 1429 & 01.03 .2017 \\
\hline $760 / 2017$ & SQ4D & charcoal & 1430 & 01.03 .2017 \\
\hline 770/2017 & SQ4D, Feature 83 & charcoal & 1431 & 01.03 .2017 \\
\hline
\end{tabular}




\begin{tabular}{|c|c|c|c|c|}
\hline $778 / 2017$ & SQ4D, Feature 83 & charcoal & 1431 & 02.03 .2017 \\
\hline $787 / 2017$ & SQ4D, Feature 83, lower part & charcoal & 1431 & 02.03 .2017 \\
\hline $767 / 2017$ & SQ4D & charcoal & 1432 & 01.03 .2017 \\
\hline $781 / 2017$ & SQ4D, Feature 85 & charcoal & 1432 & 02.03 .2017 \\
\hline $793 / 2017$ & SQ4D, Feature 85 & charcoal & 1433 & 02.03 .2017 \\
\hline $809 / 2017$ & SQ4D, Feature 85 & charcoal & 1433 & 04.03 .2017 \\
\hline $798 / 2017$ & SQ4D, Feature 83 & charcoal & 1434 & 02.03 .2017 \\
\hline $805 / 2017$ & SQ4D, Feature 83 & charcoal & 1434 & 04.03.2017 \\
\hline $819 / 2017$ & SQ4D, Feature 85 & charcoal & 1435 & 04.03 .2017 \\
\hline $815 / 2017$ & SQ4D, Feature 83 & charcoal & 1436 & 04.03 .2017 \\
\hline $825 / 2017$ & SQ4D, Feature 83 & charcoal & 1437 & 04.03 .2017 \\
\hline $857 / 2017$ & SQ4D, Feature 83, bottom surface of SU & charcoal & 1437 & 05.03 .2017 \\
\hline $844 / 2017$ & SQ4D, Feature 85 & charcoal & 1438 & 05.03 .2017 \\
\hline $835 / 2017$ & SQ4D, Feature 66/Feature 85 & charcoal & 1439 & 05.03 .2017 \\
\hline $849 / 2017$ & SQ4D, Feature 85, W-part & charcoal & 1440 & 05.03 .2017 \\
\hline $862 / 2017$ & SQ4D, Feature 85, E-part & charcoal & 1440 & 05.03 .2017 \\
\hline $879 / 2017$ & SQ4D, Feature 83 & charcoal & 1441 & 06.03 .2017 \\
\hline $897 / 2017$ & SQ4D, Feature 85 & charcoal & 1442 & 07.03 .2017 \\
\hline $911 / 2017$ & SQ4D, Feature 83 & charcoal & 1443 & 07.03 .2017 \\
\hline $921 / 2017$ & SQ4D, Feature 85 & charcoal & 1444 & 08.03 .2017 \\
\hline $929 / 2017$ & SQ4D, Feature 85 & charcoal & 1445 & 08.03 .2017 \\
\hline $936 / 2017$ & SQ4D, Feature 85 & charcoal & 1447 & 08.03 .2017 \\
\hline $940 / 2017$ & SQ4D, Feature 85 & charcoal & 1448 & 09.03.2017 \\
\hline $945 / 2017$ & SQ4D, Feature 85 & charcoal & 1449 & 09.03 .2017 \\
\hline $952 / 2017$ & SQ4D, Feature 85 & charcoal & 1452 & 09.03 .2017 \\
\hline $853 / 2017$ & SQ4C & charcoal & 1480 & 05.03 .2017 \\
\hline
\end{tabular}




\begin{tabular}{|c|c|c|c|c|}
\hline $885 / 2017$ & SQ4C & charcoal & 1481 & 06.03 .2017 \\
\hline $887 / 2017$ & SQ4C & charcoal & 1482 & 06.03 .2017 \\
\hline $900 / 2017$ & SQ4C & charcoal & 1483 & 07.03.2017 \\
\hline $918 / 2017$ & SQ4C & charcoal & 1484 & 07.03 .2017 \\
\hline $907 / 2017$ & SQ4C & charcoal & 1485 & 07.03.2017 \\
\hline $915 / 2017$ & SQ4C & charcoal & 1486 & 07.03 .2017 \\
\hline $876 / 2017$ & SQ4D, Feature 83 & clay object? yellow pigment & 1441 & 06.03 .2017 \\
\hline $948 / 2017$ & SQ4D, Feature 85 & dates (sub recent?) & 1449 & 09.03.2017 \\
\hline $730 / 2017$ & SQ4D, Feature 83 & doum nut & 1327 & 28.02 .2017 \\
\hline $546 / 2017$ & SQ4D & doum nut & 1403 & 20.02.2017 \\
\hline $528 / 2017$ & SQ4D, from ash-spot/base of SU & doum nut & 1403 & 19.02.2017 \\
\hline $553 / 2017$ & SQ4D, W-edge SU & doum nut & 1404 & 20.02.2017 \\
\hline $578 / 2017$ & SQ4D & doum nut & 1408 & 22.02 .2017 \\
\hline $588 / 2017$ & SQ4D & doum nut & 1408 & 23.02.2017 \\
\hline $653 / 2017$ & SQ4D & doum nuts (2 pieces) & 1412 & 25.02.2017 \\
\hline $671 / 2017$ & SQ4D & doum nut & 1419 & 26.02.2017 \\
\hline $681 / 2017$ & SQ4D, S-part & doum nut & 1420 & 27.02.2017 \\
\hline $698 / 2017$ & SQ4D & doum nut & 1421 & 27.02.2017 \\
\hline $739 / 2017$ & SQ4D & doum nut & 1423 & 28.02 .2017 \\
\hline $718 / 2017$ & SQ4D, Feature 83 & doum nut & 1424 & 28.02 .2017 \\
\hline $758 / 2017$ & SQ4D, Feature 83 & doum nut & 1427 & 01.03 .2017 \\
\hline $745 / 2017$ & SQ4D & doum nut & 1428 & 28.02 .2017 \\
\hline $753 / 2017$ & SQ4D & doum nut & 1429 & 01.03 .2017 \\
\hline $772 / 2017$ & SQ4D, Feature 83 & doum nut & 1431 & 01.03 .2017 \\
\hline $779 / 2017$ & SQ4D, Feature 83 & doum nut & 1431 & 02.03 .2017 \\
\hline $789 / 2017$ & SQ4D, Feature 83, lower part & doum nut & 1431 & 02.03 .2017 \\
\hline
\end{tabular}




\begin{tabular}{|c|c|c|c|c|c|}
\hline $784 / 2017$ & SQ4D, Feature 85 & doum nut & 1432 & & 02.03 .2017 \\
\hline $797 / 2017$ & SQ4D, Feature 85 & doum nut & 1433 & & 02.03 .2017 \\
\hline $812 / 2017$ & SQ4D, Feature 83 & doum nut & 1434 & & 04.03 .2017 \\
\hline $821 / 2017$ & SQ4D, Feature 85 & doum nut & 1435 & & 04.03 .2017 \\
\hline $828 / 2017$ & SQ4D, Feature 83 & doum nut & 1437 & & 04.03 .2017 \\
\hline $858 / 2017$ & SQ4D, Feature 83, bottom surface of SU & doum nuts ( 2 fragments) & 1437 & & 05.03 .2017 \\
\hline $845 / 2017$ & SQ4D, Feature 85 & doum nut & 1438 & & 05.03 .2017 \\
\hline $836 / 2017$ & SQ4D, Feature 66/Feature 85 & doum nut (1 fragment) & 1439 & & 05.03 .2017 \\
\hline $863 / 2017$ & SQ4D, Feature 85, E-part & doum nut & 1440 & & 05.03 .2017 \\
\hline $878 / 2017$ & SQ4D, Feature 83 & doum nuts (3 pieces) & 1441 & & 06.03 .2017 \\
\hline $910 / 2017$ & SQ4D, Feature 83 & doum nut & 1443 & & 07.03 .2017 \\
\hline $930 / 2017$ & SQ4D, Feature 85 & doum nut & 1445 & & 08.03 .2017 \\
\hline $947 / 2017$ & SQ4D, Feature 85 & doum nut & 1449 & & 09.03 .2017 \\
\hline $953 / 2017$ & SQ4D, Feature 85 & doum nut & 1452 & & 09.03.2017 \\
\hline $889 / 2017$ & SQ4C & doum nut & 1482 & & 06.03 .2017 \\
\hline $908 / 2017$ & SQ4C & doum nut & 1485 & & 07.03 .2017 \\
\hline $552 / 2017$ & SQ4D, S-part SU & $\begin{array}{l}\text { faience vessel? ( } 1 \text { small fragment, original form and function } \\
\text { unclear) }\end{array}$ & 1404 & SAV1E 1411 & 20.02.2017 \\
\hline $509 / 2017$ & SQ4D & figurine (1 rudimentary female figurine, clay) & 1401 & SAV1E 1065 & 18.02 .2017 \\
\hline $524 / 2017$ & SQ4D, from ash-spot/base of SU & $\begin{array}{l}\text { figurine (1 fragment, possibly rear end of a horse figurine, } \\
\text { clay) }\end{array}$ & 1403 & SAV1E 1453 & 19.02.2017 \\
\hline $687 / 2017$ & SQ4D, from pit & $\begin{array}{l}\text { figurine (1 fragment, possibly horse figurine, Christian, } \\
\text { pottery) }\end{array}$ & 1420 & SAV1E 2648 & 27.02.2017 \\
\hline $729 / 2017$ & SQ4D & figurine (1 fragment, quadruped, Post-New Kingdom, clay) & 1423 & SAV1E 2917 & 28.02 .2017 \\
\hline $859 / 2017$ & SQ4D, Feature 83, bottom surface of SU & flake (from chipping stone) & 1437 & & 05.03 .2017 \\
\hline $592 / 2017$ & SQ4D, at 'wall' & fur/hair & 1409 & & 23.02.2017 \\
\hline $593 / 2017$ & $\mathrm{SQ} 4 \mathrm{C} / 4 \mathrm{D}$ & fur & 1410 & & 23.02 .2017 \\
\hline $764 / 2017$ & SQ4D & fur/hair ball (sub recent?) & 1432 & & 01.03 .2017 \\
\hline
\end{tabular}




\begin{tabular}{|c|c|c|c|c|c|}
\hline $603 / 2017$ & SQ4D & glass vessel (1 fragment) & 1409 & SAV1E 2280 & 23.02.2017 \\
\hline $892 / 2017$ & SQ4C & glass vessel (1 fragment) & 1482 & SAV1E 2929 & 06.03 .2017 \\
\hline $670 / 2017$ & SQ4D & glazed ware (Islamic) & 1419 & & 26.02.2017 \\
\hline $706 / 2017$ & SQ4D & glazed ware (1 fragment, Islamic/Ottoman) & 1423 & SAV1E 2908 & 28.02.2017 \\
\hline $585 / 2017$ & SQ4D & leather (sub recent) & 1408 & & 23.02.2017 \\
\hline $591 / 2017$ & SQ4D, at 'wall' & leather & 1409 & & 23.02.2017 \\
\hline $711 / 2017$ & SQ4D & leather & 1425 & & 28.02 .2017 \\
\hline $722 / 2017$ & SQ4D & leather & 1426 & & 28.02.2017 \\
\hline $723 / 2017$ & SQ4D, Feature 83 & leather & 1427 & & 28.02.2017 \\
\hline $765 / 2017$ & SQ4D & leather & 1432 & & 01.03 .2017 \\
\hline $794 / 2017$ & SQ4D, Feature 85 & leather & 1433 & & 02.03 .2017 \\
\hline $811 / 2017$ & SQ4D, Feature 85 & leather? & 1433 & & 04.03 .2017 \\
\hline $891 / 2017$ & SQ4C & $\begin{array}{l}\text { mortar? (1 rim fragment from a worked stone object, } \\
\text { unspecified stone) }\end{array}$ & 1482 & SAV1E 2928 & 06.03.2017 \\
\hline $690 / 2017$ & SQ4D, from pit & $\begin{array}{l}\text { mould } \\
\text { (1 fragment of a Christian horse mould, pottery) }\end{array}$ & 1420 & SAV1E 2910 & 27.02.2017 \\
\hline $728 / 2017$ & SQ4D & $\begin{array}{l}\text { mould/figurine } \\
\text { (1 fragment of a Christian horse mould/figurine, pottery) }\end{array}$ & 1423 & SAV1E 2649 & 28.02.2017 \\
\hline $801 / 2017$ & SQ4D, Feature 83 & net weight (1 piece, clay) & 1434 & SAV1E 2646 & 02.03 .2017 \\
\hline $513 / 2017$ & SQ4D & organic material (rope ?) & 1402 & & 18.02 .2017 \\
\hline $534 / 2017$ & SQ4D & organic material & 1403 & & 19.02.2017 \\
\hline $535 / 2017$ & SQ4D & organic material & 1403 & & 19.02.2017 \\
\hline $587 / 2017$ & SQ4D & organic material (wood ?) & 1408 & & 23.02.2017 \\
\hline $621 / 2017$ & SQ4D & organic material (horn ?) & 1412 & & 25.02.2017 \\
\hline $625 / 2017$ & SQ4C/4D & organic material & 1413 & & 25.02.2017 \\
\hline $882 / 2017$ & SQ4C & $\begin{array}{l}\text { pendant } \\
\text { (1 fragment, unspecified stone) }\end{array}$ & 1481 & SAV1E 2927 & 06.03 .2017 \\
\hline $514 / 2017$ & SQ4D, NW-part & plaster (schist-plate) & 1402 & & 19.02.2017 \\
\hline $544 / 2017$ & SQ4D, W-edge SU & plaster & 1403 & & 20.02.2017 \\
\hline
\end{tabular}




\begin{tabular}{|c|c|c|c|c|}
\hline $550 / 2017$ & SQ4D, S-edge SU & plaster & 1403 & 20.02.2017 \\
\hline $573 / 2017$ & SQ4D & plaster (1 fragment) & 1407 & 22.02.2017 \\
\hline $570 / 2017$ & SQ4D, N-part & plaster & 1407 & 20.02.2017 \\
\hline $581 / 2017$ & SQ4D & plaster & 1408 & 23.02.2017 \\
\hline $596 / 2017$ & $\mathrm{SQ} 4 \mathrm{C} / 4 \mathrm{D}$ & plaster & 1410 & 23.02.2017 \\
\hline $622 / 2017$ & SQ4D & plaster & 1412 & 25.02 .2017 \\
\hline $627 / 2017$ & $\mathrm{SQ} 4 \mathrm{C} / 4 \mathrm{D}$ & plaster & 1413 & 25.02.2017 \\
\hline $634 / 2017$ & SQ4D & plaster & 1414 & 25.02 .2017 \\
\hline $639 / 2017$ & SQ4D, NE-corner SU & plaster & 1414 & 25.02 .2017 \\
\hline $645 / 2017$ & SQ4D & plaster & 1415 & 25.02 .2017 \\
\hline $650 / 2017$ & $\mathrm{SQ} 4 \mathrm{C} / 4 \mathrm{D}$ & plaster & 1416 & 25.02 .2017 \\
\hline $661 / 2017$ & SQ4D & plaster & 1417 & 26.02.2017 \\
\hline $658 / 2017$ & SQ4D & plaster & 1418 & 26.02.2017 \\
\hline $672 / 2017$ & SQ4D & plaster & 1419 & 26.02.2017 \\
\hline $680 / 2017$ & SQ4D, S-part & plaster & 1420 & 27.02.2017 \\
\hline $696 / 2017$ & SQ4D & plaster & 1421 & 27.02.2017 \\
\hline $704 / 2017$ & SQ4D & plaster & 1422 & 28.02 .2017 \\
\hline $744 / 2017$ & SQ4D & plaster & 1423 & 28.02 .2017 \\
\hline $716 / 2017$ & SQ4D, Feature 83 & plaster & 1424 & 28.02.2017 \\
\hline $713 / 2017$ & SQ4D & plaster & 1425 & 28.02 .2017 \\
\hline $741 / 2017$ & SQ4D, Feature 83 & plaster & 1427 & 28.02 .2017 \\
\hline $757 / 2017$ & SQ4D, Feature 83 & plaster & 1427 & 01.03 .2017 \\
\hline $752 / 2017$ & SQ4D & plaster & 1429 & 01.03 .2017 \\
\hline $771 / 2017$ & SQ4D, Feature 83 & plaster & 1431 & 01.03 .2017 \\
\hline $776 / 2017$ & SQ4D, Feature 83 & plaster & 1431 & 02.03 .2017 \\
\hline $786 / 2017$ & SQ4D, Feature 83, lower part & plaster & 1431 & 02.03 .2017 \\
\hline
\end{tabular}




\begin{tabular}{|c|c|c|c|c|c|}
\hline $768 / 2017$ & SQ4D & plaster & 1432 & & 01.03 .2017 \\
\hline $783 / 2017$ & SQ4D, Feature 85 & plaster & 1432 & & 02.03 .2017 \\
\hline $807 / 2017$ & SQ4D, Feature 85 & plaster & 1433 & & 04.03 .2017 \\
\hline $800 / 2017$ & SQ4D, Feature 83 & plaster & 1434 & & 02.03 .2017 \\
\hline $803 / 2017$ & SQ4D, Feature 83 & plaster & 1434 & & 04.03 .2017 \\
\hline $820 / 2017$ & SQ4D, Feature 85 & plaster & 1435 & & 04.03 .2017 \\
\hline $826 / 2017$ & SQ4D, Feature 83 & plaster & 1437 & & 04.03 .2017 \\
\hline $830 / 2017$ & SQ4D, Feature 85 & plaster & 1438 & & 04.03 .2017 \\
\hline $843 / 2017$ & SQ4D, Feature 85 & plaster & 1438 & & 05.03 .2017 \\
\hline $834 / 2017$ & SQ4D, Feature 66/Feature 85 & plaster & 1439 & & 05.03 .2017 \\
\hline $850 / 2017$ & SQ4D, Feature 85, W-part & plaster & 1440 & & 05.03 .2017 \\
\hline $864 / 2017$ & SQ4D, Feature 85, E-part & plaster & 1440 & & 05.03 .2017 \\
\hline $898 / 2017$ & SQ4D, Feature 85 & plaster & 1442 & & 07.03 .2017 \\
\hline $920 / 2017$ & SQ4D, Feature 85 & plaster & 1444 & & 08.03 .2017 \\
\hline $928 / 2017$ & SQ4D, Feature 85 & plaster & 1445 & & 08.03 .2017 \\
\hline $933 / 2017$ & SQ4D, Feature 85 & plaster (from schist pavement) & 1446 & & 08.03 .2017 \\
\hline $937 / 2017$ & SQ4D, Feature 85 & plaster & 1447 & & 08.03 .2017 \\
\hline $946 / 2017$ & SQ4D, Feature 85 & plaster & 1449 & & 09.03 .2017 \\
\hline $954 / 2017$ & SQ4D, Feature 85 & plaster & 1452 & & 09.03 .2017 \\
\hline $854 / 2017$ & SQ4C & plaster & 1480 & & 05.03 .2017 \\
\hline $888 / 2017$ & SQ4C & plaster & 1482 & & 06.03 .2017 \\
\hline $500 / 2017$ & SQ4D & pottery (31 baskets) & 1400 & & 18.02 .2017 \\
\hline $503 / 2017$ & SQ4D & $\begin{array}{l}\text { pottery } \\
\text { (11 baskets), among them scraper (1 re-used body sherd, } \\
\text { Post-New Kingdom, pottery) }\end{array}$ & 1401 & SAV1E 1815 & 18.02 .2017 \\
\hline $508 / 2017$ & SQ4D & pottery (1 basket) & 1402 & & 18.02 .2017 \\
\hline $516 / 2017$ & SQ4D & pottery ( 5 baskets) & 1402 & & 19.02.2017 \\
\hline
\end{tabular}




\begin{tabular}{|c|c|c|c|c|c|}
\hline $519 / 2017$ & SQ4D & $\begin{array}{l}\text { pottery and stone: pottery ( } 9 \text { baskets), among them } \\
\text { token/gaming piece ( } 1 \text { re-used } 18^{\text {th }} \text { Dynasty body sherd, } \\
\text { pottery), pottery object ( } 1 \text { fragment, unclear function, } \\
\text { Christian), window grille ( } 1 \text { fragment, Christian, pottery), } \\
\text { scraper ( } 1 \text { re-used sherd, pottery), model vessel stand } \\
\text { ( } 1 \text { fragment, pottery); whetstone ( } 1 \text { piece, sandstone) }\end{array}$ & 1403 & $\begin{array}{l}\text { SAV1E } 0989 \text { (token/gaming piece), SAV1E } \\
\text { 0360 (pottery object), SAV1E 0640 (window } \\
\text { grille), SAV1E 0991 (scraper), SAV1E } 1024 \\
\text { (model vessel stand), SAV1E } 1027 \\
\text { (whetstone) }\end{array}$ & 19.02.2017 \\
\hline $548 / 2017$ & SQ4D & pottery (7 baskets) & 1403 & & 20.02.2017 \\
\hline $537 / 2017$ & SQ4D, ashy area & pottery (1/2 basket) & 1403 & & 20.02.2017 \\
\hline $525 / 2017$ & SQ4D, from ash-spot/base of SU & $\begin{array}{l}\text { pottery, among it scraper (1 re-used body sherd, Christian, } \\
\text { pottery) }\end{array}$ & 1403 & SAV1E 1457 & 19.02.2017 \\
\hline $540 / 2017$ & SQ4D, pebble area & pottery (1 basket) & 1403 & & 20.02.2017 \\
\hline $558 / 2017$ & SQ4D & pottery (10 baskets) & 1404 & & 20.02.2017 \\
\hline $556 / 2017$ & SQ4D, W-edge SU & pottery (1 basket) & 1404 & & 20.02.2017 \\
\hline $561 / 2017$ & SQ4D & pottery (1 basket) & 1405 & & 20.02 .2017 \\
\hline $564 / 2017$ & SQ4D & pottery ( 1 basket) & 1406 & & 20.02.2017 \\
\hline $572 / 2017$ & SQ4D & $\begin{array}{l}\text { pottery ( } 5 \text { baskets), among them scraper ( } 1 \text { re-used Post-New } \\
\text { Kingdom sherd, pottery), scraper/token/lid? ( } 1 \text { re-used } \\
18^{\text {th }} \text { Dynasty sherd, pottery) }\end{array}$ & 1407 & $\begin{array}{l}\text { SAV1E } 1817 \text { (scraper), SAV1E } 2279 \\
\text { (scraper/token/lid?) }\end{array}$ & 22.02 .2017 \\
\hline $567 / 2017$ & SQ4D, N-part & $\begin{array}{l}\text { pottery ( } 2 \text { baskets), among them scraper ( } 1 \text { re-used } \\
18^{\text {th }} \text { Dynasty sherd, pottery) }\end{array}$ & 1407 & SAV1E 1420 & 20.02.2017 \\
\hline $582 / 2017$ & SQ4D & pottery (20 baskets) & 1408 & & 23.02.2017 \\
\hline $576 / 2017$ & SQ4D, N-part & pottery (1 basket) & 1408 & & 22.02.2017 \\
\hline $601 / 2017$ & SQ4D & pottery & 1409 & & 23.02.2017 \\
\hline $597 / 2017$ & $\mathrm{SQ} 4 \mathrm{C} / 4 \mathrm{D}$ & pottery (1 basket) & 1410 & & 23.02 .2017 \\
\hline $604 / 2017$ & SQ4C/4D & pottery & 1411 & & 23.02 .2017 \\
\hline $606 / 2017$ & SQ4D & pottery (1 basket) & 1412 & & 23.02.2017 \\
\hline $617 / 2017$ & SQ4D & pottery (7 baskets) & 1412 & & 25.02 .2017 \\
\hline $618 / 2017$ & $\mathrm{SQ} 4 \mathrm{C} / 4 \mathrm{D}$ & pottery (5 baskets) & 1413 & & 25.02 .2017 \\
\hline $631 / 2017$ & SQ4D & pottery (8 baskets) & 1414 & & 25.02 .2017 \\
\hline $637 / 2017$ & SQ4D, NE-corner SU & pottery (1 basket) & 1414 & & 25.02 .2017 \\
\hline $643 / 2017$ & SQ4D & pottery ( 2 baskets) & 1415 & & 25.02.2017 \\
\hline
\end{tabular}




\begin{tabular}{|c|c|c|c|c|c|}
\hline $647 / 2017$ & $\mathrm{SQ} 4 \mathrm{C} / 4 \mathrm{D}$ & pottery ( 1 basket) & 1416 & & 25.02.2017 \\
\hline $659 / 2017$ & SQ4D & pottery (1 basket) & 1417 & & 26.02.2017 \\
\hline $655 / 2017$ & SQ4D & pottery (2 $1 / 2$ baskets) & 1418 & & 26.02.2017 \\
\hline $666 / 2017$ & SQ4D & $\begin{array}{l}\text { pottery ( } 17 \text { baskets), among them scrapers ( } 2 \text { pieces, each re- } \\
\text { used body sherds, } 118^{\text {th }} \text { Dynasty, } 1 \text { Christian, pottery), }\end{array}$ & 1419 & SAV1E 2925, SAV1E 2924 (scrapers) & 26.02.2017 \\
\hline $677 / 2017$ & SQ4D, S-part & pottery (10 baskets) & 1420 & & 27.02.2017 \\
\hline $686 / 2017$ & SQ4D, from pit & pottery (9 baskets) & 1420 & & 27.02.2017 \\
\hline $693 / 2017$ & SQ4D & $\begin{array}{l}\text { pottery and stone: pottery ( } 5 \text { baskets), among them scraper } \\
\text { or/and lid ( } 1 \text { re-used } 18^{\text {th }} \text { Dynasty sherd, pottery), scraper } \\
\text { ( } 1 \text { re-used } 18^{\text {th }} \text { Dynasty sherd, pottery); pestle or polishing } \\
\text { stone ( } 1 \text { piece, sandstone) }\end{array}$ & 1421 & $\begin{array}{l}\text { SAV1E } 2911 \text { (scraper or/and lid), SAV1E } \\
2915 \text { (scraper), SAV1E } 2920 \text { (pestle) }\end{array}$ & 27.02.2017 \\
\hline $699 / 2017$ & SQ4D & pottery (1 basket) & 1422 & & 28.02.2017 \\
\hline $700 / 2017$ & SQ4D & $\begin{array}{l}\text { pottery ( } 6 \text { baskets), among them lid ( } 1 \text { re-used } 18^{\text {th }} \text { Dynasty } \\
\text { base sherd, pottery) }\end{array}$ & 1423 & SAV1E 2909 & 28.02.2017 \\
\hline $701 / 2017$ & SQ4D, Feature 83 & $\begin{array}{l}\text { pottery ( } 5 \text { baskets), among them scraper ( } 1 \text { re-used } \\
18^{\text {th }} \text { Dynasty sherd, pottery, traces of orange pigment) }\end{array}$ & 1424 & SAV1E 2964 & 28.02.2017 \\
\hline $710 / 2017$ & SQ4D & $\begin{array}{l}\text { pottery ( } 1 \text { basket), among it scraper ( } 1 \text { re-used sherd, } \\
\text { Christian, pottery) }\end{array}$ & 1425 & SAV1E 2914 & 28.02.2017 \\
\hline $724 / 2017$ & SQ4D & pottery (1 basket) & 1426 & & 28.02.2017 \\
\hline $731 / 2017$ & SQ4D, Feature 83 & pottery (2 baskets) & 1427 & & 28.02 .2017 \\
\hline $749 / 2017$ & SQ4D, Feature 83 & $\begin{array}{l}\text { pottery ( } 6 \text { baskets), among them scraper ( } 1 \text { re-used sherd, } \\
\text { pottery) }\end{array}$ & 1427 & SAV1E 2647 & 01.03 .2017 \\
\hline $734 / 2017$ & SQ4D & $\begin{array}{l}\text { pottery ( } 3 \text { baskets), among them scraper ( } 1 \text { re-used } \\
18^{\text {th }} \text { Dynasty sherd, pottery) }\end{array}$ & 1428 & SAV1E 2933 & 28.02 .2017 \\
\hline $748 / 2017$ & SQ4D & pottery (4 baskets) & 1429 & & 01.03 .2017 \\
\hline $759 / 2017$ & SQ4D & pottery (1 basket) & 1430 & & 01.03 .2017 \\
\hline $763 / 2017$ & SQ4D, Feature 83 & pottery (3 baskets) & 1431 & & 01.03 .2017 \\
\hline $775 / 2017$ & SQ4D, Feature 83 & pottery (1 basket) & 1431 & & 02.03 .2017 \\
\hline $762 / 2017$ & SQ4D & pottery (4 baskets) & 1432 & & 01.03 .2017 \\
\hline $780 / 2017$ & SQ4D, Feature 85 & pottery (2 baskets) & 1432 & & 02.03 .2017 \\
\hline $785 / 2017$ & SQ4D, Feature 83, lower part & pottery (1 $1 / 2$ baskets) & 1432 & & 02.03 .2017 \\
\hline $790 / 2017$ & SQ4D, Feature 85 & pottery ( 2 baskets) & 1433 & & 02.03 .2017 \\
\hline
\end{tabular}




\begin{tabular}{|c|c|c|c|c|c|}
\hline $791 / 2017$ & SQ4D, Feature 85 & pottery ( 5 baskets) & 1433 & & 02.03 .2017 \\
\hline $806 / 2017$ & SQ4D, Feature 85 & $\begin{array}{l}\text { pottery ( } 2 \text { baskets), among them scraper ( } 1 \text { re-used sherd, } \\
\text { Christian, pottery) }\end{array}$ & 1433 & SAV1E 2912 & 04.03 .2017 \\
\hline $796 / 2017$ & SQ4D, Feature 83 & pottery ( 3 baskets) & 1434 & & 02.03 .2017 \\
\hline $802 / 2017$ & SQ4D, Feature 83 & pottery & 1434 & & 04.03.2017 \\
\hline $817 / 2017$ & SQ4D, Feature 85 & pottery (6 baskets) & 1435 & & 04.03.2017 \\
\hline $813 / 2017$ & SQ4D, Feature 83 & pottery (1 basket) & 1436 & & 04.03 .2017 \\
\hline $823 / 2017$ & SQ4D, Feature 83 & pottery ( 2 baskets) & 1437 & & 04.03 .2017 \\
\hline $837 / 2017$ & SQ4D, Feature 83, bottom surface of SU & pottery (1 basket) & 1437 & & 05.03.2017 \\
\hline $829 / 2017$ & SQ4D, Feature 85 & pottery (1 basket) & 1438 & & 04.03.2017 \\
\hline $841 / 2017$ & SQ4D, Feature 85 & pottery (1 basket) & 1438 & & 05.03.2017 \\
\hline $832 / 2017$ & SQ4D, Feature 66/Feature 85 & pottery (1 basket) & 1439 & & 05.03.2017 \\
\hline $846 / 2017$ & SQ4D, Feature 85, W-part & pottery ( 1 basket) & 1440 & & 05.03 .2017 \\
\hline $865 / 2017$ & SQ4D, Feature 85, E-part & pottery (1 basket) & 1440 & & 05.03 .2017 \\
\hline $867 / 2017$ & SQ4D, Feature 83 & pottery vessel, cooking pot & 1441 & & 06.03 .2017 \\
\hline $868 / 2017$ & SQ4D, Feature 83 & pottery vessel, carinated vessel & 1441 & & 06.03.2017 \\
\hline $869 / 2017$ & SQ4D, Feature 83 & pottery vessel, lower part & 1441 & & 06.03 .2017 \\
\hline $870 / 2017$ & SQ4D, Feature 83 & pottery vessel: beer jar & 1441 & & 06.03.2017 \\
\hline $873 / 2017$ & SQ4D, Feature 83 & pottery sherds & 1441 & & 06.03 .2017 \\
\hline $874 / 2017$ & SQ4D, Feature 83 & pottery: jug & 1441 & & 06.03 .2017 \\
\hline $895 / 2017$ & SQ4D, Feature 85 & pottery (1 basket) & 1442 & & 07.03.2017 \\
\hline $912 / 2017$ & SQ4D, Feature 83 & pottery (1 basket) & 1443 & & 07.03.2017 \\
\hline $919 / 2017$ & SQ4D, Feature 85 & $\begin{array}{l}\text { pottery ( } 1 \text { basket), among it scraper or lid ( } 1 \text { re-used } \\
18^{\text {th }} \text { Dynasty sherd, pottery), scraper ( } 1 \text { re-used } 18^{\text {th }} \text { Dynasty } \\
\text { sherd, pottery) }\end{array}$ & 1444 & $\begin{array}{l}\text { SAV1E } 2970 \text { (scraper or lid), SAV1E } 2966 \\
\text { (scraper) }\end{array}$ & 08.03.2017 \\
\hline $925 / 2017$ & SQ4D, Feature 85, E-part & pottery ( $1 / 2$ basket) & 1445 & & 08.03.2017 \\
\hline $926 / 2017$ & SQ4D, Feature 85, W-part & pottery (1 basket) & 1445 & & 08.03.2017 \\
\hline $931 / 2017$ & SQ4D, Feature 85 & pottery (1 basket) & 1446 & & 08.03.2017 \\
\hline
\end{tabular}




\begin{tabular}{|c|c|c|c|c|c|}
\hline $934 / 2017$ & SQ4D, Feature 85 & pottery (1 basket) & 1447 & & 08.03 .2017 \\
\hline $938 / 2017$ & SQ4D, Feature 85 & pottery (1 basket) & 1448 & & 09.03.2017 \\
\hline $943 / 2017$ & SQ4D, Feature 85 & pottery (1 large bag) & 1449 & & 09.03 .2017 \\
\hline $941 / 2017$ & SQ4D, Feature 85 & pottery (1 basket) & 1450 & & 09.03 .2017 \\
\hline $942 / 2017$ & SQ4D, Feature 85 & pottery (1 basket) & 1451 & & 09.03 .2017 \\
\hline $950 / 2017$ & SQ4D, Feature 85 & pottery (1 basket) & 1452 & & 09.03 .2017 \\
\hline $839 / 2017$ & SQ4C & pottery (15 baskets) & 1480 & & 05.03 .2017 \\
\hline $866 / 2017$ & SQ4C & $\begin{array}{l}\text { pottery ( } 8 \text { baskets), among them lids ( } 2 \text { pieces, } 1 \text { re-used } \\
18^{\text {th }} \text { Dynasty, pottery, } 1 \text { clay) }\end{array}$ & 1481 & SAV1E 2938, SAV1E 2937 (lids) & 06.03 .2017 \\
\hline $872 / 2017$ & SQ4C & $\begin{array}{l}\text { pottery ( } 6 \text { baskets), among them window grille ( } 1 \text { fragment, } \\
\text { Christian, pottery), lid?/scraper? ( } 1 \text { piece, re-used base of a } \\
\text { pottery vessel, } 18^{\text {th }} \text { Dynasty) }\end{array}$ & 1482 & $\begin{array}{l}\text { SAV1E } 2936 \text { (window grille), SAV1E } 2946 \\
\text { (lid?/scraper?) }\end{array}$ & 06.03 .2017 \\
\hline $899 / 2017$ & SQ4C & pottery (1 basket) & 1483 & & 07.03 .2017 \\
\hline $916 / 2017$ & SQ4C & pottery (1 small bag) & 1484 & & 07.03 .2017 \\
\hline $905 / 2017$ & SQ4C & $\begin{array}{l}\text { pottery ( } 1 \text { basket), among it re-used sherd (1 piece, reworked } \\
\text { to lid) }\end{array}$ & 1485 & SAV1E 2954 & 07.03.2017 \\
\hline $913 / 2017$ & SQ4C & pottery (1 small bag) & 1486 & & 07.03 .2017 \\
\hline $515 / 2017$ & SQ4D & rope (1 fragment) & 1402 & & 19.02.2017 \\
\hline $520 / 2017$ & SQ4D & rope (1 fragment) & 1403 & & 19.02.2017 \\
\hline $584 / 2017$ & SQ4D & rope/fragment of scarf (modern) & 1408 & & 23.02.2017 \\
\hline $612 / 2017$ & SQ4D & rope (recent) & 1412 & & 25.02.2017 \\
\hline $628 / 2017$ & SQ4C/4D & rope (1 fragment) & 1413 & & 25.02 .2017 \\
\hline $615 / 2017$ & SQ4C/4D, S of 4C & rope (recent) & 1413 & & 25.02 .2017 \\
\hline $630 / 2017$ & SQ4D & rope (recent) & 1414 & & 25.02 .2017 \\
\hline $635 / 2017$ & SQ4D & rope (1 fragment) & 1414 & & 25.02 .2017 \\
\hline $708 / 2017$ & SQ4D & rope/textile (sub recent) & 1425 & & 28.02.2017 \\
\hline $721 / 2017$ & SQ4D, Feature 83 & rope? & 1427 & & 28.02 .2017 \\
\hline $827 / 2017$ & SQ4D, Feature 83 & seal? burnt brick? & 1437 & & 04.03 .2017 \\
\hline $502 / 2017$ & SQ4D, N-part & shell (1 fragment) & 1401 & & 18.02 .2017 \\
\hline
\end{tabular}




\begin{tabular}{|c|c|c|c|c|c|}
\hline $532 / 2017$ & SQ4D & shell & 1403 & & 19.02.2017 \\
\hline $589 / 2017$ & SQ4D & shell & 1408 & & 23.02.2017 \\
\hline $620 / 2017$ & SQ4D & shell & 1412 & & 25.02.2017 \\
\hline $682 / 2017$ & SQ4D, S-part & shell & 1420 & & 27.02.2017 \\
\hline $717 / 2017$ & SQ4D, Feature 83 & shell & 1424 & & 28.02.2017 \\
\hline $746 / 2017$ & SQ4D & shell & 1428 & & 28.02.2017 \\
\hline $880 / 2017$ & SQ4D, Feature 83 & shell/mollusc? (1 piece, Nile oyster) & 1441 & & 06.03.2017 \\
\hline $577 / 2017$ & SQ4D, N-part & slag (1 fragment, with small patch of brick) & 1408 & SAV1E 2223 & 22.02.2017 \\
\hline $649 / 2017$ & $\mathrm{SQ} 4 \mathrm{C} / 4 \mathrm{D}$ & slag (2 fragments) & 1416 & & 25.02.2017 \\
\hline $663 / 2017$ & SQ4D & slag & 1417 & & 26.02.2017 \\
\hline $675 / 2017$ & SQ4D & slag (8 fragments) & 1419 & SAV1E 2961 & 26.02.2017 \\
\hline $684 / 2017$ & SQ4D, from debris in pit & slag and brick ( 1 corner fragment of large brick burnt to slag) & 1420 & SAV1E 2944 & 27.02.2017 \\
\hline $736 / 2017$ & SQ4D & slag and brick (3 pieces) & 1423 & SAV1E 2923 & 28.02.2017 \\
\hline $720 / 2017$ & SQ4D, Feature 83 & slag (2 small pieces) & 1424 & SAV1E 2644 & 28.02.2017 \\
\hline $822 / 2017$ & SQ4D, Feature 85 & slag (1 piece) & 1435 & SAV1E 2913 & 04.03.2017 \\
\hline $855 / 2017$ & SQ4C & slag (3 fragments) & 1480 & SAV1E 2921 & 05.03 .2017 \\
\hline $883 / 2017$ & SQ4C & slag (1 small fragment) & 1481 & SAV1E 2931 & 06.03.2017 \\
\hline $894 / 2017$ & SQ4C & slag (4 pieces) & 1482 & SAV1E 2932 & 06.03.2017 \\
\hline $551 / 2017$ & SQ4D & $\begin{array}{l}\text { stones: hammer ( } 1 \text { piece, quartzite), polishing } \\
\text { stone/whetstone (1 fragment, sandstone) }\end{array}$ & 1403 & $\begin{array}{l}\text { SAV1E } 1208 \text { (hammer), SAV1E } 1255 \\
\text { (polishing stone/whetstone) }\end{array}$ & 20.02.2017 \\
\hline $602 / 2017$ & SQ4D & stone: pounder (1 quartzite pebble) & 1408 & SAV1E 2953 & 23.02.2017 \\
\hline $629 / 2017$ & SQ4D & $\begin{array}{l}\text { stones: pounder (1 quartzite pebble), grindstone (1 piece, } \\
\text { quartzite) }\end{array}$ & 1412 & $\begin{array}{l}\text { SAV1E } 2934 \text { (pounder), SAV1E } 2971 \\
\text { (grindstone) }\end{array}$ & 25.02.2017 \\
\hline $636 / 2017$ & SQ4D & stone: pounder (1 quartz pebble) & 1414 & SAV1E 2948 & 25.02.2017 \\
\hline $642 / 2017$ & SQ4D & stone: polishing stone (1 fragment, sandstone) & 1415 & SAV1E 2940 & 25.02.2017 \\
\hline $654 / 2017$ & $\mathrm{SQ} 4 \mathrm{C} / 4 \mathrm{D}$ & stone: pounder (1 quartz pebble) & 1416 & SAV1E 2950 & 25.02.2017 \\
\hline $664 / 2017$ & SQ4D & stone: grindstone or polishing stone (1 fragment, sandstone) & 1418 & SAV1E 2941 & 26.02.2017 \\
\hline $667 / 2017$ & SQ4D & stone: polishing stone? (1 fragment, quartzite) & 1419 & SAV1E 2942 & 26.02.2017 \\
\hline
\end{tabular}




\begin{tabular}{|c|c|c|c|c|c|}
\hline $668 / 2017$ & SQ4D & $\begin{array}{l}\text { stone, worked ( } 1 \text { large square of sandstone with drilled } \\
\text { hollow for either grinding surface or door pivot, reworked } \\
\text { several times, additional use as whetstone) }\end{array}$ & 1419 & SAV1E 2958 & 26.02.2017 \\
\hline $691 / 2017$ & SQ4D, from BS in NW-corner of pit & stone: hammer ( 1 stone ball, quartzite?) & 1420 & SAV1E 2947 & 27.02.2017 \\
\hline $697 / 2017$ & SQ4D & $\begin{array}{l}\text { stone: pounder ( } 1 \text { quartzite pebble, } \\
\text { traces of orange pigment) }\end{array}$ & 1421 & SAV1E 2939 & 27.02.2017 \\
\hline $692 / 2017$ & SQ4D & stone tool & 1421 & & 27.02.2017 \\
\hline $705 / 2017$ & SQ4D & $\begin{array}{l}\text { stone: polishing stone ( } 1 \text { block, sandstone, re-used } \\
\text { architecture?) }\end{array}$ & 1423 & SAV1E 2935 & 28.02.2017 \\
\hline $740 / 2017$ & SQ4D & stone: grindstone (1 fragment, sandstone) & 1423 & SAV1E 2960 & 28.02.2017 \\
\hline $727 / 2017$ & SQ4D & stone: grindstone (1 fragment, sandstone) & 1426 & SAV1E 2952 & 28.02.2017 \\
\hline $754 / 2017$ & SQ4D & stones: hand mill (1 piece), pounder (1 piece) & 1429 & & 01.03 .2017 \\
\hline $774 / 2017$ & SQ4D & stone: pounder (1 quartz pebble) & 1432 & SAV1E 2945 & 01.03 .2017 \\
\hline $847 / 2017$ & SQ4D, Feature 85, W-part & stone: polishing stone (1 fragment, sandstone) & 1440 & SAV1E 2969 & 05.03.2017 \\
\hline $860 / 2017$ & SQ4D, Feature 85, directly at E-wall & $\begin{array}{l}\text { stone/weight? ( } 1 \text { fragment of a stone ring, broken in half, } \\
\text { unspecified stone, schist?soapstone?) }\end{array}$ & 1440 & SAV1E 2965 & 05.03 .2017 \\
\hline $902 / 2017$ & SQ4D, Feature 85 & $\begin{array}{l}\text { stone, worked ( } 1 \text { fragment, unclear function, spout?, } \\
\text { sandstone) }\end{array}$ & 1442 & SAV1E 2972 & 07.03.2017 \\
\hline $903 / 2017$ & SQ4D, Feature 85 & $\begin{array}{l}\text { stone, worked ( } 1 \text { fragment, used as whetstone?polishing } \\
\text { stone?, sandstone) }\end{array}$ & 1442 & SAV1E 2968 & 07.03.2017 \\
\hline $851 / 2017$ & SQ4C & stone: polishing stone (1 fragment, sandstone) & 1480 & SAV1E 2949 & 05.03.2017 \\
\hline $871 / 2017$ & SQ4C & $\begin{array}{l}\text { stones: pounder (1 quartz pebble), grindstone? (1 fragment, } \\
\text { sandstone) }\end{array}$ & 1482 & $\begin{array}{l}\text { SAV1E } 2943 \text { (pounder), SAV1E } 2951 \\
\text { (grindstone?) }\end{array}$ & 06.03.2017 \\
\hline $504 / 2017$ & SQ4D, N-part & stopper or lid? (1 fragment, unclear clay object) & 1401 & SAV1E 0354 & 18.02 .2017 \\
\hline $511 / 2017$ & SQ4D & textile & 1402 & & 18.02 .2017 \\
\hline $527 / 2017$ & SQ4D, from ash-spot/base of SU & textile (recent) & 1403 & & 19.02.2017 \\
\hline $555 / 2017$ & SQ4D, W-edge SU & textile & 1404 & & 20.02.2017 \\
\hline $598 / 2017$ & SQ4D & textile & 1409 & & 23.02.2017 \\
\hline $616 / 2017$ & SQ4D & textile & 1412 & & 25.02.2017 \\
\hline $610 / 2017$ & $\mathrm{SQ} 4 \mathrm{C} / 4 \mathrm{D}$ & textile (recent) & 1413 & & 25.02 .2017 \\
\hline $707 / 2017$ & SQ4D, NW-corner SU & textile (modern?) & 1423 & & 28.02.2017 \\
\hline
\end{tabular}




\begin{tabular}{|c|c|c|c|c|c|}
\hline $893 / 2017$ & SQ4C & textile & 1482 & & 06.03 .2017 \\
\hline $549 / 2017$ & SQ4D, SW-corner SU & token/gaming piece ( 1 small clay ball) & 1403 & SAV1E 1038 & 20.02 .2017 \\
\hline $890 / 2017$ & SQ4C & weight? ( 1 almost complete ring, schist) & 1482 & SAV1E 2930 & 06.03 .2017 \\
\hline $521 / 2017$ & SQ4D, NE-part & wood & 1403 & & 19.02.2017 \\
\hline $590 / 2017$ & SQ4D & wood & 1408 & & 23.02 .2017 \\
\hline $735 / 2017$ & SQ4D & wood/reed? & 1423 & & 28.02.2017 \\
\hline
\end{tabular}

\section{List of Finds SAV1 West 2013-2017}

\begin{tabular}{|c|c|c|c|c|c|}
\hline \multicolumn{6}{|c|}{ Sai Island SAV1 West 2014} \\
\hline Find no. & Location & Description/label/material & $\begin{array}{c}\text { SU/ } \\
\text { level }\end{array}$ & Object no. & Date \\
\hline $1043 / 2014$ & $\mathrm{SQ} 1,4-6 \mathrm{~m} \mathrm{~N}-\mathrm{S} / 5-10 \mathrm{~m} \mathrm{E}-\mathrm{W}$ & architecture: door hinge (1 massive fragment, sandstone) & 536 & SAV1W 0364 & 28.01 .2014 \\
\hline $1310 / 2014$ & $\mathrm{SQ} 1,2.7 \mathrm{~m} \mathrm{~S}-\mathrm{N} / 3.4 \mathrm{~m}$ E-W & architecture: pivot stone (1 piece, sandstone) & 570 & SAV1W 0694 & 08.02 .2014 \\
\hline $701 / 2014$ & SQ1W, E-half, $5 \mathrm{~m}$ from $\mathrm{E} / 0 \mathrm{~m}$ from $\mathrm{N}$ & bead (1 spherical shaped bead, fragments of a second bead, clay) & 501 & SAV1W 0186 & 18.01 .2014 \\
\hline $713 / 2014$ & SQ1W, W-half & bead (1 spherical shaped bead, faience); bone & 501 & SAV1W 1460 & 19.01.2014 \\
\hline $628 / 2014$ & SQ1, NW-corner, $1 \mathrm{~m}$ from $\mathrm{E}, 1 \mathrm{~m}$ from $\mathrm{N}$ & bead ( 1 cylindrical bead, clay) & 502 & & 13.01 .2014 \\
\hline $830 / 2014$ & $\mathrm{SQ} 2,0.8 \mathrm{~m} \mathrm{E}-\mathrm{W} / 2.3 \mathrm{~m} \mathrm{~S}-\mathrm{N}$ & bead ( 1 cylindrical bead, faience) & 514 & SAV1W 0161 & 16.01 .2014 \\
\hline $828 / 2014$ & $\mathrm{SQ} 2,1-2 \mathrm{~m} \mathrm{E}-\mathrm{W} / 3-4 \mathrm{~m} \mathrm{~S}-\mathrm{N}$ & bead ( 1 circular bead, glass) & 516 & SAV1W 0159 & 16.01 .2014 \\
\hline $1105 / 2014$ & SQ1NW & bead (1 tubular/cylindrical bead, faience) & 545 & SAV1W 0390 & 30.01 .2014 \\
\hline $1277 / 2014$ & SQ1 & bead (1 oval/cylindrical bead, clay) & 565 & SAV1W 0508 & 06.02 .2014 \\
\hline $965 / 2014$ & SQ2 & bead (1 spherical bead, pottery) & 583 & SAV1W 0562 & 11.02 .2014 \\
\hline $1375 / 2014$ & $\mathrm{SQ} 1,2.2 \mathrm{~m} \mathrm{~N}-\mathrm{S} / 2.2 \mathrm{~m} \mathrm{E}-\mathrm{W}$ & bead (1 flat disc bead, faience) & 590 & SAV1W 0566 & 13.02 .2014 \\
\hline $516 / 2014$ & SQ1 & bone & 501 & & 06.01 .2014 \\
\hline $558 / 2014$ & SQ1 & bone & 501 & & 08.01 .2014 \\
\hline $571 / 2014$ & SQ1 & bone & 501 & & 09.01 .2014 \\
\hline $738 / 2014$ & $\begin{array}{l}\text { SQ1, adjacent to Feature } 100 \text { from E, } \\
3-5 \mathrm{~m} \mathrm{E}-\mathrm{W} / 0-4 \mathrm{~m} \mathrm{~S}-\mathrm{N}\end{array}$ & bone & 501 & & 21.01 .2014 \\
\hline $721 / 2014$ & $\begin{array}{l}\text { SQ1, adjacent to Feature } 100 \text { from E, } \\
3-5 \mathrm{~m} \mathrm{E}-\mathrm{W} / 0-5 \mathrm{~m} \mathrm{~S}-\mathrm{N}\end{array}$ & bone & 501 & & 20.01 .2014 \\
\hline
\end{tabular}




\begin{tabular}{|c|c|c|c|c|}
\hline $732 / 2014$ & $\begin{array}{l}\text { SQ1, adjacent to Feature } 100 \text { from E, } \\
3-5 \mathrm{~m} \mathrm{E}-\mathrm{W} / 0-5 \mathrm{~m} \mathrm{~S}-\mathrm{N}\end{array}$ & bone & 501 & 20.01 .2014 \\
\hline $705 / 2014$ & $\begin{array}{l}\text { SQ1, adjacent to Feature } 100 \text { from E, } \\
4-5 \mathrm{~m} \mathrm{E}-\mathrm{W} / 0-5 \mathrm{~m} \mathrm{~S}-\mathrm{N}\end{array}$ & bone & 501 & 19.01.2014 \\
\hline $666 / 2014$ & SQ1, NE-corner, 3-5m E-W/0-5m N-S & bone & 501 & 15.01 .2014 \\
\hline $669 / 2014$ & SQ1, NE-corner, 3-5m E-W/0-5m N-S & bone & 501 & 15.01 .2014 \\
\hline $672 / 2014$ & SQ1, NW-corner, 0-3m W-E/0-2m N-S & bone & 501 & 15.01 .2014 \\
\hline $680 / 2014$ & SQ1, SE-corner, 3-5m E-W/0-5m S-N & bone & 501 & 16.01 .2014 \\
\hline $689 / 2014$ & SQ1, SE-corner, 4-5m E-W/0-5m S-N & bone & 501 & 18.01 .2014 \\
\hline $1054 / 2014$ & SQ1NW & bone & 501 & 28.01 .2014 \\
\hline $716 / 2014$ & SQ1W & bone (fossilised) & 501 & 19.01.2014 \\
\hline $677 / 2014$ & SQ1W, E-half & bone & 501 & 16.01 .2014 \\
\hline $691 / 2014$ & SQ1W, E-half & bone & 501 & 18.01 .2014 \\
\hline $694 / 2014$ & SQ1W, W-half & bone & 501 & 18.01 .2014 \\
\hline $506 / 2014$ & SQ2 & bone & 501 & 05.01 .2014 \\
\hline $511 / 2014$ & SQ2 & bone & 501 & 05.01 .2014 \\
\hline $562 / 2014$ & SQ2 & bone & 501 & 08.01 .2014 \\
\hline $586 / 2014$ & SQ2 & bone & 501 & 11.01 .2014 \\
\hline $534 / 2014$ & SQ2 & bone & 501 & 07.01 .2014 \\
\hline $525 / 2014$ & SQ2, W-half & bone & 501 & 06.01 .2014 \\
\hline $538 / 2014$ & SQ1 & bone & 502 & 07.01 .2014 \\
\hline $623 / 2014$ & SQ1, NE-corner, 1-5m W-E/3-5m N-S & bone & 502 & 13.01.2014 \\
\hline $819 / 2014$ & SQ2 & bone & 504 & 16.01 .2014 \\
\hline $902 / 2014$ & SQ2 & bone & 504 & 22.01 .2014 \\
\hline $910 / 2014$ & SQ2 & bone & 504 & 23.01 .2014 \\
\hline $600 / 2014$ & SQ2, sandy area in the $\mathrm{W}$ & bone & 504 & 12.01 .2014 \\
\hline $1038 / 2014$ & SQ1 & bone & 507 & 28.01 .2014 \\
\hline $752 / 2014$ & SQ1, 0-1m E-W/0-2m S-N & bone & 507 & 23.01 .2014 \\
\hline $740 / 2014$ & SQ1, 0-2m S-N/0-4m E-W & bone & 507 & 21.01 .2014 \\
\hline $745 / 2014$ & SQ1, 0-2m S-N/0-4m E-W & bone & 507 & 22.01 .2014 \\
\hline $1046 / 2014$ & $\mathrm{SQ} 1,1.2-2.3 \mathrm{~m}$ E-W/0-5m S-N & bone & 507 & 28.01 .2014 \\
\hline $1058 / 2014$ & $\mathrm{SQ} 1,2.30-4.1 \mathrm{~m}$ E-W/0-5m S-N & bone & 507 & 28.01 .2014 \\
\hline $1065 / 2014$ & $\mathrm{SQ} 1,2.30-4.1 \mathrm{~m} \mathrm{E-W} / 0-5 \mathrm{~m} \mathrm{~S}-\mathrm{N}$ & bone & 507 & 29.01 .2014 \\
\hline
\end{tabular}




\begin{tabular}{|c|c|c|c|c|}
\hline $1084 / 2014$ & SQ1, 4-5m E-W/0-5 S-m N & bone & 507 & 29.01 .2014 \\
\hline $1092 / 2014$ & SQ1, 4-5m E-W/0-5m S-N & bone & 507 & 30.01 .2014 \\
\hline $621 / 2014$ & SQ1, NE-corner, 0-4m E-W/0-5m N-S & bone & 507 & 13.01.2014 \\
\hline $607 / 2014$ & SQ2, NE of SQ2 & bone & 508 & 12.01 .2014 \\
\hline $616 / 2014$ & $\mathrm{SQ} 2,0-5 \mathrm{~m} \mathrm{E}-\mathrm{W} / 2.5-5 \mathrm{~m} \mathrm{~S}-\mathrm{N}$ & bone & 508 & 13.01.2014 \\
\hline $632 / 2014$ & SQ2 & bone & 510 & 14.01 .2014 \\
\hline $644 / 2014$ & SQ1, NW-corner, 1-5m W-E/3-5m N-S & bone & 511 & 14.01.2014 \\
\hline $1147 / 2014$ & SQ1 & bone & 512 & 02.02 .2014 \\
\hline $1167 / 2014$ & SQ1 & bone & 512 & 03.02 .2014 \\
\hline $641 / 2014$ & SQ1, SW-corner, 0-5m W-E/0-2m S-N & bone & 513 & 14.01 .2014 \\
\hline $647 / 2014$ & SQ1, SW-corner, 2-5m S-N/0-5m W-E & bone & 513 & 14.01 .2014 \\
\hline $808 / 2014$ & SQ2 & bone & 514 & 15.01 .2014 \\
\hline $827 / 2014$ & SQ2 & bone & 514 & 16.01 .2014 \\
\hline $817 / 2014$ & SQ2 & bone & 516 & 15.01 .2014 \\
\hline $823 / 2014$ & SQ2 & bone & 516 & 16.01 .2014 \\
\hline $815 / 2014$ & SQ2 & bone & 517 & 15.01 .2014 \\
\hline $813 / 2014$ & SQ2 & bone & 518 & 15.01 .2014 \\
\hline $840 / 2014$ & SQ1W & bone & 521 & 20.01 .2014 \\
\hline $710 / 2014$ & SQ1W, E-half & bone & 521 & 19.01 .2014 \\
\hline $860 / 2014$ & SQ1W & bone & 522 & 21.01 .2014 \\
\hline $887 / 2014$ & SQ1W & bone & 522 & 22.01 .2014 \\
\hline $836 / 2014$ & $\begin{array}{l}\text { SQ1W, } \\
5-10 \mathrm{~m} \mathrm{E}-\mathrm{W}\end{array}$ & bone; shell (1 piece) & 522 & 20.01 .2014 \\
\hline $767 / 2014$ & SQ1W, W-half & bone & 522 & 25.01 .2014 \\
\hline $779 / 2014$ & SQ1W, W-half & bone & 522 & 25.01 .2014 \\
\hline $784 / 2014$ & SQ1W, W-half & bone & 522 & 26.01 .2014 \\
\hline $905 / 2014$ & SQ1W, W-half & bone & 522 & 23.01 .2014 \\
\hline $864 / 2014$ & SQ1W & bone & 523 & 21.01 .2014 \\
\hline $853 / 2014$ & SQ1W & bone & 524 & 20.01 .2014 \\
\hline $871 / 2014$ & SQ1W & bone & 524 & 21.01 .2014 \\
\hline $877 / 2014$ & SQ1W & bone & 527 & 21.01 .2014 \\
\hline $881 / 2014$ & $\begin{array}{l}\text { SQ1W, } \\
\text { surface cleaning }\end{array}$ & bone & 529 & 21.01 .2014 \\
\hline
\end{tabular}




\begin{tabular}{|c|c|c|c|c|}
\hline $1023 / 2014$ & SQ1 & bone & 530 & 27.01 .2014 \\
\hline $1051 / 2014$ & SQ1 & bone & 530 & 28.01 .2014 \\
\hline $782 / 2014$ & $\mathrm{SQ} 1,0-4 \mathrm{~m} \mathrm{~S}-\mathrm{N} / 5-10 \mathrm{~m} \mathrm{E}-\mathrm{W}$ & bone & 530 & 26.01.2014 \\
\hline $891 / 2014$ & SQ1W & bone & 530 & 22.01 .2014 \\
\hline $786 / 2014$ & $\mathrm{SQ} 1,0-4 \mathrm{~m} \mathrm{~N}-\mathrm{S} / 0-5 \mathrm{~m} \mathrm{E}-\mathrm{W}$ & bone & 533 & 26.01.2014 \\
\hline $760 / 2014$ & SQ1, 0-5m W-E/0-5m N-S & bone & 533 & 23.01 .2014 \\
\hline $915 / 2014$ & SQ1W & bone & 534 & 23.01 .2014 \\
\hline $1029 / 2014$ & $\mathrm{SQ} 1,4-6 \mathrm{~m} \mathrm{~N}-\mathrm{S} / 5-10 \mathrm{~m} \mathrm{E}-\mathrm{W}$ & bone & 536 & 27.01 .2014 \\
\hline $765 / 2014$ & SQ1, 5-10m E-W/5-7m N-S & bone & 536 & 25.01 .2014 \\
\hline $764 / 2014$ & SQ1, 0-3m E-W/0-5m N-S & bone & 537 & 25.01 .2014 \\
\hline $1011 / 2014$ & SQ1, 0-3m E-W/0-5m N-S & bone & 537 & 27.01 .2014 \\
\hline $776 / 2014$ & SQ1, 3-5m E-W/0-5m N-S & bone & 538 & 25.01 .2014 \\
\hline $796 / 2014$ & SQ1, 3-5m E-W/0-5m N-S & bone & 538 & 26.01 .2014 \\
\hline $1010 / 2014$ & SQ1, 3-5m E-W/0-5m N-S & bone & 538 & 27.01 .2014 \\
\hline $799 / 2014$ & SQ1W & bone & 539 & 26.01 .2014 \\
\hline $1006 / 2014$ & SQ1W & bone & 540 & 26.01 .2014 \\
\hline $1016 / 2014$ & SQ1W & bone & 540 & 27.01.2014 \\
\hline $1053 / 2014$ & SQ1W & bone & 541 & 28.01 .2014 \\
\hline $1067 / 2014$ & SQ1W & bone & 541 & 29.01 .2014 \\
\hline $1033 / 2014$ & SQ1W & bone & 542 & 27.01 .2014 \\
\hline $1048 / 2014$ & SQ1W & bone & 543 & 28.01 .2014 \\
\hline $1061 / 2014$ & SQ1W & bone & 544 & 28.01 .2014 \\
\hline $1094 / 2014$ & SQ1NW & bone & 545 & 30.01 .2014 \\
\hline $1151 / 2014$ & SQ1NW & bone & 545 & 02.02 .2014 \\
\hline $1162 / 2014$ & SQ1NW, 5-6.5m S-N/0-2m E-W & bone & 545 & 02.02 .2014 \\
\hline $1115 / 2014$ & SQ1NW, 5-7m S-N & bone & 545 & 30.01 .2014 \\
\hline $1096 / 2014$ & SQ1W & bone & 546 & 30.01 .2014 \\
\hline $1107 / 2014$ & SQ1, 4-7m S-N & bone & 547 & 30.01 .2014 \\
\hline $1123 / 2014$ & SQ1 & bone & 548 & 01.02 .2014 \\
\hline $1125 / 2014$ & SQ1 & bone & 549 & 01.02 .2014 \\
\hline $1141 / 2014$ & SQ1 & bone & 550 & 01.02 .2014 \\
\hline
\end{tabular}




\begin{tabular}{|c|c|c|c|c|}
\hline $1179 / 2014$ & SQ1 & bone & 551 & 03.02 .2014 \\
\hline $1185 / 2014$ & SQ1NW & bone & 552 & 03.02 .2014 \\
\hline $1194 / 2014$ & SQ1W & bone & 552 & 04.02 .2014 \\
\hline $1190 / 2014$ & SQ1W & bone & 554 & 03.02 .2014 \\
\hline $1196 / 2014$ & SQ1 & bone & 555 & 04.02 .2014 \\
\hline $1211 / 2014$ & SQ1 & bone & 555 & 04.02 .2014 \\
\hline $1224 / 2014$ & SQ1 & bone & 555 & 05.02 .2014 \\
\hline $1257 / 2014$ & SQ1 & bone & 556 & 06.02 .2014 \\
\hline $1215 \mathrm{~A} / 2014$ & SQ1W & bone & 557 & 04.02 .2014 \\
\hline $1226 / 2014$ & SQ1 & bone & 559 & 05.02 .2014 \\
\hline $1228 / 2014$ & SQ1 & bone & 560 & 05.02 .2014 \\
\hline $1246 / 2014$ & SQ1W & bone & 561 & 05.02 .2014 \\
\hline $1248 / 2014$ & SQ1 & bone & 563 & 05.02 .2014 \\
\hline $1255 / 2014$ & SQ1NW & bone & 564 & 06.02 .2014 \\
\hline $1259 / 2014$ & SQ1 & bone & 565 & 06.02 .2014 \\
\hline $1283 / 2014$ & SQ1 & bone & 567 & 08.02 .2014 \\
\hline $1285 / 2014$ & SQ1 & bone & 568 & 08.02.2014 \\
\hline $1293 / 2014$ & SQ1 & bone & 569 & 08.02 .2014 \\
\hline $1298 / 2014$ & $\mathrm{SQ} 1,8.5 \mathrm{~m} \mathrm{~S}-\mathrm{N} / 5.1 \mathrm{~m} \mathrm{E-W}$ & bone (human remains) & 569 & 08.02 .2014 \\
\hline $1303 / 2014$ & SQ1 & bone & 570 & 08.02 .2014 \\
\hline $1315 / 2014$ & SQ1 & bone & 571 & 08.02 .2014 \\
\hline $1321 / 2014$ & SQ1, 0-4m S-N/3-5m E-W & bone & 572 & 09.02.2014 \\
\hline $918 / 2014$ & SQ2, cleaning square & bone & 573 & 10.02 .2014 \\
\hline $920 / 2014$ & SQ2 & bone & 574 & 10.02 .2014 \\
\hline $923 / 2014$ & SQ2 & bone & 575 & 10.02 .2014 \\
\hline $949 / 2014$ & SQ2 & bone & 576 & 11.02 .2014 \\
\hline $934 / 2014$ & SQ2 & bones ( 3 pieces) & 576 & 10.02 .2014 \\
\hline $954 / 2014$ & SQ2 & bone & 577 & 11.02 .2014 \\
\hline $939 / 2014$ & SQ2 & bone & 578 & 10.02 .2014 \\
\hline $942 / 2014$ & SQ2 & bone & 578 & 11.02 .2014 \\
\hline $945 / 2014$ & SQ2 & bone & 579 & 11.02 .2014 \\
\hline
\end{tabular}




\begin{tabular}{|c|c|c|c|c|}
\hline $966 / 2014$ & SQ2 & bone & 580 & 11.02 .2014 \\
\hline $1332 / 2014$ & SQ1 & bone & 584 & 11.02 .2014 \\
\hline $1343 / 2014$ & SQ1 & bone & 584 & 12.02 .2014 \\
\hline $1338 / 2014$ & SQ1 & bone & 585 & 12.02 .2014 \\
\hline $968 / 2014$ & SQ2 & bone & 586 & 12.02 .2014 \\
\hline $972 / 2014$ & SQ2 & bone & 586 & 13.02 .2014 \\
\hline $1353 / 2014$ & SQ1 & bone & 587 & 12.02 .2014 \\
\hline $1365 / 2014$ & SQ1 & bone & 587 & 13.02 .2014 \\
\hline $1355 / 2014$ & SQ1 & bone & 588 & 12.02 .2014 \\
\hline $1371 / 2014$ & SQ1 & bone & 588 & 13.02 .2014 \\
\hline $1360 / 2014$ & SQ1 & bone & 590 & 13.02 .2014 \\
\hline $1079 / 2014$ & SQ1NW 4-5m N-S/5-6m E-W & bone & 592 & 29.01 .2014 \\
\hline $734 / 2014$ & $\begin{array}{l}\text { SQ1, adjacent to Feature } 100 \text { from E, 3- } \\
5 \mathrm{~m} \mathrm{E-W/0-5m} \mathrm{S-N}\end{array}$ & botanical material (2 pieces, doum nuts and acacia) & 501 & 20.01.2014 \\
\hline $724 / 2014$ & $\begin{array}{l}\text { SQ1, adjacent to Feature } 100 \text { from E, 3- } \\
5 \mathrm{~m} \text { E-W/0-5m S-N }\end{array}$ & botanical material (4 pieces, doum nuts, wood and acacia) & 501 & 20.01 .2014 \\
\hline $707 / 2014$ & $\begin{array}{l}\text { SQ1, adjacent to Feature } 100 \text { from E, } 4 \\
5 \mathrm{~m} \text { E-W/0-5m S-N }\end{array}$ & botanical remains & 501 & 19.01 .2014 \\
\hline $1060 / 2014$ & SQ1, $2.30-4.1 \mathrm{~m} \mathrm{E-W/0-5m} \mathrm{S-N}$ & botanical material ( 3 pieces, doum nuts and 1 piece palm wood) & 507 & 28.01 .2014 \\
\hline $1177 / 2014$ & SQ1 & botanical material (3 pieces, palm wood and modern acacia) & 512 & 03.02 .2014 \\
\hline $1026 / 2014$ & SQ1 & botanical material (2 pieces, doum nuts and wood) & 530 & 27.01.2014 \\
\hline $761 / 2014$ & SQ1, 0-5m W-E/0-5m N-S & botanical material (2 pieces, doum nuts and endocarp) & 533 & 23.01 .2014 \\
\hline $1117 / 2014$ & SQ1NW, $0.5 \mathrm{~m} \mathrm{E-W/0.5m} \mathrm{N-S}$ & botanical material (5 pieces), leather (1 piece) & 545 & 30.01 .2014 \\
\hline $518 / 2014$ & SQ1 & charcoal (1 bag) & 501 & 06.01 .2014 \\
\hline $556 / 2014$ & SQ1 & charcoal (1 bag) & 501 & 08.01 .2014 \\
\hline $568 / 2014$ & SQ1 & charcoal (1 bag) & 501 & 09.01 .2014 \\
\hline $589 / 2014$ & SQ1 & charcoal (1 bag) & 501 & 11.01 .2014 \\
\hline $737 / 2014$ & $\begin{array}{l}\text { SQ1, adjacent to Feature } 100 \text { from E, 3- } \\
5 \mathrm{~m} \text { E-W/0-4m S-N }\end{array}$ & charcoal (1 bag) & 501 & 21.01 .2014 \\
\hline $722 / 2014$ & $\begin{array}{l}\text { SQ1, adjacent to Feature } 100 \text { from } \mathrm{E}, 3- \\
5 \mathrm{~m} \mathrm{E}-\mathrm{W} / 0-5 \mathrm{~m} \text { S-N }\end{array}$ & charcoal (1 bag) & 501 & 20.01 .2014 \\
\hline $733 / 2014$ & $\begin{array}{l}\text { SQ1, adjacent to Feature } 100 \text { from E, 3- } \\
\text { 5m E-W/0-5m S-N }\end{array}$ & charcoal (1 bag) & 501 & 20.01 .2014 \\
\hline $706 / 2014$ & $\begin{array}{l}\text { SQ1, adjacent to Feature } 100 \text { from E, } 4 \\
5 \mathrm{~m} \text { E-W/0-5m S-N }\end{array}$ & charcoal (1 bag) & 501 & 19.01.2014 \\
\hline $667 / 2014$ & SQ1, NE-corner, 3-5m E-W/0-5m N-S & charcoal (1 bag) & 501 & 15.01 .2014 \\
\hline
\end{tabular}




\begin{tabular}{|c|c|c|c|c|}
\hline $670 / 2014$ & SQ1, NE-corner, 3-5m E-W/0-5m N-S & charcoal (1 bag) & 501 & 15.01 .2014 \\
\hline $673 / 2014$ & SQ1, NW-corner, 0-3m W-E/0-2m N-S & charcoal (1 bag) & 501 & 15.01.2014 \\
\hline $681 / 2014$ & SQ1, SE-corner, 3-5m E-W/0-5m S-N & charcoal (1 bag) & 501 & 16.01.2014 \\
\hline $688 / 2014$ & SQ1, SE-corner, 4-5m E-W/0-5m S-N & charcoal (1 bag) & 501 & 18.01 .2014 \\
\hline $1072 / 2014$ & SQ1NW & charcoal (1 bag) & 501 & 29.01.2014 \\
\hline $1069 / 2014$ & SQ1NW & charcoal (1 piece); bone & 501 & 29.01 .2014 \\
\hline $678 / 2014$ & SQ1W, E-half & charcoal (1 bag) & 501 & 16.01 .2014 \\
\hline $692 / 2014$ & SQ1W, E-half, $5 \times 5 \mathrm{~m}$ & charcoal (1 bag) & 501 & 18.01 .2014 \\
\hline $695 / 2014$ & SQ1W, W-half, $5 \times 5 \mathrm{~m}$ & charcoal (1 bag) & 501 & 18.01.2014 \\
\hline $714 / 2014$ & SQ1W, W-half, $5 \times 5 \mathrm{~m}$ & charcoal (1 bag) & 501 & 19.01.2014 \\
\hline $509 / 2014$ & SQ2 & charcoal (1 bag) & 501 & 05.01 .2014 \\
\hline $514 / 2014$ & SQ2 & charcoal (1 bag) & 501 & 06.01 .2014 \\
\hline $536 / 2014$ & SQ2 & charcoal (1 piece) & 501 & 07.01 .2014 \\
\hline $553 / 2014$ & SQ2 & charcoal (1 piece) & 501 & 08.01 .2014 \\
\hline $624 / 2014$ & SQ1, NE-corner, 1-5m W-E/3-5m N-S & charcoal & 502 & 13.01 .2014 \\
\hline $543 / 2014$ & SQ1 & charcoal (1 bag) & 503 & 07.01 .2014 \\
\hline $542 / 2014$ & SQ1 & charcoal (1 piece); bone & 503 & 07.01 .2014 \\
\hline $753 / 2014$ & SQ1, 0-1m E-W/0-2m S-N & charcoal (1 bag) & 507 & 23.01 .2014 \\
\hline $1040 / 2014$ & SQ1, 0-1m E-W/0-5m S-N & charcoal (1 bag) & 507 & 28.01 .2014 \\
\hline $742 / 2014$ & SQ1, 0-2m S-N/0-4m E-W & charcoal (1 bag) & 507 & 21.01 .2014 \\
\hline $744 / 2014$ & SQ1, 0-2m S-N/0-4m E-W & charcoal (1 bag) & 507 & 22.01 .2014 \\
\hline $1055 / 2014$ & $\mathrm{SQ} 1,1.2-2.3 \mathrm{~m} \mathrm{E}-\mathrm{W} / 0-5 \mathrm{~m} \mathrm{~S}-\mathrm{N}$ & charcoal (1 bag) & 507 & 28.01 .2014 \\
\hline $1070 / 2014$ & SQ1, 2.30-4.1m E-W/0-5m S-N & charcoal (1 bag) & 507 & 29.01 .2014 \\
\hline $1059 / 2014$ & SQ1, 2.30-4.1m E-W/0-5m S-N & charcoal (1 piece) & 507 & 28.01 .2014 \\
\hline $1085 / 2014$ & SQ1, 4-5m E-W/0-5m S-N & charcoal (1 bag) & 507 & 29.01 .2014 \\
\hline $1102 / 2014$ & SQ1, 4-5m E-W/0-5m S-N & charcoal (1 bag) & 507 & 30.01 .2014 \\
\hline $601 / 2014$ & SQ1, E-half & charcoal (1 bag) & 507 & 12.01.2014 \\
\hline $622 / 2014$ & SQ1, NE-corner, 0-4m E-W/0-5m N-S & charcoal (1 bag) & 507 & 13.01 .2014 \\
\hline $620 / 2014$ & SQ2 & charcoal (1 bag) & 508 & 13.01 .2014 \\
\hline $652 / 2014$ & SQ2 & charcoal (1 bag) & 510 & 14.01 .2014 \\
\hline $637 / 2014$ & SQ2 & charcoal (1 piece) & 510 & 14.01 .2014 \\
\hline
\end{tabular}




\begin{tabular}{|c|c|c|c|c|}
\hline $645 / 2014$ & SQ1, NW-corner, 1-5m W-E/3-5m N-S & charcoal (1 bag) & 511 & 14.01 .2014 \\
\hline $1153 / 2014$ & SQ1 & charcoal (1 bag) & 512 & 02.02 .2014 \\
\hline $1174 / 2014$ & SQ1 & charcoal (1 bag) & 512 & 03.02 .2014 \\
\hline $649 / 2014$ & SQ1, SW-corner, 0-2m S-N/0-5m W-E & charcoal & 513 & 14.01 .2014 \\
\hline $648 / 2014$ & SQ1, SW-corner, 2-5m S-N/0-5m W-E & charcoal & 513 & 14.01.2014 \\
\hline $834 / 2014$ & SQ2 & charcoal (4 pieces) & 518 & 16.01 .2014 \\
\hline $842 / 2014$ & SQ1W & charcoal (1 bag) & 521 & 20.01 .2014 \\
\hline $709 / 2014$ & SQ1W, E-half, $5 \times 5 \mathrm{~m}$ & charcoal (1 bag) & 521 & 19.01.2014 \\
\hline $838 / 2014$ & SQ1W & charcoal (1 bag) & 522 & 20.01.2014 \\
\hline $862 / 2014$ & SQ1W & charcoal (1 bag) & 522 & 21.01 .2014 \\
\hline $889 / 2014$ & SQ1W & charcoal (1 bag) & 522 & 22.01 .2014 \\
\hline $794 / 2014$ & SQ1W, bottom surface & charcoal (1 bag) & 522 & 26.01 .2014 \\
\hline $770 / 2014$ & SQ1W, W-half & charcoal (1 bag) & 522 & 25.01 .2014 \\
\hline $908 / 2014$ & SQ1W, W-half & charcoal (1 bag); leather (1 bag) & 522 & 23.01 .2014 \\
\hline $780 / 2014$ & SQ1W, W-half, bottom surface of SU & charcoal (1 bag) & 522 & 25.01 .2014 \\
\hline $787 / 2014$ & SQ1W, W-half, bottom surface of SU & charcoal (1 bag) & 522 & 26.01 .2014 \\
\hline $850 / 2014$ & SQ1W & charcoal (1 bag) & 523 & 20.01 .2014 \\
\hline $866 / 2014$ & SQ1W & charcoal (1 bag) & 523 & 21.01 .2014 \\
\hline $872 / 2014$ & SQ1W & charcoal (1 bag) & 524 & 21.01 .2014 \\
\hline $857 / 2014$ & SQ1W & charcoal (5 pieces) & 524 & 20.01 .2014 \\
\hline $1081 / 2014$ & $\mathrm{SQ} 1,4-4.5 \mathrm{~m} \mathrm{~N}-\mathrm{S} / 4.5-5 \mathrm{~m} \mathrm{E}-\mathrm{W}$ & charcoal (1 bag); bone & 526 & 29.01 .2014 \\
\hline $885 / 2014$ & SQ1W & charcoal (1 bag) & 527 & 21.01 .2014 \\
\hline $882 / 2014$ & SQ1W & charcoal & 529 & 21.01.2014 \\
\hline $1024 / 2014$ & SQ1 & charcoal (1 bag) & 530 & 27.01 .2014 \\
\hline $893 / 2014$ & SQ1W & charcoal & 531 & 22.01 .2014 \\
\hline $900 / 2014$ & SQ1W & charcoal (1 bag) & 532 & 22.01 .2014 \\
\hline $789 / 2014$ & $\mathrm{SQ} 1,0-4 \mathrm{~m} \mathrm{~N}-\mathrm{S} / 0-5 \mathrm{~m}$ E-W & charcoal (1 bag) & 533 & 26.01 .2014 \\
\hline $1030 / 2014$ & $\mathrm{SQ} 1,4-6 \mathrm{~m} \mathrm{~N}-\mathrm{S} / 5-10 \mathrm{~m} \mathrm{E}-\mathrm{W}$ & charcoal (1 bag) & 536 & 27.01 .2014 \\
\hline $769 / 2014$ & $\mathrm{SQ} 1,5-10 \mathrm{~m} \mathrm{E}-\mathrm{W} / 5-7 \mathrm{~m} \mathrm{~N}-\mathrm{S}$ & charcoal (1 bag) & 536 & 25.01 .2014 \\
\hline $768 / 2014$ & $\mathrm{SQ} 1,0-3 \mathrm{~m} \mathrm{E}-\mathrm{W} / 0-5 \mathrm{~m} \mathrm{~N}-\mathrm{S}$ & charcoal (1 bag) & 537 & 25.01 .2014 \\
\hline $1014 / 2014$ & $\mathrm{SQ} 1,0-3 \mathrm{~m} \mathrm{E}-\mathrm{W} / 0-5 \mathrm{~m} \mathrm{~N}-\mathrm{S}$ & charcoal (1 bag) & 537 & 27.01 .2014 \\
\hline
\end{tabular}




\begin{tabular}{|c|c|c|c|c|}
\hline $1015 / 2014$ & SQ1, 3-5m E-W/0-5m N-S & charcoal (1 bag) & 538 & 27.01 .2014 \\
\hline $1004 / 2014$ & SQ1, 3-5m E-W/0-5m N-S & charcoal (2 pieces) & 538 & 26.01 .2014 \\
\hline $1000 / 2014$ & SQ1W & charcoal (1 bag) & 539 & 26.01 .2014 \\
\hline $1017 / 2014$ & SQ1W & charcoal (1 bag) & 540 & 27.01 .2014 \\
\hline $1073 / 2014$ & SQ1W & charcoal (1 bag) & 541 & 29.01 .2014 \\
\hline $1099 / 2014$ & SQ1NW & charcoal (1 bag) & 545 & 30.01 .2014 \\
\hline $1159 / 2014$ & SQ1NW & charcoal (3 pieces) & 545 & 02.02 .2014 \\
\hline $1087 / 2014$ & SQ1NW 4-5m N-S/5-6m E-W & charcoal (1 bag) & 545 & 29.01 .2014 \\
\hline $1116 / 2014$ & $\begin{array}{l}\text { SQ1NW, } \\
5-7 \mathrm{~m} \text { S-N }\end{array}$ & charcoal (1 bag) & 545 & 30.01 .2014 \\
\hline $1132 / 2014$ & SQ1 & charcoal (1 bag) & 547 & 01.02 .2014 \\
\hline $1154 / 2014$ & SQ1 & charcoal (1 bag) & 547 & 02.02 .2014 \\
\hline $1172 / 2014$ & SQ1 & charcoal (1 bag) & 547 & 03.02 .2014 \\
\hline $1149 / 2014$ & SQ1 & charcoal (1 piece); bone & 547 & 02.02 .2014 \\
\hline $1169 / 2014$ & SQ1 & charcoal (1 piece); bone & 547 & 03.02 .2014 \\
\hline $1109 / 2014$ & SQ1, 4-7m S-N & charcoal (1 bag) & 547 & 30.01 .2014 \\
\hline $1135 / 2014$ & SQ1 & charcoal (1 bag) & 548 & 01.02 .2014 \\
\hline $1137 / 2014$ & SQ1 & charcoal (1 bag) & 549 & 01.02 .2014 \\
\hline $1142 / 2014$ & SQ1 & charcoal (1 bag) & 550 & 01.02 .2014 \\
\hline $1180 / 2014$ & SQ1 & charcoal (1 bag) & 551 & 03.02 .2014 \\
\hline $1186 / 2014$ & SQ1NW & charcoal (1 bag) & 552 & 03.02 .2014 \\
\hline $1201 / 2014$ & SQ1NW + W & charcoal (1 bag) & 552 & 04.02 .2014 \\
\hline $1191 / 2014$ & SQ1W & charcoal (1 bag) & 554 & 03.02 .2014 \\
\hline $1207 / 2014$ & SQ1 & charcoal (1 bag) & 555 & 04.02 .2014 \\
\hline $1215 \mathrm{~B} / 2014$ & SQ1 & charcoal (1 bag) & 555 & 04.02 .2014 \\
\hline $1229 / 2014$ & SQ1 & charcoal (4 pieces) & 555 & 05.02 .2014 \\
\hline $1274 / 2014$ & SQ1 & charcoal (1 bag) & 556 & 06.02 .2014 \\
\hline $1222 / 2014$ & SQ1W & charcoal (1 bag) & 557 & 04.02 .2014 \\
\hline $1218 / 2014$ & SQ1NW & charcoal (1 bag) & 558 & 04.02 .2014 \\
\hline $1234 / 2014$ & SQ1 & charcoal (1 bag) & 559 & 05.02 .2014 \\
\hline $1232 / 2014$ & SQ1 & charcoal (1 bag) & 560 & 05.02 .2014 \\
\hline $1245 / 2014$ & SQ1W & charcoal & 561 & 05.02 .2014 \\
\hline
\end{tabular}




\begin{tabular}{|c|c|c|c|c|c|}
\hline $1249 / 2014$ & SQ1 & charcoal (1 bag) & 563 & & 05.02 .2014 \\
\hline $1264 / 2014$ & SQ1NW & charcoal (1 bag) & 564 & & 06.02 .2014 \\
\hline $1261 / 2014$ & SQ1 & charcoal (1 bag) & 565 & & 06.02.2014 \\
\hline $1288 / 2014$ & SQ1 & charcoal (1 bag) & 567 & & 08.02.2014 \\
\hline $1289 / 2014$ & SQ1 & charcoal (1 bag) & 568 & & 08.02 .2014 \\
\hline $1294 / 2014$ & SQ1 & charcoal (1 bag) & 569 & & 08.02 .2014 \\
\hline $1305 / 2014$ & SQ1 & charcoal (1 bag) & 570 & & 08.02 .2014 \\
\hline $1322 / 2014$ & SQ1 & charcoal (1 bag) & 572 & & 09.02 .2014 \\
\hline $1319 / 2014$ & SQ1 & charcoal (1 piece); bone & 572 & & 09.02 .2014 \\
\hline $1329 / 2014$ & SQ1, 0-4m S-N/3-5m E-W & charcoal (1 bag) & 572 & & 09.02 .2014 \\
\hline $929 / 2014$ & SQ2 & charcoal (5 pieces) & 574 & & 10.02 .2014 \\
\hline $935 / 2014$ & SQ2 & charcoal (1 piece) & 575 & & 10.02 .2014 \\
\hline $950 / 2014$ & SQ2 & charcoal (1 bag) & 577 & & 11.02 .2014 \\
\hline $952 / 2014$ & SQ2 & charcoal (3 pieces) & 578 & & 11.02 .2014 \\
\hline $946 / 2014$ & SQ2 & charcoal (1 piece) & 579 & & 11.02 .2014 \\
\hline $1336 / 2014$ & SQ1 & charcoal (1 bag) & 584 & & 12.02 .2014 \\
\hline $1339 / 2014$ & SQ1 & charcoal (1 bag) & 585 & & 12.02 .2014 \\
\hline $1352 / 2014$ & SQ1 & charcoal (1 bag) & 587 & & 12.02 .2014 \\
\hline $1364 / 2014$ & SQ1 & charcoal (1 bag) & 587 & & 13.02 .2014 \\
\hline $1370 / 2014$ & SQ1 & charcoal (1 bag) & 588 & & 13.02.2014 \\
\hline $1361 / 2014$ & SQ1 & charcoal (1 bag) & 590 & & 13.02 .2014 \\
\hline $658 / 2014$ & SQ2 & clay & 516 & & 14.01 .2014 \\
\hline $873 / 2014$ & SQ1W & coprolite & 524 & & 21.01 .2014 \\
\hline $1202 / 2014$ & SQ1NW + W & coprolite & 552 & & 04.02 .2014 \\
\hline $704 / 2014$ & $\begin{array}{l}\text { SQ1, adjacent to Feature } 100 \text { from E, } 4 \\
5 \mathrm{~m} \mathrm{E}-\mathrm{W} / 0-5 \mathrm{~m} \text { S-N }\end{array}$ & $\begin{array}{l}\text { crucible? ( } 1 \text { fragment, clay), architecture/installation? } \\
\text { (1 fragment, clay), pottery }\end{array}$ & 501 & $\begin{array}{l}\text { SAV1W } 0204 \text { (crucible?), SAV1W } 0206 \\
\text { (architecture/ installation?) }\end{array}$ & 19.01 .2014 \\
\hline $1213 / 2014$ & SQ1NW & crucible? (1 fragment, clay), pottery & 558 & & 04.02 .2014 \\
\hline $1265 / 2014$ & $\mathrm{SQ} 1,1,4 \mathrm{~m} \mathrm{~N}-\mathrm{S} / 2.2 \mathrm{~m} \mathrm{E}-\mathrm{W}$ & crucible? (2 fragments, mud with copper?) & 565 & SAV1W 0572 & 06.02.2014 \\
\hline $1284 / 2014$ & SQ1 & crucibles? (2 pieces, each mud), pottery & 568 & SAV1W 0525, SAV1W 0526 (crucibles?) & 08.02 .2014 \\
\hline $630 / 2014$ & SQ2 & dates & 508 & & 14.01.2014 \\
\hline $674 / 2014$ & SQ1, NW-corner, 0-3m W-E/0-2m N-S & doum nuts ( 3 pieces) & 501 & & 15.01.2014 \\
\hline $684 / 2014$ & SQ1, SE-corner, 3-5m E-W/0-5 S-N & doum nuts ( 3 pieces) & 501 & & 16.01.2014 \\
\hline
\end{tabular}




\begin{tabular}{|c|c|c|c|c|c|}
\hline $626 / 2014$ & $\mathrm{SQ} 1,3 \mathrm{~m}$ from $\mathrm{W}, 3 \mathrm{~m}$ from $\mathrm{N}$ & doum nuts (several pieces) & 502 & & 13.01 .2014 \\
\hline $757 / 2014$ & $\mathrm{SQ} 1,0-1 \mathrm{~m}$ E-W/0-2m S-N & doum nut (1 piece) & 507 & & 23.01 .2014 \\
\hline $1042 / 2014$ & SQ1, 0-1m E-W/0-5m S-N & doum nut (1 piece) & 507 & & 28.01 .2014 \\
\hline $746 / 2014$ & SQ1, 0-2m S-N/0-4m E-W & doum nuts and wood (4 pieces) & 507 & & 22.01 .2014 \\
\hline $1076 / 2014$ & $\mathrm{SQ} 1,2.30-4.1 \mathrm{~m} \mathrm{E}-\mathrm{W} / 0-5 \mathrm{~m} \mathrm{~S}-\mathrm{N}$ & doum nut (1 piece) & 507 & & 29.01 .2014 \\
\hline $1112 / 2014$ & SQ1, 4-5m E-W/0-5m S-N & doum nut (1 piece) & 507 & & 30.01 .2014 \\
\hline $1160 / 2014$ & SQ1 & doum nuts (several pieces) & 512 & & 02.02 .2014 \\
\hline $1170 / 2014$ & SQ1 & doum nuts (5 pieces) & 512 & & 03.02 .2014 \\
\hline $856 / 2014$ & SQ1W & doum nut (1 piece) & 524 & & 20.01 .2014 \\
\hline $897 / 2014$ & SQ1W & doum nut (1 piece) & 530 & & 22.01 .2014 \\
\hline $1012 / 2014$ & $\mathrm{SQ} 1,0-3 \mathrm{~m}$ E-W/0-5m N-S & doum nuts (2 pieces) & 537 & & 27.01 .2014 \\
\hline $1152 / 2014$ & SQ1NW & doum nut (1 piece) & 545 & & 02.02 .2014 \\
\hline $1130 / 2014$ & SQ1 & doum nuts (3 pieces) & 548 & & 01.02 .2014 \\
\hline $1134 / 2014$ & SQ1 & doum nuts (4 pieces) & 549 & & 01.02 .2014 \\
\hline $1183 / 2014$ & SQ1 & doum nut (1 piece) & 551 & & 03.02 .2014 \\
\hline $1238 / 2014$ & SQ1 & doum nuts (2 pieces) & 555 & & 05.02 .2014 \\
\hline $1260 / 2014$ & SQ1 & doum nuts (several pieces) & 556 & & 06.02 .2014 \\
\hline $1267 / 2014$ & SQ1 & doum nuts (3 pieces) & 565 & & 06.02 .2014 \\
\hline $1296 / 2014$ & SQ1 & doum nuts (2 pieces) & 568 & & 08.02 .2014 \\
\hline $1295 / 2014$ & SQ1 & doum nuts (2 pieces) & 569 & & 08.02 .2014 \\
\hline $1307 / 2014$ & SQ1 & doum nuts (2 pieces) & 570 & & 08.02 .2014 \\
\hline $933 / 2014$ & SQ2 & doum nut (1 piece) & 574 & & 10.02 .2014 \\
\hline $1335 / 2014$ & SQ1 & doum nuts ( 2 pieces) & 585 & & 12.02 .2014 \\
\hline $544 / 2014$ & SQ2 & dummy brick/seal/plaque (1 part, clay) & 501 & SAV1W 0031 & 07.01 .2014 \\
\hline $937 / 2014$ & SQ2 & $\begin{array}{l}\text { dummy brick ( } 1 \text { complete piece, oval/cartouche shaped, } 18^{\text {th }} \\
\text { Dynasty, mud) }\end{array}$ & 574 & SAV1W 0532 & 10.02 .2014 \\
\hline $1164 / 2014$ & SQ1 & faience vessel ( 1 body sherd, Nun bowl) & 512 & SAV1W 0428 & 02.02 .2014 \\
\hline $1273 / 2014$ & SQ1 & faience vessel (1 body sherd, Nun bowl) & 556 & SAV1W 0529 & 06.02 .2014 \\
\hline $1287 / 2014$ & SQ1, 4.9m S-N/5.5m E-W & faience vessel ( 1 base sherd, Nun bowl) & 568 & SAV1W 0530 & 08.02 .2014 \\
\hline $1311 / 2014$ & SQ1 & $\begin{array}{l}\text { faience vessel/bowl? (1 fragment, with hole in the base - re- } \\
\text { used?) }\end{array}$ & 569 & SAV1W 0544 & 08.02 .2014 \\
\hline $803 / 2014$ & $\begin{array}{l}\text { SQ1W, 0-5m E-W/0-5m S-N, surface } \\
\text { cleaning }\end{array}$ & figurine (1 fragment, animal figurine, clay) & 500 & SAV1W 0164 & 15.01 .2014 \\
\hline
\end{tabular}




\begin{tabular}{|c|c|c|c|c|c|}
\hline $703 / 2014$ & $\begin{array}{l}\mathrm{SQ1}, \mathrm{E} \text { of Feature } 100,2 \mathrm{~m} \text { from } \mathrm{S} / 4 \mathrm{~m} \\
\text { from } \mathrm{E}\end{array}$ & figurine ( 1 fragment, animal figurine, clay) & 500 & SAV1W 0165 & 18.01 .2014 \\
\hline $756 / 2014$ & SQ1, 0-1m E-W/0-2m S-N & figurine (1 lower part of rudimentary female figurine, mud) & 507 & SAV1W 0257 & 23.01 .2014 \\
\hline $1340 / 2014$ & $\mathrm{SQ} 1,3.5 \mathrm{~m} \mathrm{~N}-\mathrm{S} / 1.8 \mathrm{~m} \mathrm{E}-\mathrm{W}$ & figurine (1 lower part of rudimentary female figurine, clay) & 585 & SAV1W 0555 & 12.02 .2014 \\
\hline $699 / 2014$ & $\mathrm{SQ1}$, SE-corner, $4 \mathrm{~m}$ from $\mathrm{E} / 3 \mathrm{~m}$ from $\mathrm{S}$ & finger ring ( 1 fragment, faience) & 501 & SAV1W 0160 & 18.01 .2014 \\
\hline $1022 / 2014$ & SQ1 & finger ring or arm ring ( 1 fragment, faience) & 530 & SAV1W 0357 & 27.01 .2014 \\
\hline $730 / 2014$ & $\begin{array}{l}\text { SQ1, adjacent to Feature } 100 \text { from E, 3- } \\
5 \mathrm{~m} \text { E-W/0-5m S-N }\end{array}$ & fossil or meteoritic material? graphite? (1 piece, sample) & 501 & & 20.01 .2014 \\
\hline $662 / 2014$ & $\mathrm{SQ} 1,3 \mathrm{~m}$ from $\mathrm{W}, 4 \mathrm{~m}$ from $\mathrm{N}$ & fruit? (1 piece) & 511 & & 14.01 .2014 \\
\hline $750 / 2014$ & $\mathrm{SQ} 1,2 \mathrm{~m}$ from $\mathrm{S} / 1 \mathrm{~m}$ from $\mathrm{E}$ & $\begin{array}{l}\text { furniture/installation/basin? (1 fragment, painted, Christian, } \\
\text { clay) }\end{array}$ & 507 & SAV1W 0268 & 22.01 .2014 \\
\hline $653 / 2014$ & $\mathrm{SQ} 2,3-5 \mathrm{~m} \mathrm{~S}-\mathrm{N} / 0-2 \mathrm{~m} \mathrm{E}-\mathrm{W}$ & glass vessel? (1 tiny fragment, modern?) & 510 & SAV1W 0184 & 14.01 .2014 \\
\hline $1113 / 2014$ & SQ1NW, 0.5m E-W/0.5m S-N & glass vessel (1 fragment) & 545 & SAV1W 0392 & 30.01 .2014 \\
\hline $1192 / 2014$ & SQ1NW & glass vessel ( 10 small fragments) & 552 & SAV1W 0427 & 03.02 .2014 \\
\hline $846 / 2014$ & SQ1W & hair, dung, date, charcoal & 521 & & 20.01 .2014 \\
\hline $847 / 2014$ & $\mathrm{SQ} 1 \mathrm{~W}, 6 \mathrm{~m}$ E-W/4.5m S-N & hair, other & 522 & & 20.01 .2014 \\
\hline $772 / 2014$ & $\mathrm{SQ1}, 3 \mathrm{~m}$ from $\mathrm{E} / 1 \mathrm{~m}$ from $\mathrm{N}$ & hair (1 piece) & 537 & & 25.01 .2014 \\
\hline $924 / 2014$ & SQ2 & hair (1 bag) & 574 & & 10.02 .2014 \\
\hline $956 / 2014$ & SQ2 & hair (1 piece) & 576 & & 11.02 .2014 \\
\hline $727 / 2014$ & $\begin{array}{l}\text { SQ1, adjacent to Feature } 100 \text { from E, 3- } \\
5 \mathrm{~m} \text { E-W/0-5m S-N }\end{array}$ & leather (1 piece) & 501 & & 20.01 .2014 \\
\hline $685 / 2014$ & SQ1, SE-corner, 3m from E/3m from $S$ & leather (several pieces); wood (several pieces) & 501 & & 16.01 .2014 \\
\hline $651 / 2014$ & SQ2 & leather, horn & 510 & & 14.01 .2014 \\
\hline $1155 / 2014$ & SQ1 & leather (several pieces) & 512 & & 02.02 .2014 \\
\hline $793 / 2014$ & SQ1W & leather (1 bag) & 522 & & 26.01 .2014 \\
\hline $868 / 2014$ & SQ1W & leather (several pieces) & 523 & & 21.01 .2014 \\
\hline $854 / 2014$ & SQ1W & leather (2 pieces) & 524 & & 20.01 .2014 \\
\hline $878 / 2014$ & SQ1W & leather (several pieces) & 527 & & 21.01 .2014 \\
\hline $1252 / 2014$ & SQ1W & leather (2 pieces) & 529 & & 05.02 .2014 \\
\hline $896 / 2014$ & SQ1W & leather (1 bag) & 530 & & 22.01 .2014 \\
\hline $774 / 2014$ & $\mathrm{SQ} 1,5-10 \mathrm{~m} \mathrm{E}-\mathrm{W} / 5-7 \mathrm{~m} \mathrm{~N}-\mathrm{S}$ & leather & 536 & & 25.01 .2014 \\
\hline $1171 / 2014$ & SQ1 & leather (2 pieces) & 547 & & 03.02 .2014 \\
\hline $1204 / 2014$ & SQ1NW + W & leather (1 bag) & 552 & & 04.02 .2014 \\
\hline $1208 \mathrm{~B} /$ & SQ1 & leather (1 bag) & 555 & & 04.02 .2014 \\
\hline
\end{tabular}




\begin{tabular}{|c|c|c|c|c|c|}
\hline 2014 & & & & & \\
\hline $1328 / 2014$ & SQ1 & leather (1 bag) & 572 & & 09.02 .2014 \\
\hline $958 / 2014$ & SQ2 & leather (1 bag) & 578 & & 11.02 .2014 \\
\hline $675 / 2014$ & $\mathrm{SQ1}$, NE-corner, $3 \mathrm{~m}$ from $\mathrm{E}, 3 \mathrm{~m}$ from $\mathrm{N}$ & linen/cloth (1 piece) & 501 & SAV1W 0127 & 15.01.2014 \\
\hline $683 / 2014$ & $\mathrm{SQ} 1, \mathrm{SE}-$ corner, $3 \mathrm{~m}$ from $\mathrm{E} / 3 \mathrm{~m}$ from $\mathrm{S}$ & linen/cloth (1 piece) & 501 & SAV1W 0155 & 16.01.2014 \\
\hline $696 / 2014$ & SQ1W, E-half, $5 \times 5 \mathrm{~m}$ & linen or cloth (conglomerate with rope) & 501 & SAV1W 0158 & 18.01 .2014 \\
\hline $627 / 2014$ & $\mathrm{SQ1}, \mathrm{NW}$-corner, $3 \mathrm{~m}$ from $\mathrm{W}, 3 \mathrm{~m}$ from $\mathrm{N}$ & linen/cloth (1 piece), linked with rope? & 502 & SAV1W 0129 & 13.01.2014 \\
\hline $911 / 2014$ & SQ2, sandy area in the $\mathrm{W}$ & linen/cloth (several pieces) & 504 & SAV1W 0263 & 23.01.2014 \\
\hline $638 / 2014$ & SQ2 & linen/cloth (2 pieces) & 510 & SAV1W 0121 & 14.01.2014 \\
\hline $634 / 2014$ & SQ2 & linen/cloth ( 2 separate pieces) & 510 & SAV1W 0126 & 13.01.2014 \\
\hline $654 / 2014$ & $\mathrm{SQ} 2,3-5 \mathrm{~m} \mathrm{~S}-\mathrm{N} / 0-2 \mathrm{~m} \mathrm{E}-\mathrm{W}$ & linen/cloth (1 piece) & 510 & SAV1W 0125 & 14.01.2014 \\
\hline $664 / 2014$ & $\begin{array}{l}\text { SQ1, SW-corner, } \\
0.5 \mathrm{~m} \mathrm{~S}, 1 \mathrm{~m} \mathrm{E}\end{array}$ & linen/cloth (1 piece) & 513 & SAV1W 0128 & 14.01 .2014 \\
\hline $832 / 2014$ & SQ2 & linen/cloth (1 piece) & 514 & SAV1W 0157 & 16.01 .2014 \\
\hline $802 / 2014$ & SQ2 & linen/cloth (1 piece) & 516 & SAV1W 0123 & 15.01.2014 \\
\hline $824 / 2014$ & SQ2 & linen/cloth (1 piece) & 516 & SAV1W 0156 & 16.01.2014 \\
\hline $814 / 2014$ & SQ2 & linen or cloth (1 piece) & 518 & SAV1W 0124 & 15.01 .2014 \\
\hline $843 / 2014$ & SQ1W & linen/cloth with rope (several pieces) & 521 & SAV1W 0193 & 20.01 .2014 \\
\hline $711 / 2014$ & SQ1W, E-half & linen/cloth (1 piece) & 521 & SAV1W 0192 & 19.01.2014 \\
\hline $879 / 2014$ & SQ1W & $\begin{array}{l}\text { linen/cloth ( } 1 \text { piece), rope ( } 2 \text { pieces, organic fibre), slag and } \\
\text { brick ( } 2 \text { fragments) }\end{array}$ & 522 & $\begin{array}{l}\text { SAV1W } 0230 \text { (linen/cloth), SAV1W } 0231 \\
\text { (rope), SAV1W } 0259 \text { (slag and brick) }\end{array}$ & 21.01 .2014 \\
\hline $844 / 2014$ & SQ1W & linen/cloth? with bundle of hair ( 2 pieces) & 522 & SAV1W 0190 & 20.01 .2014 \\
\hline $851 \mathrm{~B} / 2014$ & SQ1W & linen/cloth with rope (several pieces) & 523 & SAV1W 0191 & 20.01 .2014 \\
\hline $855 / 2014$ & SQ1W & linen/cloth (2 pieces) & 524 & SAV1W 0189 & 20.01 .2014 \\
\hline $884 / 2014$ & SQ1W & linen/cloth with rope (several pieces) & 527 & SAV1W 0227 & 21.01 .2014 \\
\hline $894 / 2014$ & SQ1W & linen/cloth (several pieces); charcoal (1 bag) & 531 & SAV1W 0249 & 22.01 .2014 \\
\hline $913 / 2014$ & SQ1W & linen and leather? (several pieces) & 534 & SAV1W 0264 & 23.01.2014 \\
\hline $719 / 2014$ & $\begin{array}{l}\mathrm{SQ1} \text {, adjacent to Feature } 100 \text { from } \mathrm{E}, 4 \mathrm{~m} \\
\text { from } \mathrm{S} / 4 \mathrm{~m} \text { from } \mathrm{E}\end{array}$ & mud object (1 fragment, covered with yellow paint/pigment) & 501 & SAV1W 0565 & 19.01.2014 \\
\hline $577 / 2014$ & SQ1, at the E of Feature 100 & $\begin{array}{l}\text { Nun bowl (1 fragment, inner body sherd with painted } \\
\text { decoration, faience, see joint 749/2014) }\end{array}$ & 501 & SAV1W 0032 & 09.01 .2014 \\
\hline $749 / 2014$ & $\mathrm{SQ1}, 1 \mathrm{~m}$ from $\mathrm{S} / 1 \mathrm{~m}$ from $\mathrm{E}$ & $\begin{array}{l}\text { Nun bowl ( } 1 \text { fragment, inner body sherd with painted } \\
\text { decoration, faience, see joint } 577 / 2014 \text { ) }\end{array}$ & 507 & SAV1W 0032 & 22.01 .2014 \\
\hline $1243 / 2014$ & SQ1 & Nun bowl? (1 body sherd, faience) & 563 & SAV1W 0506 & 05.02 .2014 \\
\hline
\end{tabular}




\begin{tabular}{|c|c|c|c|c|c|}
\hline $587 / 2014$ & SQ1 & organic material (1 piece, unclear); bone & 501 & & 11.01 .2014 \\
\hline $825 / 2014$ & SQ2 & organic material & 516 & & 16.01 .2014 \\
\hline $914 / 2014$ & SQ1W & organic material & 534 & & 23.01 .2014 \\
\hline $1019 / 2014$ & SQ1W & organic material (4 pieces, leather, bone, dung) & 540 & & 27.01 .2014 \\
\hline $1208 \mathrm{~A} / 2014$ & SQ1 & organic material & 555 & & 04.02 .2014 \\
\hline $1221 / 2014$ & SQ1W & organic material ( 1 bag, mixed, textile and hair) & 557 & & 04.02 .2014 \\
\hline $1300 / 2014$ & SQ1 & organic material & 563 & & 08.02 .2014 \\
\hline $1324 / 2014$ & SQ1, 0-4m S-N/3-5m E-W & organic material & 572 & & 09.02 .2014 \\
\hline $927 / 2014$ & SQ2 & organic material & 575 & & 10.02 .2014 \\
\hline $1341 / 2014$ & SQ1 & organic material & 584 & & 12.02 .2014 \\
\hline $1366 / 2014$ & SQ1 & organic material & 587 & & 13.02 .2014 \\
\hline $1373 / 2014$ & SQ1 & organic material & 588 & & 13.02 .2014 \\
\hline $1376 / 2014$ & SQ1 & organic material & 590 & & 13.02 .2014 \\
\hline $1182 / 2014$ & $\mathrm{SQ} 1,3.50 \mathrm{~m}$ S-N/1.1m E-W & $\begin{array}{l}\text { ostracon (1 fragment, body sherd, both sides inscribed with } \\
\text { Greek letters, Old Nubian?) }\end{array}$ & 512 & SAV1W 0450 & 03.02 .2014 \\
\hline $602 / 2014$ & SQ1, E-half & palm wood (several pieces) & 507 & & 12.01 .2014 \\
\hline $810 / 2014$ & SQ2 & palm wood and charcoal (2 pieces) & 514 & & 15.01 .2014 \\
\hline $1009 / 2014$ & $\mathrm{SQ} 1,3-5 \mathrm{~m}$ E-W/0-5m N-S & palm wood (1 piece) & 538 & & 27.01 .2014 \\
\hline $1129 / 2014$ & SQ1, 7-10m S-N/6-10m E-W & palm wood (1 piece) & 547 & & 01.02 .2014 \\
\hline $1131 / 2014$ & SQ1 & palm wood (1 piece) & 549 & & 01.02 .2014 \\
\hline $1240 / 2014$ & SQ1 & palm wood ( 3 pieces) & 559 & & 05.02 .2014 \\
\hline $1327 / 2014$ & SQ1, 0-4m S-N/3-5m E-W & palm wood (1 bag) & 572 & & 09.02 .2014 \\
\hline $1344 / 2014$ & SQ1 & palm wood (4 pieces) & 584 & & 12.02 .2014 \\
\hline $1348 / 2014$ & SQ1 & palm wood ( 3 pieces) & 585 & & 12.02 .2014 \\
\hline $1351 / 2014$ & SQ1 & palm wood (1 piece) & 587 & & 12.02 .2014 \\
\hline $502 / 2014$ & SQ1, surface & plaster (1 bag); bone & 500 & & 05.01 .2014 \\
\hline $598 / 2014$ & SQ1, E-half & plaster (1 bag); bone & 507 & & 12.01 .2014 \\
\hline $1127 / 2014$ & SQ1, 7-10m S-N/6-10m E-W & plaster (1 piece) & 547 & & 01.02 .2014 \\
\hline $579 / 2014$ & $\begin{array}{l}\text { SQ1, on the surface, just next to the SE- } \\
\text { corner of SQ1 }\end{array}$ & pottery: 1 blue painted fragment with floral motives & surface & & 09.01 .2014 \\
\hline $500 / 2014$ & SQ1, surface, $10 \times 10 \mathrm{~m}$ & $\begin{array}{l}\text { pottery ( } 26 \text { baskets), among them lid ( } 1 \text { re-used sherd, pottery), } \\
\text { lid? ( } 1 \text { re-used sherd, pottery); slag and brick ( } 1 \text { fragment) }\end{array}$ & 500 & $\begin{array}{l}\text { SAV1W } 0011 \text { (lid), SAV1W } 0034 \text { (lid?), } \\
\text { SAV1W } 0002 \text { (slag) }\end{array}$ & 05.01 .2014 \\
\hline $1037 / 2014$ & SQ1NW & pottery & 500 & & 28.01 .2014 \\
\hline
\end{tabular}




\begin{tabular}{|c|c|c|c|c|c|}
\hline $503 / 2014$ & SQ2, surface & $\begin{array}{l}\text { pottery ( } 9 \text { baskets), among them slag ( } 2 \text { pieces), lids ( } 2 \text { pieces, } \\
\text { each re-used sherds, } 1 \text { of Nubian cooking pot pottery, } 1 \text { of } \\
\text { Christian pot), re-used sherd ( } 1 \text { piece, pottery), scraper }(1 \text { re- } \\
\left.\text { used } 18^{\text {th }} \text { Dynasty sherd, pottery), weight ( } 1 \text { re-used sherd }\right)\end{array}$ & 500 & $\begin{array}{l}\text { SAV1W } 0001 \text { (slag), SAV1W 0012, SAV1W } \\
0014 \text { (lids), SAV1W 0013 (weight), SAV1W } \\
0015 \text { (re-used sherd), SAV1W } 0017 \text { (scraper) }\end{array}$ & 05.01 .2014 \\
\hline $735 / 2014$ & $\begin{array}{l}\text { SQ1, adjacent to Feature } 100 \text { from E, 3- } \\
5 \mathrm{~m} \mathrm{E}-\mathrm{W} / 0-4 \mathrm{~m} \mathrm{~S}-\mathrm{N}\end{array}$ & pottery & 501 & & 21.01 .2014 \\
\hline $720 / 2014$ & $\begin{array}{l}\text { SQ1, adjacent to Feature } 100 \text { from E, 3- } \\
5 \mathrm{~m} \mathrm{E}-\mathrm{W} / 0-5 \mathrm{~m} \mathrm{~S}-\mathrm{N}\end{array}$ & pottery, among it net weight (1 piece, complete, pottery) & 501 & SAV1W 0232 & 20.01 .2014 \\
\hline $729 / 2014$ & $\begin{array}{l}\text { SQ1, adjacent to Feature } 100 \text { from E, 3- } \\
5 \mathrm{~m} \mathrm{E}-\mathrm{W} / 0-5 \mathrm{~m} \mathrm{~S}-\mathrm{N}\end{array}$ & $\begin{array}{l}\text { pottery, among it scrapers ( } 2 \text { pieces, each re-used } 18^{\text {th }} \text { Dynasty } \\
\text { sherds, pottery) }\end{array}$ & 501 & SAV1W 0233, SAV1W 0234 (scrapers) & 20.01 .2014 \\
\hline $560 / 2014$ & SQ1, E of Feature 100 & pottery & 501 & & 08.01 .2014 \\
\hline $565 / 2014$ & SQ1, E of Feature 100 & pottery, among it re-used sherd (1 piece, Christian, pottery) & 501 & SAV1W 0024 & 09.01 .2014 \\
\hline $547 / 2014$ & SQ1, E-half & pottery & 501 & & 07.01 .2014 \\
\hline $517 / 2014$ & SQ1, E-half & $\begin{array}{l}\text { pottery ( } 7 \text { baskets), among them scraper ( } 1 \text { re-used Christian } \\
\text { sherd, pottery), slag ( } 1 \text { piece) }\end{array}$ & 501 & SAV1W 0018 (scraper), SAV1W 0055 (slag) & 06.01 .2014 \\
\hline $581 / 2014$ & SQ1, NE-corner & pottery & 501 & & 11.01 .2014 \\
\hline $564 / 2014$ & SQ1, NE-corner & pottery, among it slag ( 1 piece), slag and brick (1 piece) & 501 & $\begin{array}{l}\text { SAV1W } 0027 \text { (slag), SAV1W } 0028 \text { (slag and } \\
\text { brick) }\end{array}$ & 09.01 .2014 \\
\hline $561 / 2014$ & SQ1, NE-corner & pottery among it weight? (1 fragment, clay) & 501 & SAV1W 0057 & 08.01 .2014 \\
\hline $665 / 2014$ & SQ1, NE-corner, 3-5m E-W/0-5m N-S & pottery & 501 & & 15.01 .2014 \\
\hline $668 / 2014$ & SQ1, NE-corner, 3-5m E-W/0-5m N-S & pottery & 501 & & 15.01 .2014 \\
\hline $580 / 2014$ & SQ1, NW-corner & pottery & 501 & & 11.01 .2014 \\
\hline $604 / 2014$ & SQ1, NW-corner & pottery & 501 & & 12.01 .2014 \\
\hline $517 \mathrm{~B} / 2014$ & SQ1, NW-corner & $\begin{array}{l}\text { pottery ( } 3 \text { baskets), among them scraper ( } 1 \text { re-used Christian } \\
\text { sherd, pottery) }\end{array}$ & 501 & SAV1W 0025 & 06.01 .2014 \\
\hline $671 / 2014$ & SQ1, NW-corner, 0-3m W-E/0-2m N-S & pottery, among it scraper (1 re-used Christian sherd, pottery) & 501 & SAV1W 0168 & 15.01 .2014 \\
\hline $575 / 2014$ & SQ1, SE-corner & pottery & 501 & & 09.01 .2014 \\
\hline $679 / 2014$ & SQ1, SE-corner, 3-5m E-W/0-5m S-N & $\begin{array}{l}\text { pottery, among it crucible? ( } 1 \text { fragment, mud and copper?), } \\
\text { scraper ( } 1 \text { re-used } 18^{\text {th }} \text { Dynasty sherd, pottery), token/gaming } \\
\text { piece ( } 1 \text { re-used sherd, pottery), scraper? ( } 1 \text { piece, pottery) }\end{array}$ & 501 & $\begin{array}{l}\text { SAV1W } 0201 \text { (crucible?), SAV1W } 0205 \\
\text { (scraper), SAV1W } 0208 \text { (token/gaming piece), } \\
\text { SAV1W } 0209 \text { (scraper) }\end{array}$ & 16.01 .2014 \\
\hline $687 / 2014$ & SQ1, SE-corner, 4-5m E-W/0-5m S-N & $\begin{array}{l}\text { pottery, among it stopper? ( } 1 \text { fragment, gypsum?), stand for } \\
\text { vessels ( } 1 \text { fragment, pottery), scraper (1 re-used body sherd, } \\
\text { Christian, pottery), slag ( } 1 \text { piece) }\end{array}$ & 501 & $\begin{array}{l}\text { SAV1W } 0213 \text { (stopper?), SAV1W } 0236 \text { (stand } \\
\text { for vessels), SAV1W } 0168 \text { (scraper), SAV1W } \\
0167 \text { (slag) }\end{array}$ & 18.01 .2014 \\
\hline $548 / 2014$ & SQ1, SW-corner & pottery & 501 & & 07.01 .2014 \\
\hline $549 / 2014$ & SQ1, SW-corner & $\begin{array}{l}\text { pottery, among it oil lamp ( } 1 \text { fragment, Christian, pottery), } \\
\text { scraper (1 re-used } 18^{\text {th }} \text { Dynasty sherd, pottery) }\end{array}$ & 501 & $\begin{array}{l}\text { SAV1W } 0036 \text { (oil lamp), SAV1W } 0037 \\
\text { (scraper) }\end{array}$ & 08.01 .2014 \\
\hline $563 / 2014$ & SQ1, SW-corner & pottery, among it re-used sherd ( 1 piece, $18^{\text {th }}$ Dynasty, pottery) & 501 & SAV1W 0023 & 09.01 .2014 \\
\hline $1068 / 2014$ & SQ1NW & pottery & 501 & & 29.01.2014 \\
\hline
\end{tabular}




\begin{tabular}{|c|c|c|c|c|c|}
\hline $1039 / 2014$ & SQ1NW & $\begin{array}{l}\text { pottery, among it crucible or mould? ( } 1 \text { fragment, mud), } \\
\text { token/gaming piece ( } 1 \text { re-used sherd, pottery), lid ( } 1 \text { re-used } \\
\text { sherd, pottery) }\end{array}$ & 501 & $\begin{array}{l}\text { SAV1W } 0371 \text { (crucible or mould?), SAV1W } \\
0381 \text { (token/gaming piece), SAV1W } 0382 \text { (lid) }\end{array}$ & 28.01 .2014 \\
\hline $676 / 2014$ & SQ1W, E-half & $\begin{array}{l}\text { pottery and stone: pottery, among it weight ( } 1 \text { re-used Christian } \\
\text { sherd, pottery), scraper ( } 1 \text { re-used Post-New Kingdom sherd, } \\
\text { pottery), token/gaming piece ( } 1 \text { re-used Christian sherd, } \\
\text { pottery), figurine ( } 1 \text { fragment, animal figurine, clay); whetstone } \\
\text { (1 piece, sandstone) }\end{array}$ & 501 & $\begin{array}{l}\text { SAV1W } 0216 \text { (weight), SAV1W } 0217 \\
\text { (scraper), SAV1W 0218 (scraper), SAV1W } \\
0215 \text { (figurine), SAV1W } 0219 \text { (whetstone) }\end{array}$ & 16.01 .2014 \\
\hline $690 / 2014$ & SQ1W, E-half, $5 \times 5 \mathrm{~m}$ & $\begin{array}{l}\text { pottery, among it scraper (1 re-used Post-New Kingdom sherd, } \\
\text { pottery), token/gaming piece (1 re-used Christian, sherd, } \\
\text { pottery) }\end{array}$ & 501 & $\begin{array}{l}\text { SAV1W } 0162 \text { (scraper), SAV1W } 0163 \\
\text { (token/gaming piece) }\end{array}$ & 18.01 .2014 \\
\hline $712 / 2014$ & SQ1W, W-half, $5 \times 5 \mathrm{~m}$ & $\begin{array}{l}\text { pottery, among it scraper (1 re-used Christian sherd, pottery), } \\
\text { architecture?/window grille? (1 fragment, pottery) }\end{array}$ & 501 & $\begin{array}{l}\text { SAV1W } 0202 \text { (scraper), SAV1W } 0210 \\
\text { (architecture?/window grille?) }\end{array}$ & 19.01 .2014 \\
\hline $693 / 2014$ & SQ1W, W-half, $5 \times 5 \mathrm{~m}$ & $\begin{array}{l}\text { pottery, among it token/gaming piece (1 re-used Christian, } \\
\text { sherd, pottery) }\end{array}$ & 501 & SAV1W 0214 & 18.01 .2014 \\
\hline $529 / 2014$ & SQ2 & pottery & 501 & & 06.01 .2014 \\
\hline $512 / 2014$ & SQ2 & pottery ( 1 basket) & 501 & & 06.01 .2014 \\
\hline $507 / 2014$ & SQ2 & pottery (4 baskets), slag (1 piece) & 501 & SAV1W 0007 (slag) & 05.01 .2014 \\
\hline $570 / 2014$ & $\mathrm{SQ} 2,0$ to $2 \mathrm{~m}$ from $\mathrm{S}, 5$ to $10 \mathrm{~m}$ from $\mathrm{W}$ & pottery & 501 & & 09.01 .2014 \\
\hline $582 / 2014$ & $\mathrm{SQ} 2,0$ to $7 \mathrm{~m}$ from $\mathrm{E}$ & pottery & 501 & & 11.01 .2014 \\
\hline $594 / 2014$ & $\mathrm{SQ} 2,0$ to $7 \mathrm{~m}$ from $\mathrm{E}$ & pottery & 501 & & 12.01 .2014 \\
\hline $831 / 2014$ & $\begin{array}{l}\text { SQ2, 0-5m E-W/0-5m S-N, cleaning } \\
\text { square }\end{array}$ & pottery & 501 & & 16.01 .2014 \\
\hline $578 / 2014$ & $\mathrm{SQ} 2,1$ to $5 \mathrm{~m}$ from $\mathrm{E}, 0$ to $0.5 \mathrm{~m}$ from $\mathrm{N}$ & pottery & 501 & & 09.01 .2014 \\
\hline $555 / 2014$ & $\mathrm{SQ} 2,1$ to $5 \mathrm{~m}$ from $\mathrm{W}$ & pottery & 501 & & 08.01 .2014 \\
\hline $550 / 2014$ & $\mathrm{SQ} 2,4$ to $5 \mathrm{~m}$ from $\mathrm{E}$ & pottery & 501 & & 08.01 .2014 \\
\hline $576 / 2014$ & $\mathrm{SQ} 2,5$ to $7 \mathrm{~m}$ from $\mathrm{W}, 0$ to $3 \mathrm{~m}$ from $\mathrm{S}$ & pottery & 501 & & 09.01 .2014 \\
\hline $551 / 2014$ & $\mathrm{SQ} 2,7$ to $10 \mathrm{~m}$ from $\mathrm{E}$ & pottery & 501 & & 08.01 .2014 \\
\hline $583 / 2014$ & $\mathrm{SQ} 2,7$ to $10 \mathrm{~m}$ from $\mathrm{E}$ & pottery & 501 & & 11.01 .2014 \\
\hline $595 / 2014$ & $\mathrm{SQ} 2,7$ to $10 \mathrm{~m}$ from $\mathrm{E}$ & $\begin{array}{l}\text { pottery, among it scraper (1 re-used Post-New Kingdom sherd, } \\
\text { pottery) }\end{array}$ & 501 & SAV1W 0592 & 12.01 .2014 \\
\hline $530 / 2014$ & $\mathrm{SQ} 2$, at $1 \mathrm{~m}$ from $\mathrm{E}$ & pottery & 501 & & 07.01 .2014 \\
\hline $531 / 2014$ & $\mathrm{SQ} 2$, at $3.5 \mathrm{~m}$ from $\mathrm{E}$ & pottery & 501 & & 07.01 .2014 \\
\hline $537 / 2014$ & $\mathrm{SQ} 2$, at $5 \mathrm{~m}$ from $\mathrm{W}$ & pottery & 501 & & 07.01 .2014 \\
\hline $532 / 2014$ & $\mathrm{SQ} 2$, at $5.5 \mathrm{~m}$ from $\mathrm{E}$ & pottery & 501 & & 07.01 .2014 \\
\hline $533 / 2014$ & $\mathrm{SQ} 2$, at $7 \mathrm{~m}$ from $\mathrm{E}$ & $\begin{array}{l}\text { pottery, among it lid (1 re-used Christian sherd, pottery), slag } \\
\text { (1 piece) }\end{array}$ & 501 & SAV1W 0022 (lid), SAV1W 0004 (slag) & 07.01 .2014 \\
\hline $816 / 2014$ & SQ2, cleaning square & pottery & 501 & & 15.01 .2014 \\
\hline
\end{tabular}




\begin{tabular}{|c|c|c|c|c|c|}
\hline $655 / 2014$ & SQ2, from cleaning square & pottery & 501 & & 14.01 .2014 \\
\hline $656 / 2014$ & SQ2, from cleaning square & pottery & 501 & & 14.01 .2014 \\
\hline $527 / 2014$ & SQ2, W-half & pottery & 501 & & 06.01 .2014 \\
\hline $522 / 2014$ & SQ2, W-half & pottery (1 basket) & 501 & & 06.01 .2014 \\
\hline $539 / 2014$ & SQ1, from debris of SU & $\begin{array}{l}\text { pottery ( } 2 \text { baskets), among them token/gaming piece ( } 1 \text { re-used } \\
18^{\text {th }} \text { Dynasty sherd, pottery), scraper ( } 1 \text { re-used } 18^{\text {th }} \text { Dynasty } \\
\text { sherd, pottery) }\end{array}$ & 502 & $\begin{array}{l}\text { SAV1W } 0020 \text { (token/gaming piece), SAV1W } \\
0021 \text { (scraper) }\end{array}$ & 07.01 .2014 \\
\hline $613 / 2014$ & SQ1, NW-corner, 1-5m W-E/3-5m N-S & pottery, among it lid (1 re-used $18^{\text {th }}$ Dynasty sherd, pottery) & 502 & SAV1W 0112 & 12.01 .2014 \\
\hline $557 / 2014$ & SQ2 & pottery & 502 & & 08.01 .2014 \\
\hline $559 / 2014$ & SQ1 & pottery & 503 & & 08.01 .2014 \\
\hline $541 / 2014$ & SQ1, Pit (SU 503) & $\begin{array}{l}\text { pottery ( } 2 \text { baskets), among them figurine ( } 1 \text { lower part of } \\
\text { rudimentary female figurine, mud) }\end{array}$ & 503 & SAV1W 0029 & 07.01 .2014 \\
\hline $818 / 2014$ & SQ2 & pottery & 504 & & 16.01 .2014 \\
\hline $901 / 2014$ & SQ2 & pottery & 504 & & 22.01 .2014 \\
\hline $584 / 2014$ & $\mathrm{SQ}$, sandy area in the $\mathrm{W}$ & pottery & 504 & & 11.01 .2014 \\
\hline $596 / 2014$ & $\mathrm{SQ} 2$, sandy area in the $\mathrm{W}, 0$ to $5 \mathrm{~m}$ from $\mathrm{W}$ & $\begin{array}{l}\text { pottery, among it scraper ( } 1 \text { re-used } 18^{\text {th }} \text { Dynasty? sherd, } \\
\text { pottery); slag and brick ( } 1 \text { piece) }\end{array}$ & 504 & $\begin{array}{l}\text { SAV1W } 0593 \text { (scraper), SAV1W } 1717 \text { (slag } \\
\text { and brick) }\end{array}$ & 12.01 .2014 \\
\hline $567 / 2014$ & $\mathrm{SQ} 2, \mathrm{~W}$ sandy area, 1 to $5 \mathrm{~m}$ from $\mathrm{W}$ & pottery & 504 & & 09.01 .2014 \\
\hline $566 / 2014$ & SQ2, Feature 101 (pathway) & pottery & 505 & & 09.01 .2014 \\
\hline $751 / 2014$ & SQ1, 0-1m E-W/0-2m S-N & $\begin{array}{l}\text { pottery, among it slag in Post-New Kingdom sherd ( } 1 \text { piece), } \\
\text { scrapers ( } 2 \text { pieces, each re-used } 18^{\text {th }} \text { Dynasty sherds, pottery) }\end{array}$ & 507 & $\begin{array}{l}\text { SAV1W 0269 ( slag in Post-New Kingdom } \\
\text { sherd), SAV1W 0270, SAV1W } 0271 \\
\text { (scrapers) }\end{array}$ & 23.01 .2014 \\
\hline $743 / 2014$ & $\mathrm{SQ} 1,0-2 \mathrm{~m} \mathrm{~S}-\mathrm{N} / 0-4 \mathrm{~m} \mathrm{E}-\mathrm{W}$ & pottery, among it lid ( 1 piece, $18^{\text {th }}$ Dynasty, pottery) & 507 & SAV1W 0235 & 22.01 .2014 \\
\hline $739 / 2014$ & SQ1, 0-2m S-N/0-4m E-W & $\begin{array}{l}\text { pottery, among it lids ( } 2 \text { pieces, each re-used sherds, } 1 \text { unclear, } \\
\left.118^{\text {th }} \text { Dynasty, pottery); slag ( } 1 \text { piece }\right)\end{array}$ & 507 & $\begin{array}{l}\text { SAV1W 0260, SAV1W } 0261 \text { (lids), SAV1W } \\
0262 \text { (slag) }\end{array}$ & 21.01 .2014 \\
\hline $1036 / 2014$ & $\mathrm{SQ} 1,0-5 \mathrm{~m} \mathrm{NS} / 0-1 \mathrm{~m} \mathrm{EW}$ & $\begin{array}{l}\text { pottery, among it lid ( } 1 \text { re-used } 18^{\text {th }} \text { Dynasty sherd, pottery), } \\
\text { mould for figurine ( } 1 \text { fragment, probably for animal head, } \\
\text { pottery) }\end{array}$ & 507 & SAV1W 0359 (lid), SAV1W 0360 (mould) & 28.01 .2014 \\
\hline $1045 / 2014$ & SQ1, 1.2-2.3m E-W/0-5m S-N & pottery & 507 & & 28.01 .2014 \\
\hline $1057 / 2014$ & SQ1, 2.3-4.1m E-W/0-5m S-N & pottery & 507 & & 28.01 .2014 \\
\hline $1083 / 2014$ & $\mathrm{SQ} 1,4-5 \mathrm{~m} \mathrm{E}-\mathrm{W} / 0-5 \mathrm{~m} \mathrm{~S}-\mathrm{N}$ & pottery & 507 & & 29.01 .2014 \\
\hline $1091 / 2014$ & SQ1, 4-5m E-W/0-5m S-N & $\begin{array}{l}\text { pottery and stone: pottery, among it net weight ( } 1 \text { re-used Post- } \\
\text { New Kingdom sherd, pottery); whetstone (1 piece, sandstone), } \\
\text { grindstone or hand mill ( } 1 \text { piece, quartzite), scrapers ( } 3 \text { pieces, } \\
\text { each re-used } 18^{\text {th }} \text { Dynasty sherds, pottery), lid (1 piece, clay) }\end{array}$ & 507 & $\begin{array}{l}\text { SAV1W } 0547 \text { (net weight), SAV1W } 0385 \\
\text { (whetstone), SAV1W 0548 (grindstone or hand } \\
\text { mill), SAV1W 0384, SAV1W 0546, SAV1W } \\
\text { 0552 (scrapers), SAV1W 0524 (lid) }\end{array}$ & 30.01 .2014 \\
\hline $593 / 2014$ & SQ1, E-half & $\begin{array}{l}\text { pottery ( } 39 \text { baskets), among them scraper ( } 1 \text { re-used } 18^{\text {th }} \\
\text { Dynasty sherd, pottery), clay object/stopper? ( } 1 \text { fragment) }\end{array}$ & 507 & $\begin{array}{l}\text { SAV1W } 0581 \text { (scraper), SAV1W } 0061 \text { (clay } \\
\text { object/stopper?) }\end{array}$ & 12.01 .2014 \\
\hline $591 / 2014$ & SQ1, E-half & pottery; seashell (1 large fragment) & 507 & & 11.01 .2014 \\
\hline
\end{tabular}




\begin{tabular}{|c|c|c|c|c|c|}
\hline $612 / 2014$ & SQ1, NE-corner & $\begin{array}{l}\text { pottery, among it scraper (1 re-used sherd, pottery), lid (1 piece, } \\
\text { pottery) }\end{array}$ & 507 & SAV1W 0058 (scraper), SAV1W 0194 (lid) & 12.01.2014 \\
\hline $615 / 2014$ & $\mathrm{SQ} 2,0-5 \mathrm{~m} \mathrm{E}-\mathrm{W} / 2.5-5 \mathrm{~m} \mathrm{~S}-\mathrm{N}$ & pottery & 508 & & 13.01.2014 \\
\hline $619 / 2014$ & $\mathrm{SQ} 2,6-7 \mathrm{~m} \mathrm{E}-\mathrm{W} / 3-4 \mathrm{~m} \mathrm{~S}-\mathrm{N}$ & pottery & 508 & & 13.01.2014 \\
\hline $609 / 2014$ & $\mathrm{SQ} 2, \mathrm{~N}$ of SQ2, 5 to $10 \mathrm{~m}$ from $\mathrm{E}$ & pottery & 508 & & 12.01 .2014 \\
\hline $606 / 2014$ & SQ2, NE of SQ2 & pottery & 508 & & 12.01 .2014 \\
\hline $617 / 2014$ & SQ2 & pottery & 509 & & 13.01.2014 \\
\hline $657 / 2014$ & SQ2 & $\begin{array}{l}\text { pottery, among it lid ( } 1 \text { re-used Post New Kingdom sherd, } \\
\text { pottery), scraper ( } 1 \text { re-used Post-New Kingdom sherd, pottery) }\end{array}$ & 510 & SAV1W 0144 (lid), SAV1W 0145 (scraper) & 14.01.2014 \\
\hline $631 / 2014$ & $\mathrm{SQ} 2,3-5 \mathrm{~m} \mathrm{~S}-\mathrm{N} / 0-2 \mathrm{~m}$ E-W & pottery & 510 & & 14.01.2014 \\
\hline $633 / 2014$ & $\mathrm{SQ} 2,3-5 \mathrm{~m} \mathrm{~S}-\mathrm{N} / 2-8 \mathrm{~m}$ E-W & pottery & 510 & & 14.01 .2014 \\
\hline $643 / 2014$ & SQ1, NW-corner, 1-5m W-E/3-5m N-S & pottery, among it vessel (1 fragment, pottery) & 511 & SAV1W 0197 & 14.01 .2014 \\
\hline $1146 / 2014$ & SQ1 & $\begin{array}{l}\text { pottery and stone: pottery, among it scraper ( } 1 \text { re-used } \\
18^{\text {th }} \text { Dynasty sherd, pottery), figurine or token/gaming piece? } \\
\text { ( } 1 \text { cylindrical fragment, pottery), figurine ( } 1 \text { lower part of a } \\
\text { rudimentary female figurine, mud), lid ( } 1 \text { fragment, mud); hand } \\
\text { mill ( } 1 \text { piece, sandstone) }\end{array}$ & 512 & $\begin{array}{l}\text { SAV1W } 0410 \text { (scraper), SAV1W } 0412 \\
\text { (figurine or token/gaming piece?), SAV1W } \\
0408 \text { (figurine), SAV1W } 0414 \text { (lid), SAV1W } \\
0415 \text { (hand mill) }\end{array}$ & 02.02 .2014 \\
\hline $1166 / 2014$ & SQ1 & $\begin{array}{l}\text { pottery, among it weight ( } 1 \text { re-used } 18^{\text {th }} \text { Dynasty sherd, pottery), } \\
\text { lid ( } 1 \text { re-used } 18^{\text {th }} \text { Dynasty sherd, pottery), scraper ( } 1 \text { re-used } \\
\text { small fragment of Kerma Classique sherd, pottery) }\end{array}$ & 512 & $\begin{array}{l}\text { SAV1W } 0437 \text { (weight), SAV1W } 0438 \text { (lid), } \\
\text { SAV1W } 1533 \text { (scraper) }\end{array}$ & 03.02.2014 \\
\hline $640 / 2014$ & SQ1, SW-corner, 0-5m W-E/0-2m S-N & pottery & 513 & & 14.01.2014 \\
\hline $642 / 2014$ & SQ1, SW-corner, 2-5m S-N/0-5m W-E & pottery & 513 & & 14.01.2014 \\
\hline $660 / 2014$ & SQ2 & pottery & 514 & & 14.01.2014 \\
\hline $826 / 2014$ & SQ2 & $\begin{array}{l}\text { pottery, among it lid (1 re-used Post New Kingdom sherd, } \\
\text { pottery) }\end{array}$ & 514 & SAV1W 0166 & 16.01.2014 \\
\hline $659 / 2014$ & SQ2 & pottery, among it crucible/tuyère? (1 fragment, mud) & 515 & SAV1W 0207 & 14.01.2014 \\
\hline $821 / 2014$ & SQ2 & $\begin{array}{l}\text { pottery, among it oil lamp (1 fragment, pottery), scraper (1 re- } \\
\text { used Post-New Kingdom sherd, pottery) }\end{array}$ & 516 & $\begin{array}{l}\text { SAV1W } 0035 \text { (oil lamp), SAV1W } 0182 \\
\text { (scraper) }\end{array}$ & 16.01.2014 \\
\hline $807 / 2014$ & SQ2 & pottery & 517 & & 15.01 .2014 \\
\hline $812 / 2014$ & SQ2 & pottery & 518 & & 15.01.2014 \\
\hline $833 / 2014$ & SQ2 & pottery & 518 & & 16.01.2014 \\
\hline $839 / 2014$ & SQ1W & pottery; shell (1 piece) & 521 & & 20.01.2014 \\
\hline $708 / 2014$ & $\begin{array}{l}\text { SQ1W, E-half, } \\
5 \times 5 \mathrm{~m}\end{array}$ & $\begin{array}{l}\text { pottery, among it weight ( } 1 \text { re-used sherd, with perforation, } \\
\text { pottery), clay object/lid? ( } 1 \text { fragment, clay), crucible? } \\
\text { (1 fragment, clay); slag on brick ( } 1 \text { piece) }\end{array}$ & 521 & $\begin{array}{l}\text { SAV1W } 0265 \text { (weight), SAV1W } 0266 \text { (clay } \\
\text { object/lid?), SAV1W 0200 (slag on brick), } \\
\text { SAV1W 0211 (crucible?) }\end{array}$ & 19.01.2014 \\
\hline $886 / 2014$ & SQ1W & pottery & 522 & & 22.01.2014 \\
\hline $859 / 2014$ & SQ1W & pottery, among it lid (1 re-used sherd, Christian, pottery) & 522 & SAV1W 0220 & 21.01.2014 \\
\hline
\end{tabular}




\begin{tabular}{|c|c|c|c|c|c|}
\hline $835 / 2014$ & $\begin{array}{l}\text { SQ1W, } \\
5-10 \mathrm{~m} \mathrm{E}-\mathrm{W}\end{array}$ & pottery & 522 & & 20.01.2014 \\
\hline $766 / 2014$ & SQ1W, W-half & pottery & 522 & & 25.01 .2014 \\
\hline $778 / 2014$ & SQ1W, W-half & pottery & 522 & & 25.01.2014 \\
\hline $783 / 2014$ & SQ1W, W-half & pottery & 522 & & 26.01.2014 \\
\hline $904 / 2014$ & SQ1W, W-half & $\begin{array}{l}\text { pottery, among it re-used sherds ( } 2 \text { pieces, each disc-shaped, } \\
\text { Christian, pottery) }\end{array}$ & 522 & SAV1W 0293, SAV1W 0294 (re-used sherds) & 23.01.2014 \\
\hline $773 / 2014$ & SQ1W, W-half, 3.40m S-N/18.20m E-W & pottery (1 fragment) & 522 & & 25.01 .2014 \\
\hline $906 / 2014$ & SQ2, sandy area in $\mathrm{W}$ & pottery & 522 & & 23.01.2014 \\
\hline $863 / 2014$ & SQ1W & pottery & 523 & & 21.01 .2014 \\
\hline $848 / 2014$ & SQ1W & pottery, among it crucible? (1 fragment) & 523 & SAV1W 0267 & 20.01 .2014 \\
\hline $916 / 2014$ & SQ1W & pottery & 524 & & 23.01.2014 \\
\hline $852 / 2014$ & SQ1W & $\begin{array}{l}\text { pottery, among it token/gaming piece? (1 re-used sherd, } \\
\text { Christian, pottery) }\end{array}$ & 524 & SAV1W 0196 & 20.01.2014 \\
\hline $870 / 2014$ & SQ1W, 0-3m S-N & pottery & 524 & & 21.01 .2014 \\
\hline $869 / 2014$ & SQ1W, 3-5m S-N & pottery; linen/cloth (several pieces) & 524 & SAV1W 0228 (linen/cloth) & 21.01.2014 \\
\hline $797 / 2014$ & $\begin{array}{l}\mathrm{SQ} 1,2-2.5 \mathrm{~m} \mathrm{~N}-\mathrm{S} / 4-4.5 \mathrm{~m} \mathrm{E}-\mathrm{W} \text {, debris } \\
\text { layer adjacent to the } \mathrm{E} \text { of Feature } 100\end{array}$ & pottery & 526 & & 26.01.2014 \\
\hline $1080 / 2014$ & $\begin{array}{l}\mathrm{SQ} 1,4-4.5 \mathrm{~m} \mathrm{~N}-\mathrm{S} / 4.5-5 \mathrm{~m} \mathrm{E}-\mathrm{W} \text {, debris } \\
\text { adjacent to Feature } 100\end{array}$ & $\begin{array}{l}\text { pottery, among it token/gaming piece ( } 1 \text { re-used Christian sherd, } \\
\text { pottery) }\end{array}$ & 526 & SAV1W 0378 (token/gaming piece) & 29.01.2014 \\
\hline $876 / 2014$ & SQ1W & pottery, crucibles? ( 3 pieces, mud/clay) & 527 & $\begin{array}{l}\text { SAV1W 0221, SAV1W 0222, SAV1W } 0223 \\
\text { (crucibles) }\end{array}$ & 21.01.2014 \\
\hline $1251 / 2014$ & SQ1W & pottery & 529 & & 05.02 .2014 \\
\hline $880 / 2014$ & SQ1W, surface cleaning & pottery & 529 & & 21.01.2014 \\
\hline $1035 / 2014$ & SQ1 & pottery, among it lid (1 re-used $18^{\text {th }}$ Dynasty sherd, pottery) & 530 & SAV1W 1718 & 27.01.2014 \\
\hline $1050 / 2014$ & SQ1 & $\begin{array}{l}\text { pottery, among it re-used sherd ( } 1 \text { re-used } 18^{\text {th }} \text { Dynasty sherd, } \\
\text { pottery) }\end{array}$ & 530 & SAV1W 0586 & 28.01.2014 \\
\hline $781 / 2014$ & $\mathrm{SQ} 1,0-4 \mathrm{~m} \mathrm{~S}-\mathrm{N} / 5-10 \mathrm{~m} \mathrm{E}-\mathrm{W}$ & pottery, among it scraper (1 re-used sherd, pottery) & 530 & SAV1W 0346 & 26.01.2014 \\
\hline $777 / 2014$ & $\mathrm{SQ} 1,0-4 \mathrm{~m} \mathrm{~W}-\mathrm{E} / 0-1 \mathrm{~m} \mathrm{~S}-\mathrm{N}$ & pottery & 530 & & 25.01 .2014 \\
\hline $890 / 2014$ & SQ1W & pottery & 530 & & 22.01 .2014 \\
\hline $892 / 2014$ & SQ1W & pottery & 531 & & 22.01 .2014 \\
\hline $898 / 2014$ & SQ1W & pottery & 532 & & 22.01 .2014 \\
\hline $785 / 2014$ & $\mathrm{SQ} 1,0-4 \mathrm{~m} \mathrm{~N}-\mathrm{S} / 0-5 \mathrm{~m} \mathrm{E}-\mathrm{W}$ & $\begin{array}{l}\text { pottery, among it stopper or lid (1 piece, Post-New } \\
\text { Kingdom/Christian, mud) }\end{array}$ & 533 & SAV1W 0290 & 26.01.2014 \\
\hline $758 / 2014$ & SQ1, 0-5m W-E/0-5m N-S & $\begin{array}{l}\text { pottery, among it mould for figurine ( } 1 \text { fragment, for animal } \\
\text { figurine?, pottery), token/gaming piece ( } 1 \text { re-used } 18^{\text {th }} \text { Dynasty } \\
\text { sherd, pottery), stopper/sealing? ( } 1 \text { fragment, mud) }\end{array}$ & 533 & $\begin{array}{l}\text { SAV1W } 0295 \text { (mould), SAV1W } 0296 \\
\text { (token/gaming piece), SAV1W } 0297 \\
\text { (stopper/sealing?) }\end{array}$ & 23.01 .2014 \\
\hline
\end{tabular}




\begin{tabular}{|c|c|c|c|c|c|}
\hline $909 / 2014$ & SQ1W & pottery & 534 & & 23.01 .2014 \\
\hline $1028 / 2014$ & SQ1, 4-6m N-S/5-10m E-W & $\begin{array}{l}\text { pottery, among it scraper (1 re-used Post-New Kingdom sherd, } \\
\text { pottery) }\end{array}$ & 536 & SAV1W 0358 & 27.01 .2014 \\
\hline $762 / 2014$ & SQ1, 5-10m E-W/5-7m N-S & $\begin{array}{l}\text { pottery and stone: pottery, among it token/gaming piece ( } 1 \text { re- } \\
\text { used } 18^{\text {th }} \text { Dynasty sherd, pottery), crucible? ( } 1 \text { fragment, mud, } \\
\text { with copper layer?); hand mill? ( } 1 \text { piece, quartzite) }\end{array}$ & 536 & $\begin{array}{l}\text { SAV1W } 0329 \text { (hand mill), SAV1W } 0323 \\
\text { (token/gaming piece), SAV1W } 0339 \\
\text { (crucible?) }\end{array}$ & 25.01 .2014 \\
\hline $1144 / 2014$ & SQ1, cleaning & pottery & 537 & & 01.02 .2014 \\
\hline $763 / 2014$ & $\mathrm{SQ} 1,0-3 \mathrm{~m}$ E-W/0-5m N-S & $\begin{array}{l}\text { pottery and stones: pottery, among it scrapers ( } 2 \text { pieces, each re- } \\
\text { used sherds, } 118^{\text {th }} \text { Dynasty?, } 1 \text { Christian, pottery); hand mill } \\
\text { (1 piece, quartzite), whetstone ( } 1 \text { piece, sandstone), stone vessel } \\
\text { (1 fragment, granite, see joint } 1013 / 2014)\end{array}$ & 537 & $\begin{array}{l}\text { SAV1W 0291, SAV1W } 0292 \text { (scrapers), } \\
\text { SAV1W 0367 (stone vessel), SAV1W } 0368 \\
\text { (hand mill), SAV1W 0576 (whetstone) }\end{array}$ & 25.01 .2014 \\
\hline $1008 / 2014$ & SQ1, 0-3m E-W/0-5m N-S & $\begin{array}{l}\text { pottery, among it re-used sherd (1 piece, lid); stone palette? } \\
\text { (1 fragment, quartzite?) }\end{array}$ & 537 & SAV1W 0628 (lid), SAV1W 0362 (palette) & 27.01 .2014 \\
\hline $775 / 2014$ & SQ1, 3-5m E-W/0-5m N-S & $\begin{array}{l}\text { pottery, among it scraper (1 re-used Post-New Kingdom sherd, } \\
\text { pottery) }\end{array}$ & 538 & SAV1W 0321 & 25.01 .2014 \\
\hline $795 / 2014$ & $\mathrm{SQ} 1,3-5 \mathrm{~m}$ E-W/0-5m N-S & $\begin{array}{l}\text { pottery, among it scrapers ( } 4 \text { pieces, each re-used sherds, } 218^{\text {th }} \\
\text { Dynasty, } 1 \text { unclear, } 1 \text { Post-New Kingdom, pottery), lid (1 re- } \\
\text { used } 18^{\text {th }} \text { Dynasty sherd, pottery) }\end{array}$ & 538 & $\begin{array}{l}\text { SAV1W 0349, SAV1W 0351, SAV1W 0352, } \\
\text { SAV1W } 0354 \text { (scrapers), SAV1W } 0350 \text { (lid) }\end{array}$ & 26.01.2014 \\
\hline $1007 / 2014$ & SQ1, 3-5m E-W/0-5m N-S & pottery; grindstone (1 piece, quartzite) & 538 & SAV1W 0363 & 27.01.2014 \\
\hline $798 / 2014$ & SQ1W & $\begin{array}{l}\text { pottery, among it scraper ( } 1 \text { re-used Post-New Kingdom sherd, } \\
\text { pottery), token/gaming piece? ( } 1 \text { cylindrical object, Christian, } \\
\text { clay) }\end{array}$ & 539 & $\begin{array}{l}\text { SAV1W } 0347 \text { (scraper), SAV1W } 0348 \\
\text { (token/gaming piece?) }\end{array}$ & 26.01 .2014 \\
\hline $1005 / 2014$ & SQ1W & pottery, among it stopper/sealing? (1 fragment, mud) & 540 & SAV1W 0341 & 26.01 .2014 \\
\hline $1052 / 2014$ & SQ1W & pottery & 541 & & 28.01 .2014 \\
\hline $1066 / 2014$ & SQ1W & pottery & 541 & & 29.01.2014 \\
\hline $1077 / 2014$ & $\begin{array}{l}\text { SQ1W, } \\
4-6 \mathrm{~m} \mathrm{E}-\mathrm{W}\end{array}$ & pottery & 541 & & 29.01.2014 \\
\hline $1034 / 2014$ & SQ1W & pottery, among it oil lamp (1 fragment, Christian, pottery) & 542 & SAV1W 0578 & 27.01.2014 \\
\hline $1047 / 2014$ & SQ1W & pottery & 543 & & 28.01 .2014 \\
\hline $1056 / 2014$ & SQ1W & $\begin{array}{l}\text { pottery, among it figurine?/ mud object (1 fragment, } \\
\text { rudimentary figurine?, clay) }\end{array}$ & 544 & SAV1W 0591 & 28.01 .2014 \\
\hline $1150 / 2014$ & SQ1NW & pottery & 545 & & 02.02 .2014 \\
\hline $1093 / 2014$ & SQ1NW & $\begin{array}{l}\text { pottery, slag and stone: pottery, among it token/gaming piece (1 } \\
\text { re-used Post-New Kingdom sherd, pottery); slag and brick } \\
\text { (1 piece); hand mill (1 piece, quartzite) }\end{array}$ & 545 & $\begin{array}{l}\text { SAV1W } 0394 \text { (token/gaming piece), SAV1W } \\
0375 \text { (slag and brick), SAV1W } 0393 \text { (hand } \\
\text { mill) }\end{array}$ & 30.01 .2014 \\
\hline $1161 / 2014$ & SQ1NW, 5-6.5m S-N/0-2m E-W & pottery, among it oil lamp (1 half preserved, Christian, pottery) & 545 & SAV1W 0434 & 02.02 .2014 \\
\hline $1114 / 2014$ & SQ1NW, 5-7m S-N & pottery & 545 & & 30.01 .2014 \\
\hline $1095 / 2014$ & SQ1W & pottery & 546 & & 30.01 .2014 \\
\hline $1103 / 2014$ & SQ1W, section cleaning & pottery & 546 & & 30.01 .2014 \\
\hline
\end{tabular}




\begin{tabular}{|c|c|c|c|c|c|}
\hline $1148 / 2014$ & SQ1 & $\begin{array}{l}\text { pottery, among it scraper ( } 1 \text { re-used Christian sherd, pottery), } \\
\text { figurine/statuette ( } 1 \text { fragment, part of a foot with } 6 \text { toes?, clay), } \\
\text { crucible? ( } 1 \text { fragment, mud) }\end{array}$ & 547 & $\begin{array}{l}\text { SAV1W } 0446 \text { (scraper), SAV1W } 0409 \\
\text { (figurine/statuette), SAV1W } 0425 \text { (crucible?) }\end{array}$ & 02.02 .2014 \\
\hline $1168 / 2014$ & SQ1 & $\begin{array}{l}\text { pottery, among it scrapers ( } 2 \text { pieces, each re-used Post-New } \\
\text { Kingdom sherds, pottery), lid ( } 1 \text { re-used Post-New Kingdom } \\
\text { sherd, pottery), weight?/scraper? ( } 1 \text { re-used sherd, pottery), } \\
\text { mould?/re-used sherd? (1 pottery object), glazed ware (1 body } \\
\text { sherd) }\end{array}$ & 547 & $\begin{array}{l}\text { SAV1W 0429, SAV1W } 0431 \text { (scrapers), } \\
\text { SAV1W 0430 (lid), SAV1W } 0432 \\
\text { (weight/scraper?), SAV1W } 0433 \text { (mould?/re- } \\
\text { used sherd?), SAV1W } 0440 \text { (glazed ware) }\end{array}$ & 03.02 .2014 \\
\hline $1106 / 2014$ & SQ1, 4-7m S-N & $\begin{array}{l}\text { pottery, among it lid? (1 fragment, mud), scraper (1 re-used } \\
\text { Post-New Kingdom sherd, pottery) }\end{array}$ & 547 & SAV1W 0376 (lid?), SAV1W 0395 (scraper) & 30.01 .2014 \\
\hline $1126 / 2014$ & SQ1, 7-10m S-N/6-10m E-W & pottery & 547 & & 01.02 .2014 \\
\hline $1122 / 2014$ & SQ1 & $\begin{array}{l}\text { pottery, faience and stone: weight? ( } 1 \text { re-used sherd, pottery), } \\
\text { stopper or lid? ( } 1 \text { fragment, clay), crucible? ( } 1 \text { fragment mud } \\
\text { with copper?); faience vessel (1 body sherd, faience); hand mill } \\
\text { (1 piece, quartzite) }\end{array}$ & 548 & $\begin{array}{l}\text { SAV1W } 0582 \text { (weight?), SAV1W } 0570 \\
\text { (stopper or lid?), SAV1W 0571 (crucible?), } \\
\text { SAV1W 0574 (faience vessel), SAV1W } 0575 \\
\text { (hand mill) }\end{array}$ & 01.02 .2014 \\
\hline $1145 / 2014$ & SQ1 & pottery & 548 & & 01.02 .2014 \\
\hline $1140 / 2014$ & SQ1 & pottery & 550 & & 01.02 .2014 \\
\hline $1178 / 2014$ & SQ1 & $\begin{array}{l}\text { pottery, among it scraper (1 re-used Post-New Kingdom sherd, } \\
\text { pottery) }\end{array}$ & 551 & SAV1W 0441 & 03.02 .2014 \\
\hline $1181 / 2014$ & $\mathrm{SQ} 1,1.80 \mathrm{~m} \mathrm{~W}-\mathrm{E} / 3.9 \mathrm{~m} \mathrm{~N}-\mathrm{S}$ & $\begin{array}{l}\text { pottery, among it token/gaming piece ( } 1 \text { re-used } 18^{\text {th }} \text { Dynasty } \\
\text { sherd, pottery) }\end{array}$ & 551 & SAV1W 0439 & 03.02 .2014 \\
\hline $1279 / 2014$ & SQ1 & pottery & 552 & & 06.02 .2014 \\
\hline $1184 / 2014$ & SQ1NW & $\begin{array}{l}\text { pottery and faience: scraper ( } 1 \text { re-used Christian sherd, pottery); } \\
\text { faience vessel ( } 1 \text { body sherd, Nun bowl) }\end{array}$ & 552 & $\begin{array}{l}\text { SAV1W } 0442 \text { (faience vessel), SAV1W } 0443 \\
\text { (scraper) }\end{array}$ & 03.02 .2014 \\
\hline $1197 / 2014$ & SQ1NW, 0-5m S-N/0-2m E-W & $\begin{array}{l}\text { pottery, among it scrapers ( } 2 \text { pieces, } 1 \text { re-used Post-New } \\
\text { Kingdom sherd, } 1 \text { re-used Christian sherd, pottery) }\end{array}$ & 552 & SAV1W 0491, SAV1W 0493 (scrapers) & 04.02 .2014 \\
\hline $1193 / 2014$ & SQ1W, 0-5m S-N/0-2m E-W & $\begin{array}{l}\text { pottery, crucible? ( } 1 \text { fragment, mud), clay ball/artefact? (1 } \\
\text { almost complete piece) }\end{array}$ & 552 & $\begin{array}{l}\text { SAV1W } 0449 \text { (crucible?), SAV1W } 0470 \text { (clay } \\
\text { ball/artefact?) }\end{array}$ & 04.02 .2014 \\
\hline $1187 / 2014$ & SQ1W & pottery & 553 & & 03.02 .2014 \\
\hline $1189 / 2014$ & SQ1W & pottery & 554 & & 03.02 .2014 \\
\hline $1210 / 2014$ & SQ1 & pottery & 555 & & 04.02 .2014 \\
\hline $1195 / 2014$ & SQ1 & $\begin{array}{l}\text { pottery and stone: lid ( } 1 \text { re-used sherd, Christian, pottery), } \\
\text { token/gaming piece ( } 1 \text { re-used sherd, Christian, pottery), scraper } \\
\text { ( } 1 \text { re-used sherd, Christian, pottery), crucibles?/moulds? } \\
\text { ( } 2 \text { pieces, } 1 \text { massive fragment, } 1 \text { fragment, each mud); } \\
\text { whetstone ( } 1 \text { piece, sandstone), hand mill ( } 1 \text { piece, sandstone) }\end{array}$ & 555 & $\begin{array}{l}\text { SAV1W } 0472 \text { (lid), SAV1W } 0473 \\
\text { (token/gaming piece), SAV1W } 0475 \text { (scraper), } \\
\text { SAV1W 0435, SAV1W } 0436 \\
\text { (crucibles?/moulds? maybe related), SAV1W } \\
0476 \text { (whetstone), SAV1W } 0477 \text { (hand mill) }\end{array}$ & 04.02 .2014 \\
\hline $1223 / 2014$ & SQ1 & $\begin{array}{l}\text { pottery, among it lid or stopper ( } 1 \text { piece, mud), window grille? } \\
\text { ( } 1 \text { fragment, Christian, pottery) }\end{array}$ & 555 & $\begin{array}{l}\text { SAV1W } 0461 \text { (lid or stopper), SAV1W } 0502 \\
\text { (window grille?) }\end{array}$ & 05.02 .2014 \\
\hline $1241 / 2014$ & SQ1, cleaning section & pottery & 555 & & 05.02 .2014 \\
\hline $1256 / 2014$ & SQ1 & $\begin{array}{l}\text { pottery, faience and stone: weight? ( } 1 \text { re-used sherd, pottery), lid } \\
\left(1 \text { re-used } 18^{\text {th }} \text { Dynasty sherd, pottery); faience vessel ( } 1 \text { body }\right. \\
\text { sherd, nun bowl); grindstone (1 piece, quartzite) }\end{array}$ & 556 & $\begin{array}{l}\text { SAV1W } 0522 \text { (weight?), SAV1W } 0523 \text { (lid), } \\
\text { SAV1W } 0528 \text { (faience vessel), SAV1W } 0520 \\
\text { (grindstone) }\end{array}$ & 06.02 .2014 \\
\hline
\end{tabular}




\begin{tabular}{|c|c|c|c|c|c|}
\hline $1219 / 2014$ & SQ1 & $\begin{array}{l}\text { pottery; worked stone/part of a stone vessel? (1 fragment, } \\
\text { sandstone) }\end{array}$ & 556 & SAV1W 0482 (worked stone) & 04.02 .2014 \\
\hline $1212 / 2014$ & SQ1W & pottery & 557 & & 04.02 .2014 \\
\hline $1214 / 2014$ & SQ1NW & pottery & 558 & & 04.02 .2014 \\
\hline $1225 / 2014$ & SQ1 & $\begin{array}{l}\text { pottery, among it scraper ( } 1 \text { re-used } 18^{\text {th }} \text { Dynasty sherd, } \\
\text { pottery), figurine ( } 1 \text { fragment, animal figurine, horse?, clay) }\end{array}$ & 559 & $\begin{array}{l}\text { SAV1W } 0497 \text { (scraper), SAV1W } 0500 \\
\text { (figurine) }\end{array}$ & 05.02 .2014 \\
\hline $1227 / 2014$ & SQ1 & pottery & 560 & & 05.02 .2014 \\
\hline $1281 / 2014$ & SQ1 & pottery & 561 & & 06.02 .2014 \\
\hline $1237 / 2014$ & SQ1W & pottery, among it lid (1 re-used $18^{\text {th }}$ Dynasty? sherd, pottery) & 561 & SAV1W 0495 & 05.02 .2014 \\
\hline $1236 / 2014$ & SQ1W, cleaning section & pottery & 561 & & 05.02 .2014 \\
\hline $1242 / 2014$ & SQ1, cleaning section & pottery & 562 & & 05.02 .2014 \\
\hline $1247 / 2014$ & SQ1 & $\begin{array}{l}\text { pottery, among it re-used sherd (1 piece, disc-shaped, } \\
18^{\text {th }} \text { Dynasty?, pottery), vessel or slag? ( } 1 \text { piece of highly } \\
\text { overfired slag or sherd), figurine? (1 highly overfired fragment, } \\
\text { rudimentary human figurine?, clay), oven?/crucible? } \\
\text { (1 fragment, mud), crucibles ( } 2 \text { pieces, each fragmented, mud) }\end{array}$ & 563 & $\begin{array}{l}\text { SAV1W } 0494 \text { (re-used sherd), vessel or slag? } \\
\text { (SAV1W 0498), figurine? (SAV1W 0499), } \\
\text { SAV1W 0478 (oven?/crucible?), SAV1W } \\
\text { 0479, SAV1W 0480 (crucibles) }\end{array}$ & 05.02 .2014 \\
\hline $1301 / 2014$ & SQ1 & pottery & 563 & & 08.02 .2014 \\
\hline $1254 / 2014$ & SQ1NW & pottery & 564 & & 06.02.2014 \\
\hline $1312 / 2014$ & SQ1 & pottery & 565 & & 08.02.2014 \\
\hline $1258 / 2014$ & SQ1 & $\begin{array}{l}\text { pottery, among it net weight ( } 1 \text { re-used } 18^{\text {th }} \text { Dynasty sherd, } \\
\text { pottery); slag ( } 1 \text { piece); charcoal ( } 1 \text { bag })\end{array}$ & 565 & $\begin{array}{l}\text { SAV1W } 0481 \text { (slag), SAV1W } 0496 \text { (net } \\
\text { weight) }\end{array}$ & 06.02 .2014 \\
\hline $1280 / 2014$ & SQ1, cleaning section & pottery & 565 & & 06.02 .2014 \\
\hline $1272 / 2014$ & SQ1 & pottery & 566 & & 06.02 .2014 \\
\hline $1282 / 2014$ & SQ1 & pottery & 567 & & 08.02 .2014 \\
\hline $1292 / 2014$ & SQ1 & pottery & 569 & & 08.02 .2014 \\
\hline $1299 / 2014$ & $\mathrm{SQ} 1,8.6 \mathrm{~m} \mathrm{~S}-\mathrm{N} / 5.4 \mathrm{~m} \mathrm{E}-\mathrm{W}$ & pottery (decorated) & 569 & & 08.02 .2014 \\
\hline $1302 / 2014$ & SQ1 & pottery & 570 & & 08.02 .2014 \\
\hline $1314 / 2014$ & SQ1 & pottery & 571 & & 08.02 .2014 \\
\hline $1318 / 2014$ & SQ1 & pottery, crucible? ( 2 fragments, clay) & 572 & SAV1W 0577 (crucible) & 09.02.2014 \\
\hline $1320 / 2014$ & $\mathrm{SQ} 1,0-4 \mathrm{~m} \mathrm{~S}-\mathrm{N} / 3-5 \mathrm{~m} \mathrm{E}-\mathrm{W}$ & pottery & 572 & & 09.02 .2014 \\
\hline $917 / 2014$ & SQ2, cleaning square & pottery & 573 & & 10.02 .2014 \\
\hline $919 / 2014$ & SQ2 & pottery & 574 & & 10.02 .2014 \\
\hline $925 / 2014$ & SQ2 & pottery & 576 & & 10.02 .2014 \\
\hline $948 / 2014$ & SQ2 & pottery & 576 & & 11.02.2014 \\
\hline $932 / 2014$ & SQ2 & pottery & 577 & & 10.02 .2014 \\
\hline
\end{tabular}




\begin{tabular}{|c|c|c|c|c|c|}
\hline $947 / 2014$ & SQ2 & pottery & 577 & & 11.02.2014 \\
\hline $938 / 2014$ & SQ2 & pottery & 578 & & 10.02 .2014 \\
\hline $941 / 2014$ & SQ2 & pottery & 578 & & 11.02 .2014 \\
\hline $943 / 2014$ & SQ2, Feature 111 & $\begin{array}{l}\text { pottery, among it architecture/window grille (1 fragment, } \\
\text { Christian, clay) }\end{array}$ & 579 & SAV1W 0587 (architecture/window grille) & 11.02.2014 \\
\hline $962 / 2014$ & SQ2 & pottery & 581 & & 11.02 .2014 \\
\hline $963 / 2014$ & SQ2 & pottery & 582 & & 11.02.2014 \\
\hline $964 / 2014$ & SQ2 & pottery & 583 & & 11.02 .2014 \\
\hline $1331 / 2014$ & SQ1 & pottery & 584 & & 11.02 .2014 \\
\hline $1333 / 2014$ & SQ1 & $\begin{array}{l}\text { pottery, among it lid or scraper (1 re-used sherd), figurine? } \\
\text { (1 fragment, Post-New Kingdom - Christian?, clay), } \\
\text { architecture/window grille? (1 fragment, Christian, clay) }\end{array}$ & 584 & $\begin{array}{l}\text { SAV1W } 0561 \text { (lid or scraper), SAV1W } 0573 \\
\text { (figurine?), SAV1W } 0588 \text { (architecture/ } \\
\text { window grille?) }\end{array}$ & 12.02.2014 \\
\hline $1334 / 2014$ & SQ1 & $\begin{array}{l}\text { pottery, among it lid ( } 1 \text { fragment, mud), re-used sherd ( } 1 \text { re-used } \\
18^{\text {th }} \text { Dynasty sherd, pottery), scraper ( } 1 \text { re-used Post-New King- } \\
\left.\text { dom sherd, pottery), scraper? ( } 1 \text { re-used } 18^{\text {th }} \text { Dynasty sherd }\right)\end{array}$ & 585 & $\begin{array}{l}\text { SAV1W 0558 (lid), SAV1W } 0563 \text { (re-used } \\
\text { sherd), SAV1W } 0564 \text { (scraper), SAV1W } 0580 \\
\text { (scraper?) }\end{array}$ & 12.02.2014 \\
\hline $967 / 2014$ & SQ2 & pottery & 586 & & 12.02 .2014 \\
\hline $971 / 2014$ & SQ2 & pottery & 586 & & 13.02 .2014 \\
\hline $1349 / 2014$ & SQ1 & pottery & 587 & & 12.02 .2014 \\
\hline $1358 / 2014$ & SQ1 & $\begin{array}{l}\text { pottery, among it scraper ( } 1 \text { re-used } 18^{\text {th }} \text { Dynasty sherd, } \\
\text { pottery), stopper or lid ( } 1 \text { fragment, mud) }\end{array}$ & 587 & $\begin{array}{l}\text { SAV1W } 0584 \text { (scraper), SAV1W } 0569 \\
\text { (stopper or lid) }\end{array}$ & 13.02.2014 \\
\hline $1378 / 2014$ & SQ1, 0-1m N-S/5-6 E-W & pottery & 587 & & 13.02 .2014 \\
\hline $1374 / 2014$ & $\mathrm{SQ} 1,2-3.5 \mathrm{~m} \mathrm{~N}-\mathrm{S} / 4-5.5 \mathrm{~m} \mathrm{E}-\mathrm{W}$ & pottery & 587 & & 13.02 .2014 \\
\hline $1350 / 2014$ & SQ1 & pottery & 588 & & 12.02 .2014 \\
\hline $1368 / 2014$ & SQ1 & pottery, among it lid (1 piece, mud) & 588 & SAV1W 0568 & 13.02.2014 \\
\hline $969 / 2014$ & SQ2 & pottery & 589 & & 12.02.2014 \\
\hline $1357 / 2014$ & SQ1 & $\begin{array}{l}\text { pottery among it scraper (1 re-used Post-New Kingdom sherd, } \\
\text { pottery), }\end{array}$ & 590 & SAV1W 0579 & 13.02 .2014 \\
\hline $973 / 2014$ & SQ2 & pottery & 591 & & 13.02 .2014 \\
\hline $1078 / 2014$ & $\begin{array}{l}\mathrm{SQ1NW} 4-5 \mathrm{~m} \mathrm{~N}-\mathrm{S} / 5-6 \mathrm{~m} \mathrm{E}-\mathrm{W} \text {, debris } \\
\text { layer against remains of wall }\end{array}$ & pottery & 592 & & 29.01.2014 \\
\hline $728 / 2014$ & $\begin{array}{l}\text { SQ1, adjacent to Feature } 100 \text { from E, } \\
3-5 \mathrm{~m} \mathrm{E}-\mathrm{W} / 0-5 \mathrm{~m} \text { S-N }\end{array}$ & ropes (6 pieces, woven together, organic fibre) & 501 & SAV1W 0188 & 20.01.2014 \\
\hline $715 / 2014$ & SQ1W, W-half & rope (1 piece, organic fibre) & 501 & SAV1W 0187 & 19.01.2014 \\
\hline $545 / 2014$ & SQ1, S of SQ1 & rope? (several pieces) & 503 & SAV1W 0056 & 07.01 .2014 \\
\hline $1071 / 2014$ & $\mathrm{SQ} 1,2.30-4.1 \mathrm{~m}$ E-W/0-5m S-N & rope (4 pieces, organic fibre) & 507 & & 29.01 .2014 \\
\hline $1101 / 2014$ & $\mathrm{SQ} 1,4-5 \mathrm{~m}$ E-W/0-5m S-N & rope (several pieces, organic fibre) & 507 & SAV1W 0377 & 30.01 .2014 \\
\hline
\end{tabular}




\begin{tabular}{|c|c|c|c|c|c|}
\hline $809 / 2014$ & SQ2 & rope (1 piece), linen (1 piece) & 514 & SAV1W 0122 & 15.01.2014 \\
\hline $899 / 2014$ & SQ1W & rope (several pieces, organic fibre, with fragments of linen) & 532 & SAV1W 0248 & 22.01.2014 \\
\hline $1244 / 2014$ & SQ1 & rope & 563 & & 05.02 .2014 \\
\hline $1268 / 2014$ & SQ1 & rope (some thin fibres, organic fibre) & 565 & SAV1W 0503 & 06.02 .2014 \\
\hline $970 / 2014$ & SQ2 & rope?fibres of linen/wool? (3 thicker cords) & 589 & SAV1W 0554 & 12.02.2014 \\
\hline $1377 / 2014$ & SQ1 & rope & 590 & & 13.02.2014 \\
\hline $1308 / 2014$ & SQ1, 7.8m S-N/5.4m E-W & scarab, frog shaped (1 piece, head broken off, faience) & 569 & SAV1W 0527 & 08.02 .2014 \\
\hline $800 / 2014$ & SQ1, surface cleaning $+1.5 \mathrm{~m}$ & scraper (1 re-used Post-New Kingdom sherd, pottery) & 500 & SAV1W 0199 & 15.01.2014 \\
\hline $1176 / 2014$ & SQ1 & seashell (1 bag, fragments) & 512 & & 03.02 .2014 \\
\hline $725 / 2014$ & $\begin{array}{l}\text { SQ1, adjacent to Feature } 100 \text { from E, } \\
3-5 \mathrm{~m} \mathrm{E}-\mathrm{W} / 0-5 \mathrm{~m} \mathrm{~S}-\mathrm{N}\end{array}$ & shell & 501 & & 20.01.2014 \\
\hline $1088 / 2014$ & SQ1NW & shell & 501 & & 29.01.2014 \\
\hline $574 / 2014$ & SQ1 & shell & 506 & & 09.01.2014 \\
\hline $1041 / 2014$ & SQ1 & shell & 507 & & 28.01.2014 \\
\hline $754 / 2014$ & SQ1, 0-1m E-W/0-2m S-N & shell & 507 & & 23.01.2014 \\
\hline $748 / 2014$ & SQ1, 0-2m S-N/0-4m E-W & shell & 507 & & 22.01 .2014 \\
\hline $1082 / 2014$ & SQ1, 2.30-4.1m E-W/0-5m S-N & shell & 507 & & 29.01.2014 \\
\hline $603 / 2014$ & SQ1, E-half & shell & 507 & & 12.01.2014 \\
\hline $895 / 2014$ & SQ1W & shell & 530 & & 22.01 .2014 \\
\hline $1205 / 2014$ & SQ1NW $1.2 \mathrm{~m} \mathrm{~N}-\mathrm{S} / 0.8 \mathrm{~m}$ E-W & shell (1 very thin disc-shaped piece) & 552 & SAV1W 0469 & 04.02 .2014 \\
\hline $1239 / 2014$ & SQ1 & shell & 559 & & 05.02 .2014 \\
\hline $1266 / 2014$ & SQ1NW & shell (1 piece, used as bead, 2 perforations) & 564 & SAV1W 0504 & 06.02 .2014 \\
\hline $960 / 2014$ & SQ2 & shell & 580 & & 11.02 .2014 \\
\hline $1359 / 2014$ & SQ1 & shell & 590 & & 13.02.2014 \\
\hline $851 \mathrm{~A} / 2014$ & $\mathrm{SQ} 1 \mathrm{~W}, 1.5 \mathrm{~m} \mathrm{E}-\mathrm{W} / 2 \mathrm{~m} \mathrm{~S}-\mathrm{N}$ & skin (1 piece, animal) & 523 & & 20.01 .2014 \\
\hline $726 / 2014$ & $\begin{array}{l}\text { SQ1, adjacent to Feature } 100 \text { from E, 3- } \\
5 \mathrm{~m} \mathrm{E-W/0-5m} \mathrm{S-N}\end{array}$ & slag (1 piece) & 501 & SAV1W 0195 & 20.01 .2014 \\
\hline $540 / 2014$ & SQ1, NW-corner & slag (1 piece) & 501 & SAV1W 0006 & 07.01 .2014 \\
\hline $510 / 2014$ & SQ2 & slag (2 pieces) & 501 & SAV1W 0010 & 05.01 .2014 \\
\hline $515 / 2014$ & SQ2 & slag (2 pieces) & 501 & SAV1W 0008 & 06.01 .2014 \\
\hline $524 / 2014$ & SQ2, W-half, $5.5 \mathrm{~m}$ from $\mathrm{W}$ & slag (1 piece) & 501 & SAV1W 0009 & 06.01 .2014 \\
\hline $546 / 2014$ & SQ1 & slag (1 piece) & 503 & SAV1W 0003 & 07.01 .2014 \\
\hline $592 / 2014$ & SQ1, E-half & slag (1 piece) & 507 & SAV1W 0038 & 11.01 .2014 \\
\hline
\end{tabular}




\begin{tabular}{|c|c|c|c|c|c|}
\hline $639 / 2014$ & $\mathrm{SQ} 2,3-5 \mathrm{~m} \mathrm{~S}-\mathrm{N} / 0-2 \mathrm{~m} \mathrm{E}-\mathrm{W}$ & slag (1 piece), crucible? (1 fragment, mud); charcoal (1 piece) & 510 & SAV1W 0111 (slag), SAV1W 0203 (crucible) & 14.01 .2014 \\
\hline $636 / 2014$ & $\mathrm{SQ} 2,3-5 \mathrm{~m} \mathrm{~S}-\mathrm{N} / 2-8 \mathrm{~m}$ E-W & slag (1 piece) & 510 & SAV1W 0109 & 14.01.2014 \\
\hline $663 / 2014$ & SQ1, NW-corner, 1-5m W-E/3-5m N-S & slag (1 piece) & 511 & SAV1W 0110 & 14.01.2014 \\
\hline $811 / 2014$ & SQ2 & slag: iron (3 pieces) & 516 & SAV1W 0185 & 15.01 .2014 \\
\hline $903 / 2014$ & SQ1W & slag (1 piece); linen (1 piece); doum nut (1 piece) & 522 & SAV1W 0246 (slag), SAV1W 0247 (linen) & 22.01 .2014 \\
\hline $790 / 2014$ & SQ1W, W-half & slag (1 piece) & 522 & SAV1W 0280 & 25.01 .2014 \\
\hline $874 / 2014$ & SQ1W & $\begin{array}{l}\text { slag (1 piece); linen/cloth (several pieces), linen/cloth (1 large, } 1 \\
\text { small piece) }\end{array}$ & 524 & $\begin{array}{l}\text { SAV1W } 0224 \text { (slag), SAV1W 0226, SAV1W } \\
0229 \text { (linen/cloth) }\end{array}$ & 21.01 .2014 \\
\hline $1198 / 2014$ & SQ1NW, 0-5m S-N/0-2m E-W & slag (2 pieces) & 552 & SAV1W 0451 & 04.02 .2014 \\
\hline $1231 / 2014$ & SQ1 & slag (2 pieces) & 555 & & 05.02 .2014 \\
\hline $1317 / 2014$ & SQ1 & slag (1 large piece) & 571 & SAV1W 0507 & 08.02 .2014 \\
\hline $922 / 2014$ & SQ2 & slag (1 piece) & 575 & SAV1W 0531 & 10.02.2014 \\
\hline $953 / 2014$ & SQ2 & slag? (1 piece) & 577 & SAV1W 0556 & 11.02 .2014 \\
\hline $1346 / 2014$ & SQ1 & slag and brick (1 fragment) & 584 & SAV1W 0557 & 12.02 .2014 \\
\hline $1316 / 2014$ & SAV1W, beside SQ1 (measured) & $\begin{array}{l}\text { stone, worked/artefact ( } 1 \text { fragment with part of an inscription at } \\
\text { one side, unspecified stone) }\end{array}$ & 500 & SAV1W 0545 & 08.02 .2014 \\
\hline $501 / 2014$ & SQ1, surface, $10 \times 10 \mathrm{~m}$ & $\begin{array}{l}\text { stones: pounders ( } 2 \text { pieces, } 1 \text { quartz, } 1 \text { sandstone), whetstones } \\
\text { ( } 3 \text { pieces, each sandstone), grindstones ( } 8 \text { pieces, } 1 \text { sandstone, } \\
7 \text { quartzite), hand mills ( } 7 \text { pieces, each quartzite), worked stones } \\
\text { ( } 3 \text { pieces, each quartzite), pounders ( } 5 \text { pieces, } 3 \text { quartz, } \\
1 \text { sandstone?, } 1 \text { silicified wood), hand mills ( } 2 \text { pieces, each } \\
\text { quartzite), hand mills ( } 2 \text { pieces, each quartzite), hammer } \\
\text { (1 piece, schist), whetstone ( } 1 \text { piece, sandstone), grindstone } \\
\text { (1 piece, quartzite), blade? ( } 1 \text { piece, silex) }\end{array}$ & 500 & $\begin{array}{l}\text { SAV1W 0088, SAV1W 0089 (pounders), } \\
\text { SAV1W 0132, SAV1W 0134, SAV1W 0137 } \\
\text { (whetstones), SAV1W 0092, SAV1W 0131, } \\
\text { SAV1W 0135, SAV1W 0136, SAV1W 0148, } \\
\text { SAV1W 0150, SAV1W 0152, SAV1W 0153 } \\
\text { (grindstones), SAV1W 0090, SAV1W 0091, } \\
\text { SAV1W 0116, SAV1W 0133, SAV1W 0138, } \\
\text { SAV1W 0147, SAV1W 0151 (hand mills), } \\
\text { SAV1W 0130, SAV1W 0139, SAV1W 0149 } \\
\text { (worked stones), SAV1W: SAV1W 0076, } \\
\text { SAV1W 0083, SAV1W 0084, SAV1W 0078, } \\
\text { SAV1W 0082 (pounders), SAV1W 0085, } \\
\text { SAV1W 0075 (hand mills), SAV1W 0081 } \\
\text { (hammer), SAV1W 0077 (whetstone), } \\
\text { SAV1W 0079 (grindstone), SAV1W 0080 } \\
\text { (blade?) }\end{array}$ & 05.01 .2014 \\
\hline $804 / 2014$ & $\begin{array}{l}\text { SQ1W, 0-5m E-W/0-5m S-N, surface } \\
\text { cleaning }\end{array}$ & $\begin{array}{l}\text { stones: grindstone ( } 2 \text { pieces, each quartzite), pounder (1 piece, } \\
\text { siliceous shale?), whetstone ( } 1 \text { piece, sandstone) }\end{array}$ & 500 & $\begin{array}{l}\text { SAV1W 0250, SAV1W } 0283 \text { (grindstones), } \\
\text { SAV1W 0251 (pounder), SAV1W } 0284 \\
\text { (whetstone) }\end{array}$ & 15.01 .2014 \\
\hline $806 / 2014$ & $\begin{array}{l}\text { SQ1W, 5-10m E-W/0-5m S-N, surface } \\
\text { clearing }\end{array}$ & stones: pounder (1 piece, quartz), hammer (1 piece, quartzite) & 500 & $\begin{array}{l}\text { SAV1W } 0169 \text { (pounder), SAV1W } 0170 \\
\text { (hammer) }\end{array}$ & 15.01.2014 \\
\hline $504 / 2014$ & SQ2, surface & $\begin{array}{l}\text { stones: hammer (1 piece, quartzite), hand mill (1 piece, } \\
\text { quartzite) grindstone? (1 piece, quartzite), worked stone (1 half, } \\
\text { gneiss?) }\end{array}$ & 500 & $\begin{array}{l}\text { SAV1W } 0063 \text { (hammer), SAV1W } 0064 \text { (hand } \\
\text { mill), SAV1W } 0065 \text { (grindstone?), SAV1W } \\
0066 \text { (worked stone) }\end{array}$ & 05.01 .2014 \\
\hline
\end{tabular}




\begin{tabular}{|c|c|c|c|c|c|}
\hline $554 / 2014$ & SQ1 & $\begin{array}{l}\text { stones: abrasive stone (1 piece, quartzite), pounder (1 piece, } \\
\text { quartz), worked stone (1 piece, quartzite?) }\end{array}$ & 501 & $\begin{array}{l}\text { SAV1W } 0041 \text { (abrasive stone), SAV1W } 0042 \\
\text { (pounder), SAV1W } 0043 \text { (worked stone) }\end{array}$ & 08.01 .2014 \\
\hline- & S of SQ1, in line with enclosure wall & stone: stela (1 upper right part, sandstone) & 500 & SAV1W 0590 & 01.02 .2014 \\
\hline $588 / 2014$ & SQ1 & $\begin{array}{l}\text { stones: hammer (1 piece, granite), hand mill? ( } 1 \text { piece, } \\
\text { sandstone), grindstone (1 piece, quartzite), blade (1 piece, silex) }\end{array}$ & 501 & $\begin{array}{l}\text { SAV1W } 0068 \text { (hammer), SAV1W } 0140 \text { (hand } \\
\text { mill?), SAV1W } 0141 \text { (grindstone), SAV1W } \\
0067 \text { (blade) }\end{array}$ & 11.01.2014 \\
\hline $573 / 2014$ & SQ1 & $\begin{array}{l}\text { stones: hand mill (1 piece, quartzite), worked stone (1 piece, } \\
\text { quartzite?), stone vessel? (1 unidentified stone object, schist?), } \\
\text { whetstone or grindstone? ( } 1 \text { piece, sandstone) }\end{array}$ & 501 & $\begin{array}{l}\text { SAV1W } 0106 \text { (hand mill), SAV1W } 0107 \\
\text { (worked stone), SAV1W 0108 (stone vessel?), } \\
\text { SAV1W } 0049 \text { (whetstone or grindstone?) }\end{array}$ & 09.01 .2014 \\
\hline $736 / 2014$ & $\begin{array}{l}\text { SQ1, adjacent to Feature } 100 \text { from E, 3- } \\
5 \mathrm{~m} \mathrm{E-W/0-4m} \mathrm{S-N}\end{array}$ & $\begin{array}{l}\text { stones and architecture?: hand mill (1 piece, quartzite), pounder } \\
\text { (1 piece, quartzite); architecture? (1 large block, unspecified } \\
\text { stone) }\end{array}$ & 501 & $\begin{array}{l}\text { SAV1W } 0303 \text { (hand mill), SAV1W } 0304 \\
\text { (pounder), SAV1W } 0305 \text { ( architecture?) }\end{array}$ & 21.01 .2014 \\
\hline $723 / 2014$ & $\begin{array}{l}\text { SQ1, adjacent to Feature } 100 \text { from E, 3- } \\
5 \mathrm{~m} \text { E-W/0-5m S-N }\end{array}$ & stone: grindstone (1 piece, quartzite) & 501 & SAV1W 0272 & 20.01 .2014 \\
\hline $731 / 2014$ & $\begin{array}{l}\text { SQ1, adjacent to Feature } 100 \text { from } \mathrm{E}, 3- \\
5 \mathrm{~m} \mathrm{E}-\mathrm{W} / 0-5 \mathrm{~m} \text { S-N }\end{array}$ & $\begin{array}{l}\text { stones: hand mill ( } 1 \text { piece, sandstone), whetstone (1 piece, } \\
\text { sandstone), pounder ( } 1 \text { piece, silex) }\end{array}$ & 501 & $\begin{array}{l}\text { SAV1W } 0326 \text { (hand mill), SAV1W } 0327 \\
\text { (whetstone), SAV1W 0328 (pounder) }\end{array}$ & 20.01 .2014 \\
\hline $717 / 2014$ & $\begin{array}{l}\text { SQ1, adjacent to Feature } 100 \text { from E, } 4 \\
5 \mathrm{~m} \mathrm{E-W/0-5m} \mathrm{S-N}\end{array}$ & $\begin{array}{l}\text { stones: pounders ( } 3 \text { pieces, each quartz), whetstone ( } 3 \text { pieces, } \\
\text { each sandstone), hand mills ( } 2 \text { pieces, each quartzite) }\end{array}$ & 501 & $\begin{array}{l}\text { SAV1W 0238, SAV1W 0241, SAV1W } 0245 \\
\text { (pounders), SAV1W 0239, SAV1W 0243, } \\
\text { SAV1W 0244 (whetstones), SAV1W 0240, } \\
\text { SAV1W } 0242 \text { (hand mills) }\end{array}$ & 19.01.2014 \\
\hline $521 / 2014$ & SQ1, E-half & $\begin{array}{l}\text { stones: whetstone ( } 1 \text { piece, sandstone), abrasive stone/primarily } \\
\text { rim of stone dish? ( } 1 \text { piece, quartzite), hand mill ( } 1 \text { piece, } \\
\text { quartzite), grindstone ( } 1 \text { piece, quartzite); doum nut ( } 1 \text { piece })\end{array}$ & 501 & $\begin{array}{l}\text { SAV1W } 0098 \text { (whetstone), SAV1W } 0099 \\
\text { (abrasive stone/primarily rim of stone dish?), } \\
\text { SAV1W } 0100 \text { (hand mill), SAV1W } 0101 \\
\text { (grindstone) }\end{array}$ & 06.01 .2014 \\
\hline $519 / 2014$ & SQ1, NW-corner & $\begin{array}{l}\text { stone, worked/grindstone or architecture? (1 fragment, } \\
\text { sandstone) }\end{array}$ & 501 & SAV1W 0143 & 06.01 .2014 \\
\hline $682 / 2014$ & SQ1, SE-corner, 3-5m E-W/0-5m S-N & $\begin{array}{l}\text { stones: pounders ( } 3 \text { pieces, } 1 \text { quartz?, } 1 \text { quartz, } 1 \text { siliceous } \\
\text { shale?) hand mills ( } 2 \text { pieces, } 1 \text { quartzite, } 1 \text { sandstone) }\end{array}$ & 501 & $\begin{array}{l}\text { SAV1W 0177, SAV1W 0178, SAV1W } 0179 \\
\text { (pounders), SAV1W 0180, SAV1W } 0181 \\
\text { (hand mills) }\end{array}$ & 16.01.2014 \\
\hline $698 / 2014$ & SQ1, SE-corner, 4-5m E-W/0-5m S-N & $\begin{array}{l}\text { stones: whetstone ( } 1 \text { piece, sandstone), pounder ( } 1 \text { piece, } \\
\text { quartz), hammer ( } 1 \text { piece, quartzite), grindstone? ( } 1 \text { piece, } \\
\text { sandstone), grindstone ( } 1 \text { piece, quartzite) }\end{array}$ & 501 & $\begin{array}{l}\text { SAV1W } 0285 \text { (whetstone), SAV1W } 0286 \\
\text { (pounder), SAV1W 0287 (hammer), SAV1W } \\
0288 \text { (grindstone?), SAV1W } 0289 \text { (grindstone) }\end{array}$ & 18.01.2014 \\
\hline $517 \mathrm{~A} / 2014$ & SQ1, SW-corner & $\begin{array}{l}\text { stone, slag and pottery: whetstone (1 piece, quartzite); slag } \\
\text { (1 piece) }\end{array}$ & 501 & $\begin{array}{l}\text { SAV1W } 0048 \text { (whetstone), SAV1W } 0005 \\
\text { (slag) }\end{array}$ & 06.01 .2014 \\
\hline $686 / 2014$ & SQ1W, E-half & stone: pounder (1 piece, quartzite) & 501 & SAV1W 0282 & 16.01 .2014 \\
\hline $718 / 2014$ & SQ1W, E-half & $\begin{array}{l}\text { stones: pounders ( } 2 \text { pieces, each quartz), worked stone/stone } \\
\text { vessel ( } 1 \text { fragment, quartzite), hand mill (1 piece, quartzite) }\end{array}$ & 501 & $\begin{array}{l}\text { SAV1W 0273, SAV1W } 0274 \text { (pounders), } \\
\text { SAV1W } 0275 \text { (worked stone/stone vessel), } \\
\text { SAV1W } 0276 \text { (hand mill) }\end{array}$ & 19.01.2014 \\
\hline $700 / 2014$ & SQ1W, E-half & $\begin{array}{l}\text { stones: whetstone ( } 1 \text { piece, quartzite), grindstone (1 piece, } \\
\text { quartzite) }\end{array}$ & 501 & $\begin{array}{l}\text { SAV1W } 0277 \text { (whetstone), SAV1W } 0278 \\
\text { (grindstone) }\end{array}$ & 18.01 .2014 \\
\hline $697 / 2014$ & SQ1W, W-half, $5 \times 5 \mathrm{~m}$ & stone vessel (1 fragment, quartzite) & 501 & SAV1W 0279 & 18.01 .2014 \\
\hline $572 / 2014$ & SQ2 & $\begin{array}{l}\text { stones: grindstone? (1 piece, quartzite), whetstone (1 piece, } \\
\text { sandstone) }\end{array}$ & 501 & $\begin{array}{l}\text { SAV1W } 0104 \text { (grindstone?), SAV1W } 0105 \\
\text { (whetstone) }\end{array}$ & 09.01 .2014 \\
\hline
\end{tabular}




\begin{tabular}{|c|c|c|c|c|c|}
\hline $508 / 2014$ & SQ2 & $\begin{array}{l}\text { stones: hammer (1 piece, quartzite), grindstone (1 piece, } \\
\text { quartzite) }\end{array}$ & 501 & $\begin{array}{l}\text { SAV1W } 0059 \text { (hammer), SAV1W } 0060 \\
\text { (grindstone) }\end{array}$ & 05.01 .2014 \\
\hline $585 / 2014$ & SQ2 & $\begin{array}{l}\text { stones: hand mill (1 piece, quartzite), worked stone (1 piece, } \\
\text { granite?) }\end{array}$ & 501 & $\begin{array}{l}\text { SAV1W } 0171 \text { (hand mill), SAV1W } 0172 \\
\text { (worked stone) }\end{array}$ & 11.01 .2014 \\
\hline $552 / 2014$ & SQ2 & $\begin{array}{l}\text { stones: pounder (1 piece, quartz), hammer (1 piece, quartzite), } \\
\text { grindstone ( } 1 \text { piece, quartzite) }\end{array}$ & 501 & $\begin{array}{l}\text { SAV1W } 0045 \text { (pounder), SAV1W } 0046 \\
\text { (hammer), SAV1W } 0047 \text { (grindstone) }\end{array}$ & 08.01 .2014 \\
\hline $535 / 2014$ & SQ2 & $\begin{array}{l}\text { stones: pounder? (1 piece, quartz), whetstone (1 piece, } \\
\text { sandstone) }\end{array}$ & 501 & $\begin{array}{l}\text { SAV1W } 0102 \text { (pounder?), SAV1W } 0103 \\
\text { (whetstone) }\end{array}$ & 07.01 .2014 \\
\hline $513 / 2014$ & SQ2 & $\begin{array}{l}\text { stones: whetstones ( } 2 \text { pieces, each sandstone), stone tool? } \\
\text { (1 piece, amphibolite) }\end{array}$ & 501 & $\begin{array}{l}\text { SAV1W 0050, SAV1W } 0052 \text { (whetstones), } \\
\text { SAV1W } 0053 \text { (stone tool?) }\end{array}$ & 06.01 .2014 \\
\hline $605 / 2014$ & $\mathrm{SQ} 2,0$ to $7 \mathrm{~m}$ from $\mathrm{E}$ & stones: grindstone? (1 piece, quartzite) & 501 & SAV1W 0062 & 12.01 .2014 \\
\hline $528 / 2014$ & SQ2, $\mathrm{N}$ edge, $6 \mathrm{~m}$ from $\mathrm{W}$ & stones: blade or sickle? (1 piece, silex) & 501 & SAV1W 0154 & 06.01 .2014 \\
\hline $526 / 2014$ & $\mathrm{SQ} 2, \mathrm{~W}$-half, $5.5 \mathrm{~m}$ from $\mathrm{W}$ & $\begin{array}{l}\text { stones: pounder ( } 1 \text { piece, quartz?), hammer (1 piece, quartzite), } \\
\text { whetstones ( } 2 \text { pieces, each sandstone) }\end{array}$ & 501 & $\begin{array}{l}\text { SAV1W } 0094 \text { (pounder), SAV1W } 0095 \\
\text { (hammer), SAV1W 0096, SAV1W } 0097 \\
\text { (whetstones) }\end{array}$ & 06.01 .2014 \\
\hline $820 / 2014$ & SQ2 & stone: grindstone (1 piece, quartzite) & 504 & SAV1W 0281 & 16.01 .2014 \\
\hline $912 / 2014$ & SQ2, sandy area in the $\mathrm{W}$ & stone: hammer (1 piece, quartzite) & 504 & SAV1W 0317 & 23.01 .2014 \\
\hline $599 / 2014$ & SQ2, sandy area in the $\mathrm{W}$ & $\begin{array}{l}\text { stones: grindstone? (1 piece, sandstone), whetstone (1 piece, } \\
\text { sandstone) }\end{array}$ & 504 & $\begin{array}{l}\text { SAV1W } 0069 \text { (grindstone?), SAV1W } 0070 \\
\text { (whetstone) }\end{array}$ & 12.01.2014 \\
\hline $569 / 2014$ & SQ1, debris layer in the SW & stone: pumice stone? (1 piece) & 506 & SAV1W 0026 & 09.01 .2014 \\
\hline $755 / 2014$ & SQ1, 0-1m E-W/0-2m S-N & $\begin{array}{l}\text { stones: pounders ( } 3 \text { pieces, } 1 \text { quartzite, } 2 \text { quartz), grindstone } \\
\text { (1 piece, quartzite) }\end{array}$ & 507 & $\begin{array}{l}\text { SAV1W 0342, SAV1W 0343, SAV1W } 0344 \\
\text { (pounders), SAV1W } 0345 \text { (grindstone) }\end{array}$ & 23.01.2014 \\
\hline $741 / 2014$ & SQ1, 0-2m S-N/0-4m E-W & stones: grindstone (1 piece, quartzite), pounder (1 piece, quartz) & 507 & $\begin{array}{l}\text { SAV1W } 0311 \text { (grindstone), SAV1W } 0312 \\
\text { (pounder) }\end{array}$ & 21.01 .2014 \\
\hline $747 / 2014$ & $\mathrm{SQ} 1,0-2 \mathrm{~m} \mathrm{~S}-\mathrm{N} / 0-4 \mathrm{~m} \mathrm{E}-\mathrm{W}$ & $\begin{array}{l}\text { stones: hand mill (1 piece, quartzite), hammer (1 piece, } \\
\text { quartzite), pounder ( } 1 \text { piece, unknown stone), } \\
\text { whetstone/abrasive stone ( } 1 \text { piece, sandstone) }\end{array}$ & 507 & $\begin{array}{l}\text { SAV1W } 0306 \text { (hand mill), SAV1W } 0307 \\
\text { (hammer), SAV1W 0308 (pounder), } \\
\text { SAV1W 0310 (whetstone/abrasive stone) }\end{array}$ & 22.01 .2014 \\
\hline $1121 / 2014$ & $\mathrm{SQ} 1,2.30-4 \mathrm{~m} \mathrm{E}-\mathrm{W} / 0-5 \mathrm{~m} \mathrm{~S}-\mathrm{N}$ & stone: grindstone (1 piece, sandstone) & 507 & SAV1W 0417 & 30.01 .2014 \\
\hline $1064 / 2014$ & $\mathrm{SQ} 1,2.30-4.1 \mathrm{~m} \mathrm{E}-\mathrm{W} / 0-5 \mathrm{~m} \mathrm{~S}-\mathrm{N}$ & $\begin{array}{l}\text { stone, slag, pottery: whetstone (1 piece, sandstone); slag } \\
\text { (1 piece); crucible? ( } 1 \text { fragment with copper?, clay) }\end{array}$ & 507 & $\begin{array}{l}\text { SAV1W } 0389 \text { (whetstone), SAV1W } 0372 \\
\text { (slag), SAV1W } 0373 \text { (crucible?) }\end{array}$ & 29.01.2014 \\
\hline $1100 / 2014$ & SQ1, 4-5m E-W/0-5m S-N & $\begin{array}{l}\text { stones and wood: hand mill ( } 1 \text { piece, quartzite), whetstone } \\
\text { (1 piece, sandstone), grindstone (1 piece, quartzite); wood } \\
\text { ( } 2 \text { pieces) }\end{array}$ & 507 & $\begin{array}{l}\text { SAV1W } 0404 \text { (hand mill), SAV1W } 0405 \\
\text { (whetstone), SAV1W } 0406 \text { (grindstone) }\end{array}$ & 30.01 .2014 \\
\hline $597 / 2014$ & SQ1, E-half & $\begin{array}{l}\text { stones and architecture: weight/anchor (1 piece, sandstone), } \\
\text { hammer (1 piece, quartzite), whetstone (1 piece, sandstone), } \\
\text { hand mill (1 piece, quartzite); column base (1 fragment, } \\
\text { sandstone) }\end{array}$ & 507 & $\begin{array}{l}\text { SAV1W } 0071 \text { (weight/anchor), SAV1W } 0072 \\
\text { (hammer), SAV1W } 0073 \text { (whetstone), } \\
\text { SAV1W } 0074 \text { (hand mill), } \\
\text { SAV1W } 0146 \text { (architecture) }\end{array}$ & 12.01 .2014 \\
\hline $625 / 2014$ & SQ1, NE-corner, 0-4m E-W/0-5m N-S & $\begin{array}{l}\text { stones: pounders ( } 3 \text { pieces, } 1 \text { quartz, } 1 \text { quartzite, } 1 \text { siliceous } \\
\text { shale?) grindstone ( } 1 \text { piece, quartzite), abrasive stone (1 piece, } \\
\text { sandstone) }\end{array}$ & 507 & $\begin{array}{l}\text { SAV1W 0252, SAV1W 0253, SAV1W } 0254 \\
\text { (pounders), SAV1W } 0255 \text { (grindstone), } \\
\text { SAV1W 0256 (abrasive stone) }\end{array}$ & 13.01.2014 \\
\hline $614 / 2014$ & $\mathrm{SQ} 2,0-5 \mathrm{~m} \mathrm{E}-\mathrm{W} / 2.5-5 \mathrm{~m} \mathrm{~S}-\mathrm{N}$ & $\begin{array}{l}\text { stones: hand mill (1 piece, quartzite), grindstone (1 piece, } \\
\text { quartzite) }\end{array}$ & 508 & $\begin{array}{l}\text { SAV1W } 0117 \text { (hand mill), SAV1W } 0119 \\
\text { (grindstone) }\end{array}$ & 12.01 .2014 \\
\hline
\end{tabular}




\begin{tabular}{|c|c|c|c|c|c|}
\hline $610 / 2014$ & SQ2, $N$ of SQ2, 5 to $10 \mathrm{~m}$ from $\mathrm{E}$ & stone & 508 & & 12.01 .2014 \\
\hline $611 / 2014$ & SQ2, NE of SQ2 & stones: hand mill (1 piece, quartzite); doum nut (1 piece) & 508 & SAV1W 0174 (hand mill) & 12.01 .2014 \\
\hline $608 / 2014$ & SQ2, NE of SQ2 & $\begin{array}{l}\text { stones: whetstone (1 piece, sandstone), hand mill (1 piece, } \\
\text { quartzite) }\end{array}$ & 508 & $\begin{array}{l}\text { SAV1W } 0086 \text { (whetstone), SAV1W } 0087 \\
\text { (hand mill) }\end{array}$ & 12.01 .2014 \\
\hline $629 / 2014$ & SQ2 & stone, worked (1 piece, granite?) & 510 & SAV1W 0387 & 14.01 .2014 \\
\hline $635 / 2014$ & SQ2 & $\begin{array}{l}\text { stone: weight (1 half, semi-spherical shaped, with perforation, } \\
\text { sandstone) }\end{array}$ & 510 & SAV1W 0212 & 13.01 .2014 \\
\hline $1173 / 2014$ & SQ1 & $\begin{array}{l}\text { stones: pounders ( } 3 \text { pieces, } 2 \text { quartz, } 1 \text { siliceous shale?), } \\
\text { polishing stone ( } 1 \text { piece, diorite?), whetstones ( } 2 \text { pieces, } 1 \\
\text { sandstone, } 1 \text { quartzite), hand mills ( } 2 \text { pieces, each quartzite), } \\
\text { grindstone (1 piece, quartzite) }\end{array}$ & 512 & $\begin{array}{l}\text { SAV1W 0510, SAV1W 0511, SAV1W } 0512 \\
\text { (pounders), SAV1W 0513 (polishing stone), } \\
\text { SAV1W 0514, SAV1W } 0515 \text { (whetstones), } \\
\text { SAV1W 0535, SAV1W } 0536 \text { (hand mills), } \\
\text { SAV1W 0537 (grindstone) }\end{array}$ & 03.02 .2014 \\
\hline $1157 / 2014$ & SQ1 & $\begin{array}{l}\text { stones: pounders ( } 3 \text { pieces, each quartz), hand mill (1 piece, } \\
\text { quartzite) }\end{array}$ & 512 & $\begin{array}{l}\text { SAV1W 0602, SAV1W 0603, SAV1W } 0604 \\
\text { (pounders), SAV1W } 0605 \text { (hand mill) }\end{array}$ & 02.02 .2014 \\
\hline $661 / 2014$ & SQ1, SW-corner, 2-5m S-N/0-5m W-E & $\begin{array}{l}\text { stones: whetstone (1 piece, sandstone), grindstone (1 piece, } \\
\text { sandstone) }\end{array}$ & 513 & $\begin{array}{l}\text { SAV1W } 0175 \text { (whetstone), SAV1W } 0176 \\
\text { (grindstone) }\end{array}$ & 14.01 .2014 \\
\hline $829 / 2014$ & SQ2 & stone: pounder (1 piece, quartz) & 514 & SAV1W 0173 & 16.01 .2014 \\
\hline $822 / 2014$ & SQ2 & stone object/basin?/rim? (1 fragment, sandstone) & 516 & SAV1W 0388 & 16.01 .2014 \\
\hline $841 / 2014$ & SQ1W & stone: pounder (1 piece, silicified wood) & 521 & SAV1W 0237 & 20.01 .2014 \\
\hline $792 / 2014$ & SQ1W & $\begin{array}{l}\text { stones: grindstone (1 piece, quartzite), pounders ( } 2 \text { pieces, each } \\
\text { quartz) }\end{array}$ & 522 & $\begin{array}{l}\text { SAV1W } 0336 \text { (grindstone), SAV1W } 0337 \text {, } \\
\text { SAV1W } 0338 \text { (pounders) }\end{array}$ & 25.01 .2014 \\
\hline $861 / 2014$ & SQ1W & $\begin{array}{l}\text { stones: hand mill (1 piece, quartzite), grindstone ( } 1 \text { piece, } \\
\text { quartzite), whetstone ( } 1 \text { piece, sandstone), pounder ( } 1 \text { piece, } \\
\text { quartz) }\end{array}$ & 522 & $\begin{array}{l}\text { SAV1W } 0299 \text { (hand mill), SAV1W } 0300 \\
\text { (grindstone), SAV1W 0301 (whetstone), } \\
\text { SAV1W 0302 (pounder) }\end{array}$ & 21.01 .2014 \\
\hline $888 / 2014$ & SQ1W & $\begin{array}{l}\text { stones: pounder (1 piece, silex?), whetstone (1 piece, } \\
\text { sandstone), worked stone ( } 1 \text { piece, quartzite) }\end{array}$ & 522 & $\begin{array}{l}\text { SAV1W 0314 (pounder), SAV1W } 0315 \\
\text { (whetstone), SAV1W 0316 (worked stone) }\end{array}$ & 22.01 .2014 \\
\hline $837 / 2014$ & SQ1W, 5-10m E-W & $\begin{array}{l}\text { stones and slag: pounder (1 piece, siliceous shale?), whetstone } \\
\text { (1 fragment, sandstone); slag (1 piece) }\end{array}$ & 522 & $\begin{array}{l}\text { SAV1W } 0333 \text { (pounder), SAV1W } 0334 \\
\text { (whetstone), SAV1W } 0335 \text { (slag) }\end{array}$ & 20.01 .2014 \\
\hline $907 / 2014$ & SQ1W, W-half & stone: pivot stone? (1 piece, sandstone) & 522 & SAV1W 0340 & 23.01 .2014 \\
\hline $791 / 2014$ & SQ1W, W-half & $\begin{array}{l}\text { stones: whetstone (1 piece, sandstone), grindstone (1 piece, } \\
\text { quartzite), pounder? ( } 1 \text { piece, quartzite?) }\end{array}$ & 522 & $\begin{array}{l}\text { SAV1W } 0330 \text { (whetstone), SAV1W } 0331 \\
\text { (grindstone), SAV1W } 0332 \text { (pounder?) }\end{array}$ & 25.01 .2014 \\
\hline $849 / 2014$ & SQ1W & stone: hammer (1 piece, dolerite?); bone & 523 & SAV1W 0319 & 20.01 .2014 \\
\hline $858 / 2014$ & SQ1W & stone: raw material ( 3 pieces, carnelian? maybe flakes) & 524 & SAV1W 0198 & 20.01 .2014 \\
\hline $865 / 2014$ & SQ1W & stone: hand mill (1 piece, quartzite) & 524 & SAV1W 0318 & 21.01 .2014 \\
\hline $1089 / 2014$ & $\mathrm{SQ} 1,4-4.5 \mathrm{~m} \mathrm{~N}-\mathrm{S} / 4.5-5 \mathrm{~m} \mathrm{E}-\mathrm{W}$ & $\begin{array}{l}\text { stones and architecture?: grindstone ( } 1 \text { piece, sandstone), } \\
\text { pounder ( } 1 \text { piece, silicified wood); architecture?door } \\
\text { hinge?/worked stone (1 fragment, sandstone) }\end{array}$ & 526 & $\begin{array}{l}\text { SAV1W } 0951 \text { (grindstone), SAV1W } 0952 \\
\text { (pounder), SAV1W } 0953 \text { (architecture?door } \\
\text { hinge?/worked stone) }\end{array}$ & 29.01 .2014 \\
\hline $883 / 2014$ & SQ1W & stones: grindstones (2 pieces, each quartzite); slag (1 piece) & 527 & $\begin{array}{l}\text { SAV1W 0324, SAV1W } 0325 \text { (grindstones), } \\
\text { SAV1W } 0225 \text { (slag) }\end{array}$ & 21.01 .2014 \\
\hline $1253 / 2014$ & SQ1W & stone: pounder (1 piece, quartz) & 529 & SAV1W 0483 & 05.02 .2014 \\
\hline
\end{tabular}




\begin{tabular}{|c|c|c|c|c|c|}
\hline $759 / 2014$ & SQ1, 0-5m W-E/0-5m N-S & stone: whetstone (1 piece, sandstone) & 533 & SAV1W 0298 & 23.01 .2014 \\
\hline $771 / 2014$ & SQ1, 5-10m E-W/5-7m N-S & stone: pounder (1 piece, unknown stone) & 536 & SAV1W 0313 & 25.01 .2014 \\
\hline $1027 / 2014$ & SQ1, 0-3m E-W/0-5m N-S & stone: hand mill (1 piece, quartzite) & 537 & SAV1W 0369 & 27.01.2014 \\
\hline $1002 / 2014$ & SQ1W & $\begin{array}{l}\text { stones: worked stone (1 piece, unspecified stone), hand mill } \\
\text { (1 piece, quartzite) }\end{array}$ & 539 & $\begin{array}{l}\text { SAV1W } 0355 \text { (worked stone), SAV1W } 0356 \\
\text { (hand mill) }\end{array}$ & 26.01 .2014 \\
\hline $1021 / 2014$ & SQ1W & $\begin{array}{l}\text { stone and slag: pounder (1 pounder, silicified wood); slag? } \\
\text { (1 piece) }\end{array}$ & 540 & $\begin{array}{l}\text { SAV1W } 0370 \text { (pounder), SAV1W } 0374 \\
\text { (slag?) }\end{array}$ & 27.01.2014 \\
\hline $1165 / 2014$ & SQ1 & stone: grindstone (1 piece, quartzite) & 541 & SAV1W 0416 & 29.01 .2014 \\
\hline $1044 / 2014$ & SQ1W & $\begin{array}{l}\text { stone and architecture: hand mill? (1 piece, quartzite); door } \\
\text { hinge/architecture (1 fragment, sandstone) }\end{array}$ & 542 & $\begin{array}{l}\text { SAV1W } 0365 \text { (hand mill?), SAV1W } 0366 \\
\text { (door hinge/architecture) }\end{array}$ & 28.01.2014 \\
\hline $1158 / 2014$ & SQ1NW & $\begin{array}{l}\text { stone and wood: grindstone (1 piece, quartzite); worked wood } \\
\text { (1 fragment, horn?shaped) }\end{array}$ & 545 & $\begin{array}{l}\text { SAV1W } 0426 \text { (grindstone), SAV1W } 0567 \\
\text { (worked wood) }\end{array}$ & 02.02 .2014 \\
\hline $1097 / 2014$ & SQ1NW & $\begin{array}{l}\text { stones: hand mills ( } 4 \text { pieces, } 1 \text { sandstone, } 3 \text { quartzite), pounders } \\
(2 \text { pieces, } 1 \text { siliceous shale, } 1 \text { quartz), grindstone ( } 1 \text { piece, } \\
\text { quartzite), hammer/pounder ( } 1 \text { piece, quartzite) }\end{array}$ & 545 & $\begin{array}{l}\text { SAV1W 0396, SAV1W 0398, SAV1W 0399, } \\
\text { SAV1W } 0400 \text { (hand mills), SAV1W 0402, } \\
\text { SAV1W } 0403 \text { (pounders), SAV1W } 0397 \\
\text { (grindstone), SAV1W 0401 (hammer/pounder) }\end{array}$ & 30.01 .2014 \\
\hline $1156 / 2014$ & SQ1 & $\begin{array}{l}\text { stones and slag: stone objects ( } 3 \text { pieces, each quartzite, original } \\
\text { functions unknown), grindstone? (1 piece, quartzite), grindstone } \\
\text { (1 piece, quartzite); slag ( } 1 \text { large fragment) }\end{array}$ & 547 & $\begin{array}{l}\text { SAV1W 0670, SAV1W 0669, SAV1W 0668 } \\
\text { (stone objects), SAV1W 0666 (grindstone?), } \\
\text { SAV1W } 0667 \text { (grindstone), SAV1W 0671 } \\
\text { (slag) }\end{array}$ & 02.02 .2014 \\
\hline $1175 / 2014$ & SQ1 & $\begin{array}{l}\text { stones: grindstones ( } 3 \text { pieces, each quartzite), hand mills } \\
\text { ( } 4 \text { pieces, each quartzite), pounder ( } 1 \text { piece, quartz), whetstones } \\
\text { ( } 2 \text { pieces, } 1 \text { phyllite, } 1 \text { sandstone) }\end{array}$ & 547 & $\begin{array}{l}\text { SAV1W 0466, SAV1W 0457, SAV1W } 0459 \\
\text { (grindstones), SAV1W 0462, SAV1W 0464, } \\
\text { SAV1W 0465, SAV1W 0458 (hand mills), } \\
\text { SAV1W } 0463 \text { (pounder), SAV1W 0467, } \\
\text { SAV1W } 0460 \text { (whetstones) }\end{array}$ & 03.02 .2014 \\
\hline $1120 / 2014$ & SQ1, 4-7m S-N & stone: grindstone (1 piece, quartzite) & 547 & SAV1W 0407 & 30.01 .2014 \\
\hline $1118 / 2014$ & $\mathrm{SQ} 1,4-7 \mathrm{~m} \mathrm{~S}-\mathrm{N}$ & $\begin{array}{l}\text { stone: token/gaming piece? (1 cylindrical-conical shaped } \\
\text { artefact, unspecified stone) }\end{array}$ & 547 & SAV1W 0391 & 30.01 .2014 \\
\hline $1138 / 2014$ & SQ1, 7-10m S-N/6-10m E-W & $\begin{array}{l}\text { stones: grindstones ( } 2 \text { pieces, each quartzite), hand mill (1 piece, } \\
\text { quartzite) }\end{array}$ & 547 & $\begin{array}{l}\text { SAV1W 0418, SAV1W } 0419 \text { (grindstones), } \\
\text { SAV1W } 0420 \text { (hand mill) }\end{array}$ & 01.02 .2014 \\
\hline $1139 / 2014$ & SQ1 & $\begin{array}{l}\text { stones: pounders ( } 3 \text { pieces, } 2 \text { quartz, } 1 \text { siliceous shale?), hand } \\
\text { mill (1 piece, quartzite), stone vessel ( } 1 \text { fragment, granite?) }\end{array}$ & 548 & $\begin{array}{l}\text { SAV1W 0413, SAV1W 0453, SAV1W } 0454 \\
\text { (pounders), SAV1W 0456 (hand mill), } \\
\text { SAV1W 0455 (stone vessel) }\end{array}$ & 01.02 .2014 \\
\hline $1133 / 2014$ & SQ1 & $\begin{array}{l}\text { stones: whetstone ( } 1 \text { piece, sandstone), hand mill ( } 1 \text { piece, } \\
\text { quartzite), grindstone? ( } 1 \text { piece, quartzite), grindstone ( } 1 \text { piece, } \\
\text { quartzite) }\end{array}$ & 549 & $\begin{array}{l}\text { SAV1W } 0421 \text { (whetstone), SAV1W } 0422 \\
\text { (hand mill), SAV1W 0423 (grindstone?), } \\
\text { SAV1W 0424 (grindstone) }\end{array}$ & 01.02 .2014 \\
\hline $1200 / 2014$ & SQ1NW + W & $\begin{array}{l}\text { stones and architecture: hand mills ( } 4 \text { pieces, } 1 \text { quartzite, } \\
3 \text { sandstone), pounder ( } 1 \text { piece, quartz), grindstone ( } 1 \text { pieces, } \\
\text { quartzite), stone object ( } 1 \text { small fragment, original function } \\
\text { unknown, unspecified stone), whetstones ( } 2 \text { pieces, each } \\
\text { sandstone); door hinge?/architecture ( } 1 \text { worked stone, } \\
\text { sandstone), }\end{array}$ & 552 & $\begin{array}{l}\text { SAV1W 0484, SAV1W 0485, SAV1W 0486, } \\
\text { SAV1W 0487 (hand mills), SAV1W } 0489 \\
\text { (pounder), SAV1W 0490 (grindstone), } \\
\text { SAV1W 0686 (stone object), SAV1W 0684, } \\
\text { SAV1W 0685 (whetstones), SAV1W } 0488 \\
\text { (door hinge?/architecture) }\end{array}$ & 04.02 .2014 \\
\hline
\end{tabular}




\begin{tabular}{|c|c|c|c|c|c|}
\hline $1199 / 2014$ & SQ1NW, 1m N-S/1.2m E-W & $\begin{array}{l}\text { stone: scraper (small semi-spherical shaped stone, chert - } \\
\text { radiolarith) }\end{array}$ & 552 & SAV1W 0471 & 04.02 .2014 \\
\hline $1206 / 2014$ & SQ1 & $\begin{array}{l}\text { stones: pounders ( } 7 \text { pieces, } 5 \text { quartz, } 1 \text { flint, siliceous shale?), } \\
\text { grindstones ( } 4 \text { pieces, each quartzite), abrasive stone/hand mill } \\
\text { (1 piece, quartzite), hand mill ( } 1 \text { piece, quartzite), pestle( } 1 \text { piece, } \\
\text { quartz), polishing stone ( } 1 \text { piece, siliceous shale?), whetstone } \\
\text { (1 piece, sandstone), stone objects? ( } 3 \text { pieces, each fragments, } \\
\text { quartzite, function unknown), stone object ( } 1 \text { fragment, function } \\
\text { unclear, sandstone) }\end{array}$ & 555 & $\begin{array}{l}\text { SAV1W 0647, SAV1W 0648, SAV1W 0649, } \\
\text { SAV1W 0650, SAV1W 0651, SAV1W 0652, } \\
\text { SAV1W 0653 (pounders), SAV1W 0657, } \\
\text { SAV1W 0660, SAV1W 0661, SAV1W } 0662 \\
\text { (grindstones), SAV1W 0659 (abrasive } \\
\text { stone/hand mill), SAV1W 0658 (hand mill), } \\
\text { SAV1W 0656 (pestle), SAV1W 0655 } \\
\text { (polishing stone), SAV1W 0654 (whetstone), } \\
\text { SAV1W 0664, SAV1W 0665, SAV1W 0663 } \\
\text { (stone objects?), SAV1W 0617 (stone object) }\end{array}$ & 04.02 .2014 \\
\hline $1276 / 2014$ & SQ1 & $\begin{array}{l}\text { stones: token/gaming piece? ( } 1 \text { spherical piece, sandstone), } \\
\text { abrasive stone/grinding stone? ( } 1 \text { piece, sandstone), hand mill } \\
\text { (1 piece, quartzite), weight maybe for loom (1 piece, sandstone) }\end{array}$ & 556 & $\begin{array}{l}\text { SAV1W } 0601 \text { (token/gaming piece?), SAV1W } \\
0598 \text { (abrasive stone/grinding stone?), SAV1W } \\
0599 \text { (hand mill), SAV1W } 0600 \text { (weight } \\
\text { maybe for loom) }\end{array}$ & 06.02 .2014 \\
\hline $1220 / 2014$ & SQ1W & stone: pounder (1 piece, quartz) & 557 & SAV1W 0468 & 04.02 .2014 \\
\hline $1235 / 2014$ & SQ1 & $\begin{array}{l}\text { stones: grindstone (1 piece, quartzite), hammer (1 piece, } \\
\text { quartzite) }\end{array}$ & 559 & $\begin{array}{l}\text { SAV1W } 0594 \text { (grindstone), SAV1W } 0595 \\
\text { (hammer) }\end{array}$ & 05.02 .2014 \\
\hline $1233 / 2014$ & SQ1 & $\begin{array}{l}\text { stones: hand mill ( } 1 \text { piece, quartzite), grindstone ( } 1 \text { piece, } \\
\text { quartzite), hammers ( } 2 \text { pieces, each quartzite), pounder ( } 1 \text { piece, } \\
\text { quartz), abrasive stone ( } 1 \text { piece, quartzite) }\end{array}$ & 560 & $\begin{array}{l}\text { SAV1W } 0672 \text { (hand mill), SAV1W } 0673 \\
\text { (grindstone), SAV1W 0674, SAV1W 0675 } \\
\text { (hammers), SAV1W 0676 (pounder), SAV1W } \\
0677 \text { (abrasive stone) }\end{array}$ & 05.02 .2014 \\
\hline $1250 / 2014$ & SQ1 & $\begin{array}{l}\text { stones: grindstones ( } 2 \text { pieces, each quartzite), hand mills } \\
\text { ( } 2 \text { pieces, } 1 \text { quartzite, } 1 \text { sandstone) }\end{array}$ & 563 & $\begin{array}{l}\text { SAV1W 0516, SAV1W } 0518 \text { (grindstones), } \\
\text { SAV1W 0517, SAV1W } 0519 \text { (hand mills) }\end{array}$ & 05.02 .2014 \\
\hline $1270 / 2014$ & SQ1NW & stone: hand mill (1 piece, quartzite) & 564 & SAV1W 0521 & 06.02 .2014 \\
\hline $1262 / 2014$ & SQ1 & $\begin{array}{l}\text { stones: pounders ( } 4 \text { pieces, } 2 \text { quartz, } 2 \text { quartzite), grindstones } \\
\text { ( } 3 \text { pieces, } 2 \text { quartzite, } 1 \text { sandstone), grindstones? ( } 2 \text { pieces, each } \\
\text { quartzite), worked stone/grindstone? ( } 1 \text { piece, sandstone), stone } \\
\text { object ( } 1 \text { fragment, function unclear, sandstone), abrasive stone } \\
\text { (1 piece, sandstone), hand mill (1 piece, quartzite) }\end{array}$ & 565 & $\begin{array}{l}\text { SAV1W 0692, SAV1W 0538, SAV1W 0539, } \\
\text { SAV1W 0540 (pounders), SAV1W 0541, } \\
\text { SAV1W 0542, SAV1W 0687 (grindstones), } \\
\text { SAV1W 0688, SAV1W 0689 (grindstones?), } \\
\text { SAV1W 0543 (worked stone/grindstone?), } \\
\text { SAV1W } 0693 \text { (stone object), SAV1W 0691 } \\
\text { (abrasive stone), SAV1W 0690 (hand mill) }\end{array}$ & 06.02 .2014 \\
\hline $1291 / 2014$ & $\mathrm{SQ} 1,7.1 \mathrm{~m} \mathrm{~S}-\mathrm{N} / 2.6 \mathrm{~m} \mathrm{E}-\mathrm{W}$ & stone: blade or sickle (1 piece, chert) & 567 & SAV1W 0583 & 08.02 .2014 \\
\hline $1297 / 2014$ & SQ1 & stones: pounder (1 piece, quartz), pounder? (1 piece, basalt?) & 568 & $\begin{array}{l}\text { SAV1W } 0533 \text { (pounder), SAV1W } 0534 \\
\text { (pounder?) }\end{array}$ & 08.02 .2014 \\
\hline $1306 / 2014$ & SQ1 & $\begin{array}{l}\text { stone: whetstone ( } 1 \text { piece, sandstone), grindstone ( } 1 \text { piece, } \\
\text { sandstone), hammer ( } 1 \text { piece, quartzite), pounder (1 piece, } \\
\text { quartz), hand mill (1 piece, quartzite) }\end{array}$ & 569 & $\begin{array}{l}\text { SAV1W } 0549 \text { (whetstone), SAV1W } 0550 \\
\text { (grindstone), SAV1W 0607 (hammer), } \\
\text { SAV1W } 0608 \text { (pounder), SAV1W } 0609 \text { (hand } \\
\text { mill) }\end{array}$ & 08.02 .2014 \\
\hline $1304 / 2014$ & SQ1 & $\begin{array}{l}\text { stones: hammer (1 piece, quartzite?), grindstones ( } 2 \text { pieces, each } \\
\text { quartzite) }\end{array}$ & 570 & $\begin{array}{l}\text { SAV1W } 0643 \text { (hammer), SAV1W 0644, } \\
\text { SAV1W } 0644 \text { (grindstones) }\end{array}$ & 08.02 .2014 \\
\hline $1326 / 2014$ & SQ1 & $\begin{array}{l}\text { stones: grindstones ( } 3 \text { pieces, each quartzite), pounder (1 piece, } \\
\text { quartz) }\end{array}$ & 572 & $\begin{array}{l}\text { SAV1W 0618, SAV1W 0619, SAV1W } 06120 \\
\text { (grindstones), SAV1W } 0621 \text { (pounder) }\end{array}$ & 09.02 .2014 \\
\hline
\end{tabular}




\begin{tabular}{|c|c|c|c|c|c|}
\hline $1325 / 2014$ & $\mathrm{SQ} 1,0-4 \mathrm{~m} \mathrm{~S}-\mathrm{N} / 3-5 \mathrm{~m} \mathrm{E}-\mathrm{W}$ & $\begin{array}{l}\text { stones: pounder ( } 1 \text { piece, unknown stone), hand mill (1 piece, } \\
\text { quartzite), grindstone ( } 1 \text { piece, quartzite) }\end{array}$ & 572 & $\begin{array}{l}\text { SAV1W } 0640 \text { (pounder), SAV1W } 0641 \text { (hand } \\
\text { mill), SAV1W } 0642 \text { (grindstone) }\end{array}$ & 09.02 .2014 \\
\hline $930 / 2014$ & SQ2 & stone & 574 & & 10.02 .2014 \\
\hline $940 / 2014$ & SQ2 & stone object (1 fragment, function unknown, quartzite) & 575 & SAV1W 0646 & 10.02 .2014 \\
\hline $936 / 2014$ & SQ2 & stone & 576 & & 10.02 .2014 \\
\hline $957 / 2014$ & SQ2 & stone & 576 & & 11.02 .2014 \\
\hline $951 / 2014$ & SQ2 & stone & 578 & & 11.02 .2014 \\
\hline $959 / 2014$ & SQ2 & $\begin{array}{l}\text { stone and pottery: hand mill ( } 1 \text { piece, quartzite); oil lamp } \\
\text { (1 fragment, Christian, pottery) }\end{array}$ & 580 & $\begin{array}{l}\text { SAV1W } 0559 \text { (hand mill), SAV1W } 0560 \text { (oil } \\
\text { lamp) }\end{array}$ & 11.02 .2014 \\
\hline $1337 / 2014$ & SQ1 & $\begin{array}{l}\text { stones: grindstone ( } 2 \text { pieces, each quartzite), abrasive stone } \\
\text { (1 piece, sandstone), stone object ( } 1 \text { piece, function unclear, } \\
\text { adjoining with SAV1W } 0856-0010 / 2015 \text {, sandstone) }\end{array}$ & 584 & $\begin{array}{l}\text { SAV1W 0681, SAV1W } 0678 \text { (grindstones), } \\
\text { SAV1W } 0679 \text { (abrasive stone), SAV1W } 0682 \\
\text { (stone object) }\end{array}$ & 12.02 .2014 \\
\hline $1342 / 2014$ & SQ1 & $\begin{array}{l}\text { stone: hand mill? ( } 1 \text { piece, quartzite), hand mill ( } 1 \text { piece, } \\
\text { quartzite), polishing stone ( } 1 \text { piece, quartzite) }\end{array}$ & 585 & $\begin{array}{l}\text { SAV1W } 0630 \text { (hand mill?), SAV1W } 0631 \\
\text { (hand mill), SAV1W } 0632 \text { (polishing stone) }\end{array}$ & 12.02 .2014 \\
\hline $1354 / 2014$ & SQ1 & $\begin{array}{l}\text { stones: grindstone (1 piece, sandstone), hand mill? (1 piece, } \\
\text { quartzite) }\end{array}$ & 587 & $\begin{array}{l}\text { SAV1W } 0610 \text { (grindstone), SAV1W } 0611 \\
\text { (hand mill? }\end{array}$ & 12.02 .2014 \\
\hline $1369 / 2014$ & $\mathrm{SQ} 1,2 \mathrm{~m} \mathrm{E}-\mathrm{W} / 2.2 \mathrm{~m} \mathrm{~N}-\mathrm{S}$ & $\begin{array}{l}\text { stones: grindstone, mortar-like ( } 1 \text { piece, quartzite), grindstones } \\
\text { ( } 2 \text { pieces, each quartzite), pounder ( } 1 \text { piece, schist), whetstone } \\
\text { (1 piece, sandstone), grindstone/hand mill (1 piece, quartzite) }\end{array}$ & 587 & $\begin{array}{l}\text { SAV1W } 0606 \text { (grindstone, mortar-like), } \\
\text { SAV1W 0612, SAV1W 0614 (grindstones), } \\
\text { SAV1W 0616 (pounder), SAV1W 0615 } \\
\text { (whetstone), SAV1W 0613 (grindstone/hand } \\
\text { mill) }\end{array}$ & 13.02 .2014 \\
\hline $1356 / 2014$ & SQ1 & $\begin{array}{l}\text { stones: hand mill (1 piece, sandstone), stone object (1 fragment, } \\
\text { original function unknown, limestone) }\end{array}$ & 588 & $\begin{array}{l}\text { SAV1W } 0683 \text { (hand mill), SAV1W } 0680 \\
\text { (stone object) }\end{array}$ & 12.02 .2014 \\
\hline $1372 / 2014$ & SQ1 & $\begin{array}{l}\text { stones: hand mill/grindstone (1 piece, quartzite), abrasive stone } \\
\text { (1 piece, quartzite) }\end{array}$ & 588 & $\begin{array}{l}\text { SAV1W } 0596 \text { (hand mill/grindstone), SAV1W } \\
0597 \text { (abrasive stone) }\end{array}$ & 13.02 .2014 \\
\hline $1362 / 2014$ & SQ1 & $\begin{array}{l}\text { stones and architecture: grindstones ( } 3 \text { pieces, each quartzite), } \\
\text { grindstone? ( } 1 \text { piece, quartzite); cavetto cornice ( } 1 \text { part, } \\
\text { sandstone) }\end{array}$ & 590 & $\begin{array}{l}\text { SAV1W 0695, SAV1W 0696, SAV1W } 0697 \\
\text { (grindstones), SAV1W } 0634 \text { (grindstone?), } \\
\text { SAV1W 0698 (cavetto cornice) }\end{array}$ & 13.02.2014 \\
\hline $1013 / 2014$ & SQ1, 0-3m E-W/0-5m N-S & stone vessel (1 fragment, granite, see joint 763/2014) & 537 & SAV1W 0367 & 27.01 .2014 \\
\hline $523 / 2014$ & SQ2, W-half & stopper? (1 piece, mud) & 501 & SAV1W 0030 & 06.01 .2014 \\
\hline $1217 / 2014$ & SQ1 & $\begin{array}{l}\text { stopper ( } 1 \text { almost intact conical shaped piece, mud, enclosed by } \\
\text { a piece of linen/cloth) }\end{array}$ & 555 & SAV1W 0447 & 04.02 .2014 \\
\hline $590 / 2014$ & SQ1, NW-corner & textile (1 piece, cloth/linen?) & 501 & SAV1W 0039 & 11.01 .2014 \\
\hline $1062 / 2014$ & SQ1NW & textile (1 bag) & 501 & & 28.01 .2014 \\
\hline $1090 / 2014$ & SQ1NW & textile (1 bag) & 501 & & 29.01 .2014 \\
\hline $1063 / 2014$ & $\mathrm{SQ} 1,2.30-4.1 \mathrm{~m} \mathrm{E}-\mathrm{W} / 0-5 \mathrm{~m} \mathrm{~S}-\mathrm{N}$ & textile (1 bag) & 507 & & 28.01 .2014 \\
\hline $1086 / 2014$ & $\mathrm{SQ} 1,4-5 \mathrm{~m}$ E-W/0-5m S-N & textile (1 piece) & 507 & & 29.01 .2014 \\
\hline $618 / 2014$ & $\mathrm{SQ} 2,2-4 \mathrm{~m} \mathrm{E}-\mathrm{W} / 2.5-5 \mathrm{~m} \mathrm{~S}-\mathrm{N}$ & textile (1 piece, cloth/linen?) & 508 & SAV1W 0040 & 13.01 .2014 \\
\hline $650 / 2014$ & SQ1, NW-corner, 1-5m W-E/3-5m N-S & textile (2 pieces, probably different textiles, linen?) & 511 & SAV1W 0120 & 14.01 .2014 \\
\hline
\end{tabular}




\begin{tabular}{|c|c|c|c|c|}
\hline $788 / 2014$ & SQ1W, W-half, bottom surface of 522 & textile (1 bag) & 522 & 26.01 .2014 \\
\hline $867 / 2014$ & SQ1W & textile & 523 & 21.01 .2014 \\
\hline $875 / 2014$ & SQ1W & textile & 524 & 21.01 .2014 \\
\hline $1025 / 2014$ & SQ1 & textile (1 piece) & 530 & 27.01 .2014 \\
\hline $1031 / 2014$ & SQ1, 4-6m N-S/5-10m E-W & textile (1 bag) & 536 & 27.01 .2014 \\
\hline $1001 / 2014$ & SQ1W & textile (1 bag) & 539 & 26.01 .2014 \\
\hline $1020 / 2014$ & SQ1W & textile (1 bag) & 540 & 27.01 .2014 \\
\hline $1074 / 2014$ & SQ1W & textile (1 bag) & 541 & 29.01 .2014 \\
\hline $1075 / 2014$ & SQ1W & textile (1 bag) & 541 & 29.01 .2014 \\
\hline $1032 / 2014$ & SQ1W & textile (1 bag) & 542 & 27.01 .2014 \\
\hline $1049 / 2014$ & SQ1W & textile (1 bag) & 543 & 28.01 .2014 \\
\hline $1098 / 2014$ & SQ1NW & textile (1 bag) & 545 & 30.01 .2014 \\
\hline $1163 / 2014$ & SQ1NW, 5-6.5m S-N/0-2m E-W & textile (1 bag) & 545 & 02.02 .2014 \\
\hline $1119 / 2014$ & SQ1NW, 5-7m S-N & textile (1 bag) & 545 & 30.01 .2014 \\
\hline $1104 / 2014$ & SQ1W & textile (1 bag) & 546 & 30.01 .2014 \\
\hline $1110 / 2014$ & SQ1, 4-7m S-N & textile (1 bag) & 547 & 30.01 .2014 \\
\hline $1111 / 2014$ & SQ1, 4-7m S-N & textile (1 piece) & 547 & 30.01 .2014 \\
\hline $1128 / 2014$ & SQ1, 7-10m S-N/6-10m E-W & textile (1 bag) & 547 & 01.02 .2014 \\
\hline $1136 / 2014$ & SQ1 & textile (1 bag) & 549 & 01.02 .2014 \\
\hline $1188 / 2014$ & SQ1NW & textile (1 bag) & 552 & 03.02 .2014 \\
\hline $1203 / 2014$ & $\mathrm{SQ1NW}+\mathrm{W}$ & textile (1 bag) & 552 & 04.02 .2014 \\
\hline $1209 / 2014$ & SQ1 & textile (1 piece) & 555 & 04.02 .2014 \\
\hline $1230 / 2014$ & SQ1 & textile (1 bag) & 555 & 05.02 .2014 \\
\hline $1271 / 2014$ & $\mathrm{SQ} 1,2.3 \mathrm{~m} \mathrm{~N}-\mathrm{S} / 3.6 \mathrm{~m}$ E-W & textile (1 bag) & 565 & 06.02 .2014 \\
\hline $1309 / 2014$ & SQ1 & textile (1 bag) & 569 & 08.02 .2014 \\
\hline $1330 / 2014$ & $\mathrm{SQ} 1,0-4 \mathrm{~m} \mathrm{~S}-\mathrm{N} / 3-5 \mathrm{~m} \mathrm{E}-\mathrm{W}$ & textile (1 bag) & 572 & 09.02 .2014 \\
\hline $921 / 2014$ & SQ2 & textile (1 bag) & 574 & 10.02 .2014 \\
\hline $928 / 2014$ & SQ2 & textile (1 bag) & 575 & 10.02 .2014 \\
\hline $955 / 2014$ & SQ2 & textile (1 piece) & 578 & 11.02 .2014 \\
\hline $944 / 2014$ & SQ2 & textile (1 bag) & 579 & 11.02 .2014 \\
\hline $961 / 2014$ & SQ2 & textile (1 bag) & 580 & 11.02 .2014 \\
\hline
\end{tabular}




\begin{tabular}{|c|c|c|c|c|c|}
\hline $1345 / 2014$ & SQ1 & textile (1 bag) & 584 & & 12.02 .2014 \\
\hline $1347 / 2014$ & SQ1 & textile (2 pieces) & 585 & & 12.02 .2014 \\
\hline $1367 / 2014$ & SQ1 & textile (1 piece) & 587 & & 13.02 .2014 \\
\hline $805 / 2014$ & $\begin{array}{l}\text { SQ1W, 5-10m E-W/0-5m S-N, surface } \\
\text { clearing }\end{array}$ & token/gaming piece (1 re-used Christian sherd, pottery) & 500 & SAV1W 0113 & 15.01 .2014 \\
\hline $801 / 2014$ & SQ2 & token/gaming piece? (1 oval/egg shaped object, clay) & 516 & SAV1W 0114 & 15.01 .2014 \\
\hline $845 / 2014$ & SQ1W & token/gaming piece? (1 oval object, mud) & 521 & SAV1W 0183 & 20.01 .2014 \\
\hline $1269 / 2014$ & SQ1 & token/gaming piece? (1 small object, clay) & 556 & SAV1W 0505 & 06.02 .2014 \\
\hline $1124 / 2014$ & SQ1 & weight? (1 re-used $18^{\text {th }}$ Dynasty sherd, pottery) & 549 & SAV1W 0411 & 01.02 .2014 \\
\hline $931 / 2014$ & SQ2, 2.4m S-N $/ 5.5 \mathrm{~m} \mathrm{E-W}$ & weight (1 piece, quartzite) & 576 & SAV1W 0553 & 10.02 .2014 \\
\hline $505 / 2014$ & SQ2 & wood (1 piece); bone & 500 & & 05.01 .2014 \\
\hline $702 / 2014$ & SQ1W, E-half & wood; fish bone; bone & 501 & & 18.01 .2014 \\
\hline $646 / 2014$ & SQ1, NW-corner, 1-5m W-E/3-5m N-S & wood & 511 & & 14.01 .2014 \\
\hline $1003 / 2014$ & $\mathrm{SQ} 1,3-5 \mathrm{~m} \mathrm{E}-\mathrm{W} / 2-5 \mathrm{~m} \mathrm{~N}-\mathrm{S}$ & $\begin{array}{l}\text { wood (1 fragment of worked wood, original shape and function } \\
\text { unknown) }\end{array}$ & 538 & SAV1W 0726 & 26.01.2014 \\
\hline $1018 / 2014$ & SQ1W & wood (1 small piece, inscribed, Ottoman?) & 540 & SAV1W 0589 & 27.01 .2014 \\
\hline $1108 / 2014$ & SQ1, 4-7m S-N & $\begin{array}{l}\text { wood, worked (1 fragment, lotus flower shaped, with } \\
\text { decoration) }\end{array}$ & 547 & SAV1W 0727 & 30.01 .2014 \\
\hline $1143 / 2014$ & SQ1 & wood & 548 & & 01.02 .2014 \\
\hline $1216 / 2014$ & SQ1 & wood (1 piece) & 555 & & 04.02 .2014 \\
\hline $1275 / 2014$ & SQ1 & wood & 556 & & 06.02 .2014 \\
\hline $1263 / 2014$ & SQ1 & wood (1 piece) & 565 & & 06.02 .2014 \\
\hline $1278 / 2014$ & SQ1 & $\begin{array}{l}\text { wooden object/application?/furniture? ( } 1 \text { half spherical piece } \\
\text { with one central and four smaller perforations, some filled with } \\
\text { wood, function unclear) }\end{array}$ & 565 & SAV1W 0509 & 06.02.2014 \\
\hline $1290 / 2014$ & SQ1 & wood (2 pieces) & 567 & & 08.02 .2014 \\
\hline $1286 / 2014$ & SQ1 & wood (4 pieces) & 568 & & 08.02 .2014 \\
\hline $1323 / 2014$ & SQ1 & wood (5 pieces) & 572 & & 09.02 .2014 \\
\hline $1363 / 2014$ & SQ1 & wood & 587 & & 13.02 .2014 \\
\hline
\end{tabular}




\begin{tabular}{|c|c|c|c|c|c|}
\hline \multicolumn{6}{|c|}{ Sai Island SAV1 West 2015} \\
\hline Find no. & Location & Description/label/material & $\begin{array}{c}\text { SU } \\
\text { /level }\end{array}$ & Object no. & Date \\
\hline $0830 / 2015$ & SQ1, Feature 115, sieved material & $\begin{array}{l}\text { amulet (1 piece, lotus blossom shaped, faience); beads (various } \\
\text { forms, mostly faience, } 1 \text { unspecified); stoppers ( } 7 \text { pieces, each } \\
\text { clay); figurine ( } 1 \text { fragment, animal figurine, pottery); unidentified } \\
\text { objects ( } 6 \text { pieces, } 1 \text { glass, } 1 \text { metal, } 1 \text { stone, } 3 \text { clay); vessels } \\
\text { (2 pieces, } 1 \text { faience, } 1 \text { glazed ware); worked wood ( } 1 \text { piece); seal } \\
\text { (1 piece, clay); worked stone ( } 1 \text { piece, unspecified stone); lid } \\
\text { (1 piece, clay); token/gaming piece ( } 1 \text { re-used } 18^{\text {th }} \text { Dynasty pot, } \\
\text { pottery); disc-shaped object - token/gaming piece? (1 piece, } \\
\text { pottery); blade (1 piece, silex); flakes ( } 12 \text { flakes of various } \\
\text { stones); organic material ( } 2 \text { bags, seed); seashell ( } 2 \text { bags, } \\
\text { fragments); chalk ( } 2 \text { pieces); bones ( } 1 \text { bag); charcoal ( } 2 \text { bags); } \\
\text { doum nuts ( } 1 \text { bag); seashell ( } 1 \text { bag, fragments); wood ( } 1 \text { bag) }\end{array}$ & 731 & $\begin{array}{l}\text { SAV1W } 1436 \text { (amulet), SAV1W 1437, } \\
\text { SAV1W 1438, SAV1W 1439, SAV1W 1440, } \\
\text { SAV1W 1441, SAV1W 1442, SAV1W 1443, } \\
\text { SAV1W 1444 (beads), SAV1W 1482, SAV1W } \\
\text { 1483, SAV1W 1484, SAV1W 1485, SAV1W } \\
\text { 1486, SAV1W 1487, SAV1W 1488 (stoppers), } \\
\text { SAV1W 1491(figurine), SAV1W 1447, } \\
\text { SAV1W 1448, SAV1W 1450, SAV1W 1489, } \\
\text { SAV1W 1490 (unidentified objects), SAV1W } \\
\text { 1446, SAV1W 1494 (vessels), SAV1W 1492 } \\
\text { (worked wood), SAV1W 1495 (seal), SAV1W } \\
\text { 1496 (worked stone), SAV1W 1499 (lid), } \\
\text { SAV1W 1525 (token/gaming piece), SAV1W } \\
\text { 1445 (disc-shaped object - token/gaming } \\
\text { piece?), SAV1W 1449 (blade), SAV1W 1493 } \\
\text { (flakes) }\end{array}$ & 09.02 .2015 \\
\hline $0257 / 2015$ & SQ1S & $\begin{array}{l}\text { amulet/bead, cowroid (1 piece, flat side shows goddess Taweret, } \\
\text { faience) }\end{array}$ & 632 & SAV1W 0723 & 17.01.2015 \\
\hline $0182 / 2015$ & SQ1S & architecture: column base (1 large piece of sandstone) & 620 & SAV1W 1469 & 13.01.2015 \\
\hline $0383 / 2015$ & SQ1S & $\begin{array}{l}\text { architecture: door jamb/threshold? (1 rectangular block, } \\
\text { sandstone) }\end{array}$ & 650 & SAV1W 1135 & 21.01 .2015 \\
\hline $0513 / 2015$ & SQ1S & architecture? (1 small fragment, limestone) & 672 & SAV1W 1164 & 28.01 .2015 \\
\hline $0780 / 2015$ & SQ1/SQ1E & architecture: column base (1 large piece, sandstone) & 721 & SAV1W 1470 & 05.02 .2015 \\
\hline $0768 / 2015$ & SQ1/SQ1E & bark (1 bag) & 717 & & 05.02 .2015 \\
\hline $0803 / 2015$ & SQ1 & bead (1 circular bead, faience) & 727 & SAV1W 1397 & 08.02 .2015 \\
\hline $0804 / 2015$ & SQ1 & bead (1 circular bead, faience); petrified wood (1 piece) & 729 & SAV1W 1408 & 08.02 .2015 \\
\hline $0833 / 2015$ & SQ1, sieved material & $\begin{array}{l}\text { beads ( } 4 \text { circular beads, } 2 \text { faience, } 1 \text { stone); clay fragments, raw } \\
\text { material for sealing? ( } 3 \text { fragments); charcoal (1 bag); bones ( } 1 \\
\text { bag); organic material ( } 1 \text { bag); wood ( } 1 \text { bag); seashell } \\
\text { (1 fragment); agate ( } 1 \text { flake, reference) }\end{array}$ & 729 & $\begin{array}{l}\text { SAV1W 1409, SAV1W } 1410 \text { (beads), SAV1W } \\
\text { 1479, SAV1W 1480, SAV1W } 1481 \text { (clay } \\
\text { fragments, raw material for sealing?) }\end{array}$ & 09.02 .2015 \\
\hline $0831 / 2015$ & SQ1, Feature 115 , sieved material & $\begin{array}{l}\text { beads ( } 3 \text { circular beads, } 2 \text { faience, } 1 \text { stone); snail shell used as } \\
\text { piece of jewelry/bead ( } 1 \text { piece); pounder (1 piece, siliceous } \\
\text { stone?); faience object ( } 1 \text { fragment, original function unclear); } \\
\text { organic material (1 piece, seed); charcoal ( } 1 \text { bag); organic } \\
\text { material ( } 1 \text { bag); bones ( } 1 \text { bag) }\end{array}$ & 732 & $\begin{array}{l}\text { SAV1W 1411, SAV1W } 1412 \text { (beads), SAV1W } \\
1466 \text { (snail shell), SAV1W } 1467 \text { (pounder), } \\
\text { SAV1W } 1524 \text { (faience object) }\end{array}$ & 09.02.2015 \\
\hline
\end{tabular}




\begin{tabular}{|c|c|c|c|c|c|}
\hline $0840 / 2015$ & SQ1, Feature 115 , sieved material & $\begin{array}{l}\text { beads (numerous pieces, various forms, faience and stone); seal } \\
\text { impression ( } 1 \text { piece, with name of Thutmose III[?], clay); } \\
\text { seashell ( } 1 \text { piece, small); organic material ( } 3 \text { pieces, seeds); } \\
\text { plaster (1 piece); seal (4 fragments, undecorated); organic } \\
\text { material (1 bag); charcoal (1 bag) }\end{array}$ & 732 & $\begin{array}{l}\text { SAV1W 1413, SAV1W 1414, SAV1W 1415, } \\
\text { SAV1W 1416, SAV1W } 1417 \text { (beads), SAV1W } \\
1451 \text { (seal impression) }\end{array}$ & 10.02.2015 \\
\hline $0848 / 2015$ & $\begin{array}{l}\text { SQ1, Feature } 115 \text {, sieved material from } \\
\text { vessel } 0838 / 2015\end{array}$ & $\begin{array}{l}\text { bead ( } 1 \text { circular bead, faience); bones ( } 1 \text { bag); organic material } \\
\text { ( } 1 \text { bag); charcoal ( } 1 \text { bag) }\end{array}$ & 732 & SAV1W 1406 & 10.02.2015 \\
\hline $0244 / 2015$ & SQ1S & bead ( 1 circular bead, faience) & 600 & SAV1W 0728 & 17.01.2015 \\
\hline $0009 / 2015$ & SQ1S, $10 \times 10 \mathrm{~m}$ & $\begin{array}{l}\text { beads ( } 1 \text { ring bead, faience, } 1 \text { tubular bead, faience, } 1 \text { small ring } \\
\text { bead) }\end{array}$ & 600 & $\begin{array}{l}\text { SAV1W } 0803 \text { (ring bead), SAV1W } 0804 \\
\text { (tubular bead), SAV1W } 0805 \text { (small ring bead) }\end{array}$ & 03.01 .2015 \\
\hline $0001 / 2015$ & SQ1S, top surface & bead (1 ring bead, faience) & 600 & SAV1W 0802 & 03.01 .2015 \\
\hline $0053 / 2015$ & SQ1S & bead (1 ring bead, faience) & 606 & SAV1W 0884 & 06.01 .2015 \\
\hline $0050 / 2015$ & SQ1S & bead (1 tubular bead, faience) & 608 & SAV1W 0885 & 06.01 .2015 \\
\hline $0172 / 2015$ & SQ1S & bead (1 piece, roughly spherical in shape, clay) & 620 & SAV1W 0975 & 13.01 .2015 \\
\hline $0315 / 2015$ & SQ1S & bead (1 circular bead, faience) & 641 & SAV1W 0762 & 20.01 .2015 \\
\hline $0523 / 2015$ & SQ1S & bead (1 large piece, roughly spherical in shape, clay) & 674 & SAV1W 1169 & 28.01 .2015 \\
\hline $0548 / 2015$ & SQ1S & bead ( 1 circular bead, faience) & 678 & SAV1W 1240 & 29.01 .2015 \\
\hline $0843 / 2015$ & SQ1S, sieved material & $\begin{array}{l}\text { bead ( } 1 \text { tubular bead, faience); bones ( } 1 \text { bag); doum nuts ( } 1 \text { bag); } \\
\text { wood ( } 1 \text { bag); charcoal ( } 1 \text { bag); seal ( } 9 \text { fragments, uninscribed })\end{array}$ & 720 & SAV1W 1459 & 10.02 .2015 \\
\hline $0844 / 2015$ & SQ1S, sieved material & bead (1 circular bead, ivory); bones ( 1 bag); charcoal (1 bag) & 733 & SAV1W 1405 & 10.02 .2015 \\
\hline $0058 / 2015$ & SQ1S & beetle (1 piece) & 609 & & 06.01 .2015 \\
\hline $0334 / 2015$ & SQ1S & beetles (1 bag, fragments) & 644 & & 20.01 .2015 \\
\hline $0567 / 2015$ & SQ1 & bones (1 bag) & 664 & & 29.01 .2015 \\
\hline $0578 / 2015$ & SQ1 & bones (1 bag) & 664 & & 31.01 .2015 \\
\hline $0466 / 2015$ & SQ1 & bones ( 2 pieces $)$ & 665 & & 27.01.2015 \\
\hline $0533 / 2015$ & SQ1 & bones (1 bag) & 676 & & 28.01 .2015 \\
\hline $0712 / 2015$ & SQ1, cleaning Profile 12, sieving & bones (1 bag) & 676 & & 02.02 .2015 \\
\hline $0592 / 2015$ & SQ1 & bone (1 piece) & 684 & & 31.01 .2015 \\
\hline $0591 / 2015$ & SQ1 & bones (1 bag) & 686 & & 31.01 .2015 \\
\hline $0607 / 2015$ & SQ1 & bone (1 piece) & 687 & & 31.01 .2015 \\
\hline
\end{tabular}




\begin{tabular}{|c|c|c|c|c|}
\hline $0598 / 2015$ & SQ1 & bones (1 bag) & 688 & 31.01 .2015 \\
\hline $0616 / 2015$ & SQ1 & bones (1 bag) & 690 & 31.01 .2015 \\
\hline $0788 / 2015$ & SQ1 & bones (1 bag) & 722 & 08.02 .2015 \\
\hline $0798 / 2015$ & SQ1 & bones (1 bag) & 726 & 08.02 .2015 \\
\hline $0800 / 2015$ & SQ1 & bones (1 bag) & 727 & 08.02 .2015 \\
\hline $0807 / 2015$ & SQ1 & bones (1 bag) & 729 & 08.02 .2015 \\
\hline $0834 / 2015$ & SQ1, Feature 115 , sieved material & $\begin{array}{l}\text { bones (1 bag); charcoal (1 bag); wood (1 bag); organic material } \\
\text { (1 bag) }\end{array}$ & 725 & 09.02 .2015 \\
\hline $0793 / 2015$ & SQ1, Feature 115 & bone (1 piece) & 725 & 08.02 .2015 \\
\hline $0822 / 2015$ & SQ1, Feature 115 & bones (1 bag) & 731 & 09.02 .2015 \\
\hline $0841 / 2015$ & SQ1, Feature 115 & bones (1 bag) & 732 & 10.02 .2015 \\
\hline $0827 / 2015$ & SQ1, Feature 115 & bone (1 piece) & 732 & 09.02 .2015 \\
\hline $0077 / 2015$ & SQ1S & bones (1 bag) & 600 & 07.01 .2015 \\
\hline $0429 / 2015$ & SQ1S & bones (1 bag) & 600 & 24.01 .2015 \\
\hline $0005 / 2015$ & $\mathrm{SQ} 1 \mathrm{~S}, 10 \times 10 \mathrm{~m}$ & bones (1 bag) & 600 & 03.01 .2015 \\
\hline $0745 / 2015$ & SQ1E & bones (1 bag) & 601 & 03.02 .2015 \\
\hline $0746 / 2015$ & SQ1E & bones (1 bag) & 601 & 04.02 .2015 \\
\hline $0019 / 2015$ & SQ1S & bones & 601 & 05.01 .2015 \\
\hline $0014 / 2015$ & SQ1S, $10 \times 10 \mathrm{~m}$ & bones (1 bag); charcoal (1 bag) & 601 & 04.01 .2015 \\
\hline $0015 / 2015$ & $\mathrm{SQ} 1 \mathrm{~S}, 10 \times 10 \mathrm{~m}$ & bones (1 bag) & 601 & 04.01 .2015 \\
\hline $0028 / 2015$ & SQ1S & bone & 602 & 05.01 .2015 \\
\hline $0027 / 2015$ & SQ1S & bones (1 bag) & 603 & 05.01 .2015 \\
\hline $0032 / 2015$ & SQ1S & bone (1 piece) & 604 & 05.01 .2015 \\
\hline $0038 / 2015$ & SQ1S & bones ( 2 pieces) & 605 & 05.01 .2015 \\
\hline $0041 / 2015$ & SQ1S & bones ( 2 bags) & 606 & $\begin{array}{r}05.01 .2015 / \\
06.01 .2015\end{array}$ \\
\hline $0067 / 2015$ & SQ1/S & bones (1 bag) & 606 & 07.01 .2015 \\
\hline $0045 / 2015$ & SQ1S & bones (1 bag) & 607 & 06.01 .2015 \\
\hline
\end{tabular}




\begin{tabular}{|c|c|c|c|c|c|}
\hline $0046 / 2015$ & SQ1S & bones (1 bag) & 608 & & 06.01 .2015 \\
\hline $0052 / 2015$ & SQ1S & bones (1 bag) & 609 & & 06.01 .2015 \\
\hline $0063 / 2015$ & SQ1S & bones (1 bag) & 609 & & 07.01 .2015 \\
\hline $0086 / 2015$ & $\begin{array}{l}\text { SQ1/1S, cleaning connection between SQ1 } \\
\text { and SQ1S }\end{array}$ & bones (1 bag) & 610 & & 08.01 .2015 \\
\hline $0082 / 2015$ & SQ1S, cleaning square S of SU 610 & bones (1 bag) & 612 & & 08.01 .2015 \\
\hline $0147 / 2015$ & SQ1S & bones (1 bag) & 612 & & 12.01.2015 \\
\hline $0100 / 2015$ & SQ1S & bones (1 bag) & 613 & & 10.01 .2015 \\
\hline $0108 / 2015$ & SQ1/S & bones (1 bag) & 614 & & 10.01 .2015 \\
\hline $0125 / 2015$ & SQ1S & bones (1 bag) & 615 & & 10.01 .2015 \\
\hline $0116 / 2015$ & SQ1S & bones (1 bag) & 616 & & 10.01 .2015 \\
\hline $0121 / 2015$ & $\begin{array}{l}\text { SQ1S, } \\
\text { NW of SU }\end{array}$ & bones (1 bag) & 616 & & 10.01 .2015 \\
\hline $0128 / 2015$ & SQ1S & bones (1 bag) & 617 & & 11.01 .2015 \\
\hline $0134 / 2015$ & SQ1S & bones (1 bag) & 618 & & 11.01 .2015 \\
\hline $0152 / 2015$ & SQ1S & bones (2 pieces) & 618 & & 12.01 .2015 \\
\hline $0156 / 2015$ & SQ1S, next to metal pin (SAV1W 965) & bones (1 bag) & 618 & & 12.01 .2015 \\
\hline $0141 / 2015$ & SQ1S & bones (1 bag) & 619 & & 11.01 .2015 \\
\hline $0154 / 2015$ & SQ1S & bones (1 bag) & 619 & & 12.01 .2015 \\
\hline $0161 / 2015$ & SQ1S & bones (1 bag) & 620 & & 12.01 .2015 \\
\hline $0169 / 2015$ & SQ1S & bones (1 bag) & 620 & & 13.01 .2015 \\
\hline $0186 / 2015$ & SQ1S & bones (1 bag) & 622 & & 14.01 .2015 \\
\hline $0185 / 2015$ & SQ1S & bones (1 bag) & 624 & & 14.01 .2015 \\
\hline $0197 / 2015$ & SQ1S & bones (1 bag) & 625 & & 14.01 .2015 \\
\hline $0204 / 2015$ & SQ1S & bones (1 bag) & 626 & & 15.01 .2015 \\
\hline $0205 / 2015$ & SQ1S & bones (1 bag) & 626 & & 15.01 .2015 \\
\hline $0214 / 2015$ & SQ1S & $\begin{array}{l}\text { bone, worked ( } 1 \text { piece, shaped to be used as a piercing tool); } \\
\text { bones ( } 1 \text { bag) }\end{array}$ & 627 & SAV1W 1520 & 15.01 .2015 \\
\hline
\end{tabular}




\begin{tabular}{|c|c|c|c|c|}
\hline $0221 / 2015$ & SQ1S & bones (1 bag) & 628 & 15.01 .2015 \\
\hline $0225 / 2015$ & SQ1S & bones (1 bag) & 629 & 15.01 .2015 \\
\hline $0235 / 2015$ & SQ1S & bones (1 bag) & 630 & 17.01.2015 \\
\hline $0270 / 2015$ & SQ1S & bones (1 bag) & 632 & 18.01 .2015 \\
\hline $0243 / 2015$ & SQ1S & bones (1 bag) & 633 & 17.01.2015 \\
\hline $0263 / 2015$ & SQ1S & bones (1 bag) & 633 & 18.01 .2015 \\
\hline $0240 / 2015$ & SQ1S & bones (1 bag) & 634 & 17.01 .2015 \\
\hline $0251 / 2015$ & SQ1S & bones (1 bag) & 635 & 17.01.2015 \\
\hline $0273 / 2015$ & SQ1S & bones (1 bag) & 636 & 18.01 .2015 \\
\hline $0298 / 2015$ & SQ1S & bones (1 bag) & 636 & 19.01.2015 \\
\hline $0278 / 2015$ & SQ1S & bone & 637 & 18.01 .2015 \\
\hline $0293 / 2015$ & SQ1S & bones (1 bag) & 638 & 19.01.2015 \\
\hline $0287 / 2015$ & SQ1S & bones (1 bag); wood (1 piece) & 639 & 19.01.2015 \\
\hline $0301 / 2015$ & SQ1S & bones (1 bag) & 640 & 19.01 .2015 \\
\hline $0313 / 2015$ & SQ1S & bones (1 bag) & 641 & 20.01 .2015 \\
\hline $0343 / 2015$ & SQ1S & bones (1 bag) & 642 & 20.01 .2015 \\
\hline $0310 / 2015$ & SQ1S & bones (1 bag) & 643 & 20.01 .2015 \\
\hline $0329 / 2015$ & SQ1S & bones (1 bag) & 644 & 20.01 .2015 \\
\hline $0336 / 2015$ & SQ1S & bones (1 bag) & 645 & 20.01 .2015 \\
\hline $0345 / 2015$ & SQ1S & bones (1 bag) & 646 & 20.01 .2015 \\
\hline $0364 / 2015$ & SQ1S & bones (1 bag) & 646 & 21.01 .2015 \\
\hline $0351 / 2015$ & SQ1S & bones (1 bag) & 647 & 20.01 .2015 \\
\hline $0359 / 2015$ & SQ1S & bones (1 bag) & 647 & 21.01 .2015 \\
\hline $0363 / 2015$ & SQ1S & bones (1 bag) & 649 & 21.01 .2015 \\
\hline $0372 / 2015$ & SQ1S & bones (1 bag) & 651 & 21.01 .2015 \\
\hline $0422 / 2015$ & SQ1S & bones (1 bag) & 652 & 22.01 .2015 \\
\hline
\end{tabular}




\begin{tabular}{|c|c|c|c|c|c|}
\hline $0388 / 2015$ & SQ1S & bones (1 bag) & 653 & & 22.01.2015 \\
\hline $0393 / 2015$ & SQ1S & bones (1 bag) & 654 & & 22.01 .2015 \\
\hline $0403 / 2015$ & SQ1S & bone, worked (1 fragment, drilled hole) & 654 & SAV1W 1031 & 22.01.2015 \\
\hline $0396 / 2015$ & SQ1S & bones (1 bag) & 655 & & 22.01.2015 \\
\hline $0411 / 2015$ & SQ1S & bones (1 bag) & 657 & & 22.01.2015 \\
\hline $0413 / 2015$ & SQ1S & bones (1 bag) & 658 & & 22.01.2015 \\
\hline $0425 / 2015$ & SQ1S & bones (1 bag) & 658 & & 24.01 .2015 \\
\hline $0416 / 2015$ & SQ1S & bones (1 bag); charcoal (1 fragment) & 659 & & 22.01.2015 \\
\hline $0427 / 2015$ & SQ1S & bones (1 bag) & 659 & & 24.01.2015 \\
\hline $0439 / 2015$ & SQ1S & bones (1 bag) & 660 & & 24.01.2015 \\
\hline $0444 / 2015$ & SQ1S & bones (1 bag) & 661 & & 24.01.2015 \\
\hline $0451 / 2015$ & SQ1S & bone (1 piece) & 661 & & 24.01 .2015 \\
\hline $0454 / 2015$ & SQ1S & bones (1 bag) & 662 & & 27.01.2015 \\
\hline $0461 / 2015$ & SQ1S & bones (1 bag) & 663 & & 27.01.2015 \\
\hline $0478 / 2015$ & SQ1S & bones (1 bag) & 666 & & 27.01.2015 \\
\hline $0472 / 2015$ & SQ1S & bones (1 bag) & 667 & & 27.01.2015 \\
\hline $0482 / 2015$ & SQ1S & bones (1 bag) & 668 & & 27.01.2015 \\
\hline $0502 / 2015$ & SQ1S & bones (1 bag) & 668 & & 28.01 .2015 \\
\hline $0494 / 2015$ & SQ1S & bones (1 bag, vertebrae? with skin) & 669 & & 28.01 .2015 \\
\hline $0499 / 2015$ & SQ1S & bones (1 bag) & 669 & & 28.01.2015 \\
\hline $0492 / 2015$ & SQ1S & bones (1 bag); organic material (1 piece) & 670 & & 28.01.2015 \\
\hline $0511 / 2015$ & SQ1S & bones (1 bag) & 671 & & 28.01 .2015 \\
\hline $0510 / 2015$ & SQ1S & bones (1 bag) & 672 & & 28.01 .2015 \\
\hline $0514 / 2015$ & SQ1S & bones (1 bag) & 673 & & 28.01.2015 \\
\hline $0530 / 2015$ & SQ1S & bones (1 bag) & 674 & & 28.01.2015 \\
\hline $0544 / 2015$ & SQ1S & bones (1 bag); wood (1 piece) & 674 & & 29.01 .2015 \\
\hline
\end{tabular}




\begin{tabular}{|c|c|c|c|c|}
\hline $0521 / 2015$ & SQ1S & bones (1 bag) & 675 & 28.01 .2015 \\
\hline $0545 / 2015$ & SQ1S & bone (1 piece) & 675 & 29.01.2015 \\
\hline $0542 / 2015$ & SQ1S & bones (1 bag) & 678 & 29.01 .2015 \\
\hline 0706/2015 & $\begin{array}{l}\text { SQ1S, cleaning of bottom surface of SU } \\
678\end{array}$ & bones (1 bag) & 678 & 02.02 .2015 \\
\hline $0554 / 2015$ & SQ1S & bones (1 bag) & 679 & 29.01 .2015 \\
\hline $0562 / 2015$ & SQ1S & bones (1 bag) & 680 & 29.01.2015 \\
\hline $0564 / 2015$ & SQ1S & bone or horn? (1 piece) & 680 & 29.01.2015 \\
\hline $0577 / 2015$ & SQ1S & bones (1 bag) & 685 & 31.01 .2015 \\
\hline $0599 / 2015$ & SQ1S & bones (1 bag) & 689 & 31.01 .2015 \\
\hline $0620 / 2015$ & SQ1S & bones (1 bag) & 691 & 31.01 .2015 \\
\hline $0625 / 2015$ & SQ1S & bones (1 bag) & 691 & 01.02 .2015 \\
\hline $0632 / 2015$ & SQ1S & bones (1 bag) & 695 & 01.02 .2015 \\
\hline $0644 / 2015$ & SQ1 & bones (1 bag) & 696 & 01.02 .2015 \\
\hline $0648 / 2015$ & SQ1S & bones (1 bag) & 697 & 01.02 .2015 \\
\hline $0651 / 2015$ & SQ1S & bones (1 bag); wood (2 pieces) & 698 & 01.02 .2015 \\
\hline $0662 / 2015$ & SQ1S & bones (1 bag); charcoal (1 piece) & 701 & 01.02 .2015 \\
\hline $0675 / 2015$ & SQ1S & bones (1 bag) & 703 & 02.02 .2015 \\
\hline $0677 / 2015$ & SQ1S & bones (1 bag) & 704 & 02.02 .2015 \\
\hline $0685 / 2015$ & SQ1S & bones (1 bag) & 705 & 02.02 .2015 \\
\hline $0700 / 2015$ & SQ1S & bones (1 bag) & 706 & 02.02 .2015 \\
\hline $0725 / 2015$ & SQ1 & bones (1 bag) & 708 & 03.02 .2015 \\
\hline $0730 / 2015$ & SQ1S & bones (1 bag) & 710 & 03.02 .2015 \\
\hline $0721 / 2015$ & SQ1S & bones (1 bag) & 711 & 03.02 .2015 \\
\hline $0754 / 2015$ & SQ1E & bones (1 bag) & 713 & 04.02 .2015 \\
\hline $0813 / 2015$ & SQ1S & bone (1 piece) & 716 & 08.02 .2015 \\
\hline $0761 / 2015$ & SQ1S & bones (1 bag) & 717 & 04.02 .2015 \\
\hline
\end{tabular}




\begin{tabular}{|c|c|c|c|c|c|}
\hline $0765 / 2015$ & SQ1/SQ1E & bones (1 bag) & 717 & & 05.02 .2015 \\
\hline $0775 / 2015$ & SQ1/SQ1E & bones (1 bag) & 719 & & 05.02 .2015 \\
\hline $0786 / 2015$ & SQ1S & bones (1 bag) & 720 & & 05.02 .2015 \\
\hline $0782 / 2015$ & SQ1/SQ1E & bones (1 bag) & 721 & & 05.02 .2015 \\
\hline $0222 / 2015$ & SQ1S & botanical material? (1 bag) & 628 & & 15.01.2015 \\
\hline $0283 / 2015$ & SQ1S & botanical material (1 piece) & 636 & & 18.01 .2015 \\
\hline $0312 / 2015$ & SQ1S & botanical material (1 bag) & 643 & & 20.01 .2015 \\
\hline $0256 / 2015$ & SQ1S & bracelet (1 small fragment, siliceous shale?) & 633 & SAV1W 0729 & 17.01.2015 \\
\hline $0339 / 2015$ & SQ1S & chalk (1 piece) & 642 & & 20.01 .2015 \\
\hline $0818 / 2015$ & SQ1S & chalk (1 bag) & 723 & & 08.02 .2015 \\
\hline $0075 / 2015$ & SQ1 & charcoal (1 bag) & 600 & & 07.01 .2015 \\
\hline $0467 / 2015$ & SQ1 & charcoal (1 bag) & 664 & & 27.01 .2015 \\
\hline $0569 / 2015$ & SQ1 & charcoal (1 bag) & 664 & & 29.01 .2015 \\
\hline $0581 / 2015$ & SQ1 & charcoal (1 bag) & 664 & & 31.01 .2015 \\
\hline $0474 / 2015$ & SQ1 & charcoal (1 bag) & 667 & & 27.01 .2015 \\
\hline $0534 / 2015$ & SQ1 & charcoal (1 bag) & 675 & & 28.01 .2015 \\
\hline $0711 / 2015$ & SQ1, cleaning Profile 12, sieving & charcoal (1 bag) & 676 & & 02.02 .2015 \\
\hline $0670 / 2015$ & $\begin{array}{l}\text { SQ1, Profile 14, sampling area micro- } \\
\text { morphology }\end{array}$ & charcoal (1 bag) & 677 & & 01.02 .2015 \\
\hline $0589 / 2015$ & SQ1 & charcoal (1 bag) & 686 & & 31.01 .2015 \\
\hline $0595 / 2015$ & SQ1 & charcoal (1 bag) & 687 & & 31.01 .2015 \\
\hline $0597 / 2015$ & SQ1 & charcoal (1 bag) & 688 & & 31.01 .2015 \\
\hline $0628 / 2015$ & SQ1 & charcoal (1 bag) & 694 & & 01.02 .2015 \\
\hline $0638 / 2015$ & SQ1 & charcoal (1 bag) & 695 & & 01.02 .2015 \\
\hline $0643 / 2015$ & SQ1 & charcoal (1 bag) & 696 & & 01.02 .2015 \\
\hline $0658 / 2015$ & SQ1 & charcoal (1 bag) & 699 & & 01.02 .2015 \\
\hline $0719 / 2015$ & SQ1, SU 707 and below, sieving Profile 18 & charcoal (1 bag) & 707 & & 02.02 .2015 \\
\hline
\end{tabular}




\begin{tabular}{|c|c|c|c|c|}
\hline $0726 / 2015$ & SQ1 & charcoal (1 bag) & 708 & 03.02 .2015 \\
\hline $0791 / 2015$ & SQ1 & charcoal (1 bag) & 722 & 08.02 .2015 \\
\hline s0797/2015 & SQ1 & charcoal (2 pieces) & 726 & 08.02 .2015 \\
\hline $0801 / 2015$ & SQ1 & charcoal (1 bag) & 727 & 08.02 .2015 \\
\hline $0805 / 2015$ & SQ1 & charcoal (1 bag) & 729 & 08.02 .2015 \\
\hline $0823 / 2015$ & SQ1, Feature 115 & charcoal (1 bag) & 731 & 09.02 .2015 \\
\hline $0850 / 2015$ & $\begin{array}{l}\text { SQ1, Feature } 115 \text {, sieved material (fine } \\
\text { sampling area micro-morphology) }\end{array}$ & charcoal (1 bag) & 731 & 10.02 .2015 \\
\hline $0826 / 2015$ & SQ1, Feature 115 & charcoal (1 bag) & 732 & 09.02 .2015 \\
\hline $0424 / 2015$ & SQ1S & charcoal (1 bag); wood (1 piece) & 600 & 24.01 .2015 \\
\hline $0007 / 2015$ & SQ1S, $10 \times 10 \mathrm{~m}$ & charcoal (1 bag) & 600 & 03.01 .2015 \\
\hline $0020 / 2015$ & SQ1S, $10 \times 10 \mathrm{~m}$ & charcoal (1 bag) & 601 & 05.01 .2015 \\
\hline $0750 / 2015$ & SQ1E & charcoal (1 bag) & 601 & 04.02 .2015 \\
\hline $0055 / 2015$ & SQ1S & charcoal (3 pieces) & 606 & 06.01 .2015 \\
\hline $0065 / 2015$ & SQ1/S & charcoal (1 bag) & 606 & 07.01 .2015 \\
\hline $0049 / 2015$ & SQ1S & charcoal (3 pieces) & 607 & 06.01 .2015 \\
\hline $0047 / 2015$ & SQ1S & charcoal (6 pieces) & 608 & 06.01 .2015 \\
\hline $0070 / 2015$ & SQ1S & charcoal (1 bag) & 609 & 07.01 .2015 \\
\hline $0089 / 2015$ & $\begin{array}{l}\text { SQ1/1S, cleaning border of SU towards N, } \\
\text { connection between SQ1 and SQ1S }\end{array}$ & charcoal (1 bag) & 610 & 08.01 .2015 \\
\hline $0151 / 2015$ & SQ1S & charcoal (1 bag) & 612 & 12.01 .2015 \\
\hline $0083 / 2015$ & SQ1S, cleaning square S of SU 610 & charcoal (1 bag) & 612 & 08.01 .2015 \\
\hline $0099 / 2015$ & SQ1S & charcoal (1 bag) & 613 & 10.01 .2015 \\
\hline $0109 / 2015$ & SQ1/S & charcoal (1 bag) & 614 & 10.01 .2015 \\
\hline $0114 / 2015$ & SQ1S & charcoal (1 bag) & 616 & 10.01 .2015 \\
\hline $0131 / 2015$ & SQ1S & charcoal (1 piece) & 617 & 11.01 .2015 \\
\hline $0137 / 2015$ & SQ1S & charcoal (2 pieces) & 618 & 11.01 .2015 \\
\hline $0170 / 2015$ & SQ1S & charcoal (1 bag) & 620 & 13.01 .2015 \\
\hline
\end{tabular}




\begin{tabular}{|c|c|c|c|c|}
\hline $0187 / 2015$ & SQ1S & charcoal (1 bag) & 622 & 14.01 .2015 \\
\hline $0209 / 2015$ & SQ1S & charcoal (1 bag) & 625 & 15.01 .2015 \\
\hline $0213 / 2015$ & SQ1S & charcoal (1 bag) & 627 & 15.01 .2015 \\
\hline $0220 / 2015$ & SQ1S & charcoal (1 bag) & 628 & 15.01 .2015 \\
\hline $0233 / 2015$ & SQ1S & charcoal (1 bag) & 630 & 17.01 .2015 \\
\hline $0250 / 2015$ & SQ1S & charcoal (1 bag) & 633 & 17.01 .2015 \\
\hline $0262 / 2015$ & SQ1S & charcoal (1 bag) & 633 & 18.01 .2015 \\
\hline $0252 / 2015$ & SQ1S & charcoal (1 bag) & 635 & 17.01 .2015 \\
\hline $0282 / 2015$ & SQ1S & charcoal (1 bag) & 636 & 18.01 .2015 \\
\hline $0291 / 2015$ & SQ1S & charcoal (1 bag) & 638 & 19.01 .2015 \\
\hline $0289 / 2015$ & SQ1S & charcoal (1 bag) & 639 & 19.01 .2015 \\
\hline $0304 / 2015$ & SQ1S & charcoal (2 pieces) & 640 & 19.01 .2015 \\
\hline $0314 / 2015$ & SQ1S & charcoal (1 bag) & 641 & 20.01 .2015 \\
\hline $0317 / 2015$ & SQ1S & charcoal (1 bag) & 642 & 20.01 .2015 \\
\hline $0318 / 2015$ & SQ1S & charcoal (1 bag) & 643 & 20.01 .2015 \\
\hline $0331 / 2015$ & SQ1S & charcoal (1 bag) & 644 & 20.01 .2015 \\
\hline $0342 / 2015$ & SQ1S & charcoal (1 bag) & 645 & 20.01 .2015 \\
\hline $0346 / 2015$ & SQ1S & charcoal (1 bag) & 646 & 20.01 .2015 \\
\hline $0376 / 2015$ & SQ1S & charcoal (1 bag) & 646 & 21.01 .2015 \\
\hline $0349 / 2015$ & SQ1S & charcoal (1 bag) & 647 & 20.01 .2015 \\
\hline $0360 / 2015$ & SQ1S & charcoal (1 bag) & 647 & 21.01 .2015 \\
\hline $0433 / 2015$ & SQ1S & charcoal (1 bag) & 650 & 24.01 .2015 \\
\hline $0373 / 2015$ & SQ1S & charcoal (1 bag) & 651 & 21.01 .2015 \\
\hline $0399 / 2015$ & SQ1S & charcoal (1 bag) & 652 & 22.01 .2015 \\
\hline $0392 / 2015$ & SQ1S & charcoal (1 bag) & 653 & 22.01 .2015 \\
\hline $0394 / 2015$ & SQ1S & charcoal (1 bag) & 654 & 22.01 .2015 \\
\hline
\end{tabular}




\begin{tabular}{|c|c|c|c|c|}
\hline $0389 / 2015$ & SQ1S & charcoal (1 bag) & 655 & 22.01 .2015 \\
\hline $0408 / 2015$ & SQ1S & charcoal (1 bag) & 657 & 22.01 .2015 \\
\hline $0418 / 2015$ & SQ1S & charcoal (1 bag) & 658 & 22.01 .2015 \\
\hline $0426 / 2015$ & SQ1S & charcoal (1 bag) & 658 & 24.01 .2015 \\
\hline $0420 / 2015$ & SQ1S & charcoal (1 bag) & 659 & 22.01 .2015 \\
\hline $0428 / 2015$ & SQ1S & charcoal (1 bag) & 659 & 24.01 .2015 \\
\hline $0438 / 2015$ & SQ1S & charcoal (1 bag) & 660 & 24.01 .2015 \\
\hline $0441 / 2015$ & SQ1S & charcoal (1 bag) & 661 & 24.01 .2015 \\
\hline $0459 / 2015$ & SQ1S & charcoal (1 bag) & 662 & 27.01 .2015 \\
\hline $0457 / 2015$ & SQ1S & charcoal (1 bag) & 663 & 27.01 .2015 \\
\hline $0476 / 2015$ & SQ1S & charcoal (1 bag) & 666 & 27.01 .2015 \\
\hline $0487 / 2015$ & SQ1S & charcoal (1 bag) & 668 & 27.01 .2015 \\
\hline $0493 / 2015$ & SQ1S & charcoal (1 bag) & 668 & 28.01 .2015 \\
\hline $0501 / 2015$ & SQ1S & charcoal (1 bag) & 669 & 28.01 .2015 \\
\hline $0512 / 2015$ & SQ1S & charcoal (1 bag) & 671 & 28.01 .2015 \\
\hline $0518 / 2015$ & SQ1S & charcoal (1 bag) & 672 & 28.01 .2015 \\
\hline $0509 / 2015$ & SQ1S & charcoal (1 bag) & 673 & 28.01 .2015 \\
\hline $0557 / 2015$ & SQ1S & charcoal (1 bag) & 674 & 29.01 .2015 \\
\hline $0527 / 2015$ & SQ1S & charcoal (1 bag) & 675 & 28.01 .2015 \\
\hline $0537 / 2015$ & SQ1S & charcoal (1 bag) & 675 & 29.01 .2015 \\
\hline $0541 / 2015$ & SQ1S & charcoal (1 piece) & 678 & 29.01 .2015 \\
\hline $0552 / 2015$ & SQ1S & charcoal (1 bag) & 679 & 29.01 .2015 \\
\hline $0563 / 2015$ & SQ1S & charcoal (1 bag) & 680 & 29.01 .2015 \\
\hline $0587 / 2015$ & SQ1S & charcoal (1 bag) & 685 & 31.01 .2015 \\
\hline $0600 / 2015$ & SQ1S & charcoal (1 bag) & 689 & 31.01 .2015 \\
\hline $0631 / 2015$ & SQ1S & charcoal (1 bag) & 691 & 01.02 .2015 \\
\hline
\end{tabular}




\begin{tabular}{|c|c|c|c|c|c|}
\hline $0649 / 2015$ & SQ1S & charcoal (1 bag) & 697 & & 01.02 .2015 \\
\hline $0663 / 2015$ & SQ1S & charcoal (1 bag) & 701 & & 01.02 .2015 \\
\hline $0676 / 2015$ & SQ1S & charcoal (1 bag) & 703 & & 02.02 .2015 \\
\hline $0693 / 2015$ & SQ1S & charcoal (1 bag) & 704 & & 02.02 .2015 \\
\hline $0697 / 2015$ & SQ1S & charcoal (1 bag) & 705 & & 02.02 .2015 \\
\hline $0701 / 2015$ & SQ1S & charcoal (1 bag) & 706 & & 02.02 .2015 \\
\hline $0733 / 2015$ & SQ1S & charcoal (1 bag) & 710 & & 03.02 .2015 \\
\hline $0723 / 2015$ & SQ1S & charcoal (1 bag) & 711 & & 03.02 .2015 \\
\hline $0810 / 2015$ & SQ1S & charcoal (1 bag) & 715 & & 08.02 .2015 \\
\hline $0812 / 2015$ & SQ1S & charcoal (sample) & 716 & & 08.02 .2015 \\
\hline $0767 / 2015$ & SQ1/SQ1E & charcoal (1 bag) & 717 & & 05.02 .2015 \\
\hline $0770 / 2015$ & SQ1S & charcoal & 718 & & 05.02 .2015 \\
\hline $0785 / 2015$ & SQ1S & charcoal (1 bag) & 720 & & 05.02 .2015 \\
\hline $0816 / 2015$ & SQ1S & charcoal (1 bag) & 723 & & 08.02 .2015 \\
\hline $0847 / 2015$ & SQ1S, from sieving & charcoal (1 bag); bones (2 pieces) & 734 & & 10.02 .2015 \\
\hline $0747 / 2015$ & SQ1E & $\begin{array}{l}\text { clay object (1 piece, original function unclear, roughly spherical, } \\
1 \text { hole) }\end{array}$ & 601 & SAV1W 1323 & 04.02 .2015 \\
\hline $0194 / 2015$ & SQ1S & $\begin{array}{l}\text { clay object ( } 1 \text { piece, original function unclear, tall and relatively } \\
\text { thin with a bulbous end) }\end{array}$ & 622 & SAV1W 0985 & 14.01.2015 \\
\hline $0450 / 2015$ & SQ1S & clay object (for cooking) & 661 & & 24.01.2015 \\
\hline $0612 / 2015$ & SQ1 & clay object (1 fragment, 2 small holes, original function unclear) & 688 & SAV1W 1202 & 31.01 .2015 \\
\hline $0639 / 2015$ & SQ1S & $\begin{array}{l}\text { clay object ( } 1 \text { fragment, original function unclear, part of an } \\
\text { installation, basin/oven/crucible?) }\end{array}$ & 691 & SAV1W 1243 & 01.02 .2015 \\
\hline $0837 / 2015$ & $\begin{array}{l}\text { SQ1S, } \\
\text { sieved material }\end{array}$ & 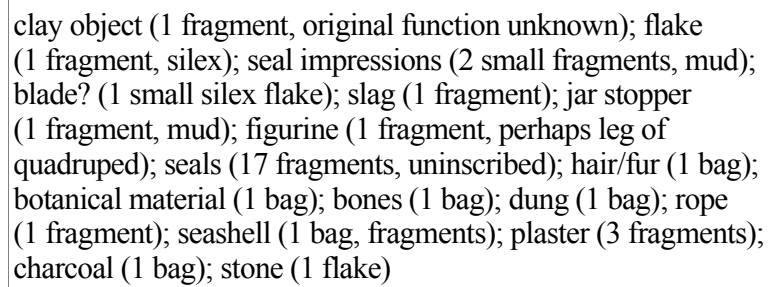 & 710 & $\begin{array}{l}\text { SAV1W } 1452 \text { (clay object), SAV1W } 1453 \\
\text { (flake), SAV1W 1454, SAV1W 1455 (seal } \\
\text { impressions), SAV1W } 1456 \text { (blade?), SAV1W } \\
1457 \text { (slag), SAV1W } 1458 \text { (jar stopper), } \\
\text { SAV1W 1523 (figurine) }\end{array}$ & 09.02 .2015 \\
\hline
\end{tabular}




\begin{tabular}{|c|c|c|c|c|c|}
\hline $0777 / 2015$ & SQ1/SQ1E & clay object & 719 & & 05.02 .2015 \\
\hline $0836 / 2015$ & $\begin{array}{l}\text { SQ1, } \\
\text { sieved material }\end{array}$ & $\begin{array}{l}\text { crucible? }(1 \text { fragment, bowl-like shape, mud); token/gaming } \\
\text { piece ( } 1 \text { re-used sherd, pottery); bead (1 small disc bead, faience); } \\
\text { worked wood, perhaps makeup applicator? ( } 1 \text { piece); bones ( } 1 \\
\text { bag); charcoal ( } 1 \text { bag); seals ( } 3 \text { fragments, uninscribed); wood ( } 1 \\
\text { bag); charcoal ( } 1 \text { piece); organic material ( } 1 \text { bag) }\end{array}$ & 726 & $\begin{array}{l}\text { SAV1W } 1461 \text { (crucible), SAV1W } 1462 \\
\text { (token/gaming piece), SAV1W } 1463 \text { (bead), } \\
\text { SAV1W } 1464 \text { (worked wood) }\end{array}$ & 09.02 .2015 \\
\hline $0579 / 2015$ & SQ1 & doum nuts (1 bag) & 664 & & 31.01 .2015 \\
\hline $0568 / 2015$ & SQ1 & doum nuts (2 pieces) & 664 & & 29.01 .2015 \\
\hline $0806 / 2015$ & SQ1 & doum nuts (1 bag) & 729 & & 08.02 .2015 \\
\hline $0752 / 2015$ & SQ1E & doum nut (1 piece) & 601 & & 04.02 .2015 \\
\hline $0136 / 2015$ & SQ1S & doum nuts (1 bag) & 618 & & 11.01 .2015 \\
\hline $0226 / 2015$ & SQ1S & doum nut (1 piece) & 627 & & 15.01 .2015 \\
\hline $0268 / 2015$ & SQ1S & doum nuts (2 pieces) & 633 & & 18.01 .2015 \\
\hline $0260 / 2015$ & SQ1S & doum nuts (1 bag) & 635 & & 17.01 .2015 \\
\hline $0333 / 2015$ & SQ1S & doum nuts (1 bag) & 641 & & 20.01 .2015 \\
\hline $0341 / 2015$ & SQ1S & doum nut (1 piece) & 645 & & 20.01 .2015 \\
\hline $0374 / 2015$ & SQ1S & doum nut (1 piece) & 646 & & 21.01 .2015 \\
\hline $0410 / 2015$ & SQ1S & doum nuts (1 bag) & 652 & & 22.01 .2015 \\
\hline $0402 / 2015$ & SQ1S & doum nut (1 piece) & 654 & & 22.01 .2015 \\
\hline $0397 / 2015$ & SQ1S & doum nuts (2 pieces) & 655 & & 22.01 .2015 \\
\hline $0407 / 2015$ & SQ1S & doum nut (1 piece) & 657 & & 22.01 .2015 \\
\hline $0417 / 2015$ & SQ1S & doum nut (1 piece) & 658 & & 22.01 .2015 \\
\hline $0430 / 2015$ & SQ1S & doum nuts (2 pieces) & 658 & & 24.01 .2015 \\
\hline $0446 / 2015$ & SQ1S & doum nuts (1 bag) & 660 & & 24.01 .2015 \\
\hline $0447 / 2015$ & SQ1S & doum nut (1 piece) & 661 & & 24.01 .2015 \\
\hline $0462 / 2015$ & SQ1S & doum nuts (1 bag) & 662 & & 27.01 .2015 \\
\hline $0460 / 2015$ & SQ1S & doum nut (1 piece) & 663 & & 27.01 .2015 \\
\hline $0516 / 2015$ & SQ1S & doum nuts (1 bag) & 671 & & 28.01 .2015 \\
\hline
\end{tabular}




\begin{tabular}{|c|c|c|c|c|c|}
\hline $0555 / 2015$ & SQ1S & doum nut (1 piece) & 679 & & 29.01 .2015 \\
\hline $0637 / 2015$ & SQ1S & doum nuts ( 2 pieces) & 691 & & 01.02 .2015 \\
\hline $0640 / 2015$ & SQ1 & doum nut (1 piece) & 694 & & 01.02 .2015 \\
\hline $0680 / 2015$ & SQ1S & doum nut (1 piece) & 703 & & 02.02 .2015 \\
\hline $0731 / 2015$ & SQ1 & doum nuts (1 bag) & 708 & & 03.02 .2015 \\
\hline $0735 / 2015$ & SQ1S & doum nuts (1 bag) & 710 & & 03.02 .2015 \\
\hline $0783 / 2015$ & SQ1/SQ1E & doum nut (1 piece) & 721 & & 05.02 .2015 \\
\hline $0142 / 2015$ & SQ1S & dung (1 bag, animal) & 618 & & 11.01 .2015 \\
\hline $0012 / 2015$ & SQ1S, surface, $10 \times 10 \mathrm{~m}$ & faience vessel (1 small rim sherd) & 600 & SAV1W 0806 & 03.01 .2015 \\
\hline $0646 / 2015$ & SQ1S & faience (1 fragment) & 691 & & 01.02 .2015 \\
\hline $0757 / 2015$ & SQ1E & faience object (1 fragment, original function unknown) & 713 & SAV1W 1426 & 04.02 .2015 \\
\hline $0590 / 2015$ & SQ1 & figurine (1 fragment of an animal figurine, horse?, pottery) & 686 & SAV1W 1206 & 31.01 .2015 \\
\hline $0808 / 2015$ & SQ1 & $\begin{array}{l}\text { figurine (fragment of an animal figurine, ibex or gazelle?, } \\
\text { pottery) }\end{array}$ & 729 & SAV1W 1396 & 08.02 .2015 \\
\hline $0025 / 2015$ & SQ1S & hair (1 bag, animal) & 603 & & 05.01 .2015 \\
\hline $0667 / 2015$ & SQ1S & hair (1 bag) & 701 & & 01.02 .2015 \\
\hline $0320 / 2015$ & SQ1S & horn (1 bag, animal) & 641 & & 20.01 .2015 \\
\hline $0618 / 2015$ & SQ1 & $\begin{array}{l}\text { ivory object ( } 1 \text { fragment, originally circular in shape, original } \\
\text { function unknown, traces of red paint) }\end{array}$ & 690 & SAV1W 1203 & 31.01 .2015 \\
\hline $0498 / 2015$ & SQ1S & $\begin{array}{l}\text { lead ( } 1 \text { small fragment, original function unclear, for sealing a } \\
\text { bag or fishing weight?) }\end{array}$ & 669 & SAV1W 1168 & 28.01 .2015 \\
\hline $0175 / 2015$ & SQ1S & leather (2 fragments) & 620 & & 13.01 .2015 \\
\hline $0241 / 2015$ & SQ1S & leather (1 piece) & 634 & & 17.01 .2015 \\
\hline $0495 / 2015$ & SQ1S & leather (1 scrap, part of pouch?) & 670 & & 28.01 .2015 \\
\hline 0690/2015 & SQ1S & leather (1 bag) & 705 & & 02.02 .2015 \\
\hline $0608 / 2015$ & SQ1S & $\begin{array}{l}\text { metal object ( } 1 \text { small fragment, thin metal, roughly v-shaped, } \\
\text { original function unknown) }\end{array}$ & 689 & SAV1W 1204 & 31.01 .2015 \\
\hline $0742 / 2015$ & SQ1S & $\begin{array}{l}\text { metal object ( } 1 \text { small fragment, copper, original function } \\
\text { unknown) }\end{array}$ & 710 & SAV1W 1322 & 03.02 .2015 \\
\hline $0129 / 2015$ & SQ1S & mud brick & 617 & & 11.01 .2015 \\
\hline
\end{tabular}




\begin{tabular}{|c|c|c|c|c|c|}
\hline $0155 / 2015$ & SQ1S & needle/pin (1 piece, bronze) & 618 & SAV1W 0965 & 12.01 .2015 \\
\hline $0387 / 2015$ & SQ1S & net weight (1 piece, pottery) & 652 & SAV1W 1475 & 22.01 .2015 \\
\hline $0695 / 2015$ & SQ1S & net weight? (1 piece, clay) & 704 & SAV1W 1267 & 02.02 .2015 \\
\hline $0201 / 2015$ & $\mathrm{SQ} 1,20 \mathrm{~m}$ E of & Nun bowl (1 small fragment, faience) & surface & SAV1W 0987 & 14.01 .2015 \\
\hline $0269 / 2015$ & SQ1S, 3m E of & Nun bowl (1 fragment with base preserved, faience) & surface & SAV1W 0771 & 18.01 .2015 \\
\hline $0820 / 2015$ & SQ1, Feature 115 & Nun bowl? (1 fragment, faience) & 731 & SAV1W 1418 & 09.02 .2015 \\
\hline $0229 / 2015$ & SQ1S & $\begin{array}{l}\text { Nun bowl ( } 1 \text { fragment with base preserved, faience, } \\
18^{\text {th }} \text { Dynasty) }\end{array}$ & 627 & SAV1W 0738 & 15.01 .2015 \\
\hline $0231 / 2015$ & SQ1S & Nun bowl? (1 small rim fragment, faience) & 631 & SAV1W 0725 & 17.01 .2015 \\
\hline $0323 / 2015$ & SQ1S & Nun bowl (1 small fragment, faience) & 642 & SAV1W 1007 & 20.01 .2015 \\
\hline $0485 / 2015$ & SQ1S & Nun bowl? (1 small rim fragment, faience) & 668 & SAV1W 1194 & 27.01 .2015 \\
\hline $0756 / 2015$ & SQ1E & Nun bowl (2 fragments, glued together, faience) & 713 & SAV1W 1424 & 04.02 .2015 \\
\hline $0772 / 2015$ & SQ1/SQ1E & Nun bowl (1 fragment, faience) & 717 & SAV1W 1425 & 05.02 .2015 \\
\hline $0338 / 2015$ & SQ1S & ochre (1 piece, yellow) & 641 & & 20.01 .2015 \\
\hline $0713 / 2015$ & SQ1, cleaning Profile 12, sieving & organic material (1 bag) & 676 & & 02.02 .2015 \\
\hline $0605 / 2015$ & SQ1 & organic material (1 bag) & 687 & & 31.01 .2015 \\
\hline $0604 / 2015$ & SQ1 & organic material (1 piece) & 688 & & 31.01 .2015 \\
\hline $0635 / 2015$ & SQ1 & organic material (1 piece) & 695 & & 01.02 .2015 \\
\hline $0832 / 2015$ & $\begin{array}{l}\text { SQ1, } \\
\text { sieved material }\end{array}$ & organic material (1 bag); wood (1 piece); charcoal (1 bag) & 722 & & 09.02 .2015 \\
\hline $0835 / 2015$ & $\begin{array}{l}\text { SQ1, } \\
\text { sieved material }\end{array}$ & organic material (1 bag); charcoal (1 bag); bones (1 bag) & 728 & & 09.02 .2015 \\
\hline $0794 / 2015$ & SQ1, Feature 115 & organic material (1 bag) & 725 & & 08.02 .2015 \\
\hline $0821 / 2015$ & SQ1, Feature 115 & organic material (1 bag) & 731 & & 09.02 .2015 \\
\hline $0031 / 2015$ & SQ1S & organic material (1 piece, unidentified) & 603 & & 05.01 .2015 \\
\hline $0061 / 2015$ & SQ1S & organic material (3 pieces, sticks) & 609 & & 06.01 .2015 \\
\hline $0069 / 2015$ & SQ1S & organic material (4 pieces, seed/nut?) & 609 & & 07.01 .2015 \\
\hline $0072 / 2015$ & SQ1S & organic material (5 pieces, sticks) & 609 & & 07.01 .2015 \\
\hline
\end{tabular}




\begin{tabular}{|c|c|c|c|c|c|}
\hline $0076 / 2015$ & SQ1S & organic material (1 piece, unclear) & 609 & & 07.01 .2015 \\
\hline $0088 / 2015$ & $\begin{array}{l}\text { SQ1/1S, cleaning border of SU towards N, } \\
\text { connection between SQ1 and SQ1S }\end{array}$ & organic material (1 bag, fibres) & 610 & & 08.01 .2015 \\
\hline $0090 / 2015$ & SQ1S & organic material (1 piece, unclear) & 612 & & 08.01 .2015 \\
\hline $0106 / 2015$ & SQ1S & organic material (1 bag, unclear) & 612 & & 10.01 .2015 \\
\hline $0150 / 2015$ & SQ1S & organic material (fibre, 1 small piece of rope?) & 612 & SAV1W 0969 & 12.01 .2015 \\
\hline $0102 / 2015$ & SQ1S & organic material (1 piece, unclear) & 613 & & 10.01 .2015 \\
\hline $0123 / 2015$ & SQ1S & organic material (1 piece, animal hide?) & 614 & & 10.01 .2015 \\
\hline $0112 / 2015$ & SQ1S & organic material (1 bag, unclear) & 615 & & 10.01 .2015 \\
\hline $0117 / 2015$ & SQ1S & organic material (1 bag, unclear) & 615 & & 10.01 .2015 \\
\hline $0132 / 2015$ & SQ1S & organic material (1 piece, seed pod?) & 617 & & 11.01 .2015 \\
\hline $0130 / 2015$ & SQ1S & organic material (1 piece, skin and fur) & 618 & & 11.01 .2015 \\
\hline $0138 / 2015$ & SQ1S & organic material (textile and hair?) & 618 & & 11.01 .2015 \\
\hline $0160 / 2015$ & SQ1S & organic material (2 pieces, leaves?) & 620 & & 12.01 .2015 \\
\hline $0163 / 2015$ & SQ1S & organic material (1 piece, unclear) & 620 & & 12.01 .2015 \\
\hline $0173 / 2015$ & SQ1S & organic material (1 bag) & 620 & & 13.01 .2015 \\
\hline $0177 / 2015$ & SQ1S & organic material (1 bag) & 621 & & 13.01 .2015 \\
\hline $0190 / 2015$ & SQ1S & organic material (1 bag) & 622 & & 14.01 .2015 \\
\hline $0195 / 2015$ & SQ1S & organic material (1 bag, unclear) & 622 & & 14.01 .2015 \\
\hline $0191 / 2015$ & SQ1S & organic material (1 piece) & 624 & & 14.01 .2015 \\
\hline $0208 / 2015$ & SQ1S & organic material (1 bag) & 625 & & 15.01 .2015 \\
\hline $0218 / 2015$ & SQ1S & organic material (1 piece, animal) & 627 & & 15.01 .2015 \\
\hline $0249 / 2015$ & SQ1S & organic material (1 piece) & 633 & & 17.01 .2015 \\
\hline $0254 / 2015$ & SQ1S & organic material (1 bag, animal hair) & 633 & & 17.01.2015 \\
\hline $0259 / 2015$ & SQ1S & organic material (1 bag) & 635 & & 17.01 .2015 \\
\hline $0280 / 2015$ & SQ1S & organic material (1 piece, unclear) & 637 & & 18.01 .2015 \\
\hline $0294 / 2015$ & SQ1S & organic material (1 bag, dung?) & 638 & & 19.01.2015 \\
\hline
\end{tabular}




\begin{tabular}{|c|c|c|c|c|c|}
\hline $0295 / 2015$ & SQ1S & organic material (1 bag) & 639 & & 19.01.2015 \\
\hline $0322 / 2015$ & SQ1S & organic material (1 bag) & 641 & & 20.01 .2015 \\
\hline $0507 / 2015$ & SQ1S & organic material (1 piece, animal) & 663 & & 28.01 .2015 \\
\hline $0471 / 2015$ & SQ1S & organic material (1 bag) & 667 & & 27.01 .2015 \\
\hline $0483 / 2015$ & SQ1S & organic material (1 bag) & 668 & & 27.01 .2015 \\
\hline $0488 / 2015$ & SQ1S & organic material $(1 \mathrm{bag})$ & 669 & & 27.01.2015 \\
\hline $0515 / 2015$ & SQ1S & organic material (1 bag) & 672 & & 28.01 .2015 \\
\hline $0529 / 2015$ & SQ1S & organic material (1 bag, seed pod) & 674 & & 28.01 .2015 \\
\hline $0526 / 2015$ & SQ1S & organic material (1 bag, unclear) & 675 & & 28.01 .2015 \\
\hline $0546 / 2015$ & SQ1S & organic material (1 bag) & 675 & & 29.01.2015 \\
\hline $0547 / 2015$ & SQ1S & organic material (1 bag) & 678 & & 29.01 .2015 \\
\hline $0705 / 2015$ & $\begin{array}{l}\text { SQ1S, cleaning of bottom surface of } \\
\text { SU } 678\end{array}$ & organic material (1 bag) & 678 & & 02.02 .2015 \\
\hline $0583 / 2015$ & SQ1S & organic material (1 bag) & 685 & & 31.01 .2015 \\
\hline $0613 / 2015$ & SQ1S & organic material (1 bag) & 689 & & 31.01 .2015 \\
\hline $0630 / 2015$ & SQ1S & organic material (1 bag) & 691 & & 01.02 .2015 \\
\hline $0645 / 2015$ & SQ1S & organic material (1 bag) & 696 & & 01.02 .2015 \\
\hline $0660 / 2015$ & SQ1S & organic material (1 bag) & 698 & & 01.02 .2015 \\
\hline $0679 / 2015$ & SQ1S & organic material (1 bag, leaves) & 703 & & 02.02 .2015 \\
\hline $0703 / 2015$ & SQ1S & organic material (1 bag) & 704 & & 02.02 .2015 \\
\hline $0696 / 2015$ & SQ1S & organic material (2 pieces) & 705 & & 02.02 .2015 \\
\hline $0734 / 2015$ & SQ1S & organic material (1 bag) & 710 & & 03.02 .2015 \\
\hline $0787 / 2015$ & SQ1S & organic material (1 bag) & 720 & & 05.02 .2015 \\
\hline $0124 / 2015$ & SQ1S & plaster (1 bag, with red paint) & 614 & & 10.01 .2015 \\
\hline $0423 / 2015$ & SQ1, cleaning central $N$ of SQ1 & pottery & 600 & & 22.01 .2015 \\
\hline $0080 / 2015$ & SQ1, cleaning SQ1 - NW-quarter & pottery & 600 & & 07.01 .2015 \\
\hline $0079 / 2015$ & SQ1, cleaning SQ1 - SE-quarter & $\begin{array}{l}\text { pottery, among it figurine ( } 1 \text { lower part of a rudimentary female } \\
\text { figurine, clay) }\end{array}$ & 600 & SAV1W 0906 & 07.01 .2015 \\
\hline
\end{tabular}




\begin{tabular}{|c|c|c|c|c|c|}
\hline $0073 / 2015$ & $\begin{array}{l}\text { SQ1, erosion part, 'trench border' to SQ1S, } \\
\text { E half (E-W 0-5m) }\end{array}$ & pottery ( 5 baskets) & 600 & & 07.01 .2015 \\
\hline $0464 / 2015$ & SQ1 & pottery & 664 & & 27.01.2015 \\
\hline $0565 / 2015$ & SQ1 & $\begin{array}{l}\text { pottery, among it figurine ( } 1 \text { fragment of animal figurine, head } \\
\text { missing, pottery), scrapers ( } 3 \text { pieces, } 2 \text { re-used } 18^{\text {th }} \text { Dynasty pot } \\
\text { sherds, } 1 \text { re-used } 18^{\text {th }} \text { Dynasty? Pot sherd, pottery) }\end{array}$ & 664 & $\begin{array}{l}\text { SAV1W } 1303 \text { (figurine), SAV1W 1304, } \\
\text { SAV1W 1305, SAV1W } 1306 \text { (scrapers) }\end{array}$ & 29.01.2015 \\
\hline $0574 / 2015$ & SQ1 & pottery & 664 & & 31.01 .2015 \\
\hline $0543 / 2015$ & SQ1 & $\begin{array}{l}\text { pottery, among it scrapers ( } 2 \text { pieces, } 1 \text { re-used } 18^{\text {th }} \text { Dynasty } \\
\text { sherd, pottery, } 1 \text { re-used base with rim, Christian ) }\end{array}$ & 674 & SAV1W 1307, SAV1W 1307 (scrapers) & 29.01.2015 \\
\hline $0535 / 2015$ & SQ1 & pottery & 675 & & 29.01.2015 \\
\hline $0532 / 2015$ & SQ1 & pottery & 676 & & 28.01 .2015 \\
\hline $0672 / 2015$ & $\begin{array}{l}\text { SQ1, Profile } 14 \text {, sampling area micro- } \\
\text { morphology }\end{array}$ & pottery & 676 & & 01.02 .2015 \\
\hline $0710 / 2015$ & SQ1, cleaning Profile 12, sieving & pottery & 676 & & 02.02 .2015 \\
\hline $0669 / 2015$ & $\begin{array}{l}\text { SQ1, Profile 14, sampling area micro- } \\
\text { morphology }\end{array}$ & pottery & 677 & & 01.02 .2015 \\
\hline $0539 / 2015$ & SQ1 & $\begin{array}{l}\text { pottery, among it figurine (lower half of female? figurine, clay), } \\
\text { token/gaming piece (1 re-used sherd, pottery) }\end{array}$ & 678 & $\begin{array}{l}\text { SAV1W } 1301 \text { (figurine), SAV1W } 1302 \\
\text { (token/gaming piece) }\end{array}$ & 29.01.2015 \\
\hline $0571 / 2015$ & SQ1 & pottery & 684 & & 29.01.2015 \\
\hline $0575 / 2015$ & SQ1 & pottery & 684 & & 31.01 .2015 \\
\hline $0584 / 2015$ & SQ1 & pottery & 686 & & 31.01 .2015 \\
\hline $0593 / 2015$ & SQ1 & pottery & 687 & & 31.01 .2015 \\
\hline $0594 / 2015$ & SQ1 & pottery & 688 & & 31.01 .2015 \\
\hline $0615 / 2015$ & SQ1 & pottery & 690 & & 31.01 .2015 \\
\hline $0623 / 2015$ & SQ1 & pottery & 694 & & 01.02 .2015 \\
\hline $0624 / 2015$ & SQ1 & pottery & 695 & & 01.02 .2015 \\
\hline $0642 / 2015$ & SQ1 & pottery, among it lid (1 fragment, clay) & 696 & SAV1W 1229 & 01.02 .2015 \\
\hline $0647 / 2015$ & SQ1S & pottery & 697 & & 01.02 .2015 \\
\hline $0650 / 2015$ & SQ1S & $\begin{array}{l}\text { pottery and stone: scrapers ( } 2 \text { pieces, each re-used sherds, } \\
\text { pottery), stopper/sealing ( } 1 \text { fragment, clay); grindstone or hand } \\
\text { mill (1 fragment, quartzite), whetstone (1 fragment, sandstone) }\end{array}$ & 698 & $\begin{array}{l}\text { SAV1W 1311, SAV1W } 1394 \text { (scrapers), } \\
\text { SAV1W } 1310 \text { (stopper/sealing), SAV1W } 1262 \\
\text { (grindstone or hand mill), SAV1W } 1272 \\
\text { (whetstone) }\end{array}$ & 01.02 .2015 \\
\hline
\end{tabular}




\begin{tabular}{|c|c|c|c|c|c|}
\hline $0653 / 2015$ & SQ1 & pottery & 699 & & 01.02 .2015 \\
\hline $0659 / 2015$ & SQ1 & pottery & 700 & & 01.02 .2015 \\
\hline $0671 / 2015$ & $\begin{array}{l}\text { SQ1, Profile 14, sampling area micro- } \\
\text { morphology }\end{array}$ & pottery & 702 & & 01.02 .2015 \\
\hline $0716 / 2015$ & SQ1, top surface, Profile 18 & pottery & 707 & & 02.02 .2015 \\
\hline $0715 / 2015$ & SQ1, Profile 18, sieving, 707 and below & pottery & 707 & & 02.02 .2015 \\
\hline $0707 / 2015$ & SQ1 & pottery & 708 & & 02.02 .2015 \\
\hline $0722 / 2015$ & SQ1 & pottery, among it lid (1 re-used $18^{\text {th }}$ Dynasty base sherd, pottery) & 708 & SAV1W 1501 & 03.02 .2015 \\
\hline $0717 / 2015$ & SQ1, top surface, Profile 18 & pottery & 709 & & 02.02 .2015 \\
\hline $0718 / 2015$ & SQ1, bottom surface, Profile 18 & pottery & 709 & & 02.02 .2015 \\
\hline $0779 / 2015$ & SQ1 & pottery & 722 & & 08.02 .2015 \\
\hline $0784 / 2015$ & SQ1 & pottery & 722 & & 05.02 .2015 \\
\hline $0795 / 2015$ & SQ1 & pottery & 726 & & 08.02 .2015 \\
\hline $0796 / 2015$ & SQ1 & pottery & 727 & & 08.02 .2015 \\
\hline $0799 / 2015$ & SQ1 & pottery & 728 & & 08.02 .2015 \\
\hline $0792 / 2015$ & SQ1, Feature 115 & pottery & 725 & & 08.02 .2015 \\
\hline $0819 / 2015$ & SQ1, Feature 115 & pottery, among it lid ( 1 re-used $18^{\text {th }}$ Dynasty pot sherd, pottery) & 731 & SAV1W 1478 & 09.02 .2015 \\
\hline $0849 / 2015$ & $\begin{array}{l}\text { SQ1, Feature } 115 \text {, sieved material } \\
\text { (sampling area morphology) }\end{array}$ & pottery & 731 & & 10.02 .2015 \\
\hline $0838 / 2015$ & SQ1, Feature 115 & pottery (complete vessel) & 732 & & 10.02 .2015 \\
\hline $0839 / 2015$ & SQ1, Feature 115 & $\begin{array}{l}\text { pottery, among it figurine (1 small fragment, animal figurine, } \\
\text { part of leg?) }\end{array}$ & 732 & SAV1W 1465 & 10.02 .2015 \\
\hline $0825 / 2015$ & SQ1, Feature 115 & $\begin{array}{l}\text { pottery, among it scraper ( } 1 \text { re-used } 18^{\text {th }} \text { Dynasty pot sherd, } \\
\text { pottery) }\end{array}$ & 732 & SAV1W 1519 & 09.02 .2015 \\
\hline $0739 / 2015$ & SQ1E, surface, extension to E, cleaning & pottery & 600 & & 03.02 .2015 \\
\hline $0035 / 2015$ & $\begin{array}{l}\text { SQ1S, trench, in front of the W-part, } \\
\text { erosion }\end{array}$ & pottery $(1 / 2$ basket $)$ & 600 & & 05.01 .2015 \\
\hline $0741 / 2015$ & SQ1E & $\begin{array}{l}\text { pottery, among it lid ( } 1 \text { re-used base sherd, Christian, pottery), } \\
\text { pottery object ( } 1 \text { re-used pot sherd, function unknown), scraper } \\
\text { ( } 1 \text { re-used } 18^{\text {th }} \text { Dynasty sherd, pottery) }\end{array}$ & 601 & $\begin{array}{l}\text { SAV1W } 1500 \text { (lid), SAV1W } 1504 \text { (pottery } \\
\text { object), SAV1W } 1518 \text { (scraper) }\end{array}$ & 03.02 .2015 \\
\hline
\end{tabular}




\begin{tabular}{|c|c|c|c|c|c|}
\hline $0728 / 2015$ & SQ1E & $\begin{array}{l}\text { pottery, among it scrapers ( } 3 \text { pieces, } 1 \text { re-used Post-New } \\
\text { Kingdom pot sherd, } 1 \text { re-used } 18^{\text {th }} \text { Dynasty pot sherd, } 1 \text { re-used } \\
\text { Ramesside pot sherd, pottery), seal impression ( } 1 \text { fragment, clay, } \\
\text { traces of hieroglyphs and cartouche) }\end{array}$ & 601 & $\begin{array}{l}\text { SAV1W 1431, SAV1W 1432, SAV1W } 1433 \\
\text { (scrapers), SAV1W } 1300 \text { (seal impression) }\end{array}$ & 04.02 .2015 \\
\hline $0008 / 2015$ & SQ1S, $10 \times 10 \mathrm{~m}$ & $\begin{array}{l}\text { pottery ( } 5 \text { baskets), among them figurine ( } 1 \text { body fragment of a } \\
\text { camel, pottery), scraper ( } 1 \text { re-used fragment of a Canaanite } \\
\text { amphora, } 18^{\text {th }} \text { Dynasty) }\end{array}$ & 600 & $\begin{array}{l}\text { SAV1W } 0800 \text { (camel figurine), SAV1W } 0801 \\
\text { (scraper) }\end{array}$ & 03.01 .2015 \\
\hline $0013 / 2015$ & SQ1S, $10 \times 10 \mathrm{~m}$ & $\begin{array}{l}\text { pottery and slag: pottery ( } 10 \text { baskets), among them re-used sherds } \\
\text { ( } 2 \text { pieces, roughly square and roughly round [lid?] in shape); slag } \\
\text { ( } 3 \text { fragments) }\end{array}$ & 600 & $\begin{array}{l}\text { SAV1W 0848, SAV1W } 0849 \text { (re-used sherds), } \\
\text { SAV1W } 0847 \text { (slag) }\end{array}$ & 03.01 .2015 \\
\hline $0017 / 2015$ & SQ1S, E-half(E-W 0-5m) & pottery & 601 & & 05.01 .2015 \\
\hline $0002 / 2015$ & SQ1S, top surface, and $1 \mathrm{~m}$ in all directions & pottery (19 baskets) & 600 & & 03.01 .2015 \\
\hline $0029 / 2015$ & SQ1S, on the enclosure wall & pottery & 601 & & 05.01 .2015 \\
\hline $0018 / 2015$ & SQ1S, W Half(E-W 5-10m) & pottery & 601 & & 05.01 .2015 \\
\hline $0022 / 2015$ & SQ1S & pottery (1 basket) & 602 & & 05.01 .2015 \\
\hline $0023 / 2015$ & SQ1S & $\begin{array}{l}\text { pottery and bones: pottery, among it oil lamp (1 small fragment, } \\
\text { pottery, Christian); bones ( } 1 \text { bag) }\end{array}$ & 603 & SAV1W 0857 (oil lamp) & 05.01 .2015 \\
\hline $0024 / 2015$ & SQ1S & pottery & 604 & & 05.01 .2015 \\
\hline $0034 / 2015$ & SQ1S & $\begin{array}{l}\text { pottery ( } 1 \text { basket), among it stopper (1 re-used Post-New } \\
\text { Kingdom pot sherd) }\end{array}$ & 605 & SAV1W 0867 & 05.01 .2015 \\
\hline $0039 / 2015$ & SQ1S & pottery, among it clay object (1 piece, original function unclear) & 606 & SAV1W 0868 & 05.01 .2015 \\
\hline $0064 / 2015$ & $\mathrm{SQ} 1 / \mathrm{S}$ & pottery (1 basket) & 606 & & 07.01 .2015 \\
\hline $0042 / 2015$ & SQ1S & pottery & 607 & & 06.01 .2015 \\
\hline $0043 / 2015$ & SQ1S & pottery & 608 & & 06.01 .2015 \\
\hline $0051 / 2015$ & SQ1S & $\begin{array}{l}\text { pottery, among it scraper ( } 1 \text { re-used sherd, pottery, Christian), } \\
\text { lid ( } 1 \text { re-used dish, broken in half, pottery, } 18^{\text {th }} \text { Dynasty) }\end{array}$ & 609 & SAV1W 0886 (scraper), SAV1W 0887 (lid) & 06.01 .2015 \\
\hline $0062 / 2015$ & SQ1S & pottery, among it window grille? (1 fragment, clay) & 609 & SAV1W 0907 & 07.01 .2015 \\
\hline $0084 / 2015$ & $\begin{array}{l}\text { SQ1/1S, cleaning connection between SQ1 } \\
\text { and SQ1S }\end{array}$ & $\begin{array}{l}\text { pottery, among it figurine? ( } 1 \text { small rectangular piece, possibly } \\
\text { part of a rudimentary female figurine, clay) }\end{array}$ & 610 & SAV1W 0908 & 08.01 .2015 \\
\hline $0087 / 2015$ & SQ1S & pottery; bone (1 piece) & 611 & & 08.01 .2015 \\
\hline $0097 / 2015$ & SQ1S & pottery & 611 & & 10.01 .2015 \\
\hline $0103 / 2015$ & SQ1S & pottery & 612 & & 10.01 .2015 \\
\hline
\end{tabular}




\begin{tabular}{|c|c|c|c|c|c|}
\hline $0091 / 2015$ & SQ1S & $\begin{array}{l}\text { pottery, among it figurine (1 quadruped figurine, head missing, } \\
\text { possibly horse, clay) }\end{array}$ & 612 & SAV1W 0910 & 08.01 .2015 \\
\hline $0081 / 2015$ & SQ1S, cleaning square S of SU 610 & pottery & 612 & & 08.01 .2015 \\
\hline $0146 / 2015$ & $\begin{array}{l}\text { SQ1S, S-part, second cleaning and sand } \\
\text { removal }\end{array}$ & $\begin{array}{l}\text { pottery and faience: pottery ( } 2 \text { baskets); Nun bowl ( } 1 \text { small } \\
\text { fragment, faience) }\end{array}$ & 612 & SAV1W 0964 (Nun bowl) & 12.01 .2015 \\
\hline $0098 / 2015$ & SQ1S & pottery ( 2 baskets) & 613 & & 10.01 .2015 \\
\hline $0107 / 2015$ & SQ1S, mud debris, partly towards SQ1 (N) & $\begin{array}{l}\text { pottery ( } 5 \text { baskets), among them scraper (1 re-used pot sherd, } \\
\text { pottery, Post-New Kingdom, Christian) }\end{array}$ & 614 & SAV1W 0947 & 10.01.2015 \\
\hline $0111 / 2015$ & SQ1S & pottery & 615 & & 10.01.2015 \\
\hline $0113 / 2015$ & SQ1S & pottery & 616 & & 10.01 .2015 \\
\hline $0119 / 2015$ & SQ1S, NW of SU & pottery & 616 & & 10.01 .2015 \\
\hline $0126 / 2015$ & SQ1S & pottery ( 2 baskets) & 617 & & 11.01.2015 \\
\hline $0127 / 2015$ & SQ1S & $\begin{array}{l}\text { pottery ( } 2 \text { baskets), among them scrapers ( } 1 \text { re-used pottery } \\
\left.\text { sherd, } 18^{\text {th }} \text { Dynasty, } 2 \text { re-used pottery sherds, Christian }\right)\end{array}$ & 618 & $\begin{array}{l}\text { SAV1W 0966, SAV1W 0967, SAV1W } 0968 \\
\text { (scrapers) }\end{array}$ & 11.01.2015 \\
\hline $0144 / 2015$ & SQ1S & pottery & 618 & & 12.01.2015 \\
\hline $0139 / 2015$ & SQ1S & pottery & 619 & & 11.01.2015 \\
\hline $0145 / 2015$ & SQ1S & pottery, among it scraper (1 re-used sherd, pottery, Christian) & 619 & SAV1W 0976 & 12.01 .2015 \\
\hline $0158 / 2015$ & SQ1S & pottery; wood (1 piece) & 620 & & 12.01 .2015 \\
\hline $0166 / 2015$ & SQ1S & pottery & 620 & & 13.01.2015 \\
\hline $0174 / 2015$ & $\begin{array}{l}\text { SQ1S, N-part of SU, } \\
\text { on remains of enclosure wall) }\end{array}$ & pottery, among it crucible or oven? (1 large fragment, clay) & 620 & SAV1W 0756 & 13.01.2015 \\
\hline $0159 / 2015$ & SQ1S & pottery & 621 & & 12.01 .2015 \\
\hline $0167 / 2015$ & SQ1S & pottery; bone ( 2 pieces, 1 horn?) & 621 & & 13.01.2015 \\
\hline $0183 / 2015$ & SQ1S & $\begin{array}{l}\text { pottery, slag and stone: pottery ( } 2 \text { baskets), among them scrapers } \\
\text { ( } 3 \text { pieces, each re-used sherds, } 1 \text { Christian, } 1 \text { Post-New Kingdom, } \\
118^{\text {th }} \text { Dynasty), lids ( } 2 \text { pieces, each re-used sherds, } 1 \text { Christian, } 1 \\
\text { not specified); slag and brick ( } 1 \text { small fragment); whetstone } \\
\text { (1 piece, sandstone) }\end{array}$ & 622 & $\begin{array}{l}\text { SAV1W 0734, SAV1W } 0758 \text { (lids), SAV1W } \\
\text { 0735, SAV1W 0736, SAV1W 0759 (scrapers), } \\
\text { SAV1W 0984 (slag and brick), SAV1W } 0993 \\
\text { (whetstone) }\end{array}$ & 14.01.2015 \\
\hline $0184 / 2015$ & SQ1S & $\begin{array}{l}\text { pottery ( } 1 \text { basket), among it clay ( } 1 \text { small fragment, possibly for } \\
\text { sealing), weight ( } 1 \text { re-used fragment of pottery, Post-New } \\
\text { Kingdom), window grille ( } 1 \text { fragment, pottery, Christian) }\end{array}$ & 624 & $\begin{array}{l}\text { SAV1W } 0986 \text { (clay object for sealing?), } \\
\text { SAV1W } 0732 \text { (weight), SAV1W } 0733 \text { (window } \\
\text { grille) }\end{array}$ & 14.01.2015 \\
\hline 0196/2015 & SQ1S & pottery & 625 & & 15.01.2015 \\
\hline $0202 / 2015$ & SQ1S & pottery & 625 & & 15.01 .2015 \\
\hline
\end{tabular}




\begin{tabular}{|c|c|c|c|c|c|}
\hline $0199 / 2015$ & SQ1S & pottery & 626 & & 14.01.2015 \\
\hline $0203 / 2015$ & SQ1S & $\begin{array}{l}\text { pottery, among it scraper (1 re-used sherd, pottery, Post-New } \\
\text { Kingdom) }\end{array}$ & 626 & SAV1W 0737 & 15.01.2015 \\
\hline $0210 / 2015$ & SQ1S & $\begin{array}{l}\text { pottery, among it slag with pottery sherd ( } 1 \text { fragment, sherd } \\
\text { Christian) }\end{array}$ & 627 & SAV1W 0989 & 15.01 .2015 \\
\hline $0211 / 2015$ & SQ1S & $\begin{array}{l}\text { pottery, among it weight ( } 1 \text { fragment, pottery, } 18^{\text {th }} \text { Dynasty); } \\
\text { wood ( } 1 \text { piece) }\end{array}$ & 628 & SAV1W 0739 & 15.01.2015 \\
\hline $0223 / 2015$ & SQ1S & pottery & 629 & & 15.01.2015 \\
\hline $0230 / 2015$ & SQ1S, E- and W-part of SU separated & pottery & 630 & & 17.01 .2015 \\
\hline $0237 / 2015$ & SQ1S & pottery & 632 & & 17.01 .2015 \\
\hline $0261 / 2015$ & SQ1S & $\begin{array}{l}\text { pottery, among it scrapers (each } 2 \text { re-used } 18^{\text {th }} \text { Dynasty pot } \\
\text { sherds, pottery) }\end{array}$ & 633 & SAV1W 0774, SAV1W 0775 (scrapers) & 18.01.2015 \\
\hline $0239 / 2015$ & SQ1S & $\begin{array}{l}\text { pottery, among it figurine ( } 1 \text { fragment of a quadruped figurine, } \\
\text { possibly cattle, pottery, } 18^{\text {th }} \text { Dynasty) }\end{array}$ & 634 & SAV1W 0772 & 17.01.2015 \\
\hline $0245 / 2015$ & SQ1S & pottery, among it oil lamp (1 fragment, pottery, Christian) & 635 & SAV1W 0773 & 17.01.2015 \\
\hline $0272 / 2015$ & SQ1S & pottery, among it lid? (1 re-used sherd, pottery) & 636 & SAV1W 0765 & 18.01 .2015 \\
\hline $0297 / 2015$ & SQ1S & pottery & 636 & & 19.01 .2015 \\
\hline $0276 / 2015$ & SQ1S & pottery & 637 & & 18.01 .2015 \\
\hline $0285 / 2015$ & SQ1S & $\begin{array}{l}\text { pottery ( } 8 \text { baskets), among them stopper/sealing? ( } 1 \text { small } \\
\text { fragment, clay), scrapers ( } 1 \text { re-used } 18^{\text {th }} \text { Dynasty pottery sherd, } \\
1 \text { re-used pot sherd, pottery, Post-New Kingdom), } \\
\text { architecture/aegis stand ( } 1 \text { fragment, pottery, } 18^{\text {th }} \text { Dynasty), oil } \\
\text { lamp ( } 1 \text { small fragment, pottery, Christian), net weights ( } 2 \text { pieces, } \\
\text { each pottery) }\end{array}$ & 638 & $\begin{array}{l}\text { SAV1W } 0778 \text { (stopper/sealing?), SAV1W } \\
\text { 1008, SAV1W 1010 (scrapers), SAV1W } 1468 \\
\text { (architecture/aegis stand), SAV1W } 1472 \text { (oil } \\
\text { lamp), SAV1W 1476, SAV1W } 1477 \text { (net } \\
\text { weights) }\end{array}$ & 19.01.2015 \\
\hline $0286 / 2015$ & SQ1S & $\begin{array}{l}\text { pottery ( } 4 \text { baskets), among them stopper/sealing? ( } 1 \text { small } \\
\text { fragment, clay), scraper ( } 1 \text { re-used } 18^{\text {th }} \text { pot sherd, pottery) }\end{array}$ & 639 & $\begin{array}{l}\text { SAV1W } 0766 \text { (stopper/sealing?), SAV1W } 1507 \\
\text { (scraper) }\end{array}$ & 19.01.2015 \\
\hline $0299 / 2015$ & SQ1S & $\begin{array}{l}\text { pottery, among it scraper ( } 1 \text { re-used pot sherd, pottery, Post-New } \\
\text { Kingdom) }\end{array}$ & 640 & SAV1W 1011 & 19.01.2015 \\
\hline $0302 / 2015$ & SQ1S & $\begin{array}{l}\text { pottery (c. } 6 \text { baskets), among them stopper/sealing? ( } 1 \text { small } \\
\text { fragment, clay), hand mill ( } 1 \text { small fragment, quartzite), scraper } \\
\text { ( } 1 \text { re-used } 18^{\text {th }} \text { Dynasty Canaanite amphora sherd, pottery), slag } \\
(1 \text { small fragment) }\end{array}$ & 641 & $\begin{array}{l}\text { SAV1W } 0767 \text { (stopper/sealing?), SAV1W } 0769 \\
\text { (hand mill), SAV1W } 1508 \text { (scraper), SAV1W } \\
0768 \text { (slag) }\end{array}$ & 19.01.2015 \\
\hline $0305 / 2015$ & SQ1S & $\begin{array}{l}\text { pottery ( } 3 \text { baskets), among them oil lamp ( } 1 \text { fragment, Christian, } \\
\text { pottery), scraper ( } 1 \text { re-used } 18^{\text {th }} \text { Dynasty pot sherd, pottery), } \\
\text { token/gaming piece ( } 1 \text { re-used sherd, pottery) }\end{array}$ & 642 & $\begin{array}{l}\text { SAV1W } 1473 \text { (oil lamp), SAV1W } 1134 \\
\text { (scraper), SAV1W } 1006 \text { (scraper) }\end{array}$ & 20.01.2015 \\
\hline
\end{tabular}




\begin{tabular}{|c|c|c|c|c|c|}
\hline $0309 / 2015$ & SQ1S & $\begin{array}{l}\text { pottery, among it scraper ( } 1 \text { re-used } 18^{\text {th }} \text { Dynasty pot sherd, } \\
\text { pottery) }\end{array}$ & 643 & SAV1W 1032 & 20.01.2015 \\
\hline $0325 / 2015$ & SQ1S & pottery ( 2 baskets) & 644 & & 20.01.2015 \\
\hline $0335 / 2015$ & SQ1S & pottery (1 basket) & 645 & & 20.01.2015 \\
\hline $0344 / 2015$ & SQ1S & pottery, among it lid (1 re-used $18^{\text {th }}$ Dynasty? sherd, pottery) & 646 & SAV1W 1035 & 20.01 .2015 \\
\hline $0356 / 2015$ & SQ1S & $\begin{array}{l}\text { pottery (c. } 7 \text { baskets), among them architecture/basin/ } \\
\text { oven/crucible? ( } 2 \text { large fragments, each clay), clay object ( } 1 \\
\text { large fragment, original function unknown, clay), } \\
\text { stopper/sealing? ( } 1 \text { small fragment, mud), scrapers ( } 3 \text { pieces, } \\
\text { each re-used sherds, pottery), lid? (re-used } 18^{\text {th }} \text { Dynasty pot } \\
\text { sherd, pottery) }\end{array}$ & 646 & $\begin{array}{l}\text { SAV1W 1001, SAV1W } 1002 \text { (architecture/ } \\
\text { basin/oven/crucible?), SAV1W } 1003 \text { (clay } \\
\text { object), SAV1W } 1005 \text { (stopper/sealing?), } \\
\text { SAV1W 1034, SAV1W 1128, SAV1W } 1129 \\
\text { (scrapers), SAV1W } 1130 \text { (lid?) }\end{array}$ & 21.01.2015 \\
\hline $0347 / 2015$ & SQ1S & $\begin{array}{l}\text { pottery, among it figurine ( } 1 \text { small figurine of a dog, clay), lid? } \\
\text { ( } 1 \text { re-used } 18^{\text {th }} \text { Dynasty pot sherd, pottery), scraper ( } 1 \text { re-used } \\
18^{\text {th }} \text { Dynasty Canaanite amphora sherd, pottery) }\end{array}$ & 647 & $\begin{array}{l}\text { SAV1W } 0764 \text { (figurine), SAV1W } 1131 \text { (lid), } \\
\text { SAV1W } 1132 \text { (scraper) }\end{array}$ & 20.01.2015 \\
\hline $0357 / 2015$ & SQ1S & pottery & 647 & & 21.01.2015 \\
\hline $0377 / 2015$ & SQ1S, SE-corner, sandy pit of SU & pottery (1 bag) & 647 & & 21.01.2015 \\
\hline $0358 / 2015$ & SQ1S & pottery & 649 & & 21.01 .2015 \\
\hline $0369 / 2015$ & SQ1S & pottery & 650 & & 21.01 .2015 \\
\hline $0431 / 2015$ & SQ1S, cleaning & pottery & 650 & & 24.01 .2015 \\
\hline $0370 / 2015$ & SQ1S & $\begin{array}{l}\text { pottery, among it architecture/basin/oven/crucible? (1 large } \\
\text { fragments, clay), clay object (1 large fragment, original function } \\
\text { unknown, clay) }\end{array}$ & 651 & $\begin{array}{l}\text { SAV1W } 1004 \text { (architecture/basin/oven/ } \\
\text { crucible?), SAV1W } 1133 \text { (clay object) }\end{array}$ & 21.01 .2015 \\
\hline $0380 / 2015$ & SQ1S & pottery & 651 & & 21.01 .2015 \\
\hline $0382 / 2015$ & SQ1S & pottery & 652 & & 21.01.2015 \\
\hline $0384 / 2015$ & SQ1S & $\begin{array}{l}\text { pottery ( } 5 \text { baskets), among them figurine ( } 1 \text { small piece, } \\
\text { rudimentary female figurine?, clay) }\end{array}$ & 652 & SAV1W 1030 & 22.01 .2015 \\
\hline $0385 / 2015$ & SQ1S & pottery & 653 & & 22.01 .2015 \\
\hline $0386 / 2015$ & SQ1S & $\begin{array}{l}\text { pottery and shell: pottery, among it clay object/basin/oven/ } \\
\text { crucible? ( } 1 \text { large fragment, clay), scraper (re-used pot sherd, } \\
\text { pottery); seashell ( } 1 \text { bag, fragments) }\end{array}$ & 654 & $\begin{array}{l}\text { SAV1W } 1036 \text { (clay object/basin/oven/ } \\
\text { crucible?), SAV1W } 1197 \text { (scraper) }\end{array}$ & 22.01 .2015 \\
\hline $0395 / 2015$ & SQ1S & $\begin{array}{l}\text { pottery, among it scraper (re-used Post-New Kingdom pot sherd, } \\
\text { pottery) }\end{array}$ & 655 & SAV1W 1509 & 22.01 .2015 \\
\hline $0404 / 2015$ & SQ1S & $\begin{array}{l}\text { pottery, among it scraper (re-used Post-New Kingdom pot sherd, } \\
\text { pottery) }\end{array}$ & 657 & SAV1W 1510 & 22.01 .2015 \\
\hline
\end{tabular}




\begin{tabular}{|c|c|c|c|c|c|}
\hline $0401 / 2015$ & SQ1S & pottery & 658 & & 24.01 .2015 \\
\hline $0412 / 2015$ & SQ1S & $\begin{array}{l}\text { pottery, among it scraper (re-used } 18^{\text {th }} \text { Dynasty pot sherd, } \\
\text { pottery) }\end{array}$ & 658 & SAV1W 1198 & 22.01 .2015 \\
\hline $0414 / 2015$ & SQ1S & pottery & 659 & & 24.01 .2015 \\
\hline $0415 / 2015$ & SQ1S & $\begin{array}{l}\text { pottery, among it scraper (re-used Post-New Kingdom base } \\
\text { sherd, pottery) }\end{array}$ & 659 & SAV1W 1716 & 22.01 .2015 \\
\hline $0437 / 2015$ & SQ1S & pottery & 660 & & 24.01 .2015 \\
\hline $0440 / 2015$ & SQ1S & pottery & 661 & & 24.01 .2015 \\
\hline $0452 / 2015$ & SQ1S & $\begin{array}{l}\text { pottery, among it scraper (re-used } 18^{\text {th }} \text { Dynasty pot sherd, } \\
\text { pottery) }\end{array}$ & 662 & SAV1W 1199 & 27.01 .2015 \\
\hline $0455 / 2015$ & SQ1S & pottery & 663 & & 27.01 .2015 \\
\hline $0473 / 2015$ & SQ1S & pottery & 665 & & 27.01 .2015 \\
\hline $0468 / 2015$ & SQ1S & pottery & 666 & & 27.01 .2015 \\
\hline $0470 / 2015$ & SQ1S & pottery & 667 & & 27.01 .2015 \\
\hline $0479 / 2015$ & SQ1S & $\begin{array}{l}\text { pottery, among it scraper ( } 1 \text { re-used } 18^{\text {th }} \text { Dynasty pot sherd, } \\
\text { pottery) }\end{array}$ & 668 & SAV1W 1512 & 27.01 .2015 \\
\hline $0490 / 2015$ & SQ1S & $\begin{array}{l}\text { pottery, among it scraper (1 re-used Post-New Kingdom pot } \\
\text { sherd, pottery) }\end{array}$ & 668 & SAV1W 1514 & 28.01 .2015 \\
\hline $0480 / 2015$ & SQ1S & $\begin{array}{l}\text { pottery and bone: pottery, among it clay object ( } 1 \text { fragment, } \\
\text { original function unclear, basin/oven/crucible?), slag attached } \\
\text { to sherd ( } 1 \text { small fragment, sherd Ottoman?); bone (1 piece) }\end{array}$ & 669 & $\begin{array}{l}\text { SAV1W } 1149 \text { (clay object), SAV1W } 1150 \\
\text { (slag) }\end{array}$ & 27.01 .2015 \\
\hline $0489 / 2015$ & SQ1S & $\begin{array}{l}\text { pottery and faience: pottery ( } 1 \text { basket), among it lid ( } 1 \text { re-used } \\
\text { Post New Kingdom sherd, pottery); Nun bowl ( } 1 \text { small fragment, } \\
\text { faience) }\end{array}$ & 669 & SAV1W 1239 (lid), SAV1W 1193 (Nun bowl) & 28.01 .2015 \\
\hline $0491 / 2015$ & SQ1S & $\begin{array}{l}\text { pottery ( } 3 \text { baskets), among it scraper ( } 1 \text { re-used Post-New } \\
\text { Kingdom pot sherd, pottery) }\end{array}$ & 670 & & 28.01 .2015 \\
\hline $0504 / 2015$ & SQ1S & pottery, among it scraper (1 re-used pot sherd, pottery) & 671 & SAV1W 1309 & 28.01 .2015 \\
\hline $0503 / 2015$ & SQ1S & pottery & 672 & & 28.01.2015 \\
\hline $0505 / 2015$ & SQ1S & pottery & 673 & & 28.01 .2015 \\
\hline $0519 / 2015$ & SQ1S & pottery, among it scraper ( 1 re-used $18^{\text {th }}$ Dynasty sherd, pottery) & 674 & SAV1W 1515 & 28.01.2015 \\
\hline $0520 / 2015$ & SQ1S & $\begin{array}{l}\text { pottery, among it token/gaming piece ( } 1 \text { re-used Post-New } \\
\text { Kingdom pot sherd, pottery) }\end{array}$ & 675 & SAV1W 1238 & 28.01.2015 \\
\hline $0704 / 2015$ & $\begin{array}{l}\text { SQ1S, cleaning of bottom surface } \\
\text { of SU } 678\end{array}$ & pottery & 678 & & 02.02 .2015 \\
\hline
\end{tabular}




\begin{tabular}{|c|c|c|c|c|c|}
\hline $0550 / 2015$ & SQ1S & pottery & 679 & & 29.01.2015 \\
\hline $0560 / 2015$ & SQ1S & $\begin{array}{l}\text { pottery ( } 1 \text { large fragment, perhaps from oven/other } \\
\text { installation?, pottery), scraper ( } 1 \text { re-used } 18^{\text {th }} \text { Dynasty pot } \\
\text { sherd, pottery) }\end{array}$ & 680 & SAV1W 1209 (oven?), SAV1W 1516 (scraper) & 29.01.2015 \\
\hline $0576 / 2015$ & SQ1S & pottery, among it stopper/sealing ( 1 large fragment, clay) & 685 & SAV1W 1237 & 31.01 .2015 \\
\hline $0619 / 2015$ & SQ1S & pottery & 691 & & 31.01 .2015 \\
\hline $0622 / 2015$ & SQ1S & pottery & 691 & & 31.01 .2015 \\
\hline $0661 / 2015$ & SQ1S & $\begin{array}{l}\text { pottery, among it scraper ( } 1 \text { re-used } 18^{\text {th }} \text { Dynasty pot sherd, } \\
\text { pottery) }\end{array}$ & 701 & SAV1W 1312 & 01.02 .2015 \\
\hline $0666 / 2015$ & SQ1S & $\begin{array}{l}\text { pottery, among it clay object ( } 1 \text { fragment, original function } \\
\text { unclear, part of an installation, basin/oven/crucible?) }\end{array}$ & 703 & SAV1W 1273 & 02.02 .2015 \\
\hline $0673 / 2015$ & SQ1S & $\begin{array}{l}\text { pottery, among it clay object ( } 1 \text { fragment, original function } \\
\text { unclear, part of an installation, basin/oven/crucible?) }\end{array}$ & 704 & SAV1W 1319 & 02.02 .2015 \\
\hline $0684 / 2015$ & SQ1S & $\begin{array}{l}\text { pottery, among it figurine ( } 1 \text { fragment of a rudimentary } \\
\text { female figurine, clay), lid ( } 1 \text { re-used } 18^{\text {th }} \text { Dynasty base sherd, } \\
\text { pottery) }\end{array}$ & 705 & SAV1W 1299 (figurine), SAV1W 1502 (lid) & 02.02 .2015 \\
\hline $0698 / 2015$ & SQ1S & pottery & 706 & & 02.02 .2015 \\
\hline $0727 / 2015$ & SQ1S & $\begin{array}{l}\text { pottery, among it stoppers/sealings ( } 2 \text { fragments, each mud), clay } \\
\text { object ( } 1 \text { fragment, original function unclear, part of an } \\
\text { installation, basin/oven/crucible?) }\end{array}$ & 710 & $\begin{array}{l}\text { SAV1W 1316, SAV1W } 1317 \\
\text { (stoppers/sealings), SAV1W } 1318 \text { (clay object) }\end{array}$ & 03.02 .2015 \\
\hline $0720 / 2015$ & SQ1S & $\begin{array}{l}\text { pottery and stone: pottery, among it stopper/sealing ( } 1 \text { fragment, } \\
\text { mud), scraper }\left(1 \text { re-used } 18^{\text {th }} \text { Dynasty sherd, pottery); hand mill }\right. \\
\text { (1 fragment, sandstone) }\end{array}$ & 711 & $\begin{array}{l}\text { SAV1W } 1422 \text { (stopper/sealing), SAV1W } 1521 \\
\text { (scraper), SAV1W } 1423 \text { (hand mill) }\end{array}$ & 03.02 .2015 \\
\hline $0753 / 2015$ & SQ1E & $\begin{array}{l}\text { pottery and charcoal: pottery (12 baskets), among them weight (1 } \\
\text { re-used pottery sherd, Christian), window grille? ( } 1 \text { fragment, } \\
\text { pottery); charcoal ( } 1 \text { piece) }\end{array}$ & 713 & $\begin{array}{l}\text { SAV1W } 1429 \text { (weight), SAV1W } 1430 \text { (window } \\
\text { grille?) }\end{array}$ & 04.02 .2015 \\
\hline $0758 / 2015$ & SQ1E & $\begin{array}{l}\text { pottery, among it figurine ( } 1 \text { fragment of a rudimentary female } \\
\text { figurine, clay) }\end{array}$ & 714 & SAV1W 1320 & 04.02 .2015 \\
\hline $0809 / 2015$ & SQ1S & pottery & 715 & & 08.02 .2015 \\
\hline $0811 / 2015$ & SQ1S & pottery & 716 & & 08.02 .2015 \\
\hline $0762 / 2015$ & SQ1/SQ1E & pottery, among it lid (1 fragment, clay) & 717 & SAV1W 1271 & 04.02 .2015 \\
\hline $0764 / 2015$ & SQ1/SQ1E & $\begin{array}{l}\text { pottery ( } 7 \text { baskets), among them hand mill ( } 1 \text { fragment, } \\
\text { sandstone), scraper ( } 1 \text { re-used fragment of an } 18^{\text {th }} \text { Dynasty } \\
\text { amphora), pottery object ( } 1 \text { fragment, original function unknown }\end{array}$ & 717 & $\begin{array}{l}\text { SAV1W } 1324 \text { (hand mill), SAV1W } 1434 \\
\text { (pounder), SAV1W } 1503 \text { (pottery object) }\end{array}$ & 05.02 .2015 \\
\hline $0769 / 2015$ & SQ1S & pottery; wood (1 bag) & 718 & & 05.02 .2015 \\
\hline
\end{tabular}




\begin{tabular}{|c|c|c|c|c|c|}
\hline $0773 / 2015$ & SQ1/SQ1E & $\begin{array}{l}\text { pottery and charcoal: pottery, among it clay } \\
\text { object/basin/oven/crucible? ( } 1 \text { fragment, clay), clay object } \\
\text { (1 fragment, original function unknown), oil lamp (1 fragment, } \\
\text { pottery, Christian), scraper (1 re-used } 18^{\text {th }} \text { Dynasty dish sherd, } \\
\text { pottery); charcoal ( } 2 \text { pieces) }\end{array}$ & 719 & $\begin{array}{l}\text { SAV1W 1296, SAV1W } 1325 \text { (clay objects), } \\
\text { SAV1W } 1474 \text { (oil lamp), SAV1W } 1522 \\
\text { (scraper) }\end{array}$ & 05.02 .2015 \\
\hline $0778 / 2015$ & SQ1S & pottery & 720 & & 05.02 .2015 \\
\hline $0781 / 2015$ & SQ1/SQ1E & pottery & 721 & & 05.02 .2015 \\
\hline $0815 / 2015$ & SQ1S & pottery & 723 & & 08.02 .2015 \\
\hline $0265 / 2015$ & $\begin{array}{l}\text { SQ1S, preparation for support wall at W- } \\
\text { border of trench }\end{array}$ & pottery & l & & 18.01.2015 \\
\hline $0057 / 2015$ & SQ1S & rope (1 small fragment, fragile) & 609 & & 06.01 .2015 \\
\hline $0846 / 2015$ & SQ1, Feature 115 & schist (1 fragment, large) & 731 & & 10.02 .2015 \\
\hline $0135 / 2015$ & SQ1S & schist (1 large fragment) & 618 & & 11.01 .2015 \\
\hline $0153 / 2015$ & SQ1S & schist (1 large, fragment) & 618 & & 12.01 .2015 \\
\hline $0200 / 2015$ & SQ1S & schist (1 large fragment) & 626 & & 14.01 .2015 \\
\hline $0802 / 2015$ & SQ1 & seashell (1 fragment) & 727 & & 08.02 .2015 \\
\hline $0011 / 2015$ & $\begin{array}{l}\text { SQ1S, } \\
10 \times 10 \mathrm{~m}\end{array}$ & seashell (2 fragments) & 600 & & 03.01 .2015 \\
\hline $0751 / 2015$ & SQ1E & seashell (1 fragment) & 601 & & 04.02 .2015 \\
\hline $0074 / 2015$ & SQ1S & seashell (1 piece) & 609 & & 07.01 .2015 \\
\hline $0120 / 2015$ & SQ1S & seashell (1 large piece) & 614 & & 10.01 .2015 \\
\hline $0236 / 2015$ & SQ1S & seashell (1 large piece) & 630 & & 17.01 .2015 \\
\hline $0281 / 2015$ & SQ1S & seashell (1 fragment) & 636 & & 18.01 .2015 \\
\hline $0319 / 2015$ & SQ1S & seashell (1 fragment) & 641 & & 20.01 .2015 \\
\hline $0409 / 2015$ & SQ1S & seashell (1 fragment) & 652 & & 22.01 .2015 \\
\hline $0445 / 2015$ & SQ1S & seashell (1 fragment) & 661 & & 24.01 .2015 \\
\hline $0585 / 2015$ & SQ1S & seashell (1 large piece) & 685 & & 31.01 .2015 \\
\hline $0610 / 2015$ & SQ1 & seashell (1 large piece) & 688 & & 31.01 .2015 \\
\hline $0629 / 2015$ & SQ1S & seashell (1 large piece) & 691 & & 01.02 .2015 \\
\hline
\end{tabular}




\begin{tabular}{|c|c|c|c|c|c|}
\hline $0654 / 2015$ & SQ1S & seashell (1 fragment) & 698 & & 01.02 .2015 \\
\hline $0738 / 2015$ & SQ1S & seashell (1 fragment) & 710 & & 03.02 .2015 \\
\hline $0845 / 2015$ & $\begin{array}{l}\text { SQ1S, } \\
\text { sieved material }\end{array}$ & sieved material & 724 & & 10.02 .2015 \\
\hline $0004 / 2015$ & SQ1S, top surface, $10 \times 10 \mathrm{~m}$ & slag (1 fragment) & 600 & SAV1W 0808 & 03.01 .2015 \\
\hline $0006 / 2015$ & SQ1S, $10 \times 10 \mathrm{~m}$ & slag (3 fragments) & 600 & SAV1W 0807 & 03.01 .2015 \\
\hline $0749 / 2015$ & SQ1E & slag (1 fragment) & 601 & SAV1W 1321 & 04.02 .2015 \\
\hline $0059 / 2015$ & SQ1S & slag (1 large fragment) & 609 & SAV1W 0883 & 06.01 .2015 \\
\hline $0105 / 2015$ & SQ1S & slag (large chunk mixed with brick) & 611 & SAV1W 0927 & 10.01 .2015 \\
\hline $0224 / 2015$ & SQ1S & slag (1 small fragment) & 628 & SAV1W 0988 & 15.01 .2015 \\
\hline $0531 / 2015$ & SQ1S & slag (1 fragment) & 674 & SAV1W 1172 & 28.01 .2015 \\
\hline $0522 / 2015$ & SQ1S & slag (1 fragment) & 675 & SAV1W 1166 & 28.01 .2015 \\
\hline $0664 / 2015$ & SQ1S & slag (1 large fragment) & 698 & SAV1W 1242 & 01.02 .2015 \\
\hline $0682 / 2015$ & SQ1S & slag (1 small fragment) & 704 & SAV1W 1314 & 02.02 .2015 \\
\hline $0759 / 2015$ & SQ1E & slag (2 large fragments) & 714 & SAV1W 1269 & 04.02 .2015 \\
\hline $0789 / 2015$ & SQ1S & slag and brick (1 small fragment) & 720 & SAV1W 1297 & 05.02 .2015 \\
\hline $0448 / 2015$ & SQ1, N-part of SQ1 & stone: pounder (1 small unspecified stone) & 600 & SAV1W 1037 & 24.01.2015 \\
\hline $0078 / 2015$ & SQ1, SW 0-5m & $\begin{array}{l}\text { stones: pounders ( } 2 \text { pieces, } 1 \text { quartzite, } 1 \text { quartz), hand mill } \\
\text { ( } 1 \text { piece, broken in half, quartzite), grindstone ( large fragment, } \\
\text { quartzite), whetstones ( } 2 \text { pieces, each sandstone), tile or paving } \\
\text { slab? ( } 1 \text { large fragment of architecture, schist or slate), polishing } \\
\text { stone? ( } 1 \text { piece of pebble, quartz) }\end{array}$ & 600 & $\begin{array}{l}\text { SAV1W } 0898 \text { (pounder, quartzite), SAV1W } \\
0904 \text { (pounder, quartz), SAV1W 0899 (hand } \\
\text { mill), SAV1W } 0900 \text { (grindstone), SAV1W } \\
\text { 0902, SAV1W } 0905 \text { (whetstones), SAV1W } \\
\text { 0901 (tile or paving slab?), SAV1W } 0903 \\
\text { (polishing stone?) }\end{array}$ & 07.01 .2015 \\
\hline $0465 / 2015$ & SQ1 & stone: Palaeolithic blade? (1 small fragment of quartz) & 664 & SAV1W 1154 & 27.01.2015 \\
\hline $0566 / 2015$ & SQ1 & $\begin{array}{l}\text { stones: grindstone ( } 1 \text { piece, quartzite), polishing stone ( } 2 \text { pieces, } \\
1 \text { quartzite, } 1 \text { unspecified stone), pounder ( } 1 \text { piece, unspecified } \\
\text { stone), hand mills ( } 1 \text { pieces, quartzite) }\end{array}$ & 664 & $\begin{array}{l}\text { SAV1W } 1185 \text { (grindstone), SAV1W 1186, } \\
\text { SAV1W } 1187 \text { (polishing stones), SAV1W } 1190 \\
\text { (pounder), SAV1W 1188, SAV1W } 1189 \text { (hand } \\
\text { mill) }\end{array}$ & 29.01.2015 \\
\hline $0580 / 2015$ & SQ1 & stone: pounder (1 quartz pebble) & 664 & SAV1W 1207 & 31.01 .2015 \\
\hline $0540 / 2015$ & SQ1 & $\begin{array}{l}\text { stones: pounder (1 fragment siliceous shale?), whetstone } \\
\text { (1 fragment, sandstone) }\end{array}$ & 678 & SAV1W 1178, SAV1W 1179 & 29.01.2015 \\
\hline
\end{tabular}




\begin{tabular}{|c|c|c|c|c|c|}
\hline $0588 / 2015$ & SQ1 & stone, worked (1 piece, quartzite) & 686 & SAV1W 1208 & 31.01 .2015 \\
\hline $0596 / 2015$ & SQ1 & $\begin{array}{l}\text { stones: pounders ( } 2 \text { pieces, } 1 \text { quartzite, } 1 \text { siliceous shale?), } \\
\text { worked stone (1 fragment, sandstone), hand mill (1 fragment, } \\
\text { quartzite) }\end{array}$ & 687 & $\begin{array}{l}\text { SAV1W 1233, SAV1W } 1234 \text { (pounders), } \\
\text { SAV1W } 1235 \text { (worked stone), SAV1W } 1236 \\
\text { (hand mill) }\end{array}$ & 31.01 .2015 \\
\hline $0601 / 2015$ & SQ1 & stone & 688 & & 31.01 .2015 \\
\hline $0611 / 2015$ & SQ1 & $\begin{array}{l}\text { stones: pounder ( } 1 \text { fragment of a quartz pebble), hand mills } \\
\text { ( } 1 \text { fragment, sandstone), worked stone ( } 1 \text { fragment, sandstone), } \\
\text { grindstone ( } 2 \text { fragments, each quartzite), pivot stone (1 large } \\
\text { piece, sandstone) }\end{array}$ & 688 & $\begin{array}{l}\text { SAV1W } 1218 \text { (pounder), SAV1W } 1219 \text { (hand } \\
\text { mill), SAV1W } 1220 \text { (worked stone), SAV1W } \\
\text { 1221, SAV1W } 1221 \text { (grindstones), SAV1W } \\
1223 \text { (pivot stone) }\end{array}$ & 31.01 .2015 \\
\hline $0626 / 2015$ & SQ1 & stone: hand mill (?) (1 fragment) & 694 & & 01.02 .2015 \\
\hline $0627 / 2015$ & SQ1 & $\begin{array}{l}\text { stones and bones: worked stones ( } 2 \text { fragments, each sandstone); } \\
\text { bones ( } 1 \text { bag) }\end{array}$ & 694 & SAV1W 1215, SAV1W 1216 (worked stones) & 01.02 .2015 \\
\hline $0633 / 2015$ & SQ1 & stone: whetstone (1 fragment, sandstone) & 695 & SAV1W 1214 & 01.02 .2015 \\
\hline $0729 / 2015$ & SQ1 & $\begin{array}{l}\text { stones and architecture?: pounders ( } 2 \text { pieces, } 1 \text { siliceous shale?, } \\
1 \text { metamorphic stone), grindstones ( } 3 \text { fragments, } 2 \text { quartzite, } \\
1 \text { sandstone), worked stone ( } 1 \text { piece, siliceous shale?), hand mill } \\
\text { (1 fragment, sandstone); architecture?/part of door jamb? ( } 1 \text { large } \\
\text { fragment, sandstone) }\end{array}$ & 708 & $\begin{array}{l}\text { SAV1W 1331, SAV1W } 1338 \text { (pounders), } \\
\text { SAV1W 1332, SAV1W 1334, SAV1W 1335 } \\
\text { (grindstones), SAV1W 1333 (worked stone), } \\
\text { SAV1W 1337 (hand mill), SAV1W } 1336 \\
\text { (architecture?/part of door jamb?) }\end{array}$ & 03.02 .2015 \\
\hline $0790 / 2015$ & SQ1 & $\begin{array}{l}\text { stone: Palaeolithic tool/blade? ( } 1 \text { mall chert [radiolith]/pebble } \\
\text { stone) }\end{array}$ & 722 & SAV1W 1407 & 08.02 .2015 \\
\hline $0824 / 2015$ & SQ1, Feature 115 & $\begin{array}{l}\text { stones: pounders ( } 3 \text { pieces, } 1 \text { siliceous shale?, } 2 \text { quartzite), } \\
\text { polishing stone ( } 1 \text { fragment, quartzite) }\end{array}$ & 731 & $\begin{array}{l}\text { SAV1W 1401, SAV1W 1402, SAV1W } 1403 \\
\text { (pounders), SAV1W } 1404 \text { (polishing stone) }\end{array}$ & 09.02 .2015 \\
\hline $0842 / 2015$ & SQ1, Feature 115 & $\begin{array}{l}\text { stones: worked stones ( } 2 \text { fragments, each sandstone), grindstone } \\
\text { (1 fragment, sandstone), }\end{array}$ & 732 & $\begin{array}{l}\text { SAV1W 1419, SAV1W } 1421 \text { (worked stones), } \\
\text { SAV1W } 1420 \text { (grindstone) }\end{array}$ & 10.02 .2015 \\
\hline $0828 / 2015$ & SQ1, Feature 115 & $\begin{array}{l}\text { stones: pounder (1 piece, quartzite), whetstone (1 fragment, } \\
\text { sandstone) }\end{array}$ & 732 & SAV1W 1399, SAV1W 1400 & 09.02 .2015 \\
\hline $0740 / 2015$ & SQ1E, surface cleaning & $\begin{array}{l}\text { stone and slag: grindstone ( } 2 \text { fragments, each sandstone), } \\
\text { whetstone ( } 1 \text { fragment, sandstone), hand mills ( } 2 \text { fragments, each } \\
\text { sandstone, pounder ( } 2 \text { fragments), flint ( } 1 \text { fragment); slag } \\
\text { (1 fragment) }\end{array}$ & 600 & $\begin{array}{l}\text { SAV1W 1328, SAV1W } 1329 \text { (grindstones), } \\
\text { SAV1W } 1330 \text { (whetstone), SAV1W 1341, } \\
\text { SAV1W } 1342 \text { (hand mills), SAV1W } 1327 \\
\text { (slag) }\end{array}$ & 03.02 .2015 \\
\hline $0010 / 2015$ & SQ1S, $10 \times 10 \mathrm{~m}$ & $\begin{array}{l}\text { stones ( } 11 / 2 \text { basket), among it pounders ( } 6 \text { pieces, } 2 \text { quartz, } 2 \\
\text { quartzite, } 1 \text { agate, } 1 \text { sandstone), cylindrical stone ( } 2 \text { adjoining } \\
\text { pieces, function unknown, sandstone) }\end{array}$ & 600 & $\begin{array}{l}\text { SAV1W 0850, SAV1W 0851, SAV1W 0852, } \\
\text { SAV1W 0853, SAV1W 0854, SAV1W 0855 } \\
\text { (pounders), SAV1W 0856 (cylindrical stone) }\end{array}$ & 03.01 .2015 \\
\hline $0228 / 2015$ & SQ1S, S of, surface & stone: stela? (1 fragment, limestone) & 600 & SAV1W 0999 & 15.01 .2015 \\
\hline $0003 / 2015$ & SQ1S, top surface, $10 \times 10 \mathrm{~m}$ & $\begin{array}{l}\text { stones and architecture: worked stone ( } 2 \text { baskets from the 19, } 38 \\
\text { pieces): pounders ( } 7 \text { pieces, } 3 \text { quartzite, } 1 \text { quartz, } 3 \text { unspecified } \\
\text { stone), hammers ( } 6 \text { pieces, } 3 \text { quartzite, quartz, } 3 \text { unspecified } \\
\text { stone), grindstones ( } 5 \text { pieces, each quartzite), hand mills } \\
\text { ( } 3 \text { pieces, each quartzite), whetstones ( } 9 \text { pieces, each sandstone), }\end{array}$ & 600 & $\begin{array}{l}\text { SAV1W 0809, SAV1W 0810, SAV1W 0811, } \\
\text { SAV1W 0812, SAV1W 0813, SAV1W 0814, } \\
\text { 0815 (pounders), SAV1W 0816, SAV1W 0817, } \\
\text { SAV1W 0818, SAV1W 0819, SAV1W 0820, } \\
\text { SAV1W 0821 (hammers), SAV1W 0822, }\end{array}$ & 03.01 .2015 \\
\hline
\end{tabular}




\begin{tabular}{|c|c|c|c|c|c|}
\hline & & $\begin{array}{l}\text { stone vessels ( } 2 \text { fragments, } 1 \text { quartzite [possibly basin], } 1 \\
\text { unspecified stone), abrasive stone ( } 1 \text { piece, quartzite), worked } \\
\text { stone (1 piece, function unknown, unspecified stone), polishing } \\
\text { stone (1 piece, unspecified stone), grindstones ( } 2 \text { fragments); } \\
\text { architecture (3 fragments, each limestone, } 2 \text { from flooring? } 1 \\
\text { door pivot?) }\end{array}$ & & $\begin{array}{l}\text { SAV1W 0823, SAV1W 0841 (hand mills), } \\
\text { SAV1W 0824, SAV1W 0825, SAV1W 0826, } \\
\text { SAV1W 0827, SAV1W 0829, SAV1W 0830, } \\
\text { SAV1W 0831, SAV1W 0832, SAV1W 0833 } \\
\text { (whetstones), SAV1W 0828 (abrasive stone), } \\
\text { SAV1W 0834, SAV1W 0837 (stone vessel), } \\
\text { SAV1W 0835, SAV1W 0836, SAV1W 0838 } \\
\text { (worked stone), SAV1W 0840 (polishing stone), } \\
\text { SAV1W 0842, SAV1W 0843, SAV1W 0844, } \\
\text { SAV1W 0845, SAV1W 0846 (grindstones), } \\
\text { SAV1W 0839 (architecture) }\end{array}$ & \\
\hline $0016 / 2015$ & SQ1S, $10 \times 10 \mathrm{~m}$ & $\begin{array}{l}\text { stones and architecture: flint ( } 1 \text { fragment, possibly from early } \\
\text { stone tool), pounders ( } 1 \text { piece, } 1 \text { quartzite, } 1 \text { unspecified stone), } \\
\text { pounder ( } 1 \text { fragment), pounder ( } 1 \text { fragment); architecture: floor } \\
\text { tile? ( } 1 \text { fragment, limestone) }\end{array}$ & 601 & $\begin{array}{l}\text { SAV1W } 0858 \text { (flint), SAV1W 0859, SAV1W } \\
0861 \text { (pounders), SAV1W } 0865 \text { (architecture, } \\
\text { floor tile?) }\end{array}$ & 04.01 .2015 \\
\hline $0021 / 2015$ & SQ1S & stone & 601 & & 05.01 .2015 \\
\hline $0744 / 2015$ & SQ1E & $\begin{array}{l}\text { stones: pounders ( } 2 \text { pieces, } 1 \text { unspecified stone, } 1 \text { quartz pebble), } \\
\text { hand mills ( } 2 \text { fragments, each sandstone), whetstone ( } 1 \text { fragment, } \\
\text { sandstone), architecture? ( } 1 \text { fragment, sandstone) }\end{array}$ & 601 & $\begin{array}{l}\text { SAV1W 1283, SAV1W } 1326 \text { (pounders), } \\
\text { SAV1W 1284, SAV1W } 1393 \text { (hand mills), } \\
\text { SAV1W 1285 (whetstone), SAV1W } 1392 \\
\text { (architecture?) }\end{array}$ & 03.02 .2015 \\
\hline $0748 / 2015$ & SQ1E & $\begin{array}{l}\text { stones: grindstone ( } 1 \text { fragment, sandstone), pounders ( } 2 \text { pieces, } \\
\text { each } 1 \text { fragment of a quartz pebble) }\end{array}$ & 601 & $\begin{array}{l}\text { SAV1W } 1374 \text { (grindstone), SAV1W 1375, } \\
\text { SAV1W } 1376 \text { (pounders) }\end{array}$ & 04.02 .2015 \\
\hline $0037 / 2015$ & SQ1S & $\begin{array}{l}\text { stone/architecture: whetstone (1 piece, possibly originally piece } \\
\text { of architecture, sandstone) }\end{array}$ & 602 & SAV1W 0872 & 05.01 .2015 \\
\hline $0026 / 2015$ & SQ1S & $\begin{array}{l}\text { stones: pounder ( } 1 \text { piece, stone), whetstone ( } 1 \text { piece, sandstone), } \\
\text { hand mill (1 half, quartzite) }\end{array}$ & 603 & $\begin{array}{l}\text { SAV1W } 0862 \text { (pounder), SAV1W } 0863 \\
\text { (whetstone), SAV1W } 0864 \text { (hand mill) }\end{array}$ & 05.01 .2015 \\
\hline $0040 / 2015$ & SQ1S & $\begin{array}{l}\text { stones and slag: hand mill ( } 1 \text { half, quartzite), hammer } \\
\text { ( } 1 \text { fragment, quartzite), grindstone ( } 1 \text { fragment, quartzite), } \\
\text { whetstone ( } 1 \text { piece), agate ( } 3 \text { adjoining pieces of raw agate, } \\
1 \text { agate pebble); slag ( } 1 \text { piece) }\end{array}$ & 606 & $\begin{array}{l}\text { SAV1W } 0873 \text { (hand mill), SAV1W } 0874 \\
\text { (hammer), SAV1W } 0888 \text { (grindstone), SAV1W } \\
0890 \text { (whetstone), SAV1W } 0875 \text { (slag) }\end{array}$ & 05.01 .2015 \\
\hline $0044 / 2015$ & SQ1S & $\begin{array}{l}\text { stones: pounders ( } 2 \text { pieces, } 1 \text { quartzite, } 1 \text { siliceous shale?), } \\
\text { grindstone or hand mill ( } 1 \text { fragment, quartzite) }\end{array}$ & 607 & $\begin{array}{l}\text { SAV1W 0880, SAV1W } 0882 \text { (pounders), } \\
\text { SAV1W } 0881 \text { (grindstone or hand mill) }\end{array}$ & 06.01 .2015 \\
\hline $0048 / 2015$ & SQ1S & $\begin{array}{l}\text { stones: whetstones ( } 2 \text { pieces, each sandstone), grindstone } \\
\text { (1 fragment, quartzite), hammer (1 fragment, quartzite) }\end{array}$ & 608 & $\begin{array}{l}\text { SAV1W 0876, SAV1W } 0877 \text { (whetstones), } \\
\text { SAV1W 0878 (grindstone), SAV1W } 0879 \\
\text { (hammer) }\end{array}$ & 06.01 .2015 \\
\hline $0054 / 2015$ & SQ1S & $\begin{array}{l}\text { stones: pounders ( } 3 \text { pieces, quartzite), whetstone ( } 1 \text { piece, } \\
\text { sandstone), } 2 \text { grindstones ( } 2 \text { large fragments, quartzite) }\end{array}$ & 609 & $\begin{array}{l}\text { SAV1W 0889, SAV1W 0894, SAV1W } 0895 \\
\text { (pounders), SAV1W } 0891 \text { (whetstone), SAV1W } \\
\text { 0892, SAV1W } 0893 \text { (grindstones) }\end{array}$ & 06.01 .2015 \\
\hline $0071 / 2015$ & SQ1S & $\begin{array}{l}\text { stones: whetstone? (1 fragment, sandstone), pounder (1 large } \\
\text { fragment, quartzite) }\end{array}$ & 609 & $\begin{array}{l}\text { SAV1W } 0896 \text { (whetstone?), SAV1W } 0897 \\
\text { (pounder) }\end{array}$ & 07.01 .2015 \\
\hline
\end{tabular}




\begin{tabular}{|c|c|c|c|c|c|}
\hline $0085 / 2015$ & $\begin{array}{l}\text { SQ1/1S, cleaning connection between } \\
\text { SQ1 and SQ1S }\end{array}$ & $\begin{array}{l}\text { stones: pounders ( } 4 \text { pieces, } 3 \text { quartzite, } 1 \text { stone), whetstones } \\
\text { ( } 4 \text { pieces, each sandstone), hand mills ( } 2 \text { pieces, quartzite), } \\
\text { grindstone ( } 1 \text { fragment) }\end{array}$ & 610 & $\begin{array}{l}\text { SAV1W 0916, SAV1W 0917 SAV1W } 0923 \\
\text { (pounders, quartzite), SAV1W 0921 (pounder, } \\
\text { stone), SAV1W 0918, SAV1W 0919, SAV1W } \\
\text { 0920 SAV1W 0924 (whetstones), SAV1W } \\
\text { 0922, SAV1W 0924 (hand mills) }\end{array}$ & 08.01 .2015 \\
\hline $0094 / 2015$ & SQ1S & stone (maybe fossil, coral) & 611 & & 08.01 .2015 \\
\hline $0092 / 2015$ & SQ1S & $\begin{array}{l}\text { stones: scraper ( } 1 \text { piece, small chert), hand mills ( } 4 \text { pieces, each } \\
\text { quartzite) }\end{array}$ & 612 & $\begin{array}{l}\text { SAV1W } 0911 \text { (scraper), SAV1W 0912, } \\
\text { SAV1W 0913, SAV1W 0914, SAV1W } 0915 \\
\text { (hand mills, quartzite) }\end{array}$ & 08.01 .2015 \\
\hline $0095 / 2015$ & SQ1S & stone: blade (1 piece, silex) & 612 & SAV1W 0909 & 08.01 .2015 \\
\hline $0104 / 2015$ & SQ1S & stone: pounder (1 fragment, stone) & 612 & SAV1W 0926 & 10.01 .2015 \\
\hline $0148 / 2015$ & SQ1S & $\begin{array}{l}\text { stones: hand mill ( } 1 \text { piece, quartzite), pounders ( } 2 \text { pieces, } 1 \text { not } \\
\text { specified stone, } 1 \text { quartz pebble), grindstone (1 large fragment, } \\
\text { quartzite) }\end{array}$ & 612 & $\begin{array}{l}\text { SAV1W } 0948 \text { (hand mill), SAV1W } 0949 \text { and } \\
\text { SAV1W } 0950 \text { (pounders), grindstone }\end{array}$ & 12.01.2015 \\
\hline $0101 / 2015$ & SQ1S & $\begin{array}{l}\text { stones and architecture: grindstone ( } 1 \text { piece, quartzite), polishing } \\
\text { stone? ( } 1 \text { fragment, stone), pounders ( } 2 \text { pieces, } 1 \text { large pebble, } \\
\text { quartz, } 1 \text { large fragment, quartzite), grindstone ( } 2 \text { fragments), } \\
\text { stone vessel? (1 fragment of quartzite); architecture? ( } 1 \text { chunk of } \\
\text { quartzite) }\end{array}$ & 613 & $\begin{array}{l}\text { SAV1W } 0928 \text { (grindstone), SAV1W } 0929 \\
\text { (polishing stones), SAV1W 0930, SAV1W } \\
\text { 0931 (pounders), SAV1W 0933 (stone vessel?), } \\
\text { SAV1W 0932, (architecture) }\end{array}$ & 10.01 .2015 \\
\hline $0110 / 2015$ & SQ1/S & $\begin{array}{l}\text { stones: polishing stone ( } 1 \text { fragment, stone), pounder ( } 1 \text { piece, } \\
\text { quartz), stone vessel ( } 1 \text { small fragment, perhaps pot stand, } \\
\text { sandstone), whetstones ( } 4 \text { pieces, each small fragments, } \\
\text { sandstone), unidentified object ( } 1 \text { small fragment, worked, } \\
\text { stone), grindstone ( } 1 \text { large fragment, quartzite) }\end{array}$ & 614 & $\begin{array}{l}\text { SAV1W } 0934 \text { (polishing stone), SAV1W } 0935 \\
\text { (pounder), SAV1W 0936 (sandstone vessel), } \\
\text { SAV1W 0937, SAV1W 0938, SAV1W 0939, } \\
\text { SAV1W 0940, SAV1W 0941 (whetstones), } \\
\text { SAV1W 0942 (unidentified stone object), } \\
\text { SAV1W } 0943 \text { (grindstone) }\end{array}$ & 10.01.2015 \\
\hline $0115 / 2015$ & SQ1S & stone & 616 & & 10.01.2015 \\
\hline $0122 / 2015$ & SQ1S, NW of SU & stones: pounders ( 3 pieces, 1 quartzite, 1 quartz, 1 agate) & 616 & $\begin{array}{l}\text { SAV1W } 0944 \text { (pounder, quartzite), SAV1W } \\
0945 \text { (pounder, quartz), SAV1W } 0946 \text { (pounder, } \\
\text { agate) }\end{array}$ & 10.01.2015 \\
\hline $0133 / 2015$ & SQ1S & $\begin{array}{l}\text { stones: hammer/pestle ( } 1 \text { piece, quartzite), pounder/polishing } \\
\text { stone ( } 1 \text { piece, siliceous shale?), whetstone ( } 1 \text { piece, sandstone), } \\
\text { hand mills ( } 2 \text { pieces, each quartzite), grindstones ( } 2 \text { pieces, each } \\
\text { quartzite) }\end{array}$ & 617 & $\begin{array}{l}\text { SAV1W } 0957 \text { (hammer/pestle), SAV1W } 0958 \\
\text { (pounder/polishing stone), SAV1W 0959 } \\
\text { (whetstone), SAV1W 0960 and SAV1W } 0961 \\
\text { (hand mills), SAV1W } 0962 \text { and SAV1W } 0963 \\
\text { (grindstones) }\end{array}$ & 11.01 .2015 \\
\hline $0140 / 2015$ & SQ1S & $\begin{array}{l}\text { stones: pounder (1 pebble, quartz), grindstone (1 fragment, } \\
\text { sandstone) }\end{array}$ & 619 & $\begin{array}{l}\text { SAV1W } 0955 \text { (pounder), SAV1W } 0956 \\
\text { (grindstone) }\end{array}$ & 11.01.2015 \\
\hline $0164 / 2015$ & SQ1S & $\begin{array}{l}\text { stones: whetstone ( } 3 \text { pieces, each sandstone), pounder ( } 1 \text { quartz } \\
\text { pebble) }\end{array}$ & 619 & $\begin{array}{l}\text { SAV1W 0971, SAV1W 0972, SAV1W } 0973 \\
\text { (whetstones), SAV1W } 0971 \text { (pounder) }\end{array}$ & 12.01 .2015 \\
\hline $0162 / 2015$ & SQ1S & stone: hand mill (1 large fragment, quartzite) & 620 & SAV1W 0970 & 12.01 .2015 \\
\hline
\end{tabular}




\begin{tabular}{|c|c|c|c|c|c|}
\hline $0168 / 2015$ & SQ1S & $\begin{array}{l}\text { stones: pounders ( } 4 \text { pieces, } 2 \text { quartzite, } 1 \text { quartz pebble, } 1 \text { not } \\
\text { specified stone), whetstone ( } 1 \text { piece, schist), whetstone } \\
\text { (1 fragment) }\end{array}$ & 620 & $\begin{array}{l}\text { SAV1W 0979, SAV1W 0980, SAV1W 0981, } \\
\text { SAV1W } 0982 \text { (pounders), SAV1W } 0983 \\
\text { (whetstone) }\end{array}$ & 13.01.2015 \\
\hline $0176 / 2015$ & SQ1S & $\begin{array}{l}\text { stones: hand mill? ( } 1 \text { fragment, quartzite), grindstone? (1 large } \\
\text { fragment, quartzite) }\end{array}$ & 621 & $\begin{array}{l}\text { SAV1W } 0977 \text { (hand mill?), SAV1W } 0978 \\
\text { (grindstone?) }\end{array}$ & 13.01.2015 \\
\hline $0188 / 2015$ & SQ1S & $\begin{array}{l}\text { stones: raw material ( } 1 \text { small chip of agate, } 1 \text { large fragment of } \\
\text { quartzite), pounder ( } 1 \text { fragment of quartz pebble), hand mills ( } 2 \\
\text { large fragments, each quartzite), whetstone ( } 1 \text { piece, sandstone), } \\
\text { grindstones ( } 2 \text { large fragments, each quartzite) }\end{array}$ & 622 & $\begin{array}{l}\text { SAV1W 0702, SAV1W 0704 (raw material), } \\
\text { SAV1W } 0703 \text { (pounder), SAV1W 0705, } \\
\text { SAV1W } 0706 \text { (hand mills), SAV1W } 0707 \\
\text { (whetstone), SAV1W 0708, SAV1W } 0709 \\
\text { (grindstones) }\end{array}$ & 14.01.2015 \\
\hline $0192 / 2015$ & SQ1S & $\begin{array}{l}\text { stones: pounder (1 piece, stone), hand mill (1 small fragment, } \\
\text { quartzite) }\end{array}$ & 624 & $\begin{array}{l}\text { SAV1W } 0991 \text { (pounder), SAV1W } 0992 \text { (hand } \\
\text { mill) }\end{array}$ & 14.01.2015 \\
\hline $0198 / 2015$ & SQ1S & stone: pounder (1 small quartz pebble) & 625 & SAV1W 0990 & 14.01.2015 \\
\hline $0207 / 2015$ & SQ1S & $\begin{array}{l}\text { stones: pounder ( } 1 \text { small fragment, quartz pebble), whetstone (1 } \\
\text { small fragment, sandstone), raw material (1 fragment, quartz) }\end{array}$ & 625 & $\begin{array}{l}\text { SAV1W } 0996 \text { (pounder), SAV1W } 0997 \\
\text { (whetstone), SAV1W } 0998 \text { (raw material) }\end{array}$ & 15.01 .2015 \\
\hline $0217 / 2015$ & SQ1S & $\begin{array}{l}\text { stones: stone vessel ( } 1 \text { fragment of rim?, quartzite), pounder } \\
\text { (1 fragment, quartz); chalk ( } 1 \text { piece) }\end{array}$ & 626 & SAV1W 0994 (vessel), SAV1W 0753 (pounder) & 15.01 .2015 \\
\hline $0212 / 2015$ & SQ1S & $\begin{array}{l}\text { stones: hand mill (1 small fragment, quartzite), grindstones } \\
\text { ( } 2 \text { fragments, each quartzite) }\end{array}$ & 627 & $\begin{array}{l}\text { SAV1W } 0699 \text { (hand mill), SAV1W 0700, } \\
\text { SAV1W } 0701 \text { (grindstones) }\end{array}$ & 15.01 .2015 \\
\hline $0216 / 2015$ & SQ1S & $\begin{array}{l}\text { stones: grindstone ( } 4 \text { pieces, each quartzite), hand mills ( } 5 \text { pieces, } \\
\text { each quartzite), } 4 \text { whetstones ( } 4 \text { pieces, each sandstone), pounder } \\
\text { (1 large quartz pebble), schist ( } 1 \text { fragment) }\end{array}$ & 628 & $\begin{array}{l}\text { SAV1W 0710, SAV1W 0711, SAV1W 0712, } \\
\text { SAV1W 0755 (grindstones), SAV1W 0713, } \\
\text { SAV1W 0714, SAV1W 0715, SAV1W 0716, } \\
\text { SAV1W 0717(hand mills), SAV1W 0718, } \\
\text { SAV1W 0719, SAV1W 0720, SAV1W } 0721 \\
\text { (whetstones), SAV1W } 0722 \text { (pounder) }\end{array}$ & 15.01 .2015 \\
\hline $0227 / 2015$ & SQ1S & stone: whetstone (1 large fragment, sandstone) & 629 & SAV1W 0995 & 15.01 .2015 \\
\hline $0234 / 2015$ & SQ1S & $\begin{array}{l}\text { stones: pounders ( } 5 \text { pieces, } 4 \text { quartz pebbles, } 1 \text { siliceous shale?), } \\
\text { hand mill ( } 1 \text { large fragment, quartzite), whetstone? ( } 1 \text { piece, } \\
\text { sandstone), grindstones ( } 2 \text { pieces, each quartzite) }\end{array}$ & 630 & $\begin{array}{l}\text { SAV1W 1080, SAV1W 1081, SAV1W 1082, } \\
\text { SAV1W 1083, SAV1W 1084 (pounders), } \\
\text { SAV1W 1085 (hand mill), SAV1W } 1086 \\
\text { (whetstone?), SAV1W 1087, SAV1W } 1088 \\
\text { (grindstones?) }\end{array}$ & 17.01.2015 \\
\hline $0271 / 2015$ & SQ1S & stone: pounder (1 medium sized quartz pebble) & 632 & SAV1W 0754 & 18.01 .2015 \\
\hline $0238 / 2015$ & SQ1S & $\begin{array}{l}\text { stone, slag, clay and faience: gaming piece?/unidentified object } \\
\text { (1 spherical stone); slag and brick (1 large fragment); clay object } \\
\text { (1 small fragment, original function unknown); Nun bowl (1 } \\
\text { small fragment, faience) }\end{array}$ & 633 & $\begin{array}{l}\text { SAV1W } 0731 \text { (gaming piece?/unidentified } \\
\text { object), SAV1W } 0730 \text { (slag and brick), SAV1W } \\
0757 \text { (clay object), SAV1W } 0724 \text { (Nun bowl) }\end{array}$ & 17.01.2015 \\
\hline $0242 / 2015$ & SQ1S & $\begin{array}{l}\text { stones: grindstone (1 piece, quartzite), polishing stone } \\
\text { (1 fragment, siliceous shale?), pounders ( } 2 \text { pieces, each quartz } \\
\text { pebbles) }\end{array}$ & 633 & $\begin{array}{l}\text { SAV1W } 0749 \text { (grindstone), SAV1W } 0750 \\
\text { (polishing stone), SAV1W 0751, SAV1W } 0752 \\
\text { (pounders) }\end{array}$ & 17.01.2015 \\
\hline
\end{tabular}




\begin{tabular}{|c|c|c|c|c|c|}
\hline $0264 / 2015$ & SQ1S & $\begin{array}{l}\text { stones: weight ( } 1 \text { piece, limestone), pounders ( } 3 \text { pieces, } 2 \text { quartz, } \\
1 \text { siliceous shale?), hand mill ( } 1 \text { small fragment, quartzite), } \\
\text { whetstone ( } 1 \text { small fragment, sandstone) }\end{array}$ & 633 & $\begin{array}{l}\text { SAV1W } 1057 \text { (weight), SAV1W 1058, } \\
\text { SAV1W 1059, SAV1W } 1060 \text { (pounders), } \\
\text { SAV1W 1061 (hand mill), SAV1W } 1062 \\
\text { (whetstone) }\end{array}$ & 18.01.2015 \\
\hline $0246 / 2015$ & SQ1S & stone: hand mill (1 fragment, quartzite) & 634 & SAV1W 0741 & 17.01.2015 \\
\hline $0255 / 2015$ & SQ1S & $\begin{array}{l}\text { stones and architecture: token/gaming piece ( } 1 \text { fragment, } \\
\text { sandstone), pounders ( } 2 \text { pieces, } 1 \text { quartz, } 1 \text { quartz pebble), } \\
\text { whetstones ( } 4 \text { pieces, each sandstone); architecture? (1 large } \\
\text { fragment, sandstone) }\end{array}$ & 635 & $\begin{array}{l}\text { SAV1W } 1098 \text { (token/gaming piece), SAV1W } \\
1099 \text {, SAV1W } 1100 \text { (pounders), SAV1W } \\
\text { 1102, SAV1W 1103, SAV1W 1104, SAV1W } \\
\text { 1105 (whetstones), SAV1W } 1101 \\
\text { (architecture?) }\end{array}$ & 17.01.2015 \\
\hline $0275 / 2015$ & SQ1S & stones: pounders ( 2 pieces, each siliceous shale?) & 636 & SAV1W 0746, SAV1W 0747 & 18.01 .2015 \\
\hline $0279 / 2015$ & SQ1S & $\begin{array}{l}\text { stones and architecture: abrasive stone ( } 1 \text { fragment, siliceous } \\
\text { shale?), hand mill ( } 1 \text { fragment, quartzite), grindstone ( } 1 \text { fragment, } \\
\text { quartzite), schist ( } 3 \text { fragments); paving slab? ( } 1 \text { small fragment, } \\
\text { limestone) }\end{array}$ & 637 & $\begin{array}{l}\text { SAV1W } 0742 \text { (abrasive stone), SAV1W } 0744 \\
\text { (hand mil), (grindstone), SAV1W } 0743 \text { (paving } \\
\text { slab?) }\end{array}$ & 18.01.2015 \\
\hline $0292 / 2015$ & SQ1S & $\begin{array}{l}\text { stones: whetstones ( } 2 \text { pieces, each sandstone), hand mills } \\
\text { ( } 2 \text { pieces, each quartzite), grindstone or hand mill ( } 1 \text { piece, } \\
\text { quartzite), grindstones ( } 3 \text { pieces, each quartzite), mortar (1large } \\
\text { fragment, sandstone), pounder ( } 1 \text { piece, quartz) }\end{array}$ & 638 & $\begin{array}{l}\text { SAV1W 0779, SAV1W } 0780 \text { (whetstones), } \\
\text { SAV1W 0781, SAV1W } 0782 \text { (hand mills), } \\
\text { SAV1W 0783 (grindstone or hand mill), } \\
\text { SAV1W 0787, SAV1W 0784, SAV1W } 0785 \\
\text { (grindstones), SAV1W 0786 (mortar), } \\
\text { SAV1W 0787 (mortar), SAV1W } 0788 \\
\text { (pounder) }\end{array}$ & 19.01.2015 \\
\hline $0288 / 2015$ & SQ1S & $\begin{array}{l}\text { stones: pounder ( } 1 \text { small fragment of a quartz pebble), worked } \\
\text { stone (1 small fragment, limestone), grindstones or hand mills } \\
\text { ( } 2 \text { pieces, each quartzite), whetstone ( } 1 \text { small fragment, } \\
\text { sandstone), schist ( } 1 \text { large fragment), grindstone or hand mill } \\
\text { (1 fragment) }\end{array}$ & 639 & $\begin{array}{l}\text { SAV1W } 1123 \text { (pounder), SAV1W } 1124 \\
\text { (worked stone), SAV1W 1125, SAV1W } 1126 \\
\text { (grindstones or hand mills), SAV1W } 1191 \\
\text { (whetstone) }\end{array}$ & 19.01.2015 \\
\hline $0316 / 2015$ & SQ1S & $\begin{array}{l}\text { stones: pounders ( } 2 \text { pieces, each quartz), whetstone ( } 1 \text { piece, } \\
\text { sandstone), hand mills ( } 2 \text { pieces, each quartzite), grindstone } \\
\text { ( } 2 \text { pieces, each quartzite), worked stones ( } 3 \text { pieces, each } \\
\text { sandstone), worked stone ( } 2 \text { fragments, function unclear) }\end{array}$ & 641 & $\begin{array}{l}\text { SAV1W 1113, SAV1W } 1114 \text { (pounders), } \\
\text { SAV1W } 1115 \text { (whetstone), SAV1W 1117, } \\
\text { SAV1W } 1119 \text { (hand mills), SAV1W 1121, } \\
\text { SAV1W } 1118 \text { (grindstones), SAV1W 1122, } \\
\text { SAV1W 1116, SAV1W } 1120 \text { (worked stones) }\end{array}$ & 20.01.2015 \\
\hline $0306 / 2015$ & SQ1S & $\begin{array}{l}\text { stones and architecture: pounder ( } 1 \text { piece, quartz), grindstone } \\
\text { (1 piece, quartzite), hand mills ( } 3 \text { pieces each quartzite), schist } \\
\text { (1 fragment); architecture? ( } 1 \text { small fragment, limestone) }\end{array}$ & 642 & $\begin{array}{l}\text { SAV1W } 1075 \text { (pounder), SAV1W } 1076 \\
\text { (grindstone), SAV1W 1077, SAV1W 1078, } \\
\text { SAV1W } 1079 \text { (hand mills),SAV1W } 1074 \\
\text { (architecture?) }\end{array}$ & 20.01 .2015 \\
\hline $0308 / 2015$ & SQ1S & stone: grindstone (1 piece, quartzite) & 643 & SAV1W 0761 & 20.01 .2015 \\
\hline $0311 / 2015$ & SQ1S & $\begin{array}{l}\text { stones: pounders ( } 5 \text { pieces, each quartz), worked stone (1 piece, } \\
\text { sandstone) }\end{array}$ & 643 & $\begin{array}{l}\text { SAV1W 1089, SAV1W 1090, SAV1W 1091, } \\
\text { SAV1W 1092, SAV1W } 1093 \text { (pounders), } \\
\text { SAV1W 1094 (worked stone) }\end{array}$ & 20.01 .2015 \\
\hline
\end{tabular}




\begin{tabular}{|c|c|c|c|c|c|}
\hline $0328 / 2015$ & SQ1S & $\begin{array}{l}\text { stones: worked stone ( } 1 \text { piece, siliceous shale), pounder ( } 1 \text { piece, } \\
\text { quartz), grindstones ( } 3 \text { pieces, each quartzite), hand mill ( } 1 \text { piece, } \\
\text { quartzite), whetstone ( } 1 \text { piece, unspecified stone) }\end{array}$ & 644 & $\begin{array}{l}\text { SAV1W } 1067 \text { (worked stone), SAV1W } 1068 \\
\text { (pounder), SAV1W 1069, SAV1W 1070, } \\
\text { SAV1W } 1071 \text { (grindstones) Stone (quartzite), } \\
\text { SAV1W } 1072 \text { (hand mill), SAV1W } 1073 \\
\text { (whetstone) }\end{array}$ & 20.01 .2015 \\
\hline $0340 / 2015$ & SQ1S & stone: hand mill (1 small fragment, quartzite) & 645 & SAV1W 0763 & 20.01 .2015 \\
\hline $0352 / 2015$ & SQ1S & $\begin{array}{l}\text { stones: pounders ( } 2 \text { pieces, each quartz pebbles), grindstone } \\
\text { (1 small fragment, quartzite), schist (1 fragment) }\end{array}$ & 646 & $\begin{array}{l}\text { SAV1W 1095, SAV1W } 1096 \text { (pounders), } \\
\text { SAV1W } 1097 \text { (grindstone) }\end{array}$ & 20.01 .2015 \\
\hline $0367 / 2015$ & SQ1S & $\begin{array}{l}\text { stones: grindstone (1 large fragment, quartzite), pounder } \\
\text { (1 fragment, siliceous shale?) }\end{array}$ & 646 & $\begin{array}{l}\text { SAV1W } 1055 \text { (grindstone),SAV1W } 1056 \\
\text { (pounder) }\end{array}$ & 21.01 .2015 \\
\hline $0348 / 2015$ & SQ1S & $\begin{array}{l}\text { stones: whetstone? ( } 1 \text { small fragment, quartzite), grindstone } \\
\text { ( } 3 \text { pieces, each quartzite), schist (1 large fragment) }\end{array}$ & 647 & $\begin{array}{l}\text { SAV1W } 1106 \text { (whetstone), SAV1W 1107, } \\
\text { SAV1W 1108, SAV1W } 1109 \text { (grindstones) }\end{array}$ & 20.01 .2015 \\
\hline $0361 / 2015$ & SQ1S & $\begin{array}{l}\text { stones and architecture: pounders ( } 3 \text { pieces, } 2 \text { quartzite, } \\
1 \text { unspecified stone), whetstones ( } 3 \text { pieces, each sandstone), } \\
\text { grindstones ( } 3 \text { pieces, each quartzite), grindstone or hand mill } \\
\text { (1 piece, quartzite), stone ( } 2 \text { imported stone fragments, } \\
\text { unworked); architecture/column base? (1 large fragment, } \\
\text { sandstone) }\end{array}$ & 647 & $\begin{array}{l}\text { SAV1W 0793, SAV1W 0794, SAV1W } 0795 \\
\text { (pounders), SAV1W 0798, SAV1W 0799, } \\
\text { SAV1W 1000 (whetstones), SAV1W 0790, } \\
\text { SAV1W 0791, SAV1W 0797 (grindstones), } \\
\text { SAV1W 0796 (grindstone or hand mill), } \\
\text { architecture/column base? (SAV1W 0792) }\end{array}$ & 21.01 .2015 \\
\hline $0355 / 2015$ & SQ1S & stone: grindstone (1 large fragment, quartzite) & 649 & SAV1W 0770 & 20.01.2015 \\
\hline $0362 / 2015$ & SQ1S & $\begin{array}{l}\text { stones: grindstone ( } 1 \text { large fragment, quartzite), grindstone or } \\
\text { hand mill ( } 1 \text { small fragment, quartzite), whetstone ( } 1 \text { small } \\
\text { fragment, sandstone), stone vessel? ( } 1 \text { small fragment, } \\
\text { unspecified stone), worked stone ( } 2 \text { fragments) }\end{array}$ & 649 & $\begin{array}{l}\text { SAV1W } 1050 \text { (grindstone), SAV1W } 1051 \\
\text { (grindstone or hand mill), SAV1W } 1052 \\
\text { (whetstone), SAV1W } 1053 \text { (vessel?) }\end{array}$ & 21.01 .2015 \\
\hline $0378 / 2015$ & SQ1S & $\begin{array}{l}\text { stones and doum: pounder ( } 1 \text { small fragment, siliceous shale?), } \\
\text { worked stone ( } 1 \text { piece, unclear function); doum nuts ( } 1 \text { bag) }\end{array}$ & 651 & SAV1W 0776 (pounder) & 21.01 .2015 \\
\hline $0421 / 2015$ & SQ1S & $\begin{array}{l}\text { stones and architecture?: pounders ( } 7 \text { pieces, } 4 \text { siliceous shale?, } \\
2 \text { quartz, } 1 \text { quartzite), grindstones or hand mills ( } 4 \text { pieces, each } \\
\text { quartzite), grindstones ( } 2 \text { pieces, each quartzite), hand mill } \\
\text { (1 piece, quartzite), whetstone ( } 1 \text { piece, sandstone), Palaeolithic } \\
\text { tool? ( } 1 \text { small fragment, unspecified stone), worked stone } \\
\text { (1 fragment, limestone, function unknown); architecture? } \\
\text { (1 small fragment, sandstone) }\end{array}$ & 652 & $\begin{array}{l}\text { SAV1W 1029, SAV1W 1012, SAV1W 1013, } \\
\text { SAV1W 1014, SAV1W 1015, SAV1W 1016, } \\
\text { SAV1W 1017 (pounders), SAV1W 1028, } \\
\text { SAV1W 1022, SAV1W 1023, SAV1W 1024 } \\
\text { (grindstones or hand mills), SAV1W 1025, } \\
\text { SAV1W 1027 (grindstones), SAV1W 1026 } \\
\text { (hand mill), SAV1W 1021 (whetstone), SAV1W } \\
\text { 1019 (Palaeolithic tool?), SAV1W 1020 (worked } \\
\text { stone), SAV1W 1018 (architecture?) }\end{array}$ & 22.01 .2015 \\
\hline $0381 / 2015$ & SQ1S & $\begin{array}{l}\text { stone and clay, stopper/sealing? ( } 1 \text { small fragment, clay); } \\
\text { grindstones ( } 4 \text { pieces, } 3 \text { quartzite, } 1 \text { sandstone), whetstone } \\
\text { (1 piece, sandstone), worked stone ( } 2 \text { fragments) }\end{array}$ & 653 & $\begin{array}{l}\text { SAV1W } 0777 \text { (stopper/sealing?), SAV1W } \\
\text { 0789, SAV1W 1063, SAV1W 1064, SAV1W } \\
\text { 1065 (grindstones), SAV1W 1066 (whetstone) }\end{array}$ & 21.01 .2015 \\
\hline $0419 / 2015$ & SQ1S & stone: grindstone (1 large fragment, quartzite) & 654 & SAV1W 1137 & 22.01 .2015 \\
\hline $0391 / 2015$ & SQ1S & $\begin{array}{l}\text { stones: pounders ( } 2 \text { pieces, } 1 \text { siliceous shale?, } 1 \text { quartz), } \\
\text { whetstone (1 piece, sandstone); schist (1 fragment) }\end{array}$ & 654 & $\begin{array}{l}\text { SAV1W 1110, SAV1W } 1111 \text { (pounders), } \\
\text { SAV1W } 1112 \text { (whetstone) }\end{array}$ & 22.01 .2015 \\
\hline
\end{tabular}




\begin{tabular}{|c|c|c|c|c|c|}
\hline $0400 / 2015$ & SQ1S & stone: grindstone (1 large fragment, quartzite) & 655 & SAV1W 1136 & 22.01.2015 \\
\hline $0436 / 2015$ & SQ1S & $\begin{array}{l}\text { stones and chalk: stone vessel? ( } 1 \text { small fragment, unidentified } \\
\text { stone), pounder ( } 1 \text { small quartz pebble), grindstones ( } 3 \text { pieces, } \\
\text { each quartzite), worked stone ( } 1 \text { fragment, unclear), schist } \\
\text { ( } 2 \text { fragments); chalk? ( } 1 \text { piece, raw material) }\end{array}$ & 658 & $\begin{array}{l}\text { SAV1W } 1045 \text { (stone vessel?), SAV1W } 1046 \\
\text { (pounder), SAV1W 1047, SAV1W 1048, } \\
\text { SAV1W } 1049 \text { (grindstones), }\end{array}$ & 24.01 .2015 \\
\hline $0442 / 2015$ & SQ1S & $\begin{array}{l}\text { stones: whetstone ( } 2 \text { pieces, each sandstone), pounder (1 quartz } \\
\text { pebble) }\end{array}$ & 660 & $\begin{array}{l}\text { SAV1W 1038, SAV1W } 1039 \text { (whetstones), } \\
\text { SAV1W } 1040 \text { (pounder) }\end{array}$ & 24.01 .2015 \\
\hline $0443 / 2015$ & SQ1S & stones: whetstone (1 small fragment, unspecified stone) & 661 & SAV1W 1054 & 24.01 .2015 \\
\hline $0449 / 2015$ & SQ1S & $\begin{array}{l}\text { stones: pounders ( } 2 \text { pieces, } 1 \text { siliceous shale?, } 1 \text { quartz), hand } \\
\text { mill ( } 1 \text { small fragment, quartzite), grindstone (1 small fragment, } \\
\text { quartzite) }\end{array}$ & 661 & $\begin{array}{l}\text { SAV1W 1041, SAV1W } 1042 \text { (pounders), } \\
\text { SAV1W } 1043 \text { (hand mill), SAV1W } 1044 \\
\text { (grindstone) }\end{array}$ & 24.01 .2015 \\
\hline $0453 / 2015$ & SQ1S & stone: grindstone or hand mill (1 fragment) & 662 & & 27.01 .2015 \\
\hline $0456 / 2015$ & SQ1S & $\begin{array}{l}\text { stones: pounders ( } 3 \text { pieces, } 2 \text { quartz, } 1 \text { siliceous shale?), } \\
\text { grindstones or hand mills ( } 4 \text { pieces, each quartzite), hand mill } \\
\text { ( } 1 \text { piece, quartzite), whetstones ( } 2 \text { pieces, each sandstone), } \\
\text { worked stone ( } 1 \text { piece, quartzite) }\end{array}$ & 663 & $\begin{array}{l}\text { SAV1W 1142, SAV1W 1143, SAV1W } 1144 \\
\text { (pounders), SAV1W 1145, SAV1W 1146, } \\
\text { SAV1W 1138, SAV1W } 1140 \text { (grindstones or } \\
\text { hand mills), SAV1W } 1139 \text { (hand mill), SAV1W } \\
1147, \text { SAV1W } 1141 \text { (whetstones), SAV1W } \\
1148 \text { (worked stone) }\end{array}$ & 27.01 .2015 \\
\hline $0469 / 2015$ & SQ1S & $\begin{array}{l}\text { stones: grindstone or hand mill ( } 1 \text { small fragment, quartzite), } \\
\text { hand mill (1 small fragment, quartzite), grindstone (1 small } \\
\text { fragment, quartzite) }\end{array}$ & 666 & $\begin{array}{l}\text { SAV1W } 1156 \text { (grindstone or hand mill), } \\
\text { SAV1W } 1157 \text { (hand mill), SAV1W } 1158 \\
\text { (grindstone) }\end{array}$ & 27.01.2015 \\
\hline $0475 / 2015$ & SQ1S & stone: pounder (1 piece, quartzite) & 667 & SAV1W 1155 & 27.01.2015 \\
\hline $0484 / 2015$ & SQ1S & $\begin{array}{l}\text { stone: worked stone (1 piece, hourglass shaped, unspecified } \\
\text { stone) }\end{array}$ & 668 & SAV1W 1184 & 27.01 .2015 \\
\hline $0496 / 2015$ & SQ1S & $\begin{array}{l}\text { stones and fossilised bone?: hand mill ( } 2 \text { pieces each quartzite), } \\
\text { grindstone ( } 2 \text { pieces, } 1 \text { quartzite, } 1 \text { sandstone), whetstone? } \\
\text { (1 fragment, sandstone), fossilised bone?( } 1 \text { fragment })\end{array}$ & 668 & $\begin{array}{l}\text { SAV1W } 1224 \text { (hand mill), SAV1W 1226, } \\
\text { SAV1W } 1228 \text { (grindstones), SAV1W } 1227 \\
\text { (whetstone) }\end{array}$ & 28.01 .2015 \\
\hline $0481 / 2015$ & SQ1S & $\begin{array}{l}\text { stones: pounder ( } 1 \text { piece, siliceous shale?), hand mill ( } 1 \text { fragment, } \\
\text { quartzite), worked stone ( } 1 \text { fragment, unspecified stone) }\end{array}$ & 669 & $\begin{array}{l}\text { SAV1W } 1151 \text { (pounder), SAV1W } 1152 \text { (hand } \\
\text { mill), SAV1W } 1153 \text { (worked stone) }\end{array}$ & 27.01 .2015 \\
\hline $0500 / 2015$ & SQ1S & $\begin{array}{l}\text { stones: grindstone ( } 2 \text { pieces, each quartzite), hand mill } \\
\text { (1 fragment, quartzite), schist ( } 2 \text { pieces) }\end{array}$ & 669 & $\begin{array}{l}\text { SAV1W 1230, SAV1W } 1232 \text { (grindstones), } \\
\text { (hand mill) }\end{array}$ & 28.01 .2015 \\
\hline $0524 / 2015$ & SQ1S & stone: grindstone (1 piece, sandstone) & 670 & SAV1W 1165 & 28.01 .2015 \\
\hline $0508 / 2015$ & SQ1S & $\begin{array}{l}\text { stones: pounders ( } 2 \text { pieces, each quartz pebbles), grindstone } \\
\text { (1 fragment, quartzite), hand mill (1 fragment, quartzite) }\end{array}$ & 671 & $\begin{array}{l}\text { SAV1W 1159, SAV1W } 1160 \text { (pounders), } \\
\text { SAV1W } 1161 \text { (grindstone), SAV1W } 1162 \\
\text { (hand mill) }\end{array}$ & 28.01 .2015 \\
\hline $0517 / 2015$ & SQ1S & stone: pounder (1 piece, quartzite) & 673 & SAV1W 1163 & 28.01 .2015 \\
\hline $0528 / 2015$ & SQ1S & $\begin{array}{l}\text { stones: hand mill (1 fragment, quartzite), whetstone (1 fragment, } \\
\text { sandstone) }\end{array}$ & 674 & SAV1W 1170, SAV1W 1171 & 28.01 .2015 \\
\hline
\end{tabular}




\begin{tabular}{|c|c|c|c|c|c|}
\hline $0558 / 2015$ & SQ1S & stone: stone vessel (1 large fragment of stone bowl, quartzite) & 674 & SAV1W 1177 & 29.01.2015 \\
\hline $0559 / 2015$ & SQ1S & $\begin{array}{l}\text { stones: pounder (1 quartz pebble), hand mill (1 fragment, } \\
\text { quartzite) }\end{array}$ & 674 & SAV1W 1180, SAV1W 1181 & 29.01.2015 \\
\hline $0525 / 2015$ & SQ1S & $\begin{array}{l}\text { stone: polishing stone? (1 piece, quartzite), worked stone } \\
\text { (1 fragment, unspecified stone), grindstone (1 fragment, } \\
\text { quartzite), }\end{array}$ & 675 & $\begin{array}{l}\text { SAV1W } 1173 \text { (polishing stone?), SAV1W } \\
1174 \text { (worked stone), SAV1W } 1175 \\
\text { (grindstone) }\end{array}$ & 28.01.2015 \\
\hline $0536 / 2015$ & SQ1S & stones: pounder (1 piece, quartzite), schist (1 fragment) & 675 & SAV1W 1176 & 29.01.2015 \\
\hline $0551 / 2015$ & SQ1S & $\begin{array}{l}\text { stones and architecture?: pounder ( } 1 \text { quartz pebble), polishing } \\
\text { stone ( } 1 \text { piece, quartzite); architecture? (1 small fragment, } \\
\text { limestone) }\end{array}$ & 679 & $\begin{array}{l}\text { SAV1W } 1182 \text { (pounder), SAV1W } 1183 \\
\text { (polishing stone), SAV1W } 1263 \text { (architecture?) }\end{array}$ & 29.01.2015 \\
\hline $0561 / 2015$ & SQ1S & $\begin{array}{l}\text { stones: grindstone ( } 1 \text { fragment, quartzite), pounders ( } 2 \text { pieces, } \\
1 \text { quartzite, } 1 \text { quartz) }\end{array}$ & 680 & $\begin{array}{l}\text { SAV1W } 1264 \text { (grindstone), SAV1W 1265, } \\
\text { SAV1W } 1266 \text { (pounders) }\end{array}$ & 29.01 .2015 \\
\hline $0586 / 2015$ & SQ1S & $\begin{array}{l}\text { stones: pumice stone ( } 1 \text { fragment), pounder ( } 1 \text { fragment, } \\
\text { quartzite), hand mill ( } 1 \text { fragment, quartzite) }\end{array}$ & 685 & $\begin{array}{l}\text { SAV1W } 1192 \text { (pumice stone), SAV1W } 1200 \\
\text { (pounder), SAV1W } 1201 \text { (hand mill) }\end{array}$ & 31.01 .2015 \\
\hline $0602 / 2015$ & SQ1S & $\begin{array}{l}\text { stone and pottery: stone vessel ( } 1 \text { small fragment, rim?, } \\
\text { unspecified stone), stopper/sealing ( } 1 \text { large fragment, clay) }\end{array}$ & 689 & $\begin{array}{l}\text { SAV1W } 1313 \text { (stone vessel), SAV1W } 1217 \\
\text { (stopper/sealing) }\end{array}$ & 31.01 .2015 \\
\hline $0621 / 2015$ & SQ1S & stone: spindle weight (1 piece, limestone) & 691 & SAV1W 1205 & 31.01 .2015 \\
\hline $0636 / 2015$ & SQ1S & $\begin{array}{l}\text { stones: polishing stone ( } 1 \text { piece, unspecified stone), grindstone or } \\
\text { hand mill ( } 1 \text { fragment, quartzite), worked stone (1 fragment, } \\
\text { sandstone), pounder ( } 1 \text { quartz pebble) }\end{array}$ & 691 & $\begin{array}{l}\text { SAV1W } 1210 \text { (polishing stone), SAV1W } 1211 \\
\text { (grindstone or hand mill), SAV1W } 1212 \\
\text { (worked stone), SAV1W } 1213 \text { (pounder) }\end{array}$ & 01.02 .2015 \\
\hline $0641 / 2015$ & SQ1S & stone: grindstone (1 fragment, quartzite) & 691 & SAV1W 1261 & 01.02 .2015 \\
\hline $0655 / 2015$ & SQ1S & stone and slag: pounder (1 fragment, quartzite); slag (1 fragment) & 697 & SAV1W 1249 (pounder), SAV1W 1244 (slag) & 01.02 .2015 \\
\hline $0652 / 2015$ & SQ1S & $\begin{array}{l}\text { stones: whetstone ( } 1 \text { fragment, sandstone), hand mills ( } 3 \text { pieces, } \\
2 \text { sandstone, } 1 \text { quartzite) }\end{array}$ & 698 & $\begin{array}{l}\text { SAV1W } 1250 \text { (whetstone), SAV1W 1251, } \\
\text { SAV1W 1252, SAV1W } 1253 \text { (hand mills) }\end{array}$ & 01.02 .2015 \\
\hline $0668 / 2015$ & SQ1S & $\begin{array}{l}\text { stones: pounder ( } 1 \text { quartz pebble), hand mills ( } 2 \text { pieces, } \\
1 \text { sandstone, } 1 \text { quartzite), worked stone (1 fragment, limestone) }\end{array}$ & 699 & $\begin{array}{l}\text { SAV1W } 1245 \text { (pounder), SAV1W 1246, } \\
\text { SAV1W } 1247 \text { (hand mills), SAV1W } 1248 \\
\text { (worked stone) }\end{array}$ & 01.02 .2015 \\
\hline $0674 / 2015$ & SQ1S & $\begin{array}{l}\text { stones and architecture: grindstones ( } 3 \text { fragments, each quartzite), } \\
\text { pounder ( } 1 \text { quartz pebble), hand mill ( } 1 \text { fragment, quartzite), } \\
\text { grindstone or hand mill ( } 1 \text { fragment, side), worked stone ( } 1 \text { piece, } \\
\text { unclear function); architecture/re-used as whetstone (1 large } \\
\text { fragment, door lintel? limestone) }\end{array}$ & 703 & $\begin{array}{l}\text { SAV1W 1254, SAV1W 1255, SAV1W } 1256 \\
\text { (grindstones), SAV1W 1258 (pounder), } \\
\text { SAV1W 1259 (hand mill); SAV1W } 12578 \\
\text { (architecture/re-used as whetstone) }\end{array}$ & 02.02 .2015 \\
\hline $0683 / 2015$ & SQ1S & stone: grindstone (1 fragment, quartzite) & 703 & SAV1W 1260 & 02.02 .2015 \\
\hline $0678 / 2015$ & SQ1S & $\begin{array}{l}\text { stones: hand mill (1 fragment, sandstone), pounder (1 quartz } \\
\text { pebble), grindstone (1 fragment, quartzite) }\end{array}$ & 704 & $\begin{array}{l}\text { SAV1W } 1275 \text { (hand mill), SAV1W } 1364 \\
\text { (pounder), SAV1W } 1365 \text { (grindstone) }\end{array}$ & 02.02 .2015 \\
\hline $0699 / 2015$ & SQ1S & stone: grindstone (1 large fragment, sandstone) & 704 & SAV1W 1339 & 02.02 .2015 \\
\hline
\end{tabular}




\begin{tabular}{|c|c|c|c|c|c|}
\hline $0686 / 2015$ & SQ1S & stone: pivot stone (1 large piece, sandstone) & 705 & SAV1W 1471 & 02.02 .2015 \\
\hline $0691 / 2015$ & SQ1S & stone: grindstone (1 piece) & 705 & & 02.02 .2015 \\
\hline $0714 / 2015$ & SQ1S & $\begin{array}{l}\text { stones: worked stones ( } 2 \text { fragments, each sandstone), grindstones } \\
\text { ( } 2 \text { fragments, each sandstone), whetstones ( } 4 \text { fragments, each } \\
\text { sandstone) }\end{array}$ & 705 & $\begin{array}{l}\text { SAV1W 1287, SAV1W worked stones), } \\
\text { SAV1W 1289, SAV1W } 1290 \text { (grindstones), } \\
\text { SAV1W 1291, SAV1W 1292, SAV1W 1293, } \\
\text { SAV1W } 1270 \text { (whetstones) }\end{array}$ & 02.02 .2015 \\
\hline $0702 / 2015$ & SQ1S & stone: blade (1 small fragment, silex) & 706 & SAV1W 1268 & 02.02 .2015 \\
\hline $0709 / 2015$ & SQ1S & stone (1 flake) & 706 & & 02.02 .2015 \\
\hline $0743 / 2015$ & SQ1S & $\begin{array}{l}\text { stones: pounder ( } 1 \text { fragment, siliceous shale?), worked stones } \\
\text { ( } 2 \text { fragments, } 1 \text { unspecified stone, } 1 \text { sandstone), grindstone } \\
\text { ( } 1 \text { fragment, sandstone) }\end{array}$ & 710 & $\begin{array}{l}\text { SAV1W } 1388 \text { (pounder), SAV1W 1389, } \\
\text { SAV1W } 1391 \text { (worked stones), SAV1W } 1390 \\
\text { (grindstone) }\end{array}$ & 03.02 .2015 \\
\hline $0737 / 2015$ & SQ1S, sieving & $\begin{array}{l}\text { stones: pounder ( } 2 \text { pieces, each siliceous shale?), whetstone } \\
\text { (1 fragment, sandstone), hand mill ( } 1 \text { fragment, sandstone), } \\
\text { worked stone ( } 1 \text { fragment, sandstone) }\end{array}$ & 710 & $\begin{array}{l}\text { SAV1W 1366, SAV1W } 1367 \text { (pounders), } \\
\text { SAV1W } 1368 \text { (whetstone), SAV1W } 1369 \text { (hand } \\
\text { mill), SAV1W } 1370 \text { (worked stone) }\end{array}$ & 03.02 .2015 \\
\hline $0755 / 2015$ & SQ1E & $\begin{array}{l}\text { stones: pounders ( } 6 \text { pieces, } 2 \text { siliceous shale?, } 4 \text { quartz), hand } \\
\text { mills ( } 3 \text { pieces, } 2 \text { sandstone, } 1 \text { quartzite), grindstones ( } 5 \text { pieces, } \\
\text { each sandstone), worked stones ( } 4 \text { pieces, each sandstone), stone } \\
\text { vessel ( } 1 \text { piece, sandstone), hammer ( } 1 \text { piece, quartzite) }\end{array}$ & 713 & $\begin{array}{l}\text { SAV1W 1344, SAV1W 1345, SAV1W 1346, } \\
\text { SAV1W 1347, SAV1W 1348, SAV1W 1349, } \\
\text { SAV1W 1350 (pounders), SAV1W 1351, } \\
\text { SAV1W 1352, SAV1W } 1353 \text { (hand mills), } \\
\text { SAV1W 1354, SAV1W 1355, SAV1W 1356, } \\
\text { SAV1W 1357, SAV1W 1358 (grindstones), } \\
\text { SAV1W 1359, SAV1W 1360, SAV1W 1362, } \\
\text { SAV1W 1363 (worked stones), SAV1W 1361 } \\
\text { (stone vessel), SAV1W } 1343 \text { (hammer) }\end{array}$ & 04.02 .2015 \\
\hline $0760 / 2015$ & SQ1E & $\begin{array}{l}\text { stones: worked stone ( } 1 \text { fragment, sandstone), pounders } \\
\text { ( } 2 \text { pieces, each unspecified stone) }\end{array}$ & 714 & $\begin{array}{l}\text { SAV1W } 1294 \text { (worked stone), SAV1W 1295, } \\
\text { SAV1W } 1371 \text { (pounders) }\end{array}$ & 04.02 .2015 \\
\hline $0763 / 2015$ & SQ1/SQ1E & stone: pounder (1 quartz pebble) & 717 & SAV1W 1372 & 04.02 .2015 \\
\hline $0766 / 2015$ & SQ1/SQ1E & $\begin{array}{l}\text { stones: pounders ( } 4 \text { pieces, } 2 \text { quartzite, } 1 \text { unspecified stone, } \\
1 \text { quartz), grindstones ( } 2 \text { pieces, each quartzite), worked stone } \\
\text { ( } 4 \text { pieces, } 3 \text { sandstone, } 1 \text { schist), hand mill (1 piece, sandstone), } \\
\text { hammer ( } 1 \text { piece, metamorphic stone) }\end{array}$ & 717 & $\begin{array}{l}\text { SAV1W 1377, SAV1W 1378, SAV1W 1380, } \\
\text { SAV1W } 1398 \text { (pounders), SAV1W 1383, } \\
\text { SAV1W } 1384 \text { (grindstones), SAV1W 1381, } \\
\text { SAV1W 1385, SAV1W 1386, SAV1W 1387 } \\
\text { (worked stones), SAV1W 1382 (hand mill), } \\
\text { SAV1W } 1379 \text { (hammer) }\end{array}$ & 05.02 .2015 \\
\hline $0771 / 2015$ & SQ1S & stone: blade? (1 small silex stone) & 718 & SAV1W 1298 & 05.02 .2015 \\
\hline $0774 / 2015$ & SQ1/SQ1E & $\begin{array}{l}\text { stones: bracelet ( } 1 \text { fragment, unspecified stone), pounders } \\
\text { ( } 6 \text { pieces, } 1 \text { unspecified stone, } 4 \text { quartz, } 1 \text { siliceous shale?), } \\
\text { whetstone ( } 1 \text { piece, sandstone), grindstone ( } 1 \text { piece, quartzite) }\end{array}$ & 719 & $\begin{array}{l}\text { SAV1W } 1276 \text { (bracelet), SAV1W 1286, } \\
\text { SAV1W 1373, SAV1W 1277, SAV1W 1278, } \\
\text { SAV1W 1279, SAV1W 1280 (pounders), } \\
\text { SAV1W 1281 (whetstone), SAV1W } 1282 \\
\text { (grindstone) }\end{array}$ & 05.02 .2015 \\
\hline
\end{tabular}




\begin{tabular}{|c|c|c|c|c|c|}
\hline $0817 / 2015$ & SQ1S & stone: blade? (1 piece, silex) & 723 & SAV1W 1428 & 08.02 .2015 \\
\hline $0657 / 2015$ & SQ1S & stopper/sealing? (1 small fragment, clay) & 697 & SAV1W 1241 & 01.02 .2015 \\
\hline $0689 / 2015$ & SQ1S & stopper/sealing? (1 small fragment, mud) & 703 & SAV1W 1274 & 02.02 .2015 \\
\hline $0060 / 2015$ & SQ1S & textile (1 bag) & 609 & & 06.01 .2015 \\
\hline $0068 / 2015$ & SQ1/S & textile (1 piece) & 609 & & 07.01 .2015 \\
\hline $0096 / 2015$ & SQ1S & textile (1 bag) & 612 & & 08.01 .2015 \\
\hline $0149 / 2015$ & SQ1S & textile (1 bag) & 612 & & 12.01 .2015 \\
\hline $0143 / 2015$ & SQ1S & textile (1 piece) & 619 & & 11.01 .2015 \\
\hline $0171 / 2015$ & SQ1S & textile (1 bag) & 620 & & 13.01 .2015 \\
\hline $0189 / 2015$ & SQ1S & textile (1 bag) & 622 & & 14.01 .2015 \\
\hline $0193 / 2015$ & SQ1S & textile (1 bag) & 624 & & 14.01 .2015 \\
\hline $0215 / 2015$ & SQ1S & textile (1 bag) & 627 & & 15.01 .2015 \\
\hline $0219 / 2015$ & SQ1S & textile (1 bag) & 628 & & 15.01 .2015 \\
\hline $0266 / 2015$ & SQ1S & textile (1 bag) & 633 & & 18.01 .2015 \\
\hline $0253 / 2015$ & SQ1S & textile (1 bag) & 635 & & 17.01 .2015 \\
\hline $0274 / 2015$ & SQ1S & textile (1 bag) & 636 & & 18.01 .2015 \\
\hline $0277 / 2015$ & SQ1S & textile (1 bag) & 637 & & 18.01 .2015 \\
\hline $0290 / 2015$ & SQ1S & textile (1 bag) & 639 & & 19.01 .2015 \\
\hline $0321 / 2015$ & SQ1S & textile (1 bag) & 641 & & 20.01 .2015 \\
\hline $0324 / 2015$ & SQ1S & textile (1 piece) & 642 & & 20.01 .2015 \\
\hline $0330 / 2015$ & SQ1S & textile (1 bag) & 644 & & 20.01 .2015 \\
\hline $0375 / 2015$ & SQ1S & textile (1 piece) & 646 & & 21.01 .2015 \\
\hline $0353 / 2015$ & SQ1S & textile (1 bag) & 647 & & 20.01 .2015 \\
\hline $0432 / 2015$ & SQ1S & textile (1 bag) & 650 & & 24.01 .2015 \\
\hline $0371 / 2015$ & SQ1S & textile (1 bag) & 651 & & 21.01 .2015 \\
\hline $0406 / 2015$ & SQ1S & textile (1 bag) & 657 & & 22.01 .2015 \\
\hline
\end{tabular}




\begin{tabular}{|c|c|c|c|c|c|}
\hline $0463 / 2015$ & SQ1S & textile (1 bag) & 662 & & 27.01.2015 \\
\hline $0570 / 2015$ & SQ1 & textile (1 bag) & 664 & & 29.01.2015 \\
\hline $0549 / 2015$ & SQ1S & textile (1 bag) & 674 & & 29.01.2015 \\
\hline $0656 / 2015$ & SQ1S & textile (1 bag, knot) & 698 & & 01.02 .2015 \\
\hline $0665 / 2015$ & SQ1S & textile (1 bag) & 701 & & 01.02 .2015 \\
\hline $0692 / 2015$ & SQ1S & textile (1 bag) & 704 & & 02.02 .2015 \\
\hline $0687 / 2015$ & SQ1S & textile (1 bag) & 705 & & 02.02 .2015 \\
\hline $0736 / 2015$ & SQ1S & textile (1 bag) & 710 & & 03.02 .2015 \\
\hline $0724 / 2015$ & SQ1S & textile (1 piece) & 711 & & 03.02 .2015 \\
\hline $0829 / 2015$ & SQ1, Feature 115 & token/gaming piece (1 piece, mud) & 731 & SAV1W 1427 & 09.02 .2015 \\
\hline $0036 / 2015$ & SQ1S & token/gaming piece (1 piece, pyramidal in shape, sandstone) & 605 & SAV1W 0869 & 05.01 .2015 \\
\hline $0033 / 2015$ & SQ1S & weight (1 re-used pottery fragment) & 603 & SAV1W 0866 & 05.01 .2015 \\
\hline $0030 / 2015$ & SQ1S & wood (1 piece) & 603 & & 05.01 .2015 \\
\hline $0066 / 2015$ & SQ1/S & wood (1 piece) & 606 & & 07.01 .2015 \\
\hline $0056 / 2015$ & SQ1S & wood (2 pieces) & 609 & & 06.01 .2015 \\
\hline $0093 / 2015$ & SQ1S & wood (1 bag) & 612 & & 08.01 .2015 \\
\hline $0165 / 2015$ & SQ1S & wood (2 pieces) & 612 & & 12.01.2015 \\
\hline $0118 / 2015$ & SQ1S & wood (1 bag) & 614 & & 10.01 .2015 \\
\hline $0157 / 2015$ & SQ1S & wood (1 bag) & 618 & & 12.01 .2015 \\
\hline $0178 / 2015$ & SQ1S & wood (1 bag) & 620 & & 13.01 .2015 \\
\hline $0206 / 2015$ & SQ1S & wood (1 bag) & 625 & & 15.01 .2015 \\
\hline $0232 / 2015$ & SQ1S & wood (2 pieces) & 630 & & 17.01 .2015 \\
\hline $0267 / 2015$ & SQ1S & wood (1 piece) & 633 & & 18.01 .2015 \\
\hline $0247 / 2015$ & SQ1S & wood (2 pieces) & 633 & & 17.01 .2015 \\
\hline $0248 / 2015$ & SQ1S & wood (1 piece) & 634 & & 17.01 .2015 \\
\hline $0258 / 2015$ & SQ1S & wood (1 bag) & 635 & & 17.01 .2015 \\
\hline
\end{tabular}




\begin{tabular}{|c|c|c|c|c|}
\hline $0284 / 2015$ & SQ1S & wood (1 bag) & 636 & 18.01 .2015 \\
\hline $0296 / 2015$ & SQ1S & wood (1 bag) & 639 & 19.01 .2015 \\
\hline $0300 / 2015$ & SQ1S & wood (1 bag) & 640 & 19.01.2015 \\
\hline $0332 / 2015$ & SQ1S & wood (1 bag) & 641 & 20.01 .2015 \\
\hline $0307 / 2015$ & SQ1S & wood (1 bag) & 642 & 20.01 .2015 \\
\hline $0326 / 2015$ & SQ1S & wood (1 piece) & 642 & 20.01 .2015 \\
\hline $0327 / 2015$ & SQ1S & wood (1 bag) & 643 & 20.01 .2015 \\
\hline $0337 / 2015$ & SQ1S & wood (1 piece) & 645 & 20.01 .2015 \\
\hline $0354 / 2015$ & SQ1S & wood (1 bag) & 646 & 20.01 .2015 \\
\hline $0368 / 2015$ & SQ1S & wood (1 bag) & 646 & 21.01 .2015 \\
\hline $0350 / 2015$ & SQ1S & wood (1 piece) & 647 & 20.01 .2015 \\
\hline $0366 / 2015$ & SQ1S & wood (2 pieces) & 647 & 21.01 .2015 \\
\hline $0365 / 2015$ & SQ1S & wood (1 piece) & 649 & 21.01 .2015 \\
\hline $0434 / 2015$ & SQ1S & wood (1 piece) & 650 & 24.01 .2015 \\
\hline $0379 / 2015$ & SQ1S & wood (1 bag) & 651 & 21.01 .2015 \\
\hline $0398 / 2015$ & SQ1S & wood (1 bag) & 652 & 22.01 .2015 \\
\hline $0390 / 2015$ & SQ1S & wood (1 piece) & 653 & 22.01 .2015 \\
\hline $0405 / 2015$ & SQ1S & wood (1 bag) & 657 & 22.01 .2015 \\
\hline $0435 / 2015$ & SQ1S & wood (1 bag) & 658 & 24.01 .2015 \\
\hline $0458 / 2015$ & SQ1S & wood (1 bag) & 662 & 27.01 .2015 \\
\hline $0477 / 2015$ & SQ1S & wood (1 piece); doum nut (1 piece) & 666 & 27.01 .2015 \\
\hline $0486 / 2015$ & SQ1S & wood (1 piece) & 668 & 27.01 .2015 \\
\hline $0497 / 2015$ & SQ1S & wood (1 piece) & 668 & 28.01 .2015 \\
\hline $0506 / 2015$ & SQ1S & wood (1 bag) & 671 & 28.01 .2015 \\
\hline $0556 / 2015$ & SQ1S & wood (1 bag) & 674 & 29.01 .2015 \\
\hline $0538 / 2015$ & SQ1S & wood (1 piece) & 678 & 29.01 .2015 \\
\hline
\end{tabular}




\begin{tabular}{|c|c|c|c|c|}
\hline $0553 / 2015$ & SQ1S & wood (1 bag) & 678 & 29.01 .2015 \\
\hline $0582 / 2015$ & SQ1S & wood (1 bag) & 685 & 31.01 .2015 \\
\hline $0606 / 2015$ & SQ1 & wood (1 piece) & 687 & 31.01 .2015 \\
\hline $0603 / 2015$ & SQ1 & wood (2 pieces) & 688 & 31.01 .2015 \\
\hline $0609 / 2015$ & SQ1 & wood (1 piece) & 688 & 31.01 .2015 \\
\hline $0617 / 2015$ & SQ1 & wood (1 bag) & 688 & 31.01 .2015 \\
\hline $0614 / 2015$ & SQ1S & wood (1 bag) & 689 & 31.01 .2015 \\
\hline $0634 / 2015$ & SQ1 & wood (1 piece) & 695 & 01.02 .2015 \\
\hline $0681 / 2015$ & SQ1S & wood (2 pieces) & 703 & 02.02 .2015 \\
\hline $0694 / 2015$ & SQ1S & wood (1 bag) & 704 & 02.02 .2015 \\
\hline $0688 / 2015$ & SQ1S & wood (2 pieces) & 705 & 02.02 .2015 \\
\hline $0708 / 2015$ & SQ1S & wood (1 bag) & 706 & 02.02 .2015 \\
\hline $0732 / 2015$ & SQ1 & wood (1 bag) & 708 & 03.02 .2015 \\
\hline $0851 / 2015$ & SQ1S, sieved material & $\begin{array}{l}\text { wood (1 piece); bones ( } 1 \text { bag); organic material ( } 1 \text { bag); charcoal } \\
\text { (1 bag) }\end{array}$ & 711 & 03.02 .2015 \\
\hline $0776 / 2015$ & SQ1/SQ1E & wood (1 piece) & 719 & 05.02 .2015 \\
\hline
\end{tabular}

\begin{tabular}{|c|c|c|c|c|c|}
\hline \multicolumn{6}{|c|}{ Sai Island SAV1 West 2016} \\
\hline Find no. & Location & Description/label/material & $\begin{array}{l}\text { SU/ } \\
\text { level }\end{array}$ & Object no. & Date \\
\hline $1521 / 2016$ & SQ1S & agate (1 piece, sample) & 878 & & 31.01 .2016 \\
\hline $1256 / 2016$ & SQ1SE & alabaster/calcite vessel (1 fragment) & 837 & SAV1W 1196 & 18.01.2016 \\
\hline $1058 / 2016$ & SQ1SE & $\begin{array}{l}\text { architecture? ( } 1 \text { schist fragment, perhaps part of a floor, remains } \\
\text { of red pigment) }\end{array}$ & 809 & SAV1W 0383 & 07.01 .2016 \\
\hline $1140 / 2016$ & SQ1SE & $\begin{array}{l}\text { architecture? ( } 1 \text { schist fragment, perhaps originally piece of } \\
\text { architecture, remains of red pigment) }\end{array}$ & 826 & SAV1W 1554 & 12.01.2016 \\
\hline $1336 / 2016$ & SQ1SE & $\begin{array}{l}\text { architecture?/stela? (1 fragment with decorative Christian design, } \\
\text { sandstone) }\end{array}$ & 853 & SAV1W 1620 & 23.01.2016 \\
\hline
\end{tabular}




\begin{tabular}{|c|c|c|c|c|c|}
\hline $1374 / 2016$ & SQ1SE & $\begin{array}{l}\text { architecture and stone: column ( } 1 \text { base or drum, sandstone), } \\
\text { architecture? ( } 1 \text { fragment, sandstone, not part of SAV1W 1009); } \\
\text { grindstone (1 piece, sandstone) }\end{array}$ & 854 & SAV1W 1009, SAV1W 1640, SAV1W 1527 & 24.01.2016 \\
\hline $1301 / 2016$ & SQ1SE & bark (1 piece) & 847 & & 20.01.2016 \\
\hline $1003 / 2016$ & SQ1SE & bead (1 piece, wood) & 800 & SAV1W 1530 & 04.01 .2016 \\
\hline $1005 / 2016$ & $\begin{array}{l}\text { SQ1SE, from } 1 \mathrm{~m} \text { around trench, surface } \\
\text { clearing }\end{array}$ & bead (1 ring bead, faience); Nun bowl (1 fragment, faience) & 800 & $\begin{array}{l}\text { SAV1W } 1529 \text { (bead), SAV1W } 1528 \text { (Nun } \\
\text { bowl) }\end{array}$ & 04.01 .2016 \\
\hline $1053 / 2016$ & SQ1SE & bead (1 tubular bead, faience) & 809 & SAV1W 0322 & 07.01 .2016 \\
\hline $1054 / 2016$ & SQ1SE & bead (1 roughly square shaped bead, glass) & 809 & SAV1W 0353 & 07.01 .2016 \\
\hline $1070 / 2016$ & SQ1SE & bead (1 cylindrical shaped bead, clay) & 811 & SAV1W 0444 & 09.01.2016 \\
\hline $1130 / 2016$ & $\begin{array}{l}\text { SQ1S, from surface cleaning at SQ1S, } \\
\text { along E profile }\end{array}$ & bead (1 small ring bead, faience) & 819 & SAV1W 1553 & 12.01.2016 \\
\hline $1310 / 2016$ & SQ1S, from surface cleaning of SQ1S & bead (1 ring bead, faience) & 819 & SAV1W 0044 & 20.01.2016 \\
\hline $1347 / 2016$ & SQ1S & bead (1 fragment, ring bead, faience) & 852 & SAV1W 1623 & 23.01.2016 \\
\hline $1418 / 2016$ & SQ1SE & bead or weight (1 large piece, clay) & 860 & SAV1W 1652 & 26.01.2016 \\
\hline $1417 / 2016$ & SQ1S & bead (1 ring bead, faience) & 861 & SAV1W 1649 & 26.01.2016 \\
\hline $1505 / 2016$ & SQ1S, sieved material & $\begin{array}{l}\text { beads ( } 3 \text { ring beads, } 1 \text { bone? } 2 \text { fragments of bone, } 1 \text { faience); } \\
\text { charcoal ( } 1 \text { bag); wood ( } 1 \text { bag); botanical material ( } 1 \text { bag, } \\
\text { mixed); doum nuts ( } 1 \text { bag); bones ( } 1 \text { bag); metal ( } 2 \text { pieces); shell } \\
\text { ( } 2 \text { pieces) }\end{array}$ & 874 & $\begin{array}{l}\text { SAV1W 1700, SAV1W 1702, SAV1W } 1701 \\
\text { (beads) }\end{array}$ & 31.01 .2016 \\
\hline $1532 / 2016$ & SQ1NW & bead (1 ring bead, faience) & 879 & SAV1W 1699 & 01.02 .2016 \\
\hline $1564 / 2016$ & SQ1S, sieved material & $\begin{array}{l}\text { beads ( } 2 \text { ring beads, faience); bones }(1 \mathrm{bag}) \text {; charcoal }(1 \mathrm{bag}) \text {; } \\
\text { wood ( } 1 \mathrm{bag}) ; \text { stone flakes ( } 4 \text { pieces, reference })\end{array}$ & 888 & SAV1W 1715 (beads) & 03.02 .2016 \\
\hline $1578 / 2016$ & SQ1, sieved material of Feature 112 & $\begin{array}{l}\text { bead ( } 1 \text { ring bead, bone); shell ( } 1 \text { piece); stone flakes ( } 7 \text { pieces, } \\
\text { reference); textile ( } 1 \text { piece); wood ( } 1 \text { bag); bones }(1 \text { bag); } \\
\text { charcoal ( } 1 \text { bag); botanical material ( } 1 \text { bag, seeds); doum nuts ( } 1 \\
\text { bag); organic material ( } 1 \text { bag, unclear) }\end{array}$ & 898 & SAV1W 1714 (bead) & 04.02 .2016 \\
\hline $1006 / 2016$ & SQ1SE & bones (1 bag) & 801 & & 05.01 .2016 \\
\hline $1014 / 2016$ & SQ1SE & bones (3 pieces) & 802 & & 05.01 .2016 \\
\hline $1028 / 2016$ & SQ1SE & bones (1 bag) & 802 & & 06.01 .2016 \\
\hline $1016 / 2016$ & SQ1SE & bones (1 bag) & 803 & & 05.01 .2016 \\
\hline $1022 / 2016$ & SQ1SE & bones (1 bag) & 803 & & 06.01 .2016 \\
\hline
\end{tabular}




\begin{tabular}{|c|c|c|c|c|}
\hline $1018 / 2016$ & SQ1SE & bones (1 bag) & 804 & 05.01 .2016 \\
\hline $1026 / 2016$ & SQ1SE & bones (1 bag) & 804 & 06.01 .2016 \\
\hline $1038 / 2016$ & SQ1SE & bones (1 bag) & 806 & 06.01 .2016 \\
\hline $1036 / 2016$ & SQ1SE & bones (1 bag) & 807 & 06.01 .2016 \\
\hline $1043 / 2016$ & SQ1SE & bones (1 bag) & 809 & 06.01 .2016 \\
\hline $1046 / 2016$ & SQ1SE & bones (1 bag) & 809 & 07.01 .2016 \\
\hline $1050 / 2016$ & SQ1SE & bones; teeth (1 bag, human, jawbone) & 809 & 07.01 .2016 \\
\hline $1055 / 2016$ & SQ1SE, 5m W-E/ 9m S-N & bones (1 bag, some human, skull and vertebrae) & 809 & 07.01 .2016 \\
\hline $1060 / 2016$ & SQ1SE & bones (1 bag) & 810 & 09.01 .2016 \\
\hline $1067 / 2016$ & SQ1SE & bones (1 bag) & 811 & 09.01.2016 \\
\hline $1075 / 2016$ & SQ1SE & bones (1 bag) & 812 & 09.01 .2016 \\
\hline $1086 / 2016$ & SQ1SE & bones ( 1 bag, partly burned) & 812 & 10.01.2016 \\
\hline $1080 / 2016$ & SQ1SE & bones (1 bag) & 813 & 09.01 .2016 \\
\hline $1089 / 2016$ & SQ1SE & bones; teeth (1 bag human, vertebrae, rib, finger) & 814 & 10.01.2016 \\
\hline $1096 / 2016$ & SQ1SE & bones (1 bag) & 815 & 10.01 .2016 \\
\hline $1103 / 2016$ & SQ1SE & bones (1 bag) & 816 & 11.01 .2016 \\
\hline $1206 / 2016$ & SQ1SE & bones ( 3 pieces) & 817 & 17.01 .2016 \\
\hline $1225 / 2016$ & SQ1SE & bones (1 bag) & 817 & 17.01.2016 \\
\hline $1132 / 2016$ & SQ1SE & bones (1 bag) & 818 & 12.01.2016 \\
\hline $1107 / 2016$ & $\begin{array}{l}\text { SQ1S and SQ1E, from surface cleaning of } \\
\text { SQ1S and SQ1E, near NW-corner of } \\
\text { SQ1SE }\end{array}$ & bones (1 bag) & 819 & 11.01.2016 \\
\hline $1129 / 2016$ & $\begin{array}{l}\text { SQ1S, from surface cleaning at SQ1S, } \\
\text { along E profile }\end{array}$ & bones (1 bag) & 819 & 12.01.2016 \\
\hline $1312 / 2016$ & SQ1S, from surface cleaning of SQ1S & bone (1 big piece) & 819 & 20.01 .2016 \\
\hline $1109 / 2016$ & SQ1SE & bones (1 bag) & 820 & 11.01 .2016 \\
\hline $1111 / 2016$ & SQ1SE & bones (1 bag) & 821 & 11.01 .2016 \\
\hline $1116 / 2016$ & SQ1SE & bones (1 bag) & 822 & 12.01.2016 \\
\hline
\end{tabular}




\begin{tabular}{|c|c|c|c|c|}
\hline $1119 / 2016$ & SQ1SE & bones (1 bag) & 823 & 12.01.2016 \\
\hline $1122 / 2016$ & SQ1SE & bones ( 3 pieces) & 824 & 12.01.2016 \\
\hline $1125 / 2016$ & SQ1SE & bones (1 bag) & 825 & 12.01 .2016 \\
\hline $1159 / 2016$ & SQ1SE & bones (1 bag) & 825 & 13.01.2016 \\
\hline $1134 / 2016$ & SQ1SE & bones (1 bag) & 826 & 12.01.2016 \\
\hline $1144 / 2016$ & SQ1SE & bones (1 bag) & 828 & 13.01 .2016 \\
\hline $1154 / 2016$ & SQ1SE & bones (1 bag, human?) & 830 & 13.01.2016 \\
\hline $1155 / 2016$ & SQ1SE & bones (1 bag) & 830 & 13.01.2016 \\
\hline $1167 / 2016$ & SQ1SE & bones (1 bag) & 831 & 14.01 .2016 \\
\hline $1169 / 2016$ & SQ1SE & bone/horn? (1 bag) & 831 & 14.01 .2016 \\
\hline $1178 / 2016$ & SQ1SE & bone (1 piece, human - child) & 831 & 14.01.2016 \\
\hline $1171 / 2016$ & SQ1SE & bones (1 bag) & 832 & 14.01 .2016 \\
\hline $1180 / 2016$ & SQ1SE & bones (1 bag) & 833 & 14.01 .2016 \\
\hline $1187 / 2016$ & SQ1SE & bones (1 bag) & 834 & 16.01 .2016 \\
\hline $1188 / 2016$ & SQ1SE & bones (1 bag, human) & 834 & 16.01 .2016 \\
\hline $1195 / 2016$ & SQ1SE & bones (1 bag) & 835 & 16.01 .2016 \\
\hline $1209 / 2016$ & SQ1SE & bones (1 bag) & 836 & 17.01.2016 \\
\hline $1210 / 2016$ & SQ1SE & bones (1 bag, human) & 836 & 17.01.2016 \\
\hline $1217 / 2016$ & SQ1SE & bones (1 bag) & 837 & 17.01 .2016 \\
\hline $1234 / 2016$ & SQ1SE & bone (1 bag, human) & 837 & 18.01 .2016 \\
\hline $1221 / 2016$ & SQ1SE & bones (1 bag) & 838 & 17.01.2016 \\
\hline $1245 / 2016$ & SQ1SE & bones (1 bag) & 840 & 18.01 .2016 \\
\hline $1250 / 2016$ & SQ1SE & bones (1 bag) & 841 & 18.01 .2016 \\
\hline $1242 / 2016$ & SQ1SE & bones (1 bag) & 842 & 18.01 .2016 \\
\hline $1261 / 2016$ & SQ1SE & bones (1 bag, human?) & 843 & 19.01.2016 \\
\hline $1262 / 2016$ & SQ1SE & bones (1 bag, human?) & 843 & 19.01 .2016 \\
\hline
\end{tabular}




\begin{tabular}{|c|c|c|c|c|}
\hline $1267 / 2016$ & SQ1SE & bones (1 bag) & 844 & 19.01.2016 \\
\hline $1283 / 2016$ & SQ1SE & bones (1 bag) & 845 & 19.01.2016 \\
\hline $1303 / 2016$ & SQ1SE & bones ( 2 pieces) & 845 & 20.01 .2016 \\
\hline $1286 / 2016$ & SQ1SE & bones (1 bag, human) & 846 & 19.01.2016 \\
\hline $1287 / 2016$ & SQ1SE & bones ( 2 pieces) & 846 & 19.01.2016 \\
\hline $1292 / 2016$ & SQ1SE & bones (1 bag) & 846 & 20.01 .2016 \\
\hline $1313 / 2016$ & SQ1SE & bones ( 3 pieces, human) & 846 & 20.01 .2016 \\
\hline $1297 / 2016$ & SQ1SE & bones (1 bag) & 847 & 20.01 .2016 \\
\hline $1305 / 2016$ & SQ1SE & bones (1 bag) & 848 & 20.01 .2016 \\
\hline $1321 / 2016$ & SQ1SE & bones (1 bag) & 849 & 21.01 .2016 \\
\hline $1330 / 2016$ & SQ1SE & bones (3 pieces, human) & 849 & 21.01 .2016 \\
\hline $1319 / 2016$ & SQ1SE & bones (1 bag) & 850 & 21.01 .2016 \\
\hline $1333 / 2016$ & SQ1SE & bones (1 bag) & 851 & 21.01 .2016 \\
\hline $1343 / 2016$ & SQ1S & bones (1 bag) & 852 & 23.01 .2016 \\
\hline $1356 / 2016$ & SQ1S & bones (1 bag) & 852 & 24.01 .2016 \\
\hline $1338 / 2016$ & SQ1SE & bones (1 bag) & 853 & 23.01 .2016 \\
\hline $1351 / 2016$ & SQ1SE & bones (1 bag) & 854 & 23.01.2016 \\
\hline $1360 / 2016$ & SQ1SE & bones (1 bag) & 854 & 24.01 .2016 \\
\hline $1366 / 2016$ & SQ1SE & bones (1 bag) & 855 & 24.01 .2016 \\
\hline $1382 / 2016$ & SQ1SE & bones (1 bag) & 856 & 25.01 .2016 \\
\hline $1385 / 2016$ & SQ1S & bones (1 bag) & 857 & 25.01 .2016 \\
\hline $1388 / 2016$ & SQ1SE & bones (1 bag) & 858 & 25.01 .2016 \\
\hline $1393 / 2016$ & SQ1S/SQ1SE & bones (1 bag) & 859 & 25.01 .2016 \\
\hline $1408 / 2016$ & SQ1SE & bones (1 bag) & 860 & 26.01 .2016 \\
\hline $1421 / 2016$ & SQ1S/SQ1SE & bones (2 pieces) & 862 & 26.01 .2016 \\
\hline $1425 / 2016$ & SQ1S/SQ1SE & bones (1 bag) & 862 & 27.01 .2016 \\
\hline
\end{tabular}




\begin{tabular}{|c|c|c|c|c|}
\hline $1435 / 2016$ & SQ1S/SQ1SE & bones ( 2 pieces) & 862 & 27.01.2016 \\
\hline $1429 / 2016$ & SQ1S & bones (1 bag) & 863 & 27.01.2016 \\
\hline $1444 / 2016$ & SQ1S & bones (1 bag) & 864 & 28.01.2016 \\
\hline $1447 / 2016$ & SQ1S & bone (1 piece)/skin & 864 & 28.01 .2016 \\
\hline $1442 / 2016$ & SQ1S/SQ1SE & bones (1 bag) & 865 & 28.01 .2016 \\
\hline $1451 / 2016$ & SQ1S & bones (1 bag) & 866 & 28.01 .2016 \\
\hline $1470 / 2016$ & SQ1S & bones (1 bag) & 867 & 30.01 .2016 \\
\hline $1480 / 2016$ & SQ1S & bone with skin (1 piece, human) & 867 & 30.01 .2016 \\
\hline $1465 / 2016$ & SQ1S & bones (1 bag) & 868 & 30.01 .2016 \\
\hline $1468 / 2016$ & SQ1S & bones (1 bag) & 869 & 30.01 .2016 \\
\hline $1476 / 2016$ & SQ1S & bones (1 bag) & 870 & 30.01 .2016 \\
\hline $1478 / 2016$ & SQ1S & bones (1 bag) & 871 & 30.01 .2016 \\
\hline $1482 / 2016$ & SQ1NW & bones (1 bag) & 872 & 30.01 .2016 \\
\hline $1502 / 2016$ & SQ1NW & bones (1 bag) & 872 & 31.01 .2016 \\
\hline $1492 / 2016$ & SQ1S & bones (1 bag) & 873 & 30.01 .2016 \\
\hline $1496 / 2016$ & SQ1S & bones (1 bag) & 873 & 31.01 .2016 \\
\hline $1483 / 2016$ & SQ1S & bones ( 1 bag, arm/hand with skin, human) & 874 & 30.01 .2016 \\
\hline $1485 / 2016$ & SQ1S & bone (1 piece) & 874 & 30.01 .2016 \\
\hline $1511 / 2016$ & SQ1S & bones (1 bag) & 877 & 31.01 .2016 \\
\hline $1519 / 2016$ & SQ1S & bones (1 bag) & 878 & 31.01 .2016 \\
\hline $1524 / 2016$ & SQ1NW & bones (1 bag) & 879 & 01.02 .2016 \\
\hline $1528 / 2016$ & SQ1S & bones (1 bag) & 880 & 01.02 .2016 \\
\hline $1536 / 2016$ & SQ1SE & bones (1 bag) & 881 & 01.02 .2016 \\
\hline $1546 / 2016$ & SQ1S/SQ1SE & bones (1 bag) & 884 & 02.02 .2016 \\
\hline $1554 / 2016$ & SQ1S/SQ1SE & bones (1 bag) & 884 & 03.02 .2016 \\
\hline $1543 / 2016$ & SQ1NW & bones (1 bag) & 885 & 02.02 .2016 \\
\hline
\end{tabular}




\begin{tabular}{|c|c|c|c|c|c|}
\hline $1549 / 2016$ & SQ1NW & bones (1 bag) & 886 & & 03.02 .2016 \\
\hline $1560 / 2016$ & SQ1NW & bones (1 bag) & 887 & & 03.02 .2016 \\
\hline $1569 / 2016$ & SQ1NW & bones (1 bag) & 891 & & 04.02 .2016 \\
\hline $1575 / 2016$ & SQ1NW & bones (1 bag) & 894 & & 04.02 .2016 \\
\hline $1580 / 2016$ & SQ1NW & bones (1 bag) & 895 & & 04.02 .2016 \\
\hline $1051 / 2016$ & SQ1SE & botanical material (2 pieces, seeds) & 809 & & 07.01 .2016 \\
\hline $1232 / 2016$ & SQ1SE & botanical material (1 bag, sticks) & 827 & & 18.01.2016 \\
\hline $1224 / 2016$ & SQ1SE & botanical material (1 piece) & 838 & & 17.01.2016 \\
\hline $1326 / 2016$ & SQ1SE & botanical material (1 bag) & 849 & & 21.01 .2016 \\
\hline $1363 / 2016$ & SQ1SE & botanical material (1 bag) & 854 & & 24.01.2016 \\
\hline $1369 / 2016$ & SQ1SE & botanical material (1 bag) & 855 & & 24.01.2016 \\
\hline $1414 / 2016$ & SQ1S & botanical material (1 bag) & 861 & & 26.01.2016 \\
\hline $1469 / 2016$ & SQ1S & botanical material (4 pieces) & 869 & & 30.01 .2016 \\
\hline $1512 / 2016$ & SQ1S & botanical material (1 small bag, seeds) & 877 & & 31.01 .2016 \\
\hline $1299 / 2016$ & SQ1SE & bracelet? (1 fragment, broken in half, unspecified stone) & 846 & SAV1W 0093 & 20.01.2016 \\
\hline $1484 / 2016$ & SQ1S & bracelet (1 fragment, unspecified stone) & 873 & SAV1W 1697 & 30.01 .2016 \\
\hline $1137 / 2016$ & SQ1SE & chalk (1 piece) & 826 & & 12.01.2016 \\
\hline $1010 / 2016$ & SQ1SE & charcoal (1 bag) & 801 & & 05.01 .2016 \\
\hline $1031 / 2016$ & SQ1SE & charcoal (1 piece) & 803 & & 06.01 .2016 \\
\hline $1027 / 2016$ & SQ1SE & charcoal (1 bag) & 804 & & 06.01 .2016 \\
\hline $1039 / 2016$ & SQ1SE & charcoal (1 piece) & 806 & & 06.01 .2016 \\
\hline $1034 / 2016$ & SQ1SE & charcoal (1 bag) & 807 & & 06.01 .2016 \\
\hline $1042 / 2016$ & SQ1SE & charcoal (4 pieces) & 808 & & 06.01 .2016 \\
\hline $1044 / 2016$ & SQ1SE & charcoal (1 bag) & 809 & & 06.01 .2016 \\
\hline $1047 / 2016$ & SQ1SE & charcoal (1 bag) & 809 & & 07.01 .2016 \\
\hline $1061 / 2016$ & SQ1SE & charcoal (1 bag) & 810 & & 09.01 .2016 \\
\hline
\end{tabular}




\begin{tabular}{|c|c|c|c|c|}
\hline $1068 / 2016$ & SQ1SE & charcoal (1 bag) & 811 & 09.01 .2016 \\
\hline $1077 / 2016$ & SQ1SE & charcoal (1 bag) & 812 & 09.01 .2016 \\
\hline $1087 / 2016$ & SQ1SE & charcoal (1 bag) & 812 & 10.01 .2016 \\
\hline $1081 / 2016$ & SQ1SE & charcoal (3 pieces) & 813 & 09.01 .2016 \\
\hline $1092 / 2016$ & SQ1SE & charcoal (1 bag) & 814 & 10.01 .2016 \\
\hline $1097 / 2016$ & SQ1SE & charcoal (1 bag) & 815 & 10.01 .2016 \\
\hline $1104 / 2016$ & SQ1SE & charcoal (1 bag) & 816 & 11.01 .2016 \\
\hline $1227 / 2016$ & SQ1SE & charcoal (1 piece) & 817 & 17.01 .2016 \\
\hline $1142 / 2016$ & SQ1SE & charcoal (1 bag) & 818 & 12.01 .2016 \\
\hline $1128 / 2016$ & $\begin{array}{l}\text { SQ1S, from surface cleaning at SQ1S, } \\
\text { along E profile }\end{array}$ & charcoal (1 bag) & 819 & 12.01 .2016 \\
\hline $1113 / 2016$ & SQ1SE & charcoal (1 bag) & 820 & 11.01 .2016 \\
\hline $1112 / 2016$ & SQ1SE & charcoal (5 pieces) & 821 & 11.01 .2016 \\
\hline $1117 / 2016$ & SQ1SE & charcoal (2 pieces) & 822 & 12.01 .2016 \\
\hline $1120 / 2016$ & SQ1SE & charcoal (5 pieces) & 823 & 12.01 .2016 \\
\hline $1123 / 2016$ & SQ1SE & charcoal (1 bag) & 824 & 12.01 .2016 \\
\hline $1126 / 2016$ & SQ1SE & charcoal (1 bag) & 825 & 12.01 .2016 \\
\hline $1160 / 2016$ & SQ1SE & charcoal (1 bag) & 825 & 13.01 .2016 \\
\hline $1135 / 2016$ & SQ1SE & charcoal (1 bag) & 826 & 12.01 .2016 \\
\hline $1231 / 2016$ & SQ1SE & charcoal (2 pieces) & 827 & 18.01 .2016 \\
\hline $1145 / 2016$ & SQ1SE & charcoal (1 bag) & 828 & 13.01 .2016 \\
\hline $1151 / 2016$ & SQ1SE & charcoal (1 bag) & 829 & 13.01 .2016 \\
\hline $1161 / 2016$ & SQ1SE & charcoal (1 bag) & 830 & 13.01 .2016 \\
\hline $1168 / 2016$ & SQ1SE & charcoal (1 bag) & 831 & 14.01 .2016 \\
\hline $1172 / 2016$ & SQ1SE & charcoal (1 bag) & 832 & 14.01 .2016 \\
\hline $1181 / 2016$ & SQ1SE & charcoal (1 bag) & 833 & 14.01 .2016 \\
\hline $1189 / 2016$ & SQ1SE & charcoal (1 bag) & 834 & 16.01 .2016 \\
\hline
\end{tabular}




\begin{tabular}{|c|c|c|c|c|}
\hline $1196 / 2016$ & SQ1SE & charcoal (1 bag) & 835 & 16.01 .2016 \\
\hline $1213 / 2016$ & SQ1SE & charcoal (1 bag) & 836 & 17.01.2016 \\
\hline $1219 / 2016$ & SQ1SE & charcoal (1 bag) & 837 & 17.01.2016 \\
\hline $1236 / 2016$ & SQ1SE & charcoal (1 bag) & 837 & 18.01.2016 \\
\hline $1222 / 2016$ & SQ1SE & charcoal (1 bag) & 838 & 17.01.2016 \\
\hline $1281 / 2016$ & SQ1SE & charcoal (1 bag) & 839 & 19.01.2016 \\
\hline $1246 / 2016$ & SQ1SE & charcoal (1 bag) & 840 & 18.01.2016 \\
\hline $1251 / 2016$ & SQ1SE & charcoal (1 bag) & 841 & 18.01.2016 \\
\hline $1243 / 2016$ & SQ1SE & charcoal (1 bag) & 842 & 18.01.2016 \\
\hline $1264 / 2016$ & SQ1SE & charcoal (1 bag) & 843 & 19.01.2016 \\
\hline $1268 / 2016$ & SQ1SE & charcoal (1 bag) & 844 & 19.01.2016 \\
\hline $1270 / 2016$ & SQ1SE & charcoal (1 bag) & 845 & 19.01.2016 \\
\hline $1306 / 2016$ & SQ1SE & charcoal (1 piece) & 845 & 20.01.2016 \\
\hline $1294 / 2016$ & SQ1SE & charcoal (1 bag) & 846 & 20.01 .2016 \\
\hline $1298 / 2016$ & SQ1SE & charcoal (1 bag) & 847 & 20.01 .2016 \\
\hline $1300 / 2016$ & SQ1SE & charcoal (1 bag) & 847 & 20.01.2016 \\
\hline $1309 / 2016$ & SQ1SE & charcoal (1 bag) & 848 & 20.01 .2016 \\
\hline $1325 / 2016$ & SQ1SE & charcoal (1 bag) & 849 & 21.01.2016 \\
\hline $1320 / 2016$ & SQ1SE & charcoal (1 bag) & 850 & 21.01.2016 \\
\hline $1334 / 2016$ & SQ1SE & charcoal (1 bag) & 851 & 21.01 .2016 \\
\hline $1344 / 2016$ & SQ1S & charcoal (1 bag) & 852 & 23.01 .2016 \\
\hline $1357 / 2016$ & SQ1S & charcoal (1 bag) & 852 & 24.01 .2016 \\
\hline $1339 / 2016$ & SQ1SE & charcoal (1 bag) & 853 & 23.01 .2016 \\
\hline $1352 / 2016$ & SQ1SE & charcoal (1 bag) & 854 & 23.01.2016 \\
\hline $1362 / 2016$ & SQ1SE & charcoal (1 bag) & 854 & 24.01 .2016 \\
\hline $1381 / 2016$ & SQ1SE & charcoal (1 bag) & 856 & 25.01 .2016 \\
\hline
\end{tabular}




\begin{tabular}{|c|c|c|c|c|}
\hline $1386 / 2016$ & SQ1S & charcoal (1 bag) & 857 & 25.01 .2016 \\
\hline $1389 / 2016$ & SQ1SE & charcoal (1 bag) & 858 & 25.01 .2016 \\
\hline $1395 / 2016$ & SQ1S/SQ1SE & charcoal (1 bag) & 859 & 25.01.2016 \\
\hline $1409 / 2016$ & SQ1SE & charcoal (1 bag) & 860 & 26.01 .2016 \\
\hline $1416 / 2016$ & SQ1S & charcoal (1 bag) & 861 & 26.01 .2016 \\
\hline $1426 / 2016$ & SQ1S/SQ1SE & charcoal (1 bag) & 862 & 27.01 .2016 \\
\hline $1430 / 2016$ & SQ1S & charcoal (1 bag) & 863 & 27.01 .2016 \\
\hline $1445 / 2016$ & SQ1S & charcoal (1 bag) & 864 & 28.01 .2016 \\
\hline $1459 / 2016$ & SQ1S/SQ1SE & charcoal (1 bag) & 865 & 28.01.2016 \\
\hline $1452 / 2016$ & SQ1S & charcoal (1 bag) & 866 & 28.01 .2016 \\
\hline $1471 / 2016$ & SQ1S & charcoal (1 bag) & 867 & 30.01 .2016 \\
\hline $1464 / 2016$ & SQ1S & charcoal (1 bag) & 868 & 30.01 .2016 \\
\hline $1467 / 2016$ & SQ1S & charcoal (1 bag) & 869 & 30.01 .2016 \\
\hline $1475 / 2016$ & SQ1S & charcoal (1 bag) & 870 & 30.01 .2016 \\
\hline $1479 / 2016$ & SQ1S & charcoal (1 bag) & 871 & 30.01 .2016 \\
\hline $1490 / 2016$ & SQ1S & charcoal (1 bag) & 873 & 30.01 .2016 \\
\hline $1495 / 2016$ & SQ1S & charcoal (1 bag) & 873 & 31.01 .2016 \\
\hline $1510 / 2016$ & SQ1S & charcoal (1 bag) & 877 & 31.01 .2016 \\
\hline $1520 / 2016$ & SQ1S & charcoal (1 bag) & 878 & 31.01 .2016 \\
\hline $1526 / 2016$ & SQ1NW & charcoal (1 bag) & 879 & 01.02 .2016 \\
\hline $1530 / 2016$ & SQ1S & charcoal (1 bag) & 880 & 01.02 .2016 \\
\hline $1537 / 2016$ & SQ1SE & charcoal (1 bag) & 881 & 01.02 .2016 \\
\hline $1541 / 2016$ & SQ1S/SQ1SE & charcoal (1 bag); leather (1 piece) & 884 & 02.02 .2016 \\
\hline $1555 / 2016$ & SQ1S/SQ1SE & charcoal (1 bag) & 884 & 03.02 .2016 \\
\hline $1544 / 2016$ & SQ1NW & charcoal (1 bag) & 885 & 02.02 .2016 \\
\hline $1552 / 2016$ & SQ1NW & charcoal (1 bag) & 886 & 03.02 .2016 \\
\hline
\end{tabular}




\begin{tabular}{|c|c|c|c|c|c|}
\hline $1563 / 2016$ & SQ1NW & charcoal (1 bag) & 887 & & 03.02 .2016 \\
\hline $1570 / 2016$ & SQ1NW & charcoal (1 bag) & 891 & & 04.02 .2016 \\
\hline $1576 / 2016$ & SQ1NW & charcoal (1 bag) & 894 & & 04.02 .2016 \\
\hline $1583 / 2016$ & SQ1NW & charcoal (1 bag) & 895 & & 04.02 .2016 \\
\hline $1577 / 2016$ & SQ1S, sieved material around Feature 112 & $\begin{array}{l}\text { charcoal ( } 1 \mathrm{bag}) \text {; wood ( } 1 \mathrm{bag}) \text {; shell (1 piece); stone flakes } \\
\text { ( } 7 \text { pieces, reference); bones ( } \mathrm{bag})\end{array}$ & 896 & & 04.02 .2016 \\
\hline $1176 / 2016$ & SQ1SE & copper (1 small fragment, from crucible?) & 831 & SAV1W 1564 & 14.01.2016 \\
\hline $1052 / 2016$ & SQ1SE & $\begin{array}{l}\text { crucible? ( } 1 \text { fragment, bowl-like shape, mud); metal artefact } \\
\text { (1 fragment, copper) }\end{array}$ & 809 & $\begin{array}{l}\text { SAV1W } 0379 \text { (crucible), SAV1W } 0380 \text { (metal } \\
\text { artefact) }\end{array}$ & 07.01 .2016 \\
\hline $1062 / 2016$ & SQ1SE & crucible? (1 fragment, copper) & 810 & SAV1W 0445 & 09.01 .2016 \\
\hline $1202 / 2016$ & SQ1SE & crucible (1 fragment, clay with traces of copper) & 834 & SAV1W 1571 & 16.01 .2016 \\
\hline $1065 / 2016$ & SQ1SE & doum nuts (2 pieces, burned) & 810 & & 09.01 .2016 \\
\hline $1078 / 2016$ & SQ1SE & doum nuts (2 pieces) & 812 & & 09.01 .2016 \\
\hline $1091 / 2016$ & SQ1SE & doum nut (1 piece) & 812 & & 10.01 .2016 \\
\hline $1100 / 2016$ & SQ1SE & doum nuts (1 bag) & 815 & & 10.01 .2016 \\
\hline $1207 / 2016$ & SQ1SE & doum nut (1 piece), date (1 piece) & 817 & & 17.01.2016 \\
\hline $1165 / 2016$ & SQ1SE & doum nut (1 piece), date (1 piece) & 825 & & 14.01.2016 \\
\hline $1139 / 2016$ & SQ1SE & doum nut (1 piece) & 826 & & 12.01 .2016 \\
\hline $1146 / 2016$ & SQ1SE & doum nuts (2 pieces) & 828 & & 13.01 .2016 \\
\hline $1152 / 2016$ & SQ1SE & doum nut (1 piece) & 829 & & 13.01.2016 \\
\hline $1162 / 2016$ & SQ1SE & doum nuts (2 pieces) & 830 & & 13.01.2016 \\
\hline $1174 / 2016$ & SQ1SE & doum nuts (1 bag) & 831 & & 14.01 .2016 \\
\hline $1173 / 2016$ & SQ1SE & doum nuts (1 bag) & 832 & & 14.01.2016 \\
\hline $1184 / 2016$ & SQ1SE & doum nuts (3 pieces) & 833 & & 14.01 .2016 \\
\hline $1192 / 2016$ & SQ1SE & doum nuts (1 bag) & 834 & & 16.01 .2016 \\
\hline $1212 / 2016$ & SQ1SE & doum nut (1 piece) & 836 & & 17.01 .2016 \\
\hline $1218 / 2016$ & SQ1SE & doum nuts (1/3 small bag) & 837 & & 17.01.2016 \\
\hline $1240 / 2016$ & SQ1SE & doum nuts (1/2 small bag) & 837 & & 18.01 .2016 \\
\hline
\end{tabular}




\begin{tabular}{|c|c|c|c|c|c|}
\hline $1248 / 2016$ & SQ1SE & doum nuts (3 pieces) & 840 & & 18.01.2016 \\
\hline $1308 / 2016$ & SQ1SE & doum nut (1 piece) & 845 & & 20.01 .2016 \\
\hline $1346 / 2016$ & SQ1S & doum nuts (1 bag) & 852 & & 23.01 .2016 \\
\hline $1358 / 2016$ & SQ1S & doum nuts (3 pieces) & 852 & & 24.01 .2016 \\
\hline $1349 / 2016$ & SQ1SE & doum nuts (1 small bag) & 853 & & 23.01 .2016 \\
\hline $1353 / 2016$ & SQ1SE & doum nuts (1 bag) & 854 & & 23.01 .2016 \\
\hline $1383 / 2016$ & SQ1SE & doum nuts (2 pieces) & 856 & & 25.01 .2016 \\
\hline $1397 / 2016$ & SQ1S & doum nuts (3 pieces) & 857 & & 25.01 .2016 \\
\hline $1390 / 2016$ & SQ1SE & doum nuts (1 bag) & 858 & & 25.01 .2016 \\
\hline $1396 / 2016$ & SQ1S/SQ1SE & doum nuts (1 bag) & 859 & & 25.01 .2016 \\
\hline $1410 / 2016$ & SQ1SE & doum nuts (1 bag) & 860 & & 26.01 .2016 \\
\hline $1433 / 2016$ & SQ1S/SQ1SE & doum nuts (3 pieces) & 862 & & 27.01 .2016 \\
\hline $1431 / 2016$ & SQ1S & doum nuts (1 bag) & 863 & & 27.01 .2016 \\
\hline $1453 / 2016$ & SQ1S & doum nuts (1 bag) & 866 & & 28.01 .2016 \\
\hline $1473 / 2016$ & SQ1S & doum nuts (2 pieces) & 867 & & 30.01 .2016 \\
\hline $1491 / 2016$ & SQ1S & doum nuts (1 bag) & 873 & & 30.01 .2016 \\
\hline $1497 / 2016$ & SQ1S & doum nuts (1 small bag) & 873 & & 31.01 .2016 \\
\hline $1514 / 2016$ & SQ1S & doum nuts (4 pieces) & 877 & & 31.01 .2016 \\
\hline $1547 / 2016$ & SQ1S/SQ1SE & doum nuts (1 bag) & 884 & & 02.02 .2016 \\
\hline $1581 / 2016$ & SQ1NW & doum nuts ( 2 pieces) & 895 & & 04.02 .2016 \\
\hline $1049 / 2016$ & SQ1SE & dung (donkey/goat?) (1 bag) & 809 & & 07.01 .2016 \\
\hline $1072 / 2016$ & SQ1SE & dung (1 piece) & 811 & & 09.01 .2016 \\
\hline $1226 / 2016$ & SQ1SE & dung (1 bag) & 817 & & 17.01.2016 \\
\hline $1400 / 2016$ & SQ1S & faience vessel or dish (1 base fragment) & 861 & SAV1W 1655 & 26.01 .2016 \\
\hline $1020 / 2016$ & SQ1SE & figurine (1 fragment, rudimentary female figurine, clay) & 802 & SAV1W 1534 & 05.01 .2016 \\
\hline $1214 / 2016$ & SQ1SE & figurine (1 small female figurine, complete, mud) & 817 & SAV1W 1498 & 17.01.2016 \\
\hline
\end{tabular}




\begin{tabular}{|c|c|c|c|c|c|}
\hline $1364 / 2016$ & SQ1SE & figurine ( 1 fragment, rudimentary female figurine, clay) & 854 & SAV1W 1624 & 24.01.2016 \\
\hline $1378 / 2016$ & SQ1S & figurine? (1 roughly rectangular block, clay) & 857 & SAV1W 1648 & 25.01.2016 \\
\hline $1391 / 2016$ & SQ1S & figurine ( 1 complete female figurine, clay) & 857 & SAV1W 1647 & 25.01 .2016 \\
\hline $1064 / 2016$ & SQ1SE & glass vessel? (1 fragment) & 810 & SAV1W 0474 & 09.01 .2016 \\
\hline $520 / 2014$ & $\mathrm{SQ} 1,3 \mathrm{~m}$ to $\mathrm{E} / 5 \mathrm{~m}$ to $\mathrm{N}$ & glazed vessel (1 fragment, glazed ware, Ottoman) & 501 & SAV1W 0033 & 06.01 .2014 \\
\hline $1275 / 2016$ & SQ1SE & glazed vessel (1 fragment, glazed ware) & 843 & SAV1W 1590 & 19.01.2016 \\
\hline $1201 / 2016$ & SQ1SE & horn (1 piece) & 834 & & 16.01.2016 \\
\hline $1367 / 2016$ & SQ1S & horn (1 piece) & 852 & & 24.01.2016 \\
\hline $1515 / 2016$ & SQ1S & horn (1 piece) & 877 & & 31.01 .2016 \\
\hline $1550 / 2016$ & SQ1NW & horn (1 bag) & 886 & & 03.02 .2016 \\
\hline $1562 / 2016$ & SQ1NW & horn (1 piece) & 887 & & 03.02 .2016 \\
\hline $1164 / 2016$ & SQ1SE & leather (1 piece) & 828 & & 13.01.2016 \\
\hline $1182 / 2016$ & SQ1SE & leather (1 piece) & 833 & & 14.01.2016 \\
\hline $1558 / 2016$ & SQ1NW & leather (1 piece) & 886 & & 03.02 .2016 \\
\hline $1493 / 2016$ & SQ1S & metal - nail? (1 fragment) & 873 & SAV1W 1704 & 31.01 .2016 \\
\hline $1507 / 2016$ & SQ1S, sieved material & $\begin{array}{l}\text { metal (1 piece); charcoal (1 bag); bones (1 bag); doum nuts (1 } \\
\text { bag), wood ( } 1 \text { bag) }\end{array}$ & 876 & & 31.01 .2016 \\
\hline $1099 / 2016$ & $\begin{array}{l}\text { SQ1SE, found on surface, } 1 \mathrm{~m} \mathrm{E} \text { from } \\
\text { trench }\end{array}$ & mineral (1 piece, graphite, sample) & 800 & & 10.01.2016 \\
\hline $1203 / 2016$ & SQ1SE & $\begin{array}{l}\text { model boat/execration figure? ( } 2 \text { fragments, joining together, } \\
\text { clay) }\end{array}$ & 834 & SAV1W 1574 & 16.01.2016 \\
\hline $1153 / 2016$ & SQ1SE & $\begin{array}{l}\text { mud object/furniture?/installation (1 fragment, semi-circular in } \\
\text { shape, mud) }\end{array}$ & 830 & SAV1W 1560 & 13.01.2016 \\
\hline $1029 / 2016$ & SQ1SE & net weight (1 piece, complete, sandstone) & 803 & SAV1W 1541 & 06.01 .2016 \\
\hline $1056 / 2016$ & SQ1SE & net weight (1 fragment, pottery) & 809 & SAV1W 0054 & 07.01 .2016 \\
\hline $1437 / 2016$ & SQ1S & net weight (1 piece, intact, clay) & 863 & SAV1W 1666 & 27.01.2016 \\
\hline $1011 / 2016$ & SQ1SE & Nun bowl? (1 fragment, faience) & 801 & SAV1W 1535 & 05.01 .2016 \\
\hline $1094 / 2016$ & SQ1SE & Nun bowl (1 base rim fragment, faience) & 815 & SAV1W 1544 & 10.01 .2016 \\
\hline $1276 / 2016$ & SQ1SE & Nun bowl (1 fragment with rim, faience) & 843 & SAV1W 1591 & 19.01.2016 \\
\hline
\end{tabular}




\begin{tabular}{|c|c|c|c|c|c|}
\hline $1370 / 2016$ & SQ1SE & Nun bowl (1 base fragment, faience) & 855 & SAV1W 1626 & 24.01.2016 \\
\hline $1440 / 2016$ & SQ1S & Nun bowl (1 rim fragment, faience) & 864 & SAV1W 1682 & 28.01 .2016 \\
\hline $1455 / 2016$ & SQ1S/SQ1SE & Nun bowl (1 fragment of body sherd, faience) & 865 & SAV1W 1681 & 28.01 .2016 \\
\hline $1516 / 2016$ & SQ1S & ochre (1 piece, yellow) & 877 & & 31.01 .2016 \\
\hline $1587 / 2016$ & SQ1NW & ochre? (1 bag, samples) & 895 & & 04.02 .2016 \\
\hline $1150 / 2016$ & SQ1SE & organic material (1 bag) & 829 & & 13.01.2016 \\
\hline $1198 / 2016$ & SQ1SE & organic material (1 bag) & 835 & & 16.01.2016 \\
\hline $1282 / 2016$ & SQ1SE & organic material (1 bag) & 839 & & 19.01.2016 \\
\hline $1265 / 2016$ & SQ1SE & organic material (1 bag) & 843 & & 19.01.2016 \\
\hline $1277 / 2016$ & SQ1SE & organic material (1 bag) & 843 & & 19.01.2016 \\
\hline $1272 / 2016$ & SQ1SE & organic material (1 bag) & 845 & & 19.01.2016 \\
\hline $1293 / 2016$ & SQ1SE & organic material ( 2 pieces) & 846 & & 20.01.2016 \\
\hline $1328 / 2016$ & $\begin{array}{l}\text { SQ1SE, } \\
\text { from edge of disturbance pit }\end{array}$ & organic material (1 piece, unclear) & 851 & & 21.01.2016 \\
\hline $1342 / 2016$ & SQ1S & organic material (1 piece, unclear) & 852 & & 23.01.2016 \\
\hline $1372 / 2016$ & SQ1SE & organic material (1 piece, unclear) & 854 & & 24.01 .2016 \\
\hline $1446 / 2016$ & SQ1S & organic material (2 pieces) & 864 & & 28.01.2016 \\
\hline $1518 / 2016$ & SQ1S & organic material (1 piece, sample) & 877 & & 31.01 .2016 \\
\hline $1522 / 2016$ & SQ1S & organic material (1 bag)/soil sample & 877 & & 31.01 .2016 \\
\hline $1534 / 2016$ & SQ1NW & organic material (1 piece) & 879 & & 01.02 .2016 \\
\hline $1000 / 2016$ & SQ1SE, surface cleaning & pottery, among it weight (1 re-used $18^{\text {th }}$ Dynasty sherd, pottery) & 800 & SAV1W 1532 & 04.01 .2016 \\
\hline $1004 / 2016$ & $\begin{array}{l}\text { SQ1SE, surface cleaning, } \\
1 \mathrm{~m} \text { strip around trench except in } \mathrm{W}\end{array}$ & pottery & 800 & & 04.01 .2016 \\
\hline $1007 / 2016$ & SQ1SE & pottery & 801 & & 05.01 .2016 \\
\hline $1013 / 2016$ & SQ1SE & pottery & 802 & & 05.01 .2016 \\
\hline $1025 / 2016$ & SQ1SE & pottery & 802 & & 06.01 .2016 \\
\hline $1015 / 2016$ & SQ1SE & pottery & 803 & & 05.01 .2016 \\
\hline $1023 / 2016$ & SQ1SE & pottery & 803 & & 06.01 .2016 \\
\hline
\end{tabular}




\begin{tabular}{|c|c|c|c|c|c|}
\hline $1017 / 2016$ & SQ1SE & pottery & 804 & & 05.01 .2016 \\
\hline $1024 / 2016$ & SQ1SE & pottery & 804 & & 06.01 .2016 \\
\hline $1033 / 2016$ & $\begin{array}{l}\text { SQ1S, from surface cleaning, begin of } \\
\text { season, SQ1 }\end{array}$ & pottery & 805 & & 06.01 .2016 \\
\hline $1037 / 2016$ & SQ1SE & pottery & 806 & & 06.01 .2016 \\
\hline $1035 / 2016$ & SQ1SE & pottery & 807 & & 06.01 .2016 \\
\hline $1040 / 2016$ & SQ1SE & pottery & 808 & & 06.01 .2016 \\
\hline $1041 / 2016$ & SQ1SE & $\begin{array}{l}\text { pottery, among it figurine ( } 1 \text { fragment, animal/quadruped } \\
\text { figurine, pottery), lid (re-used } 18^{\text {th }} \text { Dynasty pot, pottery) }\end{array}$ & 809 & SAV1W 0016 (figurine), SAV1W 0019 (lid) & 06.01 .2016 \\
\hline $1045 / 2016$ & SQ1SE & pottery & 809 & & 07.01 .2016 \\
\hline $1057 / 2016$ & SQ1SE & $\begin{array}{l}\text { pottery, among it lid (1 re-used base fragment of a bowl, } \\
18^{\text {th }} \text { Dynasty, pottery) }\end{array}$ & 809 & SAV1W 0320 & 07.01 .2016 \\
\hline $1059 / 2016$ & SQ1SE & pottery & 810 & & 09.01 .2016 \\
\hline $1066 / 2016$ & SQ1SE & $\begin{array}{l}\text { pottery, among it lid (1 fragment, pottery), artefact (1 fragment, } \\
\text { pottery) }\end{array}$ & 811 & SAV1W 0142 (lid), SAV1W 1543 (artefact) & 09.01 .2016 \\
\hline $1074 / 2016$ & SQ1SE & pottery & 812 & & 09.01 .2016 \\
\hline $1085 / 2016$ & SQ1SE & $\begin{array}{l}\text { pottery, among it lid ( } 1 \text { re-used base of an } 18^{\text {th }} \text { Dynasty dish, } \\
\text { pottery) }\end{array}$ & 812 & SAV1W 1949 & 10.01 .2016 \\
\hline $1079 / 2016$ & SQ1SE & pottery & 813 & & 09.01 .2016 \\
\hline $1090 / 2016$ & SQ1SE & pottery & 814 & & 10.01 .2016 \\
\hline $1095 / 2016$ & SQ1SE & $\begin{array}{l}\text { pottery and stone: pottery, among it figurine ( } 1 \text { fragment, } \\
\text { quadruped figurine, pottery), lid (re-used sherd, pottery); } \\
\text { whetstone ( } 1 \text { fragment, sandstone) }\end{array}$ & 815 & $\begin{array}{l}\text { SAV1W } 1546 \text { (figurine), SAV1W } 1547 \text { (lid), } \\
\text { SAV1W } 1548 \text { (whetstone) }\end{array}$ & 10.01.2016 \\
\hline $1102 / 2016$ & SQ1SE & pottery & 816 & & 11.01 .2016 \\
\hline $1205 / 2016$ & SQ1SE & pottery ( 2 baskets) & 817 & & 17.01 .2016 \\
\hline $1131 / 2016$ & SQ1SE & pottery, among it lid (1 re-used $18^{\text {th }}$ Dynasty dish, pottery) & 818 & & 12.01 .2016 \\
\hline $1114 / 2016$ & SQ1SE, from surface cleaning at SU 818 & $\begin{array}{l}\text { pottery, among it scrapers ( } 2 \text { pieces, each re-used fragments of } \\
18^{\text {th }} \text { Dynasty vessels, pottery) }\end{array}$ & 818 & SAV1W 1550, SAV1W 1551 (scrapers) & 11.01.2016 \\
\hline $1127 / 2016$ & $\begin{array}{l}\text { SQ1S, from surface cleaning along E } \\
\text { profile }\end{array}$ & pottery & 819 & & 12.01 .2016 \\
\hline $1106 / 2016$ & $\begin{array}{l}\text { SQ1S and SQ1E, from surface cleaning of } \\
\text { SQ1S and SQ1E, near NW-corner of } \\
\text { SQ1SE }\end{array}$ & pottery & 819 & & 11.01 .2016 \\
\hline
\end{tabular}




\begin{tabular}{|c|c|c|c|c|c|}
\hline $1108 / 2016$ & SQ1SE & pottery & 820 & & 11.01.2016 \\
\hline $1110 / 2016$ & SQ1SE & $\begin{array}{l}\text { pottery, among it scraper (1 re-used fragment of an } 18^{\text {th }} \text { Dynasty } \\
\text { vessel, pottery) }\end{array}$ & 821 & SAV1W 1552 & 11.01.2016 \\
\hline $1115 / 2016$ & SQ1SE & pottery & 822 & & 12.01.2016 \\
\hline $1118 / 2016$ & SQ1SE & pottery & 823 & & 12.01.2016 \\
\hline $1121 / 2016$ & SQ1SE & pottery & 824 & & 12.01 .2016 \\
\hline $1124 / 2016$ & SQ1SE & pottery & 825 & & 12.01.2016 \\
\hline $1133 / 2016$ & SQ1SE & pottery, among it window grille? (1 fragment, pottery) & 826 & SAV1W 1561 & 12.01 .2016 \\
\hline $1230 / 2016$ & SQ1SE & pottery ( 1 basket) & 827 & & 18.01 .2016 \\
\hline $1143 / 2016$ & SQ1SE & $\begin{array}{l}\text { pottery, among it lids ( } 4 \text { pieces, } 1 \text { pottery, } 2 \text { clay, } 1 \text { re-used } \\
18^{\text {th }} \text { Dynasty pottery fragment) }\end{array}$ & 828 & $\begin{array}{l}\text { SAV1W 1557, SAV1W 1558, SAV1W 1559, } \\
\text { SAV1W } 1566 \text { (lids) }\end{array}$ & 13.01.2016 \\
\hline $1148 / 2016$ & SQ1SE & pottery (1 1/2 baskets) & 829 & & 13.01.2016 \\
\hline $1166 / 2016$ & SQ1SE & pottery, among it lid (1 fragment, clay) & 831 & SAV1W 1569 & 14.01.2016 \\
\hline $1170 / 2016$ & SQ1SE & $\begin{array}{l}\text { pottery, among it lid ( } 1 \text { re-used fragment of a mid- } 18^{\text {th }} \text { Dynasty } \\
\text { pottery vessel); faience vessel ( } 1 \text { fragment with rim) }\end{array}$ & 832 & $\begin{array}{l}\text { SAV1W } 1568 \text { (lid), SAV1W } 1570 \text { (faience } \\
\text { vessel) }\end{array}$ & 14.01.2016 \\
\hline $1179 / 2016$ & SQ1SE & pottery & 833 & & 14.01.2016 \\
\hline $1186 / 2016$ & SQ1SE & $\begin{array}{l}\text { pottery, among it lid ( } 1 \text { re-used Post-New Kingdom sherd, } \\
\text { pottery), net weights ( } 2 \text { pieces, pottery) }\end{array}$ & 834 & $\begin{array}{l}\text { SAV1W } 1556 \text { (lid), SAV1W 1575, SAV1W } \\
1576 \text { (net weights) }\end{array}$ & 16.01.2016 \\
\hline $1194 / 2016$ & SQ1SE & pottery, among it clay spout (1 fragment), lid (1 piece, pottery) & 835 & SAV1W 1578 (clay spout), SAV1W 1577 (lid) & 16.01.2016 \\
\hline $1208 / 2016$ & SQ1SE & pottery ( 5 baskets) & 836 & & 17.01.2016 \\
\hline $1215 / 2016$ & SQ1SE & pottery ( 7 baskets) & 837 & & 17.01 .2016 \\
\hline $1233 / 2016$ & SQ1SE & $\begin{array}{l}\text { pottery and stone: pottery ( } 5 \text { baskets), among them figurine } \\
\text { ( } 1 \text { fragment, rear of a quadruped, pottery), lids ( } 2 \text { re-used sherds, } \\
\text { each } 18^{\text {th }} \text { Dynasty, pottery); worked stone ( } 1 \text { fragment, } \\
\text { limestone) }\end{array}$ & 837 & $\begin{array}{l}\text { SAV1W } 1586 \text { (figurine), SAV1W } 1587, \\
\text { SAV1W } 1588 \text { (lids), SAV1W } 1589 \text { (worked } \\
\text { stone) }\end{array}$ & 18.01.2016 \\
\hline $1216 / 2016$ & SQ1SE & pottery (3 baskets) & 838 & & 17.01.2016 \\
\hline $1280 / 2016$ & SQ1SE & pottery ( $1 / 2$ basket), among it lid ( 1 fragment, clay) & 839 & SAV1W 0118 & 19.01.2016 \\
\hline $1290 / 2016$ & SQ1SE & $\begin{array}{l}\text { pottery ( } 1 \text { basket), among it lid ( } 1 \text { re-used } 18^{\text {th }} \text { Dynasty Nubian } \\
\text { cooking pot sherd, pottery) }\end{array}$ & 839 & SAV1W 1599 & 20.01.2016 \\
\hline $1244 / 2016$ & SQ1SE & $\begin{array}{l}\text { pottery and stone: pottery ( } 3 \text { baskets); worked stone/polishing } \\
\text { stone? (1 piece, sandstone) }\end{array}$ & 840 & SAV1W 1584 (worked stone/polishing stone?) & 18.01.2016 \\
\hline $1249 / 2016$ & SQ1SE & pottery ( 2 baskets) & 841 & & 18.01.2016 \\
\hline
\end{tabular}




\begin{tabular}{|c|c|c|c|c|c|}
\hline $1241 / 2016$ & SQ1SE & pottery ( 2 baskets) & 842 & & 18.01.2016 \\
\hline $1260 / 2016$ & SQ1SE & $\begin{array}{l}\text { pottery ( } 8 \text { baskets), among them lid ( } 1 \text { re-used } 18^{\text {th }} \text { Dynasty } \\
\text { sherd, pottery), figurine (1 fragment, unidentified quadruped, } \\
\text { pottery) }\end{array}$ & 843 & SAV1W 1611 (lid), SAV1W 1612 (figurine) & 19.01.2016 \\
\hline $1266 / 2016$ & SQ1SE & $\begin{array}{l}\text { pottery ( } 2 \text { baskets), among them lid ( } 1 \text { re-used } 18^{\text {th }} \text { Dynasty } \\
\text { sherd, pottery) }\end{array}$ & 844 & SAV1W 1596 & 19.01.2016 \\
\hline $1302 / 2016$ & SQ1SE & pottery (1 basket) & 845 & & 20.01.2016 \\
\hline $1269 / 2016$ & SQ1SE & pottery (2 baskets) & 845 & & 19.01 .2016 \\
\hline $1285 / 2016$ & SQ1SE & pottery (1 basket) & 846 & & 19.01.2016 \\
\hline $1291 / 2016$ & SQ1SE & $\begin{array}{l}\text { pottery ( } 4 \text { baskets), among them scraper ( } 1 \text { re-used } 18^{\text {th }} \text { Dynasty } \\
\text { sherd, pottery) }\end{array}$ & 846 & SAV1W 1600 & 20.01 .2016 \\
\hline $1296 / 2016$ & SQ1SE & $\begin{array}{l}\text { pottery and stone: pottery ( } 5 \text { baskets), among them lid ( } 1 \text { re-used } \\
\text { sherd, pottery), scrapers ( } 3 \text { pieces, } 2 \text { re-used Post-New Kingdom } \\
\text { sherds, } 118^{\text {th }} \text { Dynasty); polishing stone ( } 1 \text { piece, sandstone) }\end{array}$ & 847 & $\begin{array}{l}\text { SAV1W } 1603 \text { (lid), SAV1W 1604, SAV1W } \\
\text { 1605, SAV1W } 1606 \text { (scrapers), SAV1W } 1607 \\
\text { (polishing stone) }\end{array}$ & 20.01.2016 \\
\hline $1304 / 2016$ & SQ1SE & $\begin{array}{l}\text { pottery ( } 3 \text { baskets), among them lids ( } 2 \text { re-used } 18^{\text {th }} \text { Dynasty } \\
\text { sherds, pottery) }\end{array}$ & 848 & SAV1W 1601, SAV1W 1602 (lids) & 20.01.2016 \\
\hline $1311 / 2016$ & SQ1S & $\begin{array}{l}\text { pottery ( } 9 \text { baskets), among them furniture?/installation? } \\
\text { (1 fragment, clay) }\end{array}$ & 849 & SAV1W 1613 & 21.01.2016 \\
\hline $1318 / 2016$ & SQ1SE & $\begin{array}{l}\text { pottery, faience and stone: pottery ( } 6 \text { baskets), among them } \\
\text { token/gaming piece ( } 1 \text { re-used sherd, } 18^{\text {th }} \text { Dynasty, pottery), } \\
\text { window grille ( } 1 \text { fragment, Christian, pottery), stopper } \\
\text { ( } 1 \text { fragment, mud); Nun bowl ( } 1 \text { fragment with rim, faience); } \\
\text { whetstone ( } 1 \text { piece, sandstone) }\end{array}$ & 850 & $\begin{array}{l}\text { SAV1W } 1609 \text { (token/gaming piece), SAV1W } \\
1622 \text { (window grille), SAV1W } 1614 \text { (stopper), } \\
\text { SAV1W } 1621 \text { (Nun bowl), SAV1W } 1610 \\
\text { (whetstone) }\end{array}$ & 21.01.2016 \\
\hline $1329 / 2016$ & SQ1SE & pottery ( 1 basket), among it lid (1 fragment, Christian, pottery) & 851 & SAV1W 1625 & 21.01 .2016 \\
\hline $1341 / 2016$ & SQ1S & $\begin{array}{l}\text { pottery ( } 12 \text { baskets), among them lid ( } 1 \text { re-used } 18^{\text {th }} \text { Dynasty } \\
\text { sherd), net weight ( } 1 \text { fragment, pottery) }\end{array}$ & 852 & SAV1W 1629 (lid), SAV1W 1630 (net weight) & 23.01.2016 \\
\hline $1355 / 2016$ & SQ1S & $\begin{array}{l}\text { pottery, slag and stone: pottery ( } 6 \text { baskets), among them token/ } \\
\text { gaming piece ( } 1 \text { re-used sherd, pottery), scrapers ( } 2 \text { re-used } \\
18^{\text {th }} \text { Dynasty sherds, pottery), figurine? ( } 1 \text { fragment, pottery), } \\
\text { weight ( } 1 \text { re-used sherd, pottery); slag ( } 1 \text { rim sherd, interior melted } \\
\text { to slag); grindstone or hand mill ( } 1 \text { piece, sandstone), grindstone } \\
\text { (1 piece, sandstone), paving stone? ( } 1 \text { fragment, schist) }\end{array}$ & 852 & $\begin{array}{l}\text { SAV1W } 1637 \text { (token/gaming piece), SAV1W } \\
\text { 1638, SAV1W } 1639 \text { (scrapers), SAV1W 1641 } \\
\text { (figurine?), SAV1W } 1642 \text { (weight), SAV1W } \\
\text { 1643 (slag), SAV1W } 1644 \text { (grindstone or hand } \\
\text { mill), SAV1W } 1645 \text { (grindstone), SAV1W } 1646 \\
\text { (paving stone?) }\end{array}$ & 24.01.2016 \\
\hline $1337 / 2016$ & SQ1SE & pottery (6 baskets) & 853 & & 23.01 .2016 \\
\hline $1350 / 2016$ & SQ1SE & pottery (3 baskets) & 854 & & 23.01 .2016 \\
\hline $1359 / 2016$ & SQ1SE & $\begin{array}{l}\text { pottery ( } 8 \text { baskets), among them lid ( } 1 \text { re-used } 18^{\text {th }} \text { Dynasty } \\
\text { sherd, pottery), figurine ( } 1 \text { fragment, bull?, pottery) }\end{array}$ & 854 & SAV1W 1635 (lid), SAV1W 1636 (figurine) & 24.01.2016 \\
\hline $1365 / 2016$ & SQ1SE & pottery (4 baskets); Nun bowl? (1 fragment, faience) & 855 & SAV1W 1631 (Nun bowl) & 24.01 .2016 \\
\hline
\end{tabular}




\begin{tabular}{|c|c|c|c|c|c|}
\hline $1380 / 2016$ & SQ1SE & pottery (3 baskets) & 856 & & 25.01.2016 \\
\hline $1384 / 2016$ & SQ1S & $\begin{array}{l}\text { pottery ( } 6 \text { baskets), among them lid or polishing stone ( } 1 \text { re-used } \\
18^{\text {th }} \text { Dynasty base sherd, pottery); metal ( } 1 \text { piece) }\end{array}$ & 857 & SAV1W 1654 (lid or polishing stone) & 25.01.2016 \\
\hline $1387 / 2016$ & SQ1SE & $\begin{array}{l}\text { pottery ( } 6 \text { baskets), among them scrapers ( } 3 \text { pieces, each re-used } \\
\text { sherds, } 118^{\text {th }} \text { Dynasty, } 2 \text { Post-New Kingdom/Christian, pottery), } \\
\text { window grille? ( } 1 \text { fragment, pottery) }\end{array}$ & 858 & $\begin{array}{l}\text { SAV1W 1667, SAV1W 1668, SAV1W } 1669 \\
\text { (scrapers), SAV1W } 1670 \text { (window grille?) }\end{array}$ & 25.01.2016 \\
\hline $1392 / 2016$ & SQ1S/SQ1SE & pottery ( 6 baskets); soil sample (1 large sample, termite?) & 859 & & 25.01 .2016 \\
\hline $1401 / 2016$ & SQ1S/SQ1SE & Pottery; overfired ceramic & 859 & SAV1W 1653 & 25.01.2016 \\
\hline $1407 / 2016$ & SQ1SE & $\begin{array}{l}\text { pottery ( } 5 \text { baskets), among them figurine ( } 1 \text { fragment, body of } \\
\text { quadruped, pottery) }\end{array}$ & 860 & SAV1W 1657 & 26.01.2016 \\
\hline $1412 / 2016$ & SQ1S & pottery (11 baskets), among them net weight (1 piece, pottery) & 861 & SAV1W 1665 & 26.01.2016 \\
\hline $1420 / 2016$ & SQ1S/SQ1SE & pottery ( 2 baskets) & 862 & & 26.01.2016 \\
\hline $1424 / 2016$ & SQ1S/SQ1SE & $\begin{array}{l}\text { pottery ( } 10 \text { baskets), among them weights ( } 2 \text { pieces, each re-used } \\
\text { sherds, pottery), figurine ( } 1 \text { fragment with head and partly body } \\
\text { preserved, animal, pottery), lid or stopper? ( } 1 \text { fragment, clay) }\end{array}$ & 862 & $\begin{array}{l}\text { SAV1W 1678, SAV1W } 1679 \text { (weights), } \\
\text { SAV1W } 1680 \text { (figurine), SAV1W } 1684 \text { (lid or } \\
\text { stopper?) }\end{array}$ & 27.01 .2016 \\
\hline $1428 / 2016$ & SQ1S & $\begin{array}{l}\text { pottery ( } 7 \text { baskets), among them figurine ( } 1 \text { fragment, } \\
\text { quadruped?, pottery), stand for model vessels ( } 1 \text { fragmented } \\
\text { piece, clay) }\end{array}$ & 863 & SAV1W 1683 (figurine), SAV1W 1685 (stand) & 27.01.2016 \\
\hline $1443 / 2016$ & SQ1S & pottery ( 2 baskets) & 864 & & 28.01.2016 \\
\hline $1441 / 2016$ & SQ1S/SQ1SE & $\begin{array}{l}\text { pottery ( } 9 \text { baskets), among them token/gaming piece ( } 1 \text { re-used } \\
18^{\text {th }} \text { Dynasty? sherd, pottery), scrapers ( } 3 \text { pieces, each re-used } \\
\text { sherds, } 1 \text { Christian, } 1 \text { New Kingdom, } 118^{\text {th }} \text { Dynasty, pottery), } \\
\text { weight? ( } 1 \text { re-used } 18^{\text {th }} \text { Dynasty pottery sherd), lid (1 piece, clay), } \\
\text { figurine ( } 1 \text { fragment, animal?, pottery) }\end{array}$ & 865 & $\begin{array}{l}\text { SAV1W } 1671 \text { (token/gaming piece), SAV1W } \\
\text { 1672, SAV1W 1740SAV1W 1673, SAV1W } \\
\text { 1674 (scrapers), SAV1W } 1675 \text { (weight), } \\
\text { SAV1W 1676 (lid), SAV1W } 1677 \text { (figurine) }\end{array}$ & 28.01 .2016 \\
\hline $1449 / 2016$ & SQ1S & pottery (5 baskets) & 866 & & 28.01 .2016 \\
\hline $1461 / 2016$ & SQ1S & pottery ( 2 baskets) & 867 & & 30.01 .2016 \\
\hline $1462 / 2016$ & SQ1S & pottery (1 basket) & 868 & & 30.01 .2016 \\
\hline $1463 / 2016$ & SQ1S & pottery (1 basket) & 869 & & 30.01 .2016 \\
\hline $1474 / 2016$ & SQ1S & pottery (1 basket) & 870 & & 30.01 .2016 \\
\hline $1477 / 2016$ & SQ1S & pottery (1 basket) & 871 & & 30.01 .2016 \\
\hline $1481 / 2016$ & SQ1NW & pottery (1 basket) & 872 & & 30.01 .2016 \\
\hline $1503 / 2016$ & SQ1NW & pottery ( 2 baskets) & 872 & & 31.01 .2016 \\
\hline
\end{tabular}




\begin{tabular}{|c|c|c|c|c|c|}
\hline $1489 / 2016$ & SQ1S & $\begin{array}{l}\text { pottery ( } 1 \text { basket), among them lid (1 re-used New Kingdom } \\
\text { base sherd, pottery) }\end{array}$ & 873 & SAV1W 1698 & 30.01 .2016 \\
\hline $1494 / 2016$ & SQ1S & pottery (1 basket) & 873 & & 31.01 .2016 \\
\hline $1487 / 2016$ & SQ1S & pottery (1/2 medium bag) & 874 & & 30.01 .2016 \\
\hline $1501 / 2016$ & SQ1S & pottery (1/2 basket) & 875 & & 31.01 .2016 \\
\hline $1506 / 2016$ & SQ1S & pottery (1 medium bag) & 876 & & 31.01 .2016 \\
\hline $1509 / 2016$ & SQ1S & pottery (1 basket) & 877 & & 31.01 .2016 \\
\hline $1517 / 2016$ & SQ1S & pottery (1/2 basket) & 878 & & 31.01 .2016 \\
\hline $1523 / 2016$ & SQ1NW & pottery (3 baskets) & 879 & & 01.02 .2016 \\
\hline $1527 / 2016$ & SQ1S & $\begin{array}{l}\text { pottery ( } 3 \text { baskets), among them lids ( } 2 \text { pieces, each re-used } \\
18^{\text {th }} \text { Dynasty sherds, pottery) }\end{array}$ & 880 & SAV1W 1705, SAV1W 1706 (lid) & 01.02 .2016 \\
\hline $1535 / 2016$ & SQ1SE & pottery (1 basket) & 881 & & 01.02 .2016 \\
\hline $1539 / 2016$ & SQ1S/SQ1SE & $\begin{array}{l}\text { pottery ( } 6 \text { baskets), among them figurine ( } 1 \text { fragment, almost } \\
\text { certainly head of Nubian style doll, clay), lids ( } 2 \text { pieces, each re- } \\
\text { used } 18^{\text {th }} \text { Dynasty sherds, pottery) }\end{array}$ & 884 & $\begin{array}{l}\text { SAV1W } 1708 \text { (figurine), SAV1W 1710, } \\
\text { SAV1W } 1711 \text { (lids) }\end{array}$ & 02.02 .2016 \\
\hline $1553 / 2016$ & SQ1S/SQ1SE & pottery ( 2 baskets) & 884 & & 03.02 .2016 \\
\hline $1542 / 2016$ & SQ1NW & pottery (1/2 basket) & 885 & & 02.02 .2016 \\
\hline $1548 / 2016$ & SQ1NW & pottery (3 baskets) & 886 & & 03.02 .2016 \\
\hline $1559 / 2016$ & SQ1NW & pottery (3 baskets) & 887 & & 03.02 .2016 \\
\hline $1565 / 2016$ & SQ1S & pottery (1/2 medium bag) & 888 & & 03.02 .2016 \\
\hline $1566 / 2016$ & SQ1NW & pottery (1/2 basket) & 891 & & 03.02 .2016 \\
\hline $1568 / 2016$ & SQ1NW & pottery (1/2 basket) & 891 & & 04.02 .2016 \\
\hline $1573 / 2016$ & SQ1S, W part of SU 893 in Feature 122 & pottery (1 medium bag) & 893 & & 04.02 .2016 \\
\hline $1574 / 2016$ & SQ1NW & pottery (1/2 basket) & 894 & & 04.02 .2016 \\
\hline $1579 / 2016$ & SQ1NW & pottery ( 2 baskets) & 895 & & 04.02 .2016 \\
\hline $1585 / 2016$ & SQ1S, around Feature 122 & pottery (1 medium bag) & 896 & & 04.02 .2016 \\
\hline $1584 / 2016$ & SQ1, sieved material of Feature 112 & pottery (1/2 baskets) & 898 & & 04.02 .2016 \\
\hline $1590 / 2016$ & SQ1, sieved material & pottery & 899 & & 04.02 .2016 \\
\hline $1163 / 2016$ & SQ1SE & rope (1 piece) & 825 & & 13.01 .2016 \\
\hline
\end{tabular}




\begin{tabular}{|c|c|c|c|c|c|}
\hline $1204 / 2016$ & SQ1SE & rope (1 piece) & 834 & & 16.01.2016 \\
\hline $1237 / 2016$ & SQ1SE & rope (1 piece) & 837 & & 18.01 .2016 \\
\hline $1278 / 2016$ & SQ1SE & rope (1 piece) & 843 & & 19.01.2016 \\
\hline $1012 / 2016$ & SQ1SE & shell (2 pieces) & 801 & & 05.01 .2016 \\
\hline $1063 / 2016$ & SQ1SE & shell (1 piece) & 810 & & 09.01 .2016 \\
\hline $1069 / 2016$ & SQ1SE & shell (1 piece) & 811 & & 09.01 .2016 \\
\hline $1083 / 2016$ & SQ1SE & shell (1 piece) & 812 & & 09.01 .2016 \\
\hline $1098 / 2016$ & SQ1SE & shell (2 pieces) & 815 & & 10.01 .2016 \\
\hline $1136 / 2016$ & SQ1SE & shell (1 piece) & 826 & & 12.01 .2016 \\
\hline $1149 / 2016$ & SQ1SE & shell (1 bag) & 829 & & 13.01.2016 \\
\hline $1156 / 2016$ & SQ1SE & shell (1 bag) & 830 & & 13.01 .2016 \\
\hline $1190 / 2016$ & SQ1SE & shell (1 bag) & 834 & & 16.01 .2016 \\
\hline $1197 / 2016$ & SQ1SE & shell (1 piece) & 835 & & 16.01 .2016 \\
\hline $1284 / 2016$ & SQ1SE & shell (1 piece) & 845 & & 19.01 .2016 \\
\hline $1307 / 2016$ & SQ1SE & shell (1 piece) & 845 & & 20.01 .2016 \\
\hline $1322 / 2016$ & SQ1SE & shell (2 pieces) & 849 & & 21.01 .2016 \\
\hline $1327 / 2016$ & SQ1SE & shell (1 piece) & 850 & & 21.01 .2016 \\
\hline $1368 / 2016$ & SQ1SE & shell (1 piece) & 855 & & 24.01 .2016 \\
\hline $1419 / 2016$ & SQ1SE & shell (1 piece) & 860 & & 26.01 .2016 \\
\hline $1434 / 2016$ & SQ1S/SQ1SE & shell (1 piece) & 862 & & 27.01 .2016 \\
\hline $1448 / 2016$ & SQ1S & shell (1 piece) & 866 & & 28.01 .2016 \\
\hline $1531 / 2016$ & SQ1S & shell (1 piece) & 880 & & 01.02 .2016 \\
\hline $1556 / 2016$ & SQ1S/SQ1SE & shell (1 piece) & 884 & & 03.02 .2016 \\
\hline $1572 / 2016$ & SQ1NW & shell (2 pieces) & 891 & & 04.02 .2016 \\
\hline $1032 / 2016$ & SQ1SE & slag and brick (1 fragment) & 804 & SAV1W 1542 & 06.01 .2016 \\
\hline $1259 / 2016$ & SQ1SE & slag/melted brick (1 large piece) & 837 & & 18.01.2016 \\
\hline
\end{tabular}




\begin{tabular}{|c|c|c|c|c|c|}
\hline $1406 / 2016$ & SQ1SE & slag (1 piece) & 858 & SAV1W 1650 & 25.01.2016 \\
\hline $1402 / 2016$ & SQ1S/SQ1SE & slag (1 fragment) & 859 & SAV1W 1651 & 25.01 .2016 \\
\hline $1500 / 2016$ & SQ1S & slag (1 piece) & 873 & & 31.01 .2016 \\
\hline $1538 / 2016$ & SQ1NW & stamp (1 cylindrical clay object) & 879 & SAV1W 1707 & 01.02 .2016 \\
\hline $1001 / 2016$ & SQ1SE & stone vessel (1 fragment, basin, sandstone) & 800 & SAV1W 1531 & 04.01 .2016 \\
\hline $1008 / 2016$ & SQ1SE & stones: pounders ( 3 pieces, quartzite) & 801 & $\begin{array}{l}\text { SAV1W 1538, SAV1W 1539, SAV1W } 1540 \\
\text { (pounders) }\end{array}$ & 05.01 .2016 \\
\hline $1021 / 2016$ & SQ1SE & stone: pounder (1 piece, quartzite) & 804 & SAV1W 1537 & 05.01 .2016 \\
\hline $1084 / 2016$ & SQ1SE & $\begin{array}{l}\text { stones: polishing stone (1 fragment, schist), grindstone } \\
\text { (1 fragment, sandstone) }\end{array}$ & 812 & $\begin{array}{l}\text { SAV1W } 0492 \text { (polishing stone), SAV1W } 0551 \\
\text { (grindstone) }\end{array}$ & 09.01 .2016 \\
\hline $1088 / 2016$ & SQ1SE & stone: blade? (1 fragment, silex) & 812 & SAV1W 1545 (blade?) & 10.01.2016 \\
\hline $1093 / 2016$ & SQ1SE & $\begin{array}{l}\text { stone: greenstone with quartzite, calcite crack formed (1 piece, } \\
\text { sample) }\end{array}$ & 812 & & 10.01 .2016 \\
\hline $1141 / 2016$ & SQ1SE & stone: basin (1 fragment, sandstone) & 826 & SAV1W 1555 & 12.01.2016 \\
\hline $1157 / 2016$ & SQ1SE & $\begin{array}{l}\text { stones: hammer ( } 1 \text { piece, quartz), mortars ( } 2 \text { pieces, each sand- } \\
\text { stone), pestle ( } 1 \text { piece, sandstone), hand mill ( } 1 \text { piece, sandstone), } \\
\text { sandstone ( } 1 \text { large fragment with red pigment), sandstone ( } 1 \text { large } \\
\text { fragment with yellow pigment) }\end{array}$ & 830 & $\begin{array}{l}\text { SAV1W } 0740 \text { (hammer), SAV1W } 0760 \text {, } \\
\text { SAV1W } 1497 \text { (mortars), SAV1W } 1435 \text { (pestle), } \\
\text { SAV1W } 1195 \text { (hand mill) }\end{array}$ & 13.01.2016 \\
\hline $1175 / 2016$ & SQ1SE & stone: blade (1 piece, silex) & 831 & SAV1W 1563 & 14.01.2016 \\
\hline $1185 / 2016$ & SQ1SE & stone: pounder/polishing stone (1 fragment, quartzite) & 831 & SAV1W 1565 & 14.01.2016 \\
\hline $1191 / 2016$ & SQ1SE & stones: blade (1 piece, silex), silex flake (1 piece) & 834 & SAV1W 1572 (blade) & 16.01.2016 \\
\hline $1200 / 2016$ & SQ1SE & stone: polishing stone (1 piece, schist) & 834 & SAV1W 1573 & 16.01.2016 \\
\hline $1255 / 2016$ & SQ1SE & $\begin{array}{l}\text { stones: grindstone ( } 1 \text { piece, sandstone), mortars ( } 2 \text { pieces, each } \\
\text { sandstone), weight/tethering stone ( } 1 \text { piece, quartzite), blade } \\
\text { (1 piece, silex), scraper ( } 1 \text { piece, schist) }\end{array}$ & 834 & $\begin{array}{l}\text { SAV1W } 1526 \text { (grindstone), SAV1W } 1579 \text {, } \\
\text { SAV1W } 1580 \text { (mortars), SAV1W 1581 (weight/ } \\
\text { tethering stone), SAV1W } 1582 \text { (blade), SAV1W } \\
1583 \text { (scraper) }\end{array}$ & 18.01 .2016 \\
\hline $1252 / 2016$ & SQ1SE & stone: grindstone (1 fragment, sandstone) & 835 & SAV1W 1513 & 18.01.2016 \\
\hline $1254 / 2016$ & SQ1SE & stone: grindstone (1 piece, sandstone) & 836 & SAV1W 1506 & 18.01.2016 \\
\hline $1253 / 2016$ & SQ1SE & stone: basin/vessel (1 large fragment, sandstone) & 837 & SAV1W 1585 & 18.01.2016 \\
\hline $1239 / 2016$ & SQ1SE & stone: sickle (1 piece, silex) & 840 & SAV1W 0051 & 18.01.2016 \\
\hline $1258 / 2016$ & SQ1SE & stone: pounder (1 piece, quartzite) & 840 & SAV1W 1511 & 18.01.2016 \\
\hline
\end{tabular}




\begin{tabular}{|c|c|c|c|c|c|}
\hline $1257 / 2016$ & SQ1SE & stone: scraper (1 piece, schist) & 841 & SAV1W 1315 & 18.01.2016 \\
\hline $1274 / 2016$ & SQ1SE & $\begin{array}{l}\text { stone, worked (1 pyramid shaped piece, function unclear, } \\
\text { polishing stone?/figurine?, sandstone) }\end{array}$ & 843 & SAV1W 1593 & 19.01.2016 \\
\hline $1288 / 2016$ & SQ1SE & stone: grindstone or hand mill (1 piece, sandstone) & 843 & SAV1W 1595 & 19.01.2016 \\
\hline $1289 / 2016$ & SQ1SE & stone: pounder (1 piece, unspecified stone) & 844 & SAV1W 1594 & 19.01 .2016 \\
\hline $1317 / 2016$ & SQ1SE & stone: pounder (1 piece, quartzite) & 846 & SAV1W 1598 & 20.01 .2016 \\
\hline $1316 / 2016$ & SQ1SE & stone: pounder and polishing stone (1 piece, greenstone) & 847 & SAV1W 1597 & 20.01 .2016 \\
\hline $1335 / 2016$ & SQ1SE & $\begin{array}{l}\text { stones: pounders ( } 3 \text { pieces, } 2 \text { quartz, } 1 \text { quartzite), grindstone } \\
\text { (1 piece, sandstone), polishing stone (1 piece, quartzite?) }\end{array}$ & 849 & $\begin{array}{l}\text { SAV1W 1615, SAV1W 1616, SAV1W } 1617 \\
\text { (pounders), SAV1W } 1618 \text { (grindstone), } \\
\text { SAV1W } 1619 \text { (polishing stone) }\end{array}$ & 21.01 .2016 \\
\hline $1373 / 2016$ & SQ1S & stone: silex flake (1 piece, unworked) & 852 & & 24.01 .2016 \\
\hline $1371 / 2016$ & SQ1SE & $\begin{array}{l}\text { stone tools (several) (final status unregistered stone, } 2 \text { fragments, } \\
\text { samples) }\end{array}$ & 853 & & 24.01 .2016 \\
\hline $1377 / 2016$ & SQ1SE & $\begin{array}{l}\text { stones, worked ( } 2 \text { pieces, original function unclear, each } \\
\text { limestone) }\end{array}$ & 854 & SAV1W 1632, SAV1W 1633 (worked stones) & 24.01 .2016 \\
\hline $1375 / 2016$ & SQ1SE & stone, worked (1 fragment, original function unclear, sandstone) & 855 & SAV1W 1634 & 24.01 .2016 \\
\hline $1376 / 2016$ & SQ1SE & stone: blade or scraper? (1 piece, silex) & 855 & SAV1W 1627 & 24.01 .2016 \\
\hline $1403 / 2016$ & SQ1SE & $\begin{array}{l}\text { stone vessel (1 fragment, calcite), silex (1 piece, cf. bag 1404), } \\
\text { silex (1 piece, cf. bag 1404) }\end{array}$ & 856 & SAV1W 1663 (stone vessel) & 25.01 .2016 \\
\hline $1404 / 2016$ & SQ1S & $\begin{array}{l}\text { stones: worked stone ( } 1 \text { fragment, function unclear, sandstone), } \\
\text { silex ( } 1 \text { piece, cf. bag } 1403 \text {, form same pebble ?) }\end{array}$ & 857 & SAV1W 1664 (worked stone) & 25.01 .2016 \\
\hline $1405 / 2016$ & SQ1SE & $\begin{array}{l}\text { stones: grindstone (1 piece, sandstone), pounder (1 piece, } \\
\text { unspecified stone) }\end{array}$ & 858 & $\begin{array}{l}\text { SAV1W } 1656 \text { (grindstone), SAV1W } 1662 \\
\text { (pounder) }\end{array}$ & 25.01 .2016 \\
\hline $1422 / 2016$ & SQ1SE & $\begin{array}{l}\text { stones: pestle ( } 1 \text { half, sandstone), grindstones or polishing stones } \\
\text { ( } 2 \text { pieces, each sandstone) }\end{array}$ & 860 & $\begin{array}{l}\text { SAV1W } 1658 \text { (pestle), SAV1W 1659, SAV1W } \\
1660 \text { (grindstones or polishing stones) }\end{array}$ & 26.01.2016 \\
\hline $1423 / 2016$ & SQ1S & $\begin{array}{l}\text { stone and ochre: grindstone (1 fragment, sandstone); ochre } \\
\text { (1 piece, yellow) }\end{array}$ & 861 & SAV1W 1661 & 26.01 .2016 \\
\hline $1439 / 2016$ & SQ1S/SQ1SE & $\begin{array}{l}\text { stones: hammer ( } 1 \text { quartzite pebble), worked stone (1 fragment, } \\
\text { function unclear, limestone) }\end{array}$ & 862 & $\begin{array}{l}\text { SAV1W } 1687 \text { (hammer), SAV1W } 1688 \\
\text { (worked stone) }\end{array}$ & 27.01.2016 \\
\hline $1438 / 2016$ & SQ1S & $\begin{array}{l}\text { stones: pestle ( } 1 \text { piece, unspecified stone), grindstone (1 re-used } \\
\text { floor slab?, sandstone) }\end{array}$ & 863 & $\begin{array}{l}\text { SAV1W } 1695 \text { (pestle), SAV1W } 1696 \\
\text { (grindstone) }\end{array}$ & 27.01 .2016 \\
\hline $1458 / 2016$ & SQ1S & stones: polishing stone (1 pyramid shaped fragment, sandstone) & 864 & SAV1W 1686 & 28.01 .2016 \\
\hline $1457 / 2016$ & SQ1S/SQ1SE & $\begin{array}{l}\text { stones: pounder ( } 1 \text { pebble, unspecified stone), hammer } \\
\text { (1 quartzite pebble), polishing stone ( } 1 \text { piece, unspecified stone), } \\
\text { worked stone ( } 1 \text { fragment, function unclear, sandstone) }\end{array}$ & 865 & $\begin{array}{l}\text { SAV1W } 1689 \text { (pounder), SAV1W } 1690 \\
\text { (hammer), SAV1W } 1691 \text { (polishing stone), } \\
\text { SAV1W } 1691 \text { (worked stone) }\end{array}$ & 28.01 .2016 \\
\hline
\end{tabular}




\begin{tabular}{|c|c|c|c|c|c|}
\hline $1450 / 2016$ & SQ1S & $\begin{array}{l}\text { stones: pounder ( } 1 \text { pebble, agate), basin } \\
\text { (1 fragment, red pigment inside, sandstone) }\end{array}$ & 866 & SAV1W 1693 (pounder), SAV1W 1694 (basin) & 28.01.2016 \\
\hline $1533 / 2016$ & SQ1NW & stone: token/gaming piece (1 small ball, limestone) & 879 & SAV1W 1703 & 01.02 .2016 \\
\hline $1589 / 2016$ & SQ1S/SQ1SE & stone: blade or sickle? (1 triangular flake, silex) & 880 & SAV1W 1713 & 04.02 .2016 \\
\hline $1567 / 2016$ & SQ1S/SQ1SE & $\begin{array}{l}\text { stone: token/gaming piece or stopper? ( } 1 \text { conical sandstone } \\
\text { fragment) }\end{array}$ & 884 & SAV1W 1709 & 03.02 .2016 \\
\hline $1588 / 2016$ & SQ1S & $\begin{array}{l}\text { stone: worked stone - weight? (1 small fragment, roughly } \\
\text { spherical, holes started on } 2 \text { sides, sandstone) }\end{array}$ & 884 & SAV1W 1712 & 04.02 .2016 \\
\hline $1348 / 2016$ & SQ1SE & stopper (1 piece, clay) & 853 & SAV1W 1628 & 23.01.2016 \\
\hline $1019 / 2016$ & SQ1SE & textile (1 piece, rope - woven material, linen?) & 804 & SAV1W 1536 & 05.01 .2016 \\
\hline $1073 / 2016$ & SQ1SE & textile (1 piece) & 811 & & 09.01 .2016 \\
\hline $1228 / 2016$ & SQ1SE & textile (1 bag) & 817 & & 17.01.2016 \\
\hline $1229 / 2016$ & SQ1SE & textile (1 piece) & 837 & & 17.01.2016 \\
\hline $1238 / 2016$ & SQ1SE & textile (1 bag) & 837 & & 18.01.2016 \\
\hline $1279 / 2016$ & SQ1SE & textile (1 bag) & 845 & & 19.01.2016 \\
\hline $1295 / 2016$ & SQ1SE & textile (1 piece) & 846 & & 20.01 .2016 \\
\hline $1323 / 2016$ & SQ1SE & textile (1 piece) & 849 & & 21.01 .2016 \\
\hline $1591 / 2016$ & SQ1SE & textile (1 bag) & 854 & & 23.01 .2016 \\
\hline $1379 / 2016$ & SQ1SE & textile (1 bag) & 858 & & 25.01 .2016 \\
\hline $1399 / 2016$ & SQ1S & textile (1 bag, striped) & 861 & & 26.01.2016 \\
\hline $1436 / 2016$ & SQ1S/SQ1SE & textile ( 2 pieces) & 862 & & 27.01.2016 \\
\hline $1508 / 2016$ & SQ1NW & textile/rope (1 bag) & 872 & & 31.01 .2016 \\
\hline $1498 / 2016$ & SQ1S & textile (1 bag) & 873 & & 31.01 .2016 \\
\hline $1525 / 2016$ & SQ1NW & textile (1 bag) & 879 & & 01.02 .2016 \\
\hline $1545 / 2016$ & SQ1NW & textile (1 bag) & 885 & & 02.02 .2016 \\
\hline $1551 / 2016$ & SQ1NW & textile (1 bag) & 886 & & 03.02 .2016 \\
\hline $1561 / 2016$ & SQ1NW & textile (1 bag) & 887 & & 03.02 .2016 \\
\hline $1571 / 2016$ & SQ1NW & textile (1 bag) & 891 & & 04.02 .2016 \\
\hline
\end{tabular}




\begin{tabular}{|c|c|c|c|c|c|}
\hline $1582 / 2016$ & SQ1NW & textile (1 bag) & 895 & & 04.02 .2016 \\
\hline $1271 / 2016$ & SQ1SE & token/gaming piece (1 piece, clay) & 845 & SAV1W 1592 & 19.01.2016 \\
\hline $1331 / 2016$ & SQ1SE & token/gaming piece (1 re-used sherd, $18^{\text {th }}$ Dynasty, pottery) & 850 & SAV1W 1608 & 21.01 .2016 \\
\hline $1158 / 2016$ & SQ1SE & water spout? (1 fragment, Christian, clay) & 825 & SAV1W 1562 & 13.01.2016 \\
\hline $1586 / 2016$ & SQ1, sieved material of Feature 112 & $\begin{array}{l}\text { wood, worked ( } 1 \text { small fragment); beads ( } 2 \text { pieces, each ring } \\
\text { beads, } 1 \text { bone, } 1 \text { faience); textile }(1 \text { bag); charcoal }(1 \text { bag); bones } \\
(2 \text { bags); doum nuts; organic material }(1 \text { bag, mixed); wood } \\
\text { ( } 1 \text { bag); lithic flake (1 piece, reference); stone flakes ( } 1 \text { bag, } \\
\text { reference) }\end{array}$ & 899 & $\begin{array}{l}\text { SAV1W } 1719 \text { (worked wood), SAV1W 0501, } \\
\text { SAV1W } 0585 \text { (beads) }\end{array}$ & 04.02 .2016 \\
\hline $1030 / 2016$ & SQ1SE & wood (7 fragments) & 804 & & 06.01 .2016 \\
\hline $1048 / 2016$ & SQ1SE & wood (1 bag) & 809 & & 07.01 .2016 \\
\hline $1071 / 2016$ & SQ1SE & wood (1 bag) & 811 & & 09.01 .2016 \\
\hline $1076 / 2016$ & SQ1SE & wood (1 bag) & 812 & & 09.01 .2016 \\
\hline $1082 / 2016$ & SQ1SE & wood (1 bag) & 813 & & 09.01 .2016 \\
\hline $1101 / 2016$ & SQ1SE & wood (1 bag) & 815 & & 10.01 .2016 \\
\hline $1105 / 2016$ & SQ1SE & wood (1 bag) & 816 & & 11.01 .2016 \\
\hline $1138 / 2016$ & SQ1SE & wood (1 piece, basket?) & 826 & & 12.01 .2016 \\
\hline $1147 / 2016$ & SQ1SE & wood (1 bag) & 828 & & 13.01 .2016 \\
\hline $1177 / 2016$ & SQ1SE & wood (1 piece) & 832 & & 14.01 .2016 \\
\hline $1183 / 2016$ & SQ1SE & wood (1 bag) & 833 & & 14.01 .2016 \\
\hline $1193 / 2016$ & SQ1SE & wood (1 bag) & 834 & & 16.01 .2016 \\
\hline $1199 / 2016$ & SQ1SE & wood (1 bag) & 835 & & 16.01.2016 \\
\hline $1211 / 2016$ & SQ1SE & wood (1 bag) & 836 & & 17.01.2016 \\
\hline $1220 / 2016$ & SQ1SE & wood (1 bag) & 837 & & 17.01.2016 \\
\hline $1235 / 2016$ & SQ1SE & wood (1 bag) & 837 & & 18.01 .2016 \\
\hline $1223 / 2016$ & SQ1SE & wood (3 pieces) & 838 & & 17.01.2016 \\
\hline $1247 / 2016$ & SQ1SE & wood (1 bag) & 840 & & 18.01 .2016 \\
\hline $1263 / 2016$ & SQ1SE & wood (1 bag) & 843 & & 19.01.2016 \\
\hline
\end{tabular}




\begin{tabular}{|c|c|c|c|c|}
\hline $1314 / 2016$ & SQ1SE & wood (1 bag) & 846 & 20.01 .2016 \\
\hline $1315 / 2016$ & SQ1SE & wood (1 bag) & 848 & 20.01 .2016 \\
\hline $1324 / 2016$ & SQ1SE & wood (1 bag) & 849 & 21.01 .2016 \\
\hline $1332 / 2016$ & SQ1SE & wood (1 bag) & 850 & 21.01 .2016 \\
\hline $1345 / 2016$ & SQ1S & wood (1 bag) & 852 & 23.01 .2016 \\
\hline $1340 / 2016$ & SQ1SE & wood (1 bag) & 853 & 23.01 .2016 \\
\hline $1354 / 2016$ & SQ1SE & wood (1 bag) & 854 & 23.01 .2016 \\
\hline $1361 / 2016$ & SQ1SE & wood (1 bag) & 854 & 24.01 .2016 \\
\hline $1398 / 2016$ & SQ1SE & wood (1 bag) & 858 & 25.01 .2016 \\
\hline $1394 / 2016$ & SQ1S/SQ1SE & wood (1 bag) & 859 & 25.01 .2016 \\
\hline $1411 / 2016$ & SQ1SE & wood (1 bag) & 860 & 26.01 .2016 \\
\hline $1413 / 2016$ & SQ1S & wood (1 bag) & 861 & 26.01 .2016 \\
\hline $1415 / 2016$ & SQ1S & wood (1 bag) & 861 & 26.01 .2016 \\
\hline $1427 / 2016$ & SQ1S/SQ1SE & wood (1 bag) & 862 & 27.01.2016 \\
\hline $1432 / 2016$ & SQ1S & wood (1 bag) & 863 & 27.01 .2016 \\
\hline $1456 / 2016$ & SQ1S/SQ1SE & wood (1 bag) & 865 & 28.01 .2016 \\
\hline $1454 / 2016$ & SQ1S & wood (1 bag) & 866 & 28.01 .2016 \\
\hline $1460 / 2016$ & SQ1S & wood (1 large piece) & 867 & 30.01 .2016 \\
\hline $1472 / 2016$ & SQ1S & wood (1 bag) & 867 & 30.01 .2016 \\
\hline $1466 / 2016$ & SQ1S & wood (1 bag) & 869 & 30.01 .2016 \\
\hline $1499 / 2016$ & SQ1S & wood (1 bag) & 873 & 31.01 .2016 \\
\hline $1486 / 2016$ & SQ1S & wood (1 piece) & 874 & 30.01 .2016 \\
\hline $1488 / 2016$ & SQ1S, sieved material & wood (1 bag); charcoal (1 bag); bones (1 bag) (see 1483/2016) & 874 & 30.01 .2016 \\
\hline $1513 / 2016$ & SQ1S & wood (1 bag) & 877 & 31.01 .2016 \\
\hline $1529 / 2016$ & SQ1S & wood (1 bag) & 880 & 01.02 .2016 \\
\hline $1540 / 2016$ & SQ1S/SQ1SE & wood (1 bag) & 884 & 02.02 .2016 \\
\hline $1557 / 2016$ & SQ1S/SQ1SE & wood (1 bag) & 884 & 03.02 .2016 \\
\hline
\end{tabular}




\begin{tabular}{|c|c|c|c|c|c|}
\hline \multicolumn{6}{|c|}{ Sai Island SAV1 West 2017} \\
\hline Find no. & Location & Description/label/material & $\begin{array}{l}\text { SU/ } \\
\text { level }\end{array}$ & Object no. & Date \\
\hline $133 / 2017$ & SQ1SE, Feature 151, top surface 907 (silo) & $\begin{array}{l}\text { architecture: lintel later reworked as column base (1 large } \\
\text { fragment, once inscribed, sandstone) }\end{array}$ & 907 & SAV1W 1752 & 15.01.2017 \\
\hline $129 / 2017$ & SQ1SE-E & $\begin{array}{l}\text { architecture: column drum/base } \\
\text { (1 large piece, sandstone) }\end{array}$ & 916 & SAV1W 1751 & 15.01.2017 \\
\hline $146 / 2017$ & SQ1SE-E & $\begin{array}{l}\text { architecture (1 large rectangular fragment, sandstone, Christian } \\
\text { decoration/architecture?) }\end{array}$ & 916 & SAV1W 1772 & 16.01 .2017 \\
\hline $192 / 2017$ & SQ1SE & architecture (1 rectangular block, sandstone) & 924 & SAV1W 1827 & 18.01.2017 \\
\hline $030 / 2017$ & SQ1S, N of Feature 143 & bead (1 ring bead, faience) & 903 & SAV1W 1737 & 09.01 .2017 \\
\hline $035 / 2017$ & SQ1S, N of Feature 143 & beads ( 2 ring beads, faience) & 903 & SAV1W 1738 & 09.01 .2017 \\
\hline $072 / 2017$ & SQ1SE, Feature 151 & bead (1 disc bead, faience) & 908 & SAV1W 1773 & 11.01 .2017 \\
\hline $073 / 2017$ & SQ1SE, Feature 151, from sieving & $\begin{array}{l}\text { bead ( } 1 \text { ring bead with fragment of another bead stuck to it, } \\
\text { faience) }\end{array}$ & 908 & SAV1W 1774 & 11.01 .2017 \\
\hline $078 / 2017$ & SQ1SE, Feature 151, from sieving & bead/amulet ( 1 small cowroid bead/amulet, faience) & 909 & SAV1W 1736 & 11.01 .2017 \\
\hline $087 / 2017$ & SQ1SE, Feature 151, from sieving & $\begin{array}{l}\text { bead/pendant ( } 1 \text { small tear drop shaped piece, sandstone); beads } \\
\text { ( } 7 \text { ring beads, shell/ostrich eggshell or bone; } 1 \text { clay bead; } \\
3 \text { ring/disc beads, schist?; } 1 \text { disc bead, limestone; } 2 \text { ring beads, } \\
\text { stone?/bone?/ostrich eggshell?; } 1 \text { ring bead, polished stone; } \\
1 \text { ring bead, faience; } 30 \text { ring beads, faience, bone and eggshell; } \\
2 \text { disc beads, clay; } 2 \text { fragments of ring beads, shell); agate } \\
\text { ( } 3 \text { small flakes, raw material) }\end{array}$ & 909 & $\begin{array}{l}\text { SAV1W } 1759 \text { (bead/pendant), SAV1W } 1760 \\
\text { ( } 7 \text { beads), SAV1W } 1761 \text { (clay bead), SAV1W } \\
1763 \text { (schist? beads), SAV1W 1764 (limestone } \\
\text { bead), SAV1W } 1765 \text { (stone?/bone?/ostrich } \\
\text { eggshell? beads), SAV1W } 1766 \text { (bead, polished } \\
\text { stone), SAV1W } 1767 \text { (faience bead), SAV1W } \\
1768 \text { (30 ring beads), SAV1W 1770 (disc } \\
\text { beads), SAV1W 1762 (bead fragments), } \\
\text { SAV1W } 1839 \text { (agate) }\end{array}$ & 11.01 .2017 \\
\hline $151 / 2017$ & SQ1SE-E & bead (1 ring bead, faience) & 916 & SAV1W 1775 & 16.01 .2017 \\
\hline $132 / 2017$ & SQ1SE-E and SQ1SE & beads ( 2 ring beads, faience) & 916 & SAV1W 1776 & 15.01 .2017 \\
\hline $205 / 2017$ & SQ1SE & bead (1 ring bead, faience) & 925 & SAV1W 1782 & 18.01.2017 \\
\hline $343 / 2017$ & SQ1SE and SQ1SE-E & $\begin{array}{l}\text { beads ( } 1 \text { tubular bead, composed with adjoining ring beads, } \\
\text { faience) }\end{array}$ & 947 & SAV1W 1812 & 23.01 .2017 \\
\hline $352 / 2017$ & SQ1S & bead (1 disc bead, shell) & 955 & SAV1W 1817 & 24.01 .2017 \\
\hline $382 / 2017$ & SQ1S & bead (1 ring bead, faience) & 956 & SAV1W 1818 & 24.01 .2017 \\
\hline $475 / 2017$ & SQ1S & bead (1 ring bead, bone?/faience?) & 963 & SAV1W 1822 & 25.01 .2017 \\
\hline $415 / 2017$ & SQ1S & bead (1 ball bead, faience) & 970 & SAV1W 1823 & 25.01 .2017 \\
\hline
\end{tabular}




\begin{tabular}{|c|c|c|c|c|c|}
\hline $473 / 2017$ & SQ1S, from sieving & beads ( 1 ring bead, bone; 1 ring bead, faience) & 976 & SAV1W 1825, SAV1W 1826 & 26.01 .2017 \\
\hline $433 / 2017$ & SQ1S & bead (1 disc bead, bone) & 980 & SAV1W 1821 & 26.01.2017 \\
\hline $013 / 2017$ & SQ1SE & bones & 900 & & 08.01 .2017 \\
\hline $008 / 2017$ & SQ1SE, from center of S part of SQ1SE & bones & 900 & & 07.01 .2017 \\
\hline $004 / 2017$ & SQ1SE, NE & bones; stopper (1 piece, clay) & 900 & SAV1W 1033 (stopper) & 07.01 .2017 \\
\hline $046 / 2017$ & $\begin{array}{l}\text { SQ1S, cleaning of wind-blown sand, } \\
\text { W-edge SQ1S }\end{array}$ & bones & 901 & & 10.01 .2017 \\
\hline $240 / 2017$ & SQ1SE, E part & bone & 902 & & 19.01.2017 \\
\hline $028 / 2017$ & SQ1S, S of Feature 143 & bones & 903 & & 09.01 .2017 \\
\hline $029 / 2017$ & SQ1S, N of Feature 143 & bones & 903 & & 09.01 .2017 \\
\hline $044 / 2017$ & SQ1S, N of Feature 143 & bones & 903 & & 10.01 .2017 \\
\hline $058 / 2017$ & SQ1SE, Feature 145 & bone & 904 & & 10.01 .2017 \\
\hline $052 / 2017$ & SQ1SE & bones (mummified, dog, with iron, recent) & 905 & & 10.01 .2017 \\
\hline $053 / 2017$ & SQ1SE & bones & 905 & & 10.01 .2017 \\
\hline $064 / 2017$ & SQ1SE & bones & 906 & & 11.01 .2017 \\
\hline $071 / 2017$ & SQ1SE, Feature 151, & bones & 908 & & 11.01 .2017 \\
\hline $080 / 2017$ & SQ1SE, Feature 151, & bones & 909 & & 11.01 .2017 \\
\hline $077 / 2017$ & SQ1SE, Feature 151, from sieving & $\begin{array}{l}\text { bone tool/bone, worked ( } 1 \text { small bone sherd, worked to a } \\
\text { polished point at one end) }\end{array}$ & 909 & SAV1W 1769 & 11.01 .2017 \\
\hline $092 / 2017$ & SQ1SE-E & bones & 911 & & 12.01 .2017 \\
\hline $137 / 2017$ & SQ1SE-E & bone & 911 & & 16.01 .2017 \\
\hline $100 / 2017$ & SQ1SE-E and SQ1SE & bones & 912 & & 14.01 .2017 \\
\hline $104 / 2017$ & SQ1SE-E & bones & 913 & & 14.01 .2017 \\
\hline $108 / 2017$ & SQ1SE-E and SQ1SE & bones & 914 & & 14.01 .2017 \\
\hline $114 / 2017$ & SQ1SE-E and SQ1SE & bones & 914 & & 15.01 .2017 \\
\hline $121 / 2017$ & SQ1SE-E & bones & 915 & & 15.01 .2017 \\
\hline $142 / 2017$ & SQ1SE-E & bone & 916 & & 16.01 .2017 \\
\hline $154 / 2017$ & SQ1SE-E & bone & 916 & & 17.01 .2017 \\
\hline
\end{tabular}




\begin{tabular}{|c|c|c|c|c|}
\hline $126 / 2017$ & SQ1SE-E and SQ1SE & bones & 916 & 15.01.2017 \\
\hline $177 / 2017$ & SQ1SE-E & bone & 917 & 18.01 .2017 \\
\hline $164 / 2017$ & SQ1SE-E & bone & 918 & 17.01.2017 \\
\hline $161 / 2017$ & SQ1SE & bone & 919 & 17.01.2017 \\
\hline $173 / 2017$ & SQ1SE & bone & 920 & 17.01.2017 \\
\hline $180 / 2017$ & SQ1SE & bone & 920 & 18.01 .2017 \\
\hline $191 / 2017$ & SQ1SE & bone & 921 & 18.01 .2017 \\
\hline $195 / 2017$ & SQ1SE-E & bone & 923 & 18.01.2017 \\
\hline $218 / 2017$ & SQ1SE & bone & 924 & 18.01 .2017 \\
\hline $204 / 2017$ & SQ1SE & bone & 925 & 18.01 .2017 \\
\hline $208 / 2017$ & SQ1SE & bone & 926 & 18.01 .2017 \\
\hline $216 / 2017$ & SQ1SE & bone & 927 & 18.01 .2017 \\
\hline $224 / 2017$ & SQ1 & bone & 928 & 19.01.2017 \\
\hline $230 / 2017$ & SQ1S & bone & 929 & 19.01.2017 \\
\hline $243 / 2017$ & SQ1 & bone & 931 & 19.01.2017 \\
\hline $261 / 2017$ & SQ1 & bone & 931 & 21.01 .2017 \\
\hline $246 / 2017$ & SQ1S & bone & 935 & 19.01.2017 \\
\hline $249 / 2017$ & SQ1S & bone & 936 & 19.01.2017 \\
\hline $269 / 2017$ & SQ1SE & bone & 937 & 21.01 .2017 \\
\hline $285 / 2017$ & SQ1SE, N of SU 941 & bone & 937 & 22.01 .2017 \\
\hline $290 / 2017$ & SQ1SE, S of SU 941 & bone & 937 & 22.01 .2017 \\
\hline $277 / 2017$ & SQ1SE & bone & 941 & 21.01 .2017 \\
\hline $302 / 2017$ & SQ1SE & bone & 941 & 22.01 .2017 \\
\hline $279 / 2017$ & SQ1SE & bone & 942 & 21.01 .2017 \\
\hline $300 / 2017$ & SQ1SE & bone & 943 & 22.01 .2017 \\
\hline $292 / 2017$ & SQ1SE & bone & 944 & 22.01 .2017 \\
\hline
\end{tabular}




\begin{tabular}{|c|c|c|c|c|c|}
\hline $309 / 2017$ & SQ1SE and SQ1SE-E & bone & 945 & & 22.01.2017 \\
\hline $312 / 2017$ & SQ1SE & bone & 946 & & 22.01 .2017 \\
\hline $317 / 2017$ & SQ1SE and SQ1SE-E & bone & 947 & & 23.01 .2017 \\
\hline $324 / 2017$ & SQ1S & bone & 948 & & 23.01.2017 \\
\hline $331 / 2017$ & SQ1S & bone & 950 & & 23.01.2017 \\
\hline $335 / 2017$ & SQ1S & bone & 951 & & 23.01 .2017 \\
\hline $338 / 2017$ & SQ1SE and SQ1SE-E & bone & 952 & & 23.01.2017 \\
\hline $346 / 2017$ & SQ1S & bone; weight ( 1 re-used $18^{\text {th }}$ Dynasty base sherd, pottery) & 953 & SAV1W 1833 (weight) & 24.01 .2017 \\
\hline $348 / 2017$ & SQ1S & bone & 954 & & 24.01 .2017 \\
\hline $350 / 2017$ & SQ1S & bone & 955 & & 24.01 .2017 \\
\hline $369 / 2017$ & SQ1S & bone & 956 & & 24.01 .2017 \\
\hline $354 / 2017$ & SQ1S & bone & 957 & & 24.01 .2017 \\
\hline $357 / 2017$ & SQ1S & bone & 958 & & 24.01 .2017 \\
\hline $364 / 2017$ & SQ1S & bone & 962 & & 24.01 .2017 \\
\hline $372 / 2017$ & SQ1S & bone & 963 & & 24.01 .2017 \\
\hline $392 / 2017$ & SQ1S & bone & 963 & & 25.01 .2017 \\
\hline $380 / 2017$ & SQ1S & bone & 964 & & 24.01 .2017 \\
\hline $408 / 2017$ & SQ1S & bone & 967 & & 25.01 .2017 \\
\hline $390 / 2017$ & SQ1S & bone & 968 & & 25.01 .2017 \\
\hline $419 / 2017$ & SQ1S & bone & 969 & & 25.01 .2017 \\
\hline $400 / 2017$ & SQ1S & bone & 970 & & 25.01 .2017 \\
\hline $403 / 2017$ & SQ1S & bone & 971 & & 25.01 .2017 \\
\hline $428 / 2017$ & SQ1S & bone & 976 & & 26.01 .2017 \\
\hline $439 / 2017$ & SQ1 & bone & 978 & & 26.01 .2017 \\
\hline $432 / 2017$ & SQ1S & bone & 980 & & 26.01 .2017 \\
\hline $435 / 2017$ & SQ1S & bone & 981 & & 26.01 .2017 \\
\hline
\end{tabular}




\begin{tabular}{|c|c|c|c|c|c|}
\hline $445 / 2017$ & SQ1 & bone & 982 & & 26.01 .2017 \\
\hline $450 / 2017$ & SQ1S & bone & 984 & & 26.01 .2017 \\
\hline $457 / 2017$ & SQ1S & bone & 985 & & 26.01 .2017 \\
\hline $462 / 2017$ & SQ1 & bone & 986 & & 26.01 .2017 \\
\hline $469 / 2017$ & SQ1S & bone & 988 & & 26.01 .2017 \\
\hline $481 / 2017$ & SQ1SE & bone & 992 & & 04.03 .2017 \\
\hline $483 / 2017$ & SQ1SE-E & bone & 994 & & 04.03 .2017 \\
\hline $084 / 2017$ & SQ1SE, Feature 151 & botanical material (seeds) & 909 & & 11.01.2017 \\
\hline $341 / 2017$ & SQ1SE and SQ1SE-E & botanical material & 947 & & 23.01 .2017 \\
\hline $397 / 2017$ & SQ1S & botanical material & 963 & & 25.01 .2017 \\
\hline $476 / 2017$ & SQ1S & botanical material & 970 & & 25.01 .2017 \\
\hline $472 / 2017$ & SQ1S & botanical material & 976 & & 26.01 .2017 \\
\hline $083 / 2017$ & SQ1SE, Feature 151 & bronze (some fragments) & 909 & & 11.01 .2017 \\
\hline $131 / 2017$ & SQ1SE-E and SQ1SE & $\begin{array}{l}\text { bronze object (1 small fragment, function unclear - part of } \\
\text { mould?, possibly part of SAV1W } 1756 \text { or SAV1W 1757) }\end{array}$ & 916 & SAV1W 1758 & 15.01 .2017 \\
\hline $134 / 2017$ & SQ1SE-E and SQ1SE & $\begin{array}{l}\text { bronze object (1 small fragment, function unclear - part of } \\
\text { mould?, possibly part of SAV1W } 1756 \text { or SAV1W 1758) }\end{array}$ & 916 & SAV1W 1757 & 16.01 .2017 \\
\hline $145 / 2017$ & SQ1SE-E, on stone $146 / 2017$ & $\begin{array}{l}\text { bronze object (1 small fragment, function unclear - part of } \\
\text { mould?, possibly part of SAV1W } 1757 \text { or SAV1W 1758) }\end{array}$ & 916 & SAV1W 1756 & 16.01.2017 \\
\hline $344 / 2017$ & SQ1SE and SQ1SE-E & bronze? ring (1 small ring, unclear function) & 947 & SAV1W 1815 & 23.01 .2017 \\
\hline $005 / 2017$ & SQ1SE & charcoal & 900 & & 07.01 .2017 \\
\hline $015 / 2017$ & SQ1SE & charcoal & 900 & & 08.01 .2017 \\
\hline $047 / 2017$ & $\begin{array}{l}\text { SQ1S, cleaning of wind-blown sand, W- } \\
\text { edge SQ1S }\end{array}$ & charcoal & 901 & & 10.01 .2017 \\
\hline $238 / 2017$ & SQ1SE, E part & charcoal & 902 & & 19.01.2017 \\
\hline $025 / 2017$ & SQ1S, N of Feature 143 & charcoal & 903 & & 09.01 .2017 \\
\hline $043 / 2017$ & SQ1S, N of Feature 143 & charcoal & 903 & & 10.01 .2017 \\
\hline $023 / 2017$ & SQ1S, S of Feature 143 & charcoal & 903 & & 09.01 .2017 \\
\hline $040 / 2017$ & SQ1S, S of Feature 143 & charcoal & 903 & & 10.01 .2017 \\
\hline
\end{tabular}




\begin{tabular}{|c|c|c|c|c|}
\hline $057 / 2017$ & SQ1SE, Feature 145 & charcoal & 904 & 10.01.2017 \\
\hline $054 / 2017$ & SQ1SE & charcoal & 905 & 10.01.2017 \\
\hline $065 / 2017$ & SQ1SE & charcoal & 906 & 11.01.2017 \\
\hline $068 / 2017$ & SQ1SE, Feature 151 & charcoal & 908 & 11.01 .2017 \\
\hline $076 / 2017$ & SQ1SE, Feature 151 & charcoal & 909 & 11.01.2017 \\
\hline $093 / 2017$ & SQ1SE-E & charcoal & 911 & 12.01 .2017 \\
\hline $139 / 2017$ & SQ1SE-E & charcoal & 911 & 16.01 .2017 \\
\hline $099 / 2017$ & SQ1SE-E and SQ1SE & charcoal & 912 & 14.01.2017 \\
\hline $102 / 2017$ & SQ1SE-E & charcoal & 913 & 14.01.2017 \\
\hline $107 / 2017$ & SQ1SE-E and SQ1SE & charcoal & 914 & 14.01 .2017 \\
\hline $113 / 2017$ & SQ1SE-E and SQ1SE & charcoal & 914 & 15.01 .2017 \\
\hline $120 / 2017$ & SQ1SE-E & charcoal & 915 & 15.01.2017 \\
\hline $155 / 2017$ & SQ1SE-E & charcoal & 916 & 17.01.2017 \\
\hline $141 / 2017$ & SQ1SE-E & charcoal & 916 & 16.01 .2017 \\
\hline $125 / 2017$ & SQ1SE-E and SQ1SE & charcoal & 916 & 15.01.2017 \\
\hline $181 / 2017$ & SQ1SE-E and SQ1SE & charcoal & 917 & 18.01.2017 \\
\hline $165 / 2017$ & SQ1SE-E & charcoal & 918 & 17.01 .2017 \\
\hline $162 / 2017$ & SQ1SE & charcoal & 919 & 17.01.2017 \\
\hline $174 / 2017$ & SQ1SE & charcoal & 920 & 17.01.2017 \\
\hline $179 / 2017$ & SQ1SE & charcoal & 920 & 18.01 .2017 \\
\hline $188 / 2017$ & SQ1SE-E & charcoal & 922 & 18.01 .2017 \\
\hline $194 / 2017$ & SQ1SE-E & charcoal & 923 & 18.01.2017 \\
\hline $198 / 2017$ & SQ1SE & charcoal & 924 & 18.01.2017 \\
\hline $199 / 2017$ & SQ1SE & charcoal & 924 & 18.01.2017 \\
\hline $201 / 2017$ & SQ1SE & charcoal & 925 & 18.01.2017 \\
\hline $206 / 2017$ & SQ1SE & charcoal & 926 & 18.01.2017 \\
\hline
\end{tabular}




\begin{tabular}{|c|c|c|c|c|}
\hline $215 / 2017$ & SQ1SE & charcoal & 927 & 18.01.2017 \\
\hline $222 / 2017$ & SQ1 & charcoal & 928 & 19.01.2017 \\
\hline $229 / 2017$ & SQ1S & charcoal & 929 & 19.01.2017 \\
\hline $257 / 2017$ & SQ1S & charcoal & 929 & 21.01.2017 \\
\hline $220 / 2017$ & SQ1SE & charcoal & 930 & 19.01.2017 \\
\hline $242 / 2017$ & SQ1 & charcoal & 931 & 19.01.2017 \\
\hline $259 / 2017$ & SQ1 & charcoal & 931 & 21.01.2017 \\
\hline $226 / 2017$ & SQ1S & charcoal & 932 & 19.01.2017 \\
\hline $232 / 2017$ & SQ1SE & charcoal & 933 & 19.01.2017 \\
\hline $234 / 2017$ & SQ1S & charcoal & 934 & 19.01.2017 \\
\hline $245 / 2017$ & SQ1S & charcoal & 935 & 19.01.2017 \\
\hline $255 / 2017$ & SQ1S & charcoal & 935 & 21.01.2017 \\
\hline $250 / 2017$ & SQ1S & charcoal & 936 & 19.01.2017 \\
\hline $271 / 2017$ & SQ1SE & charcoal & 937 & 21.01 .2017 \\
\hline $286 / 2017$ & SQ1SE, N of SU 941 & charcoal & 937 & 22.01 .2017 \\
\hline $289 / 2017$ & SQ1SE, S of SU 941 & charcoal & 937 & 22.01 .2017 \\
\hline $266 / 2017$ & SQ1 & charcoal & 939 & 21.01.2017 \\
\hline $303 / 2017$ & SQ1SE & charcoal & 941 & 22.01.2017 \\
\hline $280 / 2017$ & SQ1SE & charcoal & 942 & 21.01.2017 \\
\hline $297 / 2017$ & SQ1SE & charcoal & 942 & 22.01 .2017 \\
\hline $299 / 2017$ & SQ1SE & charcoal & 943 & 22.01 .2017 \\
\hline $293 / 2017$ & SQ1SE & charcoal & 944 & 22.01 .2017 \\
\hline $308 / 2017$ & SQ1SE and SQ1SE-E & charcoal & 945 & 22.01 .2017 \\
\hline $316 / 2017$ & SQ1SE and SQ1SE-E & charcoal & 947 & 23.01.2017 \\
\hline $323 / 2017$ & SQ1S & charcoal & 948 & 23.01 .2017 \\
\hline $327 / 2017$ & SQ1S & charcoal & 949 & 23.01.2017 \\
\hline
\end{tabular}




\begin{tabular}{|c|c|c|c|c|}
\hline $334 / 2017$ & SQ1S & charcoal & 951 & 23.01 .2017 \\
\hline $337 / 2017$ & SQ1SE and SQ1SE-E & charcoal & 952 & 23.01 .2017 \\
\hline $351 / 2017$ & SQ1S & charcoal & 955 & 24.01.2017 \\
\hline $367 / 2017$ & SQ1S & charcoal & 956 & 24.01 .2017 \\
\hline $358 / 2017$ & SQ1S & charcoal & 958 & 24.01 .2017 \\
\hline $363 / 2017$ & SQ1S & charcoal & 962 & 24.01 .2017 \\
\hline $373 / 2017$ & SQ1S & charcoal & 963 & 24.01 .2017 \\
\hline $393 / 2017$ & SQ1S & charcoal & 963 & 25.01 .2017 \\
\hline $378 / 2017$ & SQ1S & charcoal & 964 & 24.01.2017 \\
\hline $385 / 2017$ & SQ1S & charcoal & 965 & 25.01 .2017 \\
\hline $387 / 2017$ & SQ1S & charcoal & 966 & 25.01 .2017 \\
\hline $405 / 2017$ & SQ1S & charcoal & 967 & 25.01 .2017 \\
\hline $389 / 2017$ & SQ1S & charcoal & 968 & 25.01 .2017 \\
\hline $418 / 2017$ & SQ1S & charcoal & 969 & 25.01 .2017 \\
\hline $401 / 2017$ & SQ1S & charcoal & 970 & 25.01 .2017 \\
\hline $412 / 2017$ & SQ1S & charcoal & 974 & 25.01 .2017 \\
\hline $425 / 2017$ & SQ1S & charcoal & 976 & 26.01 .2017 \\
\hline $440 / 2017$ & SQ1 & charcoal & 978 & 26.01 .2017 \\
\hline $446 / 2017$ & SQ1S & charcoal & 980 & 26.01 .2017 \\
\hline $436 / 2017$ & SQ1S & charcoal & 981 & 26.01 .2017 \\
\hline $444 / 2017$ & SQ1 & charcoal & 982 & 26.01 .2017 \\
\hline $454 / 2017$ & SQ1 & charcoal & 983 & 26.01 .2017 \\
\hline $449 / 2017$ & SQ1S & charcoal & 984 & 26.01.2017 \\
\hline $464 / 2017$ & SQ1 & charcoal & 986 & 26.01 .2017 \\
\hline $468 / 2017$ & SQ1S & charcoal & 988 & 26.01 .2017 \\
\hline $479 / 2017$ & SQ1SE & charcoal & 992 & 04.03 .2017 \\
\hline
\end{tabular}




\begin{tabular}{|c|c|c|c|c|c|}
\hline $485 / 2017$ & SQ1SE-E & charcoal & 994 & & 04.03 .2017 \\
\hline $490 / 2017$ & SQ1S & charcoal & 996 & & 04.03 .2017 \\
\hline $493 / 2017$ & SQ1S & charcoal & 997 & & 04.03 .2017 \\
\hline $495 / 2017$ & SQ1S & charcoal & 998 & & 04.03 .2017 \\
\hline $235 / 2017$ & SQ1 & clay object/clay vessel?/crucible?/installation? (1 fragment) & 931 & SAV1W 1809 & 19.01.2017 \\
\hline $110 / 2017$ & SQ1SE-E and SQ1SE & $\begin{array}{l}\text { crucible (1 fragment of clay vessel with remains of oxidised } \\
\text { metal) }\end{array}$ & 912 & SAV1W 1748 & 14.01.2017 \\
\hline $011 / 2017$ & SQ1SE & doum nuts (burned) & 900 & & 07.01 .2017 \\
\hline $018 / 2017$ & SQ1SE & doum nuts (burned) & 900 & & 08.01 .2017 \\
\hline $049 / 2017$ & SQ1S, N of Feature 143 & doum nut & 903 & & 10.01 .2017 \\
\hline $026 / 2017$ & SQ1S, S of Feature 143 & doum nuts & 903 & & 09.01 .2017 \\
\hline $041 / 2017$ & SQ1S, S of Feature 143 & doum nut & 903 & & 10.01 .2017 \\
\hline $056 / 2017$ & SQ1SE, Feature 145 & doum nuts & 904 & & 10.01 .2017 \\
\hline $055 / 2017$ & SQ1SE & doum nut & 905 & & 10.01 .2017 \\
\hline $061 / 2017$ & SQ1SE & doum nut & 906 & & 10.01 .2017 \\
\hline $069 / 2017$ & SQ1SE, Feature 151 & doum nut & 908 & & 11.01 .2017 \\
\hline $086 / 2017$ & SQ1SE, Feature 151 & doum nuts & 909 & & 11.01 .2017 \\
\hline $097 / 2017$ & SQ1SE-E & doum nuts & 911 & & 14.01 .2017 \\
\hline $105 / 2017$ & SQ1SE-E & doum nut & 913 & & 14.01.2017 \\
\hline $117 / 2017$ & SQ1SE-E and SQ1SE & doum nuts & 914 & & 15.01 .2017 \\
\hline $122 / 2017$ & SQ1SE-E & doum nuts & 915 & & 15.01 .2017 \\
\hline $140 / 2017$ & SQ1SE-E & doum nuts & 916 & & 16.01 .2017 \\
\hline $157 / 2017$ & SQ1SE-E & doum nuts & 916 & & 17.01.2017 \\
\hline $127 / 2017$ & SQ1SE-E and SQ1SE & doum nuts & 916 & & 15.01 .2017 \\
\hline $183 / 2017$ & SQ1SE-E and SQ1SE & doum nut & 917 & & 18.01 .2017 \\
\hline $168 / 2017$ & SQ1SE-E & doum nuts & 918 & & 17.01 .2017 \\
\hline $163 / 2017$ & SQ1SE & doum nut & 919 & & 17.01 .2017 \\
\hline
\end{tabular}




\begin{tabular}{|c|c|c|c|c|}
\hline $176 / 2017$ & SQ1SE & doum nut & 920 & 17.01.2017 \\
\hline $210 / 2017$ & SQ1SE-E & doum nut & 923 & 18.01 .2017 \\
\hline $197 / 2017$ & SQ1SE & doum nut & 924 & 18.01.2017 \\
\hline $202 / 2017$ & SQ1SE & doum nuts & 925 & 18.01.2017 \\
\hline $209 / 2017$ & SQ1SE & doum nut & 926 & 18.01 .2017 \\
\hline $231 / 2017$ & SQ1S & doum nut & 929 & 19.01 .2017 \\
\hline $262 / 2017$ & SQ1 & doum nut & 931 & 21.01 .2017 \\
\hline $247 / 2017$ & SQ1S & doum nut & 935 & 19.01.2017 \\
\hline $251 / 2017$ & SQ1S & doum nut? & 936 & 19.01 .2017 \\
\hline $272 / 2017$ & SQ1SE & doum nuts & 937 & 21.01 .2017 \\
\hline $276 / 2017$ & SQ1SE & doum nuts & 941 & 21.01 .2017 \\
\hline $305 / 2017$ & SQ1SE & doum nut & 941 & 22.01 .2017 \\
\hline $281 / 2017$ & SQ1SE & doum nuts & 942 & 21.01 .2017 \\
\hline $296 / 2017$ & SQ1SE & doum nuts & 942 & 22.01 .2017 \\
\hline $310 / 2017$ & SQ1SE and SQ1SE-E & doum nuts & 945 & 22.01 .2017 \\
\hline $318 / 2017$ & SQ1SE and SQ1SE-E & doum nut & 947 & 23.01 .2017 \\
\hline $340 / 2017$ & SQ1SE and SQ1SE-E & doum nuts & 952 & 23.01 .2017 \\
\hline $368 / 2017$ & SQ1S & doum nuts & 956 & 24.01 .2017 \\
\hline $376 / 2017$ & SQ1S & doum nut & 963 & 24.01 .2017 \\
\hline $394 / 2017$ & SQ1S & doum nut & 963 & 25.01 .2017 \\
\hline $379 / 2017$ & SQ1S & doum nuts & 964 & 24.01 .2017 \\
\hline $406 / 2017$ & SQ1S & doum nuts & 967 & 25.01 .2017 \\
\hline $420 / 2017$ & SQ1S & doum nut & 969 & 25.01 .2017 \\
\hline $414 / 2017$ & SQ1S & doum nuts & 970 & 25.01 .2017 \\
\hline $426 / 2017$ & SQ1S & doum nut & 976 & 26.01 .2017 \\
\hline $442 / 2017$ & SQ1 & doum nuts & 978 & 26.01 .2017 \\
\hline
\end{tabular}




\begin{tabular}{|c|c|c|c|c|c|}
\hline $460 / 2017$ & SQ1S & doum nut & 980 & & 26.01.2017 \\
\hline $437 / 2017$ & SQ1S & doum nut & 981 & & 26.01.2017 \\
\hline $452 / 2017$ & SQ1S & doum nut & 984 & & 26.01.2017 \\
\hline $458 / 2017$ & SQ1S & doum nut & 985 & & 26.01.2017 \\
\hline $463 / 2017$ & SQ1 & doum nut & 986 & & 26.01 .2017 \\
\hline $467 / 2017$ & SQ1S & doum nut & 988 & & 26.01 .2017 \\
\hline $480 / 2017$ & SQ1SE & doum nut & 992 & & 04.03 .2017 \\
\hline $484 / 2017$ & SQ1SE-E & doum nut & 994 & & 04.03 .2017 \\
\hline $109 / 2017$ & SQ1SE-E and SQ1SE & faience vessel (1 body fragment) & 912 & SAV1W 1749 & 14.01.2017 \\
\hline $021 / 2017$ & SQ1SE & figurine (1 rudimentary female figurine, clay) & 900 & SAV1W 1733 & 08.01 .2017 \\
\hline $032 / 2017$ & SQ1S, S of Feature 143 & figurine (1 rudimentary female figurine, broken, clay) & 903 & SAV1W 1734 & 09.01 .2017 \\
\hline $048 / 2017$ & SQ1S, S of Feature 143 & figurine (1 small head of a Nubian style doll, clay) & 903 & SAV1W 1735 & 10.01 .2017 \\
\hline $186 / 2017$ & SQ1SE & figurine ( 1 fragment, rudimentary female figurine, clay) & 921 & SAV1W 1792 & 18.01.2017 \\
\hline $001 / 2017$ & SQ1SE & jar stopper (1 piece, clay/mud) & 900 & & 07.01 .2017 \\
\hline $130 / 2017$ & SQ1SE & leather & 916 & & 15.01.2017 \\
\hline $152 / 2017$ & SQ1SE-E & leather & 916 & & 16.01 .2017 \\
\hline $138 / 2017$ & SQ1SE-E & net weight (1 piece, clay) & 916 & SAV1W 1754 & 16.01.2017 \\
\hline $149 / 2017$ & SQ1SE-E & net weight (model net weight, 1 piece, clay) & 916 & SAV1W 1753 & 16.01 .2017 \\
\hline $017 / 2017$ & SQ1SE & Nun bowl? (1 small fragment, faience) & 900 & SAV1W 1732 & 08.01 .2017 \\
\hline $184 / 2017$ & SQ1SE-E and SQ1SE & plaster & 917 & & 18.01 .2017 \\
\hline $090 / 2017$ & SQ1SE-E & pottery (17 baskets) & 800 & & 12.01 .2017 \\
\hline $002 / 2017$ & SQ1SE & $\begin{array}{l}\text { pottery ( } 19 \text { baskets), among them stand for model vessels? } \\
\text { ( } 1 \text { fragment, pottery) }\end{array}$ & 900 & SAV1W 1517 & 07.01 .2017 \\
\hline $012 / 2017$ & SQ1SE & $\begin{array}{l}\text { pottery ( } 14 \text { baskets), among them clay object ( } 1 \text { piece, unclear } \\
\text { function, stopper? negative of interior of beer jar?, clay), lid ( } 1 \text { re- } \\
\text { used } 18^{\text {th }} \text { Dynasty sherd, pottery), scraper ( } 1 \text { re-used sherd, } \\
\text { pottery) }\end{array}$ & 900 & $\begin{array}{l}\text { SAV1W } 1727 \text { (clay object), SAV1W } 1730 \text { (lid), } \\
\text { SAV1W } 1731 \text { (scraper) }\end{array}$ & 08.01 .2017 \\
\hline $019 / 2017$ & $\begin{array}{l}\text { SQ1S and SQ1SE, cleaning of wind-blown } \\
\text { sand }\end{array}$ & pottery (1 basket) & 901 & & 08.01 .2017 \\
\hline
\end{tabular}




\begin{tabular}{|c|c|c|c|c|c|}
\hline $045 / 2017$ & $\begin{array}{l}\text { SQ1S and SQ1SE, W-edge of SQ1S and } \\
\text { SQ1SE, cleaning of wind-blown sand }\end{array}$ & pottery (2 bags) & 901 & & 10.01.2017 \\
\hline $037 / 2017$ & SQ1S, SE of, cleaning of wind-blown sand & pottery (1/2 basket) & 901 & & 09.01 .2017 \\
\hline $070 / 2017$ & SQ1SE, cleaning of wind-blown sand & pottery (1 basket) & 901 & & 11.01 .2017 \\
\hline $237 / 2017$ & SQ1SE, E part & pottery (1 bag) & 902 & & 19.01.2017 \\
\hline $241 / 2017$ & SQ1SE, W part & pottery (1 bag) & 902 & & 19.01 .2017 \\
\hline $024 / 2017$ & SQ1S, N of Feature 143 & pottery ( 9 baskets) & 903 & & 09.01 .2017 \\
\hline $042 / 2017$ & SQ1S, N of Feature 143 & \multirow{2}{*}{$\begin{array}{l}\text { pottery ( } 1 \text { bag) } \\
\text { pottery ( } 5 \text { baskets), among them scrapers ( } 2 \text { pieces, each re-used } \\
\text { sherds, pottery) }\end{array}$} & 903 & & 10.01 .2017 \\
\hline $022 / 2017$ & SQ1S, S of Feature 143 & & 903 & SAV1W 1739, SAV1W 1740 (scrapers) & 09.01 .2017 \\
\hline $039 / 2017$ & SQ1S, S of Feature 143 & pottery (1/2 basket) & 903 & & 10.01.2017 \\
\hline $050 / 2017$ & SQ1SE, Feature 145 & $\begin{array}{l}\text { pottery ( } 11 / 2 \text { baskets), among them unidentified piece of } \\
\text { furniture or installation/base of basin? ( } 1 \text { fragment, pottery) }\end{array}$ & 904 & SAV1W 1838 & 10.01.2017 \\
\hline $051 / 2017$ & SQ1SE & pottery (7 baskets) & 905 & & 10.01 .2017 \\
\hline $062 / 2017$ & SQ1SE & pottery (1 bag) & 906 & & 10.01 .2017 \\
\hline $063 / 2017$ & SQ1SE & pottery (2 baskets) & 906 & & 11.01 .2017 \\
\hline $066 / 2017$ & SQ1SE & pottery (1 basket) & 907 & & 11.01 .2017 \\
\hline $074 / 2017$ & SQ1SE, Feature 151 & pottery (3 1/2 baskets) & 908 & & 11.01 .2017 \\
\hline $075 / 2017$ & SQ1SE, Feature 151 & pottery (1 basket and 3 bags) & 909 & & 11.01 .2017 \\
\hline $085 / 2017$ & SQ1SE & pottery (1 basket) & 910 & & 11.01 .2017 \\
\hline $094 / 2017$ & SQ1SE-E & pottery (10 baskets) & 911 & & 12.01 .2017 \\
\hline $111 / 2017$ & SQ1SE-E & \multirow{2}{*}{$\begin{array}{l}\text { pottery ( } 21 / 2 \text { baskets) } \\
\text { pottery ( } 12 \text { baskets), among them model table? ( } 1 \text { fragment of an } \\
\text { unclear pottery object) }\end{array}$} & 911 & & 14.01 .2017 \\
\hline $136 / 2017$ & SQ1SE-E & & 911 & SAV1W 1834 & 16.01 .2017 \\
\hline $098 / 2017$ & SQ1SE-E and SQ1SE & pottery ( 1 basket), among it scraper ( 1 re-used sherd, pottery) & 912 & SAV1W 1750 & 14.01 .2017 \\
\hline $101 / 2017$ & SQ1SE-E & pottery (1 basket) & 913 & & 14.01 .2017 \\
\hline $106 / 2017$ & SQ1SE-E and SQ1SE & pottery ( 2 baskets) & 914 & & 14.01 .2017 \\
\hline $112 / 2017$ & $\begin{array}{l}\text { SQ1SE-E } \\
\text { and SQ1SE }\end{array}$ & pottery (5 $1 / 2$ baskets) & 914 & & 15.01 .2017 \\
\hline $119 / 2017$ & SQ1SE-E & pottery ( $11 / 2$ baskets) & 915 & & 15.01.2017 \\
\hline
\end{tabular}




\begin{tabular}{|c|c|c|c|c|c|}
\hline $156 / 2017$ & SQ1SE-E & pottery (2 baskets) & 916 & & 17.01.2017 \\
\hline $124 / 2017$ & SQ1SE-E and SQ1SE & pottery (18 baskets) & 916 & & 15.01.2017 \\
\hline $135 / 2017$ & SQ1SE-E, N-part & $\begin{array}{l}\text { pottery ( } 14 \text { baskets), among them window grille ( } 1 \text { corner } \\
\text { fragment, Christian, pottery), stopper ( } 1 \text { fragment, clay), lid ( } 1 \text { re- } \\
\text { used } 18^{\text {th }} \text { Dynasty sherd, pottery) }\end{array}$ & 916 & $\begin{array}{l}\text { SAV1W } 1780 \text { (window grille), SAV1W } 1781 \\
\text { (stopper), SAV1W } 1835 \text { (lid) }\end{array}$ & 16.01.2017 \\
\hline $158 / 2017$ & SQ1SE-E and SQ1SE & pottery (3 baskets) & 917 & & 17.01.2017 \\
\hline $159 / 2017$ & SQ1SE-E & pottery (2 baskets) & 918 & & 17.01.2017 \\
\hline $160 / 2017$ & SQ1SE & pottery (6 baskets) & 919 & & 17.01.2017 \\
\hline $172 / 2017$ & SQ1SE & pottery ( 3 baskets) & 920 & & 17.01.2017 \\
\hline $178 / 2017$ & SQ1SE & $\begin{array}{l}\text { pottery ( } 1 / 2 \text { basket), among it lid ( } 1 \text { re-used } 18^{\text {th }} \text { Dynasty base } \\
\text { sherd, pottery) }\end{array}$ & 920 & SAV1W 1832 & 18.01.2017 \\
\hline $190 / 2017$ & SQ1SE & $\begin{array}{l}\text { pottery ( } 2 \text { baskets), among them lids ( } 2 \text { pieces, each re-used } \\
\text { sherds, pottery) }\end{array}$ & 921 & SAV1W 1785, SAV1W 1784 (lids) & 18.01 .2017 \\
\hline $200 / 2017$ & SQ1SE & pottery ( $1 / 2$ basket $)$ & 921 & & 18.01 .2017 \\
\hline $189 / 2017$ & SQ1SE-E & pottery (1 bag) & 922 & & 18.01 .2017 \\
\hline $212 / 2017$ & SQ1SE-E & pottery (1/2 basket) & 923 & & 18.01 .2017 \\
\hline $193 / 2017$ & SQ1SE & pottery (1 bag), among it lid (1 re-used base sherd, pottery) & 924 & SAV1W 1788 & 18.01 .2017 \\
\hline $213 / 2017$ & SQ1SE & pottery (1 bag) & 926 & & 18.01.2017 \\
\hline $214 / 2017$ & SQ1SE & pottery (1 bag) & 927 & & 18.01 .2017 \\
\hline $221 / 2017$ & SQ1 & $\begin{array}{l}\text { pottery ( } 6 \text { baskets), among them lid ( } 1 \text { re-used Post-New } \\
\text { Kingdom base sherd, pottery) }\end{array}$ & 928 & SAV1W 1787 & 19.01.2017 \\
\hline $228 / 2017$ & SQ1S & pottery (1 bag) & 929 & & 19.01.2017 \\
\hline $256 / 2017$ & SQ1S & pottery ( 3 sherds) & 929 & & 21.01.2017 \\
\hline $219 / 2017$ & SQ1SE & pottery (4 sherds) & 930 & & 19.01.2017 \\
\hline $236 / 2017$ & SQ1 & $\begin{array}{l}\text { pottery ( } 5 \text { baskets), among them net weight } \\
\text { ( } 1 \text { fragment, pottery), stopper ( } 1 \text { large piece, clay) }\end{array}$ & 931 & $\begin{array}{l}\text { SAV1W } 1786 \text { (net weight), SAV1W } 1789 \\
\text { (stopper) }\end{array}$ & 19.01.2017 \\
\hline $260 / 2017$ & SQ1 & pottery ( 1 basket) & 931 & & 21.01 .2017 \\
\hline $225 / 2017$ & SQ1S & pottery (1 bag) & 932 & & 19.01.2017 \\
\hline $227 / 2017$ & SQ1SE & pottery (1 bag) & 933 & & 19.01.2017 \\
\hline $253 / 2017$ & SQ1SE & pottery (4 sherds) & 933 & & 21.01 .2017 \\
\hline
\end{tabular}




\begin{tabular}{|c|c|c|c|c|c|}
\hline $233 / 2017$ & SQ1S & pottery (1 bag) & 934 & & 19.01.2017 \\
\hline $244 / 2017$ & SQ1S & pottery (1 bag) & 935 & & 19.01.2017 \\
\hline $254 / 2017$ & SQ1S & pottery (1 small bag) & 935 & & 21.01 .2017 \\
\hline $248 / 2017$ & SQ1S & pottery (1 bag) & 936 & & 19.01.2017 \\
\hline $268 / 2017$ & SQ1S & pottery (1 small bag) & 936 & & 21.01 .2017 \\
\hline $263 / 2017$ & SQ1SE & $\begin{array}{l}\text { pottery ( } 6 \text { baskets), among them lid ( } 1 \text { re-used } 18^{\text {th }} \text { Dynasty base } \\
\text { sherd, pottery) }\end{array}$ & 937 & SAV1W 1805 & 21.01.2017 \\
\hline $287 / 2017$ & SQ1SE, N of SU 941 & $\begin{array}{l}\text { pottery ( } 1 \text { bag), among it scraper ( } 1 \text { re-used } 18^{\text {th }} \text { Dynasty base } \\
\text { sherd, pottery) }\end{array}$ & 937 & SAV1W 1836 & 22.01.2017 \\
\hline $288 / 2017$ & SQ1SE, S of SU 941 & pottery (1/2 basket) & 937 & & 22.01 .2017 \\
\hline $265 / 2017$ & SQ1 & pottery (1 bag) & 939 & & 21.01 .2017 \\
\hline $274 / 2017$ & SQ1SE & pottery ( 2 baskets) & 941 & & 21.01 .2017 \\
\hline $301 / 2017$ & SQ1SE & pottery ( 3 and 1 baskets) & 941 & & 22.01 .2017 \\
\hline $273 / 2017$ & SQ1SE & pottery (1 bag) & 942 & & 21.01 .2017 \\
\hline $295 / 2017$ & SQ1SE & pottery (1 bag) & 942 & & 22.01 .2017 \\
\hline $298 / 2017$ & SQ1SE & $\begin{array}{l}\text { pottery ( } 1 \text { basket), among it scraper ( } 1 \text { re-used } 18^{\text {th }} \text { Dynasty base } \\
\text { sherd, pottery) }\end{array}$ & 943 & SAV1W 1810 & 22.01 .2017 \\
\hline $291 / 2017$ & SQ1SE & pottery (1 basket) & 944 & & 22.01 .2017 \\
\hline $307 / 2017$ & SQ1SE and SQ1SE-E & pottery (2 baskets) & 945 & & 22.01 .2017 \\
\hline $311 / 2017$ & SQ1S & pottery (1 bag) & 946 & & 22.01 .2017 \\
\hline $315 / 2017$ & SQ1SE and SQ1SE-E & $\begin{array}{l}\text { pottery ( } 2 \text { baskets), among them token/gaming piece ( } 1 \text { re-used } \\
\text { sherd, pottery) }\end{array}$ & 947 & SAV1W 1811 & 23.01.2017 \\
\hline $321 / 2017$ & SQ1S & pottery (1 bag) & 948 & & 23.01.2017 \\
\hline $326 / 2017$ & SQ1S & pottery (1 small bag) & 949 & & 23.01 .2017 \\
\hline $329 / 2017$ & SQ1S & pottery (1 small bag) & 950 & & 23.01 .2017 \\
\hline $332 / 2017$ & SQ1S & pottery (1 bag) & 951 & & 23.01 .2017 \\
\hline $336 / 2017$ & SQ1SE and SQ1SE-E & pottery (1 bag) & 952 & & 23.01 .2017 \\
\hline $355 / 2017$ & SQ1SE and SQ1SE-E & pottery (1 small bag) & 952 & & 24.01 .2017 \\
\hline $345 / 2017$ & SQ1S & pottery (1 small bag) & 953 & & 24.01 .2017 \\
\hline
\end{tabular}




\begin{tabular}{|c|c|c|c|c|c|}
\hline $347 / 2017$ & SQ1S & pottery (1 small bag) & 954 & & 24.01.2017 \\
\hline $349 / 2017$ & SQ1S & pottery (1 small bag) & 955 & & 24.01 .2017 \\
\hline $366 / 2017$ & SQ1S & pottery (1 bag) & 956 & & 24.01 .2017 \\
\hline $353 / 2017$ & SQ1S & pottery (1 small bag) & 957 & & 24.01 .2017 \\
\hline $356 / 2017$ & SQ1S & pottery (1 small bag) & 958 & & 24.01 .2017 \\
\hline $359 / 2017$ & SQ1S & pottery (1 small bag) & 959 & & 24.01 .2017 \\
\hline $360 / 2017$ & SQ1S & pottery (1 small bag) & 960 & & 24.01 .2017 \\
\hline $361 / 2017$ & SQ1S & pottery (1 small bag) & 961 & & 24.01 .2017 \\
\hline $362 / 2017$ & SQ1S & $\begin{array}{l}\text { pottery }(1 \mathrm{bag}) \text {, among it token/gaming piece }\left(1 \text { re-used } 18^{\text {th }}\right. \\
\text { Dynasty body sherd, pottery) }\end{array}$ & 962 & SAV1W 1830 & 24.01 .2017 \\
\hline $371 / 2017$ & SQ1S & $\begin{array}{l}\text { pottery ( } 1 / 2 \text { basket), among it token/gaming piece ( } 1 \text { re-used } 18^{\text {th }} \\
\text { Dynasty body sherd, pottery) }\end{array}$ & 963 & SAV1W 1820 & 24.01 .2017 \\
\hline $377 / 2017$ & SQ1S & $\begin{array}{l}\text { pottery ( } 1 \text { bag), among it lid ( } 1 \text { re-used } 18^{\text {th }} \text { Dynasty ring base, } \\
\text { pottery) }\end{array}$ & 964 & SAV1W 1829 & 24.01 .2017 \\
\hline $384 / 2017$ & SQ1S & pottery (1 medium bag) & 965 & & 25.01 .2017 \\
\hline $386 / 2017$ & SQ1S & pottery (1 small bag) & 966 & & 25.01.2017 \\
\hline $391 / 2017$ & SQ1S & pottery (1/2 basket) & 967 & & 25.01 .2017 \\
\hline $388 / 2017$ & SQ1S & pottery (1 bag) & 968 & & 25.01 .2017 \\
\hline $416 / 2017$ & SQ1S & pottery (1/2 basket) & 969 & & 25.01 .2017 \\
\hline $398 / 2017$ & SQ1S & pottery (1 small bag) & 970 & & 25.01.2017 \\
\hline $399 / 2017$ & SQ1S & $\begin{array}{l}\text { pottery }(1 \mathrm{bag}) \text {, among it lid or scraper }\left(1 \text { re-used } 18^{\text {th }} \text { Dynasty }\right. \\
\text { base sherd, pottery) }\end{array}$ & 970 & SAV1W 1837 & 25.01 .2017 \\
\hline $402 / 2017$ & SQ1S & pottery (1 small bag) & 971 & & 25.01 .2017 \\
\hline $404 / 2017$ & SQ1S & pottery (1 small bag) & 972 & & 25.01 .2017 \\
\hline $409 / 2017$ & SQ1S & pottery (1 small bag) & 973 & & 25.01 .2017 \\
\hline $410 / 2017$ & SQ1S & pottery (1 small bag) & 974 & & 25.01 .2017 \\
\hline $417 / 2017$ & SQ1S & pottery ( 5 sherds) & 975 & & 25.01 .2017 \\
\hline $424 / 2017$ & SQ1S & pottery (1 small bag) & 976 & & 26.01 .2017 \\
\hline $422 / 2017$ & SQ1S & pottery (1 small bag) & 977 & & 26.01 .2017 \\
\hline
\end{tabular}




\begin{tabular}{|c|c|c|c|c|c|}
\hline $438 / 2017$ & SQ1 & pottery (1 basket), among it scraper (1 re-used rim sherd) & 978 & SAV1W 1831 & 26.01.2017 \\
\hline $429 / 2017$ & SQ1S & pottery (1 bag) & 979 & & 26.01.2017 \\
\hline $431 / 2017$ & SQ1S & pottery (1/2 basket) & 980 & & 26.01.2017 \\
\hline $434 / 2017$ & SQ1S & pottery (1/2 basket) & 981 & & 26.01 .2017 \\
\hline $443 / 2017$ & SQ1 & pottery (1 small bag) & 982 & & 26.01 .2017 \\
\hline $453 / 2017$ & SQ1 & pottery (4 sherds) & 983 & & 26.01 .2017 \\
\hline $448 / 2017$ & SQ1S & pottery (1 small bag) & 984 & & 26.01 .2017 \\
\hline $456 / 2017$ & SQ1S & pottery (1 bag) & 985 & & 26.01.2017 \\
\hline $455 / 2017$ & SQ1 & pottery (1 bag) & 986 & & 26.01 .2017 \\
\hline $470 / 2017$ & SQ1S & pottery (2 sherds) & 987 & & 26.01.2017 \\
\hline $466 / 2017$ & SQ1S & pottery (1 small bag) & 988 & & 26.01 .2017 \\
\hline $461 / 2017$ & SQ1 & pottery (1 bag) & 990 & & 26.01.2017 \\
\hline $471 / 2017$ & SQ1S & pottery (1 small bag) & 991 & & 26.01 .2017 \\
\hline $478 / 2017$ & SQ1SE & pottery vessel & 992 & & 04.03 .2017 \\
\hline $477 / 2017$ & SQ1SE & pottery & 993 & & 04.03 .2017 \\
\hline $482 / 2017$ & SQ1SE-E & pottery & 994 & & 04.03 .2017 \\
\hline $488 / 2017$ & SQ1SE-E & pottery & 995 & & 04.03 .2017 \\
\hline $489 / 2017$ & SQ1SE-E & pottery & 996 & & 04.03 .2017 \\
\hline $492 / 2017$ & SQ1S & pottery & 997 & & 04.03 .2017 \\
\hline $494 / 2017$ & SQ1S & pottery & 998 & & 04.03 .2017 \\
\hline $187 / 2017$ & SQ1SE & raw material /bronze?/inside of crucible? (1 fragment) & 921 & SAV1W 1791 & 18.01 .2017 \\
\hline $081 / 2017$ & SQ1SE, Feature 151, from sieving & $\begin{array}{l}\text { seals ( } 2 \text { pieces, clay); bronze?/metal fragments/raw material } \\
\text { ( } 8 \text { tiny fragments, original form and function unclear) }\end{array}$ & 909 & $\begin{array}{l}\text { SAV1W 1777, SAV1W } 1778 \text { (seals), SAV1W } \\
1779 \text { (bronze?/metal fragments/raw material) }\end{array}$ & 11.01.2017 \\
\hline $342 / 2017$ & SQ1SE and SQ1SE-E & seal (clay/mud) & 947 & & 23.01 .2017 \\
\hline $383 / 2017$ & SQ1S & seal (clay/mud) & 952 & & 24.01 .2017 \\
\hline $474 / 2017$ & SQ1S & seal (clay/mud) & 963 & & 25.01 .2017 \\
\hline $091 / 2017$ & SQ1SE-E & shell & 800 & & 12.01.2017 \\
\hline
\end{tabular}




\begin{tabular}{|c|c|c|c|c|c|}
\hline $007 / 2017$ & SQ1SE & shell (nacre?) & 900 & & 07.01 .2017 \\
\hline $020 / 2017$ & SQ1SE & shell (nacre?) & 900 & & 08.01 .2017 \\
\hline $031 / 2017$ & SQ1S, N of Feature 143 & shell (tiny and curled with white and violet) & 903 & & 09.01 .2017 \\
\hline $089 / 2017$ & SQ1SE & shell (1 fragment) & 908 & & 11.01.2017 \\
\hline $082 / 2017$ & SQ1SE, Feature 151 & shells & 909 & & 11.01.2017 \\
\hline $148 / 2017$ & SQ1SE-E & shell & 916 & & 16.01.2017 \\
\hline $167 / 2017$ & SQ1SE & shell & 919 & & 17.01.2017 \\
\hline $283 / 2017$ & SQ1 & shell & 936 & & 21.01.2017 \\
\hline $278 / 2017$ & SQ1SE & shell & 941 & & 21.01.2017 \\
\hline $325 / 2017$ & SQ1SE and SQ1SE-E & shell & 947 & & 23.01.2017 \\
\hline $375 / 2017$ & SQ1S & shell & 963 & & 24.01.2017 \\
\hline $116 / 2017$ & SQ1SE-E & slag and brick (1 fragment of heavily overfired material) & 914 & SAV1W 1794 & 15.01 .2017 \\
\hline $096 / 2017$ & SQ1SE-E & stone: polishing stone (1 piece, sandstone) & 800 & SAV1W 1797 & 12.01.2017 \\
\hline $003 / 2017$ & SQ1SE & $\begin{array}{l}\text { stones and architecture: pounders ( } 2 \text { pieces, } 1 \text { siliceous shale?, } 1 \\
\text { quartz), polishing stones ( } 3 \text { pieces, } 1 \text { quartzite, } 2 \text { sandstone), } \\
\text { whetstone ( } 1 \text { piece, sandstone); door pivot ( } 1 \text { fragment, } \\
\text { sandstone) }\end{array}$ & 900 & $\begin{array}{l}\text { SAV1W 1720, SAV1W } 1721 \text { (pounders), } \\
\text { SAV1W 1722, SAV1W 1723, SAV1W 1724 } \\
\text { (polishing stones), SAV1W } 1725 \text { (whetstone), } \\
\text { SAV1W } 1726 \text { (door pivot) }\end{array}$ & 07.01.2017 \\
\hline $016 / 2017$ & SQ1SE & $\begin{array}{l}\text { stone and architecture: grindstone (1 fragment, sandstone); } \\
\text { architecture?/floor slab? (1 fragment, quartzite) }\end{array}$ & 900 & $\begin{array}{l}\text { SAV1W } 1728 \text { (grindstone), SAV1W } 1729 \\
\text { (architecture?/floor slab?) }\end{array}$ & 08.01 .2017 \\
\hline $038 / 2017$ & SQ1S, S of Feature 143 & stone: polishing stone/whetstone (1 fragment, sandstone) & 903 & SAV1W 1741 & 09.01 .2017 \\
\hline 088/2017 & SQ1SE, Feature 151 & $\begin{array}{l}\text { stones: pounders ( } 2 \text { pieces, } 1 \text { siliceous shale?, } 1 \text { quartzite), } \\
\text { polishing stone (1 piece, quartz), grindstone (1 piece, sandstone) }\end{array}$ & 908 & $\begin{array}{l}\text { SAV1W 1744, SAV1W } 1745 \text { (pounders), } \\
\text { SAV1W } 1746 \text { (polishing stone), SAV1W } 1747 \\
\text { (grindstone) }\end{array}$ & 11.01.2017 \\
\hline $095 / 2017$ & SQ1SE-E & stone: pounder (1 piece, quartzite?) & 911 & SAV1W 1795 & 12.01.2017 \\
\hline $115 / 2017$ & SQ1SE-E, S-edge of SU 914 & stone, worked/unclear basin ( 1 corner fragment, basin) & 914 & SAV1W 1798 & 15.01.2017 \\
\hline $147 / 2017$ & SQ1SE-E & stone, worked (2 large pieces form unclear object[s], sandstone) & 916 & SAV1W 1804 & 16.01 .2017 \\
\hline $150 / 2017$ & SQ1SE-E & stone: scraper (1 large flake, silex) & 916 & SAV1W 1755 & 16.01.2017 \\
\hline $153 / 2017$ & SQ1SE-E & $\begin{array}{l}\text { stone, worked/animal tether? (1 piece with drilled hole, } \\
\text { sandstone) }\end{array}$ & 916 & SAV1W 1771 & 17.01.2017 \\
\hline $169 / 2017$ & SQ1SE-E & $\begin{array}{l}\text { stone/architecture: grindstone/re-used as door pivot (1 fragment, } \\
\text { sandstone) }\end{array}$ & 916 & SAV1W 1802 & 17.01.2017 \\
\hline
\end{tabular}




\begin{tabular}{|c|c|c|c|c|c|}
\hline $170 / 2017$ & SQ1SE-E & stone: grindstone (1 fragment, sandstone) & 916 & SAV1W 1803 & 17.01.2017 \\
\hline $171 / 2017$ & SQ1SE-E & $\begin{array}{l}\text { stone and metal: pounder ( } 1 \text { piece, unspecified stone); raw } \\
\text { material /bronze/inside of crucible? ( } 2 \text { non adjoining fragments) }\end{array}$ & 916 & $\begin{array}{l}\text { SAV1W } 1796 \text { (pounder), SAV1W } 1783 \text { (raw } \\
\text { material /bronze/inside of crucible?) }\end{array}$ & 17.01.2017 \\
\hline $185 / 2017$ & SQ1SE-E and SQ1SE & $\begin{array}{l}\text { stones: polishing stone (1 piece, sandstone), grindstones } \\
\text { ( } 2 \text { pieces, each sandstone) }\end{array}$ & 917 & $\begin{array}{l}\text { SAV1W } 1799 \text { (polishing stone), SAV1W 1800, } \\
\text { SAV1W } 1801 \text { (grindstones) }\end{array}$ & 18.01 .2017 \\
\hline $166 / 2017$ & SQ1SE & $\begin{array}{l}\text { stone: polishing stone with small rod of metal stuck to it } \\
\text { (1 fragment, sandstone) }\end{array}$ & 919 & SAV1W 1793 & 17.01.2017 \\
\hline $217 / 2017$ & SQ1SE & stone: polishing stone (1 piece, schist) & 921 & SAV1W 1790 & 18.01.2017 \\
\hline $252 / 2017$ & SQ1 & stone: pounder (1 fragment, quartzite) & 931 & SAV1W 1808 & 19.01.2017 \\
\hline $314 / 2017$ & SQ1 & stone: blade/scraper (1 small flake of the core of a silex pebble) & 931 & SAV1W 1806 & 21.01 .2017 \\
\hline $264 / 2017$ & SQ1SE & $\begin{array}{l}\text { stone: weight? (1 triangular fragment with drilled hole, } \\
\text { sandstone) }\end{array}$ & 937 & SAV1W 1807 & 21.01 .2017 \\
\hline $275 / 2017$ & SQ1SE & $\begin{array}{l}\text { stone: worked stone (1 rectangular block with drilled hole, } \\
\text { sandstone, tether stone?) }\end{array}$ & 941 & SAV1W 1828 & 21.01.2017 \\
\hline $306 / 2017$ & SQ1SE and SQ1SE-E & stone: blade (1 large flake of a silex pebble) & 945 & SAV1W 1814 & 22.01 .2017 \\
\hline $423 / 2017$ & SQ1S & stone: blade (1 small flake from the core of a silex pebble) & 977 & SAV1W 1819 & 26.01 .2017 \\
\hline $487 / 2017$ & SQ1SE-E & stone: silex & 994 & & 04.03 .2017 \\
\hline $010 / 2017$ & SQ1SE & textile (some fragments) & 900 & & 07.01 .2017 \\
\hline $036 / 2017$ & SQ1S, N of Feature 143 & textile & 903 & & 09.01 .2017 \\
\hline $059 / 2017$ & SQ1SE & textile (belongs to $052 / 2017$ ) & 905 & & 10.01 .2017 \\
\hline $319 / 2017$ & SQ1SE and SQ1SE-E & textile & 947 & & 23.01 .2017 \\
\hline $491 / 2017$ & SQ1S & tooth (animal) & 996 & & 04.03 .2017 \\
\hline $006 / 2017$ & SQ1SE & wood & 900 & & 07.01 .2017 \\
\hline $014 / 2017$ & SQ1SE & wood & 900 & & 08.01 .2017 \\
\hline $009 / 2017$ & SQ1SE, from center of S part of SQ1SE & wood (palm wood) & 900 & & 07.01 .2017 \\
\hline $239 / 2017$ & SQ1SE, E part & wood & 902 & & 19.01.2017 \\
\hline $033 / 2017$ & SQ1S, N of Feature 143 & wood & 903 & & 09.01 .2017 \\
\hline 034/2017 & $\begin{array}{l}\text { SQ1S, N of Feature 143, directly under a } \\
\text { stone }\end{array}$ & wood & 903 & & 09.01 .2017 \\
\hline $027 / 2017$ & SQ1S, S of Feature 143 & wood & 903 & & 09.01 .2017 \\
\hline
\end{tabular}




\begin{tabular}{|c|c|c|c|c|c|}
\hline $060 / 2017$ & SQ1SE & wood & 905 & & 10.01 .2017 \\
\hline $067 / 2017$ & SQ1SE, Feature 151 & wood & 908 & & 11.01 .2017 \\
\hline $079 / 2017$ & SQ1SE, Feature 151 & wood & 909 & & 11.01.2017 \\
\hline $143 / 2017$ & SQ1SE-E & wood & 911 & & 16.01 .2017 \\
\hline $103 / 2017$ & SQ1SE-E & wood & 913 & & 14.01 .2017 \\
\hline $118 / 2017$ & SQ1SE, N-part & wood & 914 & & 15.01 .2017 \\
\hline $123 / 2017$ & SQ1SE-E & wood & 915 & & 15.01 .2017 \\
\hline $144 / 2017$ & SQ1SE-E & wood & 916 & & 16.01 .2017 \\
\hline $128 / 2017$ & SQ1SE-E and SQ1SE & wood & 916 & & 15.01 .2017 \\
\hline $182 / 2017$ & SQ1SE-E and SQ1SE & wood & 917 & & 18.01.2017 \\
\hline $175 / 2017$ & SQ1SE & wood & 920 & & 17.01 .2017 \\
\hline $196 / 2017$ & SQ1SE & wood & 924 & & 18.01 .2017 \\
\hline $203 / 2017$ & SQ1SE & wood & 925 & & 18.01 .2017 \\
\hline $207 / 2017$ & SQ1SE & wood & 926 & & 18.01 .2017 \\
\hline $211 / 2017$ & SQ1SE & wood & 927 & & 18.01 .2017 \\
\hline $223 / 2017$ & SQ1 & wood & 928 & & 19.01 .2017 \\
\hline $258 / 2017$ & SQ1 & wood & 931 & & 21.01 .2017 \\
\hline $282 / 2017$ & SQ1 & wood & 936 & & 21.01 .2017 \\
\hline $270 / 2017$ & SQ1SE & wood & 937 & & 21.01 .2017 \\
\hline $267 / 2017$ & SQ1 & wood & 939 & & 21.01 .2017 \\
\hline $304 / 2017$ & SQ1SE & wood & 941 & & 22.01 .2017 \\
\hline $284 / 2017$ & SQ1SE & wood, worked (1 fragment, original function unclear) & 942 & SAV1W 1816 & 22.01 .2017 \\
\hline $294 / 2017$ & SQ1SE & wood & 944 & & 22.01 .2017 \\
\hline $313 / 2017$ & SQ1SE and SQ1SE-E & wood & 945 & & 22.01 .2017 \\
\hline $320 / 2017$ & SQ1SE and SQ1SE-E & wood & 947 & & 23.01 .2017 \\
\hline $322 / 2017$ & SQ1S & wood & 948 & & 23.01 .2017 \\
\hline
\end{tabular}




\begin{tabular}{|c|c|c|c|c|}
\hline $328 / 2017$ & SQ1S & wood & 949 & 23.01.2017 \\
\hline $330 / 2017$ & SQ1S & wood & 950 & 23.01.2017 \\
\hline $333 / 2017$ & SQ1S & wood & 951 & 23.01 .2017 \\
\hline $339 / 2017$ & SQ1SE and SQ1SE-E & wood & 952 & 23.01.2017 \\
\hline $370 / 2017$ & SQ1S & wood & 956 & 24.01 .2017 \\
\hline $365 / 2017$ & SQ1S & wood & 962 & 24.01 .2017 \\
\hline $374 / 2017$ & SQ1S & wood & 963 & 24.01.2017 \\
\hline $395 / 2017$ & SQ1S & wood & 963 & 25.01 .2017 \\
\hline $396 / 2017$ & SQ1S & wood & 963 & 25.01.2017 \\
\hline $381 / 2017$ & SQ1S & wood & 964 & 24.01.2017 \\
\hline $407 / 2017$ & SQ1S & wood & 967 & 25.01 .2017 \\
\hline $421 / 2017$ & SQ1S & wood & 969 & 25.01.2017 \\
\hline $413 / 2017$ & SQ1S & wood & 970 & 25.01 .2017 \\
\hline $411 / 2017$ & SQ1S & wood & 974 & 25.01 .2017 \\
\hline $427 / 2017$ & SQ1S & wood & 976 & 26.01.2017 \\
\hline $441 / 2017$ & SQ1 & wood & 978 & 26.01 .2017 \\
\hline $430 / 2017$ & SQ1S & wood & 979 & 26.01.2017 \\
\hline $447 / 2017$ & SQ1S & wood & 980 & 26.01.2017 \\
\hline $451 / 2017$ & SQ1S & wood & 984 & 26.01 .2017 \\
\hline $459 / 2017$ & SQ1S & wood & 985 & 26.01 .2017 \\
\hline $465 / 2017$ & SQ1 & wood & 986 & 26.01 .2017 \\
\hline $486 / 2017$ & SQ1SE-E & wood & 994 & 04.03 .2017 \\
\hline $496 / 2017$ & SQ1S & wood & 998 & 04.03 .2017 \\
\hline
\end{tabular}

